
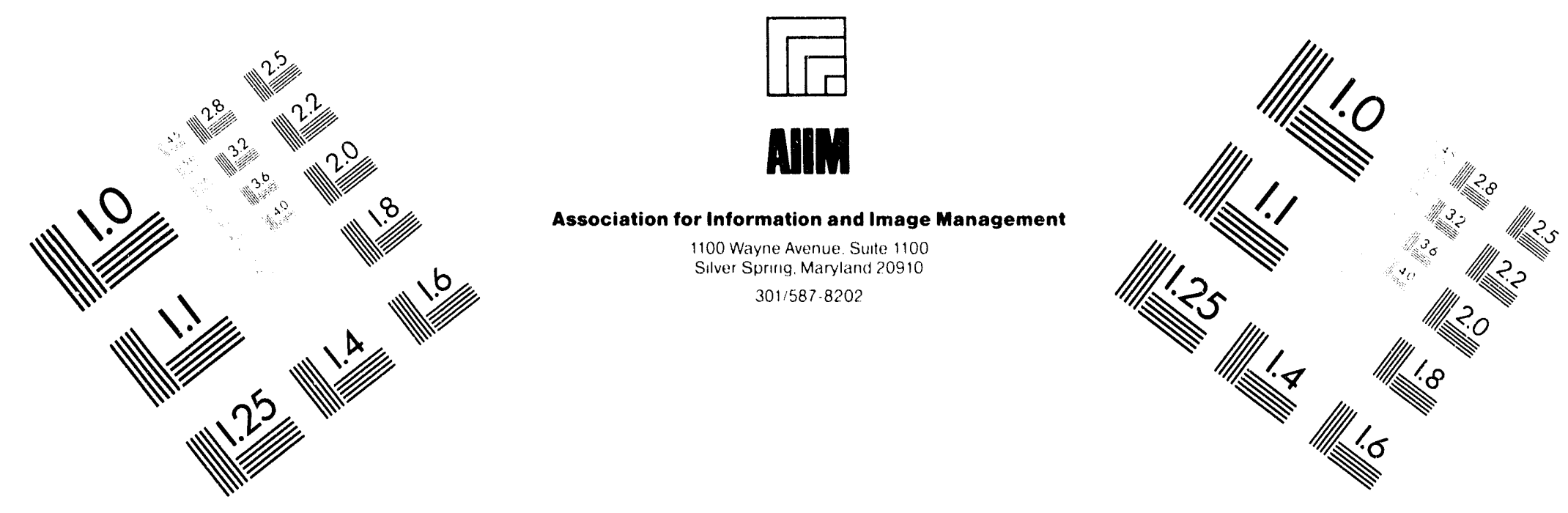

\title{
Centimeter
}

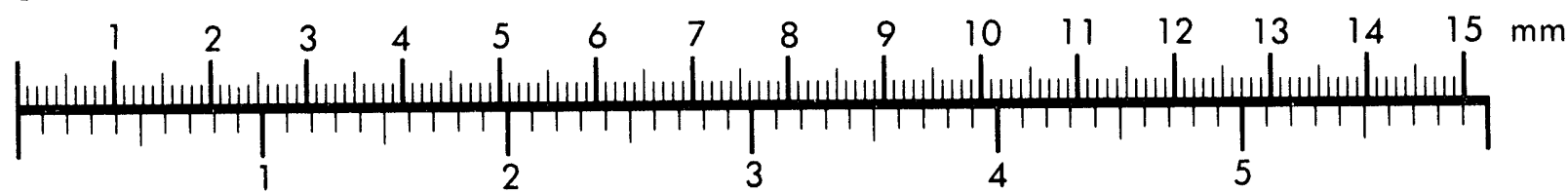

Inches
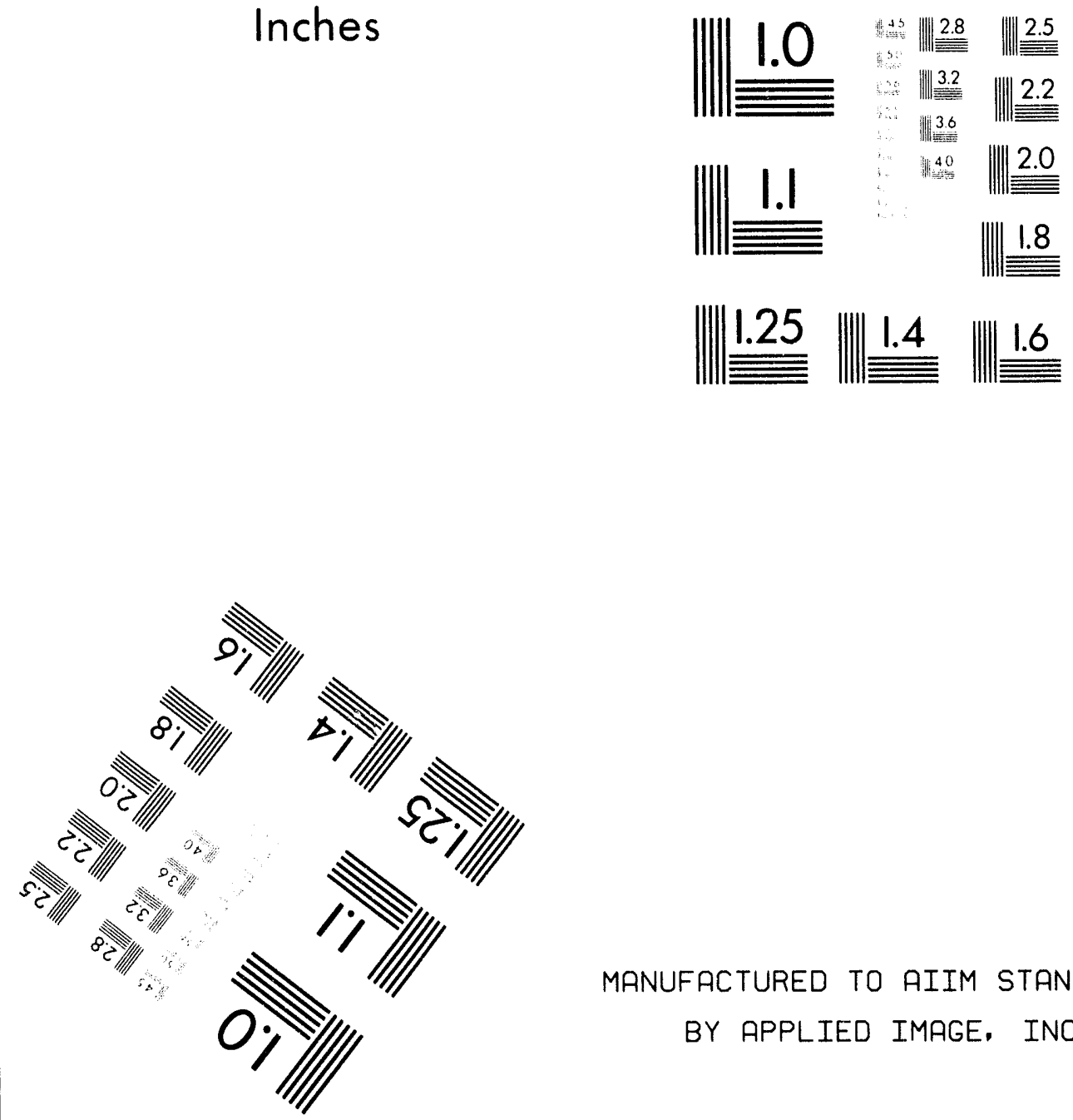

MANUFACTURED TO AIIM STANDARDS

BY APPLIED IMAGE, INC.

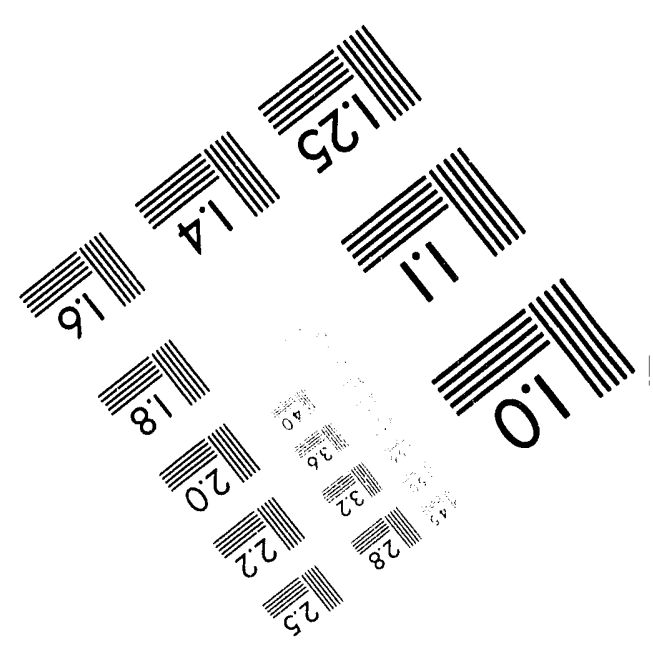



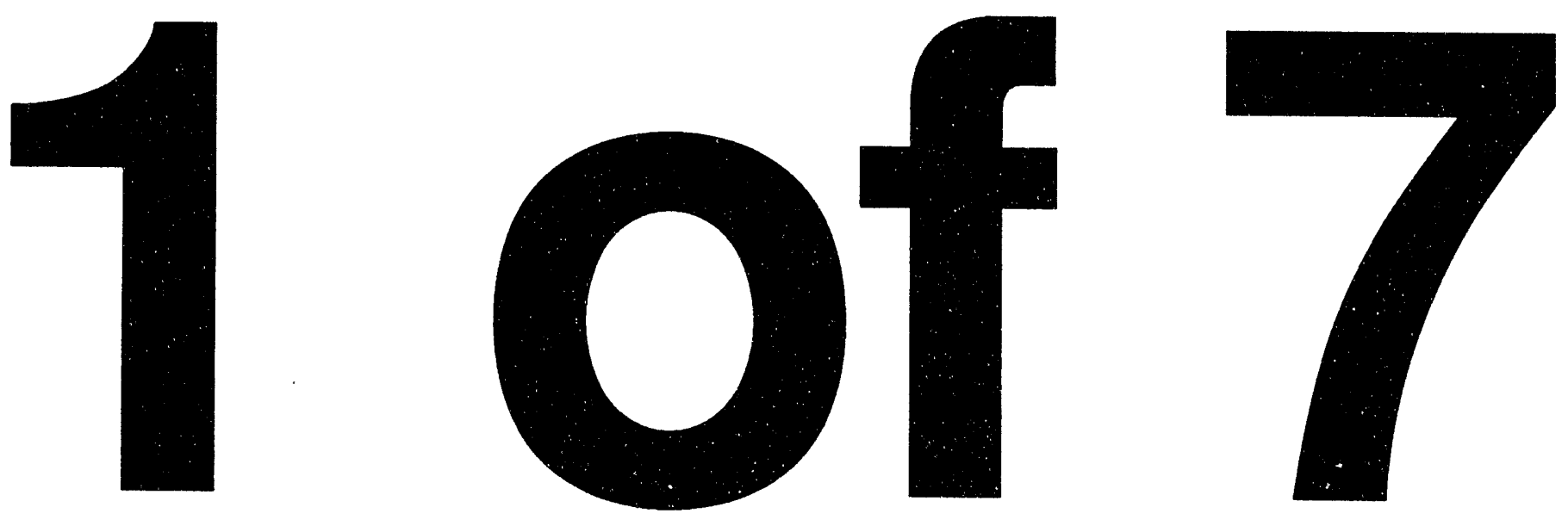


\section{Used Energy-Related Laboratory Equipment Grant Program for Institutions of Higher Learning}

Eligible Equipment Catalog

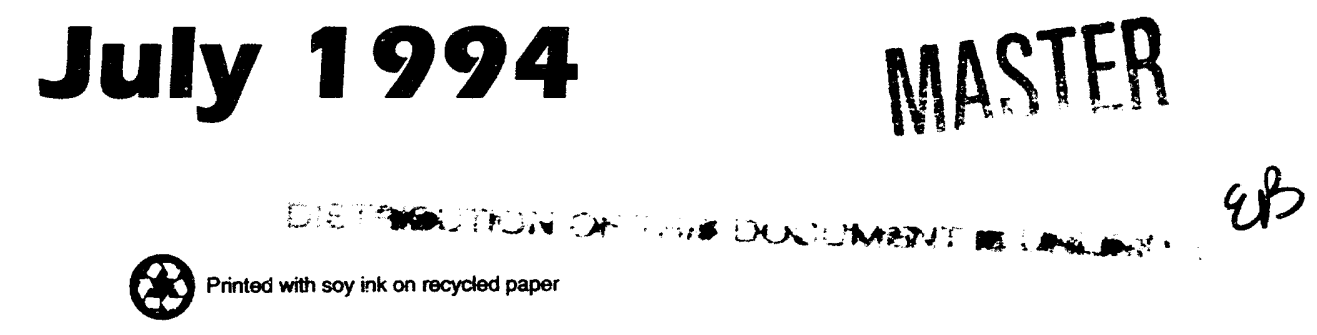




\section{Energy-Related Laboratory Equipment Grant Program}

\section{Overview}

The Energy-Related Laboratory Equipment Grant Program (ERLE) was established by the United States Depaitment of Energy (DOE) to grant available used equpment to institutions of higher education for energy-related research. The equipment listed in this catalog is available for grant: however, specific items may be recalled for DOE use and become unavallable through the program.

A hold or "freeze" must be placed on equipment desired by your institution. To freeze equipment, call the participating facility on the first working day of the month of the effeclive date of that months catalog. Provide the facility with the following information

1 vour name

2. The name of the institution you represent

3. "repcrt number (s)0" and "line item(s)" you wish to frecze for your institution

4 your telephone number

A proposal may contain several equipment items from more than one research facility as long as those facilities are served by the same DOE operations uffice. If the equipment is located at more than one facility and is served by more than one operations office. submit a proposal to each operations office. DOE operations offices and facilities are listed on Page $V$ of this catalog. To obtain information about equipment located at facilities not identified in this catalug. please contact Larry L Barker (202) 586-8947. Program Manager, for guidance on where to send proposals.

An ERLE Grant Application is provided in the back of this catalog

An institution is not required to have a currently funded project with the Department of Energy in order to participate in this program

\section{FAX "Freeze" on ERLE Equipment Prohibited}

Attempts have been made to freeze equpment using a FAX or modem before the effective date of the ERLE Catalog. Equal access in the ERLE Program is essential to help assure equitable participation for all. Because some instilutions may not have access to such equipment, the use of FAX machine to freeze equipment is not allowed. 


\section{Energy-Related Laboratory Equipment Grant Program}

\section{Guidelines}

The United States Department of Energy in accordance with its respon sibilit : encourage research and development in the energy difa. awards grants used pnerov-related laboratorv equpment to unverstles colleges and other nor proflt educa lional institutions of higher learning in the United States tor use in fnerar oriented educatuna! programs in the life. physical and environmental sciences, and engineering

Applications in the grant of avat able equipment in this program should be submitted by an eligible. non-proft educational institution to the DOE operations office responsibie for the site where the specific equipment is known to be iocated

The monthly Energy-Related Labor. atory Equipment Catalog (ERLE is available on a yearly subscription from the Government Printing Office An order form is provided on the back inside cover of this catalog

\section{Eligibility and Procedures}

Any non-profit educational institution of higher learning. such as a university. college. junior college. lechnical instutute. muscum. or hos pial located in the US and inter ested in establishing or un grading energy-orented educational programs in the the physical and envinumental sciences and enguneering is elighble

An instutum is not requared io have a current DOE grant or contract in: urder lo Danticipate on this puoram High schools grade schouls. and weational trade schools are excluded Ar energu-ciriented program is defined as an academic research activity dealing primarily or entireiy in energyrelated topics

Becaluse of space limiations cost of equmment stirage and requests by other Federal agencires fur DOE equapment. Instutum have 30 days. trom the time of 'freesing the equipmont w sut smit their grant applicition Apphration reviews and grant awards will be performed on a first-received. first qualified basis

Send requipment applicaturis to the appropriate DOE operations office $A$ list of operations offices is on Page $V$. Applications may contain multıple? equipment items from more than one
DOE facility if the facilities are served by the same DOE operations office. If the equinment is located at more than une facility. not served by the same. DOE eperatuons office an application must be submitted to fach DOE

uperatlons office

The responsibilty for identifying the locatuon of equipment rests with the irguesting institution. Specific. questums concerning equipment should be directed to the organization responsible for the item(s) under consideration. General inquiries may be made to

\section{U.S. Department of Energy}

Office of University

and Science Education. ET-31

1000 Independence Avenuse. S.W.

Wastington. D.C 20585

ATTN. Larry L Barker

Program Manager

ERLE Program

(202) 586-8947 


\section{Typical Equipment}

Tupicallems of educational traning apiallatus or equpment which may he requested are listed bolow li should be empthasized that the following

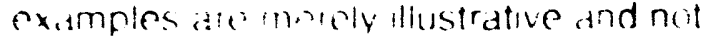
molusive

- iadiation delecturs momiturs scalers and rounters

- ecorders

- signal goni idiors

- oscilloscinfe

- amplificis

- Alsmeters suney meters iddic meters and spectroscupe

- radialion shields and reactor assiciated components

- gas and hqud chromatographs

- ampmeters. voltmeters electrometers

- mass specircimeters infrared spectrometers. and ultraviolet spectrometers

- Iinear and pulse-height analyzers

- power supplies

- catalyst test units

- distillation columns

- temperature and pressure recorders
- ion control gauges

- qas tracers and analyzers

- solar collectors and heliomelers

- personal computers

The following list is illusirative of the type of equipment which will not he provided

- general supplies

- furnifure such ds desks tables. charrs. typewriters eto (exception is such rquupment that may be an essential component of and physically attached to an eneray-related laboratory equpment system

- equipment determined to be required for uther DOE activities

- equipment intended by the institution for contractua research projects

\section{Title of Equipment}

Title to such equipment when granted by the DOE will vest with the educational institution

\section{Institutional Costs}

The cost of care and handling incident to the grant must be borne by the instltution. Such costs normally consist of packing. crating. shipping. and insuring. and are limited to actual costs Arrangements for shipment and the reimbursement of any of the aforementioned actual costs should be coordinated between the instifution and each of the DOE facilities responsible for the equpment. These. arrangements should be initiated by the requesting institution within one week after receipt and accepiance of the grant by the institution The cost of any repairs and/or modifications to any equinment will be borne by the recipif.int institution 


\section{Condition Codes}

\begin{tabular}{|c|c|c|}
\hline $\begin{array}{l}\text { Condition } \\
\text { Code }\end{array}$ & $\begin{array}{c}\text { Briet } \\
\text { Description }\end{array}$ & Expanded Detinition \\
\hline 1 & Lnuedgad & 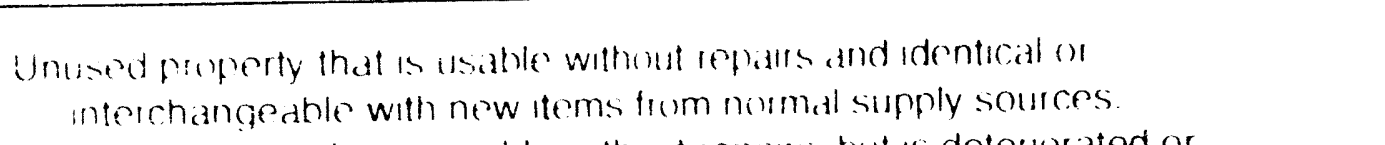 \\
\hline 2 & Unused-fall & 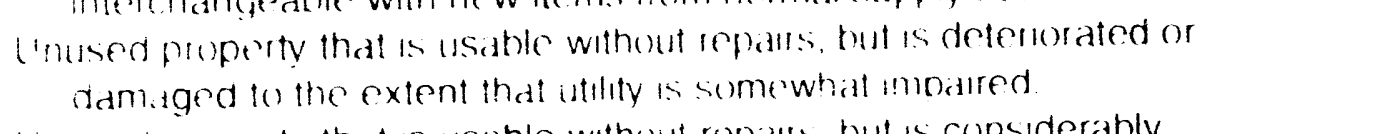 \\
\hline 3 & Unusedpoly & 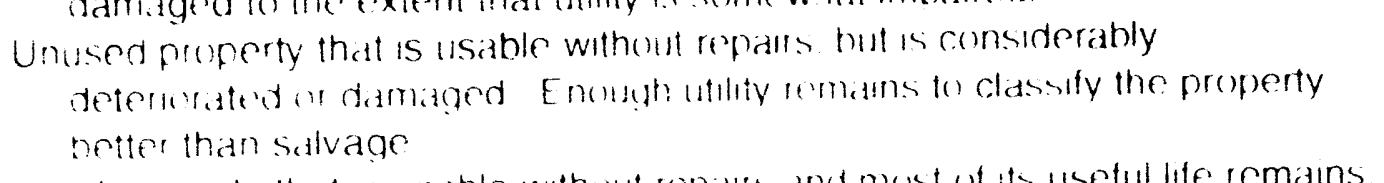 \\
\hline 4 & Used-guen & Used pooperty that is usathe without repalis and most of it use uful life inmains. \\
\hline 5 & Used-tal & 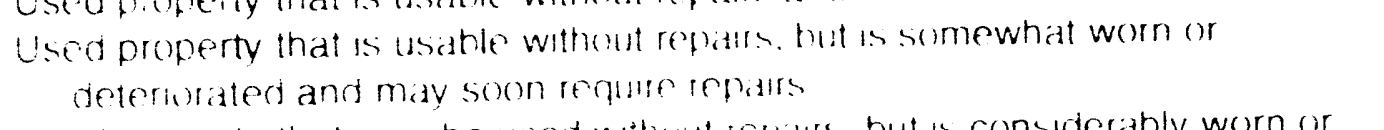 \\
\hline 6 & useanon & 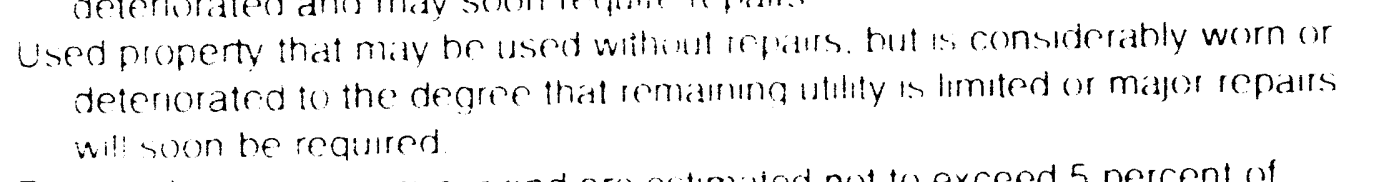 \\
\hline 7 & Repars requiled-gone & $\begin{array}{l}\text { Required repars are minor and are estimated not to exceed } 5 \text { percent of } \\
\text { ongunal acquisition cost }\end{array}$ \\
\hline 8 & Repars required fall & 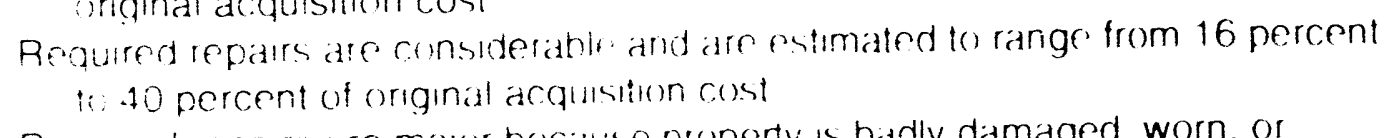 \\
\hline 9 & Repars required pui: & $\begin{array}{l}\text { Rerumed repars are majur becalise property is badly damaged, worn, or } \\
\text { deterorated and are estimated to range from } 41 \text { percent to } 65 \text { percent of } \\
\text { criginal acquisition cost }\end{array}$ \\
\hline $\mathrm{x}$ & Salvage & $\begin{array}{l}\text { Property has sume value in excess of its basic: material content, but repair or } \\
\text { echabilitation to use of the originally intended purpose is clearly }\end{array}$ \\
\hline & & $\begin{array}{l}\text { impractical Repair for any use would excend } 65 \text { percent of the original } \\
\text { arquistuon cost }\end{array}$ \\
\hline$S$ & Scrap & Matferial that has no value excrept for its basic material value. \\
\hline
\end{tabular}

Note: Condition codes assuclated with equipment are estimates and are not guaranteed to be accurate. 


\section{Where to Mail Applications}

ERLE Program

Abuquerque Operations Office (AL)

US Department of Energy

PO BOx 5400

Albuquerque NM 87:15

ATTN Corville Nohava Cmiet

(505) 845.6450

\section{ERLE Program}

\section{Chicago Operations Office $(\mathrm{CH}$}

US Department of Eneigy

9800 South Cass Avenue

Argonne. IL 60439

ATTN Gerald E Koci Chiet

(708) 252.2330

\section{ERLE Program}

Gotden Operations Office (GN)

US Department of Energy

1617 Cole Boulevard

Golden. CO 80401

ATTN Lydia Henrikson. Administ:ato

(303) $276-4765$
Allied-Signal Aerospace, Inc (MO)

ChemNuclear Geotech (CO)

$E G \& G$ Mound $(\mathrm{OH}$ )

General Electric Company (FL)

Johnson Controls World Services (NM)

Lovelace Biomedical and Environmental

Research Laboratories (NM)

Los Alamos National Laboratory (NM)

Martin Marietta Spec Components. Inc

Mason \& Hanger-Silas Mason Co inc. (TX)

Pan Am World Services. Inc (NM)

Sandia National Laboratories. Albuquerque

iNM!

Sandia National Laboratories. Livermore (CA)

Ames Laboratory ||$A \mid$

Argonne Natıonal Laboratory (IL)

Bettis Atomic Power Laboratory

Westinghouse Electric Company (PA)

Brookhaven National Laboratory (NY)

Fermi National Accelerator Laboratory (IL)

Knolls Atomic Power Laboratory General

Electric Company (NY)

Dinceton Plasma Physics Laboratory (NJ)

\section{Nationai Fenewable Energy Laboratory} iCO:

\section{ERLE Program}

daho Operations Office (ID)

US Departmient of Energy

785 DOE Place. Marl Stop 1223

Idaho Falls. ID 834021562

ATIN Glenn S Waugh. Administrator

(208) 5.6 .3712

\section{ERLE Program}

Morgantown Energy Technology Center

\section{(METC)}

U.S Department of Energy

P.O Box 880

Morgantown. WW 26507-0880

ATTN Robert Bradley. System Analyst

(304) 291.4588

\section{ERLE Program}

Nevada Operations OHfice (NV)

US. Departmen: of Energy

P.O. Box 98518

Las Vegas. NV 891938518

ATTN. Tom Senteney, Manager

(702) $295-7424$

\section{ERLE Program}

Oak Ridge Operations Office (OP)

US Department of Energy

PO Box 2001

Oak Ridge. TN 37831-8758

ATTN Charles E Crowe. Chief

(615) 576.0789
EG\&G (ID)

Morgantown Energy Technology Center MW

Reynolds Electric \& Engineering Company (NV)

EG\&G / EM Amador Valley Operations (CA)

Oak Ridge National Laboratory, Martin Marietta Energy Systems. Inc. (TN) Oak Ridge Associated Universities, Inc (TN)

Oak Ridge Gaseous Diffusion Plant/Martin Marietta Energy Systems. Inc. (TN) Portsmouth Gaseous Diffusion Plant

Martin Marietta Energy Systems. Inc. (CH) Westinghouse Materials Company of Ohio $(\mathrm{OH})$

Y-12 Plant/Martin Marietta Energy

Systerns. Inc. (TN) 


\section{Where to Mail Applications}

Mall to tollowing DOE Office

\section{ERLE Program}

Pittsburgh Energy Tochnology Center (PETC)

U.S Department of Energy

P.O Box 10940. Mall Stop 921.118

Pittsburgh. PA 15236-0940

ATTN Ralph E Bonadio. Coordinator

(412) $892-4634$

\section{ERLE Program}

\section{EG\&G Rocky Flats Plant (RF)}

PO Box 928

Golden. $\mathrm{CO}$ 80.402-0928

ATTN Jerr Adams Director

(303) $966-5128$

\section{ERLE Program}

Sen Francisco Operations Office (SF)

U.S Department of Energy

1333 Bioadway

Oakland. CA 94612

ATTN Lee Williams Administrato

(510) 637.1773

\section{ERLE Program}

Savannah River Operations Office (SR)

US Department of Energy

PO Box A

wken. SC 29802

ATTN Thomas $C$ Walker ir Cnie

(803) 725-3054

\section{ERLE Program}

Richland Operations Office (RL)

U.S. Department of Energy

P.O Box 550 . Mail Stop A7-80

Richland. WA 99352

ATTN P.E Rasmussen Chie

(509) 376-6227

\section{HEquipnient is Located at}

Pittsburgh Energy Technology Center (PA)

Mall to following DOE Otfice

ERLE Progiam

Western Area Power Administration (WAPA)

U.S Department of Energy

5555 East Crossroads Blvd

Loveland CO 80538-3003

ATrN Ted Torrez

(303) 490-7212
Equipment is Located at.

Western Area Power Administration (CO)

EG\&G Rocky Flats Plant (CO)

GA Technology (CA)

Lawrence Berkeiey Laboratory (CA

Lawrence Livermore National Laboratory (CA)

Rockwell International Corporaıı Energy Systems Group (CA)

Stanford Linear Accelerator Cente: (CA

Westinghouse Savannah River Site (SC

Westinghouse Hanford Company (WA

Battele Memorial institute (WA 


\section{Federal Supply Group Index - Numerical}

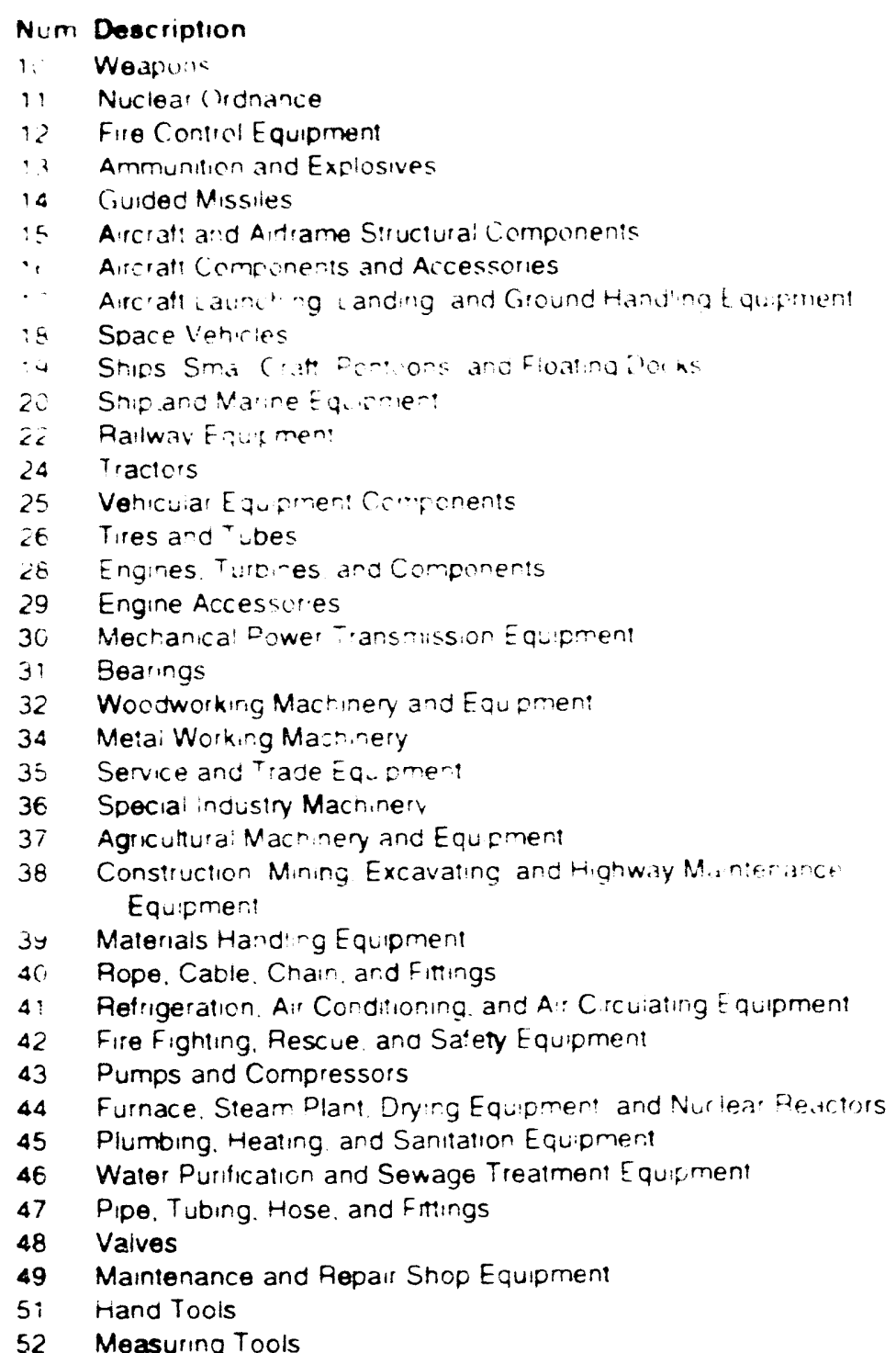

\section{Num Description}

53 Haldware and Abiasives

54 Piefabricated Structures and Scattolding

5s Lumber. Millwork Plywood, and Veneer

sti Construction and Building Materials

58 Commumication. Detection. and Coherent Fadiation Equipmen:

59 Electucial and Electronc Equpment Components

60 Fiber Optics Materials. Compononts. Assemblies, and Accessories Electric Wite and Power and Distubution F quipment

Lighting Fixtures and Lainps

Aliam and Siynal Systems

Medicil. Dental. and Veternary Equipment and Supplies

Instruments and I aboritory Equpment

Photugraphic Equpment

Chemicals and Chernical Equipment

Triining Alds and Devices

General Purpose Autornatic Data Processing f quipment including

Firmwarel. Sottware. Supplies, and Support Equpment

\section{Furniture}

Household and Commercial Furmshings and Appiances

Food Preparaion and Servirg Equipment

Office Machines. Texi Processing and Visible Record Equipment

Oftice Supplies and Devices

Books. Maps, and Oher Publications

Musical instruments. Phonographs, and Home Type Radios

Recrectional and Athletic Equipment

Cleaning Equipinent arid Supplies

Brushes Pints Sealers, and Adhesives

Containers, Packaging. ano Packing Supplies

Texiles, Leather. Furs. Apparel and Shoe Findings. Tents, and Flags Clothing, individual Equipmerit, and Insignia

iolletres

Agricultural Supplies

Live Animals

Subsistence

Fuels. Lubricants. Oils, and Waxes

Nonmetallic Fabricated Materials

Nonmetalic Crude Materials

Metal Bars. Sheets and Shapes

Miscellaneous 


\section{Federal Supply Group Index - Alphabetic}

\section{Num Description}

37 Agricultual Machinery and Equipment

Agricultural Supplies

Aircrat Components and Accessories

Alarm ano Signa: Systems

Ammunition ard Explosives

Bearings

Books. Maps and Other Pubirations

Siushes. Parrits. Seaiers, and Adhesives

Chemicais and Chemia: Equipment

Cleaning Equpment and Suppires

Cotning inswoual Equpment. and 'nsigni.z

Commuricat on Detection and Coherert Fadiation Equreme:

Consilution and Eulding Materiats

Constructer Mining Exzavating And Highway Mantename Equpren!

Contane:s. Packagng and Fack g Suppie:

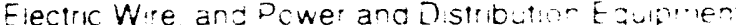

Eiectrica and Electronk Eaveren: Comporen:

Engne Accessories

Engines Turbines and Comnonerts

Fiber Optcs Materals Components Assempites and A : os mo

Fire Figring. Rescue and Sateiy Equipment

Fire Control Equipment

Food Preparation and Serving Equipment

Fueis. Lubricants O:ts. and Waxes

Furnace Siear Piari Drying Euupment ard Nuclear Rewctors

Furnture

Generai Dupose Aitumatc Daia Prccess ra Fouprrien

includrg Firmware Sottwa:e Suppiles and Subfor F fminent

Guided Missies

Hanc Toc!s

Hardware and Abrarives

Househoid and Commercia: Furrishings and Apwiances

Instruments and Laboratory Equipinent

Lighting Fixtures and Lamps

Live Animais

Lumber, Miliwork Plywood and Venee

Maintenance and Repair Shop Equipmert

Materials Handing Equpment

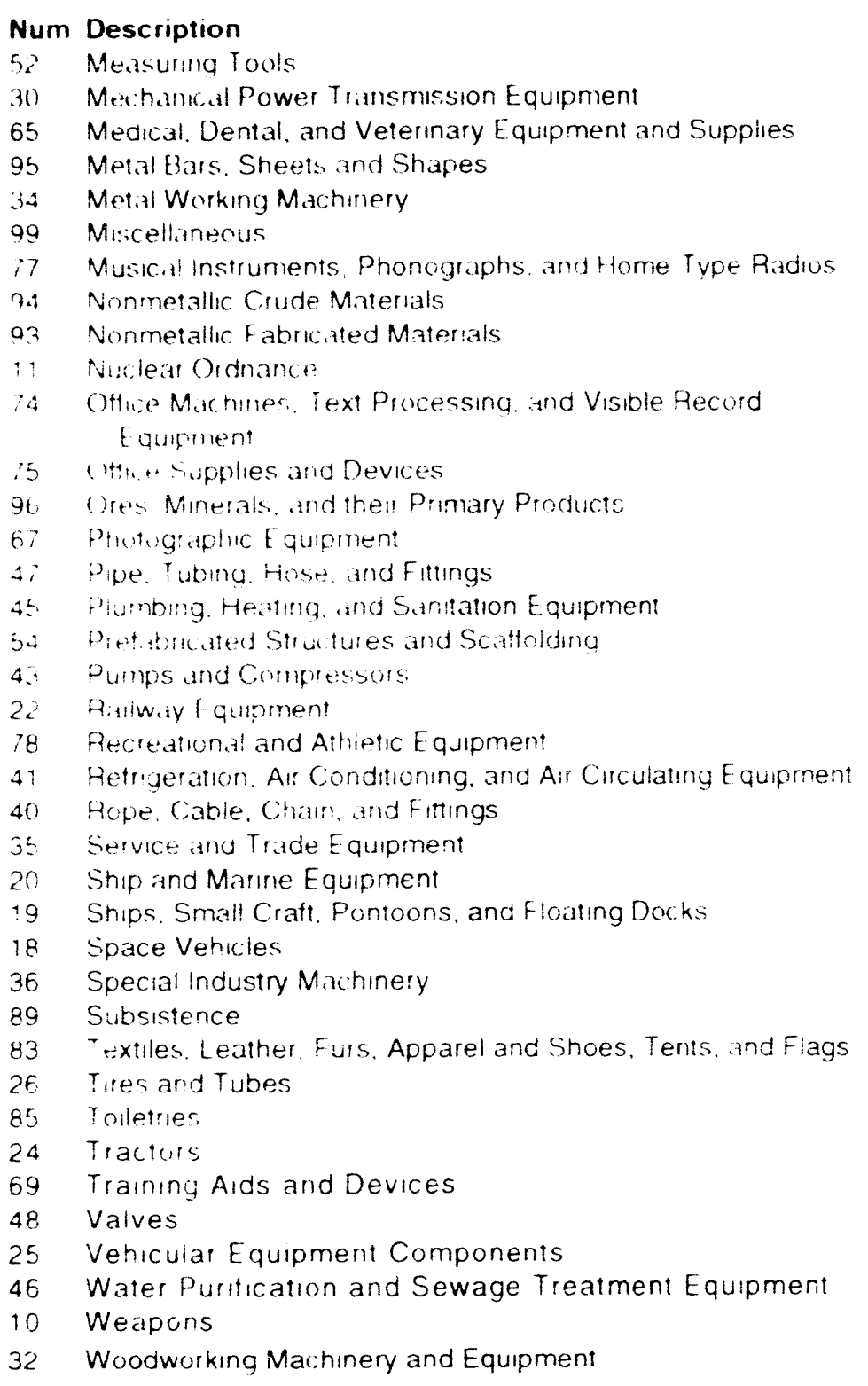




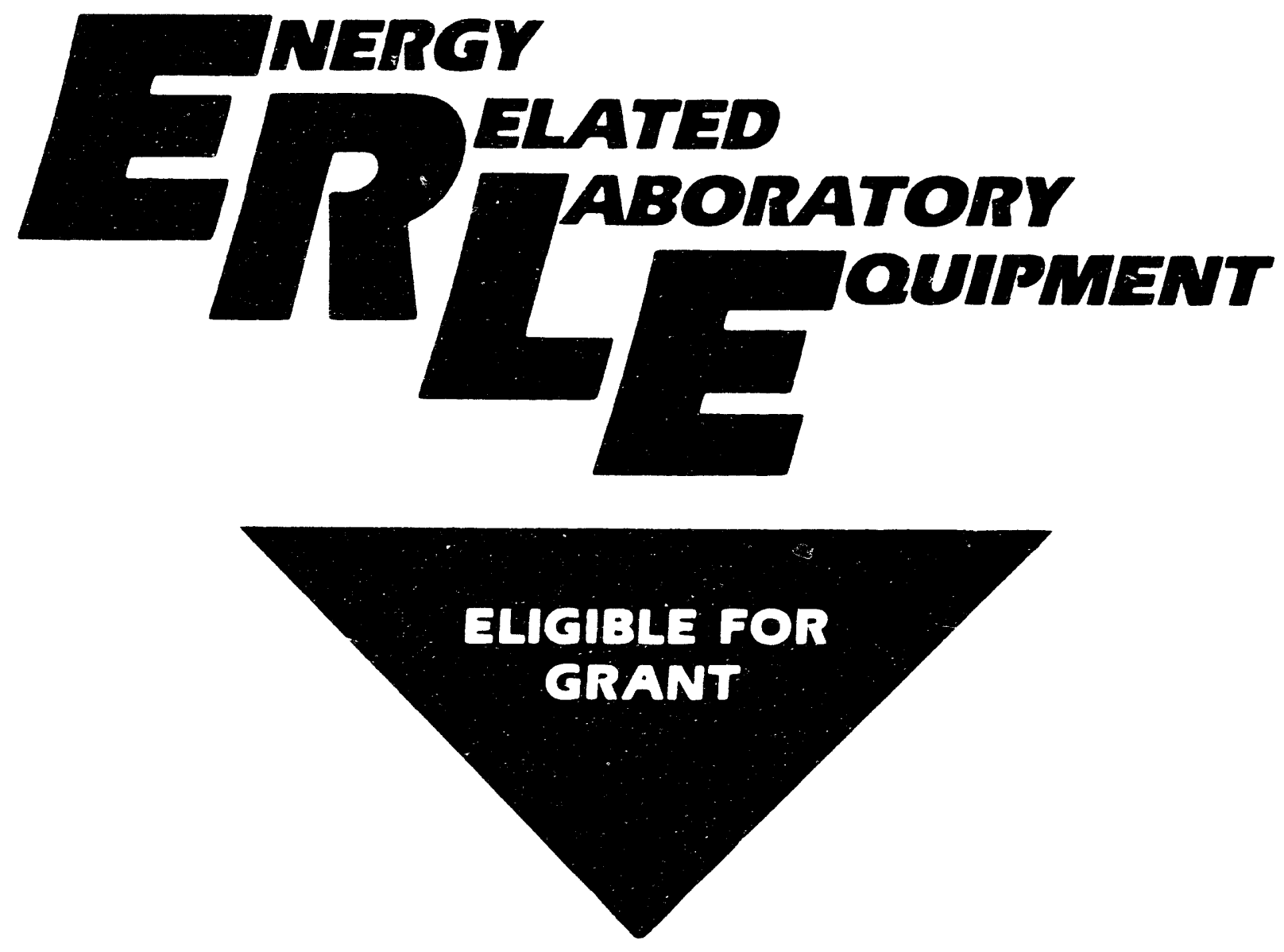




\title{
Energy-Related Laboratory Equipment Information Meeting
}

The informational meeting which was scheduled for March 1994 has been temporarily delayed. All who have expressed interest in this meeting will be advised within 30 days of the date of the meeting.

If you have not already expressed interest in this meeting, but wish to do so, please advise by providing the following information so that we may send you additional materials:

\author{
ERLE Program • U.S. Department of Energy. ET-31 \\ 1000 Independence Avenue. SW • Washington. DC 20585 \\ (202) 586-6549 Phone • (202) 586-0019 Fax
}

Name:

Title:

Organization:

City:

State:

Zip:

Phone \#:

Fax \#: 


\section{ERLE CATALOG NOW ON-LINE}

\section{Free Access to ERLE Information Via Computer/Modem or InterNet}

The listing of equipment eligible for grant under the Department of Energy's "Energy-Related Laboratory Equipment" (ERLEI Grant Program which appears in the monthly ERLE Catalog is also available on a toll-free 800 number or through InterNet on the on-line information service FEDIX.

Many institutions have reported that successful participation in the ERLE program may be attributed to utilization of a combination of the hard copy ERLE catalog and the on-lin listing of equipment. We believe that these two sources of information are complimentary and encourage their mutual usage for participation in the ERLE program. The on-line service also provides a back-up for current information in the event of a delayed ERLE Catalog arrival.

The FEDIX on-line information service contains a variety of information of interest to the academic community. The ERLE information is but one of hundreds of files available on this system. Instructions for location of the ERLE information on FEDIX follows:

\section{To Log On to the ERLE Database:}
Modem Dial Toll Free (800; 783-3349 Local 1301 : 258-0953 (Modem parameters sof at 8 data bits ' 1 'stop bit and $N$ parity at 1200.2400 or 9600 baur)

Intern't Telnet or Gopher to tedix fie com or 1.1.2:1: 22833

Howrs Avalable ¿4-tiours a day A helpline. 1301; 975-0103. is avalable weekdays tietween $8.30 \mathrm{am}$ and $500 \mathrm{rm}$ EST except Federal holidays

Costs Free no cost io, users No telephone. registration access or downioading fees

\section{To Access ERLE Information:}

- Enter UserID and other statistical information as requested.

- Select "1 - Federal Opportunities (FEDIX)" at the FEDERAL INFORMATION EXCHANGE Main Menu.

- Scroll through the "Announcements" section until the "FEDIX Main Menu" screen appears.

- Select "1-Comprehensive Agency Information" at the "FEDIX Main Menu"

- Select "1 - DOE (DOEINFO)" at the AGENCY Menu.

- Select "1 - Available Used Research Equipment (EEGADS)" at the DEPARTMENT OF ENERGY Main Menu. The ERLE information is available in the EEGADS menu.

- Screen print or download any or all of the ERLE information.

FEDIX/MOLIS is sponsored by the following particlpating Federal agencles

Def: intment of Energy - Department of Commerce - Office of Naval Research

Despartment of Ayriculture. Agency for International Development - Federal Aviation Administration National Aeronautics and Space Administration - Department of Housing and Urban Development Defense Automalion Resources Information Center - Air Force Office of Scientific Research 


\section{Notice}

Institutions operating under the statutes and constitutions of the following States are currently ineligible to participate in the ERLE program. Equipment freezes will not be accepted from these States:

\section{Arizona, Virginia and Oklahoma}

This situation arises because State laws or constitutions prevent these State institutions from signing term and condition number seven of the grant award which indemnifies the Federal Government against liability for actions related to the equipment granted under the ERLE program.

Institutions within these States that would lite to participate in the ERLE program should contact their State Attorney General's Office and request that they initiate negotiations with DOE to develop a mutually acceptable indemnification provision. The State Attorney General's Office shouli contact Mr. Christopher T. Smith of the U.S. Department of Energy's General Counsel's Office to initiate negotiations. These negotiations may only be conducted through the State Attorney General's Office. Mr. Smith may be reached at (202) 586-1526. 
EFFECTIVE DAIT: $07 / 01 / 94$ EXPIRATION DATE: $07 / 31 / 94$

CODE EXCESS PROPERTY DESCRIPIION

REFERENCE NUMBER : 1

3431 ARC FURNACE 52792, MANUF: CENTRON MODEL 5 S/N S5047

1 EACH. SIZE: 13 "W $\times 10^{\prime \prime F} / R \times 15^{\prime \prime H}$ EST WT: S LBS MFGR YR: 1980

ELIGIBLE EQUIPMEM 
EFFECIIVE DATE: $07 / 01 / 94$ EXPIRATION DATE: $07 / 31 / 94$

FSC

\section{$\operatorname{COOE}$}

EXCESS PROPERTY DESCRIPTION

REFERENCE NUMGER : 3

3694 CLEAN BENCH 43026 MANUF: DEXON MODEL HFO6ES90 S/N 9003

1 EACH LAMINAR, FLOW SIZE. 71"W $\times 3011 F / R \times$ 96"H EST WT: 1355 LES MFGR $Y R=1972$

REPORT NUMGER: $896 \div 04 \cdot 4124$

LINE ITEM NUMGER: 0038

\section{REFERENCE NUMBER : 4}

3694 CLEAN BENCH 43162, MANUF: DEXON MODEL VF56E944 S/N 9121

1 EACH. SIZE: 71 "W $\times 36^{\circ} F / R \times 96^{\prime H}$ EST WT: 950 LBS MFGR YR: 1972

REPORT NUMBER: $896404-4124$

LINE ITEM NUMBER: 0035
NO O

COND UNITS UNIT

ACQUISITION PRICE

$400001 \mathrm{EA}$

COST/UNIT:

TOTAL. COST:

$\$ 1,124.00$

$1,124.00$

KANSAS CITY
KITE REP

(816) 997-2976

MO 64141-6159

TO FREEZE EOUIPMENT

BARBARA HOLMES, REP.

KANSAS CITY

(816) $997-2976$

LOCATION OF EQUIPMENT:

ALLIEDSIGNAL, INC.

2000 EAST 95 IH STREET

KANSAS CITY

MO 64131-3095

MO 64141-6159

400001 EA

COST/UNIT:

TOTAL COST:

$\$ 1.734 .00$

$\$ 1,734.00$

FOR FURTHER INFORMATION:

BARBARA HOLMES, REP.

KANSAS CITY

(816) $997-2976$

TO FREEZE EQUIPMENT:

BARBARA HOLMES, REP.

KANSAS CITY

(816) $997-2976$

LOCATION OF EOUIPMENT:

ALLIEDSIGNAL, INC.

2000 EAST 95IH STREET

KANSAS CITY

MO 64131-3095 
EFFECTIVE DATE: $07 / 01 / 94$ EXPIRATION DATE: $07 / 31 / 94$

COOE

EXESS PROPERTY DESCRIPTION

REFERENCE NUMBER : 5

3694 CLEAN BENCH 43157 , MANUF: DEXON MODEL $7440 \mathrm{~S} / \mathrm{N} 9110$

iEACH. SIZE: 72"W $\times 36^{\prime \prime F / R} \times 96^{\prime \prime H}$ EST WT: 950 LBS MFGR YR: 1972

REPORT NUMGER: $896404-4124$

LINE ITEM NUMBER: 0036

REFERENCE NUMGER : 6

3694 CLEAN SENCH 43155 , MANUF: DEXON MODEL VF56E $944 \mathrm{~S} / \mathrm{N} 9113$

1 EACH, VERTICAL FLOW. SIZE: 72"W X 36"F/R $\times 96^{\prime \prime H ~ E S T ~ W T: ~} 950$ LBS MFGR

$Y R: 1972$
NO OF

COND UNITS UNIT

$400001 \mathrm{EA}$

COST/UNIT: TOIAL COST:

\section{$\$ 1,734.00$}

$\$ 1,734.00$
REPORT NUMBER: $896404-4124$
LINE ITEM NUMBER: 0039

\section{EA \\ COST/UNIT: \\ TOTAL COST:}

$\$ 1.734 .00$

$\$ 1,734.00$

FOR FURTHER INFORMATION:

BARBARA HOLMES, REP.

KANSAS CITY

MO $64141-6159$

TO FREEZE EQUIPMENT:

BARBARA HOLMES, REP.

KANSAS CITY

(816) $997-2976$

MO $64141-6159$

LOCATION OF EQUIPMENT:

ALLIEDSIGNAL, INC.

2000 EAST 95TH STREE

KANSAS CITY

MO $64131-3095$ 
EFFECTIVE DATE: 07/01/94 EXPIRATION DATE: 07/31/94

FSC

COOE EXCESS PROPERTY DESCRIPTION

REFERENCE NUMBER : 7

3694 CLEAN BENCH 55429 MANUF: INTEGRATED AIR MODEL LH6-36 S/N N/A 1 EACH. SIZE: $75^{\prime \prime H} \times 36^{\circ} \mathrm{F} / \mathrm{R} \times 70^{\prime \prime H}$ EST WT: 300 LBS MFGR YR: 1982

REPORT NUMBER: $896404-4124$

LINE ITEM NUMBER: 0034

REFERENCE NLMBER : 8

MANUF: INTEGRATED AIR SYSTEMS MODEL LV8-30S S/N 176334400001 EA 1 EACH. SIZE: $75^{\prime \prime W} \times 36^{\prime \prime F} / R \times 70^{\prime \prime H}$ EST WT: 300 LBS MFGR YR: 1982

REPORT NUMBER: $896404-412$

LINE ITEM NUMBER: 0040

No of

COND UNITS UNIT

ACOUISITION PRICE

COST/UNIT:

TOTAL COST

$\$ 2,818.00$

$\$ 2,818.00$

$$
\begin{aligned}
& \text { KANSAS CITY } \\
& \text { (816) } 997-2976
\end{aligned}
$$

TO FREEZE EQUIPMENT :

BARBARA HOLMES, REP.

KANSAS CITY

(816) $997-2976$

LOCATION OF EQUIPMENT

ALLIEDSIGNAL, INC.

2000 EAST 95TH STREET

KANSAS CITY

MO 64131-3095

MO 64141-6159
COST/UNIT:

TOTAL COST:
$\$ 4.720 .00$

$\$ 4,720.00$
FOR FURTHER INFORMATION:

BARBARA HOLMES, REP.

KANSAS CITY
(816) $997-2976$

TO FREEZE EQUIPMENT:

BARBARA HOLMES, REP.

KANSAS CITY

(816) $997-2976$

MO $64141-6159$

LOCATION OF EQUIPMENT :

ALLIEDSIGNAL, INC

2000 EAST 95 TH STREET

KANSAS CITY

M0 64131-3095 
EHFCTIVE DATE: 07/01/94 EXPIRATION DATE: 07/31/94

FSC

\section{CODE EXCESS PROPERTY DESCRIPIION}

REFERENCE NUMBER :

3094 CLEAN BENCH 55430, MANUF: INTEGRATED AIR MODEL LH6-36 S/N N/A

1 EACH. SIZE: 96"W $\times 36^{\circ} \mathrm{F} / R \times$ X $96^{\circ H}$ EST WT: 500 LBS MFGR YR: 1982

REPOR! NUMBER: $896404-4124$

LINE ITEM NUMBER: 004

REFERENCE NUMBER : 10

3694 CLEAN BENCH 59432, MANUF: BAKER MODEL EG6252 S/N E20899

1 EACH, LAMINAR FLOW. SIZE: $72 " \mathrm{~W} \times 36^{\prime \prime F} / R \times 60^{\prime \prime H} \mathrm{EST}$ WT: 1000 LBS MFGR

YR: 1983

REPORT NUMGER: $896404-4124$

LINE I IEM NUMBER: 0037
COST/UNIT:
TOTAL COST:

$\$ 3,302.00$

No of

COND UNIIS UNIT $\quad$ ACQUISITION PRICE

400001 EA

COST/UNIT: IOTAL COST:

\section{$\$ 2,818.00$}

$\$ 2,818.00$

KA
FOR FURTHER INFORMATION
BARBARA HOLMES, REP.
KANSAS CITY
(816) $997-2976$

MO $64141-6159$

TO FREEZE EQUIPMENT:

BARBARA HOLMES, REP

KANSAS CITY

MO $64141-6159$

(816) $997-2976$

LOCATION OF EQUIPMENT

ALL IEDSIGNAL, INC.

2000 EAST 95TH STREET

KANSAS CITY

Mo $64131-3095$

FOR FURTHER INFORMATION

BARBARA HOLMES, REP.

KANSAS CITY

(816) $997-2976$

MO $64141-6159$

TO FREEZE EQUIPMENT:

BARBARA HOLMES, REP.

KANSAS CITY
(816) $997-2976$

MO $64141-6159$

LOCATION OF EQUIPMENT

ALLIEDSIGNAL, INC.

2000 EAST 95TH STREET

KANSAS CIIYY 
FFFECIIVE DATE: 07/01/94 EXPIRATION DATE: 07/31/94

\section{CODE EXCESS PROPERTY OESCRRIPTION}

REFERENCE NUMBER : 11

3096 CLEAN BENCH $765 \%$ MANUF: ENVIROTECH MODEL CF 600 S/N 1177 I EACH. SIZE: $77^{\prime \prime W} \times 44^{" F} / R \times$ OO"H EST WT: 300 LBS MFGR YR: 1988

LINE ITEM NUMBER: 0009

RE:ERENEE NUMBER : : 2

BOC. CLEAN SENCH 74570 , MANUF: PIJRE AIRE MODEL 824 S/N N/A

CLEAN BENCH T4S70, MANUF: PIJRE AIRE MOOEL BL 1000 LBS MFGR YR: 1988

$400001 \mathrm{EA}$

COST/UNIT:

TOTAL COSI:

LINE ITEM NUMBER: 0013

$400001 \mathrm{EA}$

COST/UNIT:

TOTAL COST:

REPORT NUMBER: $896404-4124$
REPOR: NUMEER: $890+04-412$

COND UNITS UNI

ACQU:SITION PRICE

$\$ 1.076 .00$

$\$ 1,076.00$

BARBARA HOLMES, REP.
KANSAS CITY

(816) $997-2976$

MO $64141-6154$

TO FREEZE EQUIPMENT:

BARBARA HOLMES, REP.

XANSAS C! IY

(816) $997-2976$

MC 64141-6159

OCATION OF EQUIPMENT:

ALLIEDSIGNAL, INC.

95TH STREET

KANSAS CITY

MO $64131-3095$

FOR FURTHER INFORMATION:

BARBARA HOLMES, REP.

KANSAS CITY

(816) $997-2976$

MO 64141-6159

TO FREEZE EOUIPMENT:

BARBARA HOLMES, REP.

KANSAS CITY

MO $64141-6159$

(816) 997.2976

LOCATION OF EOUIPMENT:

ALLIEDSIGNAL, INC.

2000 EAST 95TH STREET

KANSAS CITY

MO 64131-3095 
EFFECIIVE DATE: 07/01/94 EXFIRATION DATE: 07/31/94

COOE EXCESS PROPERIY DESCRIPIION

REFERENCE NUMBER : 13

3694 CLEAN BENCH 7.573 MANUF: PURE AIRE MODEL $720630 \mathrm{~S} / \mathrm{N} 3406$

1 EACH. SIZE: 72"W $\times 36^{\circ} \mathrm{F} / \mathrm{RX} 60^{\prime \prime H}$ EST WT: 300 LBS MFGR YR: 1988

UNITS UNIT

$400001 E A$

COST/UNIT: TOTAL COST:

LINE ITEM NUMBER: 0014
REFORT NUMBER: $896404-4124$

(2)

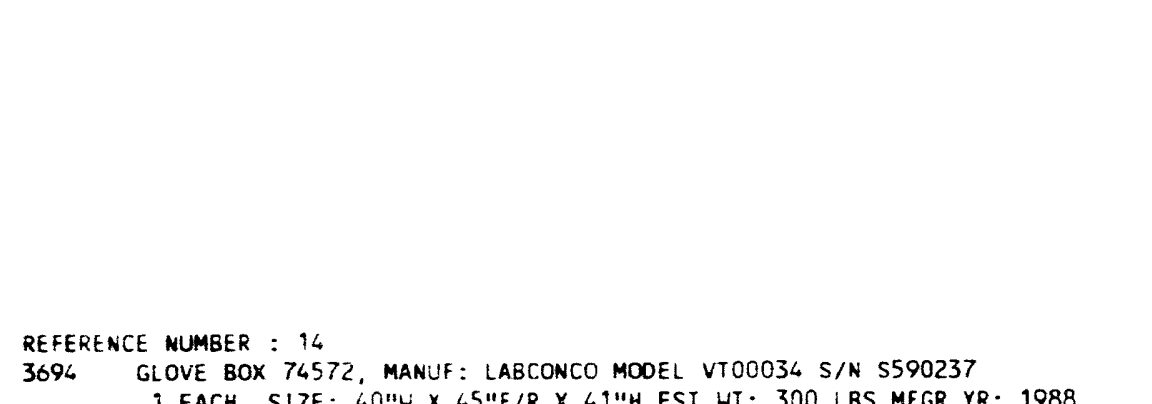

I EACH. SIZE: $60 " \mathrm{~W} \times 45$ "F/R $\times 4{ }^{11} \mathrm{H}$ EST WT: 300 LBS MFGR YR: 1988

400001 EA COSTIUNIT:
TOTAL COST:
REPORT NUMBER: $896404-4124$

LINE ITEM NUMBER: 0012
$\$ 1,768.00$

$\$ 1,768.00$

\section{COUISITION PRICE}

$\$ 1,163.00$ $\$ 1,163.00$

FOR FURTHER INFORMATION:

BARBARA HOLMES, REP.

KANSAS $C I T Y$

MO $64141-6159$

TO FREEZE EQUIPMENT:

BARBARA HOLMES, REP.

KANSAS CITY

(816) $997-2976$

MO $6 \div 141-6159$

LOCATION OF EQUIPMENT:

ALLIEDSIGNAL, INC.

2000 EAST 95TH STREET

KANSAS CITY

MO 64131-3095

FOR FURTHER INFORMATION: BARBARA HOLMES, REP. KANSAS CITY

MO $64141-6159$

(816) $997-2976$

TO FREEZE EQUIPMENT:

BARBARA HOLMES, REP.

KAYYSAS CITY

(816) $997-2976$

MO $64141-6159$

LOCATION OF EOUIPMENT:

ALLIEDSIGNAL, INC.

2000 EAST 95TH STREET

KANSAS CITY

MO $64131-3055$ 
EFFECIIVE DATE: 07/01/94 EXPIRATION DATE: $07 / 31 / 94$

FSC
COOE EXCESS PROPERTY DESCRIPTION

REFERENCE NUMBER : 15

REFERENCE NUMBER : 15
30YS VAPOR BLAST (LIOUID HONING) 63319, MANUF: VAPOR GLAST MFG. MODEL 2820

S/N O2K 1 EACH. SIZE: $72^{\prime W} \times 46^{\prime \prime F} / R \times 60^{\prime \prime H}$ EST WT: 800 LBS MFGR YR: 1985

ELIGIBLE EOUIPMENT

No of
LINË ITEM NUMBER: 0016

REPOR: NUMGER: $896404-4124$

REFERENTE NUMEER: 10

1. O" DIA FUME COLLECTOR 77163 MANUF: NEDERMAN MOOEL N/A S/N N/A

1 EACH, AFPROX. 10 FEE: OF FLEXABLE O" DUCT. SIZE: 6"W $\times 120^{\prime \prime} / R \times 6^{\prime \prime H}$

EST WI: 30 LES

MFGR YR: 1990

REPORT NUMBER: $896404-4124$

LINE ITEM NUMBER: 0022
COND UNITS UNIT

ACOUISITION PRICE

$400001 \mathrm{EA}$ COST/UNIT: TOTAL COST:

$\$ 5,000.00$ $\$ 5,000.00$

\section{FOR FURTHER INFORMATION: \\ BARBARA HOLMES, REP. \\ KANSAS CIIY \\ (816) $997-2976$}

MO 64141-6159

TO FREEZE EOUIPMENT:

BARBARA HOLMES, REP.

KANSAS CIIY

(816) $997-2976$

MO 64141-6159

LOCATION OF EQUIPMENT: ALLIEDSIGNAL, INC. KANSAS CIIY

MO 64131-3095
$400001 E A$ COST/UNIT:
TOTAL COST:
$\$ 1,600.00$

\section{FOR FURTHER INFORMATION :}

BARBARA HOLMES, REP.

KANSAS CITY

(816) $997-2976$

MO $64141-6159$

TO FREEZE EQUI PMENT: BARBARA HOLMES, REP.

KANSAS CITY

(816) $997-2976$

M0 64141-6159

LOCATION OF EOUIPMENT ALLIEDSIGNAL, INC. 2000 EAST 95TH SIREET 2000 EAST $95 T H$
KANSAS CITY 
FFFECIIVE DATE: 07/01/94 EXPIRATION DATE: 07/31/94

f SC

\section{COOE EXCESS PROPERTY DESCRIPTION}

REFERENCE NUMBER : 17

$41 \angle 0$ IMPELLER, PUMP INGERSOLL RAND W/RINGS

REPORT NLMEER: $894609-9235$

REFERENCE NUMBER : 18

-140 IMPELLER, PUMP KINNEY-VACUUM FOR VACUUM PUMP

REPORT NUMBER: $894609-9240$
MO OF

COND UNIIS UNI

ACOUISITION PRICE

100003 EA

COST/UNIT:
TOIAL COST:

LINE ITEM NUMBER: 73

100004 EA

COST/UNIT:
TOTAL COST:

LINE ITEM NUMBER: 4
CONTACT(S)

FOR FURTHER IMFORMATION:

DAVID MITCHELL

AIKEN

SC 29802

(803)

TO FREEZE EQUIPMENT:

DAVID MITCHELL

AIKEN

SC 29802

803) $715-9540$

LOCATION OF EQUIPMENT:

WESTIMGHOUSE

SAVANMAH RIVER SITE

AIKEN

SC 29802

FOR FURTHER INFORMATION:

AIKEN

SC 29802

TO FREEZE EOUI PMENT:

DAVID MITCHELL

AIKEN

(803) $725-9540$

LOCATION OF EQUIPMENT:

WESIINGHOUSE

SAVANNAH RIVER SITE

AIKEN

SC 29802 


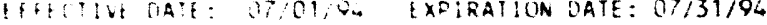

CODE EXCESS PRORERTY MESCRIPIION

REFERENCE NUMGER : 10

tiWO IMPELLER, FUMF, GOLLOS-FUMF FOR CENTRIFUGAL PUMP

REPORT NUMEER: $894609-9235$

LINE IIEM NUMBER: 72

$$
\text { No of }
$$

COND UNITS UNIT

ACOUISITION PRICE

$100004 \mathrm{EA}$

COST/UNIT: TOTAL COST:

$\$ 619.00$ $\$ 2,476.00$

DAV

DAVID MITCHEL

AiKEN $715-9540$

SC 29802

TO FREEZE EOUIPMENT :

DAVID MIICHELL

AIKEN

(803) 715.9540

LOCATION OF EQUIPMENT

WESTINGHOUSE

SAVANNAH RIVER SITE

AIKEN

SC 29802

REFEPENCE NUMGER : 20

-i,O KINNEY-VACUUM, FOR PUMF, VACUUM

REPORT NUMBER: $894609-9240$
LINE ITEM NUMBER: 3

\section{$100002 \mathrm{EA}$ \\ COST/UNIT:
TOTAL COST:}

$\$ 757.50$ $\$ 1,515.00$

FOR FURTHER INFORMATION

DAVID MITCHELL

(803) $725-9540$

IO FREEZE EQUIPMENT:

DAVID MITCHELL

AIKEN

(803) $725-9540$

LOCATION OF EOUIPMENT :

WESTINGHOUSE

SAVANNAH RIVER SITE

AIKEN

SC 29802 
EFFECTIVE DATE: $07 / 01 / 94$ EXPIRATION DATE: $07 / 31 / 94$

FSC

\section{COOE EXCESS PROPERTY DESCRIPTION}

REFERENCE NUMBER : 21

4310 PUMP VACUUM BALZER

REPORT NUMBER: $894609-9240$

REFERENCE NUMBER : 22

4310 PUMP VACUUM BALZER

REPORT NUMBER: $894609-9240$
No of

COND UNITS UNI

ACQUISITION PRICE

$600001 \mathrm{EA}$

COST/UNIT:
TOTAL COST:

LINE ITEM NUMBER: 10

600002 EA

COST/UNIT:

TOTAL COST:

$\$ 2.095 .00$

$\$ 4,190.00$

LINE ITEM NUMBER: 11
CONTACT(S)

FOR FURTHER INFORMATION:

DAVID MITCHELL

(803) $725-9540$

SC 29802

IO FREEZE EQUIPMENT

DAVID MITCHELL

AIKEN

SC 29802

LOCATION OF EQUIPMENT:

WESIINGHOUSE
SAVANNAH RIVER SITE

AIKEN

SC 29802

FOR FURTHER INFORMATION

DAVID MITCHELL

AIKEN

SC 29802

(803) $725-9540$

TO FREEZE EQUIPMENT :

DAVID MITCHELL

AIKEN

SC 29802

(803) $725-9540$

LOCATION OF EOUIPMENT:

WESIINGHOUSE

SAVANNAH RIVER SITE

AIKEN 
EFFECTIVE DATE: 07/01/94 EXPIRATION DATE: $07 / 31 / 94$

COOE EXCESS PROPERTY DESCRIPIION

REFERENCE NUMBER : 23

4310 PUMP VACUUM BALZER

REPORT NUMBER: $896609-9240$

LINE ITEM NUMBER: 9

REFERENCE NUMBER : 24

4310 PUMP VACUUM, BALZER

REPORT NUMBER: $894609-9240$
No of

COND UNITS UNIT

ACQUISITION PRICE

$600001 \mathrm{EA}$

COST /UNIT: TOTAL COST:

$\$ 2,095.00$

$\$ 2,095.00$

R INFORMATION:

DAVID MITCHELL

AIKEN $725-9540$

SC 29802

TO FREEZE EQUIPMENT :

DAVID MITCHELL

AIKEN

SC 29802

(803) $725-9540$

LOCATION OF EQUIPMENT:

ESTINGHOUSE

SAVANMAH RIVER SITE

AIKEN
$600001 \mathrm{EA}$

COST/UNIT:
TOTAL COST:

LINE ITEM NUMBER: 8
$\$ 2,095.00$
FOR FURTHER INFORMATION:

DAVID MITCHELL

AIKEN

(803) $725-$

TO FREEZE EQUIPMENT :

DAVID MITCHELL

SC 29802

(803) $725-9540$

LOCATION OF EOUIPMENT:

WESTINGHOUSE

SAVANNAH RIVER SITE

AIKEN 
EFFECTIVE DATE: $07 / 01 / 94$ EXPIRATION DATE: 07/31/94

FSC

\section{COOE EXCESS PROPERTY DESCRIPIION}

REFERENCE NUMBER : 25

4310 VACUUM PUMP, ION WITH HIGH VOLTAGE ION PUMP POWER UNIT, MOUNTED ON 20" $\times 28$ " STAND. PERKIN ULT, 20205, SER. 7230

REFERENCE NUMBER : 26

4310 VACUUM PUMP, MECH DUAL SEAL VACUUM PUMP HITH 1 HP AC MOTOR RPM 1726.

120VAC, $60 \mathrm{HZ}$. SARGENT HE, 1397B, SER. 39783

(505) $844-2746$

LOCATION OF EQUIPMENT:

SANDIA NATIONAL LABORATORIES

PROPERTY REAPPLICATION DIVISION 7617

ALBUQUERQUE
REPORT NUMBER: $898355-4088$

LINE ITEM NUMBER: 0002

500001 EA

COST/UMAT:

TOTAL COST:

$\$ 1,092.00$
$\$ 1,092.00$

ELOY N. GIRON
ALBUQUERQUE
(505) $844-2746$

TO FREEZE EQUIPMENT:

ELOY N. GIRON

ALRIIOUERQUE

NM 87185

REPART NUMRER : $898355-4088$

LINE ITEM NUMBER: 0003
NM 87185

NM 87185

ELOY N. GIRON

LOCATION OF EQUIPMENT:

DATIONAL LABORATORIES

PROPERTY REAPPLICATION DIVISION 7617

\begin{tabular}{|c|c|c|c|}
\hline UNITS UNIT & ACQUIS & ICE & CONTACTSS) \\
\hline $00001 \mathrm{EA}$ & $\begin{array}{l}\text { COST/UNIT: } \\
\text { TOTAL COST: }\end{array}$ & $\begin{array}{l}\$ 2,494.00 \\
\$ 2,494.00\end{array}$ & 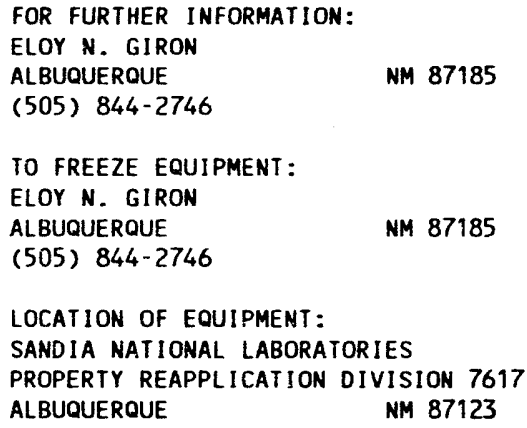 \\
\hline
\end{tabular}


EFFECTIVE DATE: 07/01/94 EXPIRATION DAIE: 07/31/94

FSC

\section{CODE EXCESS PROPERTY DESCRIPTION}

REFERENCE NUMBER : 27

4430 VACUUM OVEN 74556 MANUF: VAN WATERS MODEL 524 AE S/N OAH32633

1 EACH SIZE: $24 " W \times 24 " \mathrm{~F} / R \times 40^{\prime \prime H}$ EST WT: 200 LBS MFGR YR: 1988

ELIGIBLE EQUIPMEN

$$
\text { No } \mathrm{CF}
$$

COND UNITS UNIT

ACQUISITION PRICE

$400001 \mathrm{EA}$

COST/UNIT: TOTAL COST:

$\$ 1,642.00$

$\$ 1,642.00$

BARBARA HOLMES, REP.

KANSAS CITY

(816) $997-2976$

MO $64141-6159$

TO FREEZE EOUIPMENT:

BARBARA HOLMES, REP.

KANSAS CITY

REPORT NUMBER: $896404-4133$

LINE ITEM NUMBER: 0009

LOCATION OF EQUIPMENT:

ALLIEDSIGNAL, INC.

95TH STREE

KANSAS CITY

MO $64131-3095$

REFERENCE NUMBER : 28

4430 VACIUUM OVEN 74555 , MANUF: VAN WATERS MODEL 524AE S/N 52260004

1 EACH SIZE: $24 " W \times 24^{\prime \prime} / R \times 40^{\circ} \mathrm{H}$ EST HT: 200 LBS MFGR YR: 1988

COST/UNIT:

TOTAL COST:

$\$ 1,642.00$

$\$ 1,642.00$

FOR FURTHER INFORMATION:

BARBARA HOLMES, REP.

KANSAS CITY

(816) $997-2976$

MO 64141-6159

TO FREEZE EQUIPMENT:

BARBARA HOLMES, REP.

KANSAS CITY

(816) 997-2976

LOCATION OF EOUIPMENT

ALLIEDSIGNAL, INC.

2000 EAST 95TH STREET

KANSAS CITY

M0 64131-3095 
EFFECTIVE DATE: 07/01/94 EXPIRATION DATE: 07/31/94

\section{FSC}

\section{COOE EXCESS PROPERTY DESCRIPIION}

REFERENCE NUMBER : 29

4940 ULTRASONIC BENCHTOP CLEANER 62397, MANUF : ACCESS AND CONTROL PRODUCTS MOOELL B $92 \mathrm{H} S / \mathrm{N} N / A$

1 EACH, SIZE: 20"W X 12"F/R X 8"H EST WT: 30 LBS MFGR YR: 1984
No of

COND UNITS UNIT

$500001 \mathrm{EA}$

COST/UNIT: TOTAL COST:

LINE ITEM NUMBER: 0023
REPORT NUMBER: $896404-4124$

\section{REFERENCE NUMBER : 30}

5210 GAGE, LEVEL MFG. DANIAL IND. MOOEL \# 75RM REFLEX SER!AL \# N/A

EG\&G ID 010602-00 AUTHORIZATION MATERIAL AUTH. \$370050/N006309

YEAR ACO 1989 DOE \#
LINE ITEM NUMBER: $108 R$
100002 EA
$\$ 1,000.00$
$\$ 2,000.00$

GOR FURTHER INFORMATION:

G.A. ELVEY

GOLDEN

(303) $465-3765$

CO 80402-0464

TO FREEZE EQUIPMENT:

G.A. ELVEY

GOLDEN

(303) $465-3765$

CO 80402-0464

LOCATION OF EQUIPMENT:

EG\&G ROCKY FLATS, INC.

BUILDING 020-BROOMFIELD FACILITY

BROOMFIELD

CO 80020 
EFFECTIVE DATE: $07 / 01 / 94$ EXPIRATION DATE: $07 / 31 / 94$

FSC

COOE EXCESS PROPERTY DESCRIPTION

REFERENCE NUMBER : 31

5210 MEASUREMENT SYS TEMP. MFR. ACCU FIBER, ACQ.YR. 86, E39612

REPORT NUMBER : $899102-4163$

LINE ITEM NUMBER: 0007

REFERENCE NUMBER : 32

5210 TRANSDUCER, PRESSURE, DIFFERENTIAL ROBIN-HALPREN

REPORT NUMBER : $894609-9244$

LINE ITEM NUMBER: 24

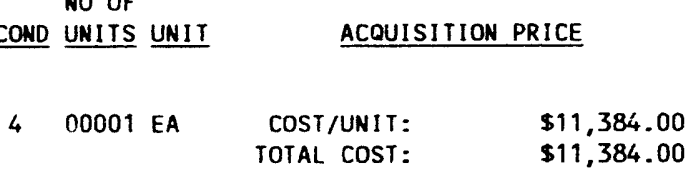

$400001 \mathrm{EA}$ TOTAL COST:

$\$ 11,384.00$

100024 EA

COST/UNIT:
YOTAL COST:

$\$ 13,824.00$

FOR FURTHER INFO
DAVID MITCHELL
AIKEN
(803) $725-9540$

SC 29802

TO FREEZE EQUIPMENT :

DAVID MITCHELL

AIKEN

(803) $725-9540$

LOCATION OF EOUIPMENT:

WESTINGHOUSE

SAVANNAH RIVER SITE

AIKEN 
EFFECIIVE DAIE: $07 / 01 / 94$ EXPIRATION DATE: 07/31/94

COOE EXCESS PROPERTY DESCRIPTION

REFERENCE NUMBER : 33

5220 COMPARATOR 74553, MANUF: EXCELLO MOOEL $30 \mathrm{~S} / \mathrm{N} 8260076$

1 EACH, 30 INCH DIA. METER. SIZE: $48 " W \times 84^{" F} / R \times 84^{\prime \prime H}$ EST WT: 4000 LBS MFGR YR: 1976

REPORT NUMEER: $896404-4124$

LINE ITEM NUMBER: 0004

REFERENCE NUMBER : 34

5220 OPTICAL COMPARATOR 74552, MANUF: MICRO PRECISION MODEL 30 S/N VTO0007

1 EACH, 30 INCHES. SIZE: 45 "W $\times$ 80"F/R $\times 83$ "H EST WT: 2000 LBS MFGR YR: 1963
$400001 E A$

TOTAL COST:

$\$ 16,167.00$

$\$ 16,167.00$
FOR FURTHER INFORMATION :

BARBARA HOLMES, REP.

KANSAS CITY

(816) $997-2976$

MO $64141-6159$

TO FREEZE EQUIPMENT:

BARBARA HOLMES, REP

KANSAS CIIY

(816) $997-2976$

MO $64141-6159$

LOCATION OF EQUIPMENT :

ALLIEDSIGNAL, INC

2000 EAST 95TH STREET

KANSAS CITY

MO $64131-3095$ 
EFFECTIVE DATE: 07/01/94 EXPIRATION DATE: 07/31/94

FSC

\section{COOE EXCESS PROPERTY DESCRIPIION}

REFERENCE NUMBER : 35

5821 AMPLIFIER, OPERATIONAL H/SOCKET

REPORT NUMBER: $894609-9247$

REFERENCE NUMBER : 36

5821 COMPARATOR BURR-6ROWN

REPORT NUMEER: $894609 \cdot 9247$

ELIGIBLE EOUIPMENT

$$
\text { NO OF }
$$

COND UNITS UNIT

ACOUISIIION PRICE

$100012 \mathrm{EA}$

COST/UNIT: TOTAL COST:

$\$ 235.00$

LINE ITEM NUMBER: 76
$\$ 75.00$

COSTIUNIT:

LINE ITEM NUMBER : 45

\section{TOTAL COST: \\ 00028 EA}

DAVID MITCHEL

AIKEN

(803) $725-9540$

TO FREEZE EQUIPMENT:

DAVID MITCHELL

AIKEN

(803) $725-9540$

LOCATION OF EQUIPMENT:

WESTINGHOUSE

SAVANNAH RIVER SITE

AIKEN

SC 29802
SC 29802

SC 29802

SC 29802

SC 29802

SC 29802 
EFFECTIVE DATE: 07/01/94 EXPIRATION DATE: $07 / 31 / 94$

FSC

\section{CODE EXCESS PROPERTY DESCRIPTION}

REFERENCE NUMBER : 37

5821 PREAMPLIFIER OXFORD-TENNELEC FOR RADIATION, GAMMA MONITOR
No of

COND UNITS UNIT

ACQUISITION PRICE

$100005 \mathrm{EA}$
COST/UNIT:
TOTAL COST:

LINE ITEM NUMBER: 51
REPORT NUMBER: $894609-9247$

REFERENCE NUMBER : 38

5821 TRANSMITTER DIALMOND-ELECTR, RACK MOUNTED FOR CCTV SYSTEM

$100010 \mathrm{EA}$
COST/UNIT:
TOTAL COST
REPORT NUMBER: $894609-9247$
LINE ITEM NUMBER: 52

\section{$\$ \$ \$ 35.00$}

CONTACT(S)

FOR FURTHER INFORMATION:

DAVID MITCHELL

(803) $725-9540$

SC 29802

TO FREEZE EQUIPMENT

DAVID MITCHELL

$$
\text { AIKEN }
$$

(803) $725-9540$

SC 29802

LOCATION OF EQUIPMENT :

WEST INGHOUSE

WESTINGHOUSE

AIKEN

$\$ 791.50$ $\$ 7,915.00$

FOR FLRTHER INFORMATION: DAVID MITCHELL

AIKEN

(803) $725-9540$

TO FREEZE EQUIPMENT:

DAVID MITCHELL

AIKEN

SC 29802

(803) $725-9540$

LOCATION OF EQUIPMENT

WESTINGHOUSE

SAVANNAH RIVER SITE

AIKEN 
EFFECIIVE DAIE: $07 / 01 / 96$ EXPIRATION DATE: $07 / 31 / 94$

COOE EXCESS PROPERTY DESCRIPTION

REFERENCE NUMGER : 39

5821 TRANSMITTER, PRESSURE, GAUGE ARTON MODEL FKGG

REPORT NUMEER: $394609-9247$

REFERENCE NUMBER : 40

5827 TRANSMITTER, PRESSURE, GAUGE FOXBORO-INSTR

REPORT NUMBER: $894609-9247$

$$
\text { No of }
$$

COND UNIIS UNIT

ACQUISITION PRICE

100002 EA

COST/UNIT:

TOTAL COST:

$\$ 1.123 .00$

LINE ITEM NUMBER: 46

\section{EA COST/UNIT: \\ TOTAL COST:}

LINE ITEM NUMBER: 47
$\$ 1.225 .25$

$\$ 4,901.00$

FOR FURTHER INFORMATION:

DAVID MITCHELL

AIKEN

SC 29802

IO FREEZE EOUIPMENT:

DAVID MITCHELL

AIKEN

(803) $725-9540$

LOCATION OF EQUIPMENT

WESTINGHOUSE

SAVANNAH RIVER SITE

AIKEN

SC 29802 
EFFECIIVE DATE: 07/01/94 EXFIRATION DATE: $07 / 31 / 94$

FSC

COOE EXCESS PROPERIY -ESCRIPTION

REFERENCE NUMBER : 41

5821 TRANSMITTER, PRESSURE, DIFFERENTIAL FOXBORO-INSTR

REPORT NUMGER: $896609-9247$
No of

COND UNIIS UNIT

$100003 \mathrm{EA}$
REFERENCE NUMBER : -2

5821 TRANSMITTER, PRESSURE, DIFFERENTIAL FOXBORO-INSIR, MOOEL 13A-MSZ FOR SYSTEM
ACOUISITION PRICE

\section{$\$ 1.444 .00$
$\$ 4,332.00$}

LINE ITEM NUMBER: 48

\section{TOTAL COST :}

\section{CONTACT(S)}

FOR FURTHER INFORMATION:

DAVID MITCHELL

AIKEN

(803) $725-9540$

SC 29802

TO FREEZE EOUIPMENT:

DAVID MITCHELL

AIKEN

SC 29802

(803) $725-9540$

LOCAIION OF EQUIPMENT:

WESTINGHOUSE

SAVANMAH RIVER SITE

AIKEN

LINE ITEM NUMBER: 49
FOR FURTHER INFORMATION:

DAVID MITCHELL

AIKEN $725-9540$

SC 29802

TO FREEZE EQUIPMENT:

DAVID MITCHELL

AIKEN

SC 29802

(803) $725-9540$

LOCATION OF EQUIPMENT:

WESTINGHOUSE

SAVANMAH RIVER SITE

AIKEN 
EFFECTIVE DAIE: OT/01/94 EXPIRAIION DATE: $07 / 31 / 94$

FSC

\section{COOE EXCESS PROPERTY DESCRIPTION}

REFERENCE NUMBER : 43

5821 IRANSMITTER, TEMPERAIURE MOORE PROO FOR CONDENSOR TANK
ELIGIBLE EOUIPMEMT wo of

COND UNITS UNIT

$100002 \mathrm{EA}$
ACOUISITION PRICE

COST/UNIT:
TOTAL COST:

LINE ITEM NUMBER: 50

REOORT NUMEER: $894609-9267$

\section{REFERENCE NUMBER : : 4}

582" VIDEO SYSTEM IN CAEINET WITH 2 25" VIDEO MONITORS AND TV CAMERA ANS

REMBRANDT, M ODEL NONE, SER. NONE. TRANSMITTE 2 . HILL TRANSMIT FROM CITY TO CITY. 12OVAC, $60 \mathrm{HZ}$.

700001 EA

REPORT NUMBER: $898355-4088$
LINE ITEM MUMBER: 0009

\section{COST/UNIT: \\ TOTAL COST:}

$\$ 166,280.00$
5684.00 $\$ 1,368.00$

CONTACT(S)

FOR FURTHER IMFORMATION:

DAVID MITCHELL

AIKEN

SC 29802

(803) $725-9540$

TO FREEZE EOUIPMENT:

DAVID MITCHELL

AIKEN

SC 29802

(803) $725-9540$

LOCATION OF EOUIPMENT:

LESIINGHOUSE

SAVAMMAH RIVER SITE

AIKEN

SC 29802

FOR FURTHER INFORMATION:

ELOY N. GIRON

ALBUOUERQUE

(505) $844-2746$

way 87185

TO FREEZE EQLIPMENT:

ELOY N. GIROW

ALBUOUEROUE

(505) $844-2746$

87185

LOCATION OF EQUIPMENT:

SANDIA MATIONAL LABORATORIES

PROPERTY REAPPLICATION DIVISION 7617

ALBUOUERQUE

HM 87123 
EFFECTIVE DATE: 07/01/94 EXP1Ration Date: 07/31/94

COOE EXCESS PROPERTY OESCRIPIION

REFERENCE NUMBER : 45

5835 RECORDER, CONFERENCE CONFER-CORDER IS SPECIALLY OESIGNED FOR RECORDING IN COURTROOM PR OCEEDINGS, CONFERENCES OR MEETIMGS. 12OVAC, GO HZ. IN COURTROOM PR OCEEDINGS, RMI G. SER. 500538

REPORT NUMBER: $898355 \cdot-038$

LINE ITEM NUMGER: 0010

$$
\text { No of }
$$

COND UNITS UNIT

ACOUISITION PRICE

$4 \quad 00001$ EA

cosi/UNIT: TOIAL COST:

$\$ 2.017 .00$ $\$ 2,017.00$

FUR FURTHER IMFORMATIOW

ELOY M. GIROW

ALBUOUEROUE

(505) $844-2746$

min 87185

TO FREEZE EOUIPNEMT

ELOY N. GIROW

ALBUOUERQUE

men 87185

(505) $844-2746$

LOCATION OF EOUIPMENT

SAMDIA MATIONAL LABORATORIES

PROPERTY REAPPLICATION DIVISION 7617

ALBUOUEROUE

Nem 87123

REFERENCE NUMBER : 40

RECORDER, VIDEO FOR STILL VIDEO RECOROING USES A 2" DISK, HAS AN 8 DIGIT

LD READO UT AMD PUSH BUTTON COWTROLS. 120VAC, $60 \mathrm{HZ}$. SONY. MVRS500, SER.

$600001 E A$

$\cos$ Tunti:

TOTAL COST:

$\$ 2,780.00$

$\$ 2,780.00$
FOR FURTHER INFORMATION:
ELOY N. GIRON
ALBUOUEROUE
(505) $844-2746$
WM 87185

TO FREEZE EQUIPMENT:

ELOY $M$. GIRON

ALBUOUEROUE

(S05) $846-2746$

87185

REPORT NUMBER: $898355-4088$

LINE ITEM MUMBER: 0011

LOCATION OF EOUIPMENT:

SAMDIA MATIONAL LABORATORIES

PROPERTY REAPPLICATION DIVISION 7617

ALBUQUEROUE

NMI 87123 
EFEECIIVE DATE: OT/01/94 EXPIRATION DATE: 0T/31/96

\section{FSC \\ COOE EXCESS PROPERTY DESCRIPIION \\ MO of}

REFERENCE NUMBER : 4

S830 TR!POO VIDEO SOOOS. MANUF: OCONNOR ENERGINEERING MOOEL $30 \mathrm{~S} / \mathrm{N} 301882105$ 1 EACH, ONE CAMERA. SITE: $45 " W \times 40^{\circ} \mathrm{F} / R \times 56^{\prime \prime H}$ EST WT: 50 IBS MFGR YR: 1982

REPURT NUMEER: $890404 \cdot 11-4$

LINE ITEM NUMBER: 0030

REFERENCE NUMBER : 48

5836 VLR PLAYER RECORDER, MOO: 519340 , SONY

REPORT NUMBER：897402 4021
COND UNIIS UNII

ACQUISITION PRICE

500001 EA

COSI/UNIT:

TOIAL COST:

51.590 .00
51.590 .00
BARBARA HOLMES, REP.
KANSAS CITY
(816) $997-2976$

BOR FURTHER INFORMATION:

M0 66169-6159

TO FREEZE EQUIPMENT :

BARBARA HOLMES, REP.

KANSAS CITY

(816) $997-2976$

190 64141-6159

LOCATION OF EOUIPMENT

ALLIEDSIGNAL. INC.

ALLIEDSIGNAL. INC.

KANSAS CIIYY

$1066131 \cdot 3095$
400001 EA
COSTAL COST:
TOTAL

\section{$\$ 1.206 .38$}

FOR FURTHER INFORMATION:

EVON DAVIS

AMARILLO

(806) $477-3870$

$1 \times 79177-0001$

TO FREEZE EQUIPMENT:

EVON DAVIS

AMARILLO

(806) $477-3870$

$7 \times 79177-0001$

LOCATION OF EQUIPMENT

MASON \& HANGER

HWY 60 AND 2373

AMARILLO

IX 79177-0001 
EFFECIIVE DAIE: 07/01/94 EXPIRATION DATE: $07 / 31 / 94$

FSC

\section{COOE EXCESS PROPERTY DESCRIPTION}

REFERENCE NUMBER : 49

5836 VCR PLAYER RECORDER MCO: VP5000, SONY
No of

COND UNITS UNI

$4 \quad 00001$ EA

\section{ACQUISITION PRICE}

$\operatorname{COSI/UNIT:~}$ TOTAL COST:

LINE ITEM NUMBER: R004
REPORT NUMEER: $897402 \quad 4021$
REFERENCE NUMBER : 50

5836 VIDEO CASS RECORDER U-MATIC VIDEO CASSETTE PLAYER HAS SEARCH OPERATION, 500001 EA STILL PICTURE, LONG PAUSE MODE, FULL AUTO REWIND, IIMER OPERATION, REMOTE CONT ROL LAND AUT SEARCH. 12OVAC, $60 \mathrm{~Hz}$. SONY.
COST/UNIT:

TOTAL COST: $\$ 2.239 .00$
$\$ 2.239 .00$

FOR FURTHER INFORMATION:

ELOY N. GIRON

ALBUDUEROUE

(505) $844-2746$

NM 87185

TO FREEZE EOUIPMENT:

ELOY N. GIRON

ALBUOUEROUE

(505) $844-2746$

NM 87185

LOCATION OF EQUIPMENT:

SANDIA NATIONAL LABORATORIES

PROPERTY REAPPLICATION DIVISION 7617 ALBUQUERQUE NM 87123 
EFFECIIVE DATE: 07/01/94 EXPIRATION DATE: 07/31/94

ELIGIBLE EQUIPMENT

FSC

\section{COOE EXCESS PROPERTY DESCRIPTION}

REFERENCE NUMBER : 51

S836 VIDEO EOUIPMENT AND ACCESSORIES SPECIAL EFFECTS GENERATOR VIDEO COLOR SYNC DISTRIBUTOR, PRINTED CIRCUIT EXIENDER BOARD, JUNCTION BOX, 12 VOLT POWER SUPPLY,

UNITS UNIT

ACOUISITION PRICE

$4 \quad 00001 \mathrm{EA}$

COSTIUNIT:
TOTAL COST:

COL OR SYNC GENERATOR, AMPLIFIER PROCESS, VIDEO SWITCHER, CORRECTOR,

EOITING CONTROL, CORRECTOR TIME BASE (REPORTED AS L LOT)

REPORT NUMEER: $897402-4021$

LINE ITEM NUMBER: R001

REFERENCE NUMBER : 52

5855 MONITOR, RADIATION RADIATION MONITOR IN BOX TO MOUNT ON HALL, WITH

SIREN, WARNING LI GHT AND 4 LED DIGIT READOUT. 12OVAC, 60 HZ. XETEX.

S10A, SER. 016866.

$600001 \mathrm{EA}$

COST/UNIT:

OTAL COST

REPORT NUMBER: $898355-4088$

LIME ITEM NUMBER: 0013
$\$ 1,450.00$

$\$ 38,199.90$ $\$ 38,199.90$

.

FOR FURTHER INFORMATION:

ELOY N. GIRON

ALBUOUEROUE

(505) $844-2746$

NM 87185

TO FREEZE EOUIPMENT:

ELOY M. GIRON

ALBUOUEROUE

MM 87185

(505) $844-2746$

LOCATION OF EQUIPMENT.

SANDIA MATIONAL LABORATORIES PROPERTY REAPPLICATION DIVISION 7617 ALBUQUERQUE NM 87123 
EFFECIIVE DATE: $07 / 01 / 94$ EXPIRATION DATE: 07/31/94

tesc

COOE EXCESS PROPERTY DESCRIPIION

REFERENCE NUMBER : 53

5860 LASER, MFR.SPECTRA PHYS, MOOEL 17117, ACQ. YR. 82, PROP. NO. 4129484
REFERENCE NUMBER : 54

5865 SATORIOUS BALANCE MODEL 2405, MFR. SATORIOUS, SER\# 2905009
No of

COMD UNITS UNI

$500001 \mathrm{EA}$

TOTAL COST:

LINE ITEM NUMBER: 0009
REPORT NUMBER : $899102-4163$
400007 EA
COST IUNIT

TOTAL COST:
LINE ITEM NUMBER: 0009

REPORT NUMBER: 899110-0125

\section{DUISITION PRICE}

$\$ 35,540.40$ $\$ 35,540.40$
$\operatorname{CONTACT}(S)$

FOR FURTHER INFORMATION:

JOANME BREZNIK

LIVERMORE

CA 94550

(510) 424-4101

TO FREEZE EQUIPMENT :

JOANNE BREZNIK

LIVERMORE

(510) $424-4101$

CA 94550

LOCATION OF EQUIPMENT:

AAURENCE LIVERMORE NATIONAL LAB

EXCESS PROPERTY SECTION, L-696

LIVERMORE

CA 94550
FOR FURTHER INFORMATION :

YOLANDA CUEVAS/MS DIV

OAKLAND

CA $94612-5208$

(510) 637-1767

TO FREEZE EQUIPMENT:

YOLANDA CUEVAS/MS DIV

OAKLAND

(510) $637-1767$

CA $94612-5208$

LOCATION OF EQUIPMENT:

GENERAL ATOMICS

3550 GENERAL ATOMICS COUR

SAN DIEGO

CA 92121 
EFFECIIVE DATE: $07 / 01 / 94$ EXPIRATION DATE: $07 / 31 / 94$

FSC NO OF

\section{COOE EXCESS PROPERTY DESCRIPTION}

REFERENCE NUMBER : 55

5865 TRIGGER AMPLIFIERS, W/PFN BOARDS ETC., ACQ. YR. 73, E38248
LINE ITEM NUMBER: 0010
COND UNITS UNIT

ACOUISITION PRICE

600001 EA

COST/UNIT: TOTAL COST: $\$ 16,595.00$
$\$ 16,595.00$
REFERENCE NUMBER : 50
5895 MOOEM

MOOEM SERIAL \# 01305018 GARCODE 850779
X94-04-074 MANUF. WESTERN DATA

$900001 \mathrm{EA}$
COST/UNIT:

IOTAL COST:
$\$ 1,180.00$ $\$ 1,180.00$

FOR FURTHER INFORMATION:

WILLIS J. MILLER

CINCINNATI

(513) $738-6630$

OH 45239-8704

TO FREEZE EQUIPMENT:

WILLIS J. MILLER

CINCINNAT!

(513) $738-6630$

OH 45239-8704

LOCATION OF EOUIPMENT :

U.S. DOE/FERMCO

7400 WILLEY ROAD

FERNALD

OH 45030 
EFFECIIVE DATE: $07 / 01 / 94$ EXPIRATION DATE: $07 / 31 / 94$

FSC

CODE EXCESS PROPERTY DESCRIPTION

COND UNITS UNIT

ACQUISITION PRICE

REFERENCE NUMBER : 57

5895 MOOEM MOOEM SERIAL \# 01305031 BARCOOE 850780

X94-04-075 MANUF. HESTERN DATA

$900001 \mathrm{EA}$

LINE ITEM NUMBER: 0028

TINE ITEM NUMBR: 0028

REFERENCE NUMBER : 58 5910 CAPACITOR, DIPPE
93. E37950

8 IPPED MICA $5 \% 500$ (1396 EA), MFR. CORNELL DUBILIER, ACO. YR. 00001 EA
COST/UNIT:

TOTAL COST:
$\$ 1,027.46$ $\$ 1,027.46$
$\$ 1,180.00$ $\$ 1,180.00$ TOTAL COST:

FOR FURTHER INFORMATION:

JOANNE BREZNIK

LIVERMORE

(510) $424-4101$

CA 94550

TO FREEZE EQUIPMENT:

JOANNE BREZNIK

LIVERMORE

CA 94550

(510) $424-4101$

LOCATION OF EQUIPMENT:

LAWRENCE LIVERMORE NATIONAL LAB

EXCESS PROPERTY SECTION, L-696

EXCESS PROPERTY SECTION, L-696
LIVERMORE
CA 94550 
EFFECIIVE DATE: $07 / 01 / 94$ EXPIRATION DATE: $07 / 31 / 94$

CODE EXCESS PROPERIY DESCRIPTION

REFERENCE NUMBER : 59

5963 COMSTANT FRACTION AMPLIFIER MODULE MODEL 437A, MFR. ORTEC (1) SER\# 588 , (2) SER\# 586, (3) SER\# 584, (4) SER\# 585

REPORT NUMBER: $899110-0125$

LINE ITEM NUMBER: 001

REFERENCE NUMBER : 60

5963 LINEAR AMPLIFIER MODULE MODEL TC205A, MFR. TENNELEC, SER\# 674

REPORT NUMBER: $899110-0125$

LINE ITEM NUMBER: 0013
NO OF

COND UNITS UNIT

ACQUISITION PRICE

COST/UNIT:

TOTAL COST:

$\$ 1,499.00$

$\$ 5,996.00$

YOLANDA CUEVAS/MS OIV

OAKLAND

CA $94612-5208$

(510) 637-1767

TO FREEZE EQUIPMENT:

YOLANDA CUEVAS/MS DIV

OAKLAND

(510) 637-1767

CA $94612-5208$

LOCATION OF EQUIPMENT

GENERAL ATOMICS

3550 GENERAL ATOMICS COURT

SAN DIEGO

CA 92121

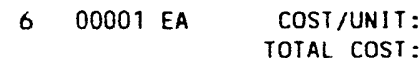

$\$ 1,102.00$

$\$ 1,102.00$
FOR FURTHER INFORMATION:

YOLANDA CUEVASIMS D

OAKLAND

CA $94612-5208$

(510) 637-1767

TO FREEZE EQUIPMENT:

YOLANDA CUEVAS/MS DIV

OAKLAND

(510) 637-1767

CA $94612-5208$

LOCATION OF EQUIPMENT :

GENERAL ATOMICS

3550 GENERAL ATOMICS COURT

SAN DIEGO

CA 92121 
EFFECTIVE DATE: $07 / 01 / 94$ EXPIRATION DATE: $07 / 31 / 96$

FSC

FSC
COOE EXCESS PROPERTY DESCRIPIION

REFERENCE NUMBER : 61

5963 TIME CORRECTOR NIM MODULE MODEL 1468A, MFR. CANBERRA, SER\# 677331

REPORT NUMBER: $890110-0125$

LINE ITEM NUMBER: 0012

REFERENCE NUMBER : 62

5963 TIME PULSE COMVETER MODULE MODEL 467, MFR. ORTEC, SER\# 1582

REPORI NUMBER: $899110-0125$

LINE ITEM NUMBER: 0011

\section{NO OF}

COND UNITS UNIT

ACOUISITION PRICE

$600001 \mathrm{EA}$

$\operatorname{COST/UNIT:}$ TOTAL COST:

$\$ 1,158.00$

$\$ 1,158.00$

YOR FURTHER INFORMATION:

YOLANDA CUEVAS/MS DIV

OAKLAND

(510) $637-1767$

TO FREEZE EQUIPMENT:

YOLANDA CUEVAS/MS DIV

OAKLAND

(510) 637-1767

LOCATION OF EQUIPMENT :

GENERAL ATOMICS

550 GENERAL ATOMICS COURT

SAN DIEGO

CA 92121

CA $94612-5208$

$\begin{array}{rrr}600001 E A & \text { COST/UNIT: } & \$ 1,075.00 \\ & \text { TOTAL COST: } & \$ 1,075.00\end{array}$

FOR FURTHER INFORMATION:

YOLANDA CUEVAS/MS DIV

OAKLAND

CA $94612-5208$

(510) 637-1767

TO FREEZE EQUIPMENT:

YOLANDA CUEVAS/MS DIV

OAKLAND

(510) $637-1767$

LOCATION OF EQUIPMENT:

GENERAL ATOMICS

3550 GEMERAL ATOMICS COURT

SAN DIEGO

CA 92121 
EFFECIIVE DATE: 07/01/94 EXPIRATION DATE: $07 / 31 / 94$

FSC

CODE EXCESS PROPERTY DESCRIPTION

REFERENCE NUMBER : 63

5975 LOGIC CARD, INLOADED, WIRE WRAP ( 45 EA), MFR. UNK., MODEL $5975-62933$, LOGIC CARD, INLOADED
ACO. YR. $93, E 38637$

INE ITEM NUMBER: 0005

REFERENCE NUMBER : 64

5975 LOGIC CARD, UNLOADED, HIRE WRAP (27 EA), MFR. UNK, MODEL 5975- 62932 ACO. YR. $93, E 38636$
REPORT NUMBER : $899102 \cdot 4169$

No OF

COND UNITS UNIT

ACQUISIIION PRICE

$400001 \mathrm{EA}$

COST /UNIT:

TOTAL COST:

$\$ 5,873.85$

$\$ 5,873.85$

JOANNE BREZNIK
LIVERMORE

(510) $424-4101$

CA 94550

TO FREEZE EQUIPMENT

JOANNE BREZNIK

LIVERMORE

CA 94550

(510) $424-410$

LOCATION OF EQUIPMENT:

LAURENCE LIVERMORE NATIONAL LAB

EXCESS SECTION, L-696

LIVERMORE

CA 94550

$\$ 4,576.92$

COST/UNIT:

TOTAL COST:

$\$ 4,576.12$

FOR FURTHER INFORMATION:

JOANNE BREZNIK

LIVERMORE

CA 94550

(510) $424-4101$

TO FREEZE EQUIPMENT

JOANNE BREZNIK

CA 94550

REPORT NUMBER: $899102-4169$

LINE ITEM NUMBER: 0006
(510) $424-410$

LOCATION OF EQUIPMENT:

LAWRENCE LIVERMORE NATIONAL LAB

EXCESS SECTION, L-696

LIVERMORE

CA 94550 
EFFECTIVE DATE: $07 / 01 / 94$ EXPIRATION DATE: 07/31/94

FSC

COOE EXCESS PROPERTY DESCRIPIION

COND UNITS UNIT

ACQUISITION PRICE

REFERENCE NUMBER : 65

5975 NUCLEAR INSTRUMENTATION GIN, TYPE B (8 EA), MFR. UNK., MODEL 5975 57233, ACO. YR. 93, PROP. NO. E38628

REPORT NUMBER: $899102-4169$

LINE ITEM NUMBER: 0003

REFERENCE NUMBER : 66

5975 RACK STORAGE WHICH HOLDS 72 IST GOARD FOR TESTING CHIPS IN A TEMP CHAMBER. 26 "WX26"DX55"H. JARVIS, ST72, SER. NONE.

$600001 \mathrm{EA}$

COST/UNIT:

TOTAL COST

$400001 \mathrm{EA}$

COST/UNIT:

IOTAL COST:

$\$ 6,103.6$

$\$ 6,103.61$

FOR FURTHER INFO
JOANNE BREZNIK

LIVERMORE

(510) $424-4101$

CA 94550

TO FREEZE EQUIPMENT:

JOANNE BREZNIK

LIVERMORE

CA 94550

(510) 424-4101

LOCATION OF EQUIPMENT:

LAWRENCE LIVERMORE NATIONAL LAB

EXCESS SECTION, L-696

LIVERMORE

CA 94550

REPORT NUMBER: $898355-4088$

LINE ITEM NUMBER: 0015
$\$ 2,300.00$

$\$ 2,300.00$
FOR FURTHER INFORMATION :

ELOY N. GIRON

ALBUQUERQUE

(505) $844-2746$

NM 87185

TO FREEZE EQUIPMENT:

ELOY N. GIRON

ALBUOUERQUE

(505) 844-2746

NM 87185

LOCATION OF EQUIPMENT:

SANDIA NAT IONAL LABORATORIES

SANDIA NATIONAL LABORATORIES
PROPERTY REAPPLICATION DIVISION 7617

PROPERTY REAPPLICATION DIVISION 7617
ALBUQUERQUE 
EFFECTIVE DATE: 07/01/96 EXPIRAIION DATE: $07 / 31 / 96$

FSC

COOE EXCESS PROPERIY DESCRIPIION

REFERENCE NUMBER : 67

S975 RFI FILTER, L SECT, 500 MILLIAMPS MX (38 EA), MFR. SPRAGUE, MO DEL 5975, 400001 EA ACO. YR. Q3, E38620

ELIGIBLE EOUIPMENT

NO OF

COND UNITS UNIT

COSTIUNIT:
TOIAL COST:

LINE ITEM NUMBER: 000 i
REPORT NUMEER : $899102-4160$
REFERENCE NUMGER : 68

5995 CABLE ASSEMBLY, CABLE M17/74-RG213 (28 EA), MOOEL 5995-33442, ACQ. YR. 4 00001 EA 93, E3852
COST/UNIT:

TOTAL COST:
$\$ 1,005.20$ $\$ 1,005.20$
$\$ 1,297.12$
$\$ 1,297.12$

INFORMATION:

JOANNE BREZNIK

LIVERMORE

(510) $424-410$

CA 94550

IO FREEZE EQUIPMENT :

JOANNE BREZNIK

LIVERMORE

(510) $424-4101$

LOCATION OF EQUIPMENT.

LAWRENCE LIVERMORE NATIONAL LAB EXCESS SECTION, L-696 EXCESS SECT

FOR FURTHER INFORMATION:

JOANNE BREZNIK

LIVERMORE

(510) $424-4101$

CA 94550

TO FREEZE EQUIPMENT:

JOANNE BREZNIK

LIVERMORE

(510) $424-4101$

CA 94550

LOCATION OF EQUIPMENT:

LAWRENCE LIVERMORE NATIONAL LAB EXCESS PROPERTY SECTION, L-696 EXCESS PROPERTY SECTION, L-696
LIVERMORE
CA 94550 
EFFECIIVE DATE: 07/01/94 EXPIRATION DATE: 07/31/94

FSC NO OF

COOE EXCESS PROPERIY DESCRIPTION COMD UNITS UNIT

REFERENCE NUMBER : 69

5999 AREA SAFETY ACCESS WARNING SIGN (46 EA), MOOEL 5999-63690, ACO, YR. 93, 400001 EA E38526

REPORT NUMBER: $899102 \cdot 4163$

LINE ITEM NUMBER: 0021

REFERENCE NUMBER: 70

5990 MAGNET-ELECTRO .5" GAP, OAD = 22" LG. $\times 13$ " DIA. (200 LBS) VARIAN V4005, 400001 EA ACO YR: 66
COST/UNIT:
TOTAL COST:
$\$ 2,153.00$
FOR FURTHER INFORMATION:

MONIE CLEVENGER

BERKELEY

(510) $486-6242$

CA 94720

TO FREEZE EQUIPMENT:

MONTE CLEVENGER

BERKELEY

CA 94720

(510) $486-6242$

LOCAIION OF EQUIPMENT:

901 MAREHOUSE

1450 64TH STREET

EMERYVILLE

CA 94608 
EFFECIIVE DATE: 07/01/94 EXPIRAIION DATE: $07 / 31 / 94$

FSC

\section{COOE EXCESS PROPERTY DESCRIPIION}

REFERENCE NUMBER : 7

5999 SAFETY BOX ASSEMBLY WILITE \& SWITCH (6 EA), MOOEL 5999-59684, ACO. YR. 93. E38525

REPORI NUMEER: $899102-4163$

LINE ITEM MUMBER: 0022

REFERENCE NUMBER : 72

5990 X-RAY SAFETY COMPNENT (8 EA), MFR. PRINT PACKAGE, MOOEL 5999-6 8262 ACO. $Y R, 93, E 38527$

REPORT NUMBER: $899102-6169$

LIME ITEM MUMBER: 0007

\section{No of}

$\$ 2.365 .54$ Cosi /UMIT: TOTAL COST: $52,365.54$ (rotal cost:

FOR FURIHER INFORMATION:

JOAMUE BREZWIK

LIVERMORE

CA 94550

10 FREEZE EOUIPWEWT:

JOANME BREZWIK

LIVERMORE

(510) $424-4101$

CA 94550

LOCAIION OF EOUIPMENT :

LALRENCE LIVERMORE MATIOUAL LAB

EXCESS PROPERTY SECTIOW, L-696

LIVERMORE CA 94550

FOR FURTHER IMFORMATION:

JOAMWE BREZWIK

LI VERMORE

TO FREEZE EOUIPMEMT

JOANME BREZMIK

LIVERMORE

(510) $424-4101$

CA 94550

LOCATION OF EQUIPMENT:

LALREMCE LIVERMORE MATIOHAL LAB

EXCESS SECIIOW. $1-696$

II IVERMORE

Ca 94550 
EFFECTIVE DATE: $07 / 01 / 94$ EXPIRATION DATE: $07 / 31 / 94$

F SC

\section{COOE EXCESS PROPERTY DESCRIPIIOM}

REFERENCE MUMGER : 73

6030 POER SUPPIY HAS CONNECTORS FOR GAUGE, COMPENSAIION, OUTPUT, TRIGGER

TAME 0 TO 90 BALANCE O TO 90 POUER SUPPL

HAVE ON/OFF CONTROLS. 120VAC, $60 \mathrm{HZ}$. DYMASEN, CK15030, SER

0107 .

REFERENCE MUMBER : 76

6110 POWER SUPPLY MOOEL SCR-300, MFR. ELECTRONIC MEASUREMENTS, SER\# 4N-7803
$500001 E A$ $\cos T /$ UN! T: TOTAL COST:
$\$ 1,000.00$ $\$ 1,000.00$

\section{$52,508.00$} $\$ 2,508.00$

\section{COST/UNIT:} TOTAL COST:

LINE ITEM MUMBER: 0004
REPORT NUMBER: $899110-0125$
FOR FURTHER IMFORMATIOW: Oakiand

CA $96612-5208$

$$
\text { (510) } 637-1767
$$

TO FREEZE EOUIPUEMT: YOLAMOA CUEVAS/MS DIV oaxiano

CA $94612-5208$ YOLAMOA CUEVAS/MS DIV

LOCATION OF EOUIPAEMT:

GEMERAL ATONICS

3550 GEMERAL ATONICS COURT

SAM DIEGO 
EFFECIIVE DAFE: OT/OY:O EXPIRATION CATE: DT/31/96

ISC
COOE EXCESS PROEERIY DESCR:DTION

REHERENCE NUMBER: 75

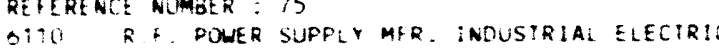

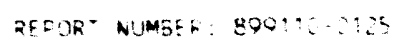

IINE ITEM NUMEER: 0003

REFERENCE NUMEER: TO

GIO SCS POWER SUPDLY MODEL STR-300-05, MFR. ELECTRONIC MEASUREMENIS. SERH
No of

COND UNITS UNIT

- $00001 \mathrm{EA}$

COST/UNIT:

TOIAL COST:

COUISIIION PRICE

$\$ 25.643 .00$

$\mathbf{s} 25,643.00$

r

YOLANOA CUEVAS/MS DIY

OAKLAMO

(510) $637-1767$

CA $96612-5208$

TO FREEZE EOUIPMENT:

YOLAMDA CUEVAS/WS DIV

OAKLAMD

(510) $637-1767$

LOCATION OF EOUIPMENT:

GEMERAL ATOMICS

3550 GEMERAL ATOMICS COURT

SAM DIEGO

CA 92121

CA $94612-5208$

\section{COST/UNIT: \\ TOIAL COST:}

$\$ 1.138 .00$

si. 138.00
FOR FURTHER INFORMATION:

YOLAMDA CUEVAS/MS DIV

OAKLAMD

(510) $637-1767$

TO FREEZE EOUIPMENT:

YOLAMDA CUEVAS/MS DIV

OAKLAND

(510) $637-1767$

CA $94612-5208$

LOCATION OF EOUIPMENT:

GEMERAL ATONICS

3550 GENERAL ATOM:CS COURT

SAM DIEGO

CA 92121 
EFHCTILF PIATE: 07/U1/96 EXPIRATION DATE: 07/31/96

\section{CURE EXCESS PROFERTY DESCR:PTION}

RE FERENCE NUMGER : 77

6130 GENERATOR, FUNCTION 30UAZ 10 3OMHZ FREO RANGE, VARIABLE RISE AND FALL

IIME, TRAPE 20 OD EL WAVESORMS, TRIGGER AND GATEO OPERATION, SWEEP UP OR

JOWN. 120 VAC, 60 HZ. WAVETEK, 16i. SER. 277087.

REPORT NUMGER: $898355-6088$

LINE ITEM NUMBER: 0022

REFERENCE NUMBER : 78

6130 GENERATOR, FLANTION 30 UHz TO 30 MHZ FRE RANGE, VARIABLE RISE AND FALI 7 00001 EA TIME. 120VAC, GO HZ. WAVETEK, 164, SER. H27927\%.

REPORT NUMBER: $898355-4088$

LINE ITEM NUMBER: 0025

$500001 \mathrm{EA}$
No of

COND UNIIS UNIT

ACQUISITION PRICE COSI/UNIT:
TOTAL COST:

$\$ 1.235 .00$ $\$ 1,235.00$

\section{ELOY N. GIRON \\ ALBUQUERQUE}

R INFORMATION:

(505) $844-2746$

IO FREEZE EOUIPMENT:

ELOY N. GIROW

ALBULUEROUE

(SO5) $846-2746$

m 87185

\section{LOCATION OF EOUIPMENT:}

SAMDIA MATIOMAL LABORATORIES

PRCPERTY REAPPLICAIION DIVISION 7617

ALBUOUEROUE
COST UNIT:
TOTAL COST:

TOTAL COST:
$\$ 1.237 .00$
$\$ 1,237.00$
FOR FURTHER INFORMATION:

ELOY N. GIRON

ALBUQUERCUE

(505) $844-2746$

MM 87185

TO FREEZE EOUIPMENT:

ELOY N. GIRON

ALBUQUEROUE

(505) $844-2746$

MM 87185

LOCATIOM OF EQUIPMENT:

SANDIA MATIONAL LABORATORIES

PROPERTY REAPPLICATION DIVISION 7617

ALBUOUEROUE NM 87123 


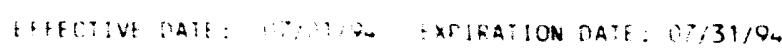

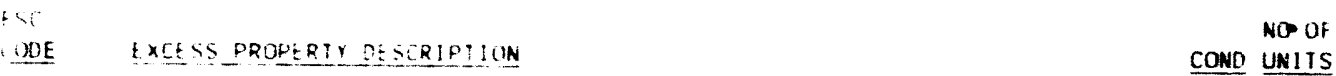

not of

LINE ITEM NUMBER: 0033

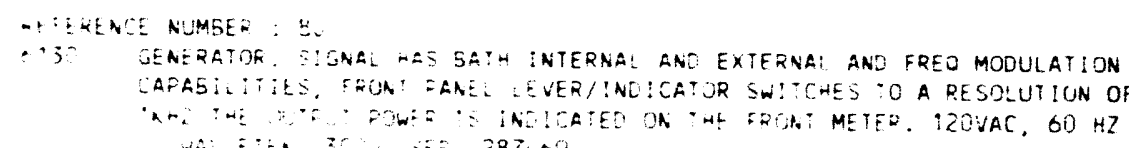

COND UNITS UN!T

ACOUISITION PRICE

$600001 \mathrm{EA}$

COST/UNIT: TOTAL COST:

$\$ 1,550.00$ $\$ 1,550.00$

\section{ELOY N. GIRON \\ ALBUOUEROLLE}

FOR FURTHER INFORMATION:

(505) $844-2746$

NM 87185

IO FREEZE EOUIPMENT:

ELOY N. GIRON

LBUOUERQUE

(505) $844-2746$

NM 87185

LOCATION OF EQUIPMENT:

SAMDIA NATIONAL LABORATORIES

PROPERTY REAPPLICATION DIVISION 7617

ALBUQUEROUE NM 87123
500001 EA COSTIUN!Y:
TOIAL COSI: $\$ 2,437.00$
FOR FURTHER INFORMATION:

ELOY N. GIRON

ALBUOUEROUE

ALBUDUEROUE
(505) $844-2746$

NM 87185

TO FREEZE EQUIPMENT:

ELOY N. GIRON

ALBUOUERQUE

(505) $844-2746$

NM 87185

LOCATION OF EQUIPMENT:

SANDIA NATIONAL LABORATORIES

PROPERTY REAPPLICATION DIVISION 7617

ALBUDUERQUE

NM 87123 
EFFECIIVE DATE: 07/01/94 EXPIRATION DATE: 07/31/94

$+S C$

CCDE EXCESS PROPERTY DESCRIPTION

REFERENCE NUMBER : 81

6130 GENERATOR, SIGNAL 606B IS A MATER OSCILLATOR, POWER AMPLIFIER HAS RANGES $50 K H Z$ TO 6 5MHZ IN 6 BANDS AND FREO RESPONSE W/OUTPUT ACCURACY. 12OVAC, $60 \mathrm{H}$ Z. HEW PACK, 606BR, 60800597.

REPORT NUMGER: $898355-4088$

LINE ITEM NUMBER: 0023

REFERENCE NUMBER : 82

6130 POWER SUPPLY DC CC OUTPUT O TO $+-16.383 \mathrm{~V}$, VOLTAGE 0 TO $+-65.532 \mathrm{~V}$. 120VAC, $60 \mathrm{H}$ Z. FLUUE, 4265A, 5055006.

$600001 \mathrm{EA}$

COST IUNIT:

TOTAL COST:

$\$ 5,768.00$
$\$ 5,768.00$

FOR FURTHER INFORMATION:

ELOY N. GIRON

ALBUQUERQUE

(505) $844-2746$

NM 87185

TO FREEZE EQUIPMENT:

ELOY N. GIRON

ALBUQUERQUE

(505) $844-2746$

NM 87185

LOCATION OF EQUIPMENT :

SANDIA NATIONAL LABORATORIES

PROPERTY REAPPLICATION DIVISION 7617

PROPERTY REAPP

NM 87123 
EFFECTIVE DATE: $07 / 01 / 94$ EXPIRATION DATE: $07 / 31 / 94$

FSC

\section{COOE EXCESS PROPERTY DESCRIPIION}

REFERENCE NUMBER : 83

6130 POWER SUPPLY, DC GP BIPOLAR OPERATIONAL POWER SUPPLY AND AMPLIFIER WITH AC OR OC VOLT METER AND - 15V TO +15V, - 20A TO +20A OUTPUT, 120VAC, 60

HZ. KEPCO, 8OP1520, SER. E112810.

REPORT NUMEER: $898355-4088$

LINE ITEM NUMBER: 0030

REFERENCE NUMBER : 84

6130 POWER SUPPLY, MFR. RAYTHEON, MODEL LPS108, ACO. YR. 73, PROP. NO. 2055761
NO OF

COND UNITS UNIT

ACQUISIIION PRICE

$\$ 1,314.00$

$\$ 1,314.00$

TOTAL COST:

ALBUOUERQUE
(505) $844-2746$

NM 87185

TO FREEZE EQUIPMENT:

ELOY N. GIRON

ALBUOUEROUE

(505) $844-2746$

LOCATION OF EQUIPMENT

SANDIA NATIONAL LABORATORIES

PROPERTY REAPPLICATION DIVISION 7617

ALBUOUERQUE

NM 87123
$\$ 7,143.00$ $\$ 7,143.00$
FOR FURTHER INFORMATION:

JOANNE BREZNIK

LIVERMORE

(510) $424-4101$

CA 94550

TO FREEZE EOUIPMENT:

JOANNE BREZNIK

LIVERMORE

CA 94550

REPORT NUMBER: $899102-4163$

LINE ITEM NUMBER: 0023
(510) $424-4101$

LOCATION OF EQUIPMENT:

LAURENCE LIVERMORE NATIONAL LAB

EXCESS PROPERTY SECTION, L-696

LIVERMORE

CA 94550 
EFFECTIVE DATE: $07 / 01 / 94$ EXPIRATION DATE: $07 / 31 / 94$

CODE EXCESS PROPERTY DESCRIPIIOH

REFERENCE NUMBER : 85 6130 POWER SUPPLY, MFR. RAYTHEON, MODEL LPSR108, ACQ. YR. 73, PROP. NO.

REPORT NUMBER: $899102-4163$

LINE ITEM NUMBER: 0024

REFERENCE NUMBER : 86
6130 POWER SUPPLY, MFR. LRL, MODEL LE745565, ACO. YR. 75, PROP. NO. 3823987, 6 00001 EA

REPORT NUMBER: $899102-4163$

LINE ITEM NUMBER: 0025

$600001 \mathrm{EA}$
No of

COND UNITS UNIT

ACOUISITION PRICE

COST/UNIT:

TOTAL COST:

$\$ 7.143 .00$

$\$ 7,143.00$

TRTHER INFORMATION:

JOANNE BREZNIK

LIVERMORE

(510) $424-4101$

TO FREEZE EQUIPMENT:

JOANNE BREZNIK

IVERMORE

(510) $424-4101$

CA 94550

LOCATION OF EQUIPMENT:

AAURENCE LIVERMORE NATIONAL LAB

EXCESS PROPERTY SECTION, L-696

LIVERMORE

CA 94550
COST/UNIT:

TOTAL COST $\$ 7,754.00$
$\$ 7,754.00$
FOR FURTHER INFORMATION:

JOANNE BREZNIK

LIVERMORE

(510) $424-4101$

CA 94550

TO FREEZE EQUIPMENT:

JOANNE BREZNIK

LIVERMORE

CA 94550

(510) $424-4101$

LOCATION OF EQUIPMENT:

LAURENCE LIVERMORE MATIONAL LAB

EXCESS PROPERTY SECTION, L-696

LIVERMORE

CA 94550 
EFFECIIVE DATE: $07 / 01 / 94$ EXPIRATION DATE: $07 / 31 / 94$

COOE EXCESS PROPERTY DESCRIPTION

UNITS UNIT

ACQUISITION PRICE

REFERENCE NUMBER : 87

6130 POWER, SUPPLY C GP RACK MOUNT AMPS 1.8 TO -1.8 VOLTS + 36 TO - 36, INPUTS

$\&$ OUTPUT JAC KS. ON/OFF CONTROLS. 120VAC, $60 \mathrm{HZ}$. KEPCO, ATE3615, SER.

110646.

$600001 \mathrm{EA}$ COST/UNIT:
TOTAL COST:

$\$ 1,054.00$ $\$ 1,054.00$

FOR FURTHER INFORMATION

ELOY N. GIRON

ALBUQUERQUE

(505) $844-2746$

NM 87185

TO FREEZE EQUIPMENT

ELOY N. GIRON

ALBUOUERQUE

NM 87185

REPORT NUMBER: $898355-4088$

LINE ITEM NUMBER: 0029

REFERENCE NUMBER : 88

6130 PULSE GENERATOR WITH CONTROLS FOR VERNIER, PULSE WIDTH, PULSE INTERVAL A 7 00001 EA 3 AMPLII UDE. 120VAC, 60 HZ. NANOFAST, NONE. SER. 2045.

COST/UNIT:

TOTAL COST:

$\$ 7,000.00$ $\$ 7,000.00$

FOR FURTHER IN
ELOY N. GIRON
ALBUQUEROUE
(505) $844-2746$

TO FREEZE EQUIPMENT

ELOY N. GIRON

ALBUQUERQUE

(505) $844-2746$

NM 87185

LOCATION OF EQUIPMENT:

SANDIA NATIONAL LABORATORIES

PROPERTY REAPPLICAIION DIVISION 7617

ALBUQUERQUE

NM 87123 
EFFECIIVE DATE: $07 / 01 / 94$ EXPIRATION DATE: $07 / 31 / 94$

FSC

\section{COOE EXCESS PROPERTY DESCRIPTION}

REFERENCE NUMBER : 89

6130 PULSE GENERATOR RACK MOUNT, CONTROLS FOR REPETITION RATE, DELAY, WIDTH, POSITIVE AMPLITUDE, RISE/FALL AND NEGATIVE AMPLITUDE. $120 \mathrm{VAC}, 60 \mathrm{~Hz}$. DAT APULSE, 110B, SER. 42279.

REPORT NUMBER: $898355-4088$

LINE ITEM NUMBER: 0028

REFERENCE NUMBER : 90

6130 SOURCE, LIGHT POWER SOURCE FOR LIGHT SET, HAS START $1 / 2$ SECOND HOLD SWITCH. 12 OVAC, $60 \mathrm{HZ}$. BAUSCH \& LOMB, 338620, SER. NONE.

LINE ITEM NUMBER: 0026
No of
COND UNITS UNIT

$700001 \mathrm{EA}$

\section{COST/UNIT: TOTAL COST:}

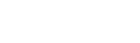

$\$ 1,244.00$
$\$ 1,244.00$

\section{ONTACT(S)}

FOR FURTHER INFORMATION:

ELOY N. GIRON

ALBUQUERQUE

(505) $844-2746$

NM 87185

TO FREEZE EQUIPMENT:

ELOY N. GIRON

ALBUQUERQUE

(505) $844-2746$

NM 87185

LOCATION OF EQUIPMENT:

SANDIA NATIONAL LABORATORIES PROPERTY REAPPLICATION DIVISION 7617 ALBUQUERQUE NM 87123
$00001 \mathrm{EA}$

COST/UNIT:
TOTAL COST:

\section{$\$ 1,030.00$ \\ $\$ 1,030.00$}

FOR FURTHER INFORMATION:

ELOY N. GIRON

ALBUQUERQUE

(505) $844-2746$

NM 87185

TO FREEZE EOUIPMENT:

ELOY N. GIRON

ALBUQUEROUE

NM 87185

(505) $844-2746$

LOCATION OF EQUIPMENT :

SANDIA NATIONAL LABORATORIES

PROPERTY REAPPLICATION DIVISION 7617

PROPERTY REAPPLICATION DIVISION 7617
ALBUOUERQUE 
EFFECIIVE DATE: $07 / 01 / 94$ EXPIRATION DATE: $07 / 31 / 94$

FSC

\section{COOE EXCESS PROPERIY DESCRIPIION}

REFERENCE NUMBER : 9

6240 ELECTRO-LUMINESCENT SACKLITE STRIP ( 40 EA), MFR. DENSITRON, MU DEL 624071160 , ACQ. YR. $93, E 38540$

REPORT NUMBER: $899102 \cdot 4160$

INE ITEM NUMBER: 0010

REFERENCE NUMGER : 92
6625 ALPHA FRESS MFR: ALPHA PRESS CO., MODEL NO. AFC 420-12, S/N 40192, ACO. 4 OCO01 EA DATE : 7/74, APPROX. WT: 2000 LBS., 60' $\mathrm{W} \times 66^{\prime} \mathrm{L} \times 116^{\prime} \mathrm{H}$

REPORT NUMBER: $899105-4081$

LINE ITEM NUMBER: 0007

NO OF
COND UNITS UNIT

00001 EA
COST/UNIT: TOTAL COST:

COST/UNIT:
TOTAL COST:
$\$ 36,660.00$ $\$ 36,660.00$
FOR FURTHER INFORMATION:

LYNN E. SHACKELFOOT

LIVERMORE

(510) 294-3067

CA $94550-0969$

TO FREEZE EQUIPMENT:

LYNN E. SHACKELFOOT

LIVERMORE

(510) $294-3067$

CA $94550-0969$

LOCATION OF EQUIPMENT:

SANDIA NATIONAL LABORATORIES

PROPERTY REAPPLICAIION DEPT 8532-1

LIVERMORE CA $94550-0969$ 
EFFECIIVE DATE: $07 / 01 / 96$ EXPIRATION DATE: $07 / 31 / 94$

FSC

\section{COOE EXCESS PROPERTY DESCRIFIION}

REFERENCE NUMBER : 93

6625 AMPLIFIER

MFR: HEWLETI PACKARD MODEL: $746 \mathrm{~A}$

S/N: 109

REPORT NUMBER: $899305-4032$

LINE ITEM NUMBER: 0002

$$
\text { NO OF }
$$

COND UNITS UNIT

ACQUISITION PRICE

$500001 \mathrm{EA}$

COST/UNIT: TOTAL COST:

\section{$\$ 2,000.00$} $\$ 2,000.00$

400001 EA

COST/UNIT:

TOTAL COST:

$\$ 1,444.11$
$\$ 1,444.11$

S/N: 2148

REPORT NUMBER: $899305-4039$
LINE ITEM NUMBER: 0001
CONTACT(S)

FOR FURTHER INFORMATION:

CYNDI DELONG

LAS VEGAS

(702) 295-6753

wV 89193-8521

TO FREEZE EOUIPMENT:

CYMDI DELONG

LAS VEGAS

(702) 295-6753

NV $89193-8521$

LOCATION OF EQUIPMENT:

REYNOLDS ELECTRICAL \& ENGINEERIMG CO

MEVADA TEST SITE

MERCURY

NV 89023

FOR FURTHER INFORMATION:

CYNDI DELONG

LAS VEGAS

(702) $295-6753$

NV $89193-8521$

TO FREEZE EQUIPMENT :

CYMDI DELONG

LAS VEGAS

(702) 295-6753

NV $89193-8521$

LOCATION OF EQUIPMENT:

REYNOLDS ELECTRICAL \& ENGIMEERING CO

NEVADA TEST SITE

MERCURY

NV 89023 
EFFECIIVE DATE: 07/01/94 EXPIRATION DATE: 07/31/94

ISC

\section{CODE EXCESS PROPERTY DESCRIPTION}

REFERENCE NUMEER : 95

6025 AMPLIFIER LINEAR MFR: TENNELEC MODEL: IC205A $S / N: \quad 339$

REPORT NUMEER : $899305 \cdot 0030$

REFERENCE NUMEER : 96

O625 AMPLIF!ER LINEAR MFR: TENNELEC MGDEL: TC205A $S / N: 215$

ELIGIBLE EQUIPMENT

\section{No of}

COND UNIIS UNII

$400001 E A$ TOIAL COST:
LINE ITEM NUMBER: 0002 COSTIUNIT:
TOTAL COST:

$400001 E A$

\section{ACQUISITION PRICE}

$\$ 1,444.10$
$\$ 1,444.10$

LINE ITEM NUMBER: 0003
CONTACISS)

FOR FURTHER INFORMATION

CYMDI DELONG

LAS VEGAS

(702) $295-6753$

NV $89193-8521$

TO FREEZE EQUIPMENT:

CYNDI DELONG

LAS VEGAS

(702) $295-6753$

NV $89193-8521$

LOCATION OF EQUIPMENT:

REYNOLDS ELECTRICAL \& ENGINEERING CO MEVADA TEST SITE

MERCURY
NV 89023

$\$ 1,444.10$
$\$ 1,444.10$

CYND! DELONG
NV $89193-8521$

(702) $295-6753$

10 FREEZE EQUIPMENT:

CYNDI DELONG

LAS VEGAS

(702) $295-6753$

NV $89193-8521$

LOCATION OF EQUIPMENT:

REYNOLDS ELECTRICAL \& ENGINEERING CO REVADA IES

NEVADA IEST SITE

NV 89023 
EFFECTIVE DATE: 07/01/94 EXPIRATION DATE: 07/31/94

SSC

COOE EXCESS PROPERTY DESCRIPTION

REFERENCE NUMBER : 97

6625 AMPLIFIER LINEAR MFR: TENNELEC MODEL: TC205A S/N: 74

REPORT NUMBER: $890305-4039$

REFERENCE NUMBER : 98

6625 AMPLIFIER LINEAR MFR: TENNELEC MODEL: TC205A S/N: 2146
No of

COND UNIIS UNI

\section{ACOUISITION PRICE}

$400001 E A$

COST/UNIT:
TOTAL COST:

LINE ITEM NUMBER: 0004

$4 \quad 00001$ EA

COST/UNIT:
TOTAL COST:
LIME ITEM NUMBER: 0005
REPORT NUMBER: $899305-4039$
CONTACT(S)

FOR FURTHER IMFORMATION:

CYMDI DELONG

LAS VEGAS

(702) $295-6753$

NV $89193-8521$

TO FREEZE EQUIPMENT

CYNDI DELONG

LAS VEGAS

(702) $295-6753$

NV $89193-852$

OCATION OF EOUIPMENT:

REYMOLDS ELECTRICAL \& ENGINEERING CO NEVADA IEST SITE

MEVADA TEST

Nv 89023

NV 89193-8521

CYNDI DELONG

LAS VEGAS

TO FREEZE EQUIPMENT:

CYMDI DELONG

LAS VEGAS

WV $89193-8521$

(702) $295-6753$

LOCATION OF EQUIPMENT

REYMOLDS ELECTRICAL \& EMGINEERING CO

MEVADA IEST SITE

NV 89023 
EFFECTIVE DATE: $07 / 01 / 94$ EXPIRATION DATE: $07 / 31 / 94$

FSC

\section{CQOE EXCESS PROPERTY DESCRIPTION}

REFERENCE MUMBER : 99

6625 AMPLIFIER LIMEAR MFR: IENNELEC MODEL: TC205A S/N: 756

REPORT NUMEER: $899305-4039$
NO OF

COMD UNITS UNIT

ACOUISITION PRICE

$400001 \mathrm{EA}$

$\operatorname{COSI} / \mathrm{N} I \mathrm{~T}:$

IOTAL COST:

$\$ 1.192 .07$

$\$ 1,192.07$

\section{CYNDI DELONG \\ LAS VEGAS}

OR FURTHER

(702) $295-6753$

wV 89193-8521

TO FREEZE EOUIPMENT:

CYMDI DELONG

LAS VEGAS

(702) $295-6753$

wV 89193-8521

LINE IIEM NUMBER: 0006

LOCATION OF EOUIPMENT:

REYMOLOS ELECTRICAL \& EMGINEERING CO REYWOLDS ELECTRICA

MEVADA TEST

in 89023
COST/UNIT:

TOTAL COST:
AMPLIFIER, DATA ONNAMICS AMPLIFIER, DATA MACHINE FILTER HZ RANGE FROM To 100K, VARIABLE ARE: GAL, GAIN, ZERO RTO. GAIN
iROM 1 io 10:0. I2OVAC, 6O HZ. DYNAM ELEC, 752
$700001 \mathrm{EA}$
LINE ITEM NUMBER: D002
REPORT NUMBER: $898355-4102$ si. 388.00
ALOU N. GIROM

(505) 846.2746

(4) 87185

TO FREEZE EOUIPMEMT:

ELOY N. GIROW

(505) $844-2746$

87185

LOCATION OF EOUIPMEMT:

SAMD I A MATIOMAL LABORATORIES PROPERTY REAPPLICAIION DIV. 7617 PROPERTY REAPPLICATION DIV. 87123 
EFFECIIVE DATE: 07/01/96 EXPIRATION DATE: 07/31/96

FSC

\section{COOE EXCESS PROPERTY DESCRIPTION}

REFERENCE NUMBER : 101

6625 AMPLIFIER, LINEAR WITH AN INPUT AND OUTPUT CONNECTION, HAS A IS DCV COMNECTION. BE NDIX ENV, LWAS104, SER. 003.

REPORT NUMBER : $898355-4102$

LINE ITEM MUMBER: 0001

\section{REFERENCE NUMBER : 102}

6625 AMPLIFIER, POWER 9.5 WATTS, 45DB, 1.7-50OMHZ, WITH AN INPUT AND OUTPUT CONMECTION. 120VAC, $60 \mathrm{HZ}$. ELECT MAVI, $510 \mathrm{~L}, 132$.

600001 EA

Cost/Unit: TOTAL COST:

\section{$\$ 2,775.00$ $\$ 2,775.00$}

FOR FURTHER IMFORMATIOA :

ELOY N. GIRO

ALBUOUEROUE

(505) $846-2746$

m 87185

TO FREEZE EOUIPUEWT:

ELOY N. GIRO

ALBUCUEROUE

(505) $844-2746$

87185

LOCATIOU OF EOUIPAENI:

SAMDIA MATIOMAL LABORATORIES

PROPERTY REAPPLICATION DIV. 7617

in 87123 
EFECIIVF DATE: CF/01/\% EXFIRAYION DATE: O7/31/96

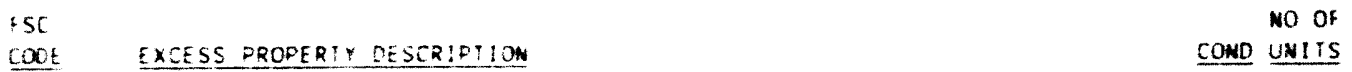

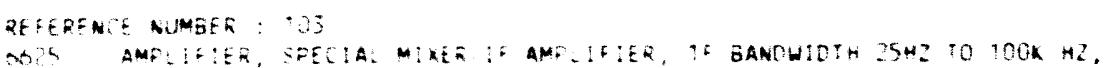

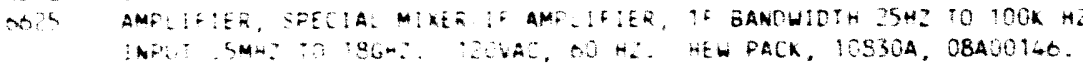

FEPOR: NUMGER: $998355 \cdots 080$

LIME ITEM NUMBER: 0004

REFERENCE NUMBER : 104

SO25 AMPLIFIER-RE POWER. MFR. ELEL NAVIGA, MOOEL 55OL, ACQ.YR. 83, PROP. NO. 5 O0001 EA $3: 026 \div 7$
COND UNITS UNIT

ACQUISIIION PRICE

- 0000 EA COSTRUNI:
TOIAL COST:

$\$ 3.705 .00$ $\$ 3.705 .00$ (2)$$
\text { AL }
$$

FOR FURTHER INFORMAIION:

ELOY $N$. GIFON

S05) $86+2740$

87185

IO FREEZE EQUIPMENT:

ELOY N. GIRON

ALBUOUEROUE

(505) $846-2746$

min 87185

LOCATION OF EQUIPMEMT:

SAMOIA MATIOMAL LABORATORIES PROPERTY REAPPLICATION DIVISION 7617

ALBUOLEROUE

4m 87123

COST/UNIT: TOTAL COST:
$\$ 5,564.08$ 55.506 .08

FOR FURTHER INFORMATION: JOAMME BRE ZNIK

IIVERMORE

CA 94550

(510) $424-6101$

TO FREEZE EOUIPMENT:

JOMUNE BREZWIK

LIVERMORE

CA 94550

REPOR: NUMEER: 8091026103

LINE ITEM NUMBER: 0029

LOCATION OF EOUIPMENT:

LAMREMCE LIVERMORE MATIOULAL LAB EXCESS PROPERTY SECTION, L-696 LIVERMORE 
EFFECTIVE DATE: 07/01/96 EXPIRATION DATE: 07/31/94

ELIGIBLE EQUIPMENT

\section{FSC \\ COOE EXCESS PROPERTY OESCRIPTION \\ REFERENCE MUMBER : 105 \\ Q625 ANALYZER \\ ANALYZER
MFR: JOBO COLOR WOOEL: 2000 \\ MFR: $\quad$ JOBO COL 006758}

REPORT MUMBER: $899305-4032$
No of

COND UNIIS UNII

\section{ACQUISITION PRICE}

$5 \quad 00001 \mathrm{EA}$

COST/UNIT:

TOTAL COST:

LINE ITEM MUMBER: 0006
$31,606.00$
$\$ 1,606.00$

\section{CONTACT(S)}

FOR FURTHER IMFORMATION:

CYMDI DELOWG

LAS VEGAS

(702) $295-6753$

Nv $89193-8521$

TO FREEZE EOUIPMEMT:

CYMDI DELLNG

LAS VEGAS

(702) $295-6753$

wv 89193-8521

OCATION OF EOUIPMENT:

REYMOLDS ELECTRICAL \& EMGIMEERIMG CO

MEVAOA TEST SITE

MERCURY

nv 89023

REFERENCE NUMBER : 106

6625 ANALYZER SIGNATURE MFR: HEWLETT PACKARO MODEL: $5004 \mathrm{~A}$ S/N: $\quad$ S24AO1711

$500001 \mathrm{EA}$

COST/UNIT:

TOTAL COST

$\$ 1,007.75$

$\$ 1.007 .75$

\section{FOR FURTHER INFORMAIION}

CYMDI DELOMG

LAS VEGAS
(702) $295-6753$

wv 89193-8521

TO FREEZE EOUIPMENT:

CYMDI DELONG

LAS VEGAS

(702) 295-6753

MV $89193-8521$

LOCATIOW OF EQUIPMENT:

REYMOLOS ELECTRICAL \& ENGIMEERIMG CO

MEVADA TEST SITE

MERCURY

NV 89023 
EFHECIIVE DATE: 07/01/94 EXPIRATION DATE: $07 / 31 / 94$

FSC

COOE EXCESS PROPERTY DESCRIPTION

REFERENCE NUMGER : 107

6625 ANALYZER SPECTRUM MFR: TEKTRONIX MODEL: LN03 S/N: B150736

REPORT NUMGER: $899305-4039$
No of COND UNITS UNIT ACOUISITION PRICE

600001 EA COST/UNIT: TOTAL COST:

LINE ITEM MUMBER: 0008
REFERENCE NUMBER : 108

6625 ANALYZER IMAGE MFR: INTERPRETATION SYSIEM INCORPORATED, MOOEL NO. VP8, S/N 092 . ACO. DATE: 9/75. APPROX. WT: $25 \mathrm{LBS}$. . $19 . \mathrm{W} \times 15^{1} \mathrm{~L} \times 5 \mathrm{H}$

600001 EA
$\operatorname{COST/UNIT:}$

TOTAL COST:
$\$ 5,185.00$

$\$ 5,185.00$
$\$ 4.433 .24$ $\$ 4.433 .24$
FOR FURTHER INFORMATION:

LYME E. SHACKELFOOT

LIVERMORE

(510) $294-3067$

CA $94550-0969$

IO FREEZE EQUIPMENT :

LYMM E. SHACKELFOOT

LIVERMORE

(510) 294-3067

CA $94550-0969$

LOCATION OF EOUIPMENi:

LOCATION OF EQUIPMENI: SAND IA MATIOMAL LABORATORIES PROPERTY REAPPLICATION DEPT. $8532-1$
LIVERMORE
CA $94550-0969$ 
EFFECIIVE DAIE: $07 / 01 / 94$ EXPIRATION DATE: $07 / 31 / 94$

$\operatorname{tsc}$

\section{CODE EXCESS PROPERTY DESCRIPTION}

DEFERENCE NUMBER -109

6625 anALYZER, LOGIC GENERAL PURPOSE LOGIC ANALYZER, THE 1230 PROVIDES 16, 25 MHZ STAT E CHANNELS OR 8, 50 MHZ OR 4, 100 MHZ TIMING CHANNEL, 120 VAC, O O HZ. TEKTRONIX, 1230, SER. B030139.

REPORT NUMBER: $898355-4089$

LINE ITEM NUMBER: 0001

REFERENCE NUMBER : 110

6625 ANALYZER, PLUG-IN FREO RANGE: 20HZ TO 620KHZ, AMPLITUDE RANGES: 3MV TO 700001 EA 3OV FULL S CALE IN 15 RANGES, RANGE HAVE LOG AND LINEAR X AND Y RECORDER

OUT PUTS. 120VAC, $60 \mathrm{HZ}$. HEW PACK, 3594A, A00610.

REPORT NUMBER: $898355-4102$

LINE ITEM NUMBER: 0005

NO OF

COND UNITS UNIT
ACQUISITION PRICE

$\$ 7,068.00$ $\$ 7,068.00$

COST/UNIT:

ALBUDUEROUE

(505) $844-2746$

NM 87185

TO FREEZE EOUI PMENT

ELOY N. GIRON

ALBUOUERQUE

(505) $844-2746$

LOCATION OF EOUIPMENT:

SANDIA NATIONAL LABORATORIES

PROPERTY REAPPLICATION DIVISION 7617

ALBUOUERQUE
COST/UNIT:

TOTAL COST:

600.00
FOR FURTHER INFORMATION:

ELOY N. GIRON

ALBUQUERQUE

(505) $844-2746$

NM 87185

TO FREEZE EQUIPMENT:

ELOY N. GIRON

ALBUQUEROUE

87185

(505) 844-2746

LOCATION OF EQUIPMENT

SANDIA NATIONAL LABORATORIES

PROPERTY REAPPLICATION DIV 7617

ALBUQUERQUE

NM 87123 
EFFECTIVE DATE: $07 / 01 / 94$ EXPIRATION DATE: $07 / 31 / 94$

\section{FSC \\ COOE EXCESS PROPERTY DESCRIPTION}

REFERENCE NUMBER : 111

6625 ANALYZER, TRACE WITH SHO-RATE TUGE SIZE IR-2-15-AA UP TO CC/MM AIR AT 70

DEG F AN D $14,7 \mathrm{PSI}$ A I2OVAC $60 \mathrm{HZ}$, BECKMAN, 340, SER 100820.

REPGRT NUMEER: $898355-4089$

LINE ITEM NUMBER: 0003

REFERENCE NUMBER : 112

O625 ANALYZER, WAVE THE MODEL 310 A HAVE FREO ANALYZER SIGNAL IS BETWEEN 1 KHZ

AND 1.5 MHZ FREO SCALE IDIV PER 200HZ, BANDWIDTH RANGE 200HZ, $1000 \mathrm{HZ}$

\& $3000 \mathrm{HZ}$. IZOVAC, $60 \mathrm{HZ}$. HEW PACK $310 \mathrm{~A}$.

LINE ITEM NUMBER: 0002
NO OF

COND UNITS UNIT

ACQUISITION PRICE

COST/UNIT: TOTAL COST:

$\$ 1,184.00$

$\$ 1.184 .00$

\section{ELOY N. GIRON \\ ALBUOUERQUE}

FOR FURTHER INFORMATION:

(505) $844-2746$

NM 87185

TO FREEZE EQUIPMENT:

ELOY N. GIRON

ALBUQUERQUE

(505) 844-2746

NM 87185

LOCATION OF EQUIPMENT:

SANDIA NATIONAL LABORATORIES

PROPERTY REAPPLICATION DIVISION 7617

ALBUQUEROUE

\section{$600001 \mathrm{EA}$
COST/UNIT:
TOTAL COST:

$\$ 2.778 .00$
$\$ 2.778 .00$
FOR FURTHER INFORMATION:

ELOY N. GIRON

ALBUQUERQUE

(505) $844-2746$

NM 87185

TO FREEZE EQUIPMENT:

ELOY N. GIRON

ALBUOEROUE

NM 87185

(505) $844-2746$

LOCATION OF EOUIPMENT:

SANDIA NATIONAL LABORATORIES

PROPERTY REAPPLICAIION DIVISION 7617

ALBUOUERQUE 
EFFECTIVE DATE: $07 / 01 / 94$ EXPIRATION DATE: $07 / 31 / 94$

FSC

\section{COOE EXCESS PROPERTY DESCRIPYION}

REFERENCE NUMBER : 113

6625 ANALYZER, HAVE FREO RANGE: 20HZ TO 620KHZ, AMPLITUDE RANGES: 3MV TO 30V FULL S CALE IN 15 RANGES. 120VAC, 60 HZ. HEW PACK, 3590A, SER. A00585.
No of

COND UNITS UNIT

700001 EA

$\operatorname{COST/UNIT:~}$

TOTAL COST:

LINE ITEM NUMBER: 0004

REFERENCE NUMBER : 114

6625 ANALYZER-PULSE HEIGHT, MFR. NUCLEAR DATA, MODEL ND100, ACQ. YR. 75, 600001 EA COST/UNIT: ANALY NO. 4191436

COST/UNIT:
TOTAL COST:
ACQUISITION PRICE

$\$ 3,200.00$

$\$ 3,200.00$

$\$ 7.275 .00$

FOR FURTHER INFORMATION:

JOANME BREZNIK

LIVERMORE

(510) $424-4101$

CA 94550

TO FREEZE EQUIPMENT :

JOANNE BREZNIK

LIVERMORE

CA 94550

REPORT NUMBER : $899102-4163$

LINE ITEM NUMBER: 0026

(510) 424-4101

LOCATION OF EQUIPMENT:

LAURENCE LIVERMORE NATIONAL LAB

EXCESS PROPERTY SECTION, L-696

LIVERMORE

CA 94550 
EFFECTIVE DATE: $07 / 01 / 94$ EXPIRATION DATE: 07/31/94

FSC

\section{COOE EXCESS PROPERTY DESCRIPTION}

REFERENCE NUMBER : 115

6625 AUTO MOTION SYSTEM MFR: HEWLETT PACKARD, MODEL NO. 98580A, S/N 1, ACQ. AUTO MOTION SYSTEM MFR: HEWLETT PACKARD, MODEL NO.
DATE: $5 / 86$, APPROX. WT: 30 LBS., $13 . \mathrm{W} \times 15^{\prime} \mathrm{L} \times 5 \mathrm{I}^{\circ} \mathrm{H}$

REPORT NUMBER: $899105-4081$

LINE ITEM NUMBER: 0009

REFERENCE NUMBER : 116

6625 BATH CIRCULATOR TANK AREA IS 12"WX12"LX13"D. TANK CAN COOL OR HEAT. 120VAC, $60 \mathrm{H} \mathrm{Z}$. FORMA SCI, 2095, SER. 20988210

REPORT NUMBER: $898355-4088$

LINE ITEM NUMBER: 0034
NO OF

T.OND UNITS UNIT

ACQUISITION PRICE

$500001 \mathrm{EA}$

COST/UNIT:

TOTAL COST

$\$ 3,900.00$

$\$ 3,900.00$

LYNN E. SHACKELFOOT

LIVERMORE

(510) 294-3067

CA $94550-0969$

TO FREEZE EQUIPMENT

LYNM

LIVERMORE

(510) 294-3067

LOCATION OF EQUIPMENT

SAMOIA MATIONAL LABORATORIES

SANDIA NATIONAL LABORATORIES

PROPERTY REAPPLICATION DEPT. $8532-1$
LIVERMORE
CA $94550-0969$
600001 EA COST/UNIT:

TOTAL COST:
$\$ 1,200.00$

$\$ 1,200.00$

FOR FURTHER INFORMATION:

ELOY N. GIRON

ALBUQUERQUE

(505) $844-2746$

NM 87185

TO FREEZE EQUIPMENT :

ELOY N. GIRON

(505) $844-2746$

NM 87185

LOCATION OF EQUIPMENT:

SAMDIA MATIONAL LABOPATORIES

SANDIA NATIONAL LABORATORIES

ALBUQUEROUE NM 87123 
EFFECTIVE DATE: $07 / 01 / 94$ EXPIRATION DATE: $07 / 31 / 94$

FSC

\section{CODE EXCESS PROPERTY DESCRIPIION}

REFERENCE NUMBER : 117

6625 BOX RESISTANCE SPECIAL IS .001 OHMS TO 11 OHMS AND 10 AMPS MAX. NEEDS

CALIBARATION. LE EDS\& NORT. SPECIAL 1772113.

REPORT NUMBER: $898355-4089$

LINE ITEM NUMBER: 0006

REFERENCE NUMBER : 118

6625 BRIDGE, CAPACITANCE METER RANGE FROM 0 TO 100, VOLTS MAX 0 TO 0.5 ,

DISSIPATION FACTOR RANGE FROM 0 TO 100 , C RANGE. I UF TO 1000MF.

$120 \mathrm{VAC}, 60 \mathrm{HZ}$. EL ECLTL SCIE, 273, SER. 226002.

$600001 \mathrm{EA}$

COST/UNIT:

TOTAL COST:

COST/UNIT:

TOTAL COST:
No of

COND UNITS UNIT ACQUISITION PRICE

$\$ 2,200.00$

$2,200.00$

INFORMATION :

ELOY N. GIRON

ALBUQUERQUE

(505) $844-2746$

NM 87185

TO FREEZE EQUIPMENT

ELOY N. GIRON

ELOY N. GIRON

(505) $844-2746$

LOCATION OF EQUIPMENT

SANDIA NATIONAL LABORATORIES

PROPERTY REAPPLICATION DIVISION 7617

PLBUQUERQUE NM 87123

REPORT NUMBER: $898355-4089$

LINE ITEM NUMBER: 0007
$1,056.00$

1.056 .00

FOR FURTHER INFORMATION:

ELOY N. GIRON
ALBUQUERQUE

(505) $844-2746$

NM 87185

TO FREEZE EQUI PMENT:

ELOY N. GIRON

ALBUQUERQUE

NM 87185

LOCATION OF EQUIPMENT:

SANDIA NATIONAL LABORATORIES

SANDIA NATIONAL LABORATORIES

PROPERTY REAPPLICATION DIVISION 7617
ALBUQUEROUE
NM 87123 
EFFECTIVE DATE: $07 / 01 / 94$ EXPIRATION DATE: $07 / 31 / 94$

FSC

COOE EXCESS PROPERTY DESCRIPIION

REFERENCE MUMBER : 119

6625 BRIDGE, IMPEDANCE RANGE 1000PF TO 1000MF. 120VAC, $60 \mathrm{HZ}$. HEW PACK, 4260A. SER. $J 08881$

REPORT NUMBER: $898355-4089$

LINE ITEM NUMBER: 0008

REFERENCE NUMBER : 120
6625 BRIDGE, INDUCTANCE WITH A FREO RANGE OF 5KHZ TO 500KHZ, 120VAC, $60 \mathrm{HZ}$. 6 00001 EA BOONTON EL, 63H, SER. 800 .

REPORT NUMBER: $898355-4089$

LINE ITEM NUMBER: 0009

COND UNITS UNIT

$600001 \mathrm{EA}$

No of

ACQUISITION PRICE

COST/UNIT:

TOTAL COST:

$\$ 1,048.00$

$\$ 1,048.00$

\section{ELOY N. GIRON \\ ALBUQUERQUE}

(505) $844-2746$

MM 87185

TO FREEZE EQUIPMENT :

ELOY N. GIRON

ALBUOUERQUE

(505) $844-2746$

NM 87185

LOCATION OF EQUIPMENT:

SANDIA NATIONAL LABORATORIES SANDIA NATIONAL LABORATORIES

PROPERTY REAPPLICATION DIVISION
ALBUQUEROUE

COST/UNIT:

TOTAL COST: $\$ 2,950.00$
$\$ 2,950.00$
FOR FURTHER INFORMATION:

ELOY N. GIRON

ALBUQUERQUE

(505) $844-2746$

NM 87185

TO FREEZE EQUIPMENT:

ELOY N. GIRON

(505) $844-2746$

NM 87185

LOCATION OF EQUIPMENT

SAMDIA MATIONAL LABORATORIES PROPERTY REAPPLICATION DIVISION 7617 PROPERTY REAPPLICATION DIVISION 87123 
EFFECTIVE DATE: $07 / 01 / 94$ EXPIRATION DATE: 07/31/94

FSC

\section{CODE EXCESS PROPERTY DESCRIPTION}

REFERENCE NUMBER : 121

6625 BURN IN SYSTEM UNIT FOR IESTING CHIPS, HILL TEST 40 TRAYS WITH SET OF CHIPS. 208 VAC, $60 \mathrm{HZ}$. KINETICON, MOD. NONE.

REPORT NUMBER: $898355-4089$

LINE ITEM NUMBER: 0010

REFERENCE NUMBER : 122

6625 BUS, INTERFACE PROVIDES A WAY TO EXTEND THE SEPARATION OF COMPONEN PARTS IN A H P-1B SYSTEM. 120VAC, $60 \mathrm{HZ}$. HEW PACK, 59403A, SER. 26400441
NO OF

COND UNITS UNIT

ACOUISITION PRICE

$\$ 167,000.00$

COST/UNIT: TOTAL CUST:

$\$ 1.539 .00$

COST/UNIT:

TOTAL COST

FOR FURTHER INFORMATION

ELOY N. GIRON

ALBUOUEROUE

(505) $844-2746$

NM 87185

TO FREEZE EOUIIPMENT:

ELOY $N$. GIRON

ALBUOUERQUE

(505) $844-2746$

A4 87185

LOCATION OF EQUIPMENT

SAMDIA NATIONAL LABORATORIES

PROPERTY REAPPLICATION DIV. 7617

HM 87123 
EFFECTIVE DATE: $07 / 01 / 94$ EXPIRATION DATE: $07 / 31 / 94$

\&SC

\section{COOE EXCESS PROPERTY DESCRIPTION}

REFERENCE NUMBER : 123

G625 BUS, INTERFACE +RTVIDES A WAY TO EXTEND THE SEPARATION OF COMPONEN PARTS IN A H I-1E SYSTEM, BY MORE THAN THE 20 METRE MAX TRANSMISSION PATH LENG TH SPECIFIED IN VARIOUS INTERFACE STDS. HEW PACK.
REFORT NUMEER: $808355-4102$

REFERENCE NUMBER : 12

6625 CALIBRATOR

MFR: VALHALLA MODEL: $2 \angle 00$ ACDC

S/N: 10152
ELIGIBLE EQUIPMENT
NO OF

CONG UNITS UNIT

6. 00001 EA
ACQUISITION PRICE

COST/UNIT

TOTAL COST:

LINE ITEM NUMBER: 0007 COST/UNIT:
TCTAL COST:
LINE ITEM NUMBER: 0005
$500001 \mathrm{EA}$
REPORT NUMBER: $899305 \cdot 4032$
$\$ 1,500.00$
$\$ 1,500.00$
CONTACT(S)
FOR FURTHER INFORMATION
ELOY N. GIRON
ALBUOUEROUE
(505) $844-2746$
NM 87185

TO FREEZE EQUI PMENT:

ELOY N. GIRON

ALBUOUERQUE

(505) $844-2746$

NM 87185

LCATION OF EQUIPMENT :

SAMDIA NATIONAL LABORATORIES

PROPERTY REAPPIICATION DIV. 7617

PROPERTY REAPPLICATION DIV

M14 87123

FOR FURIHER INFORMAIION:
CYMDI DELONG
$\begin{aligned} & \text { LAS VEGAS } \\ & \text { (702) } 295-6753\end{aligned}$

TO FREEZE EQUIPMENT

CYMDI DELONG

LAS VEGAS

(702) $295-6753$

NV 89193-8521

LOCATION OF EOUIPMENT:

REYNOLDS ELECTRICAL \& ENGINEERING CO REVADA

MEVADA TEST SITE

MERCURY

NV 89023 


\section{EFFECTIVE DATE: 07/01/94 EXPIRATION DATE: $07 / 31 / 94$}

FSC

COOE EXCESS PROPERTY DESCRIPIION

REFERENCE NUMBER : 125

6625 CALIBRATOR, DC VOLTS NULL DETECTOR VOLTAGE RANGE 10, 100 AND 1000 VOLTS OUTPUT C URRENT O TO 50 MA. 120VAC, $60 \mathrm{HZ}$. FLLUE, 3350, SER. 912.

REPORT NUMBER: $898355-4088$

LINE ITEM NUMBER: 0035

REFERENCE NUMBER : 126

6625 CALIBRATOR, DC VOLIAGE CALIBRATOR, RANGE O TO 1000 VOLTS DC. $120 \mathrm{VAC}, 60800001$ EA COST/UNIT: HZ. FLU KE, 341A, SER. 93408
No of

COND UNITS UNIT

ACQUISITION PRICE

COST/UNIT:

TOTAL COST:

$\$ 3.621 .00$

$\$ 3,621.00$

$$
\begin{aligned}
& \text { ELOY N. GIROM } \\
& \text { ALBUDUEROUE }
\end{aligned}
$$

IMFORMATION:

(505) $844-2746$

TO FREEZE EOUIPMENT

ELOY N. GIRON

AL BUDUEROUE

(505) $844-2746$

87185

LOCATION OF EQUIPMENT

SAMDIA MATIOMAL LABORATORIES

PROPERTY REAPPLICATION DIVISION 7617

ALBUDUERQUE

NM 87123
$\$ 1,271.00$

$\$ 1.271 .00$

FOR FURTHER INFORMATION:

ELOY N. GIRON

ALBUOUEROUE

(505) $844-2746$

NM 87185

TO FREEZE EQUIPMENT:

ELOY N. GIRON

ELOY N. GIROA

(505) $844-2746$

LOCATION OF EQUIPMENT:

SANDIA MATIOMAL LABORATORIES

PROPERTY REAPPLICATION DIV 7617

ALBUOUERQUE

NM 87123 
EFFECTIVE DATE: $07 / 01 / 94$ EXPIRATION DATE: $07 / 31 / 94$

\section{COOE EXCESS PROPERTY DESCRIPIION}

REFERENCE NUMBER : 127

6625 CALIBRATOR, FM IS A DIGIIAL SYSTEM CALIBRATOR 2000, FREO RANGE 10MHZ TO

$100 \mathrm{HZ}$. METER RANGE $100 \mathrm{MV}$, 10OMV, $1 \mathrm{V \%}, 10 \mathrm{~V} \%$. HAS ON/OFF CONTROLS. $60 \mathrm{HZ}$

. CEC, TSC2000, SER. 20019.

REPORT NUMGER: $898355-4089$

INE ITEM NUMBER: 0011

REFERENCE NUMBER : 128

6625 CALIBRATOR, PORTABLE THERMOCOUPLE GREATER 1OMEGOHMS, VOLTAGE - 1000

MEGOHMS. CURRENT: 5 OHMS, 120VAC, 60 HZ. RUSTCO INC, 466AIL, SER. 55188.
NO OF

COND UNITS UNIT

ACOUISITION PRICE

700001 EA

COST/UNIT:

TOTAL COST:

$\$ 5,820.00$

$\$ 5,820.00$

\section{ELOY N. GIRON \\ ALBUOUEROUE}

(505) $844-2746$

NM 87185

TO FREEZE EOUIPMENT:

ELOY N. GIRON

ALBUDUEROUE

(SUS) $844-2746$

LOCATION OF EQUIPMENT:

SANDIA MATIONAL LABORATORIES

PROPERIY REAPPLICATION DIVISION 7617
ALBUQUEROUE
$\mathbf{8}$

REPORT NUMBER: $898355-4089$

LINE ITEM NUMBER: 0012
600001 EA COSTIUNIT:

TOTAL COST:
$\$ 1,642.00$

$\$ 1,642.00$
FOR FURTHER INFORMATIOW :

ELOY N. GIRON

ALBUOUEROUE

(505) $844-2746$

MM 87185

TO FREEZE EQUIPMENT:

ELOY N. GIRO

ALBUOUEROUE

(505) $844-2746$

147185

LOCATION OF EQUIPMENT:

SAMDIA MATIONAL LABORATORIES

PROPERTY REAPPLICATION DIVISIOA 7617

AL BUQUEROUE 
EFFECIIVE DATE: $07 / 01 / 94$ EXPIRATION DATE: $07 / 31 / 94$

CODE EXCESS PROPERTY DESCRIPIION

REFERENCE NUMBER : 129

6625 CAPACITANCE BRIDGE 55067, MANUF: BOONTON MODEL 74C S/N 453

CAPACI IANCE BRIDGE 55067, MANUF: BOONTON MODEL $74 \mathrm{C}$ S/N 453
1 EACH, RANGE: 0002 TO 11000 UUF. SIZE: $15^{\prime \prime W} \times 9^{\prime \prime F} / R \times 11^{\prime \prime H}$ EST WT: 30

REPORT NUMBER: $896604-4126$

LINE ITEM MUMBER: 0002

REFERENCE MUMBER : 130

6625 CENTRIFUGE MFR: GOERZ OPTICAL INC., MODEL NO. 560E, S/H 1, ACO. DATE: 1216 9. APPROX. WT: $300 \mathrm{LBS} . .48 \mathrm{~W} \times 32 . \mathrm{L} \times 60^{\circ} \mathrm{H}$.

$400001 \mathrm{EA}$

Cost/unit:

TOTAL COST:

59.696 .00

39.696 .00

FOR FURTHER INFORMAIION:

LYWN E. SHACKELFOOT

(510) $294-3067$

CA $94550-0989$

TO FREEZE EQUIPHENT

LYWW E. SHACKELFOOT

REPORT NUMBER: $899105-408$

LINE ITEM NUMBER: 0010

LIVERMORE

(510) $294-3067$

CA $94550-0969$

LOCATION OF EOUIPMENT:

SAMOIA MATIOMAL LABORATORIES

PROPERTY REAPPLICATIOA DEPT, 8532-1

PROPERTY REAPPLICAIION DEPT. 8532-1
LIVERMCRE 
EFFECIIVE DATE: OI/01/94 EXPIRATION DATE: 07/31/94

\section{COOE EXCESS PROPERTY OESCRIPTION}

REFERENCE NUMBER : 131

O625 CENTRIFUGE SIZE IS 20 DIAX $T-1 / 2$ WUEEP, PONER BY A OHP MOTOR, CONTROL PANE: $M$ AS A 6 LEO READOLT AND A 18 KEY KEYPAD. 2OBVAC, 60 $\mathrm{HZ}$. TRIO PEC H. C103006, 57071 ?

REPORT NUMEER: $898355-6088$

LINE ITEM NUMBER: 0036

$$
\text { wo of }
$$

COND UNITS UNIT

$5 \quad 00001 \mathrm{EA}$ COSI/UNIT:
TOTAL COST: ACOUISIIIOM PRICE

$\$ 53.385 .00$ $\$ 53,385.00$

(1)

\section{FOR FURTHER IMFORMATION: \\ ELOY N. GIROM \\ 505) $864-2746$ \\ m 87185 \\ IO FREEZE EOUIPMEMT: \\ ELOY N. GIRON \\ ALBUOUEROUE \\ 87185}

(505) $844 \cdot 2746$

LOCATION OF EQUIPMENT:

SANDIA MATICAML LABORATORIES

PROPERTY REAPPLICATION DIVISION 7617

ALBUOUERQUE

im 87123

COST/UNIT: TOTAL COST:

\section{$\$ 1.595 .00$}

"EACH. SIZE: 12"W $\times 10 M / R \times 8^{\prime \prime H}$ EST WT: 2 I.BS MFGR YR: 1988
FOR FURTHER INFORMAIIOU:

BARBARA HOL MES, REP.

Kansas CITY

$1006141-6159$

TO FREEZE EQUIPMEWI

BARBARA HOL MES, REP.

Kawsas CITY

(816) $997-2976$

$19066161-6159$

LOCATION OF EOUIPMEMT :

ALLIEDSISMAL. INC.

2000 EAST OSTH STREET

Kansas CIIT

mo 66131-3095 
EFFECTIVE DATE: $07 / 01 / 94$ EXPIRATION DATE: $07 / 31 / 94$

FSC

COOE EXCESS PROPERTY DESCRIPIION

No of

COND UNITS UNIT

ACOUISITION PRICE

REFERENCE NUMBER : 133

6625 CHATIER TESTER (INIERCONNECT BOX) 74559, MAMUF: SPARTON TECHMOLOGY MODEL 400001 EA

6343316 S S/N N/A
1 EACH. SIZE: $12^{" W} \times 10^{\circ} F / R \times 8^{\circ H}$ EST WT: 2 LBS MFGR YR: 1988

COST/UNIT: TOTAL COST:

$\$ 1.659 .00$ $\$ 1.659 .00$

REPORT NUMBER: $896404-6133$

LINE ITEM MUMBER: 0016

REFERENCE MUMBER : 136
6625 CHATTER TESTER (INTERCONNECT BOX) 74560, MANUF: SPARTON TECHMOLOGY MOOEL 4 00001 EA 6343314 G S/N N/A

1 EACH. SIZE: $12^{\prime \prime W} \times 10^{\prime \prime F} / R \times 8^{\prime \prime H}$ EST WT: 2 LBS MFGR YR: 1988
COST/UNIT: TOTAL COST:
$\$ 1.595 .00$ $\$ 1.595 .00$

\section{FOR FURTHER INFORMAIION:} BARBARA HOLMES, REP. KANSAS CITY

FOR FURTHER IWFOOMATION: BARBARA HOLMES, REP. KANSAS CITY (816) $997-2976$

mo 64141-6159

TO FREEZE EOUIPNENT: BARBARA HOLMES, REP. KaNSAS C1TY
(816) $997-2976$

10 $64161-6159$

LOCATION OF ECUIPAENT: ALLIEDSIGOAL. IWC. 2000 EAST $95 T H$ STREET Kansas citr

10 64131-3095
TO FREEZE EQUIPNENT: BARBARA hOU MES, REP. KAMSAS CIIY (816) $997-2976$

LOCATION OF EQUIPMENT : ALLIEDSIGMAL, IMC. 2000 EAST 95 TH STREET KANSAS CITY 
EFFECTIVE OATE: 07/01/96 EXPIRATION DATE: 07/31/96

\section{Fse \\ COCE EXCESS FROPERTY CEESCRIPTION \\ REFERENCE NUMGER : 135}

6625 CLOCK DIGITAL TME 59309 A ASCZZ DIGITAL CLOCK DISPLAY MONTH, DAY. HOUR, MINUTE, AND SECOND: AND UPON COMMAND OUTPUT TIME VIA THE INTERFACE BUS 1 O LGGING DEVICES. I2OVAC, $60 \mathrm{HZ}$. HEW PACK.
LINE ITEM MUMBER: 0037
No of

COND UNITS UNIT

$600001 E A$
COST IUNIT:
TOTAL COST: TOTAL COST:
ACQUISITION PRICE

$\$ 1.200 .00$
Z:FOR: NUMBER: $898355-4085$
REFERENCE NUMBER : 136

6625 CLOCK, DIGITAL DISFLAY MONTH, DAY, HOUR, MINUTE, AND SECOND. WILL OUTPUT 600001 EA IME VI A THE INTE=CACE. 115/120VAC, 48-60HZ. HEW PACK., 59309A, SER. 20 A02073.
COST/UNIT: TOTAL COST: s1, 015.00 $\$ 1,015.00$

FOR FURTHER INFORMATION:

ELOY N. GIRON

ALBUDUEROUE

(505) $846-2746$

MM 87185

TO FREEZE EQUIPMENT:

ELOY $N$. GIRON

(505) 84

44 87185

LOCATION OF EQUIPMENT:

SANDIA MATIONAL LaBORATORIES PROPERIY REAPPLICAIION DIVISION 7617 ALBUDUEROUE 
EFFECTIVE DATE: 07/01/94 EXPIRATION DATE: $07 / 31 / 94$

FSC $\quad$ NO OF

COOE EXCESS PROPERTY DESCRIPIION

REFERENCE NUMBER : 137

6625 COMAC REGISTER STD ENG 25 BOARD CRATE WITH POWER + 6VDC, -6VDC, + $1200 \mathrm{C}$, $-12 \mathrm{VDC},+24 \mathrm{VDC}$, AND $-24 \mathrm{VDC}$. 120VAC, $60 \mathrm{HZ}$. PS850, SER. 5652.

COND UNITS UNIT

ACQUISITION PRICE

$600001 \mathrm{EA}$

COST/UNIT: TOTAL COST: $\$ 2,045.00$

REPORT NUMBER: $898355-4102$

LINE ITEM NUMBER: 0009

REFERENCE NUMBER : 138

6625 COMPARATOR, RF IS A NULL INDICATOR AND ANALOG MEMORY AS USED WITH

MICROPOTENTIOM ETERS AND THERMAL VOLTAGE CONVERTERS. 12OVAC, $60 \mathrm{HZ}$

BALLANTINE, 1610A, SER. 0200142

$500001 \mathrm{EA}$

COST/UNIT:

TOTAL COST:

$\$ 3,950.00$

FOR FURTHER INFORMATION:

ELOY N. GIRON

ALBUQUERQUE

FOR FURTHER INFORMATION:

ELOY $N$. GIRON

ALBUQUEROUE

NM 87185

TO FREEZE EQUIPMENT:

ELOY N. GIRON

ALBUQUERQUE
(505) $844-2746$

87185

LOCATION OF EQUIPMENT:

SAMDIA NATIONAL LABORATORIES

PROPERTY REAPPLICATION DIV. 7617

ALBUQUERQUE

NM 87123

TO FREEZE EQUIPMENT:

ELOY N. GIRON

ALBUQUEROUE

(505) $844-2746$

NM 87185

REPORT NUMBER: $898355-4089$

LINE ITEM NUMBER: 0014

LOCATION OF EQUIPMENT :

SANDIA NATIONAL LABORATORIES

PROPERTY REAPPLICATION DIVISION 7617

ALBUQUEROUE

NM 87123 
EFFECIIVE DATE: $07 / 01 / 94$ EXPIRATION DATE: $07 / 31 / 94$

FSC $\quad$ NO OF

COOE EXCESS PROPERTY DESCRIPIION

COND UNITS UNIT

O625 COMPENSATOR MFR: KARL LAMBRECHI CORP., MOOEL NO. BSD-131, S/N 180, ACO. 500001 EA

REFERENCE NUMBER : 139 DATE: $10 / 76$. APPROX. WT: 10 LBS., $12 \mathrm{~W} \times 7 . \mathrm{L} \times 5 \mathrm{H}$.

REPORT NUMBER: $899105-4081$

\section{REFERENCE NUMBER : 140}

6625 COMPUTER, INTERFACE PROVIDES A WAY TO EXTEND THE SEPARATION OF COMPONENT 6 O0001 EA PARTS INA M I-1B SYSTEM. 12OVAC, 60 HZ. KAYE INST, 59403A, SER. 26400430 .
LINE ITEM NUMBER: 0011

COST/UNIT: TOTAL COST:
REPORT NUMBER: $898355 \cdot 4102$
LINE ITEM NUMBER: 0010
COST/UNIT:

TOTAL COST:
$\$ 1,539.00$ $\$ 1,539.00$
$\$ 2,478.00$ $\$ 2,478.00$

FOR FURTHER INFORMATION:

ELOY N. GIRON

ALBUQUERQUE

FOR FURTHER INFORMATION:

LYNN E. SHACKELFOOT

CA $94550-0969$

TO FREEZE EQUIPMENT:

LYNN E. SHACKELFOOT

IVERMORE

(510) $294-3067$

CA $94550-0969$

LOCATION OF EQUIPMENT:

SANDIA NATIONAL LABORATORIES

PROPERIY REAPPLICATION DEPT. 8532-1

LIVERMORE

CA $94550-0969$

TO FREEZE EOUIPMENT:

ELOY N. GIRON

ALBUQUERQUE

(505) $844-2746$

NM 87185

LOCATION OF EQUIPMENT :

SANDIA NATIONAL LABORATORIES

PROPERTY REAPPLICATION DIV. 7617

AL BUOUERQUE

NM 87123 
EFFECTIVE DATE: 07/01/94 EXPIRATION DATE: 07/31/94

FSC

\section{CODE EXCESS PROPERTY DESCRIPTION}

REFERENCE NUMBER : 14

6625 CONDITIONERS MOOEL 2150, MFR VESHAY INSIRUMENTS, SER\# 26932
NO OF COND UNITS UNIT

$500001 \mathrm{EA}$ COST/UNIT: TOTAL COST:

ACQUISITION PRICE

$\$ 3,815.00$ $\$ 3,815.00$

CONTACT(S)

FOR FURTHER INFORMATION: YOLANDA CUEVAS/MS DIV OAKLAND

(510) $637 \cdot 1767$

TO FREEZE EQUIPMENT: YOLANDA CUEVAS/MS DIV

OAKLAND

CA $94612-5208$

(510) $637-1767$

LOCATION OF EQUIPMENT:

GENERAL ATOMICS

3550 GENERAL ATOMICS COURT

SAN DIEGO

CA 92121

REFERENCE NUMBER : 142

6625 CONTROL IONIZER HAS A 4-1/2 LED DIGITAL READOUT, CONTROLS FORM ENERGY, EXIRACTOR, LENS, PROGRAMMED ION OPIICS, AND REGULATION MODE. 12OVAC, 60

$600001 E A$

COST/UNIT:

TOTAL COST:

HZ. EXTRANUC, 020-2, SER. IC1077.

REPORT MUMBER : $898355-4088$

LINE ITEM NUMBER: 0038

FOR FURTHER INFORMATION:

ELOY N. GIRON

ALBUQUERQUE

(505) $844-2746$

NM 87185

TO FREEZE EOUIPMENT

ELOY N. GIRON

ALBUQUERQUE

(505) $844-2746$

NM 87185

LOCATION OF EQUIPMENT:

SANDIA NATIONAL LABORATORIES

PROPERTY REAPPLICATION DIVISION 7617

ALBUQUERQUE 
EFFECTIVE DATE: $07 / 41 / 94$ EXPIRATION DATE: $07 / 31 / 94$

FSC

COOE EXCESS PROPERTY DESCRIPTION

REFERENCE NUMBER : 163

6625 . WITH AN 8 DIGIT LED READOUT, 600001 EA DIAL CONTROLS, PARALLEL PORTS, PRINTER LOOF AND 9 CONNECTIONS. IZOVAC , GO HZ. ORTEC, 9320, SER. 55.

REPORT NUMBER : $898355-4089$

LINE ITEM NUMBER: 0016

REFERENCE NUMBER : 144

6625 CONTROLLER MFR: D!GITAL, MODEL NO. 11/24-BC, S/N AG05191, ACQ. DATE: 12/85. APPORX. WT: 120 LBS., $19 . \mathrm{W} \times 28^{\circ} \mathrm{L} \times 10^{\prime} \mathrm{H}$.

LINE ITEM NUMBER: 0012

NO OF

REPORT NUMBER: $899105 \cdot 4081$
COND UNITS UNIT $\quad$ ACOUISITION PRICE
COST/UNIT: TOTAL COST:

$\$ 2,741.00$ $\$ 2,741.00$
FOR FURTHER INFORMATION:
ELOY N. GIRON
ALBUQUERQUE

ALBUQUERQUE

(505) $844-2746$

NM 87185

TO FREEZE EQUIPMENT :

ELOY N. GIRON

ALBUO

(505) $844-2746$

NM 87185

LOCATION OF EQUIPMENT:

SANDIA NATIONAL LABORATORIES

PROPERTY REAPPLICATION DIVISION 7617

ALBUQUERQUE

NM 87123
COST/UNIT:

TOTAL COST:
$\$ 11,077.00$ $\$ 11,077.00$
FOR FURTHER INFORMATION:

LYNN E. SHACKELFOOT

LIVERMORE

LIVERMORE
(510) $294-3067$

TO FREEZE EQUIPMENT:

LYNN E. SHACKELFOOT

LIVERMORE

CA $94550-0969$

(510) 294-3067

CA $94550-0969$

LOCATION OF EQUIPMENT

SANDIA NATIONAL LABORATORIES

PROPERTY REAPPLICATION DEPT. 8532-1

LIVERMORE

CA 94550-0969 
EFFECTIVE DATE: $07 / 01 / 94$ EXPIRATION DATE: 07/31/94

$\mathrm{FSC}$

\section{CODE EXCESS PROPERTY DESCRIPTION}

REFERENCE NUMBER : 145

6625 CONTROLLER MFR: DIGITAL, MODEL NO. PDP 11/04, S/N 1104-WM04062, ACO DATE: $7 / 77$, APPROX. WT: 80 LBS., $19 \cdot \mathrm{W} \times 27 \cdot \mathrm{L} \times 5 \cdot \mathrm{H}$.

REPORT NUMBER: $899105-408$

LINE ITEM NUMBER: 0013

REFERENCE NUMBER : 146

6625 CONTROLLER SKP ELECTRIC, S/N 153, P/N 257757.

REPORT NUMBER: $890207-4116$

LINE ITEM NUMBER:0391
NO OF

COND UNITS UNIT

ACQUISITION PRICE

$400001 \mathrm{EA}$

COST/UNIT:

TOTAL COST:

$\$ 6,480.00$

$\$ 6,480.00$

LYNN E. SHACKELFOOT

(510) $294-3067$

CA $94550-0969$

TO FREEZE EQUIPMENT:

LYNN E. SHACKELFOOT

LIVERMORE

(510) $294-3067$

CA $94550-0969$

LOCATION OF EQUIPMENT:

SANDIA NATIONAL LABORATORIES

PROPERTYY REAPPLICATION DEPT. 8532-1

LIVERMORE CA $94550-0969$
$600001 \mathrm{EA}$

COST/UNIT:

TOTAL COST:

$\$ 3,000.00$

$\$ 3,000.00$
FOR FURTHER INFORMATION

SANDY MOON

IDAHO FALLS

(208) $526-261$

ID $83415-4100$

TO FREEZE EQUIPMENT:

SANDY MOON

IDAHO FALLS

(208) $526-2611$

ID $83415-4100$

LOCATION OF EOUIPMENT

IDAHO NATIONAL ENGINEERING LAB.

CF- 674 WAREHOUSE

SCOVILLE

ID $83415-4100$ 
EFFECIIVE DATE: $07 / 01 / 94$ EXPIRATION DATE: $07 / 31 / 94$

\section{FSC \\ No of}

COOE E.ICESS PROPERTY DESCRIPTION

REFERENCE NUMBER : 147

TYPE 5 CONTROLLER PLUG IN, 4 DIGIT LED READOUT FOR TEMP AND

CONTROLLER TYPE 5 CONTROLLER PLUG IN,

ELIG:BLE EQUIPMENT
COND UNITS UNIT

$700001 \mathrm{EA}$
ACQUISITION PRICE

$\operatorname{COST/UNIT:~}$ TOTAL COST:

LINE ITEM NUMBER: 0039
REPORT NUMBER: $898355-4088$
REFERENCE NUMBER : 148

AC/DC-LOG THE 7563A CONVERTER DYNAMICS RANGE IS I10DB, VOLTAGE DIGNALS.

12OVAC, 60 HZ. HEW PACK, 7563A, JER. 14A00522.

REPORT NUMBER: $898355-4089$

LINE ITEM NUMBER: 0018
$600001 \mathrm{EA}$

COST/UNIT:

TOTAL COST:

\section{$\$ 1,195.00$ \\ $\$ 1,195.00$}

\section{TO FREEZE EQUIPMENT: \\ ELOY N. GIRON}

ALBUQUERQUE

(505) $844-2746$

NM 87185

LOCATION OF EQUIPMENT:

SANDIA NATIONAL LABORATORIES SANDIA NATIONAL LABORATORIES PROPERTY REAPPLICATION DIVISION 7617 ALBUQUEROUE NM 87123

$\$ 1.227 .00$

FOR FURTHER INFORMATION:

ELOY N. GIRON

ALBUQUERQUE

(505) $844-2746$

NM 87185

TO FREEZE EQUIPMENT:

ELOY N. GIRON

ALBUOUERQUE

(505) $844-2746$

NM 87185

LOCATION OF EQUIPMENT:

SANDIA NATIONAL LABORATORIES

PROPERTY REAPPLICATION DIVISION 7617

PROPERTY REAPPLICATION DIVISION 7617

ALBUQUEROUE

NM 87123 
EFFECIIVE DATE: 07/01/96 EXPIRATION DATE: 07/31/94

No of

COND UNITS UNIT

ACQUISIIIION PRICE

COST/UNIT:

TOTAL COST:

$\$ 1,227.00$

$\$ 1,227.00$

RANGE: 316MV TO 100V. 120VAC, $60 \mathrm{HZ}$. HEW PACK.7563A, SER. 14A00521.

00001 EA TOTAL COST:

REPORT NUMBER : $898355-4089$

LINE ITEM NUMBER: 0017

REFERENCE NUMEER : 150

6625 COPY UNIT-HARO, MFR. TELIRONIX, MODEL 4634. ACO. YR. 83, PROP. WO.
3088072

$500001 \mathrm{EA}$

COST/UNIT:

TOTAL COST:

$\$ 6,106.98$

$\$ 6,106.98$

REPORT NUMBER: $899102-4163$

LINE ITEM NUMBER: 0030
FOR FURTHER INFORMATION:

JOANNE BREZNIK

LIVERMORE

CA 94550

(510) $424-4101$

TO FREEZE EQUIPMENT:

JOANME BREZNIK

LIVERMORE

CA 94550

NM 87185

NM 87185

ELOY N. GIRON

505) $844-2746$

SANDIA NATIONAL LABORATORIES

PROPERTY REAPPLICATION DIVISION 7617

NM 87123

(510) $424-4101$

LOCATION OF EQUIPMENT

LAURENCE LIVERMORE NATIONAL LAB

EXCESS PROPERTY SECTION, L-696

LIVERMORE

CA 94550 
EFFECIIVE DATE: $07 / 01 / 94$ EXPIRATION DATE: $07 / 31 / 94$

FSC

CODE EXCESS PROPERTY DESCRIPIION

REFERENCE NUMBER : 151 $\begin{array}{ll}0625 & \text { COPY UNIT-HARD, MFR. TEKTRONIX, MODEL 4632, ACO. YR. 86, PROP. NO. } \\ & 4395346\end{array}$
NO OF

500001 EA COST/UNIT: TOTAL COST:

$\$ 5,793.00$ $\$ 5,793.00$

LINE ITEM NUMBER: 0035
REFERENCE NUMBER : 152

O625 COPY UNIY-VIDEO HARD, MFR TEKTRONIX, MODEL 4632, ACO. YR. 85, PROP. NO. 600001 EA $\$ 159115$
REPORT NUMBER: $899102 \cdot 4163$
REPORT HUMBER: $899102-4163$

LINE ITEM NUMBER: 0034
COST/UNIT: TOTAL COST:
$\$ 5,656.87$
$\$ 5,656.87$

FOR FURTHER INFORMATION:

JOANNE BREZNIK

LIVERMORE

(510) $424-4101$

TO FREEZE EQUIPMENT

JOANNE BREZNIK

LIVERMORE

CA 94550

(510) $424-410$

LOCATION OF EQUIPMENT:

LAWRENCE LIVERMORE NATIONAL LAB

EXCESS PROPERTY SECTION, L-696

EXCESS PROPERTY SECTION, L-696
LIVERMORE
CA 94550 
EFFECTIVE DATE: $07 / 01 / 94$ EXP!RATION DATE: $07 / 31 / 94$

FSC

\section{CODE EXCESS PROPERTY DESCRIPIION}

REFERENCE NUMBER : 153

6625 COUNTER AIRBORN PARTICLE COUNTER, HAS A 6 DIGIT LCD COUNTER, 14 KEY KEYPA D CONTROL SYSTEM. 120VAC, 60 HZ. ATCOR, NET2000, SER. B2880628.

REPORT NUMBER: $898355-4088$

LINE ITEM NUMBER: 0041

\section{REFERENCE NUMBER : 154}

6625 COUNTER FREQUENCY MFR: EIP MICRO MOOLL: $350 C$ $S / N$ : 229A0153

REPORT NUMBER: $899305-4032$
NO OF

COND UNITS UNIT

ACQUISITION PRICE

$500001 E A$

COST/UNIT: TOTAL COST:

$\$ 3,739.00$ $\$ 3,739.00$

$600001 \mathrm{EA}$

COST/UNIT:
TOTAL COST:

$\$ 4.300 .00$
CONTACT(S)

FOR FURTHER IMFORMATION:

ELOY N. GIRON

ALBUQUERQUE

(505) $844-2746$

NM $87 i 85$

TO FREEZE EQUIPMENT:

ELOY N. GIRON

ALBUQUERQUE

(505) $844-2746$

NM 87185

LOCATION OF EQUIPMENT:

SAMDIA NATIONAL LABORATORIES

PROPERTY REAPPLICATION DIVISION 7617

PROPERTY REAPPLICATION DIVISION 7617
ALBUQUERQUE
NM 87123 NM 87123

FOR FURTHER INFORMATION:

CYMDI DELONG

LAS VEGAS

(702) $295-6753$

NV $89193-852$

TO FREEZE EQUIPMENT:

CYMDI DELLNG

LAS VEGAS

(702) $295-6753$

NV $89193-8521$

L ATION OF EOUIPMENT:

REYNOLDS ELECTRICAL \& ENGINEERING CO

NEVADA TEST SITE

MERCURY

NV 89023 
EFFECIIVE DATE: 07/01/9: EXPIRATION DATE: $07 / 31 / 94$

\section{COOE EXCESS PROPERIY DESCRIPTION}

REFERENCE NUMBER : 155

6625 COUNTER TIME INTERVAL MFR: HEWLETT PACKARD MODEL: $5275 \mathrm{~A}$ $S / N$ : 50400464

REPORT NUMEER: $899305-4032$

LINE ITEM NUMBER: 0001

REFERENCE NUMBER : 150

6025 COUNTER TIME INTERVAL MFR: HEWLETT PACKARD MODEL: 5275 A

LINE ITEM NUMBER: 0018

REPORT NUMBER: $899305-4032$
NO OF

COND UNITS UNIT

ACQUISITION PRICE

$500001 \mathrm{EA}$

COST/UNIT: IOIAL COST:

$\$ 2,500.00$

$\$ 2,500.00$

rotal cost:
CYNDI DELONG
LAS VEGAS
(702) $295-6753$
NV $89193-8521$

FOR FURTHER INFORMATION

TO FREEZE EOUIPMENT:

CYNDI DELONG

LAS VEGAS

NV 89193-8521

(702) $295-6753$

LOCATION OF EQUIPMENT:

REYNOLDS ELECTRICAL \& ENGINEERING CO

MEVADA TEST SITE

MERCURY

NV 89023

$500001 E A$

COST/UNIT:

TOTAL COST:
$\$ 2,450.00$
$\$ 2,450.00$

FOR FURTHER INFORMATION:

CYNDI DELONG

LAS VEGAS
(702) $295-6753$

NV $89193-8521$

TO FREEZE EQUIPMENT:

CYNDI DELONG

CYNDI DELOIG

(702) $295-6753$

NV 89193-8521

LOCATION OF EQUIPMENT:

REYNOLDS ELECTRICAL \& ENGINEERING CO

NEVADA TEST SITE

MERCURY

NV 89023 
EFFECTIVE DATE: 07/01/94 EXPIRATION DATE: 07/31/94

FSC

CODE EXCESS PROPERTY DESCRIPTION

REFERENCE NUMBER : 157

6625 COUNTER, FREO DIGITAL COUNTER RESOLUTIONS/HZ, RANGE FROM IM TO 100, INPLT CONNE CTOR 3-3 GHZ. ON/OFF CONTROLS. 12OVAC, 60 HZ. SYSTRON, 153. SER. 173 .

REPORT NUMBER: $898355-4089$

LINE ITEM NUMBER: 0021

REFERENCE NUMBER : 158

6625 COUNTER, FREO D!GITAL COUNTER RESOLUTION/HZ, RANGE FROM $1 M$ TO 100, INPUT CONMEC TOR 3-3 GHZ ON/OFF CONTROLS, 12OVAC, OD HZ SYSTRON D, 153, SER 199 .
No of

COND UNITS UNIT

ACQUISITION PRICE

$\$ 1,323.00$

\section{COSTIUNIT:}

TOTAL COST:

$600001 \mathrm{EA}$$$
\text { TO }
$$ \\ TO FREEZE EQUIPMENT: \\ ELOY N. GIRON \\ ALBUOUEROUE}

ALBUOUEROUE
(505) $844-2746$

LOCATION OF EOUIPMENT.

LOCATION OF EQUIPMENT: SANDIA MATIONAL LABORATORIES
PROPERTY REAPPLICATION DIVISION 7617 PROPERTY REAPPLICATION DIVISION 7617
ALBUOUEROUE
NM 87123

$600001 \mathrm{EA}$

COST/UNIT:

TOTAL COST:
$\$ 1,407.00$
$\$ 1,407.00$

FOR FURTHER INFORMATION:

ELOY N. GIRON

AL BUOUEROUE

(505) $844-2746$

Nat 87185

TO FREEZE EQUIPMENT:

ELOY N. GIRON

ELOY N. GIROA

(505) $844-2746$

MM 87185

LOCATION OF EQUIPMENT:

SANDIA MATIONAL LABORATORIES

SAND IA MATIONAL LABORATORIES
PROPERTY REAPPLICATION DIVISION 7617

PROPERTY REAPPLICATION DIVISION 7617
ALBUOUERQUE 
EFFECTIVE DATE: 07/01/94 EXPIRATION DATE: 07/31/94

\section{CODE EXCESS PROPERTY DESCRIPTION}

REFERENCE NUMBER : 159

O62: COUNTER, FREOUENCY HAS SINGLE INPUT 1OHZ TO 18GHZ AUTO AMPLITUDE DISCRIMINATION IS H IGH SENSITIVITY 35DBM. 120VAC, $60 \mathrm{HZ}$. HEW PACK, DISCRIMINATION IS

ELIGIBLE EQUIPMENT

No of

COND UNITS UNIT

ACQUISITION PRICE

$600001 \mathrm{EA}$

COST/UNIT: IOTAL COST:

$\$ 5.432 .00$

55.432 .00

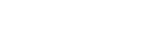

FOR FURTHER IMFORMATION:

ELOY $N$. GIRON ALBUOUEROUE

WM 87185

TO FREEZE EQUIPMENT:

ELOY N. GIROI

ALBUOUEROUE

(505) 844-2746

NM 87185

REPORT NUMBER: $898355-4102$

LINE ITEM NUMBER: 001

\section{REFERENCE NUMBER : 160}

6625 COUNTER, FREQUENCY HAS SINGIE INPUT $10 \mathrm{HZ}$ TO $18 \mathrm{GHZ}$ AUTO AMPLITUDE DISCRIMINATION IS HIGH SENSITIVITY 35 DBM. 120VAC, 60 HZ. HEW PACK 5360A, SER. 4 4A01137.
LINE ITEM NUMBER: 0012

\section{EA COST/UNIT: \\ TOTAL COST:}

$\$ 5.432 .00$

$\$ 5.432 .00$

LOCATION OF EQUIPMENT:

SANDIA MATIONAL LABORATORIES PROPERTY REAPPLICATION DIV. 7617 ALBUOUEROUE

in 87123

REPORT NUMBER: $898355-4102$

\author{
FOR FURTHER INFORMATION: \\ ELOY N. GIRO \\ ALBUOUEROUE \\ (505) $844-2746$ \\ MM 87185 \\ TO FREEZE EQUIPMENT: \\ ELOY N. GIRON \\ ALBUCUERQUE \\ (505) 844-2746 \\ Min 87185 \\ LOCATION OF EQUIPMENT \\ SAMDIA MATIOMAL LABORATORIES \\ PROPERTY REAPPLICATIOA DIV. 7617 \\ ALBUOUEROUE \\ in 8712
}


EFFECT!VE DATE: 07/01/94 EXPIRATION DATE: $07 / 31 / 94$

\section{FSC \\ COOE EXCESS PROPERTY DESCRIPIION \\ REFERENCE NUMBER : 161 \\ 6625 COUNTER, FREQUENCY DIGITIZING OSCILLOSCOPE, FULLY PROGRAMMABLE HITH FEATURES AS AUTO SCALING, AUTO TRIGGER SELF CALIBRATIOA AND DIRECT READOUT OF DEL TA VOLTAGE/DELTA TIME. HEW FACK}

REPORT NUMBER: $898355-4102$

LINE ITEM MUMBER: 0013

REFERENCE NUMBER : 162

6625 COUNTER, MULTI-FUNCT COUNTER WITH A 7 DIGIT READOUT, HAS CONTROLS FO FUNCTION TIME BA SE AND RANGE. 120VAC, 60 HZ. MONSANTO, 110A, SER. 800A224.
Mo of COND UNITS UNIT

ACOUISITION PRICE

$600001 \mathrm{EA}$ COST/UNIT: TOTAL COST

\section{$\$ 1.650 .00$ $\$ 1,650.00$}

600001 EA
$\$ 1,102,00$ $\$ 1.102 .00$
FOR FURTHER IMFORMAIION:

ELOY N. GIRO

AL BUOUERQUE

(505) $844-2746$

87185

TO FREEZE EQUIPMENT

ELOY N. GIRON

ALBUOUEROUE

(505) $844-2746$

LOCATION OF ECUIPMENT

SAMDIA MATIOMAL LABORATORIES

PROPERTY REAPPLICATION DIVISION 7617

ALBUOUERQUE 
EFFECTIVE DATE: 07/01/94 EXPIRATION DATE: 07/31/94

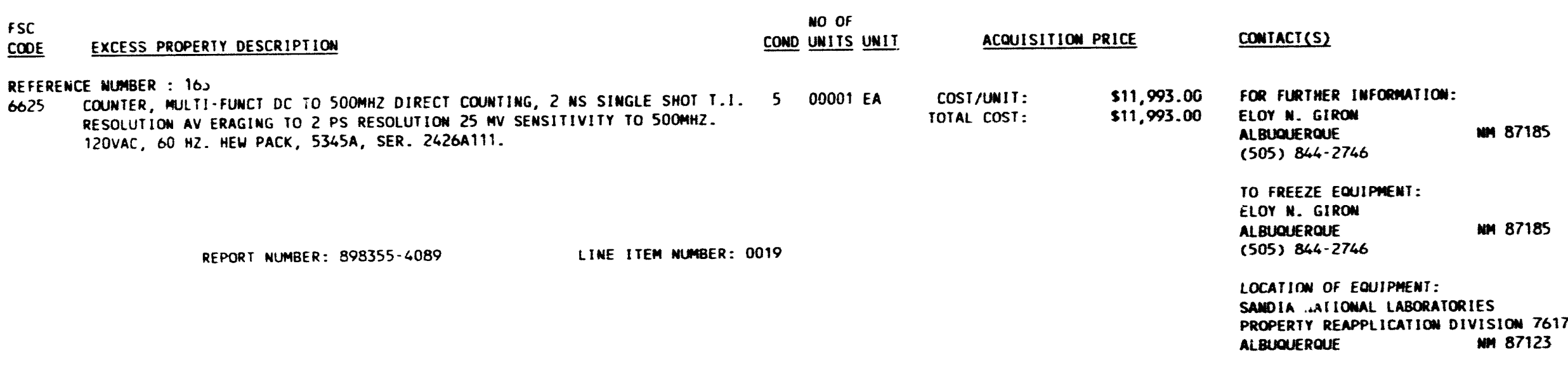

REFERENCE NUMBER : 164

6625 COUNTER, MULTI-FUNCT DC TO SOOMHZ DIRECT COUNTING, 2 NS SINGLE SHOT T.1.

$600001 \mathrm{EA}$

COST/UNIT:
TOTAL COST:

PESOLUTION A VERAGING TO 2 PS RESOLUTION, 25 MV SENSITIVITY TO $500 M H Z$.

$\$ 8,540.00$

12OVAC, $60 \mathrm{HZ}$. HEW PACK, 5345A, 2426A109.

58.540 .00

FOR FURTHER IMFORMATION:

ELOY N. GIROM

ALBUOUEROUE

(505) $844-2746$

m 87185

TO FREEZE EQUIPMENT:

ELOY N. GIROM

ALQUOUEROUE

(505) $844-2746$

4.4 87185

LOCATION OF EOUIPMENT:

SANDIA MATICWAL LABORATORIES

PROPERTY REAPPLICATIOA DIVISION 7617

ALBUOUERQUE 

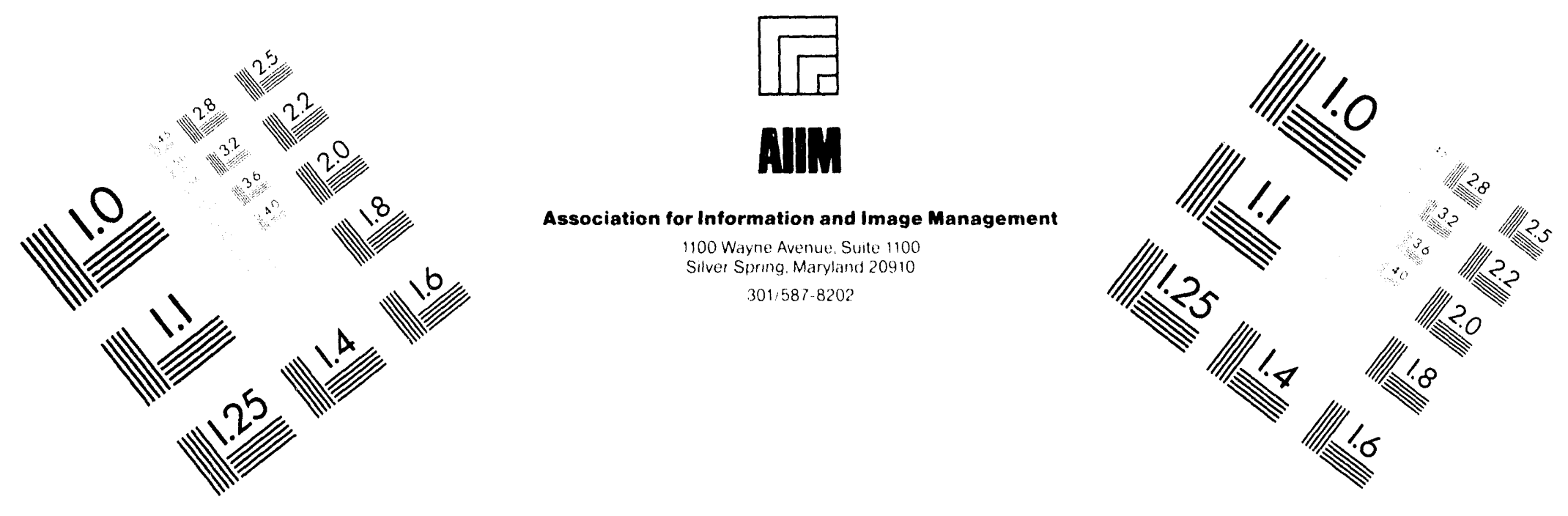

\section{Centimeter}

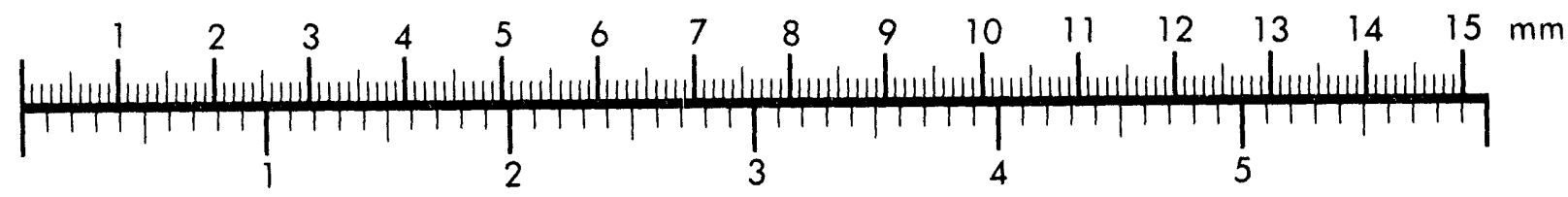
Inches
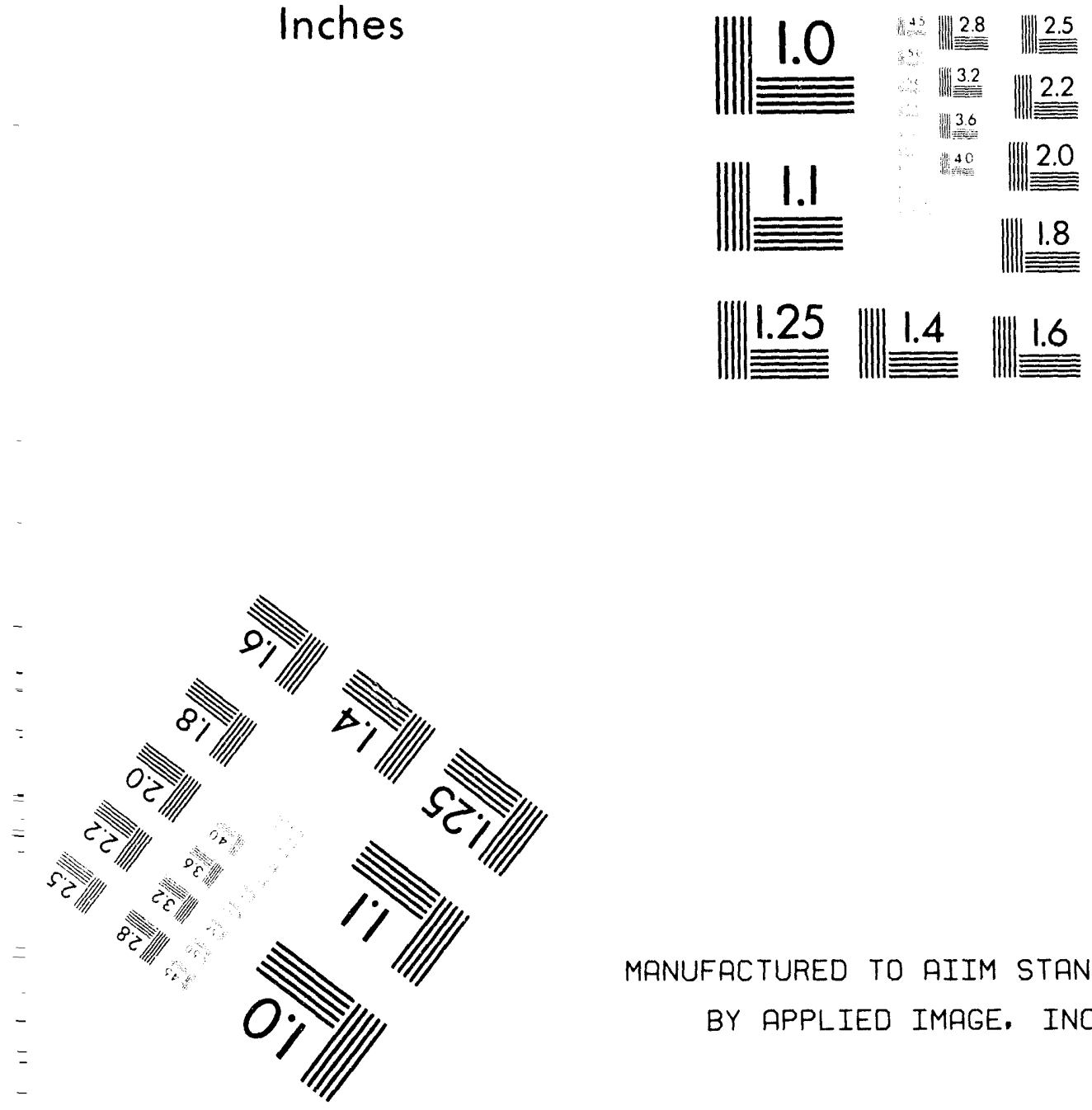

MANUFACTURED TO AIIM STANDARDS BY APPLIED IMAGE, INC.

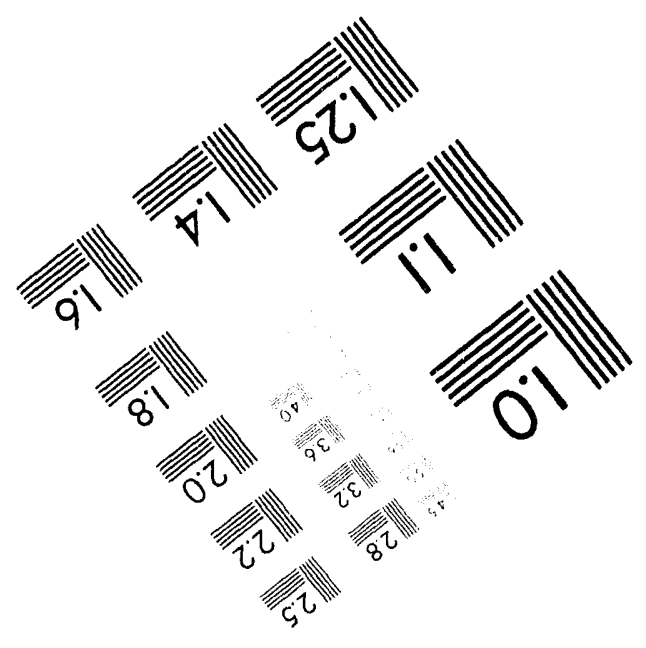



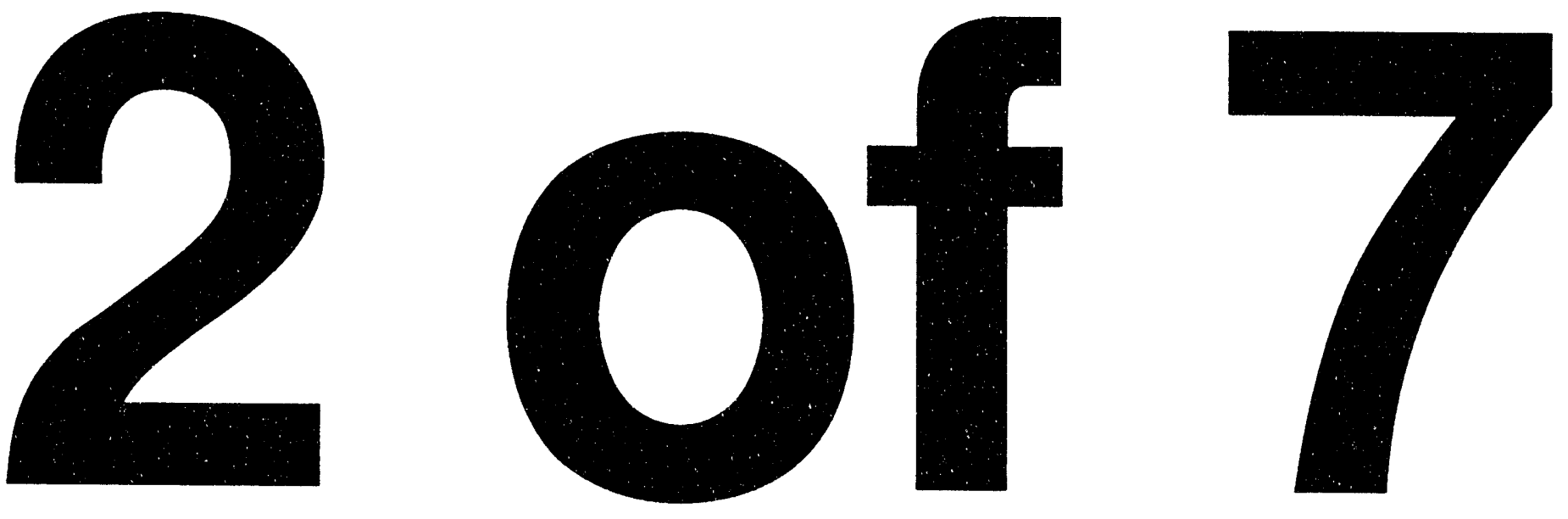
EHECTIVE DATE: 07/01/94 EXPIRATION DATE: $07 / 31 / 94$

\section{CONE EXCESS PROPERTY DESCRIPIION}

REFERENCE NUMBER : 165

O625 COUNTER, MULT! FUNCTION 2OOMHZ/2NS UNIVERSAL COUNTER BUILT IN AUTO RESETIME DUTY CYCLE, P ULSE WIDTH, SLOW RATE AND PHASE MEASUREMENT. HEW FALK, 5335A, 48A CO285.

REPORT NUMBER: $998355-4089$

LINE ITEM NUMBER: D022

REFERENCE NUMBER : 160

6625 COUNTER, MULTI-FUNCT 1 MHZ RESOLUTION, HIGH SPECTROAL PURITY PRECISION 500001 EA AMPLITUDE CONTR OL, PROGRAM STORAGE WITH HP- TB PORT. IZOVAC, 60 HZ. HEW PACK, 53 35A, SER. $2220 A 030$.
COND UNITS UNIT

$600001 \mathrm{EA}$

NO OF

ACQUISITION PRICE

COST/UNIT:
COST/UNIT: TOTAL COST:
$\$ 3,655.00$ $\$ 3,655.00$
$\$ 3,700.00$

$$
\text { FOR }
$$$$
\begin{aligned}
& \text { TO FREEZE EQUIPMENT: } \\
& \text { ELOY N. GIRON }
\end{aligned}
$$

ELOY N. GIRON

(505) $844-2746$

(505) $844-2746$

LOCATION OF EOUIPMENT:

SANDIA NATIONAL LABORATORIES

PROPERTY REAPPLICATION DIVISION 7617 ALBUOUERQUE 
EFFCIIVE DATE: OF/01/04 EXFIRATION DATE: O7/31/04

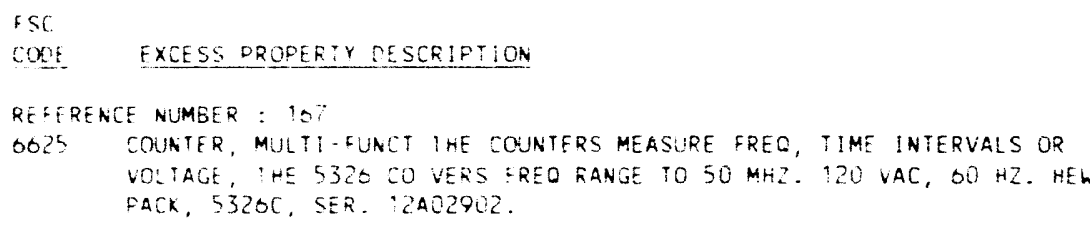

LINE ITEM NUMBER: 0025

FEF ERENE NUMGEF : 108

OQ25 COUNER, MUL:!-FUNCT RACK MOUN, D DIG READOUT, MEASURE FREO COUNT DERIOR:C OP RANDON FULSES POVAL, EO HZ. HEW PACK, S233L, SER. 00833J.

$600001 E A$ COSI/UN!T:
TOTAL COST:

LINE ITEM NUMBER: 0026

NO OF

COND UNITS UNII

6 D0001 EA

COSI/UNIT: TOTAL COST:

REPORT NUMBER: $898355-4086$
ACOUISITION PRICE

$\$ 1,482.00$
$\$ 1,482.00$

ELOY N. GIRON
ALBUOUEROUE
$(505) 844-2746$

NM 87185

TO FREEZE EOUIPMENT :

ELOY N. GIRON

ALBUQUEROUE

(505) 844.2746

NM 87185

LOCATION OF EQUIPMENT:

SANDIA NATIONAL LABORATORIES

PROPERTY REAPPLICATION DIVISION 7617

ALBUQUEROUE

NM 87123

FOR FURTHER INFORMATION:
ELOY N. GIRON

ALBUQUEROLE

(505) $844-2746$

NM 87185

TO FREEZE EQUIPMENT:

ELOY N. GIRON

ALBUQUERQUE

(505) $844-2746$

NM 87185

LOCATION OF EQUIPMENT:

SANDIA NATIONAL LABORATORIES

SANDIA NATIONAL LABORATORIES
PROPERTY REAPPLICATION DIVIC. ON 7617 PROPERTY REAPPLICATION DIVIS. ON 7617
ALBUDUEROUE 
EFFECTIVE DATE: $07 / 01 / 94$ EXPIRATION DATE: $07 / 31 / 94$

FSE

COOE EXCESS PROPERTY DESCRIPIION

REFERENCE NUMBER : 169

6025 COUNTER, MULTI-FUNCT HAS SOOMHZ DIRECT COUNT, CONVERSION PLUG-INS TO $110 \mathrm{GHZ}$. 2 NS SIN GLE SHOT RESOLUTION 2 PS AVERAGED. I2OVAC, 60 HZ. HEW PACK, 5345A, SER. $2134 A 097$

REPORT NUMBER: $898355-4102$

LINE ITEM NUMBER: 0014

REFERENCE NUMGER : 170

S625 COUNTER, MUIII-FUNCT HAS SOOMHZ DIRECT COUNT, CONVERSION PLUG-INS TO COUNTER, MULTI-FUNCT HAS 5OOMHZ DIRECT COUNT, CONVERSION
$110 \mathrm{GHZ}$. 12OVAC, 60 HZ. HEH PACK, 5345A, SER. 2134A097.

LINE ITEM NUMBER: 0015
NO OF

\section{COND UNITS UNIT $\quad$ ACQUISITION PRICE}

$600001 \mathrm{EA}$

COST/UNIT: TOIAL COST:

$\$ 7,400.00$ $\$ 7,400.00$

FOR FURTHER INFORMATION:

ELOY N. GIRON

ALBUQUERQUE

(505) $844-2746$

NM 87185

TO FREEZE EQUIPMENT

ELOY N. GIRON

ALBUQUERQUE

(505) $844-2746$

NM 87185

LOCATION OF EQUIPMENT:

SANDIA NATIONAL LABORATORIES

PROPERTY REAPPLICATION DIV. 7617

ALBUQUEROUE

NM 87123

FOR FURTHER INFORMATION:

ELOY N. GIRON

ALBUOUEROUE
(505) $844-2746$

NM 87185

TO FREEZE EQUIPMENT:

ELOY N. GIRON

ALBUQUERQUE

(505) $844-2746$

NM 87185

LOCATION OF EQUIPMENT:

SANDIA NATIONAL LABORATORIES

PROPERIY REAPPLICATION DIV. 7617

ALBUQUERQUE

NM 87123 
EFFECTIVE DATE: OT/01/94 EXPIRATION DAIE: 07/31/94

CODE EXCESS PROPERTY DESCRIPIION

REFERENCE NUMBER: $: 71$

O625 CONNTER, MULTI-FUNC: HAS $500 M H Z$ DIRECT COUNT, CONVERSION PLUG INS TO

1POGHZ. 12CVAC, OO HZ. HEW PACK, 5345A, SER. 2134 A097.
No of

COND UNITS UNIT

ACQUISITION PRICE

o $00001 \mathrm{EA}$

COST/UNIT:

TOTAL COST:

$\$ 7,400.00$ $\$ 7,400.00$

FOR FURTHER INFORMATION:

ELOY N. GIRON

ALBUQUERQUE

(505) $844-2746$

NM 87185

IO FREEZE EQUIPMENT

ELOY N. GIRON

ALBUOUERQUE

(505) $844-2746$

NM 87185

LOCATION OF ZOUIPMENT:

LABORATORIES

PROPERTY REAPPLICATION DIV. 7617

ALBUOUERQUE

REFERENCE NUMBER : $; 7 ?$

6025 COUNTER MIJT:- EUNCT HAS SOCMHZ D:RECT COUNT, CONVERSION FLUG INS TO

$110 G \mathrm{HZ}$. IZOVAC, 60 HZ. HEW PACK, 53 5AA. 2134A0S7.

$600001 E A$

COST NUNIT:

TOTAL COST:

LINE ITEM NUMBER: 0017
REPORT NUMEER: $898355 \cdot 4102$
$\$ 7,400.00$

INFORMATION:

ELOY N. GIRON

ALBUOUERQUE

(505) $844-2746$

NM 87185

IO FREEZE EQUIPMENT

ELOY N. GIRON

(505) $844-2746$

NM 87185

LOCATION OF EQUIPMENT:

SANDIA NATIONAL LABORATORIES

PROPERTY REAPPLICATION DIV. 7617

ALBUQUERQUE

NM 87123 
EFFECTIVE DATE: $07 / 01 / 94$ EXPIRATION DATE: $07 / 31 / 94$

FSC

\section{COOE EXCESS PROPERTY DESCRIPIION}

REFERENCE NUMBER : 173

6625 COUNTER, MULTI-FUNCT 200MHZ/2NS UNIVERSAL COUNTER GUILT IN AUTO

COUNTER, MULTI-FUNCT $200 M H Z$ /2NS UNIVERSAL COUNTER GUILT IN AUTO
RISETIME, DUTY CYCLE, PULSE WIDTH, SLEEVE RATE ANO PHASE. 120VAC, 60
HZ. HEW PACK, 5335 a 2838A120.

REPORT NUMBER: $898355-4102$

LINE ITEM NUMBER: 0018

\section{REFERENCE NUMBER : 176}

6625 CPU COMPUTER FLUG IN GOARD, WITH A 25 PIN PORT IN BACK. FORCE COMP.

CPUZB, SE R. AOTOT.
NO OF

\section{COND UNITS UNIT \\ ACOUISITION PRICE \\ CONTACT(S)}

$600001 \mathrm{EA}$

COST/UNIT: TOTAL COST:

$\$ 4,740.00$ $\$ 4.740 .00$

FOR FURTHER INFORMATION:

ELOY N. GIRON

ALBUQUEROUE

(505) $844-2746$

NM 87185

TO FREEZE EQUIPMENT:

ELOY N. GIRON

ALBUQUEROUE

(505) $844-2746$

NM 87185

LOCATION OF EOUIPMENT:

SANDIA NATIONAL LABORATORIES

PROPERTY REAPPI ICATION DIV 7617

PROPERTY REAPPLICATION DIV. 7617
ALBUQUEROUE

MM 87123

600001 EA

COST/UNIT:

TOTAL COST:

$\$ 18,629.00$

$\$ 18,629.00$
FOR FURTHER INFORMATION:
ELOY N. GIRON
ALBUOUEROUE
(505) $844-2746$

NM 87185

TO FREEZE EQUIPMENT:

ELOY N. GIRON

ALBUOUERQUE

NM 87185

(505) 844-2746

LOCATION OF EQUIPMENT:

SANDIA NATIONAL LABORATORIES

PROPERTY REAPPLICATION DIVISION 7617

ALBUQUERQUE 
EFFECTIVE DATE: 07/01/94 EXPIRATION DATE: 07/31/94

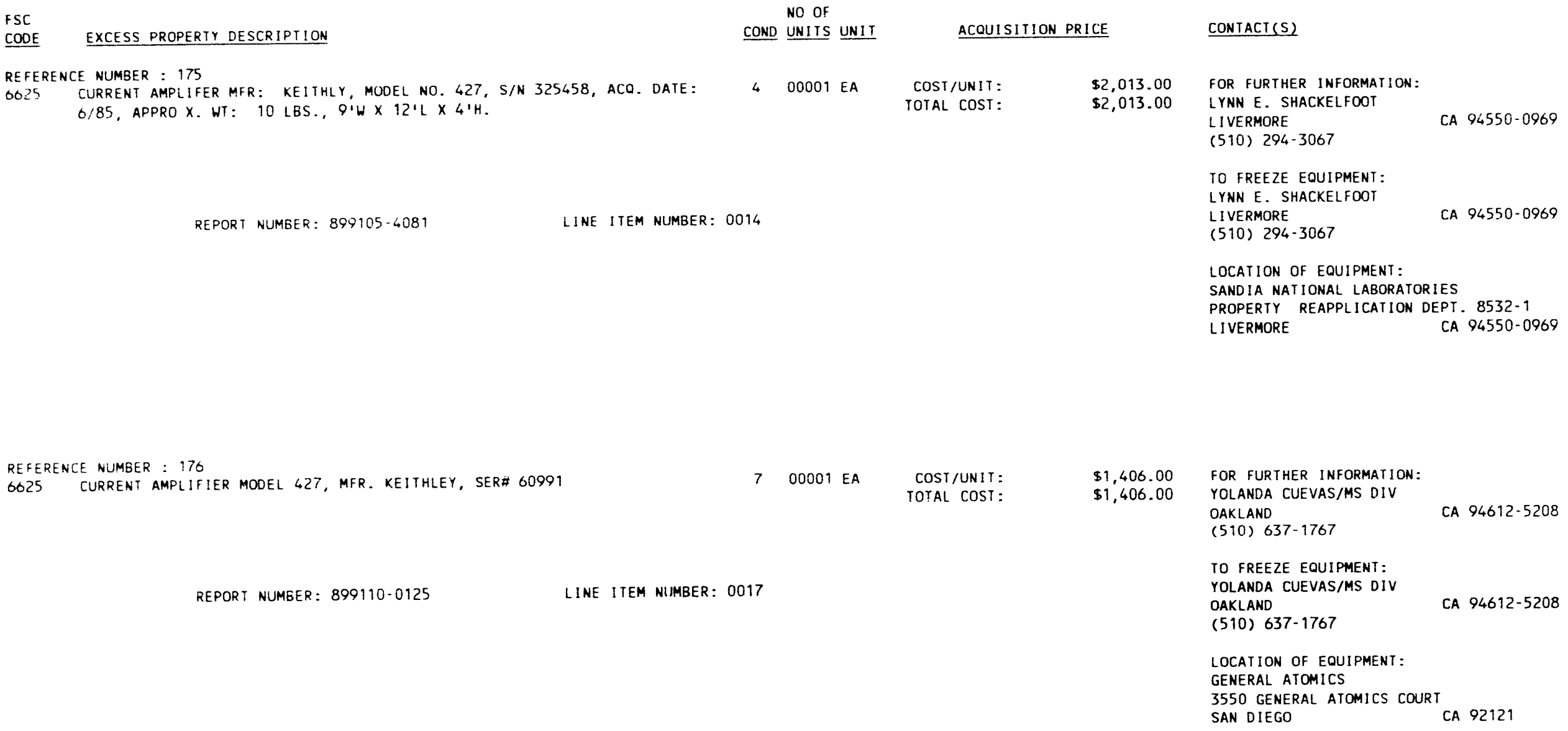


FFFECTIVE DATF: $07 / 01 / 94$ EXPIRATION DATE: $07 / 31 / 94$

FSC

CODE EXCESS PROPERIY DESCRIPTION

REFERENLE NUMBER : $1 T \%$

68:S CURVE, TRACER CAN TEST TWO AND THREE TERMINAL DISCRETE SEMI-CONDUCTORS.

POWER C APABILITY UP TO 220 WATIS. 115/230VAC, 50-60HZ. TEKTRONIX, 576,
SER. B071019.

REPORT NUMGER: $898355-4088$

LINE ITEM NUMBER: 0042

$$
\text { No OF }
$$

COND UNITS UNIT

ACQUISITION PRICE

COSI/UNIT: TOTAL COST:

\section{$\$ 2,308.00$ \\ $\$ 2,308.00$}

TOTAL COST:

(1)

REFERENCE NUMBER : 178

6625 DATA ACO ACCESSORY TRIGGER SLOPE: PROGRAM SELECTAGLE, TRANSITION EITHER LOGIC 0 TO 1 OR 100 . 12OVAC, 60 HZ. HEW PACK, 5358A, SER. $20 A 00420$.

REPORT NUMBER: $898355 \cdot 4102$
LINE ITEM NUMBER: 0019
$00001 E \hat{A}$

COST/UNIT:

TOTAL COST: $5,375.00$

FOR FURTHER INFORMATION:

ELOY N. GIRON

ALBUQUERQUE

(505) $844-2746$

NM 87185

TO FREEZE EOUIPMENT:

ELOY N. GIRON

ALBUDUEROUE

(505) $844-2746$

NM 87185

\section{LOCATION OF EOUIPMENT:}

SANDIA NATIONAL LABORATORIES

PROPERTY REAPPLICATION DIV. 7617 ALBUQUERQUE NM 87123 
EFFECTIVE DATE: $67 / 01 / 94$ EXFIRATION DATE: 07/31/0.

FSC

\section{COOE EXCESS PRUPERTY DESCRIPT:ON}

REFERENCE NUMGER: 170

GO25 OATA ACO ACCESSORY TRIGLER SLOFE. PROLRAM SEIECTABLE, IRANSIIION EITHER

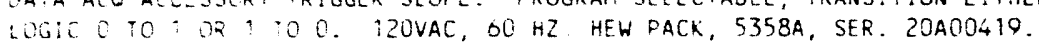

NO OF

COND UNIIS 1 .

$00001 \mathrm{EA}$

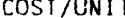

TOTAL COST:

$\$ 5,375.00$

$\$ 5,375.00$

REPORT NUMEER: $898355-41 \mathrm{C}$

INE ITEM NUMBER: 0020

REFERENCE NUMGER : 130 SO25 DATA ACQ ACEESSORY TR:GGER SLOPE: PROGRAM SELECTABLE, TRANSIYION EITHER O OOOO1 EA

COST $/ U N I T:$
TOTAL COST:

TOTAL COST:

$\$ 5,375.00$
$\$ 5,375.00$

FOR FURTHER INFORMATION
ELOY N. GIRON

(505) $844-2746$

.2746

NM 87185

TO FREEZE EOUIPMENT:

ELOY N. GIRON

ALBUQUERQUE

(505) $844-2746$

NM 37185

LOCATION OF EQUIPMENT

SANDIA NATIONAL LABORATORIES

DRODERTY REAPPLICATION DIV. 7617

ALBUOUEROUE 
EFFECTIVE DATE: 07/01/94 EXPIRATION DATE: 07/31/94

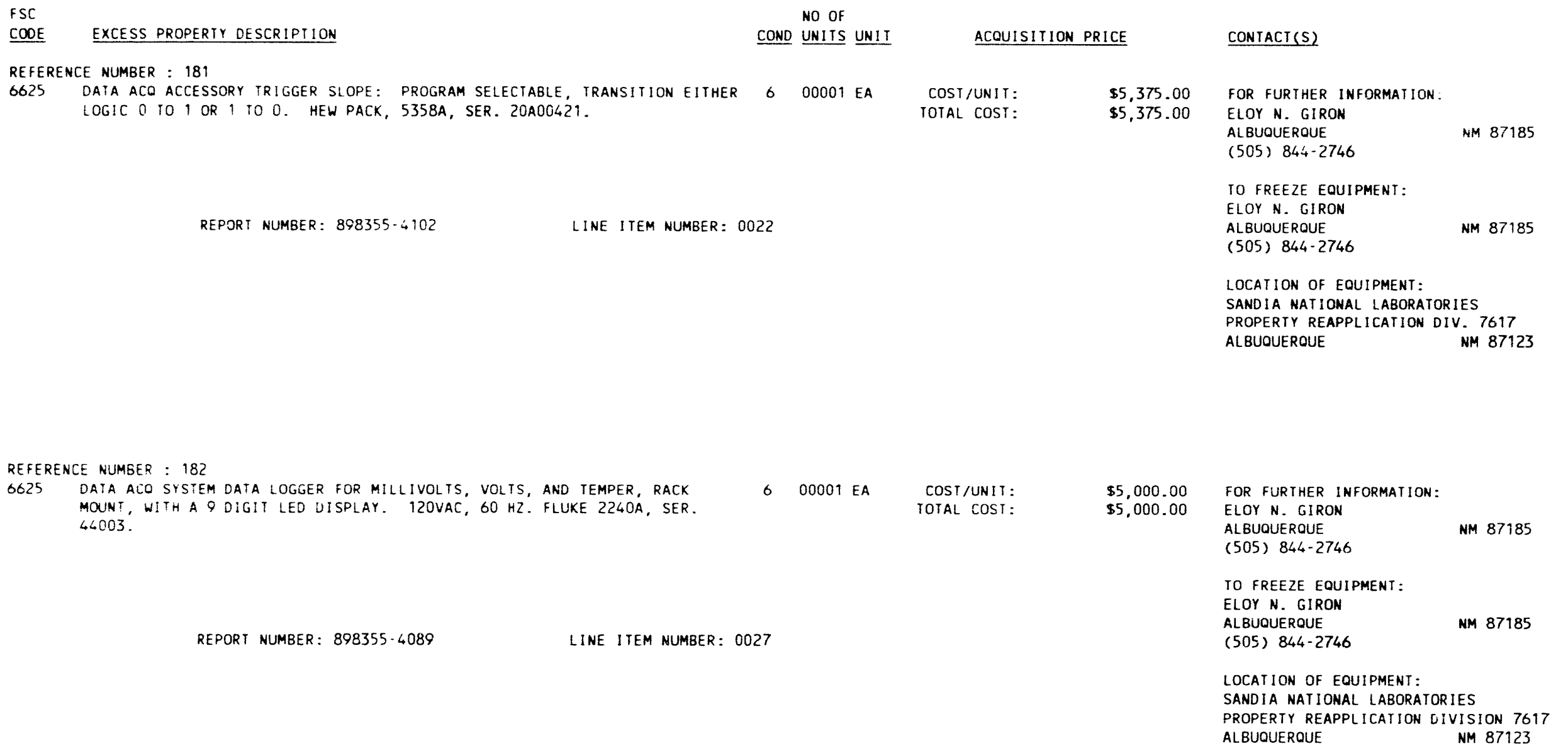


DOE USED ENERGY RELATED LABORATORY EQUIPMENT GRANT PROGRAM

EFFECTIVE DATE: $07 / 01 / 94$ EXPIRATION DATE: $07 / 31 / 94$

FSC

\section{COOE}

REFERENCE NUMBER : 183

6625 DATA LOGGER TYPE: DIG!IAL FLUKE MODEL: 224OA REF NO: 29936.02 ACO DATE: 2177

REPOR: NUMEER: $890531-6103$

LINE ITEM NLMBER: 0007

REFERENCE NUMBER : 184

6625 DATA LOGGER IYPE: DIGITAL FLUKE MODEL: 2240B ACO DATE: 10/82 REF NO: $29936-03$

REPORT NUMBER: $890531-4103$

LINE ITEM NUMBER: 0008

\section{NO OF}

COND UNIIS UNII

$500001 \mathrm{EA}$
COST/UNIT: TOTAL COST:

ACQUISITION PRICE

$\$ 6,206.00$ $\$ 6,206.00$
CONTACT(S)

FOR FURTHER INFORMATION:

MANCY J. SITTON

RICHLAND

WA 99352

TO FREEZE EQUIPMENT:

NANCY J. SITION

RICHLAND

(509) $376-5108$

WA 99352

LOCATION OF EOUIPMENT:

WEST INGHOUSE HANFORD COMPANY

ESS\&S, 1167A BLDG/1100 AREA

RICHLAND

HA 99352

COST/UNIT:

TOTAL COST:

$\$ 22,589.00$

$\$ 22,589.00$

FOR FURTHER INFORMATION:

NANCY J. SITTON

RICHLAND

WA 99352

TO FREEZE EQUIPMENT:

NANCY J. SITTON

RICHLAND

(509) $376-5108$

WA 99352

LOCATION OF EQUIPMENT:

WESTINGHOUSE HANFORD COMPANY

ESS\&S, 1167A BLDG/1100 AREA

RICHLAND

HA 90352 
EFFECTIVE DATE: $07 / 01 / 94$ EXPIRATION DATE: $07 / 31 / 94$ EXCESS PROPERTY DESCRIPIION

REFERENCE NUMBER : 185

O625 DEGAUSSER, TAPE THE DEGAUSSER IS USED FOR DEGAUSSING TAPES, AC AMPERES RANGE FROM OO 0 . H AS ONE CONIROL A POWER SWITCH TZOVAC, $60 \mathrm{HZ}$. CEC, RANGE FROM O TO 8 . H A
TD29 034 . SER. 9278.

REPORT NUMBER: $898355-4089$

LINE ITEM NUMBER: 0029

REFERENCE NUMBER : 180

6025 DENVO/NITER II MFR: TEXAS INST. INCROP., MODEL NO. PSO2WGA, S/N 7291-2, 6 00001 EA ACO. DA TE: 2/69, APPROX. WT: $25 \mathrm{LBS} ., 14 \mathrm{~W} \times 15 . \mathrm{L} \times 10^{\prime} \mathrm{H}$

REPORT NUMBER: $899105-4081$
NO OF COND UNITS UNIT ACQUISIIION PRICE

$600001 \mathrm{EA}$ COST/UNIT: TOTAL COST: $\$ 4.330 .00$
$\$ 4.330 .00$

\section{ELOY N. GIRON \\ ALBUQUERQUE}

(505) $844-2746$

NM 87185

TO FREEZE EQUIPMENT:

ELOY N. GIRON

ALBUOUEROUE

(505) $844-2746$

NM 87185

LOCATION OF EQUIPMENT:

SANDIA NATIONAL LABORATORIES

PROPERTY REAPPLICATION DIVISION 7617 ALBUOUERQUE NM 87123
COST/UNIT: TOTAL COST
$\$ 1.735 .00$
$\$ 1.735 .00$
FOR FURTHER INFORMATION:

LYNN E. SHACKEL FOOT

LIVERMORE

(510) $294-3067$

CA $94550-0969$

TO FREEZE EQUIPMENT:

LYNN E. SHACKELFOO

LIVERMORE

CA $94550-0969$

(510; $294-3067$

LOCATION OF EQUIPMENT:

SANDIA NATIONAL LABORATORIES

PROPERTY REAPPLICATION DEPT. 8532-1

LIVERMORE 
EFFECTIVE DATE: $07 / 01 / 94$ EXPIRATION DATE: $07 / 31 / 94$

$$
\text { ELIGIBLE EQUIPMENT }
$$

\section{FSC}

REFERENCE NUMBER : 187 .

6625 DIGITAL HUMIDITY ANALYZER MFR: EG+G, MOOEL NO. 911.

REPORT NUMEER: $899105-408$

INE ITEM NUMBER: 0017

\section{REFERENCE NUMBER : 188}

6625 DIGITAL MULTIMETER MODEL 6000, MFR RACAL DANA, SER\# 119573

REPORT NUMBER: $899110-0125$

LINE ITEM NUMBER: 0002
NO OF

COND UNITS UNIT

ACOUISITION PR!CE

$\$ 5,902.00$

$400001 E A$

COST/UNIT:

TOTAL COST:

$\$ 5,902.00$

LYRN ERTHER INFORMATIOH:

LYNN E. SHACKEL FOOT

CA $94550-0969$

TO FREEZE EQUIPMENT:

LYNN E. SHACKELFOOT

LIVERMORE

CA $94550-0969$

(510) $294-306$

LOCATION OF EQUIPMENT:

SANDIA NATIONAL LABORATORIES

PROPERTY REAPPLICATION DEPT. 8532- 1

LIVERMORE

CA 94550-0969$$
\text { CA } 94550-0969
$$

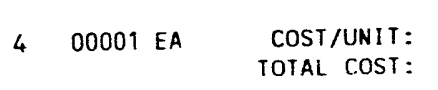

\section{$\$ 4,495.00$
$\$ 4,495.00$}

FOR FURTHER INFORMATION:

YOLANDA CUEVAS/MS DIV

OAKLAND

CA $94612-5208$

(510) 637-1767

TO FREEZE EQUIPMENT:

YOLANDA CUEVAS/MS DIV

OAKLAND

CA $94612-5208$

(510) $637-1767$

LOCATION OF EQUIPMENT:

GENERAL ATOMICS

3550 GENERAL ATOMICS COURT

SAN DIEGO

CA 92121 
EFFECTIVE DATE: 07/01/96 EXPIRATION DATE: 07/31/94

CODE EXCESS PROPERTY DESCRIPTION

REFERENCE NUMBER : 189

66.5 DIGITAL VOLTMEIER MOOEL MX-2, MFR. NON-LINEAR SYSTEM, SER\# 3508

REPORT NUMGER: $899110-0125$

LINE ITEM NUMBER: 0006

REFERENCE NUMBER : 190

DIGITAL VOLTMETER 104179, MANUF: NON-LINEAR SYSTEMS MODEL N/A S/N N/A

1 LOT, INCLUDES $104475,104642,104200$ AND 102734 POWER SUPPLIES. SIZE:

$23 " W \times 29 " F / R \times 25 " H$ EST WT $: 200$ LBS

MFGR YR: N/A

REPORT NUMBER: $896404-4133$

IINE ITEM NUMBER: 0001
NO OF

COND UNITS UNIT

ACQUISITION PRICE

$\$ 1,637.00$

$600001 \mathrm{EA}$

COST/UNIT:

TOTAL COST

$\$ 1,637.00$

YOLAMDA CUEVAS/MS DIV

OAKLAND

(510) $637-1767$

CA $94612-5208$

TO FREEZE EQUIPMENT:

YOLANDA CUEVAS/MS DIV

OAKLAND

(510) 637-1767

CA $94612-5208$

LOCATION OF EQUIPMENT:

GENERAL ATOMICS

3550 GENERAL ATOMICS COURT

SAN DIEGO

CA 92121

COST/UNIT:

TOTAL COST:

$\$ 2,300.00$

$\$ 2,300.00$

\section{FOR FURTHER INFORMATION: \\ BARBARA HOLMES, REP. \\ KANSAS CITY}

MO $64141-6159$

TO FREEZE EQUIPMENT

BARBARA HOLMES, REP.

KANSAS CITY

(816) 997-2976

MO 64141-6159

LOCATION OF EQUIPMENT

ALLIEDSIGNAL, INC.

2000 EAST 95 TH STREET

KANSAS CITY

MO 64131-3095 
EFFECIIVE DATE: 07/01/94 EXPIRATION DATE: $07 / 31 / 94$

FSC

CONE EXCESS PROPERTY DESCRIPTION

REFERENCE NUMBER : 191

O625 DIGITIZER-WAVEFORM, MFR. SEQUENCE INC., MOOEL 3100, ACO. YR, 8 8, PROP. NO. 4900139
No of

COND UNITS UNI

500001 EA
COST/UNIT: TOTAL COST:
$\$ 38,795.00$ $\$ 38,795.00$

LINE ITEM NUMBER: 0014
REFORT NUMEER: $899102-4150$
REFERENCE NUMBER : 192

SO25 DIGITREND 200 MFR: DORIC, MODEL NO. DS-200-6-K-3026-32-34-17, S/N

COST/UNIT:
TOTAL COSI
LINE ITEM NUMBER: 0018

REPORT NUMBER: $899105-4081$
CONTACTSS)

FOR FURTHER INFORMATION:

JOANNE BREZNIK

LIVERMORE

CA 94550

(510) $424-410$

TO FREEZE EQUIPMENT:

JOANNE BREZNIK

LIVERMORE

CA 94550

(510) $424-4101$

OCATION OF EQUIPMENT:

AURENCE LIVERMORE NATI IONAL LAB EXCESS SECTION, L-696 LIVERMORE

FOR FURTHER INFORMATION:

YNN E . SHACKELFOOT

LIVERMORE

(510) $294-3067$

CA $94550-0969$

TO FREEZE EQUIPMENT

LYNN E. SHACKELFOOT

LIVERMORE

(510) $294-3067$

CA $94550-0969$

LOCATION OF EQUIPMENT:

SAND IA NATIONAL LABORATORIES

PROPERTY REAPPLICATION DEPT. 8532-1

CA $94550-0969$ 
EFFECTIVE DATE: $07 / 01 / 94$ EXP!RATION DATE: $07 / 31 / 94$

\section{FSC \\ CODE EXCESS PROPERTY DESCRIPTION \\ NO OF}

REFERENCE NUMBER : 193

6625 DISCRIMINATOR AMPLIFIER DISCRIMINATOR WITH CONTROLS FOR MAIN AND MONITOR RATE D ISC. POWER FROM MAINFRAME. WORKS W/V957220. 930?, SER. 1052.

REPORT NUMGER: $898355-4102$

LINE ITEM NUMBER: 0025

REFERENCE NUMBER : 194

6025 DISCRIMINATOR OCTAL DISCRIMINATOR PLUG IN. PHILLIPS TM, TI1710, SER. 1338.
COND UNITS UNIT

ACQUISITION PRICE

$600001 E A$

COST/UNIT: TOTAL COST:

$\$ 2,204.00$ $\$ 2,204.00$

\section{FOR FURTHER INFORMATION:}

ELOY N. GIRON

(505) $844-2746$

NM 87185

TO FREEZE EQUIPMENT:

ELOY N. GIRON

ALBUQUEROUE

(505) $844-2746$

NM 87185

LOCATION OF EQUIPMENT:

SANOIA NATIONAL LABORATORIES

PROPERTY REAPPLICATION DIV. 7617

ALBUOUEROUE

NM 87123

NM 87123

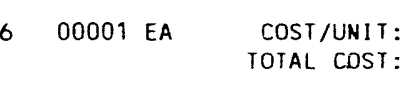

$\$ 1,102.00$ $\$ 1,102.00$
FOR FURTHER INFORMATION:

ELOY N. GIRON

ALBUQUERQUE

NM 87185

(505) $844-2746$

TO FREEZE EQUIPMENT:

ELOY N. GIRON

ALBUQUERQUE
(505) $844-2746$

NM 87185

LOCATION OF EQUIPMENT:

SANDIA NATIONAL LABORATORIES

PROPERTY REAPPLICATION DIV. 7617

ALBUOUEROUE

NM 87123 
EFFECTIVE DATE: $07 / 01 / 94$ EXPIRATION DATE: $07 / 31 / 94$

\section{COOE EXCESS PROPERIY DESCRIPTION}

REFERENCE NUMBER : 195

6625 DISCRIMINATOR OCIAL DISCRIMINATOR PLUG IN. PHILLIPS, 710, SER. 1315

REPORT NUMBER: $898355-4102$

LINE ITEM NUMBER: 0024

\section{REFERENCE NUMBER : 196}

6625 DISPLAY UNIT HAS A LARGE $9.6 \times 11.9 \mathrm{CM}$ DISPLAY FOR DIFFERENTIATING BETWEEN MANY G RAY SHADES OR FOR VIEHING IN BRIGHTLY LIGHTED AREAS. 12OVAC, 48/ 66HZ. HEW PACK, 1332A, 2252A187.

600001 EA OTAL COST
NO OF

COND UNITS UNI

\section{ACQUISITION PRICE}

$600001 E A$

COST/UNIT: TOTAL COST:

$\$ 1.102 .00$

$\$ 1,102.00$

\section{ELOY N. GIRON \\ ALBUQUERQUE}

(505) $844-2746$

NM 87185

TO FREEZE EQUIPMENT:

ELOY N. GIRON

ALBUQUERQUE

(505) $844-2746$

NM 87185

LOCATION OF EQUIPMENT :

SANDIA NATIONAL LABORATORIES PROPERTY REAPPLI ICATION DIV. 7617

PROPERTY REAPPLICATION DIV. 7617
FOR FURTHER INFORMATION:

ELOY N. GIRON

ALBUQUERQUE

(505) $844-2746$

NM 87185

TO FREEZE EQUIPMENT:

ELOY N. GIRON

ALBUQUERQUE

(505) $844-2746$

NM 87185

LOCATION OF EOUIPMENT

SANDIA NATIONAL LABURATORIES

PROPERTY REAPPLICATION DIVISION 7617

PROPERTY REAPPLICATION DIVISION 7617
ALBUDUEROUE

NM 87123 
EFFECTIVE DATE: $07 / 01 / 94$ EXPIRATION DATE: $07 / 31 / 94$

\section{COOE EXCESS PROPERTY DESCRIPTION}

REFERENCE NUMBER : 197

6625 ELECTROMETER DIGITAL ELECTROMETER WITH A 3-1/2 LED DIGIT READOUT, MEASURES VOL TS, CURRENT RESISTANCE AND CHARGE MEASUREMENTS. 12OVAC, 60 HZ. K EITHLEY, 616, SER. 25553.

REPORT NUMBER: $898355-4088$

LINE ITEM NUMBER: 0043

REFERENCE NUMBER : 198

6625 ELECTROMETER HAS A 5 DIGIT LED READOUT, MEASURES $V, 1, R$ AND $O$, AND IS A CURRENT SOURCE. 12OVAC, $60 \mathrm{HZ}$. KEITHLEY, 616, SER. 36329A.

REPORT NUMBER: $898355-4089$

LINE ITEM NUMBER: 0034
No of

COND UNITS UNIT

ACQUISITION PRICE

$\$ 1,417.00$

$00001 \mathrm{EA}$

COST/UNIT: TOTAL COST:

$\$ 1,417.00$

\section{ELOY N. GIRON \\ ALBUOUEROUE}

ELOY H. GIROM

(505) $844-2746$

NM 87185

TO FREEZE EQUIPMENT

ELOY N. GIRON

ALBUQUERQUE

(505) $844-2746$

NM 87185

LOCATION OF EOUIPMENT:

SANDIA NATIONAL LABORATORIES

PROPERTY REAPPLICATION DIVISION 7617

ALBUOIJERQUE

NM 87123

COST/UNIT:

TOTAL COST:

$\$ 1,335.00$

$\$ 1,335.00$

O0001 EA

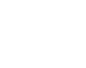

\section{FOR FURTHER INFORMATION: \\ ELOY N. GIRON \\ ALBUQUERQUE}

(505) $844-2746$

NM 87185

TO FREEZE EQUIPMENT:

ELOY N. GIRON

ALBUQUERQUE

(505) 844-2746

NM 87185

LOCATION OF EQUIPMENT:

SANDIA NATIONAL LABORATORIES

PROPERTY REAPPLICATION DIVISION 7617

ALBUQUERQUE

NM 87123 
EFFECTIVE DATE: 07/01/94 EXPIRATION DATE: 07/31/94

$F S C$

\section{COOE EXCESS PROPERIY DESCRIPTION}

REFERENCE NUMBER : 199

3025 ELECTROMETER USE FOR VOLTAGE, CURREN?, RESISTANCE AND CHARGE MEASUREMENTS AND CAN BE USED AS A CUREENT SOURCE. IZOVAC, $60 \mathrm{HZ}$. KE ITHLEY, 616, S ER. 44052A.

REFORT AUMEER: $898355-408$

LINE ITEM NUMBER: 0031

REFERENCE NUMBER : 200

DE 25 ELECTROMETER USE OOR VOLIAGE, CURREN RESISTANCE AND CHARGE MEASUREMENTS 500001 EA AND C AN BE USED FOR A CURRENT SOURCE. KEITHLEY, 616, SER. 44066A

REPORT NUMBER: $898355-4089$

LINE ITEM NUMBER: 0032

NO OF
COND UNITS UNIT

$500001 \mathrm{EA}$
ACOUISITION PRICE

COST/UNIT: TOTAL COST:
$\$ 1.417 .00$ 1.417 .00

A

CONTACT(S)

FOR FURTHER INFORMAIION: ELOY N. GIRON ALBUQUERQUE (505) $844-2746$

NM 87185

TO FREEZE EOUIPMENT:

ELOY N. GIRON

ALBUQUEROUE

(505) $844-2746$

NM 87185

LOCATION OF EQUIPMENT:

SANDIA NATIONAL LABORATORIES PROPERTY REAPPLICATION DIVISION 7617

ALBUQUEROUE NM 87123
COST/UNIT: IOTAL COST:
$\$ 1,417.00$ $\$ 1,417.00$
FOR FURTHER INFORMATION:

ELOY N. GIRON

ALBUQUERQUE

(505) $844-2746$

NM 87185

TO FREEZE EQUIPMENT :

ELOY N. GIRON

ALBUQUERQUE

(505) 844-2746

NM 87185

LOCATION OF EQUIPMENT:

SANDIA NATIONAL LABORATORIES PROPERTY REAPPLICATION DIVISION 7617 ALBUOUERQUE NM 87123 
EFFECTIVE DATE: $07 / 01 / 94$ EXPIRATION DATE: $07 / 31 / 94$

FSC

\section{COOE EXCESS PROPERTY DESCRIPTION}

REFERENCE NUMBER : 201 6625 ELECTROMETER USE FOR VOLTAGE, CURRENT, RESISTANCE AND CHARGE
MEASUREMENTS AND CAN BE USED FOR A CURRENT SOURCE. 12OVAC, $60 \mathrm{HZ}$. WILL NOT CALIBR ATE. KEITHLEY, 616, 44100A.

REPORT NUMBER: $898355-408$

LINE ITEM NUMBER: 0033

REFERENCE NUMBER : 202

6625 EXPANDER, 1/0 MFR: HEWLETT PACKARD, MODEL NO. 9878A, S/N 1625 A00251 ACQ. DATE : $3 / 77$, APPROX. WT: 35 LBS., $17 \% \mathrm{~W} \times 14^{\prime} \mathrm{L} \times 51 \mathrm{H}$.

LINE ITEM NUMBER: 0019

REPORT NUMBER: $899105-4081$
NO OF
COND UNITS UNIT

$700001 \mathrm{EA}$
ACQUISITION PRICE

COST/UNIT:

TOTAL COST:

$\$ 1,461.00$
CONTACTSS

FOR FURTHER INFORMATION

ELOY N. GIRON

ALBUOUERQUE

(505) $844-2746$

NM 87185

TO FREEZE EQUIPMENT :

ELOY N. GIRON

ALBUQUERQUE

(505) $844-2746$

NM 87185

LOCATION OF EQUIPMENT:

SANDIA NATIONAL LABORATORIES

PROPERTY REAPPLICATIOI DIVISION 7617 PROPERTY REAPPLICATIOH. DIVISION 7617
ALBUQUERQUE
NM 87123
$500001 \mathrm{EA}$

COST/UNIT:

TOTAL COST

$\$ 1,152.00$
FOR FURTHER INFORMATION:

LYNN E. SHACKELFOOT

LIVERMORE

(510) 294-3067

CA $94550-0969$

TO FREEZE EQUIPMENT:

LYNN E. SHACKELFOOT

LIVERMORE

(510) 294-3067

CA $94550-0969$

LOCATION OF EQUIPMENT:

SANDIA NATIONAL LABORATORIES

PROPERTY REAPPLIICATION DEPT. 8532-i

PROPERTY REAPPLICATION DEPT. $8532-1$
LIVERMORE
CA $94550-0969$ 
EFFECTIVE DATE: 07/01/94 EXPIRATION DATE: $07 / 31 / 94$

FSC

\section{COOE EXCESS PROPERIY DESCRIPTION}

REFERENCE NUMBER : 203

O625 FILTER MICROWAVE DIGITAL MICROWAVE FILTER WITH CALIBRATED CONTROLS. MOOES ARE DIS PLAY, SHEEP \& ON/OFF I2OVAC, 60 HZ. INTEGRA IN, IMF1800, SER. 9074263 .

REFERENCE NUMBER : 204

0625 FILTER MULTI-FUNCT DYNAMICS FILTER MULTI-FUNCT INPUT \& OUTPUT HIGH PASS CURT OFF FRE O RANGE FROM 10 TO 100. ON/OFE SWITCH. I2OVAC, $60 \mathrm{HZ}$. DYNAM ELEC, 6364AGQ, 3044
NO OF

$\$ 3,390.00$ $\$ 3,390.00$

TOIAL COST:

00001 EA

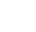

(1)


DOE USED ENERGY RELATED LABORATORY EOUIPMENT GRANT PROGRAM ELIGIBLE EQUIPMENT

EFFECIIVE DATE: OT/01/94 EXPIRATION DATE: $07 / 31 / 94$

FSC
CODE EXCESS PROPERTY OESCRIPTIION

REFERENCE NUMBER : 205

60?5 FORMATIER, TAPE REFORMATS IHE OUTPUT OF THE $7 D 10$ IN A CHOICE OF 5

DISPLAY FORMATS INCLUDING TIMING. WORKS H/V926458. POWER FROM SCOPE.
COND UNITS UNIT

$600001 E A$
ACQUISITION PRICE

COST/UNIT: TOTAL COST:
LINE ITEM NUMBER: 0036
REPORT NUMBER: $898355-4089$
REFERENCE NUMBER : 206

6625 FORMATTER, TAPE REFORMATS THE OUTPUT OF THE 7001 IN A CHOICE OF 5 DISPLAY FORMATS INCLUDING TIMING. WORK W/V926460. POWER FROM SCOPE.
$600001 \mathrm{EA}$
COST/UNIT:

TOTAL COST:
$\$ 4.390 .00$ $\$ 4,390.00$ $\$ 4,390.00$
$\$ 4,390.00$

\section{CONIACT(S)}

FOR FURTHER INFORMATION:

ELOY N. GIRON

ALBUQUERQUE

(505) $844-2746$

NM 87185

TO FREEZE EQUIPMENT:

ELOY N. GIRON

ALBUQUERQUE

(505) $844-2746$

NM 87185

LOCATION OF EQUIPMENT:

SANDIA NATIONAL LABORATORIES

SANDIA NATIONAL LABORATORILS
PROPERTY REAPPLICATION DIVISION 7617

PROPERTY REAPPLICATION DIVISION 7617
ALBUQUEROUE NM 87123

REPORY NUMBER: $898355-4089$

LINE ITEM NUMBER: 0037
FOR FURTHER INFORMATION:
ELOY N. GIRON
$\begin{aligned} & \text { ALBUQUERQUE } \\ & \text { (505) } 844-2746\end{aligned}$

TO FREEZE EQUIPMENT:

ELOY N. GIROH

ALBUOUERQUE

(505) $844-2746$

NM 87185

LOCAIION OF EQUIPMENT:

SANDIA NATIONAL LABORATORIES

PROPERTY REAPPLICATION DIVISION 7617

PROPERTY REAPPLICATION DIVISION 7617
ALBUQUERQUE 
EFFECTIVE DATE: 07/01/94 EXPIRATION DATE: 07/31/94

FSC

$\operatorname{cose}$

EXCESS PROPERTY DESCRIPIION

COND UNITS UNIT

ACOUISITION PRICE

REFERENCE NUMBER : 207

6625 FOTONIC SENSOR MFR: MECHANICAL TECH. INC., MODEL NO. KD-67, S/N 66703, 500001 EA ACO. DAT E: 1/67. APPROX. WT: $10 \mathrm{LBS}, 6 . W \times 8 . \mathrm{L} \times 9 . \mathrm{H}$.

REPORT NLMGER: $899105-408$

LINE ITEM NUMBER: 0020

REFERENCE NUMBER : 208

6025 FREO SYNTHES:ZER FREO RNAGE OF 200HZ-81 MHZ, GENERATOR HAS PERFORMANCE CHARACIER!S T!CS IHAT MAKE IT IDEALLY SUITED FOR THE TELECOMMUNICATIONS INOUS TRY. 12OVAC, 60 HZ. HEW PACK, 3325A, 25 $22 \mathrm{AZ}$

$600001 \mathrm{EA}$

TOTAL COST:
COST/UNIT:

$\$ 4,050.00$

$\$ 4,050.00$

$\$ 1,526.00$ $\$ 1,526.00$

COST/UNIT: TOTAL COST:

$$
\begin{aligned}
& \text { LYNN E. SHA } \\
& \text { LIVERMORE } \\
& (510) 294
\end{aligned}
$$

$-3067$

ELUIPMENT:

LYNN E. SHACKELFOOT

LIVERMORE

(510) $294-3067$

LOCATION OF EQUIPMENT:

SANDIA NATIONAL LABORATORIES

PROPERTY REAPPLICATION DEPT. $8532-i$

LIVERMORE CA $94550-0969$

FOR FURTHER INFORMATION:

ELOY N. GIRON

ALBUQUEROUE

(505) 844-27:4

NM 87185

TO FREEZE EQUIPMENT:

ELOY N. GIRON

ALBUOUEROUE

(505) $844-2746$

NM 87185

LOCATION OF EQUIPMENT:

SANDIA NATIONAL LABORATORIES

PROPERTY REAPPLICATION DIVISION 7617

ALBUOUERQUE

NM 87123 
EFFECTIVE DATE: 07/01/94 EXPIRATION DATE: 07/31/94

FSC

\section{COOE EXCESS PROPERTY DESCRIPTION}

REFERENCE NUMBER : 209

6625 FREO SYNTHESIZER IMHZ RESOLUTION, HIGH SPECTRAL PURITY, PRECISION AMPLITUDE CONTRO L, PROGRAM STORAGE, HAS A HPIB PLUG IN BACK. 12OVAC, $60 \mathrm{HZ}$. HEH PACK, 3335A, SER. $40 A 00112$.

REFERENCE NUMBER : 210

6625 FREQ SYNTHESIZER THE FREO SYNTHESIZER ATIENUATION -DB RANGE FROM 1 TO

4O, HAS REMO TE AND POWER SWITCH, 12OVAC, OO HZ . NEE

SWITCH IS GROKEN. ROCKLAND, 510002, SER. 51198.

REPORT NUMBER: $898355-4090$

LINE ITEM NUMBER: 0067
NO OF

COND UNITS UNIT

ACOUISITION PRICE

600001 EA

COST/UNIT: TOTAL COST:

$\$ 7,464.00$ $\$ 7.464 .00$

\section{FOR FURTHER INFORMATION:}

ELOY N. GIRON

(505) $844-2746$

NM 87185

TO FREEZE EQUIPMENT:

ELOY N. GIRON

ALBUOUERQUE

(505) $844-2746$

NM 87185

LOCATION OF EQUIPMENT:

SANDIA NATIONAL LABORATORIES

PROPERTY REAPPLICATION DIVISION 7617

ALBUOUEROUE

NM 87123

\section{EA \\ COST/UNIT: \\ TOTAL COST:} $\$ 2.629 .00$
FOR FURTHER INFORMAIION:

ELOY N. GIRON

ALBUOUEROUE

NM 87185

TO FREEZE EQUIPMENT

ELOY N. GIRON

ALBUQUERQUE

(505) $844-2746$

NM 87185

LOCATION OF EOUIPMENT:

SANDIA NATIONAL LABORATORIES

PROPERTY REAPPLICATION DIVISION 7617

ALBUQUERQUE

NM 87123 
EFFECIIVE DATE: $07 / 01 / 94$ EXPIRATION DATE: $07 / 31 / 94$

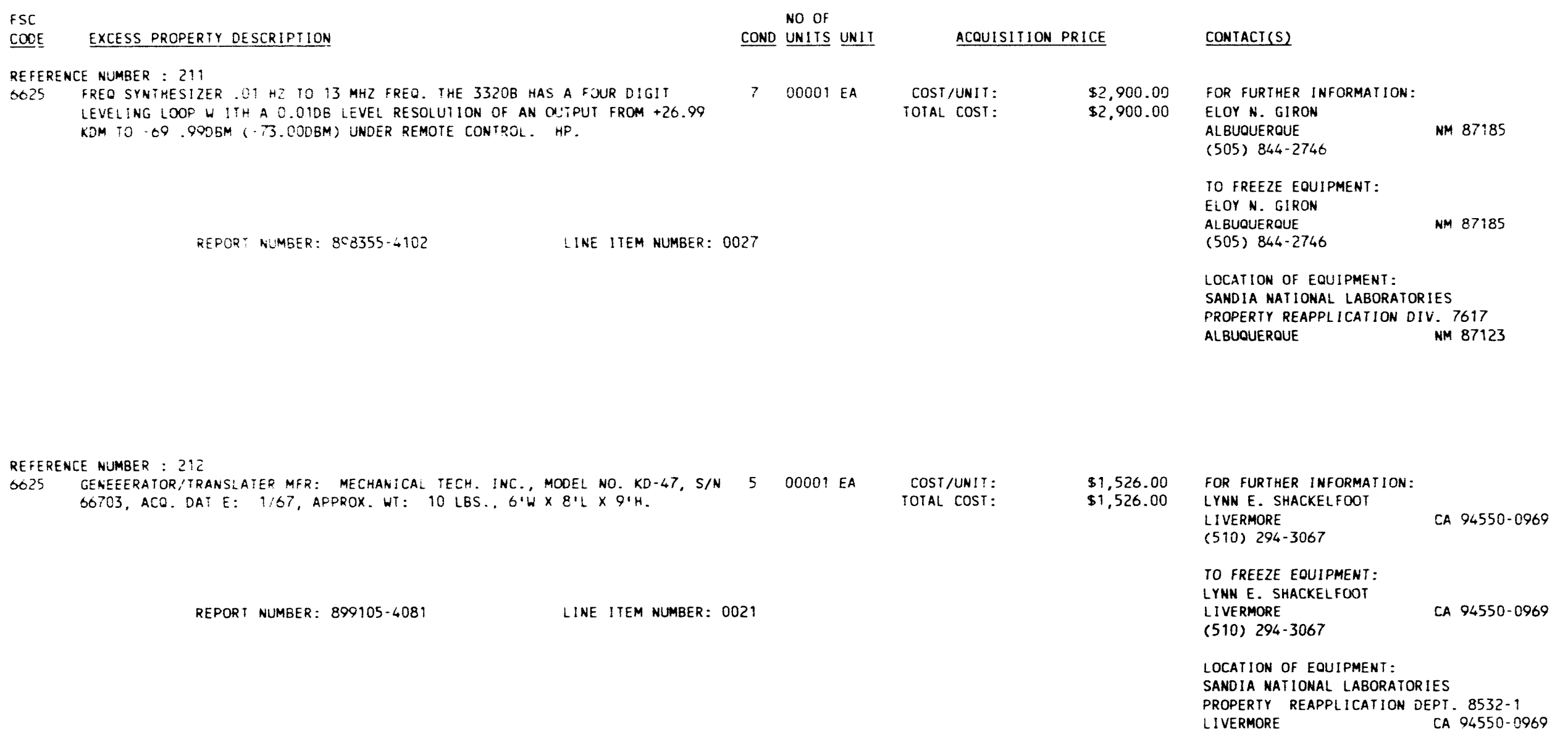


EFFECTIVE DATE: $07 / 01 / 94$ EXPIRATION DATE: $07 / 31 / 94$

\section{COOE EXCESS PROPERTY DESCRIPIION}

REFERENCE NUMBER : 213

6625 GENERAL, FUNCTION $0.002 \mathrm{HZ}$ TO $20 \mathrm{MHZ}$ UP TO $20 \mathrm{~V}$ PP FROM 50 OHMS, $10 \mathrm{NS}$ RISE/FALL $10 \%$ TO $90 \%$ VARIABLE SYMMETRY IN $1 \%$ STEPS. POWER FROM SCOPE.

REPORT NUMBER: $898355-4089$

INE ITEM NUMBER: 0038

REFERENCE NUMBER : 214

6625 GENERATOR FIXED MFR: HEWLETT PACKARD MOOEL: H021920A S/N: 265

REPORT NUMBER: $899305-4032$

LINE ITEM NUMBER: 0010
NO OF

COND UNITS UNIT

ACQUISITION PRICE

$600001 E A$

COST/UNIT: TOTAL COST:

$\$ \$, 132.00$

$\$ 4,132.00$

ELOY N. GIRON
ALBUOUEROUE

(505) $844-2746$

NM 87185

TO FREEZE EQUIPMENT:

ELOY N. GIRON

ALBUQUEROUE

(505) $844-2746$

NM 8718

LOCATION OF EQUIPMENT:

SANDIA NATIONAL LABORATORIES

PROPERTY REAPPLICATION DIVISION 7617

ALBUQUERQUE

NM 87123

COST/UNIT: TOTAL COST:

$\$ 2,079.45$

OR FURTHER INFORMATION :

CYNDI DELLNG

(702) $295-6753$

NV $89193-8521$

TO FREEZE EQUIPMENT:

CYNDI DELONG

LAS VEGAS

(702) $295-6753$

NV $89193-8521$

OCATION OF EQUIPMENT :

REYNOLDS ELECTRICAL \& ENGINEERING CO NEVADA TEST SITE

MERCURY

NV 89023 
EFFECTIVE DATE: 07/01/94 EXPIRATION DATE: $07 / 31 / 94$

FSC

\section{COOE EXCESS PROPERTY DESCRIPIION}

REFERENCE NUMBER : 215

6625 GENERATOR PLUG IN FOR THE 8690B SHEEP OSCILLATOR POWER RANGE 11 TO 4 GHZ. POWER FR OM OSCILLATOR. WORKS W/V807790. HEW PACK, 86998, SER. 91900277 .

REPORT NUMBER: $898355-4089$

LINE ITEM NUMBER: 0040

\section{REFERENCE NUMBER : 216}

6625 GENERATOR PLUG IN 1900 HP GENERATOR, 50V MAX AMPLITUDE, 2.5NS MINIMUM VARIABLE TRAM SITIONS, 100 MHZ MAX REPETIIIOM RATE. POWER COMES FROM GENERATOR , WORKS W/V926453.

REPORT NUMBER: $898355-4089$

LINE ITEM NUMBER: 0043
No of

COND UNITS UNIT

ACOUISITION PRICE

$600001 \mathrm{EA}$

COST/UNIT:

TOTAL COST:

$\$ 4,550.00$

$\$ 4,550.00$

ALBUQUERQUE

(505) $844-2746$

NM 87185

TO FREEZE EQUIPMENT:

ELOY N. GIRON

ALBUQUERQUE

(505) $844-2746$

NM 87185

LOCATION OF EQUIPMENT:

SANDIA NATIONAL LABORATORIES

PROPERTY REAPPLICAIION OIVISION 7617

ALBUDUERQUE

NM 87123

COST/UNIT:

TOTAL COST:

$\$ 3,312.00$

$\$ 3,312.00$

INFORMATION:

ELOY N. GIRON

ALBUQUEROUE

(505) $844-2746$

NM 87185

TO FREEZE EQUIPMENT:

ELOY N. GIRON

ALBUDUERQUE

(505) $844-2746$

NM 87185

OCATION OF EQUIPMENT:

SANDIA NATIONAL LABORATORIES

PROPERTY REAPPLICATION DIVISION 7617

ALBUOUERQUE

87123 
EFFECTIVE DATE: $07 / 01 / 94$ EXPIRATION DATE: $07 / 31 / 94$

FSC

COOE EXCESS PROPERTY DESCRIPTION

REFERENCE NUMBER : 217

6625 GENERATOR PULSE MFR: HEWLETI PACKARD MODEL: $8690 \mathrm{~B}$ S/N: $513 A 05430$

REPORT NUMBER: $899305-4039$

LINE ITEM NUMBER: 001

REFERENCE NUMBER : 218

6625 GENERATOR PULSE MFR: E-H RES MODEL

S/N: $\quad$ A370435

REPORT NUMBER: $899305-4032$
No of

COND UNITS UNIT

ACQUISITION PRICE

$600001 E A$ COST/UNIT:
TOTAL COST:

$\$ 2,356.20$ $\$ 2,356.20$
$600001 \mathrm{EA}$
LINE ITEM NUMBER: 0013
$\operatorname{COST/UNIT:}$

TOTAL COST:

\section{$\$ 3.450 .23$ \\ $\$ 3,450.23$}

FOR FURTHER INFORMATION:

CYNDI DELONG

LAS VEGAS
(702) $295-6753$

NV $89193-8521$

TO FREEZE EQUIPMENT:

CYNDI DELONG

LAS VEGAS

(702) $295-6753$

NV $89193-8521$

LOCATION OF EQUIPMENT:

REYNOLDS ELECTRICAL \& ENGINEERING CO NEVADA TEST SITE

MERCURY

NV 89023 
EFFECTIVE DATE: $07 / 01 / 94$ EXPIRAIION DATE: $07 / 31 / 94$

FSC

\section{CODE EXCESS PROPERTY DESCRIPTION}

REFERENCE NUMBER : 219

6625 GENERATOR PULSE MFR: E-H RES MODEL: 125A $S / N: \quad 694172$

REPORT NUMBER: $899305-4032$

REFERENCE NUMBER : 220

6625 GENERATOR PULSE MFR: E-H RES MODEL: $125 \mathrm{~A}$ $S / N: 694188$

REPORT NUMBER: $899305-4032$
ELIGIBLE EQUIPMENT
No of

COND UNITS UNIT

$600001 E A$

COST/UNIT:
TOTAL COST:

LINE ITEM NUMBER: 0014
COST/UNIT:
TOTAL COST:
ACQUISITION PRICE

$\$ 2,910.00$ $\$ 2,910.00$
FOR FURTHER INFORMATION:

CYNDI DELONG

LAS VEGAS

(702) 295-6753

NV $89193-852$

TO FREEZE EQUIPMENT:

CYNDI DELONG

LAS VEGAS

(702) $295-6753$

NV $89193-8521$

LOCATION OF EQUIPMENT:

REYNOLDS ELECTRICAL \& ENGINEERING CO

NEVADA TEST SITE

MERCURY

NV 89023

LINE ITEM NUMBER: 0015

\author{
FOR FURTHER INFORMATION: \\ CYNDI DELONG \\ LAS VEGAS
(702) $295-6753$ \\ NV $89193-852$ \\ TO FREEZE EQUIPMENT \\ CYNDI DELONG \\ LAS VEGAS \\ (702) $295-6753$ \\ NV 89193-8521 \\ LOCATION OF EQUIPMENT: \\ REYNOLDS ELECTRICAL \& ENGINEERING CO \\ NEVADA TEST SITI
}

MERCURY

NV 89023 
EFFECTIVE DATE: $07 / 01 / 94$ EXPIRATION DATE: $07 / 31 / 94$

FSC

\section{COOE EXCESS PROPERTY DESCRIPTION}

REFERENCE NUMBER : 221

6625 GENERATOR, FREQ MAR DIGITAL MARKER PROVIDES FIVE INDEPENDENT,

CONTINUOUSLY VARIALE FR EO MARKERS. 120VAC, 60 HZ. HEW PACK, 8600A, $48 \mathrm{~A} 00255$.

LINE ITEM NUMBER: 0031

REFERENCE NUMBER : 222

GENERATOR, FUNCTION DIGITIZING OSCILLOSCOPE, FULLY PROGRAMMABLE WITH FEATURES AS AUTO SCALING, AUTO TRIGGERING, SELF CALIBRATION AND DIRECT READOUT OF DELTA VOLTAGE AND DELTA TIME. HEW PACK.

REPORT NUMBER: $898355-4102$

LINE ITEM NUMBER: 0032

NO OF

COND UNITS UNIT

ACQUISITION PRICE

600001 EA COST/UNIT:
TOTAL COST:

$\$ 1,078.00$ $\$ 1,078.00$ (505) $844-2746$

NM 87185

TO FREEZE EQUIPMENT

ELOY N. GIRON

ALBUQUERQUE

(505) 844-2746

NM 87185

LOCATION OF EQUIPMENT:

SANDIA NATIONAL LABORATORIES

PROPERTY REAPPLICATION DIV. 7617

ALBUQUERQUE

NM 87123

$600001 \mathrm{EA}$

COST/UNIT:

TOTAL COST

$\$ 1,650.00$

$\$ 1,650.00$

FOR FURTHER INFORMATION :

ELOY N. GIRON

ALBUQUERQUE

(505) $844-2746$

NM 87185

TO FREEZE EQUIPMENT:

ELOY N. GIRON

ALBUQUERGUE

(505) $844-2746$

NM 87185

LOCATION OF EQUIPMENT:

SANDIA NATIONAL LABORATORIES

PROPERTY REAPPLICATION DIV. 7617

ALBUQUEROUE

NM 87123 
EFFECTIVE DATE: $07 / 01 / 94$ EXPIRATION DATE: $07 / 31 / 94$

FSC

REFERENCE NUMBER : 223

6625 GENERATOR PLUG IN 7 O TO 12.4 GHZ WITH POWER LEVEL CONTROL, HILL PLUG IN FROM THE B ACK. 12OVAC, 60 HZ. HEW PACK, 86948, 72000460.

REPORT NUMBER: $898355-4102$

LINE ITEM NUMBER: 0030

\section{REFERENCE NUMBER : 224}

GENERATOR, PULSE ATTENUATOR O TO $120 B$ IN IDB STEPS, REPETITION RATE SOURCES $100 \mathrm{HZ}$ TO 1 MHZ IN 4 RANGES. 115;230VAC, 50-60 HZ. HEW PACK, $215 A$, SER -245.

REPORT NUMBER: $898355-4088$

LINE ITEM NUMBER: 004
No OF

COND UNITS UNIT

ACOUISITION PRICE

$700001 \mathrm{EA}$

COST/UNIT:

TOTAL COST:

$\$ 2,600.00$

$\$ 2,600.00$

ALBUQUEROUE

(505) $844-2746$

TO FREEZE EQUIPMENT :

ELOY N. GIRON

(505) $844-2746$

NM 87185

LOCATION OF EQUIPMENT:

LABORATORIES

PROPERTY REAPPLICATION DIV. 76

ALBUOUERQUE

NM 87123
$600001 \mathrm{EA}$

COST/UNIT:

TOTAL COST :
$\$ 1.875 .00$

$\$ 1,875.00$
FOR FURTHER INFORMATION:

ELOY N. GIRON

ALBUQUERQUE

(505) $844-2746$

NM 87185

TO FREEZE EQUIPMENT:

ELOY N. GIRON

(505) $844-2746$

NM 87185

LOCATION OF EQUIPMENT:

SANDIA NATIONAL LABORATORIES

PROPERTY REAPPLICATION DIVISION 7617

ALBUQUERQUE

NM 87123 
EFFECTIVE DATE: 07/01/94 EXPIRATION DATE: 07/31/94
FSC

CODE EXCESS PROPERTY DESCRIPIION

REFERENCE NUMBER : 225

6625 GENERATOR, PULSE THE 1900A MAINFRAME PROVIDES HOUSING, POWER SUPPLIES

AND RFI SHEI LDING FOR ALL 1900 SYSTEM PLUG INS. 115-230V, 48-66 HZ

AND RFI SHEI LDING FOR A
HEW PACK, 1900A, 00943.

REPORT NUMBER: $898355-4102$

LINE ITEM NUMBER: 0034

REFERENCE NUMBER : 226

6625 GENERATOR, PULSE WITH CONTROLS FOR REPETITION RATE, PULSE DELAY, PUISE WIDTH AND A MPLITUDE WITH OUTPUT CONNECTIONS IN FRONT. 12OVAC, $60 \mathrm{HZ}$. DATA P ULSE. 110A, SER. 39227.
NO OF

COND UNITS UNIT

$700001 \mathrm{EA}$
COST/UNIT: TOTAL COST:

$\$ 2,850.00$

ELOY N. GIRON
ALBUQUERQUE
(505) $844-2746$

TO FREEZE EOUIPMENT

ELOY N. GIRON

ALBUQUERQUE

(505) $844-2746$

LOCATION OF EOUIPMENT:

SAMDIA MATIONAL LABORATC

SANDIA NATIONAL LABORATORIES

AL BUQUERQUE
ACQUISITION PRICE

PROPERTY REAPPLICATION DIV. 7617
COST/UNIT:

TOTAL COST:
$\$ 1.244 .00$
$\$ 1,244.00$

FOR FURTHER INFORMATION:

ELOY N. GIRON

ALBUOUEROUE

NM 87185

(SOS) $844-2746$

TO FREEZE EQUIPMENT:

ELOY N. GIRON

ALBUQUEROUE

(505) $844-2746$

NM 87185

LOCATION OF EOUIPMENT:

SAMDIA MATIONAL LABORATORIES

SANDIA MATIONAL LABORATORIES
PROPERTY REAPPLICATION DIV. 7617

PROPERTY REAPPLICATION DIV. 7617
ALBUOUERQUE 
EFFECTIVE DATE: $07 / 01 / 94$ EXPIRATION DATE: $07 / 31 / 94$

FSC

\section{CODE EXCESS PROPERTY DESCRIPTION}

REFERENCE NUMBER : 227

6625 GENERATOR, SIGNAL THE 6068 IS A MASTER OSCILLATOR-POWER AMPLIFIER AND PROVIDES $50 \mathrm{~K} \mathrm{HZ}$ TO 65MHZ FREQ RANGE IN 6 BANDS. 120VAC, $60 \mathrm{HZ}$. HEW PACK, 606B R, SER. 60800283.

REPORT NUMBER: $898355-4089$

LINE ITEM NUMBER: 0042

REFERENCE NUMBER : 228

6625 GENERATOR, SIGNAL RNGE 50KHZ TO 65MHZ FREO IN 6 BANDS, AUXILIARY RF OUTPUT ON FRNT PANEL. 12OVAC, $60 \mathrm{HZ}$. NEEDS CALIBRATION. HEW PACK, 606BR, 72400820

REPORT NUMBER: $898355-4102$

LINE ITEM NUMBER: 0029

NO OF

\section{COND UNIIS UNII ACOUISITION PRICE}

600001 EA COST/UNIT: TOTAL COST:

\section{$\$ 1,589.00$} $\$ 1,589.00$

FOR FURTHER INFORMATION:

ELOY N. GIRON

ALBUOUERQUE

(505) $844-2746$

NM 87185

TO FREEZE EQUIPMENT

ELOY N. GIRON

ALBUQUERQUE

NM 87185

(505) $844-2746$

LOCATION OF EQUIPMENT:

SANDIA NATIONAL LABORATORIES

PROPERTY REAPPLICATION DIVISION 7617

ALBUQUERQUE

\section{$00001 E A$ \\ COST/UNIT: \\ TOTAL COST:}

$\$ 1,497.00$

$\$ 1,497.00$

FOR FURTHER INFORMATION :

ELOY N. GIRON

ALBUOUERQUE

$844-2746$

TO FREEZE EQUIPMENT:

ELOY N. GIRON

ALBUQUEROUE

(505) $844-2746$

LOCATION OF EQUIPMENT

SANDIA NATIONAL LABORATORIES

PROPERTY REAPPLICATION DIV. 7617

ALBUQUERQUE 
EFFECTIVE DATE: $07 / 01 / 94$ EXPIRATION DATE: $07 / 31 / 94$

FSC

\section{CODE EXCESS PROPERTY DESCRIPTION}

REFERENCE NUMBER : 229

6625 GENERATOR, SWEEP SHEEP WIDTH RANGE 0-1400 MHZ IN 3 BANDS WITH A START AND STOP CON TROLS. 12OVAC, $60 \mathrm{HZ}$. WAVETEK, 2001 , SER. 094039.

COND UNITS UNII

$500001 E A$

COST/UNIT:
TOTAL COST:

REPORT NUMBER: $898355-4089$

LINE ITEM NUMBER: 0045
ACQUISITION PRICE

$\$ 1,636.00$ $\$ 1,636.00$

AL

\section{FOR FURTHER INFORMATION: \\ ELOY N. GIRON}

ALBUOUERQUE

NM 87185

TO FREEZE EQUIPMENT:

ELOY N. GIRON

ALBUQUERQUE

(505) $844-2746$

NM 87185

LOCATION OF EOUIPMENT:

SAMDIA NATIONAL LABORATORIES

PROPERTY REAPPLICATION DIVISION 7617

ALBUDUEROUE

MM 87123

REFERENCE NUMBER : 230

6625 GENERATOR SHEEP RACK MOUNT WITH 5 ELECTRONIC DUALS VERIER RANGE FROM 0

TO 90.12 OVAC, 60 HZ. WORKS H/949559, V948799, V949535. 5000A, SER.

$600001 \mathrm{EA}$

COST/UNIT:

1440.

$\$ 1.490 .00$

$\$ 1,490.00$

FOR FURTHER INFORMATION

ELOY N. GIRON

ALBUOUEROUE

NM 87185

TO FREEZE EQUIPMENT

ELOY N. GIRON

ALBUOUEROUE

(505) $844-2746$

NM 87185

LOCATION OF EQUIPMENT:

SANDIA NATIONAL LABORATORIES

PROPERTY REAPPLI ICATION DIV. 7617

ALBUOUEROUE

NM 87123 
EFFECTIVE DATE: 07/01/94 EXPIRATION DATE: $07 / 31 / 94$

COOE EXCESS PROPERTY DESCRIPTION

REFERENCE NUMBER : 231

6 C25 GENERATOR/TRANSLATOR RACK MOUNT HAS A 9 LED DIGII READOUT FOR DAYS GENERATOR/TRANSLATOR RACK MOUNT HAS A 9 LED DIGII READOUT FOR DAYS,
HOURS, MINUTES ANO SECONDS. 120VAC, 60 HZ. OATATRON, 3000, SER. 109.

REPORT NUMBER: $898355-4102$

LINE ITEM NUMBER: 0028
No of COND UNIIS UNIT

ACQUISIIION PRICE

COST/UNIT: TOTAL COST:

$\$ 5.979 .00$ $\$ 5,979.00$

$00001 \mathrm{EA}$

ELOY N. GIRON

AL BUOUERQUE

M 87185

TO FREEZE EOUIPMENT:

ELOY N. GIRON

AL BUOUERQUE

(505) $844-2746$

NM 87185

LOCAIION OF EQUIPMENT:

SANDIA NATIONAL LABORATOR!ES

PROPERTY REAPPLICATION DIV. 7617

ALBUOUEROUE

NM 87123

REFERENCE NUMBER : 232

REFERENCE NUMBER : 232
OO25 GRANITE TAELE MFR: TRI-SSIGMA. ACO. DATE: 1;80. APPROX WT: 3000 LBS., 400001 EA $72.4 \times 6011 \times 3614$

COSTIUNIT: TOTAL COST:

$\$ 80,000.00$ $\$ 80,000.00$

\section{FOR FURTHER INFORMATION: \\ LYNN E. SHACKEL FOOT \\ LIVERMORE \\ (510) $296-3067$}

CA $94550-0969$

TO FREEZE EOUIPMENT:

LYWN E. SHACKELFOOT

LIVERMORE

(510) $294-3067$

LOCATION OF ECUIPMENT

SAMDIA NATIONAL LABORATORIES

PROPERTY REAPPLICATION DEPT. $8532-1$

PROPERTY REAPPLICATION DEPT. $8532-1$
LIVA $94550-0969$ 
EFFECTIVE DATE: 07/01/94 EXPIRATION DATE: 07/31/94

COOE EXCESS PROPERTY DESCRIPTION

REFERENCE MUMBER : 233

6625 HD REVERSING POWER UNI! MOOEL 77750, MFR. ROCKWELL, SER\# 12040

REPORT NUMEER: $899110-0125$

LINE IIEM NUMBER: 0007

REFERENCE NUMBER : 234

6625 HYGROMETER 74569. MANUF: PANAMETRICS MODEL VT00030 S/N S590219

IEACH. SIZE: 13 "W $\times 11$ "F/R $\times$ 8"H EST WT: 10 LBS MFGR YR: 1988

COST/UNIT:

TOTAL COST:

$\$ 1,448.00$
$\$ 1.448 .00$

LINE ITEM MUMBER: 0017 no of

COND UNIIS UNIT

ACQUISITION PRICE

$\$ 1,000.00$

$\$ 1.000 .00$
REPORT NUMBER: $896404 \cdot 4124$

FOR FURTHER INFORMATION:

YOLAMDA CUEVAS/MS DIV

OAKLAND

(510) 637-1767

CA 94612-5208

TO FREEZE EQUIPMENT:

YOLANDA CUEVAS/MS DIV

OAKLAND

(510) $637-1767$

LOCATION OF EQUIPMENT:

GENERAL ATOMICS

ics cour

SAN DIEGO

CA 92121

FOR FURTHER INFORMATION :

BARBARA HOLMES, REP.

KANSAS CITY

(816) $997-2976$

MO $64141-6159$

TO FREEZE EOUIPMENT:

BARBARA HOLMES, REP.

KANSAS CIIY

(816) $997-2976$

MO $64141-6159$

LOCATION OF EQUIPMENT

ALLIEDSIGNAL, INC.

2000 EAST 95TH STREET

KANSAS CITY

MO $64131-3095$ 
EFFECTIVE DATE: $07 / 01 / 94$ EXPIRATION DATE: $07 / 31 / 94$

FSC

COOE EXCESS PROPERTY CESCRIPTION

REFERENCE MUMBER : 235

6625 IMPEDANCE BRIDGE 42636, MANUF: GENERAL RADIO MODEL GR 1683 S/N 356-A

1 EACH, THIS AUTOMATIC RLC BRIDGE HAS A RANGE OF 0 TO $0.1 \mathrm{PF}$ TO 1.9999

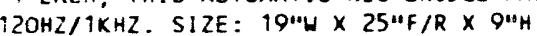

EST HT: 75 LBS MFGR YR: 1972

REPORT NUMEER: $890404-4133$

LINE ITEM NUMBER: 0023

REFERENCE NUMBER : $23 t$

6625 INDUCATOR PRESSURE HITH A $\angle$ DIGIT READOUT AND A PRESSURE CONNECTION IN 600001 EA BACK. 120 VA C, $60 \mathrm{HZ}$. DRESS IND, 7781, SER. 4392.

REPORT NUMBER: $898355-4089$

LINE ITEM NUMBER: 0039
COND UMITS UNIT ACOUISITION PRICE

COST/UNIT:

TOIAL COST:

\section{$\$ 4.872 .00$
$\$ 4,872.00$}

BARBARA HOLMES, REP.

KANSAS CITY

(816) $997-2976$

TO FREEZE EOUIPMENT:

BARBARA HOLMES, REP.

KANSAS CITY

MO $64141-6159$

(816) $997-2976$

LOCATION OF EOUIPMENT :

ALLIEDSIGMAL, INC.

2000 EAST 95IH SIREE

KAMSAS CITY

MO 64131-3095

COST/UNIT:

TOTAL COST:

$\$ 1.567 .00$

$\$ 1,567.00$

FOR FURTHER INFORMATION:

ELOY N. GIRON

ALBUOUEROUE

(505) $844-2746$

NM 87185

TO FREEZE EOUIPMENT:

ELOY $N$. GIRON

ALBUOUEROUE

(505) $844-2746$

87185

LOCATION OF EQUIPMENT:

SANDIA NATI OMAL LABORATORIES

PROPERTY REAPPLICATION DIVISION 7617

PROPERTY REAPPLICATION DIVISION 7617
ALBUOUEROUE 
EFFECTIVE DATE: 07/01/94 EXPIRATION DATE: 07/31/94

FSC

\section{COOE EXCESS PROPERTY DESCRIPIION}

\section{REFERENCE MUMBER : 237}

6625 INTENSIFIER RADIATO/TABLE LIFT SCIRROS MFR: DELCALIX/AIR TECH., MOOEL

NO. $30001 \mathrm{~S} / 2000 E \mathrm{~W}$. S/N F-197Mm/26 523, ACQ. DATE: 4/77, APPROX. WT:

300 LBS. 64'W X 32 'L $\times 42 \cdot \mathrm{H}$.

REPORT NUMBER: $899105-4081$

LINE ITEM NUMBER: 0023

REFERENCE NUMBER : 238

6625 INTERCONNECT BOX (CHATTER TESTER) 74562, MANUF: SPARTON MODEL 63433146400001 EA

STN N/A

IEACH. SIZE: 12"W $\times 10^{\prime \prime F / R} \times 8^{\prime \prime H}$ EST WT: 2 LBS MFGR YR: 1988

REPORT NUMBER: $896404-4133$

LINE ITEM NUMBER: 0011

No OF

$500001 \mathrm{EA}$
COND UNITS UNIT

ACQUISITION PRICE

COST/UNIT: TOTAL COST:

$\$ 37,714.00$ $37,714.00$

LYNN E. SHACKELFOOT

LIVERMORE

(510) $294-3067$

TO FREEZE EOUIPMENT:

LYNN E. SHACKELFOOI

LIVERMORE

(510) $294-3067$

CA $94550-0969$

LOCATION OF EQUIPMENT:

SAMDIA NATIONAL LABORATORIES

ON DEPT $8532-1$

LIVERMORE

CA $94550-0969$
COST/UNIT: TOTAL COST:
$\$ \$ 1.659 .00$ $\$ 1.659 .00$
FOR FURTHER INFORMATION:

BARBARA HOLMES, REP.

KANSAS CITY

MO $64141-6159$

(816) $997-2976$

TO FREEZE EQUIPMENT:

BARBARA HOLMES, REP.

KANSAS CITY

(816) $997-2976$

Mo $64141-6159$

LOCATION OF EQUIPMENT:

ALLIEDSIGNAL, INC.

2000 EAST 95TH STREE

KANSAS CIIT 
EFFECTIVE DATE: $07 / 01 / 94$ EXPIRATION DATE: $07 / 31 / 94$

FSC

COOE EXCESS PROPERTY DESCRIPTION

REFERENCE NUMBER : 239

6625 INTERFACE-TYPESETTER, MFR. ATEX INC. MODEL RDII, ACO. YR. 88, PROP NO 4968909

$$
\text { ELIGIBLE EQUIPMENT }
$$

NO OF

COND UNITS UNIT

$500001 \mathrm{EA}$ COST/UNIT:
TOTAL COST:

LINE ITEM NUMBER: 0013
REPORT NUMBER: $899102-4169$

REFERENCE NUMBER : 240

6625 LASER SYSTEM MFG: YAG, CW HOLLOBEAM ACD DATE: 6/91 CONSISTS OF CONTROLLERS, NEH LAMPS AND MISC PARTS

REF NO: $29913-01$

$500001 E A$
LINE ITEM NUMBER: QOOS

\section{ACQUISITION PRICE}

$\$ 9,000.00$ $\$ 9,000.00$

\section{$146,894.00$}

$\$ 146,894.00$

\section{COST/UNIT:
TOTAL COST:}

FOR FURTHER INFORMATION:

NANCY J. SITTON

WA 99352

IO FREEZE EQUIPMENT:

NANCY J. SITTOA

RICHLAND

(509) 376-5108

LOCATION OF EOUIPMENT:

WESTINGHOUSE HANFORD COMPANY

ESS\&S, 1167A BLDG/1100 AREA

RICHLAND

WA 99352 
EFFECTIVE DATE: 07/01/94 EXPIRATION DATE: 07/31/94

CODE EXCESS PROPERTY DESCRIPTION

REFERENCE NUMBER : 241

6625 LASERULER MFG: GCA CORP TYPE: LASER MOOEL: VLM200

ACO DATE: $2 / 86$ REF NO: $29936-04$

REPORT NUMBER: $890531-4103$

LINE ITEM NUMBER: 0009

REFERENCE NUMBER : 242

6625 LCR METER TEST FREO RANGE OF $100 \mathrm{HZ}$ TO $100 \mathrm{KHZ}$ IN 11 SPOT FREQUENCIES.

120VA C, 60 HZ. HEW PACK, 4274A, SER. J00502.

TOTAL COST:
ACQUISITION PRICE

COST/UNIT:

TOTAL COST:

$\$ 15,338.00$

$\$ 15,338.00$

$00001 \mathrm{EA}$

(n)

LINE ITEM NUMBER: 0036
FOR FURTHER INFORMATION:

NANCY J. SITTON

RICHLAND

(509) $376-5108$
(5ICHLAND

WA 99352

TO FREEZE EQUIPMENT:

NANCY J. SITTON

RICHLAND

WA 99352

(509) $376-5108$

LOCATION OF EQUIPMENT:

WESTINGHOUSE HANFORD COMPANY

ESS\&S, 1167A BLDG/1100 AREA

RICHLAND

WA 99352

$\$ 8,501.00$

$\$ 8,501.00$

FOR FURTHER INFORMATION:

ELOY N. GIRON

ALBUQUEROUE

(505) $844-2746$

NM 87185

TO FREEZE EQUIPMENT:

ELOY N. GIRON

ALBUDUEROUE

(505) $844-2746$

NM 87185

LOCATION OF EQUIPMENT:

SANDIA NATIOMAL LABORATORIES

PROPERTY REAPPLICATION OIV. 7617

ALBUOUERQUE

NM 8712 
EFFECTIVE DATE: $07 / 01 / 94$ EXPIRATION DATE: $07 / 31 / 94$

\section{COOE EXCESS PROPERTY DESCRIFIION}

REFERENCE NUMBER : 243

6625 LINE CONDITIONER 65423, MANUF: DELTEC MODEL GPC5060 S/N 29867

1 EACH. SIZE: $17 \mathrm{WW} \times 36^{\circ} \mathrm{F} / \mathrm{R} \times 36^{\prime H}$ EST WT: 400 LBS MFGR YR: 1984

REPORT NUMBER: $896404-4133$

LINE ITEM NUMBER: 0019

REFERENCE NUMBER : 244

6625 LINE CONDITIONER 65424, MANUF: DELTEC MODEL GPC5060 S/N 29858

1 EACH. SIZE: $26^{\prime \prime W} \times 36^{\prime \prime F} / R \times 20^{\prime \prime H}$ EST WT: 300 LBS MFGR YR: 1984

$400001 E A$

COST/UNIT:

TOTAL COST:

$\$ 1,667.00$

FOR FURTHER INFORMATION:

BARBARA HOLMES, REP.

KANSAS CITY

(816) $997-2976$

MO 64141-6159

TO FREEZE EQUIPMENT BARBARA HOLMES, REP.

KANSAS CITY

(816) $997-2976$

MO $64141-6159$

LOCATION OF EQUIPMENT

ALLIEDSIGNAL, INC.

2000 EAST 95TH STREET

XANSAS CITY

MO 64131-3095 
EFFECTIVE DATE: 07/01/94 EXPIRATION DATE: 07/31/94

FSC

\section{COOE EXCESS PROPERTY DESCRIPTION}

REFERENCE NUMBER : 245

6625 LINE CONDITIONER 1562 PD, MANUF: SOLA MODEL MCR750 S/N N/A 1 EACH SIZE: N/A EST WT: N/A MFGR YR: N/A

REPORT NUMBER: $896404-4133$

LINE ITEM NUMBER: 0026

REFERENCE NUMBER : 246

6625 LOGIC STATE ANALYZER 77028, MANUF: HEWLETT PACKARD MODEL HP1607A S/N

A162901441

1 EACH, 16 BITS. SIZE: $11 " \mathrm{~W} \times 18 " \mathrm{~F} / \mathrm{R} \times 7 " \mathrm{H}$ EST WT: 20 LBS MFGR YR: 1978
$600001 E A$ COSTUNIT: TOTAL COST:

\section{ACQUISITION PRICE}

COST/UNIT: $\quad \$ 2,000.00$

$\$ 2,000.00$
$400001 E A$

FURTHER INFORMATION:

DERBARA HOLMES, REP.

KANSAS CITY

MO 64141-6159

10 FREEZE EQUIPMENT:

BARBARA HOLMES, REP.

KANSAS CITY

(816) 997-2976

LOCATION OF EQUIPMENT :

ALLIEDSIGNAL, INC.

2000 EAST 95TH STREE

KANSAS CITY

REPORT NUMBER: $896404-4124$

LINE ITEM NUMBER: 0001

FOR FURTHER INFORMATION:

BARBARA HOLMES, REP.

KANSAS CITY

(816) $997-2976$

TO FREEZE EQUIPMENT:

BARBARA HOLMES, REP.

KANSAS CITY

(816) 997-2976

LOCATION OF EQUIPMENT:

ALLIEDSIGNAL, INC.

2000 EAST 95TH STREET

KANSAS CITY

MO $64131-3095$ 
EFFECTIVE DATE: 07/01/94 EXPIRATION DATE: $07 / 31 / 94$

FSC

\section{COOE EXCESS PROPERTY DESCRIPIION}

REFERENCE NUMBER : 247

6625 MANIPULATOR, PRECISION HAS TWO MICROMETER ADJUSTMENTS FOR $X$ AND $Y$ MOVEMENT ALSO HAS A $Z$ MOVEMENT. PERKIN ULT, 2838550.

REFORT NUMBER: $898355-4088$

LINE IIEM NUMBER: 0045
NO OF

COND UNITS UNIT

ACQUISITION PRICE

$600001 \mathrm{EA}$

$\$ 1,955.00$ TOTAL COST: $\$ 1,955.00$

FOR FURTHER INFORMATION:

ELOY N. GIRON

ALBUOUERQUE

(505) $844-2746$

NM 87185

TO FREEZE EQUIPMENT:

ELOY N. GIRON

ALBUQUEROUE

(505) $844-2746$

NM 87185

LOCATION OF EQUIPMENT:

SANDIA NATIONAL LABORATOR:ES

PROPERTY REAPPLICATION DIVISION 7617

ALBUOUERQUE

NM 87123

\section{REFERENCE NUMBER : 248}

6625 MEMORY BOARD PLUG IN BOARD WITH A 25 PIN PORT IN BACK. FORCE COMP. SRAMZ2B, S ER. 737 TC300A.

$600001 \mathrm{EA}$

COST/UNIT:

IOTAL COST:

$\$ 2,490.00$

$\$ 2,490.00$

REPORT NUMBER: $898355-4102$

LINE ITEM NUMBER: 0037

FOR FURTHER INFORMATION:

ELOY N. GIRON

ALBUQUERQUE

(505) $844-2746$

NM 87185

TO FREEZE EQUIPMENT:

ELOY N. GIRON

ALBUOUERQUE

(505) $844-2746$

NM 87185

LOCATION OF EQUIPMENT:

SANDIA NATIONAL LABORATORIES

PROPERTY REAPPLICATION DIV. 7617

ALBUOUEROUE 
EFFECTIVE DATE: $07 / 01 / 94$ EXPIRATION DATE: $07 / 31 / 94$

CODE EXCESS PROPERTY DESCR!PIION

REFERENCE NUMBER : 249

6625 METER DIGITAL MFR: HEWLETT PACKARD

S/N: 637 A04896

REPORT NUMBER: $899305-4039$

LINE ITEM NUMBER: 0009

$$
\text { NO OF }
$$

COND UNITS UNIT

ACQUISITION PRICE

$500001 E A$

COST/UNIT: TOTAL COST:

$\$ 1,965.15$ $\$ 1,965.15$

CYNDI DELONG

LAS VEGAS
(702) $295-6753$

NV 89193-8521

TO FREEZE EQUIPMENT

CYNDI DELONG

LAS VEGAS

(702) $295-6753$

NV $89193-8521$

LOCATION OF EQUIPMENT

REYNOLDS ELECTRICAL \& ENGINEERING CO

NEVADA TEST SITE

MERCURY

NV 89023

REFERENCE NUMBER : 250

6625 METER DIGITAL MFR: HEWLETT PACKARD MODEL: $3440 \mathrm{~A}$ $S / N$ : 74910605

REPORT NUMBER: $899305-4039$
$500001 \mathrm{EA}$

COST/UNIT:

TOTAL COST:

LINE ITEM NUMBER: 0024
$\$ 1.736 .53$

$\$ 1,736.53$

FOR FURTHER INFORMATION

CYNDI DELONG

LAS VEGAS

(702) $295-6753$

NV $89193-852$

TO FREEZE EQUIPMENT:

CYNDI DELONG

LAS VEGAS

(702) $295-6753$

NV 89193-8521

LOCATION OF EQUIPMENT:

REYNOLDS ELECTRICAL \& ENGINEERING CO

NEVADA TEST SITE

MERCURY

NV 89023 
EFFECTIVE DATE: $07 / 01 / 94$ EXPIRATION DATE: $07 / 31 / 94$

FSC

\section{COOE EXCESS PROPERTY DESCRIPTION}

REFERENCE NUMBER : 251

6625 METER MULII OIGITAL MFR: DANA LAB MODEL: 590012324252 S/N: 11294

ELIGIBIE EQUIPMENT

NO OF

COND UNITS UNIT

ACQUISITION PRICE

600001 EA COST/UNIT:
TOTAL COST:

LINE ITEM NUMBER: 0027
REPORT NUMBER: $899305-4039$

\section{REFERENCE NUMBER : 252}

O625 METER, CAPACITANCE METER 728 HAS A FULL SCALE RANGE FROM 1103000 CAPACITANCE RANG E FROM 0-30, CONTROL ARE ON/OFF. 120/240VAC, 50-400 HZ. 72BD, 33 9806, SER. 339806 .
600001 EA COST/UNIT:
TOTAL COST:
LINE ITEM NUMBER: 0046
CONTACT(S)

\section{$\$ 2,430.44$ $\$ 2,430.44$}

FOR FURTHER INFORMATION:

CYNDI DELONG

LAS VEGAS

(702) $295-6753$

NV $89193-8521$

TO FREEZE EOUIPMENT:

CYMDI DELONG

LAS VEGAS

(702) $295-6753$

NV 89193-8521

LOCATION OF EQUIPMENT

REYNOLDS ELECTRICAL \& ENGINEERING CO NEVADA TEST SITE

MEVADA TES

NV 89023

$\$ 1,200.00$

$\$ 1,200.00$
FOR FURTHER INFORMATION:

ELOY N. GIRON

ALBUOUERQUE

(505) $844-2746$

NM 87185

TO FREEZE EQUIPMENT:

ELOY N. GIRON

ALBUQUERQUE

(505) $844-2746$

NM 87185

LOCATION OF EQUIPMENT:

SANDIA NATIONAL LABORATORIES

SANDIA NATIONAL LABORATORIES

PROPERTY REAPPLICATION DIVISION 7617
ALBUOUEROUE 
EFFECTIVE DATE: $07 / 01 / 94$ EXPIRATION DATE: 07/31/94

CODE EXCESS PROPERTY DESCRIPTION

REFERENCE NUMBER : 253

6625 METER, CAPACITANCE $72 B$ HAS A FULL SCALE RANGE FROM 1 TO 3000 CAPACITANGE RANGE FROM 0-30. CONTROLS ARE: ON/OFF, 120/240VAC, 50-400HZ. BOONION RANGE FROM $0-30$. CONTROL

REPORT NUMBER : $898355-4088$

LINE ITEM NUMBER: 0047

REFERENCE NUMBER : 254

METER, NULL ELECTRONIC NULL DETECTOR MAY BE USED INSTEAD OF A

VALVANOMETER IN PHOTENTIOMETER AND BRIDGE MEASUREMENTS. 120VAC, $60 \mathrm{HZ}$ LEEDS \& NORT, 9828, SER. 1893182

LINE ITEM NUMBER: 0050

$$
\text { NO OF }
$$

$60001 \mathrm{EA}$

COST/UNIT: TOTAL COST:

$\$ 1,200.00$ $\$ 1,200.00$

FOR FURTHER INFORMATION:

ELOY N. GIRON

ALBUOUEROUE

(505) $844-2746$

NM 87185

TO FREEZE EQUIPMENT:

ELOY N. GIRON

ALBUQUERQUE

(505) $844-2746$

NM 87185

LOCATION OF EQUIPMENT:

SANDIA NATIONAL LABORATORIES

PROPERTY REAPPLICATION DIVISION 7617 PROPERTY REAPPLICATION DIVISION 7617
ALBUQUERQUE
NM 87123

COST/UNIT:

TOTAL COST

$\$ 2,010.00$

$\$ 2,010.00$

\section{FOR FURTHER INFORMATION:}

ELOY N. GIRON

ALBUQUERQUE

(505) $844-2746$

NM 87185

TO FREEZE EQUIPMENT

ELOY N. GIRON

ALBUQUERQUE

NM 87185

(505) 844-2746

LOCATION OF EQUIPMENT:

SANDIA NATIONAL LABORATORIES

PROPERTY REAPPLICATION DIVISION 7617

ALBUQUERQUE 
EFFECTIVE DATE: $07 / 01 / 94$ EXPIRATION DATE: $07 / 31 / 94$

FSC

\section{COOE EXCESS PROPERTY DESCRIPIION}

REFERENCE NUMBER : 255

6625 METER, NULL NANOVOLT DETECTOR, W/CONTROLS FOR BATTERY AC LINE, OFF,

CHARGE, B AITERY IEST, SENSITIVITY AND ZERO SUPPRESSION. 120VAC, $60 \mathrm{HZ}$

LE EDS \& NORTH. 9838, SER. NONE.

REFERENCE NUMBER : 256

6625 METER, POWER 9 DIGIT LED READOUT FOR AMPERS. $120 \mathrm{VAC}, 60 \mathrm{HZ}$. MATROL, 4610 , SE R. 90231

No of

COND UNIIS UNIT

ACQUISITION PRICE

$600001 \mathrm{EA}$

COST/UNIT:

TOTAL COST:

$\$ 6,143.00$

A

\section{TO FREEZE EQUIPMENT: \\ ELOY N. GIRON \\ ALBUQUERQUE}

(505) 844-2746

NM 87185

LOCATION OF EQUIPMENT:

SANDIA NATIONAL LABORATORIES

PROPERTY REAPPLICATION DIVISION 7617

ALBUOUERQUE

600001 EA COST/UNIT:

TOTAL COST:
$\$ 3,076.00$ $\$ 3,076.00$
FOR FURTHER INFORMATION :

ELOY N. GIRON

ALBUQUERQUE

(505) 844-2746

NM 87185

TO FREEZE EQUIPMENT:

ELOY N. GIRON

ALBUQUERQUE

NM 87185

LOCATION OF EOUIPMENT:

SANDIA NATIONAL LABORATORIES
PROPERTY REAPPLICATION DIVISION 7617

PROPERTY REAPPLICATION DIVISION 7617
ALBUQUEROUE
MM 87123 
DOE USED EMERGY RELATED LABORATORY EQUIPMENT GRANT PROGRAM

EFFECTIVE DATE: $07 / 01 / 94$ EXPIRATION DATE: $07 / 31 / 94$

FSC

\section{COOE EXCESS PROPERTY DESCRIPTION}

REFERENCE NUMBER : 257

6625 METER, PONER 9 DIGIT LED READOUT FOR AMPERES VOLTS AND WATTS. CONTROLS FOR 2 TO 50 AMPS AND 15 TO 600 VOLTS. 120VAC, 60 HZ. MAGTROL, 4610, SE R. $8 \mathrm{C} 67$.
COND UNITS UNIT

$600001 \mathrm{EA}$

\section{ACQUISITION PRICE} COST/UNIT:
TOTAL COST:

REPORT NUMBER: $898355-4089$

LINE ITEM NUMBER: 0049
REFERENCE NUMBER : 258

SPEED MFR: ADVANCED KINETICS INC, MODEL NO CM10/VSM, ACO. DATE: 9/ 84, APPROX WI: 15 LBS., $19 \cdot \mathrm{W} \times 141 \mathrm{~L} \times 5 \mathrm{H}$.

$4 \quad 00001$ EA

COST/UNIT:

TOTAL COST:

$\$ 1,595.00$
$\$ 1,595.00$
$\$ 2,134.00$

\section{CONTACT(S)}

FOR FURTHER INFORMATION:

ELOY N. GIRON

ALBUQUERQUE

(505) $844-2746$

NM 87185

TO FREEZE EOUIPMENT:

ELOY N. GIRON

ALBUQUERQUE

(505) $844-2746$

NM 87185

LOCATION OF EOUIPMENT:

SAMDIA NATIONAL LABORATORIES

PROPERTY REAPPLICATION DIVISION 7617

PROPERTY REAPPLICATION DIVISION 7617
ALBUQUERQUE
NM 87123

REPORT NUMBER: $899105-408$

LINE ITEM NUMBER: 0024

FOR FURTHER INFORMATION:

LYMN E. SHACKELFOOT

LIVERMORE

(510) $294-3067$

CA $94550-0969$

TO FREEZE EQUIPMENT:

LYMN E. SHACKELFOOT

LIVERMORE

(510) $294-3067$

LOCATION OF EQUIPMENT

SANDIA NATIONAL LABORATORIES

PROPERTY REAPPLICATION DEPT. 8532-1

PROPERIY REAPPLICATION DEPT. $8532-1$
LIVERMORE
CA $94550-0969$ 
EFFECTIVE DATE: 07/01/94 EXPIRATION DATE: 07/31/94

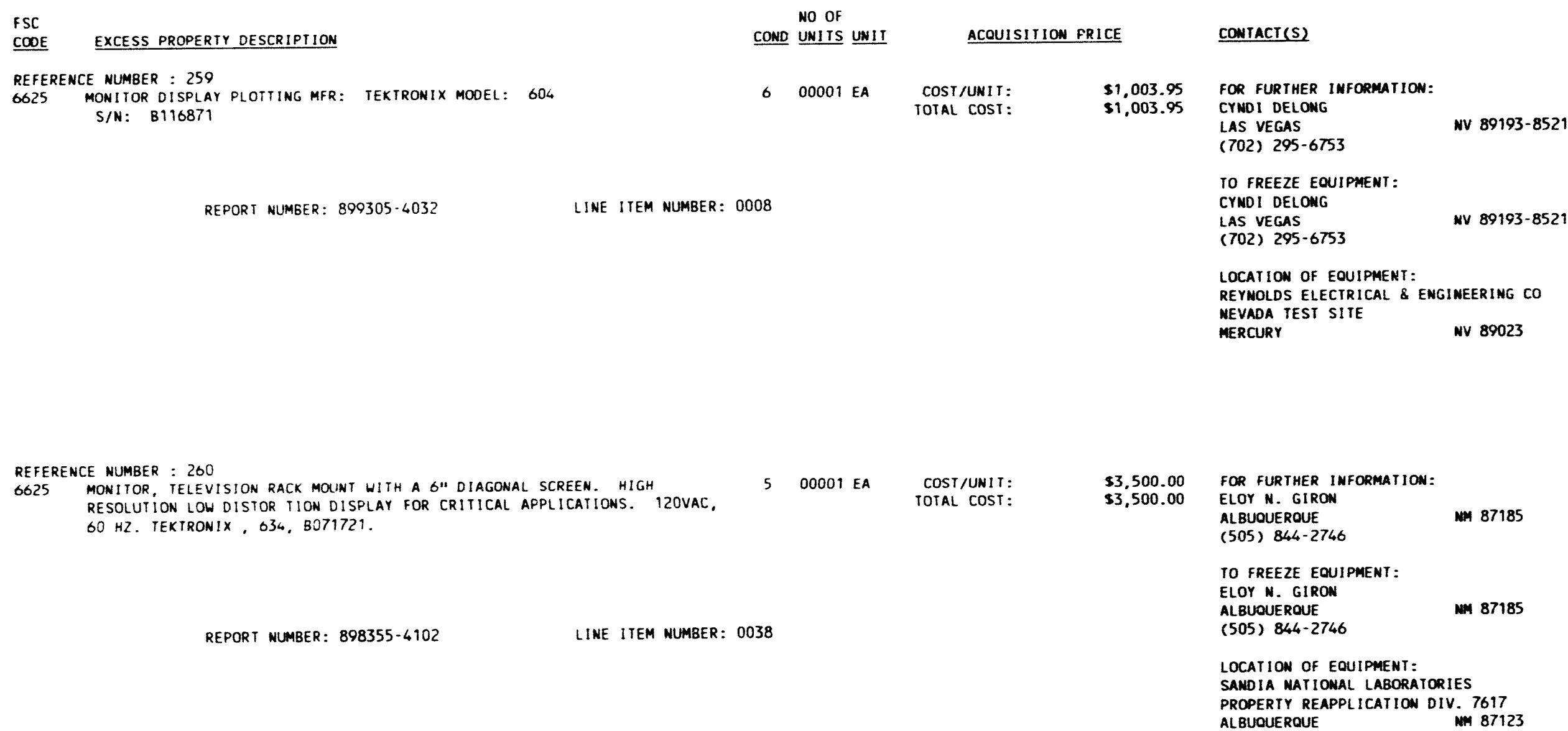


EFFECTIVE DAIE: 0i/01/94 EXPIRATION DATE: 07/31/94

FSC
COOE EXCESS PROPERTY DESCRIPTION

\section{REFERENCE NUMBER : 26}

6625 MULTICHANNEL ANALYER ND100 MFE: NUCLEAR DATA INC. MODEL NO. 880552 , S/N 74-164, ACO. DATE: 2/75, APPROX. WT: $60 \mathrm{LBS} ., 18 . \mathrm{W} \times 25 . \mathrm{L} \times 111 \mathrm{H}$. COST/UNIT:
IOTAL COST: ACOUISITION PRICE

$\$ 10,095.00$ $\$ 10.095 .00$

REPORT NUMBER: $899105-4081$

LINE ITEM NUMBER: 0025

$$
\text { No of }
$$

The

COND UNIIS UNIT

FOR FURTHER INFORMATION:

LYMN E. SHACKELFOO

LIVERMORE

CA $96550-0969$

TO FREEZE EQUIPMENT:

LYWN E. SHACKEL FOOT

LIVERMORE

(510) $294-3067$

CA $94550-0969$

LOCATION OF EOUIPMENT:

SAMDIA MATIONAL LABORATORIES

PROPERTY REAPPLICATION DEPT . 8532-1

LIVERMORE

CA $94550-0969$

\section{REFERENCE NUMBER : 262}

6625 MULTIMETER WITH AN IEE-LI88 BUS, VOLT RANGE .2 VOLTS TO 2000 VOLTS, OHM RANG E IS UP TO 20 M-OHM. 120VAC, 60 HZ. KEITHLEY, 192. SER. 34215.

$500001 \mathrm{EA}$
LINE ITEM NUMBER: 0054
COST/UNIT: TOTAL COST:
$\$ 1.503 .00$ $\$ 1.503 .00$

FOR FURTHER INFORMATION:

ELOY N. GIROW

ALBUOUEROUE

(505) $844-2746$

MM 87185

TO FREEZE EOUIPMENT :

ELOY N. GIRON

ALBUOUEROUE

(505) $844-2746$

MM 87185

LOCATION OF EQUIPMENT:

SAMDIA MATIOMAL LABORATORIES PROPERTY REAPPLICATION DIVISION 7617 ALBUOUEROUE 
EFFECIIVE DAIE: 07/01/94 EXPIRAIION DAIE: 07/31/94

FSC

\section{COOE EXCESS PRGPERTY DESCRIPTION}

REFERENCE NUMBER : 263

OO25 MULTIMETER, DIGITAL HAS O DIGIT READOUT. HILL MEASURE I TO $1000 \mathrm{VAC}, 0.1600001$ EA TO 1000 VDC, AND UF TO $100 \mathrm{M}-\mathrm{OHM}$. 120/2 $60 \mathrm{VAC}, 50-60 \mathrm{HZ}$. RACAL-DANA.

5900125, SER. 110063 .

REFORT NUMSER: $898355-4088$

LINE ITEM NUMBER: 0048
REFERENCE NUMBER : $26 \%$

O625 MULTIMETER, DIGITAL HAS A 5-1/2 DIGIT READOUT, FUNCTION ARE VDC, VAC,

MAUC ANO OHM, $\checkmark$ OLT RANGE, VOLT TO 101000 VOLTS. OHMS UP TO $10 \mathrm{~m}$-OHM.

IZOVAC, 6O HZ. FAIRCHILD, 7000, SER. 2090.

REPORT NUMEER: $898355-4088$
No of

\section{ACQUISITION PRICE}

COST/UNIT:

TOTAL COST:

$4,316.00$
COND UNITS UNi

\section{COMTACr(S)}

FOR FURTHER INFORMATION:

ELOY N. GIRON

ALBUOUEROUE

(505) $844 \cdot 2746$

87185

TO FREEZE EOUIPMENT:

ELOY N. GIRON

AL BUOUEROUE

(505) $8464-2746$

87185

LOCATION OF EQUIPMENT:

SAMD IA MATIONAL LABORATORIES PROPERTY REAPPLICATION DIVISION 7617 SROPERTY REAPPLication DIVI 87123
$600001 E A$

TOTAL COST:
$\$ 1,895.00$
OR FLRTHER INFDRMATION:

ELOY N. GIRON

ALBUQUEROUE

(505) $844-2746$

87185

TO FREEZE EOUIPMENT

ELOY N. GIRON

ALBUQUEROUE

87185

(505) $844-2746$

LOCATION OF EOUIPMENT

SAMDIA NAIIONAL LABORATORIES PROPERIY REAPPLICAIION DIVISION 7617

PROPERTY REAPPLICATION DIVISION 7617 
EFFECTIVE DATE: 07/01/96 EXPIRATION DATE: 07/31/94

FSC

COOE EXCESS PROPERTY DESCRIPIIOW

REFERENCE NUMBER : 265

6625 MULTIMETER DIGITAL HAS A 5-1/2 DIGITAL READOUT MEASURES DC VOLTS AND OHMS ON 5 RANGe $S$. 120VAC, $60 \mathrm{HZ}$. FLUKE, 8400A, SER. 52427.

REPORT NUMBER: $898355-4088$

LINE ITEM NUMBER: 0050

REFERENCE NUMBER : 266

6625 MULTIMETER, DIGITAL ACV RANGE $0-1000$ VOLTS, DCV RANGE 0.1 TO 1000 VOLTS MULTIMETER, DIGITAL ACV RANGE $0-1000$ VOLTS, DCV RANGE 0.1 TO 1000
OHM RANGE UP TO $100 \mathrm{M}$-OHM. ICOVAC, $60 \mathrm{HZ}$. RACAL DANA, 5900 , SER. 122340.

LINE ITEM MUMBER: 0051

$$
\text { NO OF }
$$

COMD UMITS UNIT

$600001 \mathrm{EA}$
ACOUISITION PRICE

COST/UNIT: TOTAL COST:

$\$ 2,619.00$ $\$ 2,619.00$
CONTACT(S)

FOR FURTHER INFORMATION:

ELOY N. GIRO

AL BUOUEROUE

(505) $844-2746$

87185

TO FREEZE EQUIPMENT

ELOY N. GIROM

ALBUOUEROUE

(505) 844-2746

87185

LOCAIION OF EOUIPMENT:

SAMDIA MATIOMAL LABORATORIES

PROPERTY REAPPLICATION DIVISION 7617

ALBUOUERQUE
COST/UNIT:

OTAL COST
$\$ 2.000 .00$

$\$ 2,000.00$

\section{FOR FURTHER INFORMAIION:}

ELOY N. GIRO

ALBUOUEROUE

(505) $844-2746$

87185

TO FREEZE EQUIPMENT:

ELOY N. GIRON

ALBUOUEROUE

(505) $844-2746$

87185

LOCATION OF EQUIPMENT:

SAMDIA MATIOMAL LABORATORIES

PROPERTY REAPPLICATION DIVISIOM 7617

ALBUOUEROUE 
EFFECIIVE DATE: $07 / 01 / 94$ EXPIRATION DATE: $07 / 31 / 94$

FSC

\section{COOE EXCESS PROPERTY DESCRIPTION}

REFERENCE NUMBER : 267

6625 MULTIMETER, DIGITAL GPPM DC ACCURACY 6.1/2 DIGIT READOUT 500 READING.

PER SEC SYSTEM SPEED MOOULAR CONSTRUCTION FOR CONFIGURABILITY. 12OVAC,

GO HZ. N EEDS RECALIBRATION. FLUKE.

REPORT NUMBER: $898355-4089$

LINE ITEM MUMBER: 0051

\section{REFERENCE NUMBER : 268}

6625 MULIIMETER DIGITAL THIS PLUGIN PROVIDES FOUR RANGES OF BOTH AC AND DC PLUS SIZE RANG ES OF OHMS. 120 VAC, 60 HZ. HEW PACK, 34703A, SER. 51 A00065.
No of

COND UNITS UNIT

ACQUISITION PRICE

$600001 \mathrm{EA}$

COST/UMIT:

TOTAL COST:
$\$ 5,399.00$
$\$ 5,399.00$

ELOY N. GIRON
ALBUQUEROUE
(505) $844-2746$

87185

TO FREEZE EQUIPMENT:

ELOY N. GIRON

ALBUQUEROUE

(505) $844-2746$

m4 87185

LOCATION OF EOUIPMENT:

SAMDIA MATIOMAL LABORATORIES

PROPERTY REAPPLICATION DIVISION 7617

PROPERTY REAPPLICATION DIVISIOW 7617
ALBUOUEROUE

$600001 \mathrm{EA}$

COST/UNIT:

TOTAL COST:

$\$ 1.334 .00$

$\$ 1,334.00$

REPORT NUMBER: $898355 \cdot 4089$

INE ITEM NUMBER: OC52
FOR FURTHER INFORMATION:
ELOY N. GIRON
ALBUOUEROUE
87185
(505) $844-2746$
TO FREEZE EQUIPMENT
ELOY N. GIRO
ALBUOUEROUE
MM 87185
(505) $844-2746$
LOCATION OF EQUIPMENT:
ABORATORIES
PROPERTY REAPPLICATION DIVISION 7617
ALBUOUEROUE NM 87123 
EFFECTIVE DATE: 07/01/94 EXPIRATION DATE: 07/31/94

COOE EXCESS PROPERIY DESCRIPIION

REFERENCE NUMBER : 209

6625 MULTIMETER, DIGITAL WITH ACV, DCV AND MSZ ALSO HAS A RANGE CONTROL KNOB PLUS RATIO, F ILIER, DATA AND PROGRAM CONTROLS. 120VAC, $60 \mathrm{HZ}$. RACALDANA, 590 0, SER. 113804.

REPORT NUMBER: $898355-4089$

LINE ITEM NUMBER: 0053

\section{REFERENCE NUMBER : 270}

6625 MULTIMETER, DIGITAL WITH AN 8 OIGIT LED READOUT, AC AVERAGE CONVERTER

CURRENT SHUNTS, OHM CONVERTER, CALIBRATION MEMORY, AC-DC RMS CONVERTER.

I20VAC, $60 \mathrm{HZ}$. FLUKE, 8502A, SER. 2965025

REPORT NUMBER: $898355-4089$

LINE ITEM NUMBER: 0055
No of

COND UNITS UNIT

ACQUISITION PRICE

$700001 \mathrm{EA}$

COST/UNIT:

TOTAL COST:

$\$ 1,500.00$

$\$ 1,500.00$

ELOY N. GIRON
ALBUQUEROUE

(505) $844-2746$

NM 87185

TO FREEZE EQUIPMENT:

ELOY N. GIRON

ALBUQUERQUE

(505) $844-2746$

NM 87185

LOCATION OF EQUIPMENT:

SANDIA NATIONAL LABORATORIES

PROPERTY REAPPLICATION DIVISION 7617

ALBUOUERQUE

00001 EA

COST/UNIT:

OTAL COST:

$\$ 2,832.00$

FOR FURTHER INFORMAIION:

ELOY N. GIRON

ALBUDUERQUE

(505) $844-2746$

NM 87185

TO FREEZE EQUIPMENT :

ELOY N. GIRON

ALBUQUEROUE

(505) $844-2746$

NM 87185

LOCATION OF EOUIPMENT:

SANDIA NATIONAL LABORATORIES

PROPERTY REAPPLICATION DIVISION 7617

ALBUOUEROUE 
EFFECIIVE DATE: $07 / 01 / 94$ EXPIRATION DATE: $07 / 31 / 94$

FSC

\section{CODE EXCESS PROPERTY DESCRIPTION}

REFERENCE MUMBER : 271

6625 MULTIMETER, DIGITAL FOR ACV DCV AND OHMX VOLT RANGE 0.1 TO 1,000 DCV AND 1 TO $1000 S$ CV HITH A 6 DIGIT LED READOUI T2OVAC $60 \mathrm{HZ}$ RACALDANA, 5900, SER. 120962.

REPORT NUMBER: $898355-4089$

LINE ITEM NUMBER: 0056

REFERENCE NUMBER : 272

6625 O/SCOPE PLUG-IN DUAL TRACE MFR: TEKTRONIX

SER: 2292 PCO7075 PRC-40013

REPORT NUMBER : $899109-4101$

INE ITEM NUMBER: 0014
No of

COND UNITS UNIT

$600001 \mathrm{EA}$ COST/UNIT: TOTAL COST:

\section{$\$ 1,500.00$}

$\$ 1,500.00$

\section{CONTACT(S)}

FOR FURTHER INFORMATION:

ELOY N. GIRON

ALBUQUERQUE

(505) $844-2746$

NM 87185

TO FREEZE EQUIPMENT:

ELOY N. GIRON

ALBUQUERQUE

(505) $844-2746$

NM 87185

LOCATION OF EQUIPMENT:

SANDIA NATIONAL LABORATORIES

PROPERTY REAPPLICAIION DIVISION 7617

PROPERTY REAPPLICATION DIVISION
ALBUDUEROUE
NM 87123

NM 87123
$600001 \mathrm{EA}$

TOTAL COST:
$\$ 600.56$ $\$ 600.56$
FOR FURTHER INFORMATION:

MATTIE PACE

STANFORD

(415) $926-2231$

CA 94309

TO FREEZE EQUIPMENT:

MATTIE PACE

STANFORD

(415) $926-2231$

CA 94309

LOCATION OF EQUIPMENT:

STANFORD LINEAR ACCELERATOR CENTER 2575 SAND HILL ROAD

MENLO PARK

CA 94025 
FFFECTIVE DATE: 07/01/94 EXPIRATION DATE: $07 / 31 / 94$

FSC:

\section{CODE EXCESS PROPERTY DESCRIPIION}

REFERENCE NUMBER : 273

6625 OSCILLOGRAPH (LIGHT) RECORDING OSCILLOGRAPH, HITH CONTROL FOR LIGHT

INTENSITY AND PAPE R SPEED. 120VAC, $60 \mathrm{HZ}$. CEC, 5-124, SER. 3022.

REPORT NUMBER: $898355-4102$

LINE ITEM NUMBER: 0039

REFERENCE NUMBER : 274

0625 OSCILLOGRAPH (LIGHT) VISICORDER, PAPER SPEED IN/SEC, 1,2,4 AND 8 WITH

MULTIPLIER . 1 , 1 AND 10 , WITH CONTROLS FOR TRACE GRID, TIME INTERVAL AND POWER. I2OVAC, 6O HZ. HONEYWELL, 1508C, GEG75.

REPORT NUMBER: $898355-4102$

LINE ITEM NUMBER: 0040

NO OF

COND UNITS UNIT

ACQUISITION PRICE

CONTACT(S)

$700001 \mathrm{EA}$

COST/UNIT: TOTAL COST:

$\$ 7,925.00$ $\$ 7,925.00$

FOR FURTHER INFORMATION :

ELOY N. GIRON

ALBUQUERQUE

NM 87185

844-2746

TO FREEZE EQUIPMENT:

ELOY N. GIRON

ALBUQUERQUE

(505) $844-2746$

NM 87185

LOCATION OF EQUIPMENT:

SAMDIA NATIOMAL LABORATORIES

PROPERTY REAPPLICATION DIV. 7617

ALBUQUERQUE

COST/UNIT:

TOTAL COST
$\$ 2,813.00$ $\$ 2,813$
FOR FURTHER INFORMATION

ELOY N. GIRON

ALBUQUERQUE

(505) $844-2746$

NM 87185

TO FREEZE EQUIPMENT:

ELOY N. GIRON

ALBUOUERQUE

(505) $844-2746$

NM 87185

LOCATION OF EQUIPMENT

SANDIA NATIONAL LABORATORIES

PROPERTY REAPPLICATION DIV. 7617

ALBUQUERQUE

NM 87123 


\section{EFFECTIVE DATE: $07 / 01 / 94$ EXPIRATION DATE: $07 / 31 / 94$}

FSC

\section{COOE EXCESS PROPERTY DESCRIPTION}

REFERENCE NUMBER : 275

6625 OSCILLOGRAPH 18 CHANNEL RECORDER, USES PLUG INS FOR THE TYPE OF RECORDING. 12 OVAC, $60 \mathrm{HZ}$. HONEYWELL, 1858, SER. 1906JD74.

REPORT NUMBER: $898355-4089$

LINE IIEM NUMBER: 0057

\section{REFERENCE NUMBER : 270}

6625 OSCILLLGRAPH 1962 HONEYHELL MODEL 906-C, S/N 9387 (N,BC,0000801)
No of

CONO UNITS UNIT

$600001 \mathrm{EA}$
ACQUISIIION PRICE

COST/UNIT:

TOTAL COST:

$\$ 10,482.00$

$\$ 10,482.00$

$$
\begin{aligned}
& \text { ELOY N. GIRON } \\
& \text { ALBUQUEROUE }
\end{aligned}
$$

(505) $844-2$

NM 87185

TO FREEZE EQUIPMENT:

ELOY N. GIRON

ALBUDUEROUE

(505) $844-2746$

LOCATION OF EQUIPMENT:

SANDIA MATIOMAL LABORATORIES

PROPERTY REAPPLICATION DIVISION 7617 ALBUDUERQUE

LINE ITEM NUMBER: 0001
500001 EA COST/UNIT: TOTAL COST: $\$ 4,400.00$

FOR FURTHER INFORMATION :

TOM JENKS

HURON

(605) $353-9217$

SD 57350

IO FREEZE EOUIPMENT

TOM JENKS

TOM JENK

(605) 353-9217

SD 57350

LOCATION OF EOUIPMENT: WESTERN AREA POWER ADMIHISTRATION 110 IOWA N.E.

SD 57350 
EFFECTIVE DATE: $07 / 01 / 94$ EXPIRATION DATE: $07 / 31 / 94$

COOE EXCESS PROPERTY DESCRIPTION

REFERENCE NUMBER : 277

6625 OSCILLOGRAPH 1965 HONEYHELL MODEL 906-C, S/N 9-10366 (N,BC,0000802)

REPORT NUMBER : $898814-4125$

LINE ITEM NUMBER: 0002

REFERENCE NUMBER : 278

R625 OSCILLOSCOPE

MFR: TEKTRON1X MOOEL: 546

$S / N: 255$

REPORT NUMBER : $899305-4032$
NO OF

COND UNITS UNIT

ACOUISITION PRICE

$500001 E A$

COST/UNIT:

TOTAL COST

$\$ 1,052.25$

$\$ 1.052 .25$

TOM JENKS

HURON

(605) $353-9217$

SD 57350

TO FREEZE EQUIPMENT:

TOM JENKS

HURON

SD 57350

(605) $353-9217$

LOCATION OF EQUIPMENT:

WESTERN AREA POUER ADMINISTRATION 110 IOWA N.E.

HURON

SD 57350
600001 EA

COST/UNIT:
TOTAL COST:
$\$ 3,512.40$
$\$ 3,512.40$
FOR FURTHER INFORMATION:

CYNDI DELONG

LAS VEGAS

(702) $295-6753$

NV 89193-8521

TO FREEZE EQUIPMENT:

CYNDI DELONG

LAS VEGAS

(702) $295-6753$

NV $89193-8521$

LOCATION OF EQUIPMENT:

REYNOLDS ELECTRICAL \& ENGINEERING CO NEVADA TESI SITE \& ENGINEERING CO NEVADA TEST SITE

NV 89023 
EFFECTIVE DATE: 07/01/94 EXPIRATION DATE: $07 / 31 / 94$

FSC

COOE EXCESS FROPERTY DESCRIPIION

REFERENCE NUMBER : 279

O625 OSCILLOSCOPE

MFR: TEKTRONIX MOOEL: 546

S/N: 275

REPORT NUMEER: $899305-4032$

REFERENCE NUMBER : 280

6625 OSCILLOSCOPE

MFR: TEKTRON IX MODEL: 546

$S / N: 226$

REPORT NUMBER : $899305-4032$

$$
\text { ELIGIBLE EQUIPMENT }
$$

NOND UNITS UNIT
COF

$600001 \mathrm{EA}$

COST/UNIT:

TOTAL COST:

LINE ITEM NUMBER: 0026

600001 EA

COST/UNIT:
TOTAL COST:

LINE ITEM NUMBER: 0027

TOTAL COST:

CQUISITION PRICE

$\$ 4.101 .52$

54.101 .52

CYNDI DELONG

LAS VEGAS

(702) $295-6753$

NV 89193-8521

IO FREEZE EQUIPMENT :

CYNDI DELONC

CYNDI DELONG

(702) $295-6753$

LOCATION OF EQUIPMENT

REYNOLDS ELECTRICAL \& ENGINEERING CO

NEVADA TEST SITE

MERCURY

NV 89023

$\$ 3,353.40$
$\$ 3,353.40$

FOR FURTHER INFORMATION:

CYNDI DELLNG

LAS VEGAS

(702) $295-6753$

NV $89193-8521$

TO FREEZE EQUIPMENT

CYNDI DELONG

LAS VEGAS

(702) $295-6753$

NV 89193-8521

LOCATION OF EQUIPMENT:

REYNOLDS ELECTRICAL \& ENGINEERING CO

REVADA TEST SITE

MERCURY

NV 89023 
EFFECIIVE DATE: $07 / 01 / 94$ EXPIRATION DATE: $07 / 31 / 94$

FSC

\section{CODE EXCESS PROPERTY DESCRIPTION}

REFERENCE NUMBER : 281

6625 OSCILLOSCOPE

MFR: TEKTRONIX MODEL: 546

$S / N: 239$

REPORT NUMBER: $899305-4032$

REFERENCE NUMBER : 28

O625 OSCILLOSCOPE

MFR: TEKTRONIX MODEL: 546

S/N: 233

REPORT NUMBER: $899305-4032$
NO OF

COND UNITS UNI

ACQUISITION PRICE

$600001 \mathrm{EA}$

COST/UNIT: TOTAL COST:

$\$ 3,353.40$ $\$ 3,353.40$

LINE ITEM NUMBER: 0028
$600001 \mathrm{EA}$
LINE I TEM NUMBER: 0029
CONTACT(S)

FOR FURTHER INFORMATION:

CYNDI DELONG

LAS VEGAS

NV $89193-8521$

TO FREEZE EQUIPMENT :

CYNDI DELONG

LAS VEGAS

(702) $295-6753$

NV $89193-8521$

LOCATION OF EQUIPMENT

REYNOLDS ELECTRICAL \& ENGINEERING CO NEVADA TEST SITE

NV 89023
$\$ 4.101 .51$ FOR FURTKER INFORMATION:

$\$ 4,101.51$ CYNDI DELONG

LAS VEGAS

(702) $295-6753$

NV $89193-852$

TO FREEZE EQUIPMENT:

CYNDI DELONG

LAS VEGAS

(702) $295-6753$

NV 89193-8521

LOCATION OF EQUIPMENT:

REYNOLDS ELECTRICAL \& ENGINEERING CO

NEVADA TEST SITE

MERCURY

NV 89023 
EFFECTIVE DATE: 07/01/94 EXPIRATION DATE: $07 / 31 / 94$

FSC

COOE EXCESS PROPERTY DESCRIPTION

REFERENCE NUMBER : 283

6625

MFR: TEKTRONIX MOOEL: 540

S/N : 546

REPORT NUMBER: $899305-4032$

REFERENCE NUMBER : 284

6625 OSCILLOSCOPE

MFR: TEKTRONIX MOOEL: 546

$S / N: 249$

REPORT NUMBER: $899305-4032$

$$
\text { ELIGIBLE EQUIPMENT }
$$

No OF

COND UNITS UNIT

ACQUISITION PRICE

600001 EA

COST $/ U N I T$ :
TOTAL COST

LINE ITEM NUMBER: 0030

$600001 \mathrm{EA}$ COST/UNII:
TOTAL COST:

LINE ITEM NUMBER: 0031
$\$ 4,534.28$
$\$ 4,534.28$

CYNDI DELONG

LAS VEGAS

(702) $295-6753$

NV 89193-8521

TO FREEZE EQUIPMENT:

CYND1 DELONG

LAS VEGAS

(702) $295-6753$

NV 89193-8521

LOCATION OF EQUIPMENT:

REYNOLDS ELECTRICAL \& ENGINEERING CO REYNOLDS ELECTRI

MERCURY

NV 89023

$\$ 3.991 .90$

FOR FURTHER INFORMATION:

CYNDI DELONG

LAS VEGAS

(702) $295-6753$

NV $89193-8521$

TO FREEZE EOUIPMENT:

CYNDI DELONG

LAS VEGAS

(702) 295-6753

NV 89193-8521

LOCATION OF EQUIPMENT

REYNOLDS ELECTRICAL \& ENGINEERING CO NEVADA TEST SITE

NEVADA TES
MERCURY

NV 89023 
EFFECIIVE DATE: $07 / 01 / 94$ EXPIRAIION DATE: $07 / 31 / 94$

FSC
COOE EXCESS PROPERIY DESCRIPTION

REFERENCE NUMBER : 285

6625 OSCILLOSCOPE 75443, MANUF: TEKTRONIX MODEL $466 \mathrm{~S} / \mathrm{N}$ B010237

1 EACH. SIZE: $12^{\prime \prime} \times 22^{\prime \prime F} / R \times 6 "$ H EST UT: 25 LBS MFGR YR: 1988

REPORT NUMBER: $896404-4124$

LINE ITEM NUMBER: 0015

\section{REFERENCE NUMBER : 286}

6625 OSCILLOSCOPE CAMERA MFR: TEXTRONIX, MOOEL NO. C.53, S/M B097701, ACO. OSCILLOSCOPE CAMERA MFR: TEXTRONIX, MOOEL NO.
DATE: 4/84, A PPROX. WT: 15 LBS., $8 . \mathrm{W} \times 10 . \mathrm{L} \times 8 . \mathrm{H}$.

REPORT NUMBER: $899105-4081$

LINE ITEM NUMBER: 0028
NO OF

COND UNITS UNIT

ACOUISITION PRICE

$400001 \mathrm{EA}$

COSI/UNII:
TOIAL COSI

$\$ 3,800.00$

$\$ 3,800.00$
$\$ 3,800.00$

(B)

BARBARA HOLMES, REP.
KANSAS CIIY

MO $84141-6159$

TO FREEZE EOUIPMENT:

BARBARA HOLMES, REP.

KANSAS CITY

(816) $997-2976$

M0 64141-6159

LOCATION OF EQUIPMENT:

ALL IEDSIGNAL, INC.

2000 EAST 95TH STREET

KANSAS CITY

MO $64131-3095$

COST/UNIT: TOTAL COST:
$\$ 1.926 .00$ $\$ 1,926.00$

FOR FURTHER INFORMATION:

LYNN E. SHACKEL FOOT

LIVERMORE

CA $94550-0969$

TO FREEZE EQUIPMENT:

IYNN E. SHACKELFOOT

LIVERMORE

(510) $294-3067$

CA $94550-0969$

LOCATION OF EOUIPMENT:

SANDIA MATIONAL LABORATORIES

PROPERTY REAPPLICATION DEPT. 8532-1

LIVERMORE

CA $94550-0969$ 
EFFECIIVE DAIE: $07 / 01 / 94$ EXPIRATION DATE: $07 / 31 / 94$

FSC
CODE EXCESS PROPERTY DESCRIPIION
REFERENCE NUMBER : 287
S625 OSCILLOSCOPE DUAL BEAM MFR: TEKTRON1X, MOOEL NO. R7844, S/N B1040824,

S625 OSCILLOSCOPE DUAL BEAM MFR: TEKTRON1X, MOOEL NO. R7844,
ACO. DATE: 4/79, APPROX WT: 40 LBS., 19'W X $23 . \mathrm{L} .7 \cdot \mathrm{H}$.

REPORT NUMEER: $899105-4081$

INE ITEM MUMBER: 0032

\section{REFERENCE NUMBER : 288}

6625 OSCILLOSCOPE MODEL R7704, MFR. TEKTRONIX, SER\# EMI

REPORT NUMBER: $899110-0125$

LIME ITEM MUMBER: 0014

\section{No of}

COND UNITS UNIT

$60001 \mathrm{EA}$

TOTAL COST:

$\$ 7,933.00$

$\$ 7.933 .00$

\section{LYWN E. SHACKELFOOT}

LYMM E. SHACKELFOOT

LIVERMORE

(510) $294-3067$

CA $94550-0969$

TO FREEZE EOUIPMENT:

LYNN E. SHACKELFOOT

LIVERMORE

CA $94550-0969$

LOCATION OF EOUIPMENT:

SAMDIA MATIONAL LABORATORIES

PROPERTY REAPPLICATION DEPT 8532.1

LIVERMORE CA $94550-0969$
$800001 E A$

COST/UNIT:

TOTAL COST:

$\$ 3,100.00$

$\$ 3,100.00$

FOR FURTHER INFORMAIION:
YOLANDA CUEVAS/MS DIV

OAKLAND

(510) $637-1767$

CA $94612-5208$

TO FREEZE EOUIPMENT:

YOLANDA CUEVAS/MS DIV

DAKLAMD

(510) $637-1767$

CA $94612-5208$

LOCATION OF EOUIPMENT:

GEMERAL ATOMICS

3550 GEMERAL ATOMICS COURT

SAN DIEGO

CA 9212 
EFFECIIVE DATE: $07 / 01 / 94$ EXPIRAIION DATE: $07 / 31 / 94$

FSC

\section{COOE EXCESS PROPERTY DESCRIPTION}

REFERENCE MUMBR : 280

6S25 OSCILLOSCOPE MODEL D40, MFR. TEKTRONIX, SERIAL \#'S: (1) 8021572, (2) OSCILLOSCOPE MODEL D D
B021906, (3) B021571

REPORT NUMBER: 899110-0125

LINE ITEM NUMBER: 0015

\section{REFERENCE NUMBER : 290}

6625 OSCILLOSCOPE MOOEL 5110, MFR. TEKTRONIX, SER\# B123117

REPORI NUMBER: 899110-0125

LINE ITEM NUMBER: 0016
No of
COND UMITS UNIT

700003 EA
COST/UNIT: TOTAL COST:
ACQUISITION PRICE

$\$ 1,315.00$ $\$ 3.945 .00$
FOR FURTHER IMFORMATIOW:

YOLAMDA CUEVAS/MS DIV

CA $94612-5208$

(510) $637-1767$

TO FREEZE EOUIPMENT:

YOLANDA CUEVAS/MS DIV

OAKLAND

(510) $637-1767$

CA $94612-5208$

LOCATION OF EOUIPMEMT

GEMEREL ATOMICS

3550 GEMERAL ATOUICS COURT

SAN DIEGO

CA 92121
7 00001 EA
FOR FURTHER INFORMATIOW

YOLAMDA CUEVAS/MS DIV

OAKLAND

(510) 637-i767

TO FREEZE EQUIPMENT:

YOLAMDA CUEVAS/MS DIV

OAKLAND

CA $94612-5208$

(510) $637-1767$

LOCATION OF EOUIPMENT:

GENERAL ATOMICS

3550 GEMERAL ATOMICS COURT

SAN DIEGO

CA 92121 
EFFECTIVE DATE: 07/01/94 EXPIRATION DATE: 07/31/94

FSC

COOE EXCESS PROPERTY DESCRIPTION

REFERENCE MUMBER : 291

6625 OSCILLOSCOPE NICOLET $4094 \mathrm{C}$ SCOPE, HAS A 5" DIAGONAL SCREEN, 120VAC, 60

HZ. NIC OLET IN, LO94C SER, 88503145.

REPORT NUMBER: $898355-4090$

LIME ITEM MUMBER: 002צ

REFERENCE NUMBER : 292

6625 OSCILLOSCOPE W/ RECPLAY ADIO DISC MFR: NICOLET, MODEL NO. 4094B/XF44,

S/N 87B02840/85C00813, ACO. DATE: $10 / 87$ APPROX HT: OO LABS . 17 IU X

$18^{\prime} \mathrm{L} \times 14^{\circ} \mathrm{H}$

no of

COND UNITS UNI

ACOUISITION PRICE

600001 EA

COST/UNIT:

TOTAL COST

$\$ 7.900 .00$

$\$ 7.900 .00$

\section{ALAOTEROE}

ELOY $\boldsymbol{~ W . ~ G I R O M ~}$

(505) $844-2746$

87185

TO FREEZE EOUIPMEMT:

ELOY N. GIRON

ALBUOUEROUE

(505) $844-2746$

87185

LOCATION OF EOUIPMENT:

SANo Ia MATIOMaL LaBoratoriES

PROPERTY REAPPLICATION DIVISION 7617

AL BUCUEROUE

i14 87123

COST/UNIT:

TOTAL COST:
$\$ 20.375 .00$ $320,375.00$
FOR FURTMER IMFORMATION:

IYUM E. SHACKELFOOT

LIVERTORE

(510) $294-3067$

CA $94550-0969$

IO FREEZE EQUI PMENT.

I YMU E. SHACKELFOOT

LIVERMORE

(510) $294-3067$

LOCATION OF EOUIPMENT:

SADDIA MATIOMAL LaBORATORIES

PROPERTY REAPPLICATIOA DEPT. 8532-1

LIVERHORE

CA $94550-0969$ 
EFFECIIVE DATE: 07/01/96 EXPIRATION DATE: 07/31/94

$\operatorname{SSC}$

COOE EXCESS PROPERTY DESCRIPTION

REFERENCE NUMBER : 293

6625 OSCILLOSCOPE W/ RECPLAY AUDIO DISC MFR: NICOLET, MODEL NO. 4094A/XF44, S/N 85801505/87C01613, ACO. DATE: 10/87, APPROX. WT: 90 LABS 1714 $18 \cdot \mathrm{L} \times 14 \cdot \mathrm{H}$.
REPOPT MUMEER : $899105-4081$

LINE ITEM MUMBER: 0027

REFERENCE MUMBER : 296

6625 OSCILLOSCOPE, DIGITAL DC TO 40OMHZ BANDWIDTH AT 1OMV/DIV CALIBRATED SUEEP RATES TO 500 PS/DIV. 120VAC, $60 \mathrm{HZ}$. TEKTRONIX, 7854, SER.

500001 EA B010204.
No of

COND UNITS UWIT

ACOUISITION PRICE

COST/UNIT:

TOTAL COST:

$\$ 10,830.00$

$\$ 10,830.00$

400001 EA

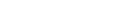

(2)

(

LYMA E. SHACXEL FOOT

LIVERMORE

(510) $294-3067$

CA $94550-0969$

TO FREEZE EOUIPMENT:

LYMM E. SHACKELFOOT

LIVERMORE

(510) $294-3067$

CA $94550-0969$

LOCATION OF EQUIPMENT:

SAMDIA MATIOAAL LABORATORIES

PROPERTY REAPPLICATION DEPT. 8532-1

LIVERMORE

CA $94550-0969$

REPORT MUMBER: $898355-4090$

LIME ITEM MUMBER: 0027

\section{$\cos T / U N 1 T:$ \\ TOTAL COST:}
$\$ 12.324 .00$ $\$ 12,324.00$

FOR FURTHER IMFORMATIOM:
ELOY N. GIRON
ALBUOUEROUE

(505) $864-2746$

TO FREEZE EOUIPMEHT:

ELOY $N$. GIRON

ALBUOUEROUE

(505) $844-2746$

LOCATIOW OF EQUIPMENT:

SAMDIA MATIOWAL LABORATORIES

PROPERTY REAPPLICATION DIVISION 7617

ALBUOUEROUE 
EFFECTIVE DATE: 07/01/94 EXFIRATION DATE: $07 / 31 / 94$

COOE EXCESS PROPERTY DESCRIPIION

REFERENCE NUMBER : 295

GO25 OSCILLOSCOPE DIGITAL SCOPE HAS A 5" DIAGONAL CRT, TWO PLUG INS, ONE WITH A 5-1/4" D:SK DR!VE STORAGE. I2OVAC, $60 \mathrm{HZ}$. 20903C, SER. 301178 WITH A $5-1 / 4$ M: D:SK

REFERENCE NUMBER : 290

FOR FURTHER INFORMATION:

JOANNE BREZNIK

LIVERMORE

CA 94550

(510) $424-4101$

TO FREEZE EOUIPMENT:

JOAMME BREZNIK

LIVERMORE

CA 94550

LINE ITEM NUMBER: 0027
(510) $424-4101$

OCATION OF EOUIPMENT:

IALRENCE LIVERMORE NATIOMAL LAB

EXCESS PROPERTY SECTION, L-696

LIVERMORE

CA 94550 
EFFECTIVE DATE: 07/01/94 EXPIRATION DATE: 07/31/94

FSC

COOE EXCESS PROPERTY DESCRIPIION

REFEREMCE MUMBER : 297

6625 OSCILLOSCOPE, MFR. TEKTRONIX, MOOEL 7104, ACO. YR. 80, PROP. N O. 3417926

REPORT MUMBER: $899102 \cdot 4163$

LINE ITEM NUMBER: 0032

REFERENCE NUMBER : 298

6625 OSCILLOSCOPE-STORAGE, MFR. TEKTRON1X, MOOEL 7834, ACO. YR. 85, E39314

600001 EA

$\cos$ TUN1T: TOIAL COST:
$\$ 13,201.74$ $\$ 13,201.74$

FOR FURTHER INFORMATION:

JOANWE BREZMIK

LIVERMORE

(510) $424-410$

CA 94550

TO FREEZE EOUIPMENT:

JOANME BREZNIK

LIVERMORE

(510) $424-4101$

CA 94550

JOAMME BREZMIK

(510) $424-4 i 01$

TO FREEZE EOUIPMENT:

JOANWE BREZMIK

LIVERMORE
$-10) 424-4101$

A 94550

LOCATION OF EQUIPMENT:

LALREMCE LIVERMORE MATIOMAL LAB

EXCESS PROPERTY SECTION, L-696

LIVERMORE

Ca 94550

REPORT NLMBER: $899102-4163$

LINE ITEM NUMBER: 0031

LOCATION OF EOUIPMENT

LAURENCE LIVERMORE MATIOMAL LAB EXCESS PROPERTY SECTION L L-696

LIVERMORE

A 94550 
EFFECTIVE DATE: $07 / 11 / 94$ EXPIRATION DATE: $07 / 31 / 94$

FSC
COOE EXCESS PROPERTY DESCRIPTION

REFERENCE NUMBER : 299

OS625 OSCILLOSCOPE/ON CART MFR: TEKTRONIX, MODEL NO. 7 A26/7633, S/N

B096768/B121475, ACQ. D ATE: 7/75, APPROX. WT: 120 LBS., 16 ' W X 32 'L $\mathrm{L}$ 46 ' H.

REPORT NUMBER: $899105-408$

LINE ITEM NUMBER: 0029

REFERENCE NUMBER : 300

作 DATE: $10 / 82$, APPROX. WT: 100 LBS., $19 . \mathrm{W} \times 28 . \mathrm{L} \times 48^{\prime} \mathrm{H}$.

REPORT NUMBER : $899105-408$

LINE ITEM NUMBER: 0030

No of

COND UNITS UNIT

$500001 E A$

ACQUISITION PRICE

COST/UNIT:

TOTAL COST

$\$ 4,821.00$

$\$ 4,821.00$

\section{TO FREEZE EQUIPMENT: \\ LYNN E. SHACKELFOOT}

LIVERMORE

(510) 294-3067

CA $94550-0969$

LOCATION OF EOUIPMENT:

SANDIA NATIONAL LABORATORIES

PROPERTY REAPPLICATION DEPT. 8532-1

LIVERMORE

CA $94550-0969$

\section{COST/UNIT: \\ TOTAL COST:}

$\$ 11,782.00$

$\$ 11,782.00$
FOR FURTHER INFORMATION:

LYNN E. SHACKELLFOOT

LIVERMORE

(510) 294-3067

CA $94550-0969$

TO FREEZE EQUIPMENT:

LYNN E. SHACKELFOOT

IVERMORE

CA $94550-0969$

(510) $294-3067$

LOCATION OF EQUIPMENT:

SANDIA NATIONAL LABORATORIES

PROPERTY REAPPLICATION DEPT. 8532-1

LIVERMORE

CA $94550-0969$ 
EFFECTIVE DATE: $07 / 01 / 94$ EXPIRATION DATE: $07 / 31 / 94$

FSC

\section{COOE EXCESS PROPERTY DESCRIPIION}

REFERENCE NUMBER : 301

6625 OSCILLOSCOPE/ON CART MFR: TEKTRONIX, MODEL NO. 7844/7B92/7B53, S/N

OSCILLOSCOPE/ON CART MFR: TEKTRONIX, MODEL NO. 7844/7B92/7B53, S/N
B141699/B010411, A CQ. DATE: 5/79, APPROX. WT: 100 LBS., 19' W X $28 . \mathrm{L} \times$ $48 \cdot \mathrm{H}$.

REPORT NUMBER: $899105-4081$

LINE ITEM NUMBER: 0031
NO OF
COND UNITS UNII

$600001 \mathrm{EA}$
ACOUISITION PIICE

COST/UNIT: OTAL COST

$\$ 10,203.00$ $\$ 10,203.00$

\section{REFERENCE NUMBER : 302}

6625 PA SYSTEM FREEDOMIKE SOUND SYSTEM HAS RECHARGEABLE BATTERIES AUX MIC/H LEV EL VOLUME AND WIRELESS VOLUME. BROKEN CORNER ON BOX. EDUCAT DEV . VPT31, SER. 20495

\section{EA \\ COST/UNIT:
TOTAL COST:}

$\$ 1,656.00$

$\$ 1,656.00$
LINE ITEM NUMBER: 0058

FOR FURTHER INFORMATION:

ELOY N. GIRON

ALBUOUERQUE

(505) $844-2746$

NM 87185

TO FREEZE EQUIPMENT:

ELOY N. GIRON

ALBUOUERQUE

(505) $844-2746$

NM 87185

LOCATION OF EOUIPMENT:

SANDIA NATIONAL LABORATORIES

PROPERTY REAPPLICATION DIVISION 7617

ALBUQUEROUE 
EFFECTIVE DATE: $07 / 01 / 94$ EXPIRATION DATE: $07 / 31 / 94$

FSC

COOE EXCESS PROPERTY DESCRIPIION

REFERENCE NUMBER : 303

6625 PHASE SHIFTER 1978 STATES S/N 1471

REPORT NUMBER: $898277-4090$

REFERENCE NUMBER : 304

6625 PLUG IN TIME BASE MFR: TEKTRONIX MODEL: 7892 $S / N: \quad B 063584$
ELIGIBLE EOUIPMENT

NO OF

COND UNITS UNIT

ACQUISITION PRICE

$400001 \mathrm{EA}$

COST/UNIT:

TOTAL COST:

LINE ITEM NUMBER: 0007

\section{EA COST/UNIT: \\ TOTAL COST:}

LINE ITEM NUMBER: 0032
CONTACT(S)

$\$ 3,005.91$ FOR FURTHER INFORMATION:

$\$ 3,005.91$ TED TORREZ

LOVELAND

(303) $490-7212$

CO 80539-3003

TO FREEZE EQUIPMENT :

TED TORREZ

LOVELAND

CO $80539-3003$

LOCATION OF EQUIPMENT:

NER ADMINISTRATION

5555 EAST CROSSROADS BLVD

LOVELAND

CO $80538-8986$

REPORT NUMBER: $899305-4032$
FOR FURTHER INFORMATION:

CYNDI DELONG

LAS VEGAS

(702) $295-6753$

NV $89193-8521$

TO FREEZE EOUIPMENT

CYNDI DELONG

LAS VEGAS

NV 89193-8521

(702) 295-6753

LOCATION OF EQUIPMENT

REYNOLDS ELECTRICAL \& ENGINEERING CO

REYNOLDS ELECTRICAL
MEVADA TEST SITE

MERCURY

NV 89023 
EFFECTIVE DATE: $07 / 01 / 94$ EXPIRATION DATE: $07 / 31 / 94$

FSC

\section{COOE EXCESS PROPERTY DESCRIPTION}

REFERENCE NUMBER : 305

6625 POROSIMETER, MERCURY UNIT HAS A DUAL SEAL VACUUM PUMP AND FILTERS. HITH WARNING LIGHT S FOR MERCURY AND PRESSURE. PART OF V840448. MICROMERIT.

9200 . SER. 271.

REPORT NUMBER: $898355-4089$

REFERENCE NUMBER : 306

6625 PORTABIN

PORTABIN BNC MODEL: AP3

S/N: 11464

NO OF

COND UNITS UNIT

ACQUISITION PRICE

LINE ITEM NUMBER: 0059

COST/UNIT: TOTAL COST: $\quad \$ 58,992.00$

$00001 \mathrm{EA}$

$600001 \mathrm{EA}$

COST /UNIT:

OTAL COST:

LINE ITEM NUMBER: 0012
REPORT NUMBER: $899305-4032$ $\$ 58,992.00$

\section{FOR FURTHER INFORMATION:}

ELOY N. GIRON

ALBUQUERQUE

(505) $844-2746$

NM 87185

TO FREEZE EQUIPMENT:

ELOY N. GIRON

ALBUQUERQUE

(505) 844-2746

NM 87185

LOCATION OF EQUIPMENT:

SAND IA NATIONAL LABORATORIES

PROPERTY REAPPLICATION DIVISION 7617

ALBUOUERQUE

NM 87123

$\$ 1,093.22$

$\$ 1,093.22$

FOR FURTHER INFORMATION:

CYNDI DELONG

LAS VEGAS
(702) $295-6753$

NV $89193-8521$

TO FREEZE EQUIPMENT:

CYNDI DELONG

LAS VEGAS

(702) 295-6753

NV 89193-8521

LOCATION OF EQUIPMENT:

REYNOLDS ELECTRICAL \& ENGINEERING CO NEVADA TEST SITE

NV 89023 
FSC

\section{CODE EXCESS PROPERTY DESCRIPIION}

REFERENCE NUMBER : 307

6625 PROCESSOR, FOURIER THIS INSTRUMENT HAS DISPLAY MODES FOR INPUT, MEASUREMENT, REFEREN CE, FREQ AND PULSE. RESPONSE IT ALSO HAS TIME/FREQ AND SWEEP AVERA GE DISPLAYS. 12OVAC, $60 \mathrm{HZ}$.

REPORT NUMBER: $898355-4089$

LINE ITEM NUMBER: 0060

REFERENCE NUMBER : 308

6625 PROGRAMMER DIGELEC MODEL EP804, S/N 1270, P/N 257502.

REPORT NUMBER: $890207-4116$

LINE IIEM NUMBER: 0382
NO OF

COND UNITS UNIT

ACQUISITION PRICE

$600001 E A$

COST/UNIT: TOTAL COST:

\section{$\$ 13,636.00$}

$\$ 13,636.00$

\section{ELOY N. GIRON \\ ALBUQUERQUE}

(505) $844-2746$

TO FREEZE EQUIPMENT :

ELOY N. GIRON

ALBUOUEROUE

(505) $844-2746$

NM 87185

LOCATION OF EQUIPMENT:

SANDIA NATIONAL LABORATORIES

PROPERTY REAPPLICATION DIVISION 7617

ALBUQUERQUE NM 87123
$700001 \mathrm{EA}$ COST/UNIT:
$\$ 1,495.00$ $\$ 1,495.00$
FOR FURTHER INFORMATION:

SANOY MOON

DDAHO FALLS

(208) $526-2611$

ID $83415-4100$

TO FREEZE EQUIPMENT:

SANDY MOON

IDAHO FALLS

(208) 526-2611

$1083415-4100$

LOCATION OF EQUIPMENT:

IDAHO NATIONAL ENGINEERING LAB.

CF-674 WAREHOUSE

SCOVILLE

ID $83415-4100$ 
EFFECTIVE DATE: $07 / 01 / 94$ EXPIRATION DATE: $07 / 31 / 94$

FSC

6625 PULSE GENERATOR 50 MHZ PULSE GENERATOR, WITH CONTROLS FOR MOOE, TRIGGER PERIOO, D ELAY, HIDTH, TRANSITION, LEADING EDGE TRAILING EDGE, OUTPUT, AND LOWER LEVEL. 12OVAC, $60 \mathrm{HZ}$. WAVETEK.

REPORT NUMBER: $898355-4089$

LINE ITEM NUMBER: 0046

REFERENCE NUMBER : 310

6625 RECORDER MFR: MOORE PRODUCT

MOO: $5321 T 2$

SER: NONE TYPE: SIRIP-CHART DOE 39715M PRC-40014

REPORT NUMBER : $899109-4101$

INE ITEM NUMBER: 0019
TOTAL COST

NO OF

COND UNITS UNIT

ACQUISITION PRICE

COST/UNIT:

TOTAL COST:

$\$ 1,500.00$
$\$ 1,500.00$

\section{ELOY N. GIRON \\ ALBUOUERQUE}

INFORMATION:

(505) $844-2746$

NM 87185

TO FREEZE EZUIIPMENT:

ELOY N. GIRON

ALBUOUERQUE

(505) $844-2746$

NM 87185

LOCATION OF EQUIPMENT:

SANDIA NATIONAL LABORATORIES

A MAT REAPPLICATION DIVISION 7617

ALBUQUERQUE

NM 87123

685.46

MATTIE PACE

STANFORD

(415) $926-223$

CA 94309

TO FREEZE EQUI PMENT:

MATTIE PACE

STANFORD

(415) $926-2231$

CA 94309

LOCATION OF EQUIPMENT:

STANFORD LINEAR ACCELERATOR CENTER 2575 SAND HILL ROAD

MENLO PARK

CA 94025 
EFFECTIVE DATE: $07 / 01 / 94$ EXPIRATION DATE: $07 / 31 / 94$

FSC

COOE EXCESS PROPERTY DESCRIPIION

REFERENCE NUMBER : 311

6625 RECORDER SIRIP CHART MFR: HEWLETT PACKARD MODEL: $7100 B 019$ S/N: A09046

REPORT NUMGER: $899305-4032$

LINE ITEM NUMBER: 0016

REFERENCE NUMBER : 312
6625 RECORDER STRIP CHART MFR: HEWLETT PACKARD MODEL: $7100 B R$ S/N: 02962

REPORT NUMBER : $899305-4032$

LINE ITEM NUMBER: 0017
No o

COND UNITS UNIT

ACQUISITION PRICE

800001 EA

COST/UNIT: TOTAL COST:

\section{$\$ 2,321.55$}

$\$ 2,321.55$

CONTACT(S)

FOR FURTHER INFORMATION:

CYNDI DELONG

AS VEGAS

(702) 295-6753

NV $89193-8521$

TO FREEZE EQUIPMENT:

CYNDI DELONG

AS VEGAS

(702) $295-6753$

NV 89193-8521

LOCATION OF EOUIPMENT:

REYNOLDS ELECTRICAL \& ENGINEERING CO NEYADA TEST SITE

MEVADA TES

NV 89023
$800001 \mathrm{EA}$

COST/UNIT:
TOTAL COST:

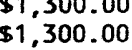

FOR FURTHER INFORMATION:

CYNDI DELONG

AS VEGAS

(702) $295-6753$

NV 89193-8521

TO FREEZE EQUIPMENT :

CYNDI DELONG

LAS VEGAS

(702) $295-6753$

NV $89193-8521$

LOCATION OF EQUIPMENT:

REYNOLDS ELECTRICAL \& ENGINEERING CO MEVADA TEST SITE

MERCURY

NV 89023 
EFFECTIVE DATE: 07/01/96 EXPIRATIDN DATE: 07/31/94

FSC

COOE EXCESS PROPERTY DESCRIPIION

REFERENCE NUMBER : 313

6625 RECORDER X-Y MFR: MOSELY FL MODEL: 202

REPORT NUMBER: $899305-4039$

LINE ITEM NUMBER: 0007

REFERENCE NUMBER : 314

6625 RECORDER, STRIP CHART 8 CHANNEL RECORDER, INPUTS IN BACK FOR CHANNELS.

HOMEYUELL, 1800 , SER, VOLTAGE

REPORT NUMBER: $898355-4090$

LINE ITEM NUMBER: 0002
No OF

COND UNITS UNIT

ACQUISITION PRICE

600001 EA

COSTIUNIT:
TOTAL COST:

$\$ 2,285.00$

$\$ 2,285.00$

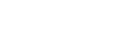

\section{FOR FURTHER IMFORMATION: \\ CYMDI DELONG}

LAS VEGAS

(702) $295-6753$

NV 89193-8521

TO FREEZE EOUIPMENT:

CYHDI DELONG

LAS VEGAS

(702) $295-6753$

NV 89193-852

LOCATION OF EOUIPMENT

REYMOLDS ELECTRICAL \& ENGINEERIMG CO NEVADA TEST SITE

MERCURY

NY 89023
$600001 \mathrm{EA}$
COST/UNIT: $\$ 8.879 .00$
FOR FURTHER IUFORMATION:

ELOY N. GIRON

ALBUOUEROUE

(505) $844-2746$

TO FREEZE EOUIPMENT:

ELOY N. GIRON

ALBUOUEROUE

(S05) $844-2746$

NM 87185

LOCATION OF EQUIPMENT:

SAMDIA NATIONAL LABORATORIES

PROPERTY REAPPLICATION DIVISION 7617

ALBUOUERQUE

NM 87123 
EFFECTIVE DATE: $07 / 01 / 94$ EXPIRATION DATE: $07 / 31 / 94$

FSC

\section{COOE EXCESS PROPERTY DESCRIPTION}

REFERENCE NUMBER : 315

6625 RECORDER, STRIP CHART 8 CHANNEL RECORDER, INPUT IN BACK FOR CHANNELS

CHART SPEED, TO 100,120 VAC, $60 \mathrm{HZ}$. HONEYWELL, 1400, SER. 00 00 AD8.

REPORT NUMEER: $898355-4090$

INE ITEM NUMBER: 0003
No of

COND UNITS UNIT

ACQUISITION PRICE

COST/UNIT:

TOTAL COST:

$00001 \mathrm{EA}$
REFERENCE NUMBER : 310

6625 RECORDER STRIP CHART 8 CHANNEL RECORDER, INPUTS IN BACK FOR CHANNELS.

I2OVAC, $60 \mathrm{HZ}$. HONEYHELL, 1800. SER. 00403AD8.
600001 EA

COST/UNIT:
TOTAL COST:
$\$ 8,879.00$
$\$ 8,879.00$

\author{
FOR FURTHER INFORMATION: \\ ELOY N. GIRON \\ ALBUOUEROUE \\ (505) $844-2746$ \\ NM 87185 \\ TO FREEZE EQUIPMENT \\ ELOY N. GIRON \\ ALBUOUEROUE \\ (505) $844-2746$ \\ Mim 87185 \\ LOCATION OF EOUIPMENT: \\ SAMD IA NATIONAL LABORATORIES \\ PROPERTY REAPPLICATION DIVISION 7617 \\ PROPERTY REAPPLICATION DII \\ NM 87123
}

REPORT NUMBER: $898355 \cdot 4090$

LINE ITEM NUMBER: 0004 
EFFECTIVE DATE: 07/01/96 EXPIRATION DATE: 07/31/94

\section{COOE EXCESS PROPERTY DESCRIPTION}

REFERENCE NUMBER : 317

6625 RECORDER, STRIP CHART 8 CHANNEL CHART RECORDER, CHART SPEED 1 TO 100 IN $\mathrm{MM} / \mathrm{SEC}$, MM/MIN AND MM/HR RECORDING RANGE .5 MV TO $50 \mathrm{MV} / \mathrm{MM} .120 \mathrm{VAC}, 60$ HZ. MFE CORF. 1800, SER. 221648.

REPORT NUMBER: $898355-4090$

LINE ITEM NUMBER: 0005

REFERENCE NUMBER : 318

6625 RECORDER, STRIP CHART ONE PEN RECORDER, GRAPH SPAN RANGE O TO 100 ,

TIME/IN IS 1 SEC TO $40 \mathrm{SEC}, 1 \mathrm{MIN}$ TO $10 \mathrm{MIN}$. ON/OFF CONTROLS. 12OVAC

$60 \mathrm{HZ}$. HONEYW ELL, 1951100, SER. O02.

REPORT NUMBER: $898355-4090$

LINE ITEM NUMBER: 0006
NO OF

COND UNITS UNIT

\section{ACOUISITION PRICE}

$\$ 8,764.00$ $\$ 8,764.00$ TOTAL COST:

\section{FOR FURTHER ' FORMATION:}

ELOY N. GIRON

ALBUOUEROUE

(505) $844-2746$

NM $8: 185$

TO FREEZE EQUIPMENT:

ELOY N. GIRON

ALBUOUEROUE

(505) $844-2746$

NM 87185

LOCATION OF EOUIPMENT:

SAMDIA NATIOMAL LABORATORIES

SAMDIA NATIONAL LABORATORIES
PROPERTY REAPPLICATION DIVISION 7617

PROPERTY REAPPLICATIOW DIVISION 7617
ALBUOUEROUE
87123

$600001 \mathrm{EA}$

COST/UNIT:

TOTAL COST:
$\$ 1,539.00$ $\$ 1.539 .00$

\section{FOR FURTHER INFORMATION \\ ELOY N. GIRON}

ALBUOUEROUE

(505) $844-2746$

87185

TO FREEZE EQUIPMENT :

ELOY $N$. GIRON

ALBUQUEROUE

(505) $844-2746$

MM 87185

LOCATION OF EOUIPMENT:

SAMDIA MATIONAL LABORATORIES

PROPERTY REAPPLICATION DIVISION 7617

PROPERTY REAPPLICATION DIVISIOW 7617 
EFFECTIVE DATE: $07 / 01 / 94$ EXPIRATION DATE: $07 / 31 / 94$

FSC

COOE EXCESS PROPERTY DESCRIPTION

REFERENCE NUMBER : 319

6625 RECORDER, STRIP CHART ONE PEN RECORDER, GRAPH SPAN RANGE FROM 0 TO 100 TIMEIIN IS I SE C TO 40 SEC I MIN TO $60 \mathrm{HZ}$. H ONEYWELL. 193111, SER. 003.

ELIGIBLE EOUIPMENT

COND UNITS UNIT

ACQUISITION PRICE

600001 EA COST/UNIT: TOTAL COST:

LINE ITEM NUMBER: 0007

REFERENCE NUMBER : 320

6625 RECORDER, STRIP CHART ONE PEN RECORDER, GRAPH RANGE 0 TO 100, CAL VOLTS IS $0.1 M V$ SPAN RANGE 0.2 TO 2 AND -5 TO -10.120 VAC, $60 \mathrm{HZ}$. HONEYHELL.

$600001 \mathrm{EA}$

$\$ 1.058 .00$

$\$ 1,058.00$

$$
\text { ALBUOUEROUE }
$$

ELOY N. GIRON

TO FREEZE EQUIPMENT :

ELOY N. GIRON

ALBUOUEROUE

(505) $844-2746$

M7185

LOCATION OF EOUIPMEMT:

SAMDIA MATIOUAL LABORATORIES

PROPERTY REAPPLICATION DIVISION 7617

ALBUOUEROUE
COST/UNIT:

TOTAL COST:
$\$ 1.058 .00$

$\$ 1,058.00$
FOR FURTHER INFORMATION:

ELOY N. GIROW

ALBUOUEROUE

(505) $846-2746$

4. 87185

TO FREEZE EQUIPMENT:

ELOY N. GIROA

(505) $844-2746$

87185

LOCATIOW OF EQUIPMENT:

SAMDIA MATIOMAL LABORATORIES

PROPERTY REAPPLICATION DIVISICN 7617

ALBUOUEROUE 
EFFECTIVE DATE: 07/01/94 EXPIRATION DATE: $07 / 31 / 94$

\section{FSC
COOE EXCESS PROPERTY DESCRIPIION}

REFERENCE MUMBER : 321

6625 RECORDER, STRIP FOR TEMPERATURE, RANGE - 100 DEG F 10250 DEG F. 120VAC, 7 00001 EA $60 \mathrm{HZ}$. H ONEYHELL, 3020112, SER. 13729001.

LINE ITEM NUMBER: 0009

REFERENCE HUMBER : 322

RECORDER, STRIP CHART ONE PEN RECORDER HAS GRAPH RANGE FROM 0 TO 100 CAL VOL TS IS 0.1 MV
194222 . SER. 022.

$$
\text { No of }
$$

\section{COND UNITS UNII ACOUISIIION PRICE}

COST/UNT:

TOTAL COST:

$\$ 1.845 .00$

$\$ 1.845 .00$
COMTACT(S)

\author{
FOR FURTHER IMFORMAIION \\ ELOY N. GIRON \\ AL BUOUEROUE \\ (505) $844-2746$ \\ 4M 87185 \\ TO FREEZE EQUIPMENT : \\ ELOY N. GIRON \\ ALBUQUEROUE \\ (4) 87185 \\ (505) $844-2746$ \\ LOCATION OF EOUIPMENT: \\ SAMDIA MATIOMAL LABORATORIES \\ PROPERTY REAPPLICATION DIVISION 7617 \\ AL BUDUEROUE

600001 EA

COST/UNIT:
TOTAL COST:

$\$ 2,298.00$

$\$ 2.298 .00$

\section{FOR FURTHER IMFORMATIOW: \\ ELOY M. GIROW}

ALBUOUEROUE

(505) $844-2746$

MM 87:85

IO FREEZE EQUIPMEMT:

ELOY N. GIRON

ALBUOUEROUE

(505) $864-2746$

87185

\section{LOCATION OF EOUIPMENT:}

SANDIA MAIIOMAL LABORATORIES

PROPERTY REAPPLICATIOA DIVISION 7617

ALBUOUEROUE 
EFFECTIVE DATE: $07 / 01 / 94$ EXPIRATION DATE: $07 / 31 / 94$

FSC

COOE EXCESS PROPERTY DESCRIPTION

\section{REFERENCE NUMBER : 323}

6625 RECORDER, STRIP CHART ONE PEN RECORDER HAS GRAPH RANGE FROM 0 TO 100. CAL VOLTS IS $0.1 \mathrm{MV}$, SPAN RANGE 0.2 TO 2 AND 5 TO 100 . 120VAC, $60 \mathrm{HZ}$ HONEYWELL, 1 94222, SER. 023.

REPORT NUMBER: $898355-4090$

LINE ITEM MUMBER: 0012

REFERENCE NUMBER : 326

6625 RECORDER, STRIP CHART USE WITH TYPE K THERMOCOUPLE, CHART DRIVE, HIGH AND LOW SPEED WIT H RANGES FROM 0-300 DEGREES FAHRENHEIT. 12OVAC, $60 \mathrm{HZ}$ HONEYWELL, $158 \times 890$, SER. R364216.

REPORT NUMBER : $898355-4090$

LINE ITEM MUMBER: 0013 wo of
COND UNITS UNIT

$60001 E A$
Cost/Unit: TOTAL COST:
ACOUISITION PRICE

\section{$\$ 2.298 .00$} $\$ 2,298.00$
FOR FURTHER IMFORMATION:

ELOY N. GIRO

ALBUOUEROUE

(505) $844-2746$

m 87185

TO FREEZE EOUIPMENT:

ELOY N. GIROM

ALBUOUEROUE

(505) $8464-2746$

87185

LOCAIION OF EQUIPMENT:

SAMDIA MATIOMAL LABORATORIES

PROPERTY REAPPLICATION DIVISION 7617

ALBUOUEROUE

87123
$\$ 1,923.00$ $\$ 1.923 .00$
TOTAL COST:

\section{FOR FURTMER INFORMATIOW:}

ELOY M. GIRU

AL BUOUEROUE

MM 87185

TO FREEZE EOUIPMENT:

ELOY N. GIROM

ALBUOUEROUE

(505) $846-2746$

LOCATIOW OF EQUIPMENT:

SAMDIA MATIOMAL LABORATORIES

PROPERTY REAPPLICATION DIVISION 7617

ALBUOUEROUE

in 87123 
EFFECTIVE DATE: 07/01/94 EXPIRATION DATE: 07/31/94

FSC

\section{COOE EXCESS PROPERTY DESCRIPTION}

REFERENCE MUMBER : 325

6625 RECORDER, STRIP CHART ONE PEN RECORDER, GRAPH SPAN RANGE 0 TO 100 IIME/IN IS TSEC TO 40 SEC, 1 MIN TO 10 MIN. HAS ON/OFF CONTROLS.

IZOVAC, 60 HZ. HOWEYN ELL, 19511, SER. 004.

REPORT NUMBER: $898355-4090$

LIME ITEM NUMBER: 0014

REFERENCE NUMBER : 326

G625 RECORDER, STRIP CHART ONE PEN RECORDER, GRAPH RANGE FROM 0 TO 100, CAL VOLIS IS 0.1 MV, SPAN RANGE 0.2 TO 2 AND 5 TO 100 , VERNIER RANGE: -1 TO VOLIS IS 0.1 MV. SPAN RANGE 0.2 TO 2 AND S TO 100 , VERH
NO of

COND UNITS UNIT

ACOUISITION PRICE

600001 EA

COST/UNIT:

TOTAL COST:

$\$ 1,529.00$

$\$ 1,529.00$

(2)

(505) $844-2746$

TO FREEZE EOUIPMENT:

ELOY N. GIRON

(505) $844-2746$

LOCATION OF EOUIPMENT:

SAMDIA MATIOMAL LABORATORIES

PROPERTY REAPPLICAIION DIVISION 7617

ALBUOUEROUE

im 87123
COST/UNIT:

TOTAL COST:
$\$ 2,397.00$
$\$ 2,397.00$
FOR FURTHER IMFORMATION:

ELOY N. GIRO

ALBUQUEROUE

(505) $844-2746$

MM 87185

TO FREEZE EOUIPMENT:

ELOY N. GIROW

ALBUOUEROUE

87185

(505) 844-2746

LOCATION OF EOUIPMENT

SAMOIA MATIOMAL LABORATORIES

PROPERTY REAPPLICATION DIVISION 7617

AL BUQUEROUE

1. 87123 
EFFECIIVE DATE: $07 / 01 / 94$ EXPIRATION DATE: $07 / 31 / 94$

FSC

CODE EXCESS PROPERTY DESCRIPTION

REFERENCE NUMBER : 327

6625 RECORDER, STRIP CHART ONE PEN RECORDER, GRAPH SPAN RANGE 0 TO 100, TIME IN-IS 1 SEC TO 40 SEC, 1 MIN TO $10 \mathrm{MIN}$. ON/OFF CONTROLS. 120VAC, $60 \mathrm{HZ}$ HONEYWE LL, 1951100, SER. 003195.

REPORT NUMBER: $898355-4090$

LINE ITEM NUMBER: 0016

REFERENCE NUMBER : 328

6625 RECORDER, STRIP CHART ONE PEN RECORDER GRAPH SPAN RANGE FROM 0 TO 100.

12OVAC, $60 \mathrm{HZ}$. HONEYWELL, 1951100, SER. 139002.
No of

COND UNITS UNI

ACQUISITION PRICE

COST/UNIT:

TOTAL COST:

$600001 \mathrm{EA}$

$\$ 1,385.00$

$$
\text { ALBUQUERQUE }
$$

ELOY N. GIRON

(505) $844-2746$

TO FREEZE EQUIPMENT:

ELOY N. GIROM

ELOY N. GIROH

(505) $844-2746$

LOCATION OF EQUIPMENT:

SANDIA NATIONAL LABORATORIES

PROPERTY REAPPLICATION DIVISION 7617

ALBUQUERQUE

REPORT NUMBER: $898355-4090$

LINE ITEM NUMBER: 0017
COST/UNIT:

TOTAL COST:
$\$ 1,380.00$ $\$ 1,380.00$
FOR FURTHER INFORMATION:

ELOY N. GIRON

ALBUQUERQUE

(505) 844-2746

NM 87185

TO FREEZE EQUIPMENT:

ELOY N. GIRON

NM 87185

(505) $844-2746$

LOCATION OF EOUIPMENT:

SANDIA NATIONAL LABORATORIES

SANDIA NATIONAL LABORATORIES
PROPERTY REAPPLICATION DIVISION 7617

ALBUQUERQUE

NM 87123 
EFFECTIVE DATE: 07/01/94 EXPIRATION DATE: 07/31/94

\section{COOE EXCESS PROPERTY DESCRIPTION}

REFERENCE :UUMBER : 320

6625 RECORDER SIRIP CHART ONE PEN RECORDER, GRAPH RANGE FROM 0 TO 100, CAL VOLTS IS $0.1 \mathrm{MV}$, SPAN RANGE 0.2 TO 2 AND 5 TO 100. 120VAC, $60 \mathrm{H} Z$

HONEYHELL, 193 i11, SER. 4726013.

REPORT NUMBER: $898355-4090$

LINE ITEM NUMBER: 0018

REFERENCE NUMBER : 330

RECORDER, STRIP CHART TWO PEN CHART RECORDER, WITH RANGE FROM 1 TO 100

MV, AND SPEED CA N BE SET IN MINUTE OR HOUR. 12OVAC, $60 \mathrm{HZ}$. HONEYWELL,

AFB200A, S ER. NONE.

REPORT MUMBER: $898355-4090$

LINE ITEM NUMBER: 001
NO OF

COND UNITS UNIT

ACOUISITION PRICE

$600001 \mathrm{EA}$

COST/UNIT: TOTAL COST: $\$ 1,129.00$
$\$ 1,129.00$

FURTHER INFORMATION:

ELOY N. GIRON

ALBU

NM 87185

TO FREEZE EQUIPMENT:

ELOY N. GIRON

ALBUQUERQLE

(505) $844-2746$

NM 87185

LOCATION OF EQUIPMENT :

SANDIA MATIONAL LABORATORIES

PROPERTY REAPPLICATION DIVISION 7617

ALBUQUERQUE

NM 87123

$600001 \mathrm{EA}$

COST/UNIT:

TOTAL COST:

$\$ 1,152.00$

$\$ 1,152.00$

FOR FURTHER INFORMATION:

ELOY N. GIRON

ALBUQUERQUE

NM 87185

IO FREEZE EQUIPMENT:

ELOY N. GIRON

ALBUOUERQUE

(505) $844-2746$

NM 87185

LOCATION OF EQUIPMENT:

SANDIA NATIONAL LABORATORIES

PROPERTY REAPPLICATION DIVISION 7617

ALBUQUERQUE 
EFFECTIVE DATE: 07/01/94 EXPIRATION DATE: $07 / 31 / 94$

\section{FSC}

COOE EXCESS PROPERTY DESCRIPIION

REFERENCE NUMBER : 33

6625 RECORDER, STRIP CHART TWO CHANNEL CHART RECORDER, VOLT RANGE IMV TO 100 RECORDER, STRIP CHART TWO CHANNEL CHART RECORDER, VOLT RANGE IMV TO 100
VOLTS. SPEED S ELECTION IS IN TIME PER INCH 0.1 MIN TO 60 MIN. 12OVAC, $60 \mathrm{HZ}$. 6 06222, SER. 23001.

REPORT NUMBER: $898355-4102$

LINE ITEM NUMBER: 0042

\section{REFERENCE NUMBER : 332}

RECORDER, STRIP CHART 2 CHANNEL RECORDER VOLT RANGE $10 M V$ TO 100 VOLTS, USES ROLL PAPER 11" WIDE. 12OVAC, $60 \mathrm{HZ}$. HOUST INST, D521712, SER. D1353111.

$600001 \mathrm{EA}$

COST/UNIT: TOTAL COST :

ALB

\section{FOR FURTHER INFORMATION:}

ELOY N. GIRON

ALBUQUERQUE
(505) $844-2746$

NM 87185

TO FREEZE EQUIPMENT :

ELOY N. GIRON

ALBUQUEROUE

(505) $844-2746$

NM 87185

LOCATION OF EOUIPMENT:

SANDIA NATIONAL LABORATORIES

PROPERTY REAPPLICATION DIV - 7617

ALBUOUERQUE

NM 87123 
EFFECTIVE DATE: $07 / 01 / 94$ EXPIRATION DATE: $07 / 31 / 94$

FSC

\section{CODE EXCESS PROFERTY DESCRIPIION}

REFERENCE NUMBER : 333

6625 RECORDER, STRIP CHART HAS A SPEED OF 10 MIN TO 1 SEC. TIME/IN AND IS A TWO CHANNEL WITH SPAN AND ZERO RANGE CONTROLS AND CAN MEASURE FROM . IMV TO 10OMV. 120VAC, SO HZ. HONEYWELL, 194, SER. 2578.

REPORT NUMBER: $898355-4102$

LINE ITEM NUMBER: 0044

REFERENCE NUMBER : 334

6625 RECORDER, TEMPERATURE RANGE IS O DEG F TO 400 DEG F. 120VAC, $60 \mathrm{~Hz}$. HONEYWELL, 1124018 ，SER. 001.

REPORT NUMBER: $898355-4090$

LINE ITEM NUMBER: 0010
No of

COND UNITS UNIT

ACQUISITION PRICE

$600001 \mathrm{EA}$

COST/UNIT:

TOTAL COST:

\section{$\$ 2,279.00$}

$\$ 2,279.00$

ELOY N. GIRON
ALBUQUERQUE
(505) $844-2746$

NM 87185

TO FREEZE EQUIPMENT:

ELOY N. GIRON

ALBUQUERQUE

(505) $844-2746$

NM 87185

LOCATION OF EQUIPMENT:

SANDIA NATIONAL LABORATORIES

PROPERTY REAPPLICATION DIV. 7617

PROPERTY REAPPLICATION DIV.
ALBUQUERQUE
NM 87123
$600001 \mathrm{EA}$

TOTAL COST:

$\$ 1,917.00$

$\$ 1,917.00$

FOR FURTHER INFORMATION:

ELOY N. GIRON

ALBUQUEROUE

(505) $844-2746$

NM 87185

TO FREEZE EQUIPMENT:

ELOY N. GIRON

ALBUQUERQUE

(505) $844-2746$

NM 87185

LOCATION OF EOUIPMENT:

SANDIA NATIONAL LABORATORIES

PROPERTY REAPPLICATION DIVISION 7617

ALBUQUERQUE 
EFFECIIVE DATE: $07 / 01 / 94$ EXPIRATION DATE: $07 / 31 / 94$

REFERENCE NUMBER : 335

6625 RECORDER, WAVEFORM DIGITIZES AND STORES SINGLE SHOT OR REPETITIVE SIGNALS. 120VAC, $60 \mathrm{HZ}$. HEW PACK, 5180A, SER. 2044 A001.

$500001 \mathrm{EA}$ COST/UNIT:
TOTAL COST:

\section{$\$ 18,110.00$ $\$ 18,110.00$}

LINE ITEM NUMBER: 0001
REPORT NUMBER: $898355-4090$
REFERENCE NUMBER : 336

6625 RECORDER, WAVEFORM HAS 16K MEMORY RECORDS UP TO 32 WAVEFORMS, HIGH SPEED SIGNAL ACOU ISITION, ACCURATELY DIGITIZES TRANSIENT SIGNALS AND UP TO 1 MILLI ON WORDS DATA TRANSFER. 12OVAC, $60 \mathrm{HZ}$. HP.

REPORT NUMBER: $898355-4102$
LINE ITEM NUMBER: 0045

\section{$00001 \mathrm{EA}$ TOTAL COST:}

$\$ 16,000.00$ $16,000.00$

FOR FURTHER INFORMATION:

ELOY N. GIRON

ALBUQUEROUE

NM 87185

TO FREEZE EQUIPMENT:

ELOY N. GIRON

ALBUQUERQUE

(505) $844-2746$

NM 87185

LOCATION OF EOUIPMENT:

SANDIA NATIONAL LABORAYORIES

PROPERTY REAPPLICATION DIV. 7617

ALBUOUERQUE

NM 87123 
EFFECTIVE DATE: $07,1 / 94$ EXPIRATION DATE: $07 / 31 / 94$

FSC

COOE EXCESS PROPERTY DESCRIPTION

REFERENCE NUMBER : 337

6625 RECORDER XY HORIZONTAL SENSITIVITY 1MV/IN TO 10V/IN, VERTICAL SEISSITIVITY IMV /IN TO 1OV/IN AND RATE .01 IN/SEC. TO 2 IN/SEC. 12OVAC, $60 \mathrm{HZ}$. EL ECTRO-IN, 530T, 2013CD71.

REPORT NUMBER: $898355-4090$

LINE ITEM NUMBER: 0020

REFERENCE NUMBER : 338

6625 RECORDER, XY HP XY RECORDER IS A GENERAL USE X-Y RECORDER AND CAN RECORD A SIG NAL FROM DC TO 1OHZ AT 2 CM PEAK TO PEAK AMPLITUDE ON EITHER AXIS AND HAS A CHART. IZOVAC, $60 \mathrm{HZ}$. HEW PACK

LINE ITEM NUMBER: 0046

NO OF

\section{COND UNITS UNIT $\quad$ ACOUISITION PRICE}

$500001 \mathrm{EA}$ COST/UNIT:
TOTAL COST:

$\$ 1,353.00$ $\$ 1,353.00$

ELOY N. GIRON

ALBUQUEROUE

(505) $844-2746$

NM 87185

TO FREEZE EOUIPMENT

ELOY N. GIRON

ALBUQUERQUE

(505) $844-2746$

NM 87185

LOCATION OF EQUIPMENT:

SANDIA NATIONAL LABORATORIES

PROPERTY REAPPLICATION OIVISION 7617

ALBUQUERQUUE

NM 87123

COST/UNIT:

TOTAL COST:

$\$ 3,665.00$

$\$ 3,665.00$

$00001 \mathrm{EA}$ TOTAL

FOR FURTHER INFORMATION:

ELOY N. GIRON

ALBUQUEROUE

(505) 844-2746

NM 87185

TO FREEZE EQUIPMENT:

ELOY N. GIRON

ALBUQUERQUE

(505) $844-2746$

NM 87185

LOCATION OF EQUIPMENT:

SANDIA NATIONAL LABORATORIES

PROPERTY REAPPLICATION DIV. 7617

ALBUQUERQUE

. 8717 
EFFECTIVE DATE: 07/01/94 EXPIRATION DATE: $07 / 31 / 94$

FSC

\section{CODE EXCESS PROPERTY DESCRIPTION}

REFERENCE NUMBER : 339

6625 RECORDER, XY TIME BASE RANGE FROM $0.5,110,50$ 100/IN, TYPE OF INPUT FLOATING WITH INPUTS THRU POST OR REAR CONNECTOR. 12OVAC, 60 HZ. HEW PAC K, 7015B, SER. 11A02922.

REPORT NUMBER: $898355-4090$

LINE ITEM NUMBER: OQ??

REFERENCE NUMBER : 340

6625 RECORDER-DIGITAL LOGIC, MFR. BIOMATION, MODEL 81000, ACQ. YR. 78, PROP. 7 00001 EA NO. 3611591

\section{NO OF}

$600001 E A$
COND UNITS UNIT

ACQUISITION PRICE

COST/UNIT: TOTAL COST:

$\$ 1,504.00$ $\$ 1,504.00$

\section{ELOY N. GIRON
ALBUQUERQUE \\ (505) $844-2746$}

TO FREEZE EQUIPMENT:

ELOY N. GIRON

ALBUQUERQUE

(505) $844-2746$

NM 87185

LOCATION OF EQUIPMENT

SANDIA NATIONAL LABORATORIES

PROPERTY REAPPLICATION DIVISION 7617

PROPERTY REAP

MM 87123

COST/UNIT:

TOTAL COST:

$\$ 8,584.50$

FOR FURTHER INFORMATION:

JOANNE BREZNIK

LIVERMORE

(510) $424-4101$

CA 94550

TO FREEZE EOUIPMENT:

JOANNE BREZNIK

LIVERMORE

CA 94550

(510) 424-4101

LOCATION OF EQUIPMENT:

LAWRENCE LIVERMORE NATIONAL LAB

EXCESS SECTION, L-696

LXCESS SECT 
EFFECTIVE DATE: $07 / 01 / 94$ EXPIRAIION DATE: $07 / 31 / 94$

FSC

CCOE EXCESS PROPERTY DESCRIPIION

REFERENCE NUMBER : 341

6625 RECTOR, ACQ. YR. 86, E39613

REPORT NUMBER: $899102-4163$

$$
\text { No of }
$$

COND UNITS UNIT

ACOUISITION PRICE

400001 EA

COST/UNIT: TOTAL COST:

LINE ITEM NUMBER: 0028

\section{$\$ 2,926.00$
$\$ 2,926.00$}

FOR FURTHER INFORMATION:

JOANNE BREZNIK

LIVERMORE

CA 94550

TO FREEZE EQUIPMENT:

JOANME BREZNIK

LIVERMORE

(510) $424-4101$

CA 94550

LOCATION OF EQUIPMENT

LAURENCE LIVERMORE NATIONAL LAB

EXCESS PROPERTY SECTION, L-696

LIVERMORE

REFERENCE NUMBER : 342

6625 REED SHUTTLE LOOM 81119, MANUF: TEXTRO (PYRMID) MODEL N/A S/N N/A

1 ASSY., APPROX. 8 PIECES, MOVABLE FRONT SIZE: $12^{\prime \prime} \mathrm{W} \times 14^{\prime \prime F} / \mathrm{R} \times 8^{\prime \prime} \mathrm{H}$ EST

$400001 \mathrm{EA}$

COST/UNIT:

TOTAL COST:

$\$ 8,358.00$

$\$ 8,358.00$

FOR FURTHER INFORMATION:

BARBARA HOLMES, REP.

KANSAS CITY

(816) $997-2976$

MO $64141-6159$

TO FREEZE EQUIPMENT:

BARBARA HOLMES, REP.

KANSAS CITY

(816) $997-2976$

MO $64141-6159$

LOCATION OF EQUIPMENT

ALLIEDSIGNAL, INC.

2000 EAST 95TH STREET

KANSAS CITY

M0 64131-3095 
EFFECTIVE DATE: 07/01/94 EXPIRATION DATE: 07/31/94

FSC

COOE EXCESS PROPERTY DESCRIPTION

REFERENCE NUMBER : 343

6625 REFLECTOMETER TIME DOMAIN MFR: HEWLETT PACKARD MODEL $1415 \mathrm{~A}$

S/N: 45000390

REPORT MUMBER: $899305-4032$

LINE ITEM NUMBER: 0019

REFERENCE NUMBER : 344

6625 REFLECTOMETER TIME DOMAIN MFR: HEWLETT PACKARD MODEL

$1415 A$

1415A
$S / N=81702055$

REPORT NIMBER: $899305-4032$

LINE ITEM NUMBER: 0020
No of

COND UNITS UNIT

ACQUISITION PRICE

$600001 \mathrm{EA}$

COST/UNIT:

TOTAL COST:

\section{$\$ 1,212.09$
$\$ 1,212.09$}

$\$ 1,212.09$

CYNDI DELONG

LAS VEGAS

(702) $295-6753$

TO FREEZE EOUIPMENT:

CYNDI DELONG

LAS VEGAS

(702) $295-6753$

NV $89193-8521$

LOCATION OF EQUIPMENT:

REYNOLDS ELECTRICAL \& ENGINEERING CO REVADA TEST SITE

MERCURY

NV 89023

600001 EA

COST/UNIT: TOTAL COST:

$\$ 1,050.00$

HER INFORMATION:

CYNDI DELONG

(702) $295-6753$

NV $89193-8521$

TO FREEZE EQUIPMENT:

CYNDI DELONG

LAS VEGAS

(702) 295-6753

NV $89193-8521$

LOCATION OF EQUIPMENT:

REYNOLDS ELECTRICAL \& ENGINEERING CO NEVADA TEST SITE

MERCURY NV 89023 
EFFECTIVE DATE : $07 / 01 / 94$ EXPIRAIION DATE: $07 / 31 / 94$

\section{FSC \\ COOE EXCESS PROPERTY OESCRIPIION \\ REFERENCE MUMBER : 345 \\ 6625 REFLECTOMETER TIME DOMAIN MFR: HEHLETT PACXARD MODEL}

$$
\text { ELIGIBLE EQUIPMENT }
$$

PAGE 17

NO of

COND UNITS UNIT

ACQUISITION PRICE

600001 EA COST UNIT:
TOTAL COST:

LINE ITEM NUMBER: 0021
600001 EA REFLECTOMETER TIME DOMAIN MFR: HEWLETT PACKARD MODEL: $1415 \mathrm{~A}$
S/N: 2429
REPORT MUMBER : $899305-4032$
LINE ITEM NUMBER: 0022

\section{COST/UNIT:} TOTAL COST:
$\$ 1,020.00$
$\$ 1,020.00$ $\$ 1,212.09$
$\$ 1,212.09$

FOR FURTHER INFORMATION:

CYMDI DELONG

LAS VEGAS

(702) $295-6753$

NV $89193-8521$

TO FREEZE EQUIPMENT

CYNDI DELONG

LAS VEGAS

(702) $295-6753$

NV 89193-8521

LOCATION OF EQUIPMENT:

REYMOLDS ELECTRICAL \& ENGINEERIMG CO NEVADA TEST SITE

NV 89023

FOR FURTHER INFORMATION

CYMDI DELLNG

LAS VEGAS
(702) 295-6753

NV $89193-8521$

TO FREEZE EQUIPMENT:

CYNDI DELONG

(702) $295-6753$

NV $89193-8521$

LOCATION OF EQUIPMENT

REYNOLDS ELECTRICAL \& ENGINEERING CO MEVADA TEST SITE

NV 89023 
EFFECTIVE DATE: $07 / 01 / 94$ EXPIRATION DATE: $07 / 31 / 94$

\section{FSC \\ NO OF}

COOE EXCESS PROPERTY DESCRIPTION

REFERENCE NUMBER : 347

6625 REFLECTROMETER TEST SET REFLECTOMETER COUPLER FREQ RANGE 1.7 TO $4.2 \mathrm{GHZ}$. WITH HIGH DIRECT IVITY COUPLER. NARDA, 3073, 2071.

REPORT NUMBER : $898355-4090$

LINE ITEM NUMBER: 0068

REFERENCE NUMBER : 348

ROBOT MFG: SEIKO MODEL: TR3000 ACO DATE: $6 / 87$

REF NO: 56310-01 INCLUDES MISC. PARTS. NO FURTHER DETAILS AVAILABLE AS

$400001 E A$

TO THE FUNCTION AND TYPE OF ROBOT THIS IS.

REPORT NUMBER : $890531-4103$

LIME ITEM NUMBER: 0010
COND UNITS UNIT

ACOUISITION PRICE
COST/UNIT: TOTAL COST:

\section{$\$ 1.375 .00$ \\ $\$ 1,375.00$} TO FREEZE EQUIPMENT:
ALBUOUEROUEN
ALBUQUEROUE
(505) $844-2746$
LOCATION OF EQUIPMENT:
SANDIA MATIONAL LABORATORIES PROPERTY REAPPLICATION DIVISION 7617
ALBUQUERQUE In 87123
URTHER INFORMATION:
FOR FURTHER
ELOY N. GIROI
ALBUQUEROUE
87185
MM 87185

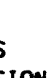

$34,500.00$

COST/UNIT:

TOTAL COST:

$\$ 34,500.00$

FOR FURTHER INFORMATION:
NANCY J. SITTON

RICHLAND

(509) $376-5108$

HA 99352

TO FREEZE EOUIPMENT :

NANCY J. SITTON

RICHLAND

m 99352

(509) 376-5108

LOCATION OF EQUIPMENT

UESTINGHOUSE HAMFORD COMPANY

ESS\&S, 1167A BLDG/1100 AREA

RICHLAND

HA 99352


EFFECTIVE DATE: $07 / 01 / 94$ EXPIRATION DATE: $07 / 31 / 94$

FSC

COOE EXCESS PROPERTY OESCRIPIION

REFERENCE NUMBER : 349

6625 SAMPLER

MFR: HEWLETT PACKARD MODEL: 1432A

S/N : 80100171

REPORT NUMBER: $899305-4032$
NO OF

COND UNITS UNIT

ACOUISITION PRICE

500001 EA

COSTIUNIT:
TOTAL COST:
$\$ 1,000.00$ $\$ 1,000.00$

LINE ITEM NUMBER: 0009

REFERENCE HUMBER : 350

6625 SCANNER MFR: HEWLETT PACKARD, MODEL NO. 3495A, S/N 1428A00661, ACO.

DATE : 6/76, APPROX. WT: 45 LBS. $19 . \mathrm{W} \times 20.1 \times 71 \mathrm{H}$.

500001 EA

COST/UNIT: TOTAL COST:

$\$ 3,432.00$ $\$ 3,432.00$

FOR FURTHER INFORMATION

LYMN E. SHACKEL FOOT

CA $94550-0969$

(510) $294-3067$

TO FREEZE EQUIPMENT:

LYWN E. SHACKELFOOT

(510) $294-3067$

CA $94550-0969$

LOCATION OF EQUIPMENT:

SAMDIA MATIOMAL LABORATORIES

PROPERTY REAPPLICATION DEPT. 3532-1

LIVEAMTORE

CA $94550-0969$ 
EFFECTIVE DATE: $07 / 01 / 94$ EXPIRATION DATE: $07 / 31 / 94$

FSC

\section{COOE EXCESS PROPERTY OESCRIPIION}

REFERENCE MUMBER : 351

O625 SCANMER, DATA SWITCHES ANAL OG INPUT SIGNALS TO AN APPROPRIATE MEASURING DEVICE. 12OVAC, 60 HZ. HEW PACK, 3495A, 28 A06011.

\section{REFERENCE NUMGER : 352}

6025 SCANNER, DATA SWITCHES ANALOG INPUT SIGNALS TO AN APPROPRIATE MEASURING 700001 EA DROPRIATE MEASURIMG DOVAC, GO HZ HEU PACK, 3495A, 28900721.

$7 \quad 00001 \mathrm{EA}$

\section{No of}

COND UNITS UNIT

ACOUISITION PRICE

CONTACT(S) COST/UNIT: TOTAL COST: $\$ 2,236.00$
$\$ 2,236.00$

IMFORMATION :

ELOY N. GIRON

ALBUOUEROUE

(505) $844-2746$

N*4 87185

IO FREEZE EOUIPMENT :

ELOY M. GIRON

ALBUOUEROUE

(505) $844-2746$

Mm 87185

LOCATION OF EOUIPMENT

SANDIA MATIONAL LABORATORIES

PROPERTY REAPPLICATION DIV. 7617

ALBUOUEROUE

im 87123

COST/UNIT:

TOTAL COST:

$\$ 3.618 .00$

$\$ 3,618.00$

ER INFORMATION:

ELOY N. GIRON

ALBUOUEROUE

NM 87185

(505) $844-2746$

TO FREEZE EOUIPMENT:

ELOY N. GIRON

(505) $844-2746$

N4 87185

LOCATION OF EOUIPMENT :

SAMDIA MATIOMAL LABORATORIES

PROPERTY REAPPLICATION DIV. 7617

alBUOUERQUE 
EFFECIIVE DATE: 07/01/94 EXPIRATION DATE: 07/31/94

COOE EXCESS PROPERTY OESCRIPTION

REFEREMCE MUMBER : 353

6625 SCAMHER, DATA SWIICHES ANALOG INPUT SIGMALS TO AN APPROPRIATE MEASURING DEVICE. 120VAC, $60 \mathrm{HZ}$. HEW PACK, 3495A, 28A00731.

REPORT NUMBER: $898355-4102$

LINE ITEM MUMBER: 0049
No OF COMD UNITS UNIT

$00001 \mathrm{EA}$

OOOOI EA

COST/UNIT:
TOTAL COST:

ACOUISITIOA PRICE
$\$ 3.618 .00$ $\$ 3,618.00$

ELOY N. GIROM
ALBUOUEROUE
(505) $844-2746$

TO FREEZE EQUIPMEMT:

ELOY N. GIRON

ALBUOUEROUE

(505) $844-2746$

Mm 87185

LOCATION OF EOUIPMENT:

SANDIA MA - MIIL LABORATORIES

PROPERTY REAPPLICATION DIV. 7617

PROPERTY REAPPLICATION DIV. 7617
ALBUQUERQUE

\section{REFERENCE MUMBER : 354}

6625 STAMNER, DATA SUITCHES ANALOG INPUT SIGNALS TO AN APPROPRIATE MEASURING DEVICE. POWER 120VAC, $60 \mathrm{HZ}$., HEW PACK, 3495A, SER. $28 A 00727$.

00001 EA

COST/UNIT:

TOTAL COST:

$\$ 3,618.00$
$\$ 3,618.00$

FOR FURTHER INFORMATION:
ELOY N. GIRON
ALBUOUEROUE
(505) $844-2746$

TO FREEZE EOUIPMENT:

ELOY N. GIRON

ALBUOUERQUE

(505) $844-2746$

87185

LOCATION OF EQUIPMEMT:

SANDIA MATIOWAL LABORATORIES

PROPERTY REAPPLICATION DIV. 7617

PROPERTY REAPPLICATION DIV. 7617
ALBUOUEROUE 

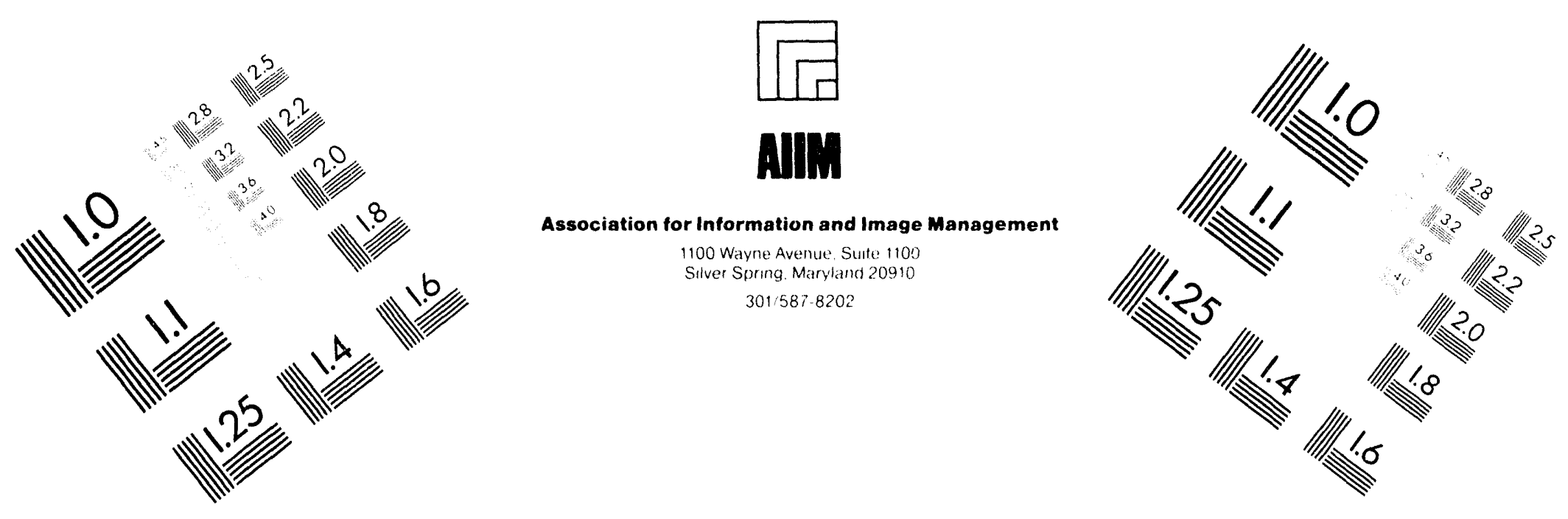

\section{Centimeter}

$\mid \begin{aligned} & 2 \\ & \mid\end{aligned}$

Inches
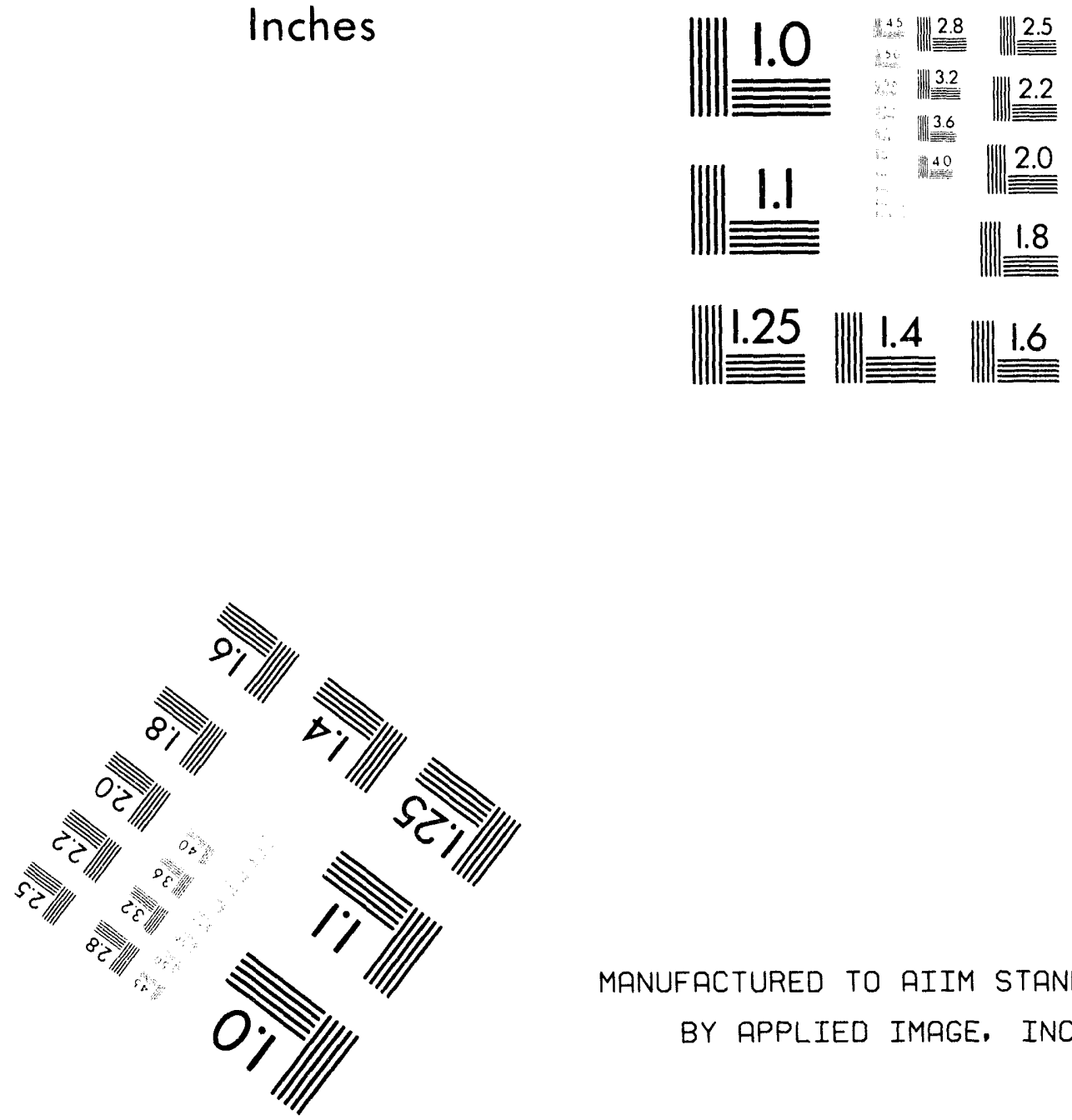

MANUFACTURED TO AIIM STANDARDS

BY APPLIED IMAGE. INC.

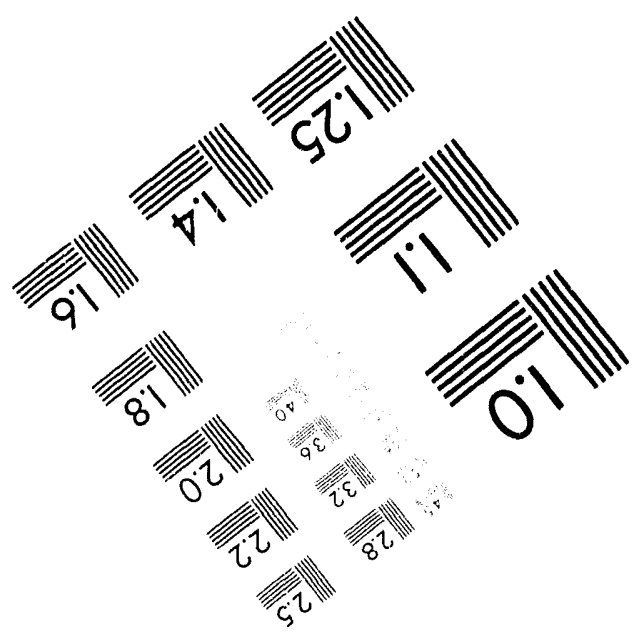



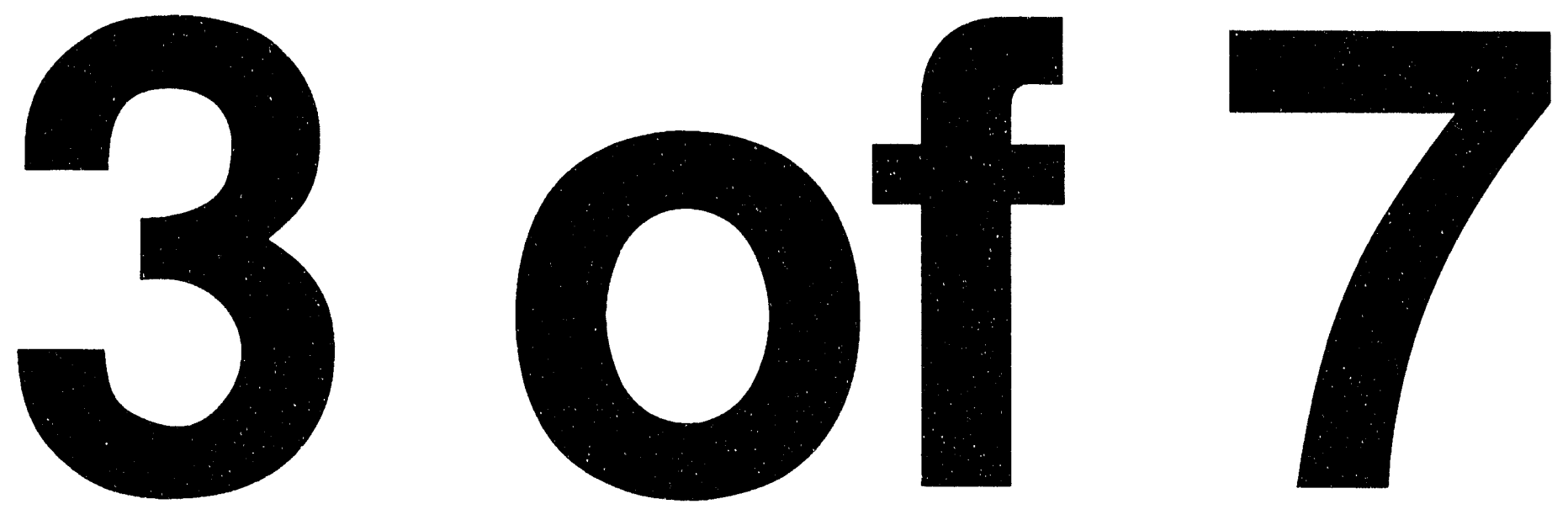
EFFECTIVE DATE: 07/01/94 EXPIRATION DATE: $07 / 31 / 94$

FSC

\section{COOE EXCESS PROPERTY DESCRIPIION}

REFERENCE NUMBER : 355

6625 SCANER DATA SUITCHES ANALOG INPUT SIGNALS TO AN APPROPRIATE MEASURING DEVICE. 120VAC, $60 \mathrm{HZ}$. HEW PACK, 3495A, 28A00726.

REPORT NUMBER: $898355-4102$

LINE ITEM NUMBER: 005

REFERENCE NUMBER : 356 6625 SCANNER, DATA SWITCHES ANALOG INPUT SIGNALS TO AN APPROPRIATE MEASURING 7 00001 EA
DEVICE. 120VAC, 60 HZ. HEW PACK, 3495A, 28A00724.

REPORT NUMBER: $898355-4102$

LINE ITEM NUMBER: 0052

NO OF
COND UNITS UNIT

ACQUISITION PRICE

$\$ 3,168.00$

COST/UNIT:

TOTAL COST:

RER INFORMATION:

ELOY N. GIRON

ALBUQUERQUE

(505) $844-2746$

NM 87185

TO FREEZE EQUIPMENT :

ELOY N. GIRON

ALBUQUEROUE

(505) $844-2746$

NM 87185

LOCATION OF EOUIPMENT

PROPERTY REAPPLICATION DIV. 7617

ALBUOUERQUE
COST/UNIT:

TOTAL COST:
$\$ 3,618.00$

$\$ 3,618.00$
FOR FURTHER INFORMATION :

ELOY N. GIRON

ALBUOUERQUE

(505) $844-2746$

NM 87185

TO FREEZE EQUIPMENT:

ELOY N. GIRON

ALBUQUEROUE

NM 87185

(505) $844-2746$

LOCATION OF EQUIPMENT:

SANDIA NATIONAL LABORATORIES

PROPERTY REAPPLICATION DIV. 7617

ALBUOUERQUE

NM 87123 
EFFECTIVE DATE: $07 / 01 / 94$ EXPIRATION DATE: $07 / 31 / 94$

$\mathrm{FSC}$

\section{CODE EXCESS PROPERTY DESCRIPTION}

REFERENCE NUMBER : 357

6625 SCANNER, DATA SWITCHES ANALOG INPUT SIGNALS TO AN APPROPRIATE MEASURING DEVICE, I2OVAC, $60 \mathrm{HZ}$. HEW PACK, 3495A, 28 A00722.

REPORT NUMBER: $898355-4102$

LINE ITEM NUMBER: 0053

\section{REFERENCE NUMBER : 358}

6625 SCANNER, DATA SH! TCHES ANALOG INPUT SIGNALS TO AN APPROPRIATE MEASURING DEVICE. 120VAC, 60 HZ. HEW PACK, 3495A, 28A00729.
NO OF

COND UNITS UNIT

ACQUISITION PRICE

$\$ 3,618.00$

COST/UNIT: TOIAL COST:

$\$ 3,618.00$

$00001 \mathrm{EA}$

NM 8718

ELOY N. GIRON

(505) $844-2746$

TO FREEZE EQUIPMENT:

ELOY N. GIRON

ALBUOUERQUE

(505) $844-2746$

NM 87185

LOCATION OF EQUIPMENT:

SANDIA NATIONAL LABORATORIES

PROPERTY REAPPLICATION DIV. 7617

ALBUQUERQUE
COST/UNIT: TOTAL COST:
$\$ 3,618.00$ $\$ 3,618.00$

FOR FURTHER INFORMATION:

ELOY N. GIRON

ALBUOUERQUE

87185

(505) $844-2746$

TO FREEZE EQUIPMENT:

ELOY N. GIRON

ALBUQUERQUE

(505) 844-2746

NM 87185

LOCATION OF EQUIPMENT

SANDIA NATIONAL LABORATORIES

PROPERTY REAPPLICATION DIV. 7617

ALBUQUERQUE 
EFFECTIVE DATE: $07 / 01 / 94$ EXPIRATION DATE: $07 / 31 / 94$

\section{FSC \\ NO OF}

COOE EXCESS PROPERTY DESCRIPTION

REFERENCE NUMBER : $350^{\circ}$

6625 SCOPE P-1, 1 TRACE DC TO 75 MHZ DUAL TRACE AMPLIFIER 5 MV/DIV TO 5 V/DIV SCOPE P-1, 1 TRACE DC TO 75 MHZ DUAL TRACE AMPLIFIER 5 MV/DIV TO CALIBRATED DEFLECTION FACTORS,
TEKTRONIX. 7AIGA, SER. B137143.

REPORT NUMBER: $898355-4102$

LINE ITEM NUMBER: 0061

\section{REFERENCE NUMBER : 360}

6625 SCOPE P.1 1 TRACE DC TO 225 MHZ BANDWIDTH, 5 MV/DIV TO 5V/DIV CALIBRATED DEFLECTION FACTORS, 1 M OHM INPUT. POWER FROM SCOPE. TEKTRONIX, 7A16A, SER. B 137147
COND UNITS UNIT

ACQUISITION PRICE

COST/UNIT:

TOTAL COST:

\section{$\$ 1,018.00$}

$\$ 1.018 .00$

$600001 \mathrm{EA}$

ELO

CONTACT(S)

FOR FURTHER INFORMATION:

ELOY N. GIRON

ALBUQUERQUE

505) $844-2746$

NM 87185

TO FREEZE EOUIPMENT:

ELOY N. GIRON

ALBUOUERQUE

(505) $844-2746$

NM 87185

LOCATION OF EQUIPMENT:

SANDIA NATIONAL LABORATORIES

PROPERTY REAPPLICATION DIV. 7617

ALBUQUEROUE

COST/UNIT:

TOTAL COST:
$\$ 1,018.00$
$\$ 1,018.00$

FOR FURTHER INFORMATION:

ELOY N. GIRON

ALBUQUERQUE

NM 87185

(505) $844-2746$

TO FREEZE EQUIPMENT:

ELOY N. GIRON
ALBUQUERQUE

(505) $844-2746$

NM 87185

LOCATION OF EQUIPMENT:

LOCATION OF EQUIPMENT:
SANDIA NATIONAL LABORATORIES

SANDIA NATIONAL LABORATORIES
PROPERTY REAPPLICATION DIV. 7617

PROPERTY REAPPLICATION DIV. 7617
ALBUQUERQUE 
EFFECTIVE DATE: $07 / 01 / 94$ EXPIRATION DATE: $07 / 31 / 94$

FSC

\section{CODE EXCESS PROPERTY DESCRIPTION}

REFERENCE NUMBER : 361

6625 SCOPE P-1, 1 IRACE DC 1075 MHZ DUAL TRACE AMPLIFIER, 5 MV/DIV TO 5 VIDIV CALIBRATED DEFLECTION FACTORS, IM OHM INPUT. POWER FROM SCOPE.

TEKTRONIX, 7A16A, SER. B137120.

REPORT NUMBER: $898355-4102$

LINE ITEM NUMBER: 0063

REFERENCE NUMBER : 362

6625 SCOPE P-I, ANALYZER FREO RANGE IS $0.01-40 G \mathrm{GZ}$, TUNING RANGE WITH INTERNAL 600001 EA MIXER 0 01-1 8.0 SHZ WITH EXTERNAL MIXER 12.4-40GHZ - POWER COMES FROM

MAINFRA ME. HEW PACK, 8555A, SER. 31A06448.

REPORT NUMBER: $898355-4102$

LINE ITEM NUMBER: 0055

NO OF

COND UNITS UNIT

$600001 \mathrm{EA}$

ACQUISITION PRICE

CONTACT(S)

COST/UNIT: TOTAL COST: $\$ 1,018.00$
$\$ 1,018.00$

FOR FURTHER INFORMATION:

ELOY N. GIRON

ALBUQUERQUE

NM 87185

TO FREEZE EQUIPMENT

ELOY N. GIRON

ALBUQUERQUE

(505) $844-2746$

NM 87185

LOCATION OF EQUIPMENT:

SANDIA NATIONAL LABORATORIES

PROPERTY REAPPLICATION DIV. 7617

ALBUUUEROUIE

NM 87123

COST/UNIT: TOTAL COST:

$\$ 7,445.00$ $\$ 7,445.00$ $\$ 7,445.00$

ALBU$$
\text { ELOY N. GIRON }
$$$$
\begin{aligned}
& \text { ALBUOUERQUE } \\
& \text { (505) } 844-2746
\end{aligned}
$$

FOR FURTHER INFORMATION:
ELOY N. GIRON

ELOY N. GIRON

LOCATION OF EQUIPMENT

SANDIA NATIONAL LABORATORIES PROPERTY REAPPLICATION DIV. 7617 ALBUQUERQUE 
EFFECTIVE DATE: $07 / 01 / 94$ EXPIRATION DATE: $07 / 31 / 94$

FSC

CODE EXCESS PROPERTY DESCRIPTION

REFERENCE NUMBER : 363

S625 SCOPE P-1. ANALYZER PLUG IN FOR THE SPECIRUM ANALYZER, FREO RANGE 10 30OKHZ AND $0.30 \mathrm{~K}$ HZ FOR BETTER RESOLUT!ON AT LOW FREQUENCIES. WORKS W/V944654 AND V920568. HEW PACK, 8556A, SER. 2148 A052.

REPORT NUMBER: $898355-4102$

LINE ITEM NUMBER: 0056

\section{REFERENCE NUMBER : 364}

6625 SCOPE P-1, ANALYZER HIGH RESOLUTION TO 100HZ, FLAT FREO RESPONSE +-10B,

HIGH SENSITIV ITY TO - 12ZDBM VARIABLE PERSISIENCE DISPLAY, EXTERNAL

COUNTER CAP ABILITY. POWER FROM MAINFRAME. HEW PACK.

REPORT NUMBER: $898355-4102$

LINE ITEM NUMBER: 005
NO OF

COND UNITS UNIT

ACQUISITION PRICE

600001 EA

COST/UNIT:

TOTAL COST:

$\$ 8,107.00$

$88,107.00$

(20)

ELOY N. GIRON

ALBUQUERQUE

(505) $844-2746$

NM 87185

TO FREEZE EQUIPMENT:

ELOY N. GIRON

ALBUOUERQUE

(505) $844-2746$

NM 87185

LOCATION OF EQUIPMENT:

SANDIA NATIONAL LABORATORIES

PROPERTY REAPPLICATION DIV. 7617

ALBUQUERQUE

NM 87123

$\$ 3,802.00$

$\$ 3,802.00$

COST/UNIT:
TOTAL COST:

FOR FURTHER INFORMATION:

ELOY N. GIRGN

ALBUQUERQUE

(505) $844-2746$

NM 87185

TO FREEZE EQUIPMENT:

ELOY N. GIRON

ALBUQUERQUE

NM 87185

(505) $844-2746$

LOCATION OF EQUIPMENT

SANDIA NATIONAL LABORATORIES

PROPERTY REAPPLICATION DIV. 7617

ALBUQUERQUE

NM 8712 
EFFECTIVE DATE: $07 / 01 / 94$ EXPIRATION DATE: $07 / 31 / 94$

\section{COOE EXCESS PROPERTY DESCRIPTION}

REFERENCE NUMBER : 365

6625 SCOPE P-1, DIFF DC TO 105MHZ BANDWIDTH, 1MV/DIV TO 5V/DIV CALIBRATED DEFLECTION F ACTORS, 20,000:1 CMRR, 10,000 CM EFFECTIVE SCREEN HEIGHT. 12OVAC, 60 HZ. TEKTRONIX, 7A 13, SER. B225318.

REPORT NUMBER: $898355-4090$

LINE IIEM NUMBER: 0050

\section{REFERENCE NUMBER : 366}

6625 SCOPE P-1, DIFF DC TO 105 MHZ BANDWIDTH, IMV/DIV TO 5V/DIV CALIBRATED DEFLECTION FACTORS, 20,000:1

CMRR, 10,000 CM EFFECTIVE SCREEN HEIGHT. POWE R FROM SCOPE, TEKTRONIX, 7A13, B240139.
NO OF
$600001 E A$

\section{COND UNITS UNIT}

\section{ACQUISITION PRICE}

\section{COST/UNIT:} TOTAL COST:

\section{$\$ 1,450.00$ \\ $\$ 1,450.00$}

INFORMATION:

ELOY N. GIRON

ALBUQUERQUE

(505) $844-2746$

NM 87185

TO FREEZE EQUIPMENT:

FLOY N. GIRON

ALBUQUERQUE

(505) $844-2746$

NM 87185

LOCATION OF EOUIPMENT:

SANDIA NATIONAL LABORATORIES

PROPERTY REAPPLICATION DIVISION 7617

ALBUQUERQUE

$600001 \mathrm{EA}$

COST/UNIT:

TOTAL COST:

$\$ 1,983.00$
$\$ 1,983.00$

FOR FURTHER INFORMATION:

ELOY N. GIRON

ALBUQUERQUE

(505) $844-2746$

NM 87185

TO FREEZE EQUIPMENT

ELOY N. GIRON

ALBUQUERQLE

(505) $844-2746$

NM 87185

LOCATION OF EQUIPMENT:

SANDIA NATIONAL LABORATORIES

PROPERTY REAPPLICATION DIVISION 7617

ALBUDUERQUE NM 87123 
EFFECTIVE DAIE: $07 / 01 / 94$ EXPIRATION DATE: $07 / 31 / 94$

FSC

COOE EXCESS PROPERTY OESCRIPIION

REFERENCE NUMBER : 367

6625 SCOPE P-1, DIFF DC TO 105 MH2 BANDWIDTH IMV/DIV TO 5 V/DIV CALIBRATED DEFLECTION FACTORS. POWER FROM SCOPE. TEKTRONIX, 7A13, SER. B257478.

NO OF

ACQUISITION PRICE

REPORT NUMBER: $898355-4090$

LINE ITEM NUMBER: 0054

REFERENCE NUMBER : 368

O625 SCOPE P-1, DIGITIZER PLUG IN FOR NICOLET SCOPE, HAS CONTROLS FOR STORAGE CONTROL TIME PER POINT, VOLIS FULL SCALE FOR CHANNELS A\&B. POWER COMES FROM S COPE. NICOLET IN, 4562 .

REPORT NUMBER: $898355-4090$

LINE ITEM NUMBER: 0060
$600001 E A$

COST/UNIT:

TOTAL COST:

$\$ 4,360.00$

$\$ 4,360.00$

CONTACISS)

FOR FURTHER INFORMATION:

ELOY N. GIRON

ALBUOUEROUE

(505) $844-2746$

NM 87185

TO FREEZE EQUIPMENT :

ELOY N. GIRON

ALBUQUERQUE

(505) $844-2746$

NM 87185

LOCATION OF EQUIPMENT:

SAMDIA NATIONAL LABORATORIES

PROPERTY REAPPLICATION DIVISION 7617

ALBUQUEROUE NM 87123
COST/UNIT: TOTAL COST:

$700001 \mathrm{EA}$

$\$ 4,900.00$

ALC

FOR FURTHER INFORMATION:
ELOY N. GIRON

ALBUQUEROUE

(505) $844-2746$

NM 87185

TO FREEZE EQUI PMENT :

ELOY N. GIRON

ALBUQUERQUE

(505) $844-2746$

NM 87185

LOCATION OF EQUIPMENT:

SANDIA NATIONAL LABORATORIES

PROPERTY REAPPLICATION DIVISION 7617

ALBUQUERQUE

NM 87123 
EFFECTIVE DATE: $07 / 01 / 94$ EXPIRATION DATE: $07 / 31 / 94$

FSC

COOE EXCESS PROPERTY DESCRIPTION

REFERENCE NUMBER : 369

6625 SCOPE P-I, DIGITIZER PLUG IN FOR NICOLET SCOPE, HAS CONIROLS FOR STORAGE CONTROL, TIME PER POINT, VOLTS FULL SCALE FOR CHANNELS A\&B. POWER COMES

FROM SCOPE. NICOLET IN, 4562 .

REPORT NUMEER: $898355-4090$

LINE ITEM NUMBER: 0061

REFERENCE NUMBER : 370

6625 SCOPE P-1, DIGITIZER DIGITIZING OSCILLOSCOPE, FULLY PROGRAMMABLE. HEW 600001 EA PACK, G1016A, S ER. 2522A007.

REPORT NUMBER: $898355-4102$

LINE ITEM NUMBER: 0060

NO OF

COND UNITS UNIT $\quad$ ACQUISITION PRICE

O0001 EA COST/UNIT: TOTAL COST:

$\$ 4,900.00$ $\$ 4,900.00$

FOR FURTHER INFORMATION:

ELOY N. GIRON

AL BUOUEROUE

(505) $844-2: 46$

NM 87185

TO FREEZE EQUIPMENT:

ELOY N. GIRON

ALBUQUERQUE

(505) $844-2746$

NM 87185

LOCATION OF EQUIPMENT:

SANDIA NATIONAL LABORATORIES

PROPERTY REAPPLICATION DIVISION 7617

ALBUQUERQUE

NM 87123

COST/UNIT: TOTAL COST:

$\$ 1,650.00$ $\$ 1,650.00$
FOR FURTHER INFORMATION:

ELOY N. GIRON

ALBUOUEROUE

(505) $844-2746$

TO FREEZE EQUIPMENT

ELOY N. GIRON

ALBUQUERQUE

(505) $844-2746$

NM 87185

LOCATION OF EQUIPMENT:

SANDIA NATIONAL LABORATORIES

PROPERTY REAPPLICATION DIV. 7617

ALBUQUEROUE NM 87123 
EFFECTIVE DATE: 07/01/94 EXPIRATION DATE: 07/31/94

FSC

COOE EXCESS PROPERTY DESCRIPTION

REFERENCE NUMBER : 371

6625 SCOPE P-1, DIGITIZER DIGITIZING OSCILLOSCOPE, FULLY PROGRAMMABLE WITH FEATURES AS AUTO SCALING. HEW PACK, 61016A, $2522 A 003$.

REPORT NUMBER: $898355-4102$

LINE ITEM NUMBER: 0069

REFERENCE NUMBER : 372

6625 SCOPE P-1, DIGITIZER DIGITIZING OSCILLOSCOPE, FULLY PROGRAMMABLE, HEW PACK, 61016A, SE R. 2522A002

REPORT NUMBER: $898355-4102$

LINE ITEM NUMBER: 0070
NO OF

COND UNITS UNIT ACQUISITION PRICE CONTACTSS
600001 EA COST/UNIT: TOTAL COST:

\section{$\$ 1,500.00$ $\$ 1,500.00$}

FOR FURTHER INFORMATION:

ELOY N. GIRON

ALBUQUEROUE

(505) $844-2746$

NM 87185

TO FREEZE EQUIPMENT:

ELOY N. GIRON

ALBUQUEROUE

(505) $844-2746$

NM 87185

LOCATION OF EQUIPMENT:

SANDIA NAIIONAL LABORATORIES

PROPERTY REAPPLICATION DIV. 7617

ALBUQUERQUE
6 O0001 EA COST/UNIT:

TOTAL COST:
$\$ 1.650 .00$

$\$ 1.650 .00$
FOR FURTHER INFORMATION:

ELOY N. GIRON

ALBUQUERQUE

(505) 844-2746

TO FREEZE EQUIPMENT

ELOY N. GIRON

ALBUQUERQUE
(505) $844-2746$

NM 87185

LOCATION OF EOUIPMENT:

SANDIA NATIONAL LABORATORIES

PROPERTY REAPPLICATION DIV. 7617

ALBUQUERQUE

NM 87123 


\section{EFFECTIVE DATE: $07 / 01 / 94$ EXPIRATION DATE: $07 / 31 / 94$}

FSC

COOE EXCESS PROPERTY DESCRIPTION

REFERENCE NUMBER : 373

6625 SCOPE P-I, DUAL TRACE DC TO 60OMHZ BANDWIDTH, 5MV/DIV TO TV/DIV CALIBRATED DEFLECTION F ACTORS, 5OOM INPUT. POWER COMES FROM SCOPE.

TEKTRONIX. 7A24, S ER. B010105.

REPORT NUMBER: $898355-4102$

LINE ITEM NUMBER: 0066

REFERENCE NUMBER : 376

6625 SCOPE P-1, LOGIC SAMPLING RATES CAN BE SELECTED UP TO 100 MHZ IN THE 4 CH MOOE. P OWER FROM SCOPE.

REPORT NUMBER: $898355-4090$

LINE ITEM NUMBER: 0056

NO OF

COND UNITS UNIT

ACQUISITION PRICE

CONTACT(S)

$600001 \mathrm{EA}$

COST/UNIT: TOTAL COST:

\section{$\$ 1,050.00$}

$\$ 1,050.00$

FOR FURTHER INFORMATION:

ELOY N. GIRON

ALBUQUERQUE

(505) $844-2746$

NM 87185

TO FREEZE EQUIPMENT:

ELOY N. GIRON

ALBUQUERQUE

(505) $844-2746$

NM 87185

LOCAT!ON OF EQUIPMENT:

SANDIA NATIONAL LABORATORIES

PROPERTY REAPPLICATION DIV. 7617

ALBUOUEROUE

$\$ 4,247.00$

COST/UNIT:

TOTAL COST:

$\$ 4,247.00$

FOR FURTHER INFORMATION:

ELOY N. GIRON

ALBUQUERQUE

(505) $844-2746$

NM 87185

TO FREEZE EOUIPMENT:

ELOY N. GIRON

ALBUOUEROUE

(505) $844-2746$

NM 87185

LOCATION OF EQUIPMENT:

SANDIA NATIONAL LABORATORIES

PROPERTY REAPPL ICATION DIVISION 7617

ALBUOUEROUE

NM 87123 
EFFECTIVE DATE: $07 / 01 / 94$ EXPIRATION DATE: $07 / 31 / 94$

\section{FSC}

$\underline{C O D E}$

EXCESS PROPERTY DESCRIPIION

REFERENCE NUMBER : 375

6625 SCOPE P.I, SAMPLING 2MV/DIV TO 200 MV/DIV CALIBRATED DEFLECTION FACTORS,

TAKES PLUG I N HEADS. IZOVAC, $60 \mathrm{HZ}$. TEKTRONIX. 7S11, SER. B148807.

REPORT NUMBER: $898355-4090$

LINE ITEM NUMBER: 0053
NO OF

COND UNITS UNIT

$00001 \mathrm{EA}$ TOTAL COST:

COUISITION PRICE

$\$ 2,033.00$ $\$ 2,033.00$
REFERENCE NUMBER : 376

6625 SCOPE P-1. SPEC PUR THE UNIT PROVIDES FRONT PANEL KEYBOARD OPERATION FOR 6 O0001 EA CONVENIENT A CCESS TO THE CRT READOUT CHARACTERS. POWER FROM SCOPE. TEKTRONI X, 7M!3, SER. BO72976.
REPORT NUMBER: $898355-4090$

LINE ITEM NUMBER: 0052

\section{COST/UNIT: \\ TOTAL COST:}

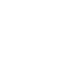

\section{CONTACT(S)}

FOR FURTHER INFORMATION:

ELOY N. GIRON

ALBUQUERQUE

(505) $844-2746$

NM 87185

TO FREEZE EQUIPMENT:

ELOY N. GIRON

ALBUQUERQUE

(505) $844-2746$

NM 87185

LOCATION OF EOUIPMENT:

SANDIA NATIONAL LABORATORIES

PROPERTY REAPPLICATION DIVISION 7617

ALBUQUERQUE NM 87123

$\$ 1,496.00$

$\$ 1,496.00$

FOR FURTHER INFORMATION:

ELOY N. GIRON

ALBUQUERQUE

(505) $844-2746$

NM 87185

TO FREEZE EQUIPMENT.

ELOY N. GIRON

ALBUQUERQUE

(505) $844-2746$

NM 87185

LOCATION OF EQUIPMENT

SANDIA NATIONAL LABORATORIES

PROPERTY REAPPLICAIION DIVISION 7617

PROPERTY REAPPLICATION DIVISION 7617
ALBUOUEROUE NM 87123 
EFFECTIVE DATE: $07 / 01 / 94$ EXPIRATION DATE: 07/31/94

FSC

\section{COOE EXCESS PROPERTY DESCRIPTION}

REFERENCE MUMBER : 377

6625 SCOPE P-1, SWEEP DELAYED SWEEP, SHEEPS TO 1OPS/DIV TRIGGERING TO 1 GHZ. POWER FROM MAINFRAME. HEW PACK, 1425A, SER. 71600430

REPORT NUMBER: $898355-4102$

LINE ITEM NUMBER: 0058

\section{REFERENCE NUMBER : 378}

6625 SCOPE P-1, SWEEP TRIGGERING TO 5GHZ, SWEEPS TO 1OPS/DIV, DIRECT READOUT

ON ALL SWE EPS. POWER COMES FROM MAINFRAME. HEW PACK, 1424A, SER.

$600001 \mathrm{EA}$ 80300229 .

REPORT NUMBER : $898355-4102$

LINE ITEM NUMBER: 0059

NO OF
COND UNITS UNIT

\section{ACQUISITION PRICE}

COST/UNIT: TOTAL COST:

00001

\section{$\$ 1,600.00$ \\ $\$ 1,600.00$}

FOR FIRTHER INFORMATION:

ELOY N. GIRON

(505) $844-2746$

NM 87185

TO FREEZE EQUIPMENT :

ELOY N. GIRON

ALBUQUERQUE

(505) $844-2746$

NM 87185

LOCATION OF EQUIPMENT

SANDIA MAIIONAL LABORATORIES

PROPERTY REAPPLICATION DIV. 7617

ALBUQUERQUE
COST/UNIT:

TOTAL COST:
$\$ 1,200.00$
$\$ 1,200.00$
FOR FURTHER INFORMATION:

ELOY N. GIRON

ALBUQUEROUE

(505) $844-2746$

NM 87185

TO FREEZE EQUIPMENT:

ELOY N. GIRON

ALBUQUERQUE

(505) $844-2746$

NM 87185

LOCATION OF EQUIPMENT.

SANDIA NATIONAL LABORATORIES

SANDIA NATIONAL LABORATORIES
PROPERTY REAPPLICATION DIV. 7617

PROPERTY REAPPLICATION DIV. 7617
ALBUQUERQUE 
EFFECTIVE DATE: $07 / 01 / 94$ EXPIRATION DATE: $07 / 31 / 94$

FSC

COOE EXCESS PROPERTY DESCRIPIION

REFERENCE NUMBER : 379

6625 SCOPE P-1. TIME BASE 1 NS/DIV TO 5S/DIV CALIBRATED TIME BASES

IRIGGERING TO $400 M H Z, \checkmark$ ARIABLE TRIGGER HOLDOFF. POWER FROM SCOPE, 7880 , SER. B064058. T EKTRONIX.

REPORT NUMBER: $898355-4102$

LINE ITEM NUMBER: 006
NO OF

COND UNITS UNIT

ACQUISITION PRICE

$600001 \mathrm{EA}$

COST/UNIT: TOTAL COST:

$\$ 1,580.00$

$\$ 1,580.00$

(n)

CONTACTSS)

FOR FURTHER INFORMATION :

ELOY N. GIRON

ALBUQUERQUE

(505) $844-2746$

NM 87185

TO FREEZE EQUIPMENT

ELOY N. GIRON

(505) $844-2746$

LOCATION OF EOUIPMENT

OF EOUIPMENT:

PROPERTY REAPPLICATION DIV. 7617

ALBUQUEROUE

NM 87123

\section{REFERENCE NUMBER : 380}

625 SCOPE P.I TIME BASE 0.5NS/DIV TO 0.2 S/DIV CALIBRATED TIME BASE,

TRIGGERING TO 50OMHZ ALTERMATE DISPLAY

600001 EA

COST/UNIT:

TOTAL COST:

$\$ 1,400.00$
$\$ 1,400.00$

FOR FURTHER INFORMATION:

ELOY N. GIRON

ALBUOUERQUE

(505) $844-2746$

NM 87185

TO FREEZE EQUIPMENT :

ELOY N. GIRON

ALBUQUERQUE

(S05) $844-2746$

NM 87185

LOCATION OF EQUIPMENT:

SANDIA NATIONAL LABORATORIES

PROPERTY REAPPLICATION DIV. 7617

ALBUQUEROUE 
EFFECTIVE DATE: $07 / 01 / 94$ EXPIRATION DATE: $07 / 31 / 94$

FSC

CODE EXCESS PROPERTY DESCRIPTION

REFERENCE NUMBER : 381

6625 SCOPE P-1, TIME BASE DUAL TIME BASE, 0.5NS/DIV TO 0.2 S/DIV CALIBRATED TIME BASE. POW ER FROM SCOPE. TEKTRONIX, 7B92, SER. B042067.

REPORT NUMBER: $898355-4102$

LINE IIEM NUMBER: 0068

REFERENCE NUMBER : 382

6625 SCOPE, CAMERA FASTEST WRITING SPEED, AUTOMATIC SINGLE SWEEP MOOE, REDUCE 600001 EA IMAGE SIZE, WITH A F/1.2 LENS AND SHUTTER SPEED 4 SEC TO $1 / 60$ SEC. POWER FROM SCOPE. TEKTRONIX.

COND UNITS UNIT

$600001 \mathrm{EA}$ no of

ACQUISITION PRICE

COST/UNIT: TOTAL COST:

$\$ 1,402.00$
$\$ 1,402.00$

ELOY N. GIRON

ALBUQUERQUE

(505) $844-2746$

NM 87185

TO FREEZE EOUIPMENT

ELOY N. GIRON

(505) $844-2746$

NM 87185

LOCATION OF EQUIPMENT:

SANDIA NATIONAL LABORATORIES

PANDIA NATIONAL LABORATORIES 7617

PROPERTY REAPPLICATION DIV. 7617
ALBUQUERQUE

COST/UNIT:

TOTAL COST

$\$ 2,250.00$

$\$ 2,250.00$

FOR FURTHER INFORMATION:

ELOY N. GIRON

ALBUQUERQUE

(505) $844-2746$

NM 87185

TO FREEZE EQUIPMENT:

ELOY N. GIRON

ALBUOUERQUE

(505) $844-2746$

NM 87185

LOCATION OF EQUIPMENT:

SANDIA NATIONAL LABORATORIES

SANDIA NATIONAL LABORATORIES

PROPERTY REAPPLICATION DIV 
EFFECTIVE DATE: $07 / 01 / 94$ EXPIRATION DATE: $07 / 31 / 94$

\section{FSC \\ COOE EXCESS PROPERTY DESCRIPTION}

REFERENCE NUMBER : 383

6625 SCOPE CAMERA FOR LARGER CRT, MECHANICAL SHUTTER, PHOTOMETER EXPOSURE SCOPE, CAMERA FOR LARGER CRT, MECHAN BY BATTERY OR POUER FROM SCOPE.

IEKIRO NIX, C-59-A, SER. B011791.

REPORT NUMBER: $898355-4090$

LINE ITEM NUMBER: 0035

\section{REFERENCE NUMBER : 384}

SCOPE, CAMERA FASIEST WRITING SPEED, AUTO SINGLE SWEEP MOOE, REDUCED IMACE SIZE WITH A F/1.2 LENS SHUTTER SPEEDS 4 SEC TO $1 / 60$ SEC. POWER FROM 1 ENS. TEKTRONIX.
NO O

COND UNITS UNIT

$600001 \mathrm{EA}$ COST/UNIT:
TOTAL COST:

UISITION PRICE

$\$ 1,315.00$ $\$ 1,315.00$ ELOY N. GIRON ALBUQUERQUE
(505) $844-2746$

NM 87185

TO FREEZE EQUIPMENT:

ELOY N. GIRON

ALBUQUERQUE

(505) $844-2746$

NM 87185

LOCATION OF EQUIPMENT :

SANDIA NATIONAL LABORATORIES

PROPERTY REAPPLICATION DIVISION 7617

ALBUOUERQUE

NM 87123

FOR FURTHER INFORMATION:

ELOY N. GIRON

ALBUQUERQUE

(505) $844-2746$

NM 87185

TO FREEZE EQUIPMENT

ELOY N. GIRON

ALBUQUEROUE

(505) $844-2746$

NM 87185

LOCATION OF EQUIPMENT:

SANDIA NATIONAL LABORATORIES

PROPERTY REAPPLICATION DIVISION 7617

ALBUQUERQUE

NM 87123 
EFFECTIVE DATE: $07 / 01 / 94$ EXPIRATION DATE: $07 / 31 / 94$

CODE EXCESS PROPERTY DESCRIPIION

REFERENCE NUMBER : 385

6625 SCOPE, CAMERA FOR LARGER CRT, MECHANICAL SHUTTER PHOTOMETER EXPOSURE AID, RANGE FINDER FOCUSING. POWER BY BATTERY OR SCOPE. TEKTRONIX, C S9-A, SER. BO11790.

REPORT NUMBER: $898355-4090$

LINE ITEM NUMBER: 0038

REFERENCE NUMBER : 386

6625 SCOPE, CAMERA FASTEST WRITING SPEED, AUTOMATIC SINGLE SHEEP MODE,

SCOPE, CAMERA FASTEST WRITING SPEED, AUTOMATIC SINGLE SWEEP MODE,
REDUCED IMAGE SIZE, WITH A F 11.2 LENS AND SHUTTER SPEED 4 SEC TO $1 / 60$

REDUCED IMAGE SIZE, WITH A F
SEC. PO WER FROM SCOPE. TEKTRONIX.

REPORT NUMBER: $898355-4090$

LINE ITEM NUMBER: 0039
NO OF

COND UNITS UNIT

ACQUISITION PRICE

$\$ 1,315.00$

$600001 E A$

COST/UNIT:

TOTAL COST:

$\$ 1,315.00$

ELOY N. GIRON

ALBUQUERQUE

(505) $844-2746$

NM 87185

TO FREEZE EQUIPMENT:

ELOY N. GIRON

ALBUOUEROUE

(505) $844-2746$

NM 87185

LOCATION OF EQUIPMENT

SANDIA NATIONAL LABORATORIES PROPERTY REAPPLICATION DIVISION 7617

ALBUQUERQUE

NM 87123
$600001 \mathrm{EA}$

COST/UNIT: TOTAL COST : $\$ 2,250.00$
FOR FURTHER INFORMATION:

ELOY N. GIRON

ALBUQUERQUE

(505) $844-2746$

NM 87185

TO FREEZE EOUIPMENT:

ELOY N. GIRON

ALBUQUERQUE

(505) $844-2746$

NM 87185

LOCATION OF EOUIPMENT:

SANDIA NATIONAL ABORATORIES

PROPERTY REAPPLICAIION DIVISION 7617

AL.BUQUERQUE

NM 87123 
EFFECTIVE DATE: $07 / 01 / 94$ EXPIRATION DATE: $07 / 31 / 94$

ESC

COOE EXCESS PROPERTY DESCRIPTION

REFERENCE NUMBER : 387

6625 SCOPE, CAMERA FOR LARGER CRT, MECHANICAL SHUTTER, RANGE-FINDER FOCUSING. 600001 EA POWER FROM BATTERY OR SCOPE. TEKTRONIX, C-59-A, SER. B011795.

REPORT NUMGER: $898355-4090$

LINE ITEM NUMBER: 0040

REFERENCE NUMBER : 388

6625 SCOPE, CAMERA FASTEST URITING SPEED, AUTOMATIC SINGLE SWEEP MODE. POWER 600001 EA FROM S COPE. TEKTRONIX, MOOEL [-51, SER. B095990.

LINE ITEM NUMBER: 0041

NO OF

REPORT NUMEER: $898355-4090$
COND UNITS UNIT

ACQUISITION PRICE

$\$ 1,315.00$

$\$ 1,315.00$

TOTAL COST:

ELOY N. GIRON

ALBUOUERQUE

NM 87185

TO FREEZE EOUIPMENT

ELOY N. GIRON

ALBUOUEROUE

(505) $844-2746$

NM 87185

LOCATION OF EQUIPMENT

SANDIA NATIONAL LABORATORIES

PROPERTY REAPPLICAIION DIVISION 7617

ALBULUERQUE NM 87123
FOR FURTHER INFORMATION:

ELOY N. GIRON

ALBUQUEROUE

(505) $844-2746$

NM 87185

TO FREEZE EQUIPMENT :

ELOY N. GIRON

ALBUOUEROUE

505) $844-2746$

NM 87185

LOCATION OF EQUIPMENT:

SANDIA NATIONAL LABORATORIES

SANDIA NATIONAL LABORATORIES PROPERTY REAPPLICATION DIVISION 7617
ALBUOUEROUE
NM 87123 
EFFECTIVE DATE: $07 / 01 / 94$ EXPIRAIION DATE: $07 / 31 / 94$

FSC

\section{COOE EXCESS PROPERTY DESCRIPTION}

REFERENCE NUMBER : 389

6625 SCOPE, CAMERA FASTEST WRITING SPEED, AUTOMATIC SINGLE SWEEP MODE

REDUCED IMAGE SIZE, WITH A F/1.2 LENS, SHUTTER SPEED 4 SEC TO $1 / 60 \mathrm{SEC}$.

POWER FROM SCOPE. TEKTRONIX.

REPORT NUMBER: $898355-4090$

LINE ITEM NUMBER: 0043

REFERENCE NUMBER : 390

6625 SCOPE, CAMERA IS ELECTRICALLY ACTUATED, OPEN AND CLOSE, BY SHUTTER

BUTTON OR RE MOTELY THROUGH A REMOTE-INPUT CONNECTOR LOCATED ON THE SIDE

CONTR OL PANEL. POWER COMES FROM SCOPE. TEKTRONIX, CS3, SER. BOS

$78: 3$.
NO OF

COND UNITS UNIT

ACQUISITION PRICE

$\$ 2,250.00$

$60001 \mathrm{EA}$

COST/UNIT:

TOTAL COST:

$\$ 2,250.00$

\section{FOR FURTHER INFORMATION}

ELOY N. GIRON

ALBUQUERQUE

(505) $844-2746$

NM 87185

TO FREEZE EQUIPMENT:

ELOY N. GIRON

ALBUQUERQUE

(505) $844-2746$

NM 87185

LOCATION OF EQUIPMENT:

SANDIA NATIONAL LABORATORIES

PROPERTY REAPPLICATION DIVISION 7617

ALBUOUERQUE

NM 87123

$600001 E A$

COST/UNIT:

TOTAL COST:

$\$ 1,855.00$

$\$ 1,855.00$

\section{FOR FURTHER INTORMATION}

ELOY N. GIRON

ALBUQUERQUE

(505) $844-2746$

NM 87185

TO FREEZE EOUIPMENT:

ELOY N. GIRON

ALBUOUEROUE

(505) $844-2746$

NM 87185

LOCAIION OF EOUIPMENT:

SANDIA NATIONAL LABORATORIES

PROPERTY REAPPLICATION DIVISION 7617

ALBUQUERQUE

NM 87123 
EFFECTIVE DATE: 07/01/94 EXPIRATION DATE: $07 / 31 / 94$

\section{FSC \\ COOE EXCESS PROPERTY DESCRIPIION \\ NO OF \\ COND UNITS UNI}

\section{REFERENCE NUMBER : 391}

002.5 SCOPE, CAMERA MEDIUM SPEED GENERAL PURPOSE CAMERA AUTOMATIC SINGLE SWEEP MODE W ITH A F/1.9 LENS AND SHUTTER SPEEO 4 SEC TO 1/60 SEC. POWER FROM SCOPE. TEKTRONIX.
$600001 \mathrm{EA}$

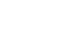

ro

\section{ACQUISITION PRICE}

COSTIUNIT:
TOTAL COST:

LINE ITEM NUMBER: 0032
REPOR I NUMBER: $898355 \cdot 6090$
REFERENCE NUMBER : 392

6025 SCOPE, CAMERA MEDIUM SPEED, GeNeRAL PURPOSE CAMERA AUTO SINGLE SWEEP SC OPE. TEKTRONIX.

REPORT NUMBER: $898355-4090$

\section{$600001 E A$

TOTAL COST:

$\$ 1,161.00$
$\$ 1,161.00$

CONTACTSS)

\section{FOR FURTHER INFORMATION: \\ ELOY N. GIRON}

ALBUQUERQUE

(505) $844-2746$

NM 87185

TO FREEZE EQUIPMENT:

ELOY N. GIRON

ALBUQUEROUE

(505) $844-2746$

NM 87185

LOCATION OF EOUIPMENT:

SANDIA NATIONAL LABORATORIES

ROPERTY REAPPLICATION DIVISION 7617

ALBUQUERQUE

LINE ITEM NUMBER: 0034 
EFFECTIVE DATE: 07/01/94 EXPIRATION DATE: $07 / 31 / 94$

FSC

COOE EXCESS PROPERTY DESCRIPTION

REFERENCE NUMBER : 393

6625 SCOPE, CAMERA MEDIUM SPEED, GENERAL PURPOSE CAMERA AUTOMATIC SINGLE SUEEP MOOE, WITH A 1.9 LENS SHUTIER SPEED 4 SEC TO $1 / 60$ SEC. POWER FROM SCOP E. TEKTRONIX.

REPORT NUMBER: $898355-4090$

LINE ITEM NUMBER: 0036
COND UNITS UNIT

ó $00001 \mathrm{EA}$
COST/UNIT:
TOTAL COST: TOTAL COST:

COST/UNIT: TOTAL COST: SCOPE, CAMERA MEDIUM SPEED GENERAL PURPOSE CAMERA AUTOMATIC SINGLE SHEEP MOOE, WITH F/1.9 LENS SHUTTER SPEED 4 SEC TO 1/60 SEC. 120VAC, 60 HZ. TEKTRONIX.
00001 EA

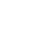

\section{ACQUISITION PRICE}

$\$ 1,855.00$ $\$ 1,855.00$

$\$ 1,285.00$ $\$ 1,285.00$

REPORT NUMBER: $898355-4090$

LINE I IEM MUMBER: 0042

\section{CONTACT(S)}

FOR FURTHER INFORMATION:

ELOY $N$. GIRON

ALBUOUEROUE

(505) $844-2746$

NM 87185

TO FREEZE EQUIPMENT:

ELOY N. GIRON

ALBUQUERQUE

(505) $844-2746$

NM 87185

LOCATION OF EOUIPMENT:

SANDIA MATIONAL LABORATORIES

PROPERTY REAPPLICATION DIVISION 7617

ALBUQUERQUE

NM 87123

FOR FURTHER INFORMATION:

ELOY N. GIRON

ALBUDUEROUE

NM 87185

TO FREEZE EOUIPMENT:

ELOY N. GIRON

ALBUQUERQUE

(505) $844-2746$

NM 87185

LOCATION OF EQUIPMENT:

SANDIA NATIONAL LABORATORIES

PROPERTY REAPPLICATION DIVISION 7617

ALBUQUEROUE

NM 87123 
EFFECTIVE DATE: $07 / 01 / 94$ EXPIRATION DATE: $07 / 31 / 94$

\section{COOE EXCESS PROPERTY DESCRIPTION}

\section{REFERENCE NUMBER : 395}

6025 SCOPE, CAMERA MEDIUM SPEED, GENERAL PURPOSE CAMERA WITH 0.85 MAG,

REMOIELY CONT ROLLED SHUTIER 1.9 APERTURE SHUTTER SPEEDS 4 SEC TO $1 / 60$

SEC. POW ER FROM SCOPE. TEKTRONIX, C-53, B099445.

REPORT NUMGER: $898355-4090$

LINE ITEM NUMBER: 0046

REFERENCE NUMBER : 396

6625 SCOPE, DUAL BEAM 400 MHZ BANDWIDTH, 900 PS RISETIME TRUE DUAL BEAM FULL

VERTICAL C ROSSOVER SHITCHING BCMXIOCM DISPLAY CRT READOUT INS/DIV MAX

CALIB RATED SWEEP. 115VAC, 60 HZ. TEK, R7844, B100.

6 O0001 EA

COST/UNIT:

TOTAL COST:

$\$ 5,800.00$

$\$ 5,800.00$ COND UNITS UNIT

ACQUISITION PRICE
NO OF

\section{COST/UNIT:}

TOTAL COST:

\section{$\$ 1,845.00$ \\ $\$ 1.845 .00$}

ELOY N. GIRON
ALBUDUEROUE

505) $844-2746$

IO FREEZE EQUIPMENT:

ELOY N. GIRON

ALBUOUERQUE

(505) $844-2746$

LOCATION OF EQUIPMENT:

SANDIA NATIONAL LABORATORIES

PROPERTY REAPPLICATION DIVISION 7617

ALBUQUERQUE
FOR FURTHER INFORMATION:

ELOY N. GIRON

ALBUOUERQUE

(505) $844-2746$

NM 87185

TO FREEZE EQUIPMENT:

ELOY N. GIRON

ALBUOUEROUE

(505) $844-2746$

NM 87185

LOCATION OF EOUIPMENT:

SANDIA NATIONAL LABORATORIES

PROPERTY REAPPLICATION DIVISION 761?

ALBUQUERQUE 
EFFECTIVE DATE: $07 / 01 / 94$ EXPIRATION DATE: $07 / 31 / 94$

FSC
COOE EXCESS PROPERTY DESCRIPTION

REFERENCE NUMBER : 397

6625 SCOPE, DUAL BEAM FOR MEASUREMENTS FROM OC TO 50 MHZ RANGE WITH VERTICAL AND HORIZO NTAL DEFLECIION SYSTEMS. 115/230VAC, $60 \mathrm{HZ}$. TEKTRONIX, R556, 003471.

REPORT NUMBER: $898355-4102$

LINE ITEM NUMBER: 0065

No of

COND UNITS UNIT

ACQUISITION PRICE

COST/UNIT: TOTAL COST:

$\$ 3,813.00$ $\$ 3,813.00$

FOR FURTHER INFORMATION:

ELOY N. GIRON

ALBUQUERQUE

(505) $844-2746$

NM 87185

TO FREEZE EQUIPMENT:

ELOY N. GIRON

ALBUQUERQUE

(505) $844-2746$

NM 87185

LOCATION OF EQUIPMENT:

SANDIA NATIONAL LABORATORIES

PROPERTY REAPPLICATION DIV. 7617

ALBUDUERQUE

NM 87123

COST/UNIT: TOTAL COST:

SCOPE, DUAL BEAM 40OMHZ BANDWIDTH, 900 PS RISETIME, TRUE DUAL BEAM (OUAL 600001 EA GUN) FULL VERTICAL CROSSOVER SHITCHING $8 \mathrm{CM} / \times 10 \mathrm{CM}$ DISPLAY CRT READOUT

INSID IV MAINFRAME. 115V, $60 \mathrm{HZ}$. TEKTRONIX.

REPORT NUMBER: $898355-4102$
LINE ITEM NUMBER: 0071
$\$ 8,007.00$

$\$ 8,007.00$

FOR FURTHER INFORMATION:

(505) $844-2746$

NM 87185

TO FREEZE EQUIPMENT:

ELOY N. GIRON

ALBUQUERQUE

(505) $844-2746$

NM 87185

LOCATION OF EQUIPMENT:

SANDIA NATIONAL LABORATORIES

PROPERTY REAPPLICATION DIV. 7617

ALBUQUEROUE 
EFFECIIVE DATE: 07/01/94 EXPIRATION DATE: 07/31/94

$$
\text { ELIGIBLE EQUIPMEN T }
$$

FSC

\section{COOE EXCESS PROPERTY DESCRIPTION}

REFERENCE NUMBER : 399

6625 SCOPE, GENERAL PURF 5OOMHZ AT 1OMV/DIV, 0.8 NS RISETIME 500 PS/DIV

B282594.
FASTEST CALIBRATEO SUEEP RATE, 120VAC, 60 HZ TEXTRONIX,7904, SER.

NO OF

COND UNITS UNIT

ACQUISITION PRICE

$6 \quad 00001 E A$ COST/UNIT:

TOTAL COST:

LINE ITEM NUMBER: 0022

REFERENCE NUMBER : 400

SE25 SCOPE, GENERAL PURP 5OOMHZ AT 10 MV/DIV, 0.8 NS RISETIME 500 PS/DIV

FASTEST CALIBRATE D SHEEP RATE GREATER THAN 15 CM/NS, $120 \mathrm{VAC}, 60 \mathrm{HZ}$.

VERTICAL MOOE SWITCHES NEED KEPAIR, TEK, 7904, B282586.

$7 \quad 00001$ EA

COST/UNIT:

TOTAL COST:

$\$ 8.572 .00$
$\$ 8.572 .00$

$\$ 8,572.00$

$\$ 8,572.00$
ELOY N. GIRON

(505) $844-2746$

NM 87185

TO FREEZE EOUIPMENT:

ELOY N. GIRON

ALBUQUERDUE

(505) $844-2746$

LOCATION OF EOUIPMENT:

SANDIA NATIONAL LABORATOR!ES

PROPERTY REAPPLICATION DIVISION 7617

ALBUOUEROUE

NM 87123

REPORT NUMBER: $898355-4090$

LINE ITEM NUMBER: 0023
FOR FURTHER INFORMATION:

ELOY N. GIRON

$(505) 844-$

NM 87185

TO FREEZE EOUIPMENT:

ELOY N. GIRON

(505) $844-2746$

NM 87185

LOCATION OF EQUIPMENT:

SANDIA NATIONAL LABORATORIES

PROPERTY REAPPLICATION DIVISION 7617

ALBUOUEROUE

NM 87123 
EFFECTIVE DATE: 07/01/94 EXPIRATION DATE: $07 / 31 / 94$

\section{EOOE EXESS PROPERTY DESCRIPIION}

REFERENCE NUMBER : 401

6625 SCOPE, GENERAL PURP BRIGHT VISUAL DISPLAY AND FAST PHOTOGRAPHIC WRITING SPEEDS WITH T HE 2OKV CATHOLE RAY TUBE WRITING SPEEDS OF $4 \mathrm{CM} / \mathrm{MS}$. 120VAC, 60 H Z. HEW PACK, 183A, SER. 1235A.

REPORI NUMBER: $898355-4090$

LINE ITEM NUMBER: 0030

REFERENCE NUMBER : 402

6625 SCOPE, GENERAL PURPOSE PORTABLE OSCILLLSCOPES, DUAL TRACE 15 MHZ BANDWIDTH, CALIBRATED F ACTORS FROM 1MV TO 2OV/DIV SWEEP SPEEDS RANGE FROM $0.5 \mathrm{~S}$ TO $50 \mathrm{NS} / \mathrm{OIV}$. 120VAC, $60 \mathrm{HZ}$. TEK, 422, 01179.
NO OF

CONTACISS)

$00001 E A$

COST/UNIT:

TOTAL COST:

$\$ 1,850.00$

FOR FURTHER INFORMATION:

ELOY N. GIRON

(505) $844-2746$

NM 87185

TO FREEZE EQUIPMENT :

ELOY N. GIRON

ALBUQUERQUE

(505) $844-2746$

NM 87185

LOCATION OF EQUIPMENT

SANDIA NATIONAL LABORATORIES

PROPERTY REAPPLICATION DIVISION 7617 ALBUQUERQUE

\section{COST/UNIT:}

TOTAL COST:

$\$ 1,750.00$
$\$ 1,750.00$

FOR FURTHER INFORMATION:

ELOY N. GIRON

ALBUQUEROUE

NM 87185

(505) $844-2746$

TO FREEZE EQUIPMENT:

ELOY N. GIRON

ALBUOUEROUE

(505) $844-2746$

NM 87185

LOCATION OF EQUIPMENT:

SANDIA MATIONAL LABORATORIES

PROPERTY REAPPLICATION DIVISION 7617

ALBUQUERQUE
NM 87123 
EFFECIIVE DATE: $07 / 01 / 94$ EXPIRATION DATE: $07 / 31 / 94$

FSC

\section{REFERENCE NUMBER : 403} SER. B 150677

\section{COOE EXCESS PROPERIY DESCRIPIION}

6625 SCOPE, GENERAL PURPOSE HAS LARGE DISPLAY 6.5" CRT, 5 NS/DIV DELAYING SLEEP, 0.5 MV VERTI CAL SENSITIVITY. 120VAC, $60 \mathrm{HZ}$. TEKTRONIX, R7903,

No of

COND UNIIS UNIT

$00001 \mathrm{EA}$ COST/UNIT: TOTAL COST:

ACQUISITION PRICE

\section{$\$ 3,389.00$}

$\$ 3,389.00$

FOR FURTHER INFORMAIION:

ALBUOUEROUE

(505) $844-2746$

NM 87185

TO FREEZE EQUIPMENT

EL.OY N. GIRON

ALBUQUERQUE

(505) $844-2746$

NM 87185

LOCATION OF EQUIPMENT

SANDIA NATIONAL LABORATORIES

PROPERTY REAPPLICATION OIVISION 7617

PROPERTY REAPPLICATION OIVISION 7617
ALBUQUEROUE NM 87123

\section{REFERENCE NUMBER : 404}

6625 SCOPE, GENERAL PURPOSE BRIGHT VISUAL DISPLAY AND FAST PHOTOGRAPHIC

WRITING SPEEDS ARE AS SURED WITH THE 2OKV CATHODE TUBE, WRITING SPEEDS

$600001 \mathrm{EA}$

COST/UNIT:

TOTAL COST:

OF $4 \mathrm{CM} / \mathrm{NS}, 120 \mathrm{VAC}, 60 \mathrm{HZ}$. HEW PACK, 183C, SER. $20 A 00120$.

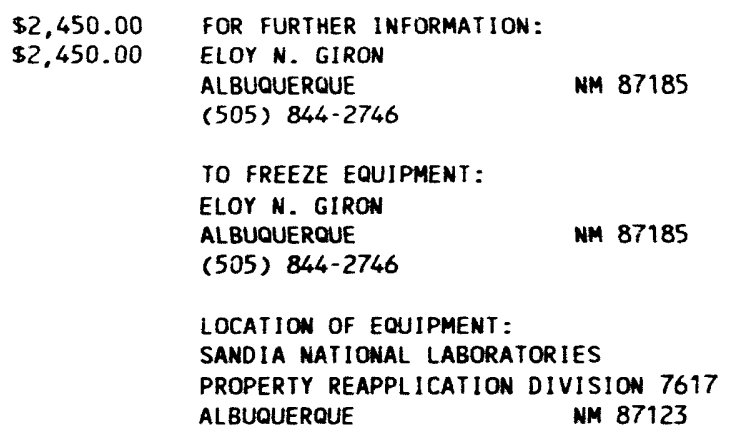


EFFECTIVE DATE: $07 / 01 / 94$ EXPIRATION DATE: $07 / 31 / 94$

FSC

\section{COOE EXCESS PROPERTY DESCRIPTION}

REFERENCE NUMBER : 405

6625 SCOPE, GENERAL PURPOSE 350 MHZ AT 5MY/DIV, 1 NS/DIV SUEEP RATE, 2.0 SCOPE, GENERAL PURPOSE 350 MHZ AT 5 MV/DIV, 1 NS/DIV SWEEP RATE, 2.0
DIV/NS WRITING SPEED. 1 M-OHM AND 50 OHM INPIJT IMPEDANCES, PUSHBUTTON DIV/NS WRITING SPEED 1 M-OHM AND
EXT TRIGGER VIE W. 12OVAC, $60 \mathrm{HZ}$.

REPORT NUMBER: $898355-4090$

LINE ITEM NUMBER: 0055

REFERENCE NUMBER : 406

6625 SCOPE, GENERAL PURPOSE 250 MHZ AND 200 MHZ DUAL TRACE SCOPE WITH INS/DIV SWEEP RATE WITH 10X SWEEP MAGNIFIER, TRIGGER VIEW, VERSATILE TRIGGER SELECTION. 12OVAC, $60 \mathrm{HZ}$.

REPORT NUMBER: $898355-4090$

LINE ITEM NUMBER : 0059
No of
COND UNITS UNIT

$60001 \mathrm{EA}$
COST/UNIT: TOTAL COST:

\section{$\$ 4.750 .00$
$\$ 4.750 .00$}

FOR FURTHER INFORMATION:

ELOY $N$. GIRON

ALBUQUERQUE

(505) 844-2746

NM 87185

TO FREEZE EQUIPMENT

ELOY N. GIRON

ALBUQUERQUE

NM 87185

(505) $844-2746$

LOCATION OF EQUIPMENT:

SANDIA NATIONAL LABORATORIES

PROPERTY REAPPLICATION DIVISION 7617

ALBUQUERQUE

NM 87123

00001 EA

COST/UNIT:

IOTAL COST:

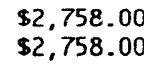

FOR FURTHER INFORMATION:

ELOY N. GIRON

ALBUQUERQUE

(505) $844-2746$

NM 87185

TO FREEZE EQUIPMENT:

ELOY N. GIRON

ALBUQUERQUE

MM 87185

(505) 844-2746

LOCATION OF EQUIPMENT

SANDIA NATIONAL LABORATORIES

PROPERTY REAPPLICATION DIVISION 7617

ALBUQUEROUE 
EFFECTIVE DATE: $07 / 01 / 94$ EXPIRATION DATE: $07 / 31 / 94$

FSC

\section{COOE EXCESS PROPERTY DESCRIPTION}

REFERENCE NUMBER : 407

6625 SCOPE, GENERAL PURP HAS LARGE DISPLAY 6.5" CRT 5NS/DIV DELAYING SWEEP, 0.5 MV VERTICA L SENSITIVITY, TAKE 3 PI. 120VAC, 60 HZ. TEKTRONIX, 7603, SER. B 204282.

REPORT NUMBER: $898355-4102$

LINE ITEM NUMBER: 0072

$$
\text { NO OF }
$$

COND UNITS UNIT

ACQUISITION PRICE

COST/UNIT: TOTAL COST:

$00001 \mathrm{EA}$

\section{$\$ 1,666.00$
$\$ 1,666.00$}

ELOY N. GIRON

(505) $844-2746$

TO FREEZE EQUIPMENT

ELOY N. GIRON

ALBUQUERQUE

(505) $844-2746$

LOCATION OF EQUIPMENT:

SANDIA NATIONAL LABORATORIES

PROPERTY REAPPLICATION DIV. 7617

ALBUQUEROUE

NM 87123

NM 87185

MM 87185
$\$ 2.892 .00$

COST/UNIT:

CALIBRATED SHE EP RATE GREATER THAN 15CM/NS ENHANCED
READOUT. I2OVAC, $60 \mathrm{HZ}$. TEKTRONIX, 7904, B031267.

REPORT NUMBER: $898355-4102$
LINE ITEM NUMBER: 0073
FOR FURTHER INFORMATION:

ELOY N. GIRON

ALBUQUERQUE

(505) $844-2746$

NM 87185

TO FREEZE EQUIPMENT:

ELOY N. GIRON

ALBUQUERQUE

(505) $844-2746$

LOCATION OF EQUIPMENT:

SANDIA NATIONAL LABORATORIES

PROPERTY REAPPLICATION DIV. 7617

ALBUOUERQUE

NM 8712 
EFFECTIVE DATE: 07/01/94 EXPIRATION DATE: 07/31/94

FSC

\section{COOE EXCESS PROPERTY DESCRIPTION}

REFERENCE NUMBER : 409

6625 SCOPE, GENERAL PURP $350 M H Z$ AT 5MV/DIV, 1 NS/DIV SWEEP RATE, 3.0 DIV/NS WRITING SPEED. 120VAC, $60 \mathrm{HZ}$. R485, SER. B 166809.

REPORT NUMBER: $898355-4102$

LINE ITEM NUMBER: 0074

REFERENCE NUMBER : 410

6625 SCOPE, GENERAL PURP $350 M H Z$ AT 5MV/DIV INS/DIV SUEEP RATE 3.0 DIV/NS

WRITING SPEED. 12OVAC, $60 \mathrm{HZ}$. 485, B191773.

REPORT NUMBER: $898355-4102$

LINE ITEM NUMBER: 0075
No of

COND UNITS UNIT

ACQUISITION PRICE

$\$ 5,751.00$

$600001 \mathrm{EA}$

COST/UNIT: TOTAL COST:

$\$ 5,751.00$

FOR FURTHER INFORMATION:

ELOY N. GIRON

ALBUQUEROUE

(505) 844-2746

NM 87185

TO FREEZE EQUIPMENT :

ELOY N. GIRON

ALBUOUEROUE

(505) $844-2746$

NM 87185

LOCATION OF EQUIPMENT:

SANDIA NATIONAL LABORATORIES

PROPERTY REAPPLICATION DIV. 7617

ALBUOUERQUE

NM 87123

COST/UNIT:

TOTAL COST:

$\$ 8.127 .00$

$\$ 8,127.00$

FOR FURTHER INFORMATION:

ELOY N. GIRON

ALBUSOUERQUE

(505) $844-2746$

NM 87185

TO FREEZE EOUIPMENT:

ELOY $N$. GIRON

ALBUOUERQUE

(505) 844-2746

NM 87185

LOCATION OF EQUIPMENT:

SANDIA NATIONAL LABORATORIES

PROPERTY REAPPLICATION DIV. 7617

ALBUQUERQUE

NM 87123 
EFFECTIVE DATE: $07 / 01 / 94$ EXPIRATION DATE: $07 / 31 / 94$

FSC

\section{CODE EXCESS PROPERTY DESCRIPTION}

REFERENCE NUMBER : 411

6625 SCOPE, GENERAL PURP RACK MOUNT, 1 GHZ AT 10MV/DIV, 200 PS/DIV FASTEST CALIBRATED SHEE P RATE. 120VAC, 60 HZ. R7103, SER. B051006.

REPORT NUMBER: $898355-4102$

REFERENCE NUMBER : 412

6625 SCOPE, GENERAL PURP 100MHZ AT 5MV/DIV, 2 NS/DIV SWEEP RATE WITH $\times 10$ SWEEP MAGNIFIED. TRIGGER VIEW, VERSATILE TRIGGER SELECTION, AND ALTERNATE SWEEP. 12OVAC, $60 \mathrm{HZ}$. NEEDS NEW CORD. TEKTRONIX.
LINE ITEM NUMBER: 0076

7 O0001 EA COST/UNIT:

TOTAL COST:

\section{$\$ 2,495.00$} $\$ 2,495.00$

$\$ 34,459.00$

TOSTAL COS

$$
\begin{aligned}
& \text { ELOY N. GIRON } \\
& \text { ALBUQUEROUE }
\end{aligned}
$$

(505) $844-2746$

NM 87185

TO FREEZE EQUIPMENT :

ELOY N. GIRON

ALBUQUEROUE

(505) $844-2746$

NM 87185

LOCATION OF EQUIPMENT:

SANDIA NATIONAL LABORATORIES

PROPERTY REAPPLICATION DIV. 7617

ALBUQUEROUE

NM 87123

LINE ITEM NUMBER: 0077
REPORT NUMBER: $898355-4102$
FOR FURTHER INFORMATION:

ELOY N. GIRON

ALBUQUERQUE

(505) $844-2746$

TO FREEZE EQUIPMENT:

ELOY N. GIRON

ALBUQUEROUE

NM 87185

(505) 844-2746

LOCATION OF EQUIPMENT:

SANDIA NATIONAL LABORATORIES

PROPERTY REAPPLIICATION DIV. 7617

ALBUQUERQUE

NM 8712 
EFFECTIIVE DATE: 07/01/94 EXPIRATION DATE: $07 / 31 / 94$

\section{FSC \\ COOE EXCESS PROPERTY DESCRIPIION \\ NO OF \\ COND UNITS UNIT}

REFERENCE NUMBER : 413

6625 SCOPE, P-1, 1 TRACE FULLY PROGRAMMABLE PLUG IN FOR 7912 AD OR 76120 DIGITIZER ONLY. POWER COMES FROM SCOPE, TEKTRONIX, 7A16P, SER. B043944.

REPORT NUMBER: $898355-4090$

LINE ITEM NUMBER: 0044

REFERENCE NUMBER : 414

6625 SCOPE, STORAGE 1000 CM/NS STORED WRITING SPEED, LONG VIEW TIME, MULTIMODE STORAG E, OC TO $100 \mathrm{MHZ}$ BANDWIDTH. 120VAC, $60 \mathrm{HZ}$. TEKTRONIX, 7633. SER. B215058.

$600001 \mathrm{EA}$
ACQUISITION PRICE

COST/UNIT:

TOTAL COST:

$\$ 2,399.00$
$\$ 2,399.00$

ELOY N. GIRON

ALBUQUERQUE

(505) $844-2746$

NM 87185

TO FREEZE EOUIPMENT :

ELOY N. GIRON

ALBUQUERQUE

(505) $844-2746$

NM 87185

LOCATION OF EQUIPMENT:

SANDIA NATIONAL LABORATORIES

PROPERTY REAPPLICATION DIVISION 7617 ALBUQUEROUE

COST/UNIT

TOTAL COST:

$\$ 5,031.00$

FOR FURTHER INFORMATION

ELOY N. GIRON

ALBUOUERQUE

(505) $844-2746$

NM 87185

TO FREEZE EQUIPMENT

ELOY N. GIRON

ALBUQUERQUE

(505) $844-2746$

NM 87185

LOCATION OF EOUIPMENT:

SANDIA MATIONAL LABORATORIES

PROPERTY REAPPLICATION DIVISIOM 7617

PROPERTY REAPPLICATION DIVISION 7617
ALBUOUEROUE 
EFFECTIVE DATE: $07 / 01 / 94$ EXPIRATION DATE: $07 / 31 / 94$

\section{FSC
CODE EXCESS PROPERTY DESCRIPTION}

REFERENCE NUMBER : 415

6625 SCOPE STORAGE 2500 CM/NS STORED HRITING SPEED, STORES SINGLE SHOT SCOPE, STORAGE 2500 CM/NS STORED WRITING SPEED, STORES SINGLE SHOT
RISETIME AS F AST AS 1.4 NS DC TO 400 MHZ BANDWIDTH MULTIMODE STORAGE. 12OVAC, $60 \mathrm{HZ}$. TEKTRONIX, 7834, B035931.

REPORT NUMBER: $898355-4090$

LINE ITEM NUMBER: 0026

\section{REFERENCE NUMBER : 416}

6625 SCOPE, STORAGE CATHODE-RAY TUBE AND CONTROLS, TYPE POST ACCELERATOR STORAGE TUBE 8.5KV BEAM FINDER RETURNS TRACE TO CRT SCREEN. 12OVAC, $60 \mathrm{HZ}$. HEW PACK, 181A, SER 3504.

REPORT NUMBER: $898355-4090$

LINE IYEM NUMBER: 0047
No of

COND UNITS UNII

ACQUISITION PRICE

COST/UNIT:

TOTAL COST:

$\$ 12,415.00$

$\$ 12,415.00$

(1)
TO FREEZE EOUIPMENT:
ELOY N. GIRON

ALBUOUEROUE

CONTACT(S)

FOR FURTHER INFORMATION:

ELOY N. GIRON

ALBUOUEROUE

NM 87185

(505) $844-2746$

NM 87185

LOCATION OF EQUIPMENT:

SANDIA NATIONAL LABORATORIES

PROPERTY REAPPLICAIION DIVISION 7617

ALBUQUEROUE

NM 87123

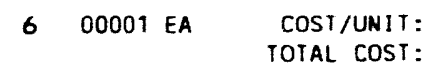

$\$ 1.934 .00$

$\$ 1.934 .00$
FOR FURTHER INFORMATION:

ELOY N. GIRON

ALBUQUEROUE

ALBUOUERQUE
(505) $844-2746$

NM 87185

IO FREEZE EQUIPMENT:

ELOY N. GIRON

ALBUQUEROUE

MM 87185

(505) $844-2746$

LOCATION OF EOUIPMENT:

SAMD IA MATIONAL LABORATORIES

PROPERTY REAPPLICATION DIVISION 7617

ALBUOUERRUE NM 87123 
EFFECIIVE DATE: $07 / 01 / 94$ EXPIRATION DATE: $07 / 31 / 94$

FSC

\section{CODE EXCESS PROPERTY DESCRIPTION}

REFERENCE NUMBER : 417

6625 SCOPE, STORAGE CATHODE-RAY TUBE AND CONTROLS, TYPE POST-ACCELERATOR STORAGE TUBE , 8.5 KV, BEAM FINDER RETURNS TRACE TO CRT SCREEN. HEW PACK, 184A, 455

REPORT NUMBER: $898355-4090$

LINE ITEM NUMBER: 0048

REFERENCE NUMBER : 418

6625 SCOPE, STORAGE 100MHZ AT SMV/DIV, 5NS/DIV SWEEP RATE WITH X10 SWEEP

MAGNIFIER, V ARIABLE PERSISTANCE AND FAST MESH TRANSFER STORAGE MOOES,

3000 DI V/NS STORED WRITING SPEED. $115 / 230 \mathrm{~V}, 50-400 \mathrm{HZ}$, TEKIRONIX,

$600001 \mathrm{EA}$

COST/UNIT:

TOTAL COST:

No of

COND UNITS UNIT

COST/UNIT:

TOTAL COST:
LINE ITEM NUMBER: 0078

ACQUISITION PRICE

$\$ 3,081.00$

$\$ 3,081.00$

ELOY N. GIRON
ALBUOUEROUE

(505) $844-2746$

TO FREEZE EOUIPMENT:

ELOY N. G!RON

ALBUOUERQUUE

(505) $844-2746$

NM 87185

LOCATION OF EQUIPMENT

SAMDIA NATIONAL LABORATORIES

PROPERTY REAPPLICATION DIVISION 7617

ALBUQUERQUE NM 87123

REPORT NUMBER : $898355-4102$ 
EFFECTIVE DATE: 07/01/94 EXPIRAIION DATE: 07/31/94

FSC

COOE EXCESS PROPERTY DESCR!PTION

REFERENCE NUMBER : 410

6625 SCOPE, STORAGE 1000CM/NS STORED HRITING SPEED. 12OVAC, GOHZ. NEEDS

REPORT NUMBER: $898355-6102$

LINE ITEM NUMBER: 007 SCOPE, STORAGE $1000 \mathrm{CM} / \mathrm{NS}$ SIORED
REPAIR SCOP E IS MAKING NOISE.

NO OF

COND UNITS UNIT

$700001 \mathrm{EA}$

$\cos$ /UNIT:

TOTAL COST:

ACOUISITION PRICE

$\$ 4,074.00$

$\$ 4.074 .00$

FOR FURTHER

ELOY N. GIROM

(505) $844-2746$

MM 87185

TO FREEZE EQUIPMENT:

ELOY N. GIRON

ALBUOUEROUE

(SOS) $844-2746$

LOCATION OF EOUIPMENT:

SANDIA NATIONAL LABORATORIES

PROPERTY REAPPLICATION DIV. 7617

ALBUQUEROUE

NM 87123

REFERENCE NLMBER : 420

6025 SCOPE, STORAGE RACK MOUNT WITH A 5" CRT, USES THE 1300 SERIES PLUG INS, 7 00001 EA THE 141 TA GIVES STORAGE FOR SIDE-BY-SIDE COMPARISON OF HAVEFORMS.

COST/UNIT:

TOTAL COST:

$\$ 1,395.00$

$\$ 1,395.00$

120VAC, 60 HZ. HEW PACK, 141A. 84403458.

REPORT NUMBER: $898355-4102$

LINE ITEM NUMBER: 0080

FOR FURTHER INFORMATION:

ELOY N. GIRON
AL BUOUEROUE

(505) $844-2746$

MM 87185

TO FREEZE EOUIPMENT

ELOY N. GIRON

ALBUOUERQUE

(505) $844 \cdot 2746$

MM 87185

LOCATION OF EQUIPMENT :

SAMDIA NATIONAL LABORATORIES

PROPERTY REAPPLICATION DIV. 7617

ALBUOUEROUE

NM 87123 
EFFECTIVE DATE: 07/01/94 EXPIRATION DATE: $07 / 31 / 94$

FSC

COOE EXCESS PROPERTY DESCRIPTION

REFERENCE MUMBER : 421

G625 SERVO COUTOL LER MFR. GEMERAL SCAMHING INC. MODEL NO. GDG1001, S/N 100393, ACO. DATE: 2/86. APPROX. WT: $10 \mathrm{LBS}$. . 19.W $\times 14 . \mathrm{L} \times 4 \mathrm{H}$.

REFERENCE NUMBER : 422

GENERAL SCANHING INC., MOOEL NO. DX2005, S/N

100269, ACO. D ATE: 2/86, APPROX. WT: 25 LBS., $19 \cdot \mathrm{W} \times 14 . \mathrm{L} \times 44^{1} \mathrm{H}$.

$\$ 2,976.00$
REPORT MUMBER: $899105-408$

LINE ITEM MLMBER: 003

$400001 E A$

COST/UNIT:

TOTAL COST:
LINE ITEM MUMBER: 0035
No of

400001 EA
COST/UNIT:
TOTAL COST:
REPORT MUMBER : $899105-408$
FOQ FURTHER INFORMAIIO :

LYMN E. SHACKELFOOT

LIVERMORE

CA $94550-0969$

(510) $294-3067$

TO FREEZE EQUIPMENT:

LYMM E. SHACKEL FOOT

LIVERMORE

(510) $294-3067$

Ca $94550-0969$

LOCATION OF EQUIPMENT :

SAMDIA NATIONAL LABORATORIES

PROPERTY REAPPLICATION DEPT. 8532-1

LIVERMORE

CA $94550-0969$ 
EFFECTIVE DATE: 07/01/94 EXPIRATION DATE: $07 / 31 / 94$

ACOUISITION PRICE

COST/UNIT: TOTAL COSI: SPECTROGRAPH MFR: JARRELL-ASH, MOOEL NO. 19.300,
$4 / 61$. APPROX. WT: 2000 LBS., $16 . W \times 32 . L \times 67 \%$.

REPORT NUMBER: $899105-408$

LINE ITEM NUMBER: 0036

REFERENCE NUMBER : 424

O625 SPECTROMETER, MFR. ENGIS EOUIP, MODEL 1700, ACO. YR 64, PROP. NO. 437896
$600001 \mathrm{EA}$

\section{COST/UNIT: TOTAL COST:}

$\$ 9.198 .00$ $\$ 9,198.00$
FOR FURTHER INFORMATION:

JOANNE BREZNIK

LIVERMORE

(510) $424-4101$

TO FREEZE EQUIPMENT:

JOANME BREZNIK

LIVERMORE

CA 94550

(510) $424-4101$

LOCATION OF EOUIPMENT

LAURENCE LIVERMORE MATIONAL LAB

EXCESS PROPERTY SECTION, L-696

LIVERMORE 
EFFECTIVE DATE: $07 / 01 / 94$ EXPIRATION DATE: $07 / 31 / 94$

FSC
COOE EXCESS PROPERTY DESCRIPIION

REFERENCE MUMBER : 425

6625 SPECTROMETER, MASS CONTROL PANEL HAS ION SOURCE CONTROLS, GAS CONTROLS, PONER CONTRO LS AND MASS RANGE SELECT, IN BACK THERE ARE CONNECTION FOR WATER, AIR, GAS AND HYDROGEN. 208VAC, DUPONT.

REPORT NUMBER: $898355-4102$

LIME ITEM NUMBER: 0081

\section{REFERENCE NUMBER : 426}

6625 SPECTRORADIOMETER RADIOMETER INDICATOR UNIT WITH AMPERE MULTIPLIER,

COULOMB MULTIPL IER AND CURRENT DAMPING CONTROLS HITH DC INDICATOR.

$120 \mathrm{VAC}, 60 \mathrm{~Hz}$. POWER CORD NEEDS REPLACING. EG\&G, 58012

COST/UNIT:
TOTAL COST:

TOTAL COST:

REPORT NUMBER: $898355-\div 102$

LINE ITEM NUMBER: 0082

No of

COND UNITS UNIT
COST/UNIT:

TOTAL COST:

CONTACT(S)

$\$ 18,795.00$

$\$ 18,795.00$
FOR FURTHER INFORMATION:

ELOY N. GIRON

ALBUOUEROUE

(505) $844-2746$

MM 87185

TO FREEZE EOUIPMENT :

ELOY N. GIRON

ALBUOUEROUE

(505) $844-2746$

MM 87185

LOCATION OF EQUIPMENT:

SANDIA MATIONAL LABORATORIES

PROPERTY REAPPLICATION DIV. 7617

ALBUOUEROUE

im 87123 
EFFECIIVE DATE: 07/01/94 EXPIRATION DAIE: 07/31/94

\section{FSC \\ COOE EXCESS PROPERTY DESCRIPTION \\ Mo of}

REFERENCE NUMBER : 427

6625 STATION THE STATION IS AN ACCURATE CHECKER WITH CONNECTIONS IN FRONT AND BACK. TESC INC., T812HBD, SER. 41160076.

REPORT NUMBER: $398355-6088$

LINE ITEM NUMBER: 0052
COND UNITS UN!

$00001 \mathrm{EA}$ SWEEP PLUG IN SAMPLER MFR: TEKTRONIX MOOEL: 7514 $S / N: B 070863$

\section{COST/UNIT:} TOTAL COST:

\section{$\$ 2.100 .00$ $\$ 2,100.00$}

500001 EA

$$
\begin{aligned}
& \text { COST/UNIT: } \\
& \text { TOTAL COST: }
\end{aligned}
$$

LINE IIEM NUMBER: 0026
CONTACT(S)

FOR FURTHER INFORMATION:

ELOY N. GIRON

ALBUOUEROUE

505) $844-2746$

NM 87185

TO FREEZE EQUIPMENT:

ELOY N. GIRON

ALBUOUEROUE

(505) $844-2746$

NM 87185

OCATION OF EOUIPMENT:

SAMDIA MATIONAL LABORATORIES

PROPERTY REAPPLICATION DIVISION 7617 ALBUOUERQUE NM 87123

REPORT NUMBER: $899305-4039$
FOR FURTHER INFORMATION:

CYNDI DELONG

LAS VEGAS

(702) $295-6753$

TO FREEZE EOUIPMENT:

CYNDI DELONG

LAS VEGAS

(702) $295-6753$

NV $89193-8521$

LOCATION OF EOUIPMENT:

REYMOLDS ELECTRICAL \& ENGINEERING CO

MEVADA TEST SITE

NV 89023 
EFFECIIVE DATE: $07 / 01 / 94$ EXPIRATION DATE: $07 / 31 / 94$

COOE EXCESS PROPERTY DESCRIPTION

REFERENCE NUMBER : 429

6625 SUEEP PLUG IN SAMPLER MFR: TEKTRONIX MODEL: 7514 S/N: $B 091013$

REPORT NUMBER: $899305-4039$

LINE ITEM NUMBER: 0028

REFEREMCE MUMBER : 430

6625 TELEMETRY ACCESS EMR PLUG IN WITH CONTROLS FOR OFFSET, FULL SCAL VERIER, CENTER F REQ, ZERO, OUTPUT AND ON/OFF. POWER COMES FROM MAINFRAME. EMR T ELEM, 237A01M, SER. G6.

\section{FOR FURTHER INFORMATION}

ELOY N. GIRON

ALBUDUEROUE

(505) $844-2746$

87185

TO FREEZE EQUIPMENT:

ELOY N. GIRON

ALBUOUEROUE

(505) $844-2746$

87185

REPORT MUMBER: $898355-4090$

LINE ITEM MUMBER: 0069
LOCATION OF EQUIPMENT:

SAMDIA MATIONAL LABORATORIES PROPERTY REAPPLICATION DIVISION 7617 ALBUQUEROUE 
EFFECTIVE DATE: $07 / 01 / 94$ EXPIRATION DATE: $07 / 31 / 94$

FSC

COOE EXCESS PROPERTY DESCRIPIIION

REFERENCE NUMBER : 431

6625 TEST CABINET W/SPECTROMETER MFR. PHYSICAL ELECTRONICS

ELIGIBLE EQUIPMENT

NO OF

COND UNITS UNIT

ACQUISITION PRICE

$500001 \mathrm{EA}$

COST/UNIT:

TOTAL COST:

$\$ 37.295 .00$ $\$ 37.295 .00$

LINE ITEM NUMBER: 0018
REPORT NUMBER: 899110-0125
REFERENCE NUMBER : 432

6625 TESTER TENSIL OR COMPRESSION LOAD, LOAD WEIGHING SYSTEM PROVIDES SELEC IION OF FULL SCALE SENSITIVITIES FORM 0-20,000 POUNDS. MODEL $1115=$ A4,

S/N 3377. MFG. INSTRON CORP. P/N P28981.
LINE ITEM NUMBER: 0383
$600001 E A$

COST/UNIT: TOTAL COST:

$\$ 15,465.00$ $\$ 15,465.00$
FOR FURTHER INFORMATION:

SANDY MOON

IDAHO FALLS

(208) 526-2611

$1083415-4100$

TO FREEZE EOUIPMENT

SAMDY MOON

IDAHO FALLS

ID $83415-4100$

LOCATION OF EQUIPMENT:

IDAHO NATIONAL ENGIMEERING LAB.

CF-674 WAREHOUSE

SCOVILLE 
EFFECTIVE DATE: $07 / 01 / 94$ EXPIRATION DATE: $07 / 31 / 94$

FSC

COOE EXCESS PROPERTY DESCRIPTION

REFERENCE NUMBER : 433

6625 TESTER-NETHORK NMC2, MFR. NETHORK SYS, MODEL 300758, ACQ. YR. 79, PROP. 700001 EA NO. 3207558

NO OF

COND UNITS UNIT

ACQUISITION PRICE

$\$ 13,319.16$ $\$ 13,319.16$

COST/UNIT: TOTAL COST:

LINE ITEM NUMBER: 0011
REPORT NUMBER: $899102-4169$

REFERENCE NUMBER : 434

6625 TORQUE MONITORING SYS 3-1/2 DIGIT LED READOUT, 3 CHANNEL SELECT, 160 KHZ 600001 EA TORQUE MONITORING SYS $3-1 / 2$ DIGIT LED READOUT, 3 CHANNEL SELECT,
OUTPUT CONNECT ION IN GACK. 120VAC, 60 HZ. ACUREX, 1200A, $1-195$.

REPORT NUMBER: $898355-4090$

LINE ITEM NUMBER : 0070
COST/UNIT: TOTAL COST:
$\$ 8,550.00$ $\$ 8,550.00$
FOR FURTHER INFORMATION:

ELOY N. GIRON

ALBUQUERQUE

(505) $844-2746$

NM 87185

TO FREEZE EQUIPMENT:

ELOY N. GIRON

ALBUQUERQUE

(505) $844-2746$

NM 87185

LOCATION OF EQUIPMENT:

SANDIA NATIONAL LABORATORIES

PROPERTY REAPPLICATION DIVISION 7617

ALBUQUERQUE

NM 87123 
EFFECTIVE DATE: $07 / 01 / 94$ EXPIRATION DATE: $07 / 31 / 94$

\section{COOE EXCESS PROPERTY DESCRIPTION}

REFERENCE NUMBER : 435

6625 TRANSFORMER MFR: IOPAZ, MODDEL NO. 91905-66, S/N 118560, ACQ. DATE:

3/86, A PPROX. WT: 100 LBS., 8.W $\times 17 \cdot \mathrm{L} \times 7 \cdot \mathrm{H}$.

REPORT NUMBER: $899105-4081$

LINE ITEM NUMBER: 0037

REFERENCE NUMBER : 436

6625 TRANSFORMER MFR: XENTEK INC., MODEL NO. 5410, ACQ. DATE: 3/86, APPROX. 600002 EA

$W T: 100$ LABS., 8.W $\times 17 . \mathrm{L} \times 7 . \mathrm{H}$. no of

COND UNITS UNIT

$600001 \mathrm{EA}$

COST/UNIT:

TOTAL COST:
REPORT NUMBER: $899105-4081$
LINE ITEM NUMBER: 0038
COST/UNIT: TOTAL COST:

\section{$\$ 2,000.00$}

FOR FURTHER INFORMATION

LYNN E. SHACKELFOOT

LIVERMORE

(510) $294-3067$

CA $94550-0969$

TO FREEZE EQUIPMENT:

LYNN E. SHACKELFOOT

LIVERMORE

(510) $294-3067$

CA $94550-0969$

LOCATION OF EQUIPMENT:

SANDIA NATIONAL LABORATORIES

PROPERTY REAPPLICATION DEPT 8532-1

PROPERTY REAPPLICATION DEPT. 8532-1 
EFFECTIVE DATE: $07 / 01 / 94$ EXPIRATION DATE: $07 / 31 / 94$

COOE EXCESS PROPERTY DESCRIPIION

UNITS UNIT

ACQUISITION PRICE

6625 TRANSLATOR IN A CABINET $22-1 / 2$ "WX31"DX71"H, WITH A CNC MOTION CONTROLLER WIT $\mathrm{H} X$ AND $Y$ READING. 120VAC, $60 \mathrm{HZ}$. WFCA 15 , SER. 0028

$600001 \mathrm{EA}$ COST/UNIT: TOTAL COST

$\$ 2.781 .00$ $\$ 2,781.00$

REPORT NUMBER: $898355-4090$

LINE ITEM NUMBER: 0071

\section{REFERENCE NUMBER : 438}

1 EACH. SIZE: 8"W $\times 12^{\prime \prime F / R} \times 8^{\prime \prime H}$ EST WT: 20 LBS MFGR YR: 1985

$4 \quad 00001 \mathrm{EA}$

COST/UNIT:

TOTAL COST:

$\$ 1.648 .00$

$\$ 1,648.00$

FOR FURTHER INFORMATION

BARBARA HOLMES, REP.

KANSAS CITY

MO $64141-6159$

TO FREEZE EQUIPMENT:

BARBARA HOLMES, REP.

KANSAS CITY

(816) $997-2976$

MO 64141-6159

OCATION OF EQUIPMENT

ALLIEDSIGNAL, INC.

2000 EAST 95TH STREE

KANSAS CITY

MO $64131-3095$ 
EFFECTIVE DATE: $07 / 01 / 94$ EXPIRATION DATE: $07 / 31 / 94$

\section{FSC \\ COOE EXCESS PROPERTY DESCRIPTION \\ REFERENCE NUMBER : 439 \\ 6625 TUMABLE MICRO FILTER THE TUMABLE MICROWAVE FILIER IS A HIDEBAND YIG} FILTER. 12OVAC, $60 \mathrm{HZ}$. INTEGRA, TMF 1800, SER. 4091502.

REPORT NUMBER: $898355-4090$

LINE ITEM NUMBER: 0072

\section{NO OF}

COND UNITS UNIT

$600001 \mathrm{EA}$
ACQUISITION PRICE

\section{COST/UNIT:}

TOTAL COST :

$\$ 5,100.00$
$\$ 5,100.00$

CONTACT(S)

FOR FURTHER INFORMATION:

ELOY N. GIRON

ALBUQUEROUE

(505) $844-2746$

NM 87185

TO FREEZE EQUI PMENT

EIOY N. GIRON

ALBUQUEROUE

NM 87185

(505) $844-2746$

LOCATION OF EQUIPMENT

SANDIA NATIONAL LABORATORIES

PROPERTY REAPPLICATION DIVISION 7617

ALBUQUEROUE

COST/UNIT:

TOTAL COST:
$\$ 9.488 .00$ $\$ 9,488.00$
FOR FURTHER INFORMATION:

LYNN E. SHACKELFOOT

LIVERMORE

(510) 294-3067

TO FREEZE EOUIPMENT:

LYNN E. SHACKELFOOT

LIVERMORE

(510) 294-3067

LOCATION OF EQUIPMENT:

TRATORIES

PROPERTY REAPPLICATION DEPT. 8532-1

LIVERMORE

CA $94550-096$ 
EFFECTIVE DATE: 07/01/94 EXPIRATION DATE: $07 / 31 / 94$

FSC

\section{COOE EXCESS PROPERTY DESCRIPTION}

REFERENCE NUMBER : 441

6625 ULTRASONIC FLAW DETECTOR 62965, MANUF: SONATEST MODEL 026 S/N 843314 1 ASSY., INCLUDES CE 64955 ULTRASONIC FLAW DETECTOR, MFG. BALTEAU, MOOEL UFD-S, S/N 0235 SIZE: $13^{\prime \prime W} \times 16^{\prime \prime F} / R \times 11^{\prime \prime H}$

EST HT: 25 LBS MFGR YR: 1984

REPORT NUMBER: $896404-4124$

LINE ITEM NUMBER: 0003

REFERENCE NUMBER : 442

6625 VACUUM ACCESSORY BASIC MANIPULATOR COMBINES A DIRECT DRIVE ROTARY

MANIPULATOR WITH A MICROMETER DRIVE TRANSLATOR MECHANISM TO GIVE HIGH

PRECISION S AMPLE POSITIONING. VARIAN, $9810523,274$.

REPORT NUMBER: $898355-4088$

LINE ITEM NUMBER: 0053
NO OF

COND UNITS UNIT

ACQUISITION PRICE

$400001 \mathrm{EA}$ COST/UNIT:
TOTAL COST:

$\$ 14,857.00$ $\$ 14,857.00$

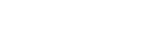

(1)


EFFECTIVE DATE: $07 / 01 / 94$ EXPIRATION DATE: $07 / 31 / 94$

REFERENCE NUMBER : 443

O625 VACUUM SYSTEM MFG: GRANVILLE TYPE: HIGH VACUUM ACQ DATE: 8/67 REF NO: $29936-01$

\section{$600001 \mathrm{EA}$ COST/UNIT:} TOTAL COST:
LINE ITEM NUMBER: 0006

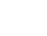

REPORT NUMBER: $890531-4103$

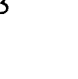


EFFECTIVE DATE: $07 / 01 / 94$ EXPIRATION DATE: $07 / 31 / 94$

FSC

COOE EXCESS PROPERTY DESCRIPTION

REFERENCE MUMBER : 445

6625 VIBRATION GENERATOR MFR: WILCOXON RESEARCH, MODEL NO. F4 DRIVER, S/N 228, ACQ. DATE: $1 / 75$, APPROX. WT: $20 \mathrm{LBS} ., 5 \mathrm{~W} \times 5 . \mathrm{L} \times 8 . \mathrm{H}$

REPORT MUMBER: $899105-4081$

LINE ITEM NUMBER: 0040
NO OF

COND UNITS UNIT

ACQUISITION PRICE

$500001 \mathrm{EA}$

\section{COST/UNIT:}

TOTAL COST:

\section{$\$ 1,958.00$}

$\$ 1,958.00$

\section{Couract(s)}

FOR FURTHER INFORMATION:

(510) $294-3067$

CA $94550-0969$

TO FREEZE EQUIPMENT:

LYNN E. SHACKELFOOT

LIVERMORE

(510) $294-3067$

CA $94550-0969$

LOCATION OF EQUIPMENT:

SAMDIA MATIOMAL LABORATORIES

PROPERTY REAPPLICATION DEPT. 8532-1

LIVERMORE RA $94550-0969$

REFERENCE MUMBER : 446

VIBRATICN TESTER 80584, MANUF: UNHOLTZ-DICKIE MOOEL TA145A-1301AR S/N

1 ASSY., INCLUDES CE 80584 A CONTROL PAMNEL AND CE 80585 CONTROLLER.

SIZE: 98"W X 36"F/R X 97"H EST WT: 12000 LBS

MFGR YR: 1992
$4 \quad 00001$ EA

COST/UNIT:

TOTAL COST:

$\$ 385,555.00$

$\$ 385,555.00$
FOR FURTHER INFORMATION:

BARBARA HOLMES, REP.

KANSAS CITY

(816) $997-2976$

MO $64141-6159$

TO FREEZE EQUIPMENT:

BARBARA HOLMES, REP

KANSAS CITY

MO 64141-6159

REPORT NUMBER: $896404-4124$

LINE ITEM NUMBER: 0008
(816) $997-2976$

LOCATION OF EQUIPMENT:

ALLIEDSIGMAL, INC

2000 EAST 95TH STREET

XANSAS CITY 
EFFECTIVE DATE: $07 / 01 / 94$ EXPIRAIION DATE: $07 / 31 / 94$
FSC

\section{COOE EXCESS PROPERTY DESCRIPTION}

REFERENCE NUMBER : 447

6625 VOLTMETER DIG!TAL MFR: HEWLETT PACKARD

$\begin{array}{ll}\text { MOD: } & 2401 C \\ \text { SER: } & 605-00773 \text { PC08332 PRC-40014 }\end{array}$

REFERENCE NUMBER : 448
6625 VOLTMETER DIGITAL MFR: HEWLETT PACKARD MOD: 24010

SER: $616-00873$ PC08335 PRC-40014

REPORT NUMBER: $899109-4101$ ELIGIBLE EQUIPMENT

\section{COND UNITS UNIT \\ ACQUISITION PRICE}

$600001 \mathrm{EA}$

LINE ITEM NUMBER: 0015

60001 EA

LINE ITEM NUMBER: 0016

STAMFORD

MATTIE PACE

STANFORD
COST/UNIT:
TOTAL COST:

COST/UN1T:
TOTAL COST:

$\$ 4,335.00$
$\$ 4,335.00$

MUTTIE PACE

STAMFORD

(415) $926-2231$

CA 94309

TO FREEZE EQUIPMENT:

MAITIE PACE

STANFORD

(415) $926-2231$

CA 94309

LOCATION OF EQUIPMENT:

STAMFORD LIMEAR ACCELERATOR CENTER 2575 SAND HILL ROAD

MENLO PARK

CA 94025

$\begin{array}{ll} & \$ 4.335 .00 \\ \text { TOTAL COST: } & \$ 4,335.00\end{array}$

FOR FURTHER INFORMATION:

MATTIE PACE
(415) $926-2231$

(415) $926-2231$

CA 94309

TO FREEZE EOUIPMENT:

(415) $926-2231$

LOCATION OF EQUIPMENT:

STANFORD LIMEAR ACCELERATOR CENTER 2575 SAND HILL ROAD

MENLO PARK

CA 94025 
EFFECTIVE DATE: $07 / 01 / 94$ EXPIRATION DATE: 07/31/94

FSC

COOE EXCESS PROPERTY DESCRIPIION

REFERENCE NUMBER : 640

6625 VOLTMETER DIGITAL MFR: HEULETT PACKARD MOD: $2401 C$

$\begin{array}{ll}\text { MOO: } & 2401 C \\ \text { SER: } & 637-01599 \text { PC06547 PRC-40014 }\end{array}$

REPORT NUMBER: $899109 \cdot 6101$

REFERENCE NUMBER : 450

6625 VOLTMETER DIGITAL MFR: HEWLETT PACKARD

MOO: $2401 C$

YOM 1966 PC01802 PRC-40014

REPORT NUMBER: $899109-4101$
NO OF

COND UNITS UNIT

ACOUISITION PRICE

$600001 \mathrm{EA}$

COST/UNIT: IOTAL COST:

$\$ 1,372.00$ $\$ 1,372.00$

FOR FURTHER IMFORMATION:

MATIIE PACE

STANFORD

CA 94309

TO FREEZE EQUIPMENT:

MATIIE PACE

STAMFORD

CA 94309

(415) $926-223$

LOCATION OF EQUIPMENT:

STAMFORD LIMEAR ACCELERATOR CENTER

2575 SAND HILL ROAD

MENLO PARK

CA 94025

\section{$600001 \mathrm{EA}$ \\ COST/UNIT: \\ TOTAL COST:}

$\$ 4.200 .00$

FOR FURTHER INFORMAIION:

MATTIE PACE

SIANFORD

CA 94309

TO FREEZE EOUIPMENT

MATIIE PACE

STANFORD

(415) $926-2231$

CA 94309

LOCATION OF EQUIPMENT:

STAMFORD LIMEAR ACCELERATOR CENTER 2575 SAMD HILL ROAD

CA 94025 
EFFECTIVE DATE: $07 / 01 / 96$ EXPIRATION DATE: $07 / 31 / 96$

FSC

\section{COOE EXCESS PROPERTY DESCRIPTION}

REFERENCE NUMBER : 45

6625 VOLTMETER, DIFF AC/DC HAS NULL SELECT SWITCH, i TO 1000 RANGE SHITCH +1 TO 1 VOLTMETER. 120VAC, $60 \mathrm{HZ}$. FLUKE, 887AB, SER. 985003.

REPORT NUMBER: $898355-4090$

LINE ITEM NUMBER: 0078

\section{REFERENCE NUMBER : 452}

6625 VOLTMETER, DIGITAL IT IS A 3-1/2 DIGIT HIGH SPEED DC VOLTMETER WITH SAMPLE AND HOLD. I2OVAC, $60 \mathrm{HZ}$. HEW PACK, DC3437A, SER. 25164090.

REPORT NUMBER: $898355-4090$

LINE IIEM MLMBER: 0079
$600001 \mathrm{EA}$ COST/UNIT:
TOTAL COST:

no of

COND UNITS UNIT

ACQUISITION PRICE

COST/UNIT:

TOTAL COST:

$\$ 2,266.00$
$\$ 2.266 .00$

FOR FURTHER INFORMAT!OW
ELOY N. GIROW

ALBUDUEROUE

(505) 844-2746

m 87185

TO FREEZE EOUIPMENT:

ELOY N. GIRON

ALBUOUEROUE

(505) $844-2746$

N 87185

LOCATION OF EQUIPMENT.

SAMDIA MATIOALL LABORATORIES

PROPERTY REAPPLICATIOU DIVISIOY 7617

PROPERTY REAPPLICATIOW DIVISIOW 7617

ALBUOUEROUE

IM 87123

$\$ 2,000.00$

$\$ 2,000.00$

FOR FURTHER IMFORMAIION:

ELOY N. GIRON

ALBUQUEROUE

(505) 844-2746

87185

TO FREEZE EQUIPNENT:

ELOY N. GIRON

ALBUOUERONE

Mn 87185

LOCATION OF EQUIPMENT:

SAMDIA MATIOWAL LABORATORIES

PROPERTY REAPPLICATION OIVISION 7617

PROPERTY REAPPLICATION DIVISION 7617

im 87123 
EFFECTIVE DATE: 07/01/94 EXPIRATION DATE: $07 / 31 / 94$

FSC

\section{COOE EXCESS PROPERTY DESCRIPTION}

REFERENCE MUMBER : 453

6625 VOLTMETER, DIGITAL THE 3440K AMD IIS ASSOCIATED PLUG INS ARE DESIGMED

FOR GENERAL PU RPOSE MEASUREMENTS OF DC VOLTS, AC VOLTS, DC CURRENT AMD

FOR GENERAL PU RPOSE MEASUREMENTS OF DC VOLTS, AC
OHMS. 1 2OVAC, $60 \mathrm{HZ}$. HEW PACK, 3440A, 63707793.

REPORT MUMBER: $898355-4090$

LINE ITEM MUMBER: 0080

REFERENCE NUMBER : 454

6625 VOLTMETER, RMS IS USABLE FOR DC, LON FREQ, AUDIO, RF AND IF MEASUREMENTS

WITH FA ST AND SLOW FREO RESPONSE TIME PERFORMANCE. 120VAC, $60 \mathrm{HZ}$. HEW

$P$ ACK, 3403C, SER. 03A00296.

LINE ITEM MUMBER: 0077

No of

COND UNITS UNIT

ACQUISITION PRICE

COATACT(S)

$700001 \mathrm{EA}$

COST/UNIT: TOTAL COST:

\section{$\$ 1,132.00$ $\$ 1,132.00$}

FOR FURTHER IMFORMATION
ELOY N. GIROW

ALBUOUERCUE

TO FREEZE EQUIPMEMT:

ELOY N. GIROM

ALBUOUERCUE

(505) $844-2746$

87185

LOCATION OF EOUIPMENT

SAMDIA MATIOMAL LABORATORIES PROPERTY REAPPLICATION DIVISION 7617 ALBUOUERQVE

4. 87123

$500001 \mathrm{EA}$

COST/UNIT:

TOTAL COST:

$\$ 1,595.00$

FOR FURTHER IMFORMATION:

ELOY N. GIROM

ALBUDUEROUE

(505) 844.2746

N40 87185

IO FREEZE EQUIPMENT:

ELOY N. GIROW

ALBUCUEROUE

(505) $844-2746$

87185

LOCATION OF EQUIPMENT

SAMOIA MATIOMAL LABORATORIES

PROPERTY REAPPLICATION DIVISION 7617

ALBUOUEROUE

in 87123 
FSC

\section{COOE EXCESS PROPERTY DESCRIPIION}

REFERENCE NUMBER : 455

6025 VOLTMETER, IRUE RMS IS USABLE FOR DC, LON FREO, AUDIO, RF AND IF MEASUREMENTS PLUS FA ST AND SLOU RESPONSE TIME PERFORMANCE. 120VAC, 60 HZ. HEW PACK, 3403C, 03A00510

REFERENCE NUMBER : 456

O625 VOLTMETER, TRUE RMS IS USABLE FOR DC, LOW FREO, AUDIO, RF AND IF MEASUREMENTS, WITH F AST AND SLOW RESPOLSE TIME PERFORMANCE. IZOVAC. 6OHZ. HEW PACK, 3403C, 03A00907.
No of

COND UNITS UNIT

ACQUISITION PRICE

$5 \quad 00001 \mathrm{EA}$

\section{COST/UNIT:} TOTAL COSI:

\section{$\$ 1.595 .00$ \\ $\$ 1.595 .00$}

ELOY N. GIROW
ALBUCUEROUE
(505) $844-2746$

WM 87185

TO FREEZE EQUIPMENT:

ELOY M. GIKON

AL BUOUEROUE

(505) $846-2746$

NM 87185

LOCATION OF EOUIPMENT:

SAMDIA MRTIONAL LABORATORIES

PROPERIY REAPPLICATION DIVISION 7617

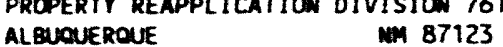

$\$ 2.15+.00$
$\$ 2.194 .00$

COTAL COST:

$500001 E A$

iOTAL COST:

FOR FURTHER INFORMATION:
ELOY N. GIRON
ALBUOUEROUE
(505) $844-2746$
TO FREEZE EQUIPMENT:
ELOY N. GIRON
ALBUOUEROUE
(5OS) $864-2746$
LOCATION OF EQUIPMENT:
SAMDIA MATIONAL LABORATORIES
PROPERTY REAPPLICATION DIVISION 7617
ALBUOUEROUE


EFFECTIVE DATE: 07/01/94 EXPIRATION DATE: 07/31/94

FSC

COOE EXCESS PROPERTY DESCRIPTION

EEFERENCE MUMBER : 457

6625 VOLTMETER, TRUE RMS IS USABLE FOR DC, LOU FREO, AUDIO, RF AND IF MEASUREMENT WITH FAS T AMD SLOW FREO RESPONSE TIME PERFORMANCE. 120VAC, MEA HZ. HEW PA CK, 3403C, SER. 03A00969.

REPORT MUMBER: $898355-4090$

LINE ITEM MUMBER: 0076

REFERENCE MUMBER : 458

6630 ANALYZER OUANTA CHROME 087 ACO YR: 76

REPORT MUABER: $899101-9413$ no of

COND UNITS UNIT

ACQUISITIOA PRICE

$500001 \mathrm{EA}$

COST/UNIT

TOTAL COST:

$\$ 2,194.00$

$\$ 2,194.00$

ELOY N. GIROM
ALBUOUEROUE
(505) $844-2746$

TO FREEZE EQUIPMENT:

ELOY N. GIRON

ALBUCUEROUE

(505) $844-2746$

M4 87185

LOCATION OF EQUIPMENT :

SAMD IA MATIOMAL LABORATORIES

PROPERTY REAPPLICATION DIVISION 7617

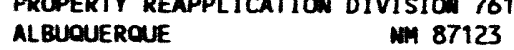

$500001 \mathrm{EA}$

cost/UNIT:

TOTAL COST:

$\$ 8,820.00$
$\$ 8,820.00$

$58,820.00$

FOR FURTHER IMFORMATION:

MONTE CLEVENGER

BERKELEY

(510) 486-6242

CA 94720

TO FREEZE EQUIPMENT:

MONTE CLEVEMGER

BERKELEY

(510) $486-6242$

CA 94720

LOCATION OF EQUIPMENT:

901 MAREHOUSE

1450 64TH STREET

EMERYVILLE

CA 94608 
EFFECTIVE DATE: 07/01/94 EXPIRATION DATE: $07 / 31 / 94$

COOE EXCESS PROPERTY DESCRIPIION

REFERENCE MUMBER : 459

6630 CONTROL-FIOY (DUAL) OUANTA CHROME LMFC-4 ACQ YR: 76

REPORT NUMBER: $899101-9613$

LINE ITEM NUMBER: 0009

REFERENCE NUMBER : 460

6635 AMPLIFIER-VIBRATING REED CARY 36, ACO YR: 64

REPORT NUMBER: $899101-9413$
No of

COND UNITS UNIT

ACQUISITION PRICE

$500001 \mathrm{EA}$

COST/UN $1 \mathrm{~T}$

TOTAL COST:

$\$ 3,478.00$

$\$ 3,478.00$

THER INFORMATION :

MONTE CLEVENGER

BERKELEY

CA 94720

(510) $486-6242$

OO FREEZE EOUIPMENT:

MONTE CLEVENGER

BERKELEY

CA 94720

(510) $486-6242$

LOCATION OF EQUIPMENT:

901 WAREHOUSE

145064 TH STREET

EMERYVILLE

CA 94608
$500001 \mathrm{EA}$

LINE ITEM NUMBER: 0026

$$
\begin{aligned}
& \text { COST/UNIT: } \\
& \text { TOTAL COST: }
\end{aligned}
$$

TOTAL COST:

$\$ 4.600 .00$
$\$ 4,600.00$
FOR FURTHER INFORMATION :

MOWTE CLEVENGER

BERKELEY

(510) $486-6242$

CA 94720

TO FREEZE EOUIPMENT:

MONTE CLEVENGER

BERKELEY

CA 94720

(510) 486-6242

LOCATION OF EQUIPMENT:

901 WAREHOUSE

1450 64TH STREET

EMERYVILLE 
EFFECTIVE DATE: $07 / 01 / 94$ EXPIRATION DATE: $07 / 31 / 94$

\section{FSC \\ COOE EXCESS PROPERTY DESCRIPTION}

REFERENCE NUMBER : 461

6635 DATA ACOUISITION, CONTROL UNIT MFR: HEWLETT PACKARD, MODEL NO. 3497A S/N 2448A15500, ACD. DATE : 2/85, APPROX. WT: 50 LABS., 19'WX $20^{\prime} L$ 'X

REPORT NUMBER: $899105-4081$

LINE ITEM NUMBER: 004
NO OF

COND UNITS UNIT

ACQUISITION PRICE

$500001 E A$

COST/UNIT: TOTAL COST:

$\$ 4,630.00$ $\$ 4,630.00$

FOR FURTHER INFORMATION:

LYNN E. SHACKELFOOT

LIVERMORE

(510) $294-3067$

CA $94550-0969$

TO FREEZE EQUIPMENT:

LYNN E. SHACKELFOOT

LIVERMORE

(510) $294-3067$

CA $94550-0969$

LOCATION OF EOUIPMENT:

SANDIA NATIONAL LABORATORIES

PROPERTY REAPPLICATION DEPT. 8532-1

LIVERMORE REAPPLICATION DEPT $94550-0969$

REFERENCE NUMBER : 462

6635 DATA ACOUISITION SYSTEM MFR: FLUKE, MODEL NO, 2200B, S/N 2150027, ACO. 4 00001 EA DATE: $7 / 79$, APPR OX. WT: 35 LAABS., $17 \cdot \mathrm{W} \times 21^{\prime} \mathrm{L} \times 8^{\prime} \mathrm{H}$.

COST/UNIT:

TOTAL COST:

$\$ 3,584.00$

$\$ 3,584.00$

FOR FURTHER INFORMATION:

LYNN E. SHACKELFOOT

IVERMORE

(510) $294-3067$

CA $94550-0969$

TO FREEZE EQUIPMENT:

LYNN E. SHACKELFOOT

LIVERMORE

(510) $294-3067$

CA $94550-0969$

LOCATION OF EQUIPMENT:

SANDIA NATIONAL LABORATORIES

PROPERTY REAPPLICATION DEPT 8532-1

PROPERTY REAPPLICATION DEPI. 8532-1 
EFFECTIVE DATE: $07 / 01 / 94$ EXPIRATION DATE: $07 / 31 / 94$

COOE EXCESS PROPERTY DESCRIPIION

REFERENCE NUMBER : 463

6635 FURNACE BLUE M MOOEL POM7-206C, S/N P38-1711, P/N 221473.

REPORT NUMBER: $890207-4116$

LINE ITEM NUMBER: 0372

RE FERENCE NUMBER : 464

6635 TRANSDUCER PRESSURE MFG. CEC, MOOEL 1500LB, S/N 1016, P/N 703339.

$600001 \mathrm{EA}$

COST/UNIT:

TOTAL COST:
ACQUISITION PRICE

$\$ 3,500.00$

$\$ 3,500.00$

COST/UNIT:
TOTAL COST:

53.500 .00

SON

CONTACT(S)

FOR FURTHER INFORMATION:

SANDY MOON

DAHO FALLS

ID $83415-4100$

TO FREEZE EQUIPMENT:

SANDY MOON

IDAHO FALLS

(208) $526-2611$

ID $83415-4100$

LOCATION OF EQUIPMENT:

IDAHO NATIONAL ENGINEERING LAB.

CF-674 WAREHOUSE

SCOVILLE

ID $83415-4100$

$\$ 1.000 .00$

$\$ 1,000.00$

FOR FURTHER INFORMATION:

SANDY MOON

IDAHO FALLS

(208) $526-2611$

ID $83415-4100$

TO FREEZE EQUIPMENT:

SANDY MOON

IDAHO FALLS

(208) $526-2611$

ID $83415-4100$

LOCATION OF EQUIPMENT:

IDAHO NATIONAL ENGINEERING LAB.

CF-674 WAREHOUSE

SCOVILLE

ID $83415-4100$ 
EFFECTIVE DATE: $07 / 01 / 94$ EXPIRATION DATE: $07 / 31 / 94$

FSC

\section{CODE EXCESS PROPERTY DESCRIPTION}

REFERENCE MUMBER : 465

6635 TRANSDUCER PRESSURE MFG. CEC, MODEL 0-50 PSI, S/N 1672, P/N 702471.

REPORT NUMBER: $890207-4116$

LINE ITEM NUMBER: 037

REFERENCE NUMBER : 466

$6635 X$-RAY DENSITY GAGE 66799, MANUF: CONNECTICUT GAUGING MODEL $191179001 \mathrm{~S} / \mathrm{N} 600001$ EA

1 ASSY., INCLUDES CE 74041 GAGE TABLE. SIZE: $72^{\prime \prime W} \times 102 " \mathrm{~W} / \mathrm{R} \times 72^{\prime \prime H}$ EST WT : 1000 LBS MFGR YR: 1987

NO OF

$60001 \mathrm{EA}$
COND UMITS UNIT

ACQUISITION PRICE

$\$ 1,000.00$

COST/UNIT:

TOTAL COST:

$\$ 1,000.00$

FOR FURTHER INFORMATION:

SAMDY MOON

IDAHO FALLS

(208) $526-261$

ID $83415-4100$

TO FREEZE EQUIPMENT:

SANDY MOON

IDAHO FALLS

(208) $526-261$

ID $83415-4100$

LOCATION OF EQUIPMENT:

IDAHO MATIOMAL ENGINEERING LAB.

CF-674 HAREHOUSE

SCOVILLE

ID $83415-4100$
COST/UNIT:

TOTAL COST:

$\$ 47,398.00$

$\$ 47.398 .00$
FOR FURTHER INFORMATION

BARBARA HOLMES, REP.

KANSAS CITY

(816) $997-2976$

MO $64141-6159$

TO FREEZE EOUIPMENT : BARBARA HOLMES, REP.

KANSAS CITY

MO $64141-6159$

(816) $997-2976$

LOCATION OF EQUIPMENT:

ALLIEDSIGNAL, INC.

2000 EAST 95TH STREET

KANSAS CITY

MO 64131-3095 
EFFECTIVE DATE: $07 / 01 / 94$ EXPIRATION DATE: $07 / 31 / 94$

FSC ELIGIBLE EQUIPMENT

EXCSS PROPERTY DESCRIPTION

CONTACT(S)

REFERENCE NUMBER : 467

6636 ANALYZER-LOGIC, MFR. GOULD, MOOEL K1000, ACQ. YR. 80, PROP. NO . 3496549600001 EA

COST/UNIT:

REPORT NUMBER: $899102-4163$

LINE ITEM NUMBER: 0037

REFERENCE NUMBER : 468

6636 CHAMBER, TEMPERATURE TEMP RANGE -73 DEG C TO 315 DEG C. 120VAC, $60 \mathrm{HZ} .500001$ EA DELTA DES, 640 OCN, SER. $0.56-4$

REPORT NUMBER: $898355-4088$

LINE ITEM NUMBER: 0054
$\$ 10,185.00$

$\$ 10,185.00$

FOR FURTHER INFORMATION:

JOANHE BREZNIK

LIVERMORE

CA 94550

$4-4101$

TO FREEZE EQUIPMENT:

JOANNE BREZNIK

LIVERMORE

CA 94550

(510) $424-4101$

LOCATION OF EQUIPMENT:

LALRENCE LIVERMORE NATIONAL LAB

EXCESS PROPERTY SECTION, L-696

LIVERMORE

CA 94550

COST/UNIT:

TOTAL COST:

$\$ 3,670.00$

$\$ 3.670 .00$

FOR FURTHER INFORMATION:

ELOY N. GIRON

ALBUQUEROUE

(505) $844-2746$

NM 87185

TO FREEZE EQUIPMENT:

ELOY N. GIRO

ALBUDUEROUE

NM 87185

LOCATION OF EQUIPMENT:

SANDIA NATI ONAL LABORATORIES

PROPERTY REAPPLICATION DIVISION 7617

ALBUQUERQUE

NM 87123 
DOE USED ENERGY RELATED LABORATORY EQUIPMENT GRANT PROGRAM

EFFECTIVE DATE: 07/01/94 EXPIRATION DATE: 07/31/94

FSC

COOE EXCESS PROPERTY DESCRIPIION

REFERENCE NUMBER : 469

6636 CHAMBER, TEMPERATURE OVER WITH TEMP SETIING GAS CONNECTION IN BACK, CHAMBER, TEMPERATURE OVER WITH TEMP SETTING GAS CONNECTION IN BACK,
INSIDE DIM: 16"WX 7"HX8"D. OUTSIDE DIM: 28-3/4"WX12-1/4"HX16"D. 120VAC, $60 \mathrm{HZ}$. D ELTA DES, 2850LS, SER. 156.

REPORT NUMBER: $898355-4102$

LINE ITEM NUMBER: 0084
NO OF

COND UNITS UNIT

ACQUISITION PRICE

$700001 \mathrm{EA}$

COST/UNIT:
TOTAL COST:

$\$ 1,240.00$

FOR FURTHER INFORMATION:

ELOY N. GIRON

ALBUQUEROUE

(505) $844-2746$

NM 87185

TO FREEZE EQUIPMENT:

ELOY N. GIRON

ALBUQUERQUE

(505) $844-2746$

NM 87185

LOCATION OF EQUIPMENT:

SANDIA NATIONAL LABORATORIES

PROPERTY REAPPLICATION DIV. 7617

ALBUQUERQUE

MM 87123

REFERENCE NUMBER : 470

6636 GLOVE BOX/COMBUSION CHAMGBER, MFR. LLNL FABRICATION, MODEL NON E, ACQ. 500001 EA YR. 86, E38113

COST/UNIT:
TOTAL COST:
$\$ 1,000.00$

$\$ 1,000.00$

FOR FURTHER INFORMATION:

JOANNE BREZNIK

LIVERMORE

(510) $424-4101$

CA 94550

TO FREEZE EQUIPMENT :

JOANNE BREZNIK

LIVERMORE

REPORT NUMBER: $899102-4163$

LINE ITEM NUMBER: 37

(510) $424-4101$

LOCATION OF EQUIPMENT:

LAWRENCE LIVERMORE NATIONAL LAB
EXCESS PROPERTY SECTION, L-696

EXCESS PROPERTY SECTION, L-696 
EFFECTIVE DATE: $07 / 01 / 94$ EXPIRATION DATE: $07 / 31 / 94$

FSC

\section{COOE EXCESS PROPERTY DESCRIPTION}

REFERENCE NUMBER : 471

6636 MACHINE SHOCK TEST ITEMS FOR SHOCK HAS A TRAVEL OF UP AND DOWN IS 90"

MOR SHOCK TEST SET UP AREA IS 18"X18". 120VAC,60 HZ. MONTEREY, 1818.

REPORT NUMBER : $898355-4102$

LINE ITEM NUMBER: 0085

REFERENCE NUMBER : 472

6636 MACHINE SHOCK TEST ITEMS FOR SHOCK, HAS A TRAVEL OF UP AND DOWN IS 85" FOR SHOC X TEST SET UP AREA IS 7" $\times 7 "$ "120VAC, $60 \mathrm{HZ}$. MONTEREY, IMPAC66.

LINE ITEM NUMBER: 0086
COND UNITS UNI

ACOUISITION PRICE

7 ? UUV01 EA

COST/UNIT:

TOTAL COST:

$\$ 6,545.00$
$\$ 6,545.00$

FOR FURTHER

ELOY H. GIROM

ALBUQUERQUE

(505) $844-2746$

NM 87185

TO FREEZE EQUIPMENT:

ELOY N. GIRON

ALBUOUERQUE

(505) $844-2746$

MM 87185

OCATION OF EQUIPMENT :

SANDIA NATIONAL LABORATORIES

SANDIA NATIONAL LABORATORIES

PROPERTY REAPPLICATION DIV. 7617

COST/UNIT: $\quad \$ 22,993.00$

TOTAL COST:

$\$ 22,993.00$
FOR FURTHER INFORMATION:

ELOY N. GIRON

AL BUOLLEROUE

(505) $844-2746$

NM 87185

TO FREEZE EOUIPMENT:

ELOY N. GIRON

ALBUQUERQUE

(505) $844-2746$

NM 87185

LOCATION OF EQUIPMENT:

SANDIA MATIONAL LABORATORIES

PROPERTY REAPPLICATION DIV. 7617

PROPERTY REAPPLICATION DIV. 7617
ALBUDUERQUE
NM 87123 
EFFECTIVE DATE: $07 / 01 / 94$ EXPIRATION DATE: $07 / 31 / 94$

FSC

COOE EXCESS PROPERTY DESCRIPTION

\section{REFEREMCE MUMBER : 473}

6636 MACHINE SHOCK TEST ITEMS FOR SHOCK HAS A TRAVEL OF UP AMD DOH IS 120" FOR SHOC $K$ TEST SET UP AREA IS $15 " \times 14-1 / 2 "$ ". SANDIA LABS.

REPORT MUMBER: $898355-4102$

REFERENCE MUMBER : 474

6636 TEMPERATURE CHAMBER 75446, MANUF: THERMOTRON MOOEL EL $8 \mathrm{CH} 11 \mathrm{~S} \mathrm{~S} / \mathrm{M}$ 1 EACH, -45 DEGREES TO 160 DEGREES C. SIZE: 32"W $\times 37 " F / R \times 79 " H$ EST WT : 500 LBS MFGR YR: 1988
LINE ITEM NUMBER: 0087

\section{NO OF}

COND UNITS UNIT

ACOUISITION PRICE

$700001 \mathrm{EA}$

\section{COST/UNIT:}

TOTAL COST:

\section{$\$ 3.600 .00$}

$\$ 3,600.00$

0.600 .00

10

CONTACT(S)

FOR FURTHER INFORMATION:

ELOY N. GIRO

ALBUQUEROUE

(505; $844-2746$

NM 87185

TO FREEZE EOUIPMENT:

ELOY N. GIRON

ALBUOUERQUE

(505) $844-2746$

LOCATION OF EQUIPMENT:

SAMOIA MATIOMAL LABORATORIES

PROPERTY REAPPLICATION DIV. 7617

ALBUDUEROUE

N⿴囗十 87123

$800001 \mathrm{EA}$

$$
\begin{aligned}
& \text { COST/UNIT: } \\
& \text { TOTAL COST: }
\end{aligned}
$$

$\$ 2,859.00$

$\$ 2,859.00$
FOR FURTHER INFORMATION:

BARBARA HOLMES, REP.

KANSAS CITY

(816) $997-2976$

MO $64141-6159$

TO FREEZE EOUIPMENT:

BARBARA HOLMES, REP.

KANSAS CITY

REPORT NUMBER: $896404-4124$

LINE ITEM MUMBER: D011
(816) $997-2976$

LOCATION OF EOUIPMENT

ALLIEDSIGNAL, INC

2000 EAST 95TH STREET

KANSAS CITY 
EFFECTIVE DATE: $07 / 01 / 96$ EXPIRATION DATE: $07 / 31 / 94$

CODE EXCESS PROPERTY DESCRIPTION

REFERENCE NUMBER : 475

6636 TEMPERATURE CHAMBER 65756, MANUF: BEMCO MOOEL FB8-2-2 S/N N/A 1 EACH SIZE: $60^{\prime \prime W} \times 36^{\prime \prime F} / R \times 72^{\prime \prime H}$ ESI WT: 700 LBS MFGR YR: 1957

ELIGIBLE EQUIPMENT

NO OF

ACQUISITION PRICE

$500001 \mathrm{EA}$

COST/UNIT:

TOTAL COST:
LIME ITEM NUMBER: 0006

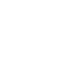


EFFECTIVE DATE: $07 / 01 / 94$ EXPIRATION DATE: $07 / 31 / 94$

FSC

COOE EXCESS PROPERTY DESCRIPTION

REFERENCE NUMBER : 477

6640 BATH, CIRCULATOR HITH TEMP CONTROL SYSIEM FOR HEATING AMD COOLING.

120VAC, $60 \mathrm{HZ}$. LED READOUT BAD. FORMA SCl, 2067, 20686179.

LINE ITEM NUMBER: 0088

REFERENCE NUMBER : 478

6640 CENTRI FUGE-REFRIGERATED $(-20$ rO $+40 \mathrm{~F}), 115$ VOLI, 8 AMP, 1 PH, $3 / 4$ HP MOTOR INTERNATIONAL EOUIPMENT PR-2 ACO YR: 57

600001 EA

COST/UNIT:

TOTAL COST:
ACQUISITION PRICE

$51,384.00$

$\$ 1,384.00$
TOTAL COS

(5)

TO FREEZE EOUIPMENT:

ELOY M. GIRON

ALBUOUEROUE

LOCATION OF EOUIPMENT:

SAMDIA MATIOMAL LABORATORIES

PROPERTY REAPPLICATION DIV. 7617

ALBUOUEROUE

in 87123

REPORT NUMBER: $899101-9413$

LINE ITEM NUMBER: 0023

$\$ 2.195 .00$

$\$ 2,195.00$

FOR FURTHER INFORMATION:

MONTE CLEVEMGER

BERKELEY
$(510) 486-6242$

CA 94720

TO FREEZE EQUIPMENT:

MOWTE CLEVENGER

BERKELEY

CA 94720

(510) $486-6242$

LOCATIOW OF EQUIPMENT:

901 HAREHOUSE

1450 64TH STREET

EMERYVILLE

CA 94608 
EFFECTIVE DATE: $07 / 01 / 94$ EXPIRATION DATE: $07 / 31 / 94$

COOE EXCESS PROPERTY DESCRIPTION

REFERENCE NUMBER : 479

6640 CRYOSTAT DEWAR MODEL D 100910, MFR. CRYENCO, SER\# 7001

REPORT NUMBER: $899110-0125$

LINE ITEM NUMBER: 0019

$$
\text { No of }
$$

COND UNITS UNIT

ACOUISITION PRICE

$4 \quad 00001 \mathrm{EA}$

COST/UNIT:
TOTAL COST:
$\$ 48,610.00$ $48,610.00$

LINE ITEM MUMBER: 0029
REPORT MUMBER: $899101-9413$
REFERENCE NUMBER : 480

6640 FURNACE-DIFFUSION THERMCO 3000 , ACO YR: 84

\section{$500001 E A$ \\ COST/UNIT: \\ S TOTAL COST:}


EFFECTIVE DATE: 07/01/94 EXPIRATION DATE: 07/31/94

FSC

\section{CODE EXCESS PROPERTY DESCRIPTION}

$: 481$

6640 INSTRLMENT CABINET MFG. ELECTRONIC RACK PROOUCTS, MODEL 10520449, DUAL BAY, 36" X 48 " WIDE, S/M 8748, P/M P20878.

REPORT MUABER: $890207-4116$

LIME ITEM MUABER: 0384
No of

COND UNITS Uwit

800001 EA
ACOUISITIOA PRICE

cost/unit: TOTAL COST:

\section{$\$ 1.349 .00$
$\$ 1,349.00$}

CONTACT(S)

FOR FURTHER INFORMATIOA:

Savor moOn

IDAHO FALLS
(208) $526-2611$

10 $83415-4100$

TO FREEZE EOUIPMENT:

SMDOr MOOW

ICAHO FALLS
(208) $526-2611$

$1083415-4100$

LOCATIOM OF ECUIPAENT:

IDAHO MATIOMLL EMGIMEERIMG LAB.

CF-674 HAREHOUSE

SCOVILLE

10 $83415-4100$

REFERENCE MUMBER : 482

6640 LABORATORY EQUIPMENT SPECTROMETER, CONSISIING: MANUF: MICOLET,

ML:7199A, SN:79132, DOE TAG* 110249 PLOTTER,

$400001 \mathrm{EA}$

COSTRUIT:

TOTAL COST:

$\$ 118,163.15$

$\$ 118,163.15$

MANUF: ZETA, MOL: 160 , SW: 2071. DOE TAGH 11024901 TELETYPE, WLWF :

MDL: 1180, SN: 80359, DOE TAG\# 110

24903 IT USES LARGE RIGID DISK "PLATTERS" RATHER THAN COMPACT FLOPPY

DISKS AS REMOVABLE STORAGE MEDIA ANO IT CAMMOT COMMUICATE WITH PC'S OR

CAN IT BE UPGRADED TO PC CONTROL OF THE SPECTROMETER. COST OF EOUIPMEMT

IS REFLECTED IN LINE ITEM 1.

FOR FURTHER INFORMATIOA:

TIMA JARAMILLO

(303) $384-6156$

co $80401-3393$

TO FREEZE EOUIPMENT :

TIMA JARAMILLO

COLDEM

CO 8040:-3393

LOCATION OF EQUIPMENT

1607 COLE BLVD

GOLDEM

co $80401-3393$ 
EFFECTIVE DATE: $07 / 01 / 94$ EXPIRATION DATE: 07/31/94

FSC
COOE EXCESS PROPERTY DESCRIPTION

\section{REFERENCE NUMBER : 483}

6640 LAMP-ADJUSTABLE HOUSING WITH PONER SUPPLY IIYOOA, ACO YR: 75 wo of COND UnITs Ualt ACOUISIIIOU PRICE

$4 \quad 00001$ EA COSI/UNIT: TOTAL COST:

$\$ 1,005.00$ $\$ 1,005.00$

LINE ITEM MUMBER: 0014
REPORT MUMBER: $899101-9413$
REFERENCE NUMBER : 486

6640 MANIPULATOR, MASTER SLAVE MFG. SARGENT, MODEL B-LD, S/M 5922, INCLLDES BALANCE. COUNTER FOR L/1 47. HANGER ARM. MANIPULATOR FOR L/1 47. HOLDER, HANGER ARM FOR L/1 47 (2) MANIPULATOR MOOEL B-LD, S/N 5923.

REPORT MUMBER: $890207-4116$
LINE ITEM MUMBER: 0376 $\cos T / \lim _{11}:$
TOTAL $\cos 1:$

00001 EA
$\$ 13,272.00$ $\$ 13.272 .00$

\section{FOR FURTHER INFORMATION:}

SANOY MOOM

(208) $526-2611$

$1083415-4100$

TO FREEZE EOUIPMEWT:

SAMOY MOON

IOAHO FALLS

(208) $526-2611$

$1083415-4100$

LOCATION OF EQUIPNEWT:

IDAHO MATIOWAL EMGIMEERIMG LAB.

CF- 674 MAREHOUSE

SCOVILLE

$1083415-4100$ 
EFFECTIVE DATE: 07/01/94 EXPIRATION DATE: 07/31/94

FSC
COOE EXCESS PROPERTY DESCRIPTION

R6.0 ROBOT GCA/CIMROC CONTROLLER SN-12650137

REPORT MUMBER: $894609-9090$

INE ITEM MUMBER: 1
REFEREMCE MUMBER : 485

no of

COND UnITS unI

ACQUISITIOU PRICE

$500001 \mathrm{EA}$

COST/UNIT:

TOTAL COST:

$\$ 24,000.00$ $\$ 24,000.00$

DAVID MITCHELL

(803) $725-9540$

SC 29802

TO FREEZE EQUIPHENT:

DAVID MITCHELL

AIKE

(803) $725-9540$

LOCATION OF EOUIPMENT:

LESTIMGHOUSE

SAVAMMAH RIVER SITE

AIKEN

SC 29802

$1,605.00$

REFEREMCE NUMBER : 486

6655 PROGPAMER FOR TURMACES EQUIPMENT HAS HEATING AND COOLING CONTRLS AND TIME 12OVAC, $60 \mathrm{HZ}$. THERMCO, IPCEHCP, 773950.

O0001 EA

COST/UNIT:

TOTAL COST:

$\$ 1,605.00$

FOR FURTHER INFORMATION:

ELOY N. GIRON

ALBUOUEROUE

(505) $844-2746$

m 87185

TO FREEZE EQUIPMEMT

ELOY N. GIROM

ALBU

(505) $844-2746$

LOCATION OF EOUIPMENT

SAMOIA MATIOAA

PROPERTY REAPPLICATION DIV. 7617

ALBUOUEROUE

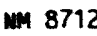


EFFECIIVE DATE: 07/01/94 EXPIRATION DATE: $07 / 31 / 94$

\section{FSC}

COOE EXCESS PROPERTY DESCRIPTION

REFERENCE NUMBER : 487

6650 MICROSCOPE, 200M HAS ONE SET OF EYEPIECES, 200M IS 1.5 TO 4 MICROSCOPE, 20OM HAS ONE SET OF EYEPIECES, 200M IS 1.5 TO 4 UST MAGNIFICATION OF EYEPI ECES IS 12.2 . THE EYEPIECES ARE ADJUST
DISTANCE BETWEEN E YES BASE IS $1-1 / 2 "$. OLYMPUS, SZ, 304463 .

REPORT NUMBER: $898355-4088$

LINE ITEM NUMBER: 0056

REFERENCE NUMBER : 488

6660 RECORDER HIMD SPEED AND DIRECTION 0-100 MPH MFR: QUALIMETRICS INC MOOEL: W224R S/N: 1220
No of

COND UAITS UNIT

$600001 \mathrm{EA}$

COST/UNIT:

TOTAL COST:

ACOISITION PRICE

\section{$\$ 1,300.00$
$\$ 1,300.00$}

$\$ 1,300.00$

$\operatorname{rog}$

\section{TO FREEZE EOUIPMENT:}

ELOY N. GIROW

ALBUOUEROUE

(505) $844-2746$

MM 87185

LOCATION OF EOUIPMENT:

LOCATION OF EOUIPMENT: SANDIA MATIONAL LABORATORIES PROPERTY REAPPLICATION DIVISION
ALBUOUEROUE

COST/UNIT: TOTAL COST:
$\$ 1,074.06$

$\$ 1.074 .06$
FOR FURTHER INFORMATION:

CYNDI DELONG

LAS VEGAS

(702) $295-6753$

NV $89193-852$

TO FREEZE EOUIPMENT:

CYMDI DELONG

LAS VEGAS

(702) $295-6753$

NV 89193-8521

LOCATION OF EQUIPMENT:

REYWOLDS ELECTRICAL \& ENGINEERING CO MEVADA TEST SITE

MERCURY

WV 89023 
EFFECTIVE DATE: $07 / 01 / 94$ EXPIRATION DATE: $07 / 31 / 94$

\section{FSC \\ COOE EXCESS PROPERTY DESCRIPTION}

REFERENCE NUMBER : 489

6665 CHROMATOGRAPH-GAS VARIAN MOOULINE SERIES 2700 ACQ YR: 75

ELIGIBLE EQUIPMENT

NO OF

COND UNITS UNIT

$500001 \mathrm{EA}$

\section{ACQUISITION PRICE}

COST/UNIT: TOTAL COST:

LINE ITEM NUMBER: 0006
REPORT NUMBER: $899101-9413$
REFERENCE NUMBER : 490

6665 DETECTOR-OXYGEN, MFR. ORBISHPERE, MOOEL 2611, ACO. YR. 86, PRO P. NO. 4361167
$600001 \mathrm{EA}$
$\$ 6,800.00$ $\$ 6,800.00$

FOR FURTHER INFORMATION:

JOANNE BREZNIK

LIVERMORE
(510) $424-4101$

CA 94550

TO FREEZE EOUIPMENT:

JOANNE BREZNIK

LIVERMORE

REPORT NUMBER: $899102-4163$

LINE ITEM NUMBER: 0038

(510) $424-4101$

LOCATION OF EQUIPMENT:

LAWRENCE LIVERMORE NATIONAL LAB

LAWRENCE LIVERMORE NATIONAL LAB
EXCESS PROPERTY SECTION, L-696

EXCESS PROPERTY SECTION, L-696
LIVERMORE 
EFFECTIVE DATE: $07 / 01 / 94$ EXPIRATION DATE: $07 / 31 / 94$

COOE EXCESS PROPERTY DESCRIPTION

REFERENCE NUMBER : 491

6665 PHOTODIODE-PARTICLE SCINTILLATION DETECT., MFR. FABRICATED, AC Q. YR.

92, E39616
LINE ITEM NUMBER: 0039
No of

COND UNITS UNIT

$400001 \mathrm{EA}$

COST/UNIT:

TOTAL COST:

REPORI NUMBER: $899102-4163$

REFERENCE NUMBER : 492

6670 BALANCE, ANALYTICAL BALANCE MEASURE UP TO 1000MG. HAS CONTROL DIALS.

11 "HX10"W. 120 VAC, $00 \mathrm{HZ}$ METTLER, B5.
$00001 \mathrm{EA}$ COST/UNIT:

TOTAL COST:
$\$ 1,450.00$

$\$ 1,450.00$

FOR FURTHER INFORMATION:

ELOY N. GIRON

ALBUDUERQUE

NM 87185

CA 94550

R BREZNI

510) $424-410$

FREEZE EQUIPMENT

OANNE BREZNIK

LIVERMORE

LOCATION OF EQUIPMENT:

LAURENCE LIVERMORE NATIONAL LAB

EXCESS PROPERTY SECTION, L-696

LIVERMORE

TO FREEZE EQUI PMENT

ELOY N. GIRON

ALBUOUEROUE

(505) $844-2746$

NM 87185

OCATION OF EOUIPMENT:

SANDIA NATIONAL LABORATORIES

PROPERTY REAPPLICATION DIV. 7617

ALBUDUEROUE 
EFFECTIVE DRTE: $07 / 01 / 94$ EXPIRATION DATE: $07 / 31 / 94$

FSC

CODE EXCESS PROPERTY DESCRIPTION

REFERENCE NUMBER : 493

6675 BERNOULLI BOX 10+10 MFR: IOMEGA CORP., MODEL A210H, S/N ATHMS1658, ACQ. 500001 EA DATE: 5/86, APPROX. WT: 30 LBS., $133^{\prime} \mathrm{H} \times 16^{\prime} \mathrm{L} \times 6^{\prime} \mathrm{H}$.

REPORT NUMBER: $899105-4082$

LINE ITEM NUMBER: 0015

REFERENCE NUMBER : 494

6675 BERNOULLI BOX 20+20, DISC DRIVE MFR: IOMEGA CORP., MOOEL A220H, S/N O5RXV, ACO. DATE: 8/87, APP ROX. WT: $30 \mathrm{LBS} ., 13 \mathrm{~W} \times 16^{\prime} \mathrm{L} \times 6^{\prime} \mathrm{H}$.

600001 EA

REPORT NUMBER: $899105-4082$

LINE ITEM NUMBER: 0016
COST/UNIT: TOTAL COST:

$\$ 1.765 .00$ $\$ 1,765.00$

NO OF

COND UNITS UNIT

ACQUISITION PRICE

$\$ 2,650.00$ $\$ 2,650.00$

COST/UNIT: TOTAL COST:

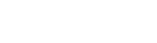

To

LYMU E. SHACKELFOTIO

TYNN E. SHACKEL FOO

ERMORE

(510) $294-3067$

CA $94550-0969$

TO FREEZE EQUIPMENT:

LYNM E. SHACKELFOOT

LIVERMORE

(510) 294-3067

CA $94550-0969$

LOCATION OF EQUIPMENT:

SARDIA NATIONAL LABORATORIES

PROPERTY REAPPLICATION DEPT. 8532-1

LIVERMORE

CA $94550-0969$

FOR FURTHER INFORMATION:

LYNA E. SHACKELFOOT

LYNA E. SHAC

LIVERMORE
(510) $294-3067$

CA $94550-0969$

TO FREEZE EQUIPMENT :

LYMN E. SHACKELFOOT

(510) $294-3067$

CA $94550-0969$

LOCATION OF EQUIPMENT:

SANDIA MATIONAL LABORATORIES

PROPERTY REAPPLICATION DEPT. $8532-1$

LIVERMORE

CA $94550-0969$ 
EFFECTIVE DATE: $07 / 01 / 94$ EXPIRATION DATE: $07 / 31 / 94$

TSC

COOE EXCESS PROPERTY DESCRIPTION

COND UNITS UNIT

ACQUISITION PRICE

REFERENCE NUMBER : 495

REFERENCE NUMBER : 495
6675 BERNOULLI BOX MFR: IOMEGA CORP., MODEL NO. B120X, S/N 0439160009, ACQ. 400001 EA DATE: 6/89, APPROX. WT: 20 LBS., $14 . \mathrm{W} \times 15 . \mathrm{L} \times 3^{\prime} \mathrm{H}$

COST/UNIT: TOTAL COST:

REPORT NUMBER: $899105-4082$

LINE ITEM NUMBER: 0013

REFERENCE NUMBER : 496

6675 BERNOULI DATE: 6/89, APPROX. WT: 20 LBS., $14 . \mathrm{W} \times 15^{\prime} \mathrm{L} \times 3^{\prime} \mathrm{H}$

TOIAL COST:

REPORT NUMBER: $899105-4082$

LINE ITEM NUMBER: 0014
$\$ 1,900.00$

$\$ 1,900.00$

$\$ 1,900.00$

$\$ 1,900.00$

LYNN E. SHACKEL FOOT
LIVERMORE
$(510) 294-3067$

TO FREEZE EQUIPMENT:

LYNN E. SHACKELFOOT

LIVERMORE

(510) $294-3067$

LOCATION OF EQUIPMENT:

SANDIA NATIONAL LABORATORIES

PROPERTY REAPPLICATION DEPT. 8532-1

LIVERMORE

CA $94550-0969$

FOR FURTHER INFORMATION:

LYNN E. SHACKELFOOT

LIVERMORE

(510) $294-3067$

TO FREEZE EQUIPMENT:

LYNM E. SHACKELFOOT

LIVERMORE

CA $94550-0969$

(510) $294-3067$

LOCATION OF EQUIPMENT:

SANDIA NATIONAL LABORATORIES

PROPERTY REAPPLICATION DEPT. 8532-1

LIVERMORE

CA $94550-0969$ 


\section{EFFECTIVE DATE: 07/01/94 EXPIRATION DATE: $07 / 31 / 94$}

FSC

\section{COOE EXCESS PROPERTY DESCRIPIION}

\section{REFERENCE MUMBER : 497}

6675 DUAL DISK DRIVE 3-1/2" EXTERNAL MFR: MICROMAC TECH. INC., MOOEL NO T1015-AT, S/N 8122DL75, ACQ. DATE: 9/91, APPROX WT: 10 LBS., 12'W $12^{\prime} \mathrm{L} \times \mathrm{B}^{\prime} \mathrm{H}$
REPORT NUMBER: $899105-4082$

LINE ITEM NUMBER: 0017
REFERENCE NUMBER : 498

6675 PLOTTER GRAPHICS 6 PEN 81/2X11-11X17 IN CHART MFR: HELLETT PACKARD MOOEL: 7475A S/N: 20624

REPORT HUMBER: $899305-4053$

LINE ITEM MUMBER: 0001

\section{No of}

COND UNITS UNIT

\section{ACQUISITION PRICE}

$400001 \mathrm{EA}$

COST/UNIT: TOTAL COST:

$\$ 1,900.00$

$\$ 1,900.00$

LYNN E. SHACKELFOOT
LIVERMORE

(510) $294-3067$

CA $94550-0969$

TO FREEZE EQUIPMENT

LYNN E. SHACKELFOOT

LIVERMORE

(510) $294-3067$

PROPERTY REAPPLICATION DEPT 8532-1

LIVERMORE

CA $94550-0969$
LOCATION OF EQUIPMENT

SAMDIA NATIONAL LABORATORIES
$\$ 1.345 .83$ $\$ 1,345.83$
FOR FURTHER INFORMATION:

CYNDI DELONG

LAS VEGAS

(702) $295-6753$

NV 89193-8521

TO FREEZE EQUIPMENT:

CYNDI DELONG

LAS VEGAS

NV 89193-8521

LOCATION OF EQUIPMENT:

REYNOLDS ELECTRICAL \& ENGINEERING CD NEVADA TEST SITE

MERCURY

NV 89023 
EFFECTIVE DATE: $07 / 01 / 94$ EXPIRATION DATE: $07 / 31 / 94$

\section{NO OF}

COOE EXCESS PROPERTY DESCRIPTION

REFERENCE NUMBER : 499

6675 PLOTTER MFR: HEWLEII PACKARD, MODEL NO. $7221 \mathrm{C}, \mathrm{S} / \mathrm{N} 2036 \mathrm{~A} 00360$, ACO PLOTTER MFR: HEWLEII PACKARD, MODEL NO.
DATE : 5/91, APPROX. WT: 40 LBS., $20^{\prime} \mathrm{W} \times 19^{\prime} \mathrm{L} \times 81 \mathrm{H}$.

REPORT NUMBER: $899105-4082$

LINE ITEM NUMBER: 0001

REFERENCE NUMBER : 500

6675 PLOTTER MFR HEWLETT PACKARD, MODEL NO. 7475A, S/N 2541A59136, ACO. DATE : 8/86, APPROX. WT: 25 LBS., $22, \mathrm{~W} \times 15^{\prime} \mathrm{L} \times 5 \cdot \mathrm{H}$

REPORT NUMBER: $899105-4082$

LINE ITEM NUMBER: 0002
COND UNITS UNIT

ACQUISITION PRICE

COST/UNIT:

IOTAL COST:

$\$ 4.254 .00$

$\$ 4,254.00$

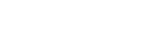

CONTACT(S)

FOR FURTHER INFORMATION:

LYNN E. SHACKELFOOT

(510) 294-3067

CA $94550-0969$

TO FREEZE EQUIPMENT:

IYNN E. SHACKELFOOT

LIVERMORE

(510) $294-3067$

CA $94550-0969$

LOCATION OF EQUIPMENT:

SAMDIA MAIIONAL LABORATORIES

PROPERTY REAPPLICATION DEPT. $8532-1$

LIVERMORE

CA $94550-0969$

400001 EA

\section{COST/UNIT: \\ TOTAL COST:}

$\$ 1,400.00$

$\$ 1,400.00$
FOR FURTHER INFORMATION:

LYNN E. SHACKELFOOT

LIVERMORE

(510) 294-3067

CA $94550-0969$

TO FREEZE EQUIPMENT :

LYNN E. SHACKELFOOT

LIVERMORE

(510) $294-3067$

CA $94550-0969$

LOCATION OF EQUIPMENT :

SANDIA NATIONAL LABORATORIES

PROPERTY REAPPLICATION DEPT. $8532-1$

LIVERMORE

CA $94550-0969$ 
EFFECTIVE DATE: 07/0i/94 EXPIRATION DATE: $07 / 31 / 94$

FSC
COOE EXCESS PROPERTY DESCRIPTION

REFERENCE NUMBER : 501

6675 PLOTTER MFR: HEWLETT PACKARD, MOOEL NO. 7475A, S/N 2641V19730, ACO. DATE : 8/87, APPROX. WT: 25 LBS., 22 'W $\times 15, \mathrm{~L} \times 5 \cdot H$.

REPORT NUMBER : $899105-4082$

LINE ITEM NUMBER: 0003

REFERENCE NUMBER : 502

6675 PLOTTER MFR: HEWLETT PACKARD, MOOEL NO. 7475A, S/N 2325A38222, ACQ. DATE : 4/86, APPROX. WT: 25 LBS., 22 'W $\times 15^{\prime} \mathrm{L} \times 5^{\prime} \mathrm{H}$.

REPORT NUMBER: $899105-4082$

LINE ITEM NUMBER: 0004
NO OF

COND UNITS UNIT

ACQUISITION PRICE

$400001 \mathrm{EA}$

COST/UNIT: TOTAL COST:

$\$ 1,400.00$ $\$ 1,400.00$

TOTAL COST:

CONTACT(S)

FOR FURTHER INFORMATION:

LYNN E. SHACKELFOOT

LIVERMORE

(510) $294-3067$

CA $94550-0969$

TO FREEZE EQUIPMENT:

LYNN E. SHACKEL FOOT

LIVERMORE

(510) $294-3067$

CA $94550-0969$

LOCATION OF EOUIPMENT:

SAMD IA NATIONAL LABORATORIES

PROPERTY REAPPLICATION DEPT. 8532-1

LIVERMORE

CA $94550-0969$
COST/UNIT: TOTAL COST:
$\$ 1,200.00$ $\$ 1,200.00$
FOR FURTHER INFORMATION:

LYNN E. SHACKEL FOOT

LIVERMORE
(510) $294-3067$

CA $94550-0969$

TO FREEZE EQUIPMENT:

IYNN E. SHACKELFOOT

LIVERMORE

(510) $294-3067$

CA $94550-0969$

LOCATION OF EOUIPMENT

SANDIA NATIONAL LABORATORIES

SANDIA NATIONAL LABORATORIES

LIVERMORE

CA $94550-0969$ 
EFFECTIVE DATE: $07 / 01 / 94$ EXPIRATION DATE: $07 / 31 / 94$

FSC

\section{COOE EXCESS PROPERTY DESCRIPTION}

REFERENCE NUMBER : 503

6675 PLOTTER MFR: HEWLETT PACKARD, MOOEL NO. 7475A, S/N 2641V71662, ACQ. DATE : 6/88, APPROX. WT: 20 LBS., $22 ' \mathrm{~W} \times 14^{\prime} \mathrm{L} \times 5^{\prime} \mathrm{H}$.

REPORT NUMBER: $890105-4082$

LINE ITEM NUMBER: 0005

REFERENCE NUMBER : 504

6675 PLOTTER MFR: VERSATEC, MODEL NO. V80-811, S/N B621215, ACQ. DATE:

8/85. APPROX. WT: 80 LBS., $23 . \mathrm{W} \times 24 . \mathrm{L} \times 10^{\prime} \mathrm{H}$

COST/UNIT:

TOTAL COST:

$\$ 3,000.00$

FOR FURTHER INFORMATION:

LYMN E. SHACKELFOOT

LIVERMORE

CA $94550-0969$

(510) $294-3067$

TO FREEZE EQUIPMENT:

LYMU E. SHACKELFOOT

LIVERMORE

(510) $294-3067$

CA $94550-0969$

LOCATION OF EQUIPMENT

SANDIA MATIONAL LABORATORIES

PROPERTY REAPPLICATION DEPT. 8532-1

LIVERMORE

CA $94550-0969$ 
EFFECTIVE DATE: $07 / 01 / 94$ EXPIRATION DATE: $07 / 31 / 94$

\section{FSC}

COOE EXCESS PROPERTY DESCRIPTION

REFERENCE NUMBER : 505

6675 PLOTTER MFR: VERSATEC, MOOEL NO. V80-311, S/N 0596300, ACQ. DATE: 10/83. APPROX. WT: 100 LBS., $23 . \mathrm{W} \times 24 . \mathrm{L} \times 10^{\circ} \mathrm{H}$

REPORT NUMBER: $899105-4082$

LINE ITEM NUMBER: 0007

\section{REFERENCE NUMBER : 506}

6675 PLOTTER MFR

HEWLETT PACKARD, MCDEL NO. 7470A, S/N 2308A80128, ACO. DATE: $11 / 84$,

APPROX. HT: 20 LBS., 17'W $\times 131 \mathrm{~L} \times 5 \cdot \mathrm{H}$.

REPORT NUMBER: $899105-4082$

LINE ITEM NLMBER: 0008
NO OF
COND UNITS UNIT

$500001 \mathrm{EA}$

\section{COST/UNIT:} TOTAL COST:

COUISITION PRICE

$\$ 10,828.00$ $\$ 10,828.00$
CONTACT(S)

FOR FURTHER INFORMATION:

IYHM E. SHACKELFOOT

IVERMORE

(510) $294-3067$

CA $94550-0969$

TO FREEZE EQUIPMEMT:

LYNN E. SHACKELFOOT

LIVERMORE

(510) $294-3067$

CA $94550-0969$

LOCATION OF EQUIPMENT:

SANDIA NATIONAL LABORATORIES

PROPERTY REAPPLICATION DEPT. 8532-1

LIVERMORE

CA $94550-0969$
400001 EA COST/UNIT:
TOTAL COST:
$\$ 1.500 .00$ $\$ 1.500 .00$
FOR FURTHER INFORMATION:

LYMN E. SHACKELFOOI

IVERMORE

(510) $294-3067$

CA $94550-0969$

TO FREEZE EQUIPMENT:

LYWN E. SHACKELFOOT

LIVERMORE

(510) 294-3067

CA $94550-0969$

LOCATION OF EQUIPMENT:

SAMDIA MATIONAL LABORATORIES

PROPERTY REAPPLICATION DEPT. 8532-1

LIVERMORE CA 94550-0969 
EFFECIIVE DATE: $07 / 01 / 94$ EXPIRATION DATE: $07 / 31 / 94$

FSC

\section{COOE EXCESS PROPERTY DESCRIPTION}

REFERENCE MUMBER : 507

6675 PLOTTER MFR

HEWLETT PACKARD, MODEL NO. 7475A, S/N 2325A45401, ACO. DATE : 4/84,

APPROX. WT: 30 LBS., $22 . \mathrm{W} \times 15^{\circ} \mathrm{L} \times 5^{\circ} \mathrm{H}$.

REPORT NUMBER: $899105-4082$

LINE ITEM NUMBER: 0009

\footnotetext{
REFERENCE NUMBER : 508

6675 PLOTIER MFR

HEULET PACKARD, MODEL NO. 7475A, S/N 2451A64617, ACQ. DATE: 6/87,

APPROX WT: 30 LBS. $22 . \mathrm{W} \times 15 . \mathrm{L} \times 5 . \mathrm{H}$
}

REPORT NUMBER: $899105-4082$

LINE ITEM NUMBER: 0010
$400001 E A$

COST/UNIT:
TOTAL COST:

No of

COND UNITS UNIT

ACQUISITION PRICE

$\$ 11,400.00$

$400001 \mathrm{EA}$

COST/UNIT:

$\$ 11,400.00$

$\$ 1,400.00$
$\$ 1,400.00$

FOR FURTHER INFORMATION:
LYNN E. SHACKELFOOT
LIVERMORE
(510) $294-3067$

TO FREEZE EQUIPMENT:

LYNM E. SHACKELFOOT

LIVERMORE

CA $94550-0969$

(510) $294-3067$

LOCATION OF EQUIPMENT:

SAMDIA MATIOMAL LABOPATORIES

PROPERTY REAPPLICATION DEPT - 8532-1

PRDERTY REAPPLICATION DEPT. 8532-1 
DOE USED EMERGY RELATED LABORATORY EOUIPMENT GRANT PROGRAM

EFFECTIVE DATE: $07 / 01 / 94$ EXPIRATION DATE: $07 / 31 / 94$

COOE EXCESS PROPERTY DESCRIPIION

REFERENCE MLMBER : 509

HEWLETT PACKARD, MOOEL NO. 9872B, S/N 2032A03201, ACO. DATE: 4/81, APPROX. WT: 40 LBS., $20 . \mathrm{W} \times 18^{\prime} \mathrm{L} \times 8 . \mathrm{H}$.

REPORT NUMBER: $899105-4082$

LINE ITEM NUMBER: 0011

REFERENCE NUMBER : 510
6675 PLOTTER MFR

HEWLETT PACKARD, MOOEL NO. 7550A, S/N 2725A95691, ACO. DATE: 12/88,

APPROX. WT: $40 \mathrm{LBS} ., 26^{\prime} \mathrm{W} \times 17^{\circ} \mathrm{L} \times 9^{\prime} \mathrm{H}$.

COST/UNIT:

TOTAL COST:

$\$ 3.900 .00$

$\$ 3,900.00$
FOR FURTHER INFORMATION:

LYUM E. SHACKELFOOT

(510) $294-3067$

CA $94550-0969$

TO FREEZE EOUIPMENT:

LYUM E. SHACKELFOOT

LIVERICRE

(510) $294-3067$

CA $94550-0969$

LOCATION OF EQUIPRENT:

SAMDIA MATIOUAL LABORATORIES

PROPERTY PEAPPLICATION DEPT

LIVERMORE CA $94550-0969$ 
EFFECTIVE DATE: 07/C1/94 EXPIRATION DATE: 07/31/94

COOE EXCESS PROPERTY DESCRIPTION

REFERENCE NUMBER : 511

6675 SURVEY SYSTEM GLOBAL POSITIONING MFR: ISTAC CORP MOOEL: 2002 S/N: 19

REPORT NUMBER: $899305-4069$

LINE ITEM MUMBER: 0001

REFERENCE NUMBER : 512

6675 SURVEY SYSTEM GLOBAL POSITIONING MFR: ISTAC CORP MOOEL: 2002 SURVEY SYSTE

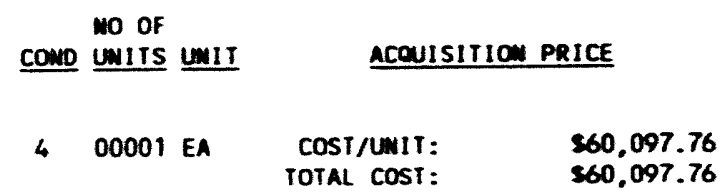
TOTAL COST: $60,097.76$

Cominact(s)

FOR FURTHER IMFORMAIIOW:

CrMDI DELOUE

LAS VEGAS

(702) $295-6753$

wv 89193-8521

TO FREEZE EOUIPMENT:

CYMCI DELONG

LAS VEGAS

(702) $295-6753$

MV 89193-8521

LOCATION OF ECUIPMENT:

REYUOD ELECTRICAL \& EMGIMEERIMG CO MEVADA TEST SITE

nercurr

my 89023

$400001 E A$

Cost/UNIT: TOTAL COST:

$\$ 60,097.76$

FOR FURTHER IMFORMATION:

CYMOI DELOWG

(702) $295-6753$

wV $89 ! 93-8521$

TO FREEZE EQUIPMEMT:

CYMDI DELONG

LAS VeGaS

(702) $295-6753$

wV 89193-8521

LOCATIOW OF EOUIPMENT:

REYHOLOS ELECTRICAL \& EMGIMEERIMG CO MEVAOA TEST SITE

MERCURY

wv 89023 
EFFECTIVE DATE: 07/01/94 EXPIRATION DATE: $07 / 31 / 94$

FSC

CODE EXCESS PROPERTY DESCRIPTION

REFERENCE MUMBER : 513

6675 SURVEY SYSTEM GLOBAL POSITIONING MFR: ISTAC CORP MODEL: 2002 S/N: 18
LIME ITEM MUMBER: 0003
No of

COWD WNITS UNIT

ACOUISITION PRICE

400001 EA

COST/UNIT: TOTAL COST:

$\$ 60.182 .56$ $\$ 60,182.56$

REPORT MUMBER: $899305-4069$

REFERENCE NUMBER : 514

ONE LOT CONSISTING OF COMPUTER,DUPLICATOR, FLUOROMETER, LASER COMPUTER,

SYSTEM ORTHO MOOEL NO 12150 AML NO P11795 ACO DATE 6/80. LOC: D-6

$600001 \mathrm{EA}$

(N.EAST) NOMINAL LOADING CHARGES MAY BE INCURRED BY RECIPIENT

REPORT MUMBER: $895140-2282$

LINE ITEM MUMBER: 0001
COST/UNIT:

TOTAL COST:
$\$ 26,515.00$
$\$ 26,515.00$
FOR FURTHER IMFORMATION:

IIM CAROTHERS

LEMONT

(708) $252 \cdot 2786$

TO FREEZE ECUIPMEMT :

IIM CAROTHERS

LEMON

(708) $252-2786$

IL $60439-4812$

LOCRTION OF ECUIPMEWT

ARGOWWE MATIOMAL LABORATORY

9700 SOUTH Cass avenUe

LEMONT

IL $60439-4812$ 
EFFECTIVE DATE: 07/01/94 EXPIRAIION DATE: 07/31/96

FSC

\section{COOE EXCESS PROPERTY DESCRIPIION}

REFERENCE NUMBER : 515

O680 PERSONAL GAS MONITORS VENDOR: GASTEC CORP MOL: $N D-2 B$

SN: 2388,3232

TAG $\# 57958,57959$

REPORT NUMBER: $893604-4137$

REFERENCE MUMBER : 516

6680 RECORDER VENDOR: LEEDS \& NORTHRUP

MOL: SPEEDOMAX

SN: 78-60331-2-1

TAG \# F6596

ACO: JAN 91

$$
\text { ELIGIBLE EOUIPMENT }
$$

No of

COMD UNITS UMIT

ACOUISITION PRICE

500002 EA

COSTIUNIT:
TOTAL COST:

$\$ 2,500.00$

$\$ 5,000.00$
LYOIA A KLEINHENZ
PIITSBURGH

MFODMATION:

(412) $892-5895$

PA 15236

TO FREEZE EQUIPMENT

LYDIA A KLEINHEMZ

PIITSBURGH

PA 15236

LINE ITEM MUMBER: 000

LOCATIOW OF EQUIPMENT:

US DEPARTMEMT OF EMERG

PITISBURGH ENERGY TECHMOLOGY CTR

BRUCETON

PA 15236
$500001 \mathrm{EA}$ COST/UNIT:

$\$ 2,475.00$
$\$ 2,475.00$
FOR FURTHER INFORMATIOM:

LYDIA A KLEINHENZ

PITTSBURGH

PA 15236

(412) $892-5895$

TO FREEZE EOUIPMENT

LYOIA A KLEINHENZ

PITTSBURGH

PA 15236

(412) 892-5895

LINE ITEM NUMBER: 0002
LOCATION OF EOUIPMENT:

US DEPARTMEMT OF ENERGY

PIITSBURGH EMERGY TECHMOLOGY CTR

BRUCETON

PA 15236 
EFFECTIVE DATE: 07/01/94 EXPIRATION DATE: 07/31/94

FSC

\section{COOE EXCESS PROPERTY DESCRIPTIO}

\section{REFERENCE MUMBER : 517}

6685 CHART RECORDER SINGLE PEN CHART RECORDER, TEMP RANGE O DEG TO 1200 DEG C. I2OVA C, $60 \mathrm{HZ}$. HONEYUELL.

REPORT NUMBER: $898355-4102$

LINE ITEM NUMBER: 0091

REFERENCE MUMBER : 518

6685 HUMIDITY AMD TEMPERATURE METER 57550, MANUF: HONEYWELL MODEL W809A S/N N/A

1 EACH, PORTABLE WITH BATTERY BACKUP. SIZE: $10 " \mathrm{H} \times 4.5 " \mathrm{~F} / \mathrm{R} \times 8.5 " \mathrm{H}$ EST $W T: 2$ LBS MFGR YR: 1982
NO OF

COND UNITS UNIT

ACQUISITION PRICE

$600001 \mathrm{EA}$

COST/UNIT: TOTAL COST:

$\$ 3,786.00$ $\$ 3.786 .00$

A

\section{CONTACT(S)}

FOR FURTHER INFORMATION:

ELOY N. GIRON

ALBUQUERQUE

(505) 844-2746

MM 87185

TO FREEZE EQUIPMENT:

ELOY N. GIRON

ALBUQUEROUE

(505) $844-2746$

NM 87185

LOCATION OF EQUIPMENT:

SANDIA NATIONAL LABORATORIES

SANDIA NAT IONAL LABORATORIES
PROPERTY REAPP: ICATION DIV. 7617

PROPERTY REAPP:ICATION DIV. 7617
ALBUQUERQUE

LINE ITEM NUMBER: 0024
400001 EA

COST/UNIT:

TOTAL COST:

$\$ 1,471.00$
$\$ 1,471.00$
FOR FURTHER INFORMATION

BARBARA HOLMES, REP.

KANSAS CITY

(816) $997-2976$

TO FREEZE EQUIPMENT:

BARBARA HOLMES, REP

KANSAS CITY

(816) $997-2976$

MO $64141-6159$

MO $64141-6159$

LOCATION OF EQUIPMENT:

ALLIEDSIGNAL, INC

ALLIEDSIGNAL, INC.
2000 EAST 95TH STREET

2000 EAST 95 
EFFECTIVE DATE: 07/01/94 EXPIRATION DATE: $07 / 31 / 94$

FSC

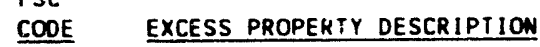

CODE EXCESS PROPERTY DESCRIPTION

REFERENCE NUMBER : 519

6685 HYGTOMETER MFR: PAMAMETRICS, MOOEL NO. 700-09H-04YBWD-08-10H, S/N 1546, 600001 EA ACO. DATE: $1 / 82$, APPROX. WT: 10 LBS., $81 \mathrm{H} \times 10^{\circ} \mathrm{L} \times 5$ 'H.

REPORT NUMBER: $899105-4081$

LINE ITEM NUMBER: 0043
No of

COND UNITS UNIT

ACQUISITION PRICE

COST/UNIT:

TOTAL COST:

$\$ 3,321.00$ $\$ 3,321.00$

LYWN E. SHACKELFOOT
LIVERMORE
(510) $294-3067$

CA $94550-0969$

TO FREEZE EQUIPMENT:

LYNN E. SHACKEL FOOT

LIVERMORE

(510) $294-3067$

LOCATION OF EQUIPMENT

SANDIA MATIONAL LABORATORIES

SANDIA NATIONAL LABORATORIES

PROPERTY REAPPLICATION DEPT. $8532-1$
LIVERMCRE
CA $94550-0969$

REFERENCE NUMBER : 520

6685 HYGTOMETER MFR: PANAMETRICS, MODEL NO. 700-09H-04YBWD-05-10H, S/N 1549, 600001 EA ACO. DATE: $1 / 82$, APPROX. WT: 10 LBS., $81 \mathrm{~W} \times 10^{\prime} \mathrm{L} \times 5^{\prime} \mathrm{H}$.
COST/UNIT:

TOTAL COST: $\$ 3,321.00$

REPORT NUMBER: $899105-408$
LINE ITEM NUMBER: 0044
FOR FURTHER INFORMATION:

LYMN E. SHACKELFOOT

LIVERMORE

(510) $294-3067$

CA $94550-0969$

TO FREEZE EQUIPMENT:

LYNA E. SHACKELFOOT

LIVERMORE

(510) $294-3067$

CA $94550-0969$

LOCATION OF EQUIPMENT:

SANDIA NATIONAL LABORATORIES

PROPERTY REAPPLICATION DEPT. 8532-1

PROPERTY REAPPLICATION DEPT. $8532-1$
LIVERMORE
CA $94550-0969$ 
EFFECTIVE DATE: 07/01/94 EXPIRATION DATE: $07 / 31 / 94$

FSC

COOE EXCESS PROPERTY DESCRIPTION

REFERENCE MUMBER : 521

REFERENCE NUMBER : S21 PAMAMETRICS, MODEL NO, 700-09H-04YBWD-05-10H, S/N 1544, 600001 EA ICO. DATE: $1 / 82$, APPROX. WT: $10 \mathrm{LBS}$., $81 \mathrm{~W} \times 10^{\prime} \mathrm{L} \times 5^{\prime} \mathrm{H}$.

REPORT NUMBER: $899105-408$

LINE ITEM NUMBER: 0045

REFERENCE NUMBER : 522

6685 PRESSURE CALLRATOR AUTO PRESSURE CONTROLLER PROVIDES PROGRAMMABLE TEST

PRESSURE CALIBRATOR AUTO PRESSURE CONTROLLER PROVIDES PROGRAMMABLE
SEDUENCES, AU TO MEASURE OR CONTROL PRESSURE UP TO 5000 PSI USE FOR

CALIBRATION - IZOVAC, $60 \mathrm{HZ}$. NEEDS POWER CORD. CONS C

REPORT NUMBER: $898355-4102$

LINE ITEM NUMBER: 0092

NO OF
COND UNITS UNIT $\quad$ ACQUISITION PRICE

COST/UNIT:

TOTAL COST:

$\$ 3,321.00$

$\$ 3,321.00$

LYNN E. SHACKELFOOT

LI VERMORE

(510) $294-3067$

CA $94550-0969$

TO FREEZE EQUIPMENT:

LYNN E. SHACKELFOOT

LIVERMORE

(510) $294-3067$

LOCATION OF EQUIPMENT:

SANDIA NATIONAL LABORATORIES

SANDIA NATIONAL LABORATORIES $8532-1$

PROPERTY REAPPLICATION DEPT. $8532-1$

COST/UNIT:

TOTAL COST:

$\$ 7,546.00$

$\$ 7.546 .00$

FOR FURTHER INFORMATION:

ELOY N. GIRON

ALBUQUERQUE

(505) $844-2746$

NM 87185

TO FREEZE EOUIPMENT

ELOY N. GIRON

ALBUQUERQUE

(505) $844-2746$

LOCATION OF EQUIPMENT:

SANDIA NATIONAL LABORATORIES

PROPERTY REAPPLICATION DIV. 7617

ALBUQUERQUE 
EFFECTIVE DATE: 07/01/94 EXPIRATION DATE: $07 / 31 / 94$

FSC

COOE EXCESS PROPERTY DESCRIPIION

REFERENCE NUMBER : 523

MCTOR 10 H.P. MFG: INGERSOLL RAND MDL: $2 \times 11 / 2 \times 8 \mathrm{HC}$

SN: $0481-4057$

TAG \# 57957

REPORT NUMBER: $893604-4126$ ELIGIBLE EQUIPMENT

\begin{tabular}{|c|c|c|c|}
\hline COND & $\begin{array}{l}\text { NO OF } \\
\text { UNITS UNIT }\end{array}$ & \multicolumn{2}{|c|}{ ACQUISITION PRICE } \\
\hline 4 & 00001 EA & $\begin{array}{l}\text { COST/UNIT: } \\
\text { TOTAL COST: }\end{array}$ & $\begin{array}{l}\$ 3,970.00 \\
\$ 3,970.00\end{array}$ \\
\hline
\end{tabular}

LINE ITEM NUMBER: 0001

REFERENCE NUMBER : 524

6695 SYSTEM COOLING COOLS EQUIP

CONTROLS - IROVAC, $60 \mathrm{HZ}$.

LINE ITEM NUMBER: 0057

CONTACT(S)

FOR FURTHER INFORMATION:

LYDIA A KLEINHENZ

PITTSBURGH

(412) $892-5895$

PA 15236

TO FREEZE EQUIPMENT: LYD!A A KLEINHENZ

PITTSBURGH

(412) $892-5895$

PA 15236

LOCATION OF EQUIPMENT:

US DEPARTMENT OF ENERGY

PITTSBURGH ENERGY TECHNOLOGY CTR
BRUCETON

$2,928.00$

TOTAL COST

FOR FURTHER INFORMATION:

ELOY N. GIRON

ALBUQUERQUE

(505) $844-2746$

NM 87185

TO FREEZE EQUIPMENT:

ELOY N. GIRON

ALBUQUERQUE

(505) $844-2746$

NM 87185

LOCATION OF EQUIPMENT:

SANDIA NATIONAL LABORATORIES

PROPERTY REAPPLICATION DIVISION 7617

PROPERIY REAPPLICATION DIVISION 7617

NM 87123 
EFFECTIVE DATE: $07 / 01 / 94$ EXPIRATION DATE: $07 / 31 / 94$

FS

\section{COOE EXCESS PROPERTY DESCRIPTION}

REFERENCE NUMBER : 525

6695 VIBRATION TABLE 75903 MANUF: KINETIC SYSTEMS MOOEL 12110211 S'A 124225

1 EACH, INCLUDES STAND \& TABLETOP SIZE: $36^{\prime \prime} \mathrm{W} \times 36^{\prime \prime} \mathrm{F} / \mathrm{R} \times 36^{\prime \prime H}$ EST WT:

400 LBS MFGR YR: 1989

REPORT NUMBER: $896404-4133$

LINE ITEM NUMBER: 0028

REFERENCE NUMBER : 526

6695 VIBRATION TABLE 75904, MANUF: KINETIC SYSTEMS MODEL 12110211 S/N 124226

1 EACH, INCLUDES STAND \& TABLETOP SIZE: $36^{\prime \prime W} \times 36^{\prime \prime F} / R \times 36^{\prime \prime H ~ E S T ~ W T: ~}$

400 LBS MFGR YR: 1989

REPORT NUMBER: $896404-4133$

LINE ITEM NUMBER: 0029
NO OF

COND UNITS UNIT

ACQUISITION PRICE

$400001 \mathrm{EA}$

COST/UNIT: TOTAL COST:

$\$ 1,300.00$ $\$ 1,300.00$

FOR FURTHER INFORMATION

BARBARA HOLMES, REP.

KANSAS CITY

MO 64141-6159

TO FREEZE EQUIPMENT:

BARBARA HOLMES, REP.

KANSAS CITY

(816) $997-2976$

M0 64141-6159

LOCATION OF EQUIPMENT

ALLIEDSIGNAL, INC.

2000 EAST 95TH STREET

KANSAS CITY

MO $64131-3095$
$\$ 1,300.00$ $\$ 1,300.00$
COST/UNIT: TOTAL COST:
FOR FURTHER INFORMATION : BARBARA HOLMES, REP

(816) $997-2976$

MO 64141-6159

TO FREEZE EQUIPMENT: BARBARA HOLMES, REP. KANSAS CITY

(816) $997-2976$

M0 $64141-6159$

LOCATION OF EQUIPMENT: ALLIEDSIGNAL, INC. 2000 EAST 95TH STREET KANSAS CITY

MO 64131-3095 
EFFECTIVE DATE: $07 / 01 / 94$ EXPIRATION DAYE: $07 / 31 / 94$

FSC

\section{COOE EXCESS PROPERTY DESCRIPIION}

REFERENCE NUMBER : 527

6695 VIBRATION TABLE 75908, MANUF: KINETIC SYSTEMS MODEL $12110211 \mathrm{~S} / \mathrm{N} 124228$

1 EACH INCLUDES STAND \& TABLETOP SIZE: 35"W X 36"F/R X 36"H EST WT:

400 LBS MFGR YR: 1989

REPORT NUMBER: $896404-4133$

LINE ITEM NUMBER: 0030

\section{REFERENCE NUMBER : 528}

6695 VIBRATION TABLE 75905 MANUF: 1989 MODEL $12110211 \mathrm{~S} / \mathrm{N} 124227$

1 EACH INCLUDES STAND \& TABLETOP SIZE: $36^{\prime \prime} \mathrm{W} \times 36^{\prime \prime} \mathrm{F} / \mathrm{R} \times 36^{\prime \prime H}$ EST WT:

400 LBS MFGR YR: 1989 no of

COND UNITS UNIT

ACQUISITION PRICE

COST/UNIT: TOTAL COST:

$400001 \mathrm{EA}$

$\$ 1,300.00$
$\$ 1,300.00$
REPORT NUMBER: $896404-4133$

LINE ITEM NUMBER: 0031

\section{$4 \quad 00001$ EA}

TOTAL COST:

$\$ 1,300.00$

FOR FURTHER INFORMATION:

BARBARA HOLMES, REP.

KANSAS CITY

(816) $997-2976$

MO $64141-6159$

TO FREEZE EOUIPMENT:

BARBARA HOLMES, REP.

KANSAS CITY

MO 64141-6159

(816) $997-2976$

LOCATION OF EQUIPMENT

ALLIEDSIGNAL, INC.

2000 EAST 95TH STREET

KANSAS CITY

M0 64141-6159

MO 64141-6159

M0 64131-3095
MO $64131-3095$ 
EFFECTIVE DATE: 07/01/94 EXPIRATION DATE: 07/31/94

FSC

COOE EXCESS PROPERTY DESCRIPTION

REFERENCE NUMBER : 529

6695 VIBRATION TABLE 74245, MANUF: KINETIC SYSTEMS MOOEL $1201 \mathrm{~S} / \mathrm{N} 120833$ 9 EACH, INCLUDES STAND \& TABLETOP SIZE: $36^{\prime W} \times 36^{\prime \prime} / R$ X 36 "H EST WT. 9 EACH, INCLUDES STAND
400 LBS MFGR YR: 1989

REPORT NUMBER: $896404-4133$

LINE ITEM NUMBER: 0032
NO OF

COND UNITS UNIT

4. $00001 \mathrm{EA}$ TOTAL COST:

$\$ 1,300.00$ $\$ 1,300.00$

CONTACT(S)

FOR FURTHER INFORMATION :

BARBARA HOLMES, REP.

KANSAS CITY

(816) $997-2976$

Mo 64141-6159

TO FREEZE EQUIPMENT:

BARBARA HOLMES, REP.

KANSAS CITY

Mо 64141-6159

(816) $997-2976$

LOCATION OF EQUIPMENT

ALLIEDSIGNAL, INC.

ALLIEDSIGNAL, INC.

KANSAS CITY

MO 64131-3095

$400001 \mathrm{EA}$

COST/UNIT

TOTAL COST:

120833

1 EACH, INCLUDES STAND \& TABLETOP SIZE: $36^{\mathrm{mW}} \times 36^{\mathrm{mF} / R} \times 36^{\mathrm{NH}} \mathrm{EST}$ WT:

400 LBS MFGR YR: 1989
LINE I TEM NUMBER: 0033
REPORT NUMBER : $896404-4133$
FOR FURTHER INFORMATION:

BARBARA HOLMES, REP.

KANSAS CITY

(816) $997-2976$

MO 64141-6159

TO FREEZE EQUIPMENT:

BARBARA HOLMES, REP.

KANSAS CITY

(816) $997-2976$

MO 64141-6159

LOCATION OF EQUIPMENT

ALLIEDSIGMAL, INC.

2000 EAST 95TH STREET

KANSAS CITY

MO 64131-3095 
EFFECIIVE DATE: $07 / 01 / 94$ EXPIRATION DATE: $07 / 31 / 94$

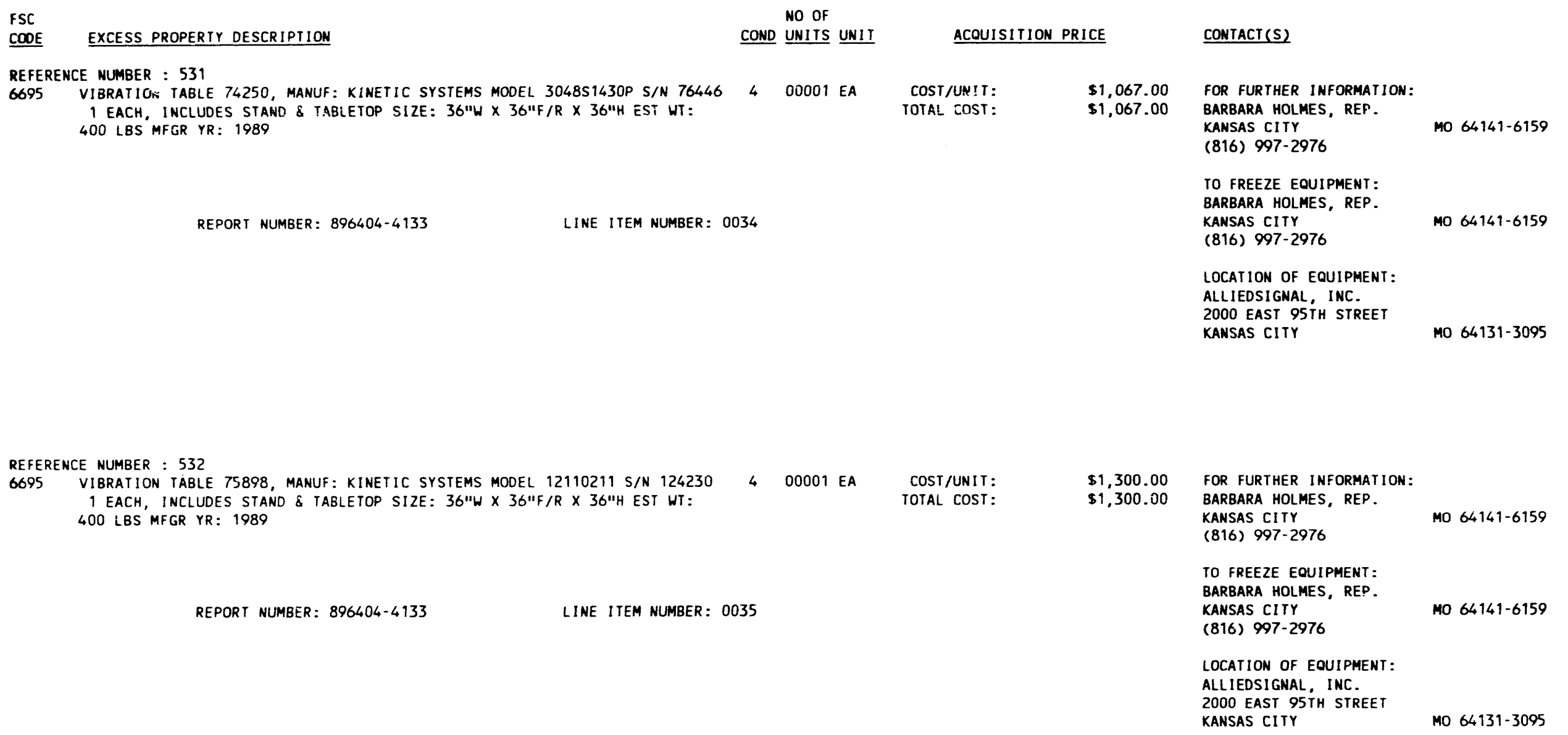


EFFECIIVE DATE: $07 / 01 / 94$ EXPIRATION DATE: $07 / 31 / 94$

FSC

FOOE EXCESS PROPERTY OESCRIPTION

REFERENCE NUMBER : 533

6695 VIBRATION TABLE 75900 , MANUF: KINETIC SYSTEMS MODEL 12110211 S/N 124229 1 EACH, INCLUDES STAND \& TABLETOP SIZE: $36^{\prime \prime} \mathrm{W} \times 36^{\prime \prime F} / \mathrm{R} \times 36^{\prime \prime H} \mathrm{HST}$ WT: 400 LBS MFGR YR: 1989

REPORT NUMBER: $896404-4133$

LINE ITEM NUMBER: 0036

\section{REFERENCE NUMBER : 534}

6710 CAMERA, EQUIPMENT MF'L: DICOMED CORP., ACQ. DATE: 1/82, APPROX WT: 300400001 EA LBS., $38^{\prime} \mathrm{W} \times 36^{\prime} \mathrm{L} \times 24^{\circ} \mathrm{H}$

REPORT NUMBER: $899105-4081$

LINE ITEM NUMBER: 0046
NO OF

COND UNITS UNIT

ACQUISITION PRICE

COST/UNIT: TOTAL COST:

\section{$\$ 1,300.00$}

$\$ 1,300.00$

00001 EA

(a)

BARBARA HOLMES, REP.

KANSAS CIIY

816) $997-2976$

TO FREEZE EOUIPMENT:

BARBARA HOLMES, REP.

KANSAS CITY

(816) $997-2976$

LOCATION OF EQUIPMENT:

ALLIEDSIGNAL, INC.

2000 EAST 95TH SIREET

KANSAS CITY

Mo 64131-3095
COST/UNIT: TOTAL COST: $\$ 2,070.00$
$\$ 2,070.00$
FOR FURTHER INFORMATION:

LYNN E. SHACKELFOOT

LIVERMORE

(510) 294-3067

CA $94550-0969$

TO FREEZE EQUIPMENT:

LYNN E - SHACKELFOOT

LIVERMORE

(510) 294-3067

CA $94550-0969$

LOCATION OF EQUIPMENT

SANDIA NATIONAL LABORATORIES

PROPERTY REAPPLICATION DEPT. 8532-1

LIVERMORE

CA $94550-0969$ 
EFFECTIVE DATE: $07 / 01 / 94$ EXPIRATION DATE: $07 / 31 / 94$

FSC

CODE EXCESS PROPERTY DESCRIPTION

REFERENCE NUMBER : 535

6710 MODULAR VIDEO SYS USE FOR SPECIAL PURPOSES, MODULAR VIDEO SYSTEM. MILETUS, SER. 007
NO OF

COND UNITS UNIT

$700001 \mathrm{EA}$ COST/UNIT:
TOTAL COST:

LINE ITEM NUMBER: 0093
REPORT NUMBER: $898355-4102$
REFERENCE NUMBER : 536

6710 POLARISCOPE VISTRAY MFR: PHOTOLASTIC INC., MODEL NO. 131, S/N 107606, ACO. DATE: 1/77, APPROX. WT: $40 \mathrm{LBS}, 13^{\prime} \mathrm{W} \times 14^{\prime} \mathrm{L} \times 8^{\prime} \mathrm{H}$
$500001 \mathrm{EA}$

COST/UNIT: TOTAL COST:
$\$ 2,253.00$ $\$ 2,253.00$

FOR FURTHER INFORMATION:

LYNN E. SHACKELFOOT

LIVERMORE

(510) 294-3067

CA $94550-0969$

TO FREEZE EQUIPMENT:

LYNN E. SHACKELFOOT

LIVERMORE

(510) $294-3067$

CA $94550-0969$

REPORT NUMBER: $899105-4081$

LINE ITEM NUMBER: 0047

LOCATION OF EQUIPMENT:

SANDIA NATIONAL LABORATORIES

PROPERTY REAPPLICATION DEPT . 8532-1

LIVERMORE CA $94550-0969$ 
EFFECTIVE DATE: 07/01/94 EXPIRATION DATE: $07 / 31 / 94$

FSC

\section{COOE EXCESS PROPERTY DESCRIPTION}

REFERENCE NUMBER : 537

6710 TELESCOPE VISTRAY MFR: PHOTOLASTIC INC., MODEL NO. 137, S/N 017711,

ACQ. DATE: $1 / 77$, APPROX. WT: 10 LBS., $13, \mathrm{~W} \times 13^{\prime} \mathrm{L} \times 5^{\prime} \mathrm{H}$

REPORT NUMBER: $899105-4081$

LINE ITEM NUMBER: 0048

REFERENCE NUMBER : 538

6710 VIDEO QUATIZER 606 MFR

COLORADO VIDEO INC., MODEL NO. 606, S/N 161, ACO. DATE: 6/ 86 APPROX. WT: 20 LBS., $19 \cdot \mathrm{W} \times 13 . \mathrm{L} \times \mathrm{T}^{\prime} \mathrm{H}$.
$400001 \mathrm{EA}$

COST/UNIT:
TOTAL COST:

TOTAL COST:
$\$ 5,000.00$
$\$ 5,000.00$

FOR FURTHER INFORMATION:

LYNN E. SHACKELFOOT

LIVERMORE

(510) $294-3067$

CA $94550-0969$

TO FREEZE EQUIPMENT:

LYNW E. SHACKEL FOOT

CA $94550-0969$
(510) $294-3067$

LOCATION OF EQUIPMENT:

SANDIA NATIONAL LABORATORIES

PROPERTY REAPPLICATION DEPT. 8532-1

LIVERMORE 


\section{EFFECIIVE DATE: 07/01/94 EXPIRATION DATE: $07 / 31 / 94$}

\section{FSC \\ COOE EXCESS PROPERTY OESCRIPIION \\ REFERENCE NUMBER : 539 \\ 6710 VIDEOGRAPH PRINTER MFR: ADVANCED IMAGING DEVICES INC., MODEL NO. C} 1500, S/N 15000651 , ACO. DATE: 3/85, APPROX. WT: 80 LBS., 17'W $\times 231 \mathrm{~L}$ $\times 11 \cdot H$
No of

COND UNITS UNIT

400001 EA
REPORT NUMBER: $899105-6081$

LINE ITEM NUMBER: 0049

\section{ACQUISITION PRICE}

\section{COST/UNIT:} TOTAL COST:

\section{$\$ 9.330 .00$ $\$ 9,330.00$}

CONTACTSS)

FOR FURTHER INFORMATION:

LYNN E. SHACKELFOOT

LIVERMORE

(510) $294-3067$

CA $94550-0969$

TO FREEZE EQUIPMENT:

LYNN E. SHACKELFOO

LIVERMORE

(510) 294-3067

CA $94550-0969$

LOCATION OF EOUIPMENT

SANDIA NATIONRL LABORATORIES

PROPERTY REAPPLICATION DEPT, $8532-1$

LIVERMORE CA $94550-0969$

REFERENCE NUMBER : 540

6720 ADAPTER, CAMERA BACK TAKES $8 \times 10$ PICTURES, HAS AN ELECTRONIC SHUTTER. MATRIX INS, 6881,7690138
$600001 \mathrm{EA}$ TOTAL COST: $\$ 2,495.00$
$\$ 2,495.00$

FOR FURTHER INFORMATION:
ELOY N. GIRON
ALBUOUEROUE
(505) $844-2746$
IO FREEZE EOUIPMENT:
ELOY N. CIRON
ALBUOUEROUE
(505) $844-2746$
LOCATION OF EQUIPMENT:
SANDIA NATIONAL LABORATORIES
PROPERTY REAPPLICATION DIV. 7617
ALBUDUEROUE
MM 87123

REPORT NUMBER: $898355-4102$

LINE ITEM NUMBER: 009 
EFFECTIVE DATE: $07 / 01 / 94$ EXPIRATION DATE: $07 / 31 / 94$

FSC

\section{COOE EXCESS PROPERTY DESCRIPIION}

REFERENCE MUMBER : 541

6720 CAMERA IS A POLAROID 35MM CAMERA THAT IS ATTACHED TO A VIDEO PRINT 5000 AMD IS USED FOR PHOTOGRAPHIC VIDEO IMAGES. POLAROID, $8400,83080001$.

REPORT MUMBER: $898355-4102$

LIME ITEM MLMBER: 0095
No of

COND UNITS UWIT

$600001 \mathrm{EA}$

COST/UNIT: TOTAL COST:

$\$ 1,074.00$ $\$ 1.074 .00$

FOR FURTHER IMFOOMATION:

ELOY N. GIROM

ALBUOUEROUE

(505) $844-2746$

(1) 87185

TO FREEZE EOUIPMENT

ELOY N. GIROW

ALBUOUEROUE

(505) $844-2746$

87185

LOCATION OF EQUIPMEMT:

SAMDIA MATIOUAL LABORATORIES

PROPERTY REAPPLICAIION DIV. 7617

ALBUOUEROUE

87123

$\$ 1.195 .00$ $\$ 1,195.00$

COST/UNIT: TOTAL COST:

FOR FURTHER INFORMAIION

ELOY M. GIROW

ALBUOUEROUE

(505) $844-2746$

147185

TO FREEZE EOUIPMEMT

ELOY N. GIRON

ALBUOUEROUE

(505) 844-2746

4.4 87185

LOCATION OF EQUIPMENT:

SAMDIA MATICMAL LABORATORIES

PROPERTY REAPPLICATION DIVISION 7617

ALBUOUEROUE

.4. 87123 
EFFECTIVE DATE: $07 / 01 / 94$ EXPIRATION DATE: $07 / 3 i / 94$

FSC

\section{COOE EXCESS PROPERTY DESCRIPIION}

REFERENCE MUMBER : 543

6720 CAMERA, HIGH SPEED ON STAND, USES 35MM FILM, WITH FRAME COUNTER AND VIEH FINDER. PO WER BY POWER SUPPLY, DECK\&WHIT, 189A, 101.

REFERENCE NUMBER : 544

6720 COPY, CAMERA IS $20^{\prime \prime}$ BY $24^{\prime \prime}$ LIGHT TABLE, CAMERA TRAVEL UP AND DOWN IS 44". 120 VAC, $60 \mathrm{HZ}$. POLAROID. wo of

COND UNITS UNIT

ACQUISITIOM PRICE

$700001 \mathrm{EA}$ COST/UMIT:

TOTAL COST:

$\$ 43,463.00$

(2)

Contact(s)

FOR FURTHER IMFORMATION:

ELOY N. GIRON

ALBUQUEROUE

(505) $844-2746$

4m 87185

TO FREEZE EOUIPHENT :

ELOY N. GIRON

ALBuaueraut

(505) $844-2746$

LOCATION OF EQUIPNEMT

SAMDIA MATIOULL LABORATORIES

PROPERTY REAPPLICATION DIVISION 7617

ALBUOUEROUE

COST/UNIT: TOTAL COST:
$\$ \$ 1,000.00$

FOR FURTHER IMFORMATION:

ELOY N. GIROW

ALBUOUERROUE

(505) $844-2746$

TO FREEZE EOUIPMENT :

ELOY N. GIROW

ALBUOUEROUE

(505) $864-2746$

87185

LOCATION OF EOU! PMENT

SAMDIA MAT IONAL LABORATORIES PROPERTY REAPPLICATION DIVISION 7617 ALBUOUEROUE 

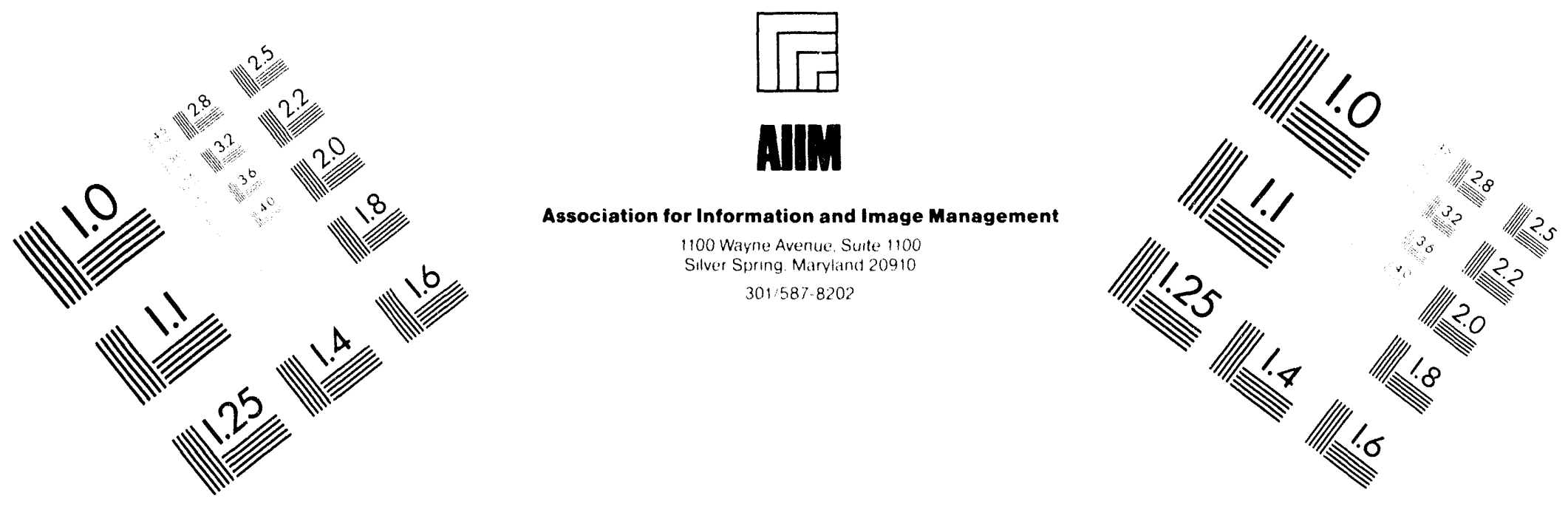

\section{Centimeter}

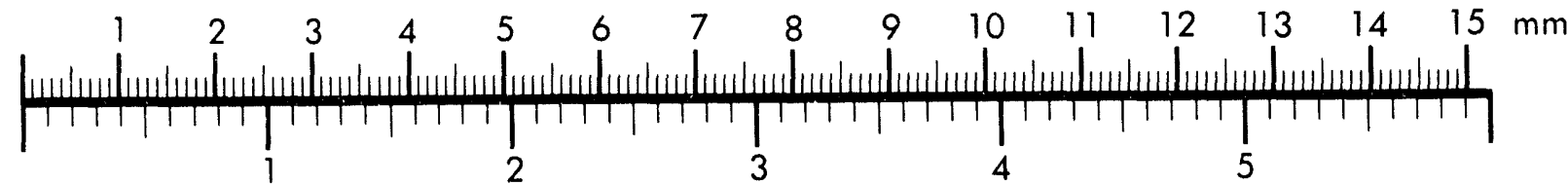

Inches
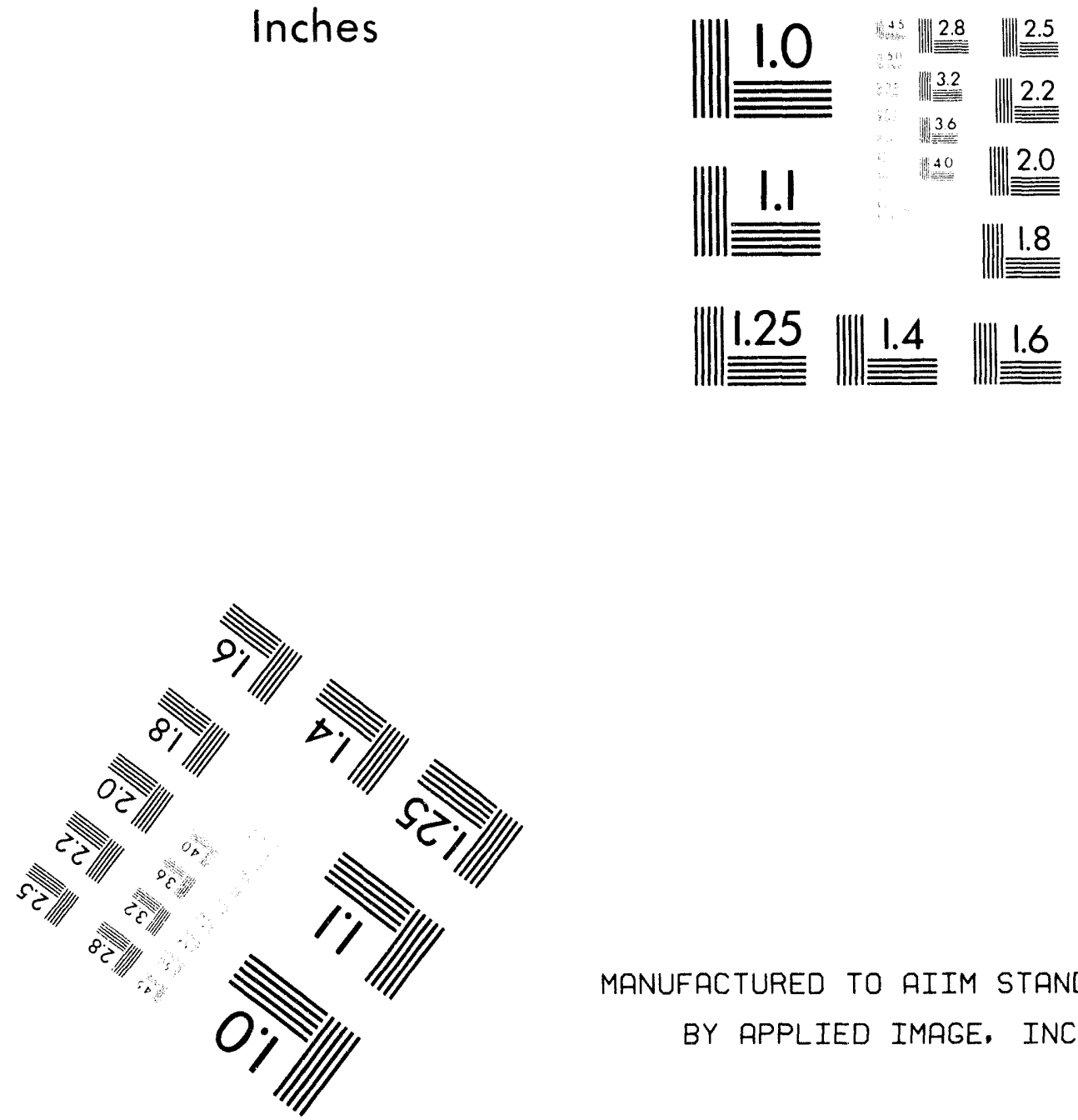

MANUFACTURED TO AIIM STANDARDS

BY APPLIED IMAGE. INC.

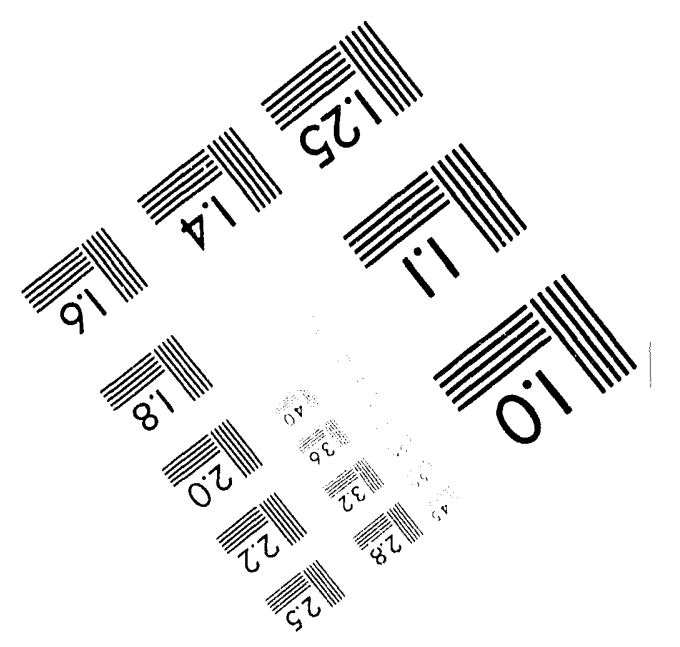



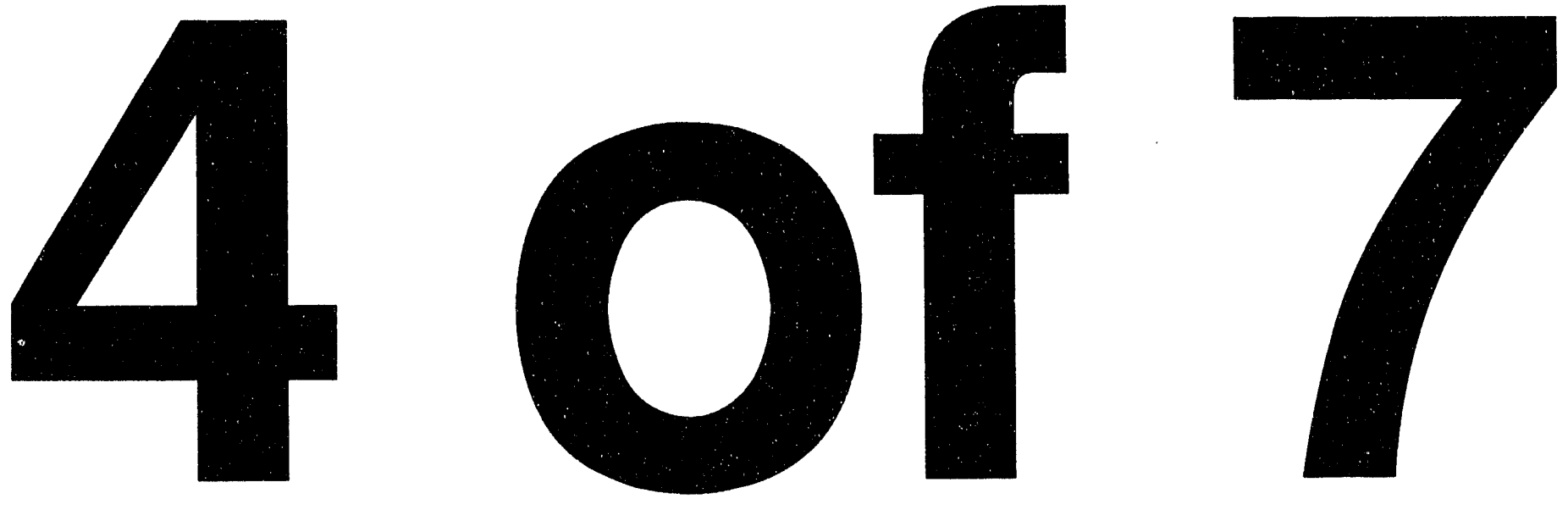
EFFECTIVE DATE: $07 / 01 / 94$ EXPIRATION DATE: $07 / 31 / 94$

FSC

CODE EXCESS PROPERTY DESCRIPIION

REFERENCE NUMBER : 545

6720 SCOPE CAMERA MFR: TEKTRONIX MODEL: C51 S/N: B096767

REPORT NUMBER: $899305-4039$

INE ITEM NUMBER: 0012

REFERENCE NUMBER : 546

6720 SCOPE CAMERA MFR: TEKTRONIX MOOEL: C51 $S / N: B 094812$

REPORT NUMBER: $899305-4039$
NO OF

COND UNITS UNIT

ACQUISITION PRICE

$500001 \mathrm{EA}$

COST/UNIT:

TOTAL COST:

$\$ 2,506.86$ $\$ 2,506.86$

CYNDI DELONG

(702) $295-6753$

TO FREEZE EQUIPMENT:

CYNDI DELONG

LAS VEGAS

(702) 295-6753

LOCATION OF EOUIPMENT:

REYNOLDS ELECTRICAL \& ENGINEERING CO

NEVADA TEST SITE

MERCURY

NV 89023
$500001 \mathrm{EA}$

\section{COST/UNIT: \\ TOTAL COST:}

LINE ITEM NUMBER: 0013
$1,934.83$ $\$ 1,934.83$
FOR FURTHER INFORMATION:

CYNDI DELONG

NV $89193-8521$

(702) $295-6753$

TO FREEZE EQUIPMENT :

CYNDI DELONG

AS VEGAS

NV 89193-8521

OCATION OF EQUIPMENT:

REYNOLDS ELECTRICAL \& ENGINEERING CO NEVADA TEST SITE

MERCURY

NV 89023 
EFFECTIVE DATE: $07 / 01 / 94$ EXPIRATION DATE: $07 / 31 / 94$

FSC

\section{COOE EXCESS PROPERTY DESCRIPTION}

REFERENCE NUMBER : 547

6720 SCOPE CAMERA MFR: TEKTRONIX MODEL: C51 $S / N: \quad B 096360$

REPORT NUMBER: $899305-4030$

REFERENCE NUMBER : 548

6720 SCOPE CAMERA MFR: TEKTRONIX MODEL: C51 $S / N: \quad B 095876$

REPORT NUMBER: $899305-4,039$

ELIGIBLE EQUIPMENT

No of

COND UNITS UNIT

ACQUISITION PRICE

$500001 E A$

COST/UNIT:

LINE ITEM NUMBER: 0014

$500001 \mathrm{EA}$

COST/UNIT:
TOTAL COST:

LINE ITEM NUMIER: 0015

\section{$\$ 2,399.08$}

$\$ 2,399.08$
CONTACT(S)

FOR FURTHER INFORMATION

CYNDI DELONG

LAS VEGAS

(702) $295-6753$

TO FREEZE EQUIPMENT:

CYNDI DELONG

LAS VEGAS

(702) $295-6753$

NV $89193-8521$

LOCATION OF EQUIPMENT:

REYNOLDS ELECTRICAL \& ENGINEERING CO

NEVADA TEST SITE

NEVADA TES

NV 89023

FOR FURTHER INFORMATION:

CYNDI DELONG

LAS VEGAS

(702) $295-6753$

NV $89193-8521$

TO FREEZE EQUIPMENT:

CYNDI DELONG

LAS VEGAS

(702) $295-6753$

NV $89193-8521$

LOCATION OF EQUIPMENT:

REYNOLDS ELECTRICAL \& ENGINEERING CO

NEVADA TEST SITE

MERCURY

NV 89023 
EFFECTIVE DATE: $07 / 01 / 94$ EXPIRATION DATE: $07 / 31 / 94$

\section{FSC}

\section{COOE EXCESS PROPERTY DESCRIPIION}

REFERENCE NUMBER : 549

6720 SCOPE CAMERA MFR: TEKTRONIX MODEL: C51 $S / N: \quad B 094752$

REPORT NUMBER: $899305-4039$

REFERENCE NUMBER : 550

6720 SCOPE CAMERA MFR: TEKTRONIX MODEL: C51 S/N: $B 095674$

REPORT NUMBER: $899305-4039$

ELIGIBLE EQUIPMENT

No of

COND UNITS UNIT

ACQUISITION PRICE

$500001 \mathrm{EA}$

COST/UNIT:
LINE ITEM NUMBER: 0016

$500001 E A$

COST/UNIT:
TOTAL COST:

LINE ITEM NUMBER: 0017
$\$ 2,097.95$ $\$ 2,097.95$
CONTACTSS)

FOR FURTHER INFORMATION:

CYNDI DELONG

AS VEGAS

(702) $295-6753$

IO FREEZE EQUIPMENT:

CYNDI DELONG

LAS VEGAS

NV $89193-8521$

(702) $295-6753$

LOCATION OF EQUIPMENT:

REYNOLDS ELECTRICAL \& ENGINEERING CO

NEVADA TEST SITE

NV 89023

MERCURY

$\$ 2,088.00$

$\$ 2,088.00$

FOR FURTHER INFORMATION:

CYNDI DELONG

LAS VEGAS

(702) $295-6753$

NV 89193-8521

TO FREEZE EQUIPMENT:

CYNDI DELONG

LAS VEGAS

NV $89193-8521$

(702) $295-6753$

LOCATION OF EQUIPMENT:

REYNOLDS ELECTRICAL \& ENGINEERING CO NEVADA TEST SITE

MERCURY

NV 89023 
DOE USED ENERGY RELATED LABORATORY EQLIPMENT GRANT PROGRAM

EFFECTIVE DATE: $07 / 01 / 94$ EXPIRATION DATE: 07/31/94

FSC

CODE EXCESS PROPERTY DESCRIPIION

REFERENCE NUMBER : 551

6720 SCOPE CAMERA MFR: TEKTRONIX MOOEL: C5 S/N: B096053

REPORT NUMBER: $899305-4039$

REFERENCE NUMBER : 552

6720 SCOPE CAMERA MFR: TEKTRONIX MODEL: C51 $S / N: B 096262$

REPORT NUMBER: $899305-4039$
ELIGIBLE EQUIPMENT

$$
\text { NO OF }
$$

$500001 \mathrm{EA}$
ACQUISITION PRICE

COST/UNIT: TOTAL COST:

LINE ITEM NUMBER: 0018
COST/UNIT:

TOTAL COST:
$\$ 2,393.36$
$\$ 2,393.36$
FOR FURTHER INFORMATION:

CYNDI DELONG

LAS VEGAS

(702) 295.6753

TO FREEZE EQUIPMENT:

CYNDI DELONG
LAS VEGAS

(702) $295-6753$

NV $89193-8521$

LOCATION OF EOUIPMENT:

REYNOLDS ELECTRICAL \& ENGINEERING CO NEVADA TEST SITE

MERCURY

NV 89023 
EFFECTIVE DATE: $07 / 01 / 94$ EXPIRATION DATE: $07 / 31 / 94$

FSC

\section{COOE EXCESS PROPERTY DESCRIPTION}

REFERENCE NUMBER : 553

6720 SCOPE CAMERA MFR: TEKTRONIX MOOEL: C51 S/N: B095051

REPORT NUMBER: $899305-4039$

LINE ITEM NUMBER: 0020

$$
\text { No OF }
$$

COND UNITS UNIT

ACQUISITION PRICE

$5 \quad 00001 \mathrm{EA}$

COST/UNIT: TOTAL COST:

\section{$\$ 1.892 .37$ \\ $\$ 1,892.37$}

CYNDI DELONG

LAS VEGAS

(702) $295-6753$

NV $89193-8521$

TO FREEZE EQUIPMENT:

CYNDI DELONG

(702) 295-6753

NV $89193-8521$

LOCATION OF EQUIPMENT:

REYNOLDS ELECTRICAL \& ENGINEERING CO

NEVADA TEST SITE

MERCURY

NV 89023

$500001 E A$

6720 SCOPE CAMERA MFR: TEKTRONIX MODEL: CS S/N: $\quad$ B096047

REPORT NUMBER: $899305-4039$
LINE ITEM NUMBER: 0021

\section{COST/UNIT: \\ TOTAL COST:}

$\$ 2.210 .44$ $\$ 2,210.44$

CYNDI DELON
LAS VEGAS
(702) $295-6753$

(702) 295-6753

TO FREEZE EOUIPMENT:

CYNDI DELONG

LAS VEGAS

(702) 295-6753

NV $89193-852$

LOCATION OF EQUIPMENT:

REYNOLDS ELECTRICAL \& ENGINEERING CO

NEVADA TEST SITE

MERCURY

NV 89023 
EFFECTIVE DATE: $07 / 01 / 94$ EXPIRATION DATE: $07 / 31 / 94$ FSC

\section{CODE EXCESS PROPERTY DESCRIPTION}

REFERENCE NUMBER : 555

6720 SCOPE CAMERA MFR: TEKTRONIX MODEL: C51 $S / N: \quad B 096116$

REPORT MUMBER: $899305-4039$

REFERENCE NUMBER : 556

6720 SCOPE CAMERA MFR: TEKTRONIX MODEL: C51 $S / N$ : BO96114

REPORT NUMBER : $899305-4039$
ELIGIBLE EQUIPMENT
NO OF

COND UNITS UNIT

500001 EA

COST/UNIT:

TOTAL COST:

LINE ITEM NUMBER: 0022

$500001 E A$

COST/UNIT:
TOTAL COST:

LINE ITEM NUMBER: 0023

\section{ACUISITION PRICE}

$\$ 2,756.09$ $\$ 2,756.09$
FOR FURTHER INFORMATION:

CYNDI DELONG

LAS VEGAS

NV $89193-8521$

TO FREEZE EQUIPMENT:

CYNDI DELONG

LAS VEGAS

(702) $295-6753$

LOCATION OF EQUIPMENT:

REYMOLDS ELECTRICAL \& ENGINEERING CO

NEVADA TEST SITE

MERCURY

NV 89023
FOR FURTHER INFORMATION:

CYNDI DELONG

LAS VEGAS

NV $89193-8521$

TO FREEZE EQUIPMENT:

CYNDI DELONG

LAS VEGAS

(702) $295-6753$

NV $89193-8521$

LOCATION OF EQUIPMENT:

REYNOLDS ELECTRICAL \& ENGINEERING CO NEVADA TEST SITE

NEVADA
MERCURY

NV 89023 
EFFECIIVE DATE: $07 / 01 / 94$ EXPIRATION DATE: $07 / 31 / 94$

FSC

COOE EXCESS PROPERIY DESCRIPIION

REFERENCE NUMBER : 557

6720 SCOPE CAMERA MFR: TEKTRONIX MODEL: C51 $S / N: B 094767$

REPORT NUMBER: $899305-4039$

REFERENCE NUMBER : 558

6720 SCOPE CAMERA MFR: TEKTRONIX MODEL: C51 S/N: $B 094889$

ELIGIBLE EOUIPMENT

279

COND UNIIS UNIT

ACQUISITION PRICE

$500001 E A$ COST/UNIT:
TOTAL COST: TOTAL COST:

LINE ITEM NUMBER: 0025

$500001 \mathrm{EA}$ COST/UNIT:
TOTAL COST:

LINE ITEM NUMBER: 0029
$\$ 1,824.48$
$\$ 1,824.48$

$\$ 1,824.48$
CONTACT(S)

FOR FURTHER INFORMATION

CYNDI DELONG

LAS VEGAS

(702) $295-6753$

NV 89193-8521

TO FREEZE EQUIPMENT:

CYNDI DELONG

LAS VEGAS

(702) $295-6753$

NV 89193-8521

LOCATION OF EQUIPMENT:

REYNOLDS ELECTRICAL \& ENGINEERING CO NEVADA TEST SITE

MERCURY

NV 89023
FOR FURTHER INFORMATION:

CYNDI DELONG

LAS VEGAS

(702) $295-6753$

NV $89193-8521$

TO FREEZE EQUIPMENT :

CYNDI DELONG

LAS VEGAS

(702) $295-6753$

NV $89193 \cdot 8521$

LOCATION OF EOUIPMENT

REYNOLDS ELECTRICAL \& ENGINEERING CO NEVADA TEST SITE

MERCURY

NV 89023 
EFFECTIVE DATE: $07 / 01 / 94$ EXPIRATION DATE: $07 / 31 / 94$ 
EFFECTIVE DATE: $07 / 01 / 94$ EXPIRATION DATE: $07 / 31 / 94$ FSC

\section{COOE EXCESS PROPERTY DESCRIPTION}

REFERENCE NUMBER : 561

6720 SCOPE CAMERA MFR: TEKTRONIX MODEL: C51

PEPORT NUMBER : $899305-4039$

\section{REFERENCE NUMBER : 562}

6720 SCOPE CAMERA MFR: TEKTRONIX MODEL: C51 S/N: $B 095154$
ELIGIBLE EOUIPMENT

\section{No of \\ COND UNITS UNIT}

$500001 \mathrm{EA}$

COST/UNIT:

TOTAL COST:

LINE ITEM NUMBER: 0032
$500001 E A$

\section{COST/UNIT: \\ TOTAL COST:}

LINE ITEM NUMBER: 0033

\section{ACQUISITION PRICE}

$\$ 2.041 .87$

$\$ 2,041.87$

FOR FURTHER INFORMATION:

CYNDI DELONG

LAS VEGAS

NV $89193-8521$

(702) $295-6753$

TO FREEZE EQUIPMENT:

CYNDI DELONG

LAS VEGAS

(702) $295-6753$

NV $89193-8521$

LOCATION OF EQUIPMENT

REYNOLDS ELECTRICAL \& ENGINEERING CO

NEVADA TEST SITE

MERCURY

NV 89023

$\$ 1,892.00$

$\$ 1,892.00$

FOR FURTHER INFORMATION:

CYNDI DELONG

LAS VEGAS

NV $89193 \cdot 8521$

TO FREEZE EQUIPMENT:

CYNDI DELONG

(702) $295-6753$

NV $89193-8521$

OCATION OF EQUIPMENT: REYNOLDS ELECTRICAL \& ENGINEERING CO NEVADA TEST SITE

NV 89023 
DOE USED ENERGY RELATED LABORATORY EQUIPMENT GRANT PROGRAM

EFFECIIVE DATE: $07 / 01 / 94$ EXPIRATION DATE: $07 / 31 / 94$

\section{FSC
COOE EXCESS PROPERTY DESCRIPTION}

REFERENCE NUMBER : 563

6720 SCOPE CAMERA MFR: TEKTRONIX MODEL: C5 $S / N: \quad B 096909$

REPORT NUMBER: $899305-4039$

REFERENCE NUMEER : 564

6720 SCOPE CAMERA MFR: TEKTRONIX MODEL: C5 $S / N$ : $B 094751$

REPORT NUMBER: $899305-4039$
ELIGIBLE EQUIPMENT
NO OF
COND UNITS UNIT ACOUISITION PRICE

500001 EA COST/UNIT: TOTAL COST: $\$ 2,620.99$
$\$ 2,620.99$

CYNDI DELONG

CYND! DELON

(702) $295-6753$

NV $89193-8521$

TO FREEZE EQUIPMENT:

CYNDI DELONG

LAS VEGAS

LAS VEGAS -6753

NV $89193-8521$

LINE ITEM NUMBER: 0034

LOCATION OF EQUIPMENT:

REYNOLDS ELECTRICAL \& ENGINEERING CO REYNOLDS ELECTRICAL \& ENGINEERING

NEVADA TEST SITE

NV 89023
$\$ 2,097.95$
$\$ 2,097.95$

$500001 E A$

FOR FURTHER INFORMATION :

CYNDI DELONG

LAS VEGAS

NV $89193-852$

TO FREEZE EQUIPMENT:

CYNDI DELONG

CYNDI DELONG

(702) $295-6753$

NV $89193-8521$

LINE ITEM NUMBER: 0035

LOCATION OF EQUIPMENT:

REYNOLDS ELECTRICAL \& ENGINEERING CO REYNOLDS ELECTRICAL \& ENGINEERING TEST SITE

NV 89023 
EFFECTIVE DATE: $07 / 01 / 94$ EXPIRATION DATE: $07 / 31 / 94$

\section{CODE EXCESS PROPERTY DESCRIPIION}

REFERENCE NUMBER : 565

6720 SCOPE CAMERA MFR: TEKTRONIX MOOEL: C51 S/N: B094815

REPORT NUMBER: $899305-4039$

LINE ITEM NUMBER: 0036

No of

COND UNITS UNIT

ACOUISITION PRICE

$500001 \mathrm{EA}$

COST/UNIT: TOTAL COST:

\section{$\$ 1,695.92$}

$\$ 1,695.92$ $\$ 2,620.98$

LINE ITEM NUMBER: 0037

REPORT NUMBER: $899305-4039$
REFERENCE NUMBER : 566

6720 SCOPE CAMERA MFR: TEKTRONIX MODEL: CS S/N: $\quad$ B096918

\section{$00001 \mathrm{EA}$ COST/UNIT: \\ TOTAL COST:}

FOR FURTHER INFORMATION:

CYNDI DELONG

LAS VEGAS

NV $89193-8521$

(702) $295-6753$

TO FREEZE EQUIPMENT:

CYNDI DELONG

LAS VEGAS

(702) 295-6753

NV 89193-8521

LOCATION OF EQUIPMENT

REYNOLDS ELECTRICAL \& ENGINEERING CO

NEVADA TEST SITE

MERCURY

NV 89023 
EFFECTIVE DATE: $07 / 01 / 94$ EXPIRATION DATE: $07 / 31 / 94$

FSC

\section{COOE EXCESS PROPERTY DESCRIPIION}

\section{REFERENCE NLMBER : 567}

6720 SCOPE CAMERA MFR: TEKTRONIX MOOEL: CS1 S/N: $B 096910$

REPORT NUMBER: $899305-4039$

REFERENCE NUMBER : 568

720 SCOPE CAMERA MFR: TEKTRONIX MOOEL: C51 $S / N$ : BO95882

REPORT NUMBER : $899305-4039$

ELIGIBLE EOUIPMENT

NO OF

COND UNITS UNIT

$500001 \mathrm{EA}$

COST/UNIT: TOTAL COST:

LINE ITEM NUMBER: 0038

$500001 E A$

COST/UNIT:

TOTAL COST

$\$ 2,324.79$
$\$ 2,324.79$

LINE ITEM NUMBER: 0039
$\operatorname{CONTACT(S)}$

FOR FURTHER INFORMATION:

CYNDI DELONG

LAS VEGAS

TO FREEZE EOUIPMENT:

CYNDI DELONG

LAS VEGAS

(702) $295-6753$

NV 89193-8521

OCATION OF EQUIPMENT: REYNOLDS ELECTRICAL \& ENGINEERING CO NEVADA TEST SITE

MERCURY

NV 89023

FOR FURTHER INFDRMATION:

CYNDI DELONG

LAS VEGAS

(702) $295-6753$

NV $89193-8521$

TO FREEZE EQUIPMENT :

CYNDI DELONG

LAS VEGAS

(702) $295-6753$

NV $89193-8521$

LOCATION OF EQUIPMENT:

REYNOLDS ELECTRICAL \& ENGINEERING CO NEVADA TEST SITE

MERCURY

NV 89023 
EFFECTIVE DATE: $07 / 01 / 94$ EXPIRATION DATE: $07 / 31 / 94$

FSC

CODE EXCESS PROPERTY DESCRIPTION

REFERENCE NUMBER : 569

6720 SCOPE CAMERA MFR: TEKTRONIX MODEL: C51 $S / N:$ B096102

REPORT NUMBER: $899305-4039$

REFERENCE NUMBER : 570

6720 SCOPE CAMERA MFR: TEKTRON1X MODEL: C51 S/N: $B 096214$
No of

COND UNITS UNIT

ACOUISITION PRICE

$500001 E$

COST/UNIT:

TOTAL COST:

LINE ITEM NUMBER: 0040

500001 EA

COST/UNIT:
TOTAL COST:

LINE ITEM NUMBER: 0041
CONTACTSS)

$\$ 2,246.37$

$\$ 2,246.37$

FOR FURTHER INFORMATION:

CYNDI DELONG

LAS VEGAS

(702) $295-6753$

NV $89193-8521$

TO FREEZE EQUIPMENT:

CYNDI DELONG

LAS VEGAS

AS VEGAS

NV 89193-8521

LOCATION OF EQUIPMENT:

REYNOLDS ELECTRICAL \& ENGINEERING CO NEVADA TEST SITE

NV 89023

REPORT NUMBER: $899305-4039$

FOR FURTHER INFORMATION:

CYNDI DELONG

LAS VEGAS

NV $89193-8521$

(702)

TO FREEZE EQUIPMENT:

CYNDI DELONG

LAS VEGAS

NV $89193-8521$

(702) $295-6753$

LOCATION OF EQUIPMENT:

REYNOLDS ELECTRICAL \& ENGINEERING CO NEVADA TEST SITE

NV 89023 
EFFECTIVE DATE: 07/01/96 EXPIRATION DATE: 07/31/94

FSC

\section{CODE EXCESS PROPERTY DESCRIPIION}

REFERENCE NUMBER : 571

6720 SCOPE CAMERA MFR: TEKTRONIX MOCEL: C5 $S / N: B 097225$

REPORT NUMBER: $899305-4039$

REFERENCE NUMBER : 572

6720 SCOPE CAMERA MFR: TEKTRONIX MODEL: C51 $S / N: \quad 8096730$

REPORT NUMBER : $899305-4039$
NO OF

COND UNITS UNIT

ACQUISITION PRICE

$500001 E A$
COST/UNIT:
TOTAL COST:

LINE ITEM NUMBER: 0042

$500001 E A$

COST UNIT:
TOTAL COST:

LINE ITEM NUMBER: 0043
CONTACTSS)

FOR FURTHER INFORMAIION:

CYNDI DELONG

LAS VEGAS

(702) $295-6753$

NV $89193-8521$

TO FREEZE EOUIPMENT:

CYND1 DELONG

AS VEGAS

(702) $295-6753$

NV $89193-8521$

LOCATION OF EQUIPMENT:

REYNOLDS ELECTRICAL \& ENGINEERING CO NEVADA TEST SITE

NV 89023
$\$ 2,506.86$ $\$ 2,506.86$
FOR FURTHER INFORMATION

CYNDI DELONG

LAS VEGAS

NV $89193-8521$

(702) $295-6753$

TO FREEZE EQUIPMENT:

CYNDI DELONG

LAS VEGAS

(702) 295-6753

NV $89193-8521$

LOCATION OF EQUIPMENT:

REYNOLDS ELECTRICAL \& ENGINEERING CO NEVADA TEST SITE

MERCURY

NV 89023 
EFFECTIVE DATE: $07 / 01 / 94$ EXPIRATION DATE: $07 / 31 / 94$

FSC CODE EXCESS PROPERTY DESCRIPIION

REFERENCE NUMBER : 573

REFERENCE NUMBER : 573
6720 SCOPE CAMERA MFR: TEKTRONIX MODEL: C51 S/N: 8096605

REPORT NUMBER: $899305-4039$

REFERENCE NUMBER : 574

6720 SCOPE CAMERA MFR: TEKTRONIX MOOEL: C51 S/N : $B 096087$

REPORT NUMBER: $899305-4039$

ELIGIBLE EQUIPMENT

NO OF COND UNITS UNIT

ACQUISITION PRICE

$500001 E A$

COST/UNIT: TOTAL COST :

LINE ITEM NUMBER: 0044

$500001 \mathrm{EA}$

COST/UNIT:

TOTAL COST:

LINE ITEM NUMBER: 0045
$\$ 2,532.38$

$\$ 2,532.38$

CYNDI DELONG

LAS VEGAS

(702) $295-6753$

NV $89193-8521$

TO FREEZE EQUIPMENT:

CYNDI DELONG

LAS VEGAS

(702) $295-6753$

NV $89193-8521$

OCATION OF EQUIPMENT:

REYNOLDS ELECTRICAL \& ENGINEERING CO REYNOLDS ELECTRICAL

MEVADA TES

NV 89023
$\$ 2,246.38$
$\$ 2,246.38$
FOR FURTHER INFORMATION:

CYNDI DELONG

(702) $295-6753$

NV $89193-852$

TO FREEZE EQUIPMENT

CYNDI DELONG

LAS VEGAS

(702) 295-6753

LOCATION OF EQUIPMENT:

REYNOLDS ELECTRICAL \& ENGINEERING CO REYNOLDS ELECTRICAL

NEVADA TEST
MERCURY

NV 89023 
EFFECTIVE DATE: $07 / 01 / 94$ EXPIRATION DATE: $07 / 31 / 94$

FSC

COOE EXCESS PROPERTY DESCRIPTION

REFERENCE NUMBER : 575

6720 SCOPE CAMERA MFR: TEKTRONIX MOOEL: C51 $S / N$ : $B 094825$

REPORT NUMBER: $899305-4039$

REFERENCE NUMBER : 576

6720 SCOPE CAMERA MFR: TEKTRONIX MODEL: C51 S/N: $B 096920$

REPORT NUMBER: $899305-4039$

ELIGIBLE EQUIPMENT

No OF

COND UNITS UNIT

ACQUISITION PRICE

$500001 \mathrm{EA}$

COST/UNIT:

TOTAL COST:

$\$ 1,695.92$
$\$ 1,695.92$

LINE ITEM NUMBER: 0046

$500001 \mathrm{EA}$

COST/UNIT:
TOTAL COST:

:INE ITEM NUMBER: 0047
CONTACT(S)

FOR FURTHER INFORMATION

CYNDI DELONG

LAS VEGAS

NV $89193-8521$

(702) $295-6753$

TO FREEZE EQUIPMENT

CYNDI DELONG

LAS VEGAS

(702) $295-6753$

NV $89193-8521$

LOCATION OF EQUIPMENT

REYNOLDS ELECTRICAL \& ENGINEER ING CO

NEVADA TEST SITE

NV 89023

MERCURY

OR FURTHER INFORMATION:

CYNDI DELONG

LAS VEGAS
(70?) $295-6753$

NV $89193-8521$

TO FREEZE EQUIPMENT:

CYNDI DELONG

LAS VEGAS

(702) $295-6753$

NV $89193-8521$

LOCATION OF EQUIPMENT:

REYNOLDS ELECTRICAL \& ENGINEERING CO

NEVADA TEST SITE

MERCURY

NV 89023 
EFFECIIVE DATE: 07/01/94 EXPIRATION DATE: $07 / 31 / 94$

FSC

CODE EXCESS PROPERTY DESCRIPIION

REFERENCE MUMBER : 577

6720 SCOPE CAMERA MFR: TEKTRONIX MOOEL: C51 S/N: 8096372

REPORT NUMBER: $899305 \cdot 4039$

LINE ITEM NUMBER: 0048

\section{REFERENCE NUMBER : 578}

6720 SCOPE CAMERA MFR: TEKTRONIX MOOEL: C51 S/N: B096762

REPORT NUMBER: $899305-4039$

No of

COND UNITS UNIT

ACQUISITION PRICE

$500001 \mathrm{EA}$

COST/UNIT:
TOTAL COST:

$\$ 2,399.08$ $\$ 2,399.08$

(702) $295-6753$

TO FREEZE EQUIPMENT:

CYNDI DELONG

LAS VEGAS

(702) $295-6753$

NV 89193-8521

LOCATION OF EQUIPMENT :

REYNOLDS ELECTRICAL \& ENGINEERING CO

NEVADA TEST SITE

MERCURY

NV 89023

$500001 \mathrm{EA}$

COST/UNIT:

LINE ITEM NUMBER: 0049
$\$ 2.506 .86$ $\$ 2,506.86$
FOR FURTHER INFORMATION:

CYNDI DELONG

LAS VEGAS

NV $89193-8521$

TO FREEZE EQUI PMENT :

CYNDI DELONG

LAS VEGAS

(702) 295-6753

NV 89193-8521

LOCATION OF EQUIPMENT:

REYNOLDS ELECTRICAL \& ENGINEERING CO

NEVADA TEST SITE

MERCURY

NV 89023 
EFFECTIVE DATE: $07 / 01 / 94$ EXPIRATION DATE: $07 / 31 / 94$

FSC

\section{COOE EXCESS PROPERTY DESCRIPTION}

REFERENCE NUMBER : 579

6720 SCOPE CAMERA MFR: IEKTRONIX MOOEL: C51 SCOPE CAMERA M R
S/N: B096275

REPORT NUMBER: $899305-4039$

REFERENCE NUMBER : 580

6720 SCOPE CAMERA MFR: TEKTRONIX MODEL: $\quad 51$ $S / N: \quad B 094947$

REPORT NUMBER: $899305-4039$
ELIGIBLE EQUIPMENT
$500001 \mathrm{EA}$ TOTAL COST:

LINE ITEM NUMBER: 0050

$500001 E A$ COST.UNIT:
TOTAL COST:

LINE ITEM NUMBER: 0051

\begin{tabular}{clr}
$\begin{array}{c}\text { NO OF } \\
\text { COND UNITS UNIT }\end{array}$ & \multicolumn{2}{c}{ ACQUISITION PRICE } \\
500001 EA & $\begin{array}{r}\text { COST/UNIT: } \\
\text { TOTAL COST: }\end{array}$ & $\$ 2,506.86$ \\
$\$ 2,506.86$
\end{tabular}

CONTACTSS)

FOR FURTHER INFORMATION:

CYNDI DELONG

LAS VEGAS

(702) 295-6753

NV $89193-8521$

TO FREEZE EQUIPMENT:

CYMOI DELONG

LAS VEGAS

NV $89193-8521$

(702) $295-6753$

LOCATION OF EQUIPMENT:

REYNOLDS ELECIRICAL \& ENGINEERING CO REYNOLDS ELECTRICAL

NEVADA TEST SITE

NV 89023
FOR FURTHER INFORMATION:

CYNDI DELONG

LAS VEGAS

(702) $295-6753$

NV 89193-8521

TO FREEZE EQUIPMENT:

CYNDI DELONG

LAS VEGAS

(702) 295-6753

NV $89193-852$ ?

LOCATION OF EQUIPMENT:

REYNOLDS ELECTRICAL \& ENGINEERING CO REYNOLA TEST

NEVADA TEST
MERCURY

NV 89023 
FHFETIIVF DATI: O7/01/94 EXPIRATION DATE: 07/31/94

fSL:

FOOE EXIESS PROPERTY DESCRIPTION

REFERENCE NUMBER : 581

67:0 SCOPE CAMERA MFR: TEKIRONIX MODEL: C51 $S / N: \quad 6095178$

REFOR: NUMEER: $899305-4030$

REFERENCE NUMEER : 592 6720 SLOFE CAMERA MFR: TEKTRONIX MOOEL: CS1
SIN: BOQSOS

REPORT NUMBER: $899305-4039$
NO OF

COND UNITS UNIT

ACQUISITION PRICE

$5 \quad 00001 \mathrm{EA}$

COST/UNIT:

TOIAL COST:

$\$ 1,943.34$

$\$ 1,943.34$

LINE ITEM NUMBER: DOS?

$500001 \mathrm{EA}$

COST/UNIT:
TOTAL COST:

$\$ 1,892.37$

$\$ 1,892.37$

LINE ITEM NUMBER: 0053
FOR FURTHER INFORMATION:

CYNDI DELONG

LAS VEGAS

NV $89193-8521$

TO FREEZE EQUIPMENT

CYNDI DELONG

LAS VEGAS
(702) $295-6753$

NV $89193-8521$

NV $89193-8521$

NV $89193-8521$

LOCATION OF EQUIPMENT:

REYNOLDS ELECTRICAL \& ENGINEERING CO

NEVADA TEST SITE
NV 89023

MERCURY 


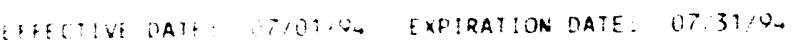

isc

COOE EXCESS PROFERIY DESCR!FIION

REFERENCS AUMBER : 995

SIZ SCOPE CAMERA MFR: TEKTFONIX MOOEL: WS S/N. 5005,5

PFPOE: AMEER: $890305 \cdot 4030$

RE ERENCE NUMEEF : SOD

672. SCOPE CAMERA MFR: TEKTRONIX MODEL: C5, SiA: 5 QSOC?
ELIGIBLE EQUIPMENT
No of

CUND UNIIS UNII

ACOUISIIIION PRICE

$500001 E A$

COSITUNIT:

TOTAL COST:

$\$ 2.227 .20$

$\$ 2,227.20$

LINE ITEM NUMBER: 005-
COST/UNIT: TOTAL COST:

LAE IIEM NUMBER: 0055
$\$ 2,530.77$ $\$ 2,530.77$
FOR FURTHER INFORMATION:

CYNDI DELONG

(702) $295-6753$

TO FREEZE EQUIPMENT:

CYNDI DELONG

LAS VEGAS

NV 89193-8521

(702) $295-6753$

LOCATION OF EOUIPMENT

REYNOLDS ELECTRICAL ¿̊ ENGINEERING CO

NEVADA TEST SITE

MERCURY

NV 89023 
EFFECIIVE DATE: 07/01/92 EXP:RATION DATE: 07/31/94

FSC
COOL EXCESS PROPFRTY OESCRIPTION

REFERENCE NUMBER : 585

6720 SCOPE CAMERA MFR: TEKIRONIX MODEL: 251 $S / N:$ BOOSTS:

REPORT NUMBER: $800305-4030$

REFERENCE NUMBER : 586

TEKTRONIX MOOEL: CS $S / N$ : $B 094869$

REPORT NUMBER: $899305-4039$
ELIGIBLE EQUIPMENI

NO OF
COND UNITS UNIT

500001 EA COST/UNIT:
TOIAL COST:

LINE ITEM NUMBER: 0056
COST/UNIT:
TOTAL COST:
$\$ 2.088 .00$ $\$ 2,088.00$

$\$ 1,746.42$
FOR FURTHER INFORMATION:

CYNDI DELONG

LAS VEGAS

(702) $295-6753$

NV $89193-8521$

TO FREEZE EQUIPMENT:

CYNDI DELONG

LAS VEGAS

(702) 295.6753

LOCATION OF EOUIPMENT

REYNOLDS ELECTRICAL \& ENGINEERING CO NEVACA TEST SITE

MERCURY

NV 89023 
fFFETIVE DATE: 07/01/04 EXFiRATION DATE: 07/31/94

isc

\section{CODE EXCESS DROFERTY DESTRIPIION}

REFERENCE NUMGER : 587

GT2 SCDPE CAMERA MFR: TEKTRON:X MOOEL: CS SIN: BOOSOT8

REPORT NUMEER: $899305 \div 039$

LINE ITEM NUMBER: 005
NO OF

COND UNIIS UNII

$500001 E A$

COST/UNIT: TOTAL COST:

$\$ 2,210.44$ $\$ 2.210 .44$
CYNOI DELONG
LAS VEGAS

LAS VEGAS
(702) $295-6753$

IO FREEZE EQUIPMENT:

CYNDI DELONG

LAS VEGAS

(702) 295-6753

LOCAIION OF EQUIPMENT:

REYNOLDS ELECTRICAL \& ENGINEERING CO

NEVADA TEST SITE

MERCURY

NV $890 \div 3$
COST UNIT:

TOTAL COST:
$\$ 1.198 .00$ $\$ 1,198.00$
RE:ERENCE NUMGFF: 588

SCOLE. CAMERA ELECTRONIC CON DOLLO SHCTER DHOTOMETER EXPOSURE AID RANCE F:NDE = BOUSING AUPO S:NULE-SWEEF
ROLI F :LM. OWLR FROM SCOFE. TEKTRONIX

- 00001 iA

rotal cost:

REPORT NUMBER: $898355-4088$

LINE :TEM NUMBER: D062
FOR FURTHER INFORMATION:

ELOY N. GIRON

ALBUOUERQUE

(505) $844-2746$

NM 87185

TO FREEZE EQUIPMENT:

ELOY N. GIRON

ALBUOUEROUE

(505) $844-2746$

NM 87185

LOCATION OF EOUIPMENT:

SANDIA NATIONAL LABORATORIES PROPERTY REAPPLICATION DIVISION 7617 ALBUOUEROUE 
IFFECTIVE DATE: 07/01/04 EXPIRATION DATE: 07/31/96

\section{COOE EXCESS PROPERIY DESCRIPTION}

REFEREMCE HUMEER : 589

O720 TERMINAL GRAPHILS IHE COLOR GRAPHIC RECORDER SYSIEM IS DESIGNED FOR PECORDING FHOTO GRACHIC HARD COFY FROM THE OUTPUT OF RASTER SCAN TERMINALS I2OV AC, OO HZ. WORKS W/V930752. MATRIX INS, 3000, 314

ELIGIBLE EOUIPMENT

\section{No of}

REPOR: NLMBER: $398355-4038$

LINE ITEM NUMGER: 0060

RFFFRFNCF NUMEER: 590

T73 EIECTRISCREEN IS 10 HWXO'LONG SCREEN IS ELCTPIC WILL COME AUTD is MADE -O HANS GENEFAL USED ON CONFERENCE ROOMS SCFEEN :S STILL IN BOX. SIEHA RT FS FSA-B.
COND UNITS UNI

ACOUISITION PRICE

$600001 \mathrm{EA}$ COST/UNIT:
TOTAL COST:

$\$ 9.755 .00$
$\$ 9.755 .00$

FOR FURTHER

ELOY N. GIRON

ALBUQUEROUE

(505) $844 \cdot 2746$

NM 87185

TO FREEZE EQUIPMENT:

ELOY N. GIRON

ALBUOUERQUE

(505) $844-2746$

NM 87185

LOCAIION OF EQUIPMENT:

SANDIA NATIONAL LABORATORIES

PROPERTY REAPPLICATION DIVISION 7617

PROPERTY REAPPLICATION DIV
COST/UNIT:

TOTAL COST:

$00001 \mathrm{EA}$

$\$ 2,197.00$
FOR FURTHER INFORMATION:

ELOY N. GIRON

ALBUDUEROUE

(505) $844-2746$

NM 87185

TO FREEZE EQUIPMENT

ELOY N. GIRON

ALBUQUEROUE

NM 87185

(505) $844-2746$

LOCATION OF EQUIPMENT:

SAMDIA MATIONAL LABORATORIES

PROPERTY REAPPLICATION DIVISION 7617

PROPERTY REAPPLICATION DIVISION 7617

NM 87123 
EFFECTIVE DATE: 07/0:/0, EXFIRAIION DATE: 07;31/94

FSC

COOE EXCESS PROPERIY DESCRIPIION

RE FERENCE NUMBER : 591

O730 ELECTRISCREEN SCREEN IS 10LT X 9FT LONG SCREEN IS ELECTRIC. WILL COME

IN BOX. IOVVAC, OO HZ. STEWART ES, A-B.

REPOR: NUMBER: $898355-4088$

LINE ITEM NUMBER: 0064

REFERENCE NUMBER : 592
G730 PROJECTOR, MOTION FIX EIKI PHOTO ANALYZER: 16 MMM SOUND PROJECTOR WITH

FIIM SPEED OF ZO TRAMES/SEC STANDARD SOUND SPEEO ANO SIILL PICTURE.

I2OVAC, SO HZ . I.W !NIL, 224SMKV, 27680.

REPORT NUMBER: $898355-4088$

LINE ITEM NUMBER: 0066
No of

COND UNITS UNII

ACQUISITION PRICE

$\$ 2,197.00$

$100001 \mathrm{EA}$

COST/UNIT:
TOTAL COST:

$\$ 2,197.00$

\section{ELOY N. GIRON \\ ALBUQUEROUE}

(505) $844-2740$

NM 87185

TO FREEZE EOUIPMENT:

ELOY N. GIRON

ELOY N. GIRON

(505) $844-2746$

NM 87185

LOCATION OF EQUIPMENT:

SANDIA NATIONAL LABORATORIES

PROPERTY REAPPLICATION DIVISION 7617

PROPERTY REAPPLICATION DIVISION 7617
ALBUQUERQUE
NM 87123

$500001 \mathrm{EA}$

COSI/UNIT:

TOTAL COST:

$\$ 2.795 .00$

INFORMATION

ELOY N. GIRON

ALBUOUERQUE

(505) $844-2746$

NM 87185

TO FREEZE EQUIPMENT:

ELOY N. GIRON

(505) $844-2746$

LOCATION OF EQUIPMENT:

SANDIA NATIONAL LABORATORIES

PROPERTY REAPPLICATION DIVISION 7617

PROPERTY REAPPLICATION DIVISION 7617
ALBUQUEROUE 


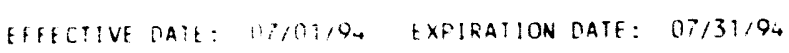

$+\mathrm{si}:$

\section{CODE EXCESS PROFERIY DESCR!FIION}

RE IERENCE NUMGER : 593

OTSO PROJECTOR, MOIION FIX EIK! FHOTO ANALYZER: IOMM SOUND FROJECTOR WITH

IILM SPEED OF 24 FRAMES/SECUND S TO SPEED AND STILL PICTURE. 12OVAC, 60

H2. 10 : N NTI. 226 SMKV. 27679.

REFOR! NUMAER: $898355-4088$

LINE ITEM NUMBER: 0067

REFERENCE NUMBER : 596

673O PROJECTOR, MOTION PIX HAS REMOTE WITH STILL F:CTUJE, FORWARD AND REVERSE 7 D0001 EA WITH SINGLE CO NTROL. ZZOVAC, GO HZ. LAMP DOES NO: COME ON. NAC,

DF $16 C, 81150$.
NO OF

CUND UNITS UNIT

ACOUISITION PRICE

$\$ 2,795.00$

U0001 EA

COSI/UMIT:

TOTAL COST:
COST/UNIT:

TOTAL COST

$\$ 2.795 .00$
$\$ 2.795 .00$

FOR FURTHER INFORMATION:

ELOY N. GIRON

ALBUOUERQUE

(505) $844-2746$

TO FREEZE EOUIPMENT

ELOY N. GIRËN

ALBUOUERQUE

(505) $844-2746$

NM 87185

LOCATION OF EQUIPMENT:

SANDIA NATIONAL LABORATORIES

PROPERTY REAPPLICATION DIV. 7617

AL GUQUERQUE

NM 87123 


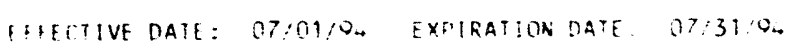

CONE EXCESS PROPERTY DESCRIPIION

QE SERENCE NUMBER : 405

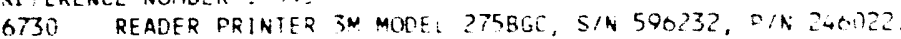

NO OF

CONO UNITS UNII

600001 EA
ACQUISITION PRICE

COST/UNIT:
TOTAL COST:

INE :TEM NUMBER: 0380

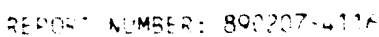

REFERENCE NUMEEF: : SES

OTS. DENSITOMETEK

MFR: MACSET+ MOOE: : TESZ-

$=1 \mathrm{~N}: 2290 \mathrm{CE}$
$50000: E A$

REFUET NUMEER: $899305-403$
LINE :TEM NUMGER: 0007
$\$ 2,500.00$ $\$ 2,500.00$
TOTAL COST

$\$ 2,322.00$
FOR FURTHER INFORMATICN:

CYNDI DELONG

LAS VEGAS

(702) $295-6753$

NV $89193-8521$

TO FREEZE EQUIPMENT:

CYNDI DELONG

LAS VEGAS

NV 89193-8521
ID $83415-4100$

ID $83415 \cdot 4100$

LOCATION OF EOUIPMENT :

IDAHO NATIONAL ENGINEERING LAB.

CF- 674 WAREHOUSE

$1083415-4100$

LOCATION OF EQUIPMENT:

REYNOLDS ELECTRICAL \& ENGINEERING CO REYNOLDS ELECTRICA

MERCURY

NV 89023 
EFFECTIVE DATE: $07 / 01 / 94$ EXPIRATION DATE: $07 / 31 / 94$
FSC

COOE EXCESS PROPERTY DESCRIPTION

REFERENCE NUMBER : 597

6760 DENSITOMETER

MFR: MACBETH MOOEL: TD903

S/N: 1152

REPORT NUMGER: $899305-4032$

REFERENCE NUMBER : 598

6760 DENSITOMETER

MFR: MACBETH MOOEL: TD903

S/N: 1182

REPORT NUMGER: $899305-4032$
No of

COND UNITS UNIT

ACQUISITION PRICE

$500001 E A$

COST/UNIT:

LINE ITEM NUMBER: 0011

$500001 \mathrm{EA}$

COST/UNIT:
TOTAL COST:

LINE ITEM NUMBER: C023
CONTACTSS)

\section{$\$ 2,410.80$} $\$ 2,410.80$

CYNDI DELONG

LAS VEGAS

(702) $295-6733$

NV $89193-8521$

TO FREEZE EQUIPMENT:

CYNDI DELONG

LAS VEGAS

(702) $295-6753$

LOCATION OF EQUIPMENT NEVADA TEST SITE

MERCURY

NV 89023
REYNOLDS ELECTRICAL \& ENGINEERING CO $\$ 2,410.80$
$\$ 2,410.80$
FOR FURTHER INFORMATION:

CYNDI DELONG

LAS VEGAS

(702) $295-6753$

NV $89193-8521$

TO FREEZE EQUIPMENT:

CYNDI DELONG

LAS VEGAS

(702) $295-6753$

NV $89193-8521$

LOCATION OF EQUIPMENT:

REYNOLDS ELECTRICAL \& ENGINEERING CO NEVADA TEST SITE

MERCURY

NV 89023 
EHECIIVE DATE: 0i/11/94 EXPIRATION DATE: 07/31/94

+SC

\section{CODE EXCESS PROPERTY DESCRIPIION}

REFERENCE NUMBER : 599

6760 GRAPHICS MANER

MFR: $3 M$ MODEL:

REPORT NLMEER: $899305-4032$

LINE ITEM NUMGER: 0024

REFERENCE NUMBER : 600

6910 PORIABLE POWER MANAGEMENI SIMULATOR (2 STEEL CASES) INCLUDES 1-TV

MONITOR AND 1 -PORTABLE VCR UNIT PSN: 7090.0003 YR. 1986

MFG: MONTANA STATE UNIV. ACO: 4/29/86 AA NO: CGG000002
REPORT NUMBER: $899401-4123$

LINE ITEM NUMBER: GOO2
COST/UNIT:

TOTAL COST:

$\$ 16,000.00$

NO OF

COND UNITS UNIT

COST/UNIT:

TOTAL COST:

\section{$\$ 4.228 .33$}

CYNDI DELONG
LAS VEGAS

LAS VEGAS
(702) $295-6753$

TO FREEZE EQUIPMENT :

CYNDI DELONG

LAS VEGAS

(702) 295-6753

NV $89193-8521$

LOCATION OF EQUIPMENT:

REYNOLDS ELECTRICAL \& ENGINEERING CO

NEVADA TEST SITE

MERCURY

NV 89023

FOR FURTHER INFORMATION:

JIM POPE

PHOENIX

(602) $352-2734$

AZ 85009

TO FREEZE EQUIPMENT:

JIM POPE

PHOENIX
(602) $352-2734$

A2 85009

OCATION OF EQUIPMENT:

WESTERN AREA POWER ADMINISTRATION PHOENIX AREA OFFICE

PHOENIX

AZ 85009 
EFFECTIVE DATE: 07/01/94 EXFIRATION DATE: 07/31/94

FSC

CODE EXCESS PROPERTY DESCRIPTION

REFERENCE NUMBER : 601

6910 POWER SIMULATOR SYSTEM ( 4 GOXES) INCLUDING 1 TV MONITOR AND 1 PORTABLE VCR UNIT. PSN: 7090.0002 YR. 1984

MFG: MONTANA STATE UNIVERSITY ACO: 3/26/84 AA NO: CGG0000017
NO OF

COND UN!TS UNI

$500001 E A$

\section{COST/UNIT:}

TOTAL COST:

CQUISITION PRICE

$\$ 17,000.00$
REPORT NUMBER: $899401-4123$

LINE ITEM NUMBER: G001
REFERENCE NUMBER : 602

7010 ANALYYZER, OPTICAL EGSG OPTICAL ANALYZER HAS SINGLE PRECISION ADDITION INPUT, LINE S YNCHRONOUS FRAMES, ONE START PULSE AND EXTRNAL START.

12OVAC, $60 \mathrm{HZ}$. CRT SHOWS INSTABILITY. 1215, 29101.
LINE ITEM NUMBER: 0001
$700001 \mathrm{EA}$ TOTAL COST:

\section{ELOY N. GIRON \\ ALBUOUERQUE}

(505) $844-2746$

NM 87185

TO FREEZE EQUIPMENT :

ELOY N. GIRON

ALBUOUEROUE

(505) $844-2746$

NM 87185

LOCATION OF EQUIPMENT:

SANDIA NATIONAL LABORATORIES

PROPERTY REAPPLICATION DIV. 7617

PROPERTY REAPPLICATION DIV. 7617
ALBUOUERQUE
NM 87123 
EFFECIIVE DATF: 07/01/9 EXIRATION DATE: O7/31/49

$\mathrm{FSC}$

\section{COOE EXCESS PROPERTY DESCRIPIION}

REFERENCE NUMBER : 003

7010 GERNOULL! 6OX WIIH TWO 20+20 DRIVES AND IT IS AN EXTERNAL SUBSYSTEM WHICH CONNE CIS IO A COMPUTER THROUGM ONE OF IOMEGAS HOST INTERFACE BOARDS. $120: \because A C$, BO HZ. IOMEGA, B220X, 1282402. no of

COND UNITS UNI

60001 EA

COST/UNIT:

TOTAL COST:

LINE ITEM NUMBER: 0003
REDORT NUMBER: $898355-4097$

REFERENCE NUMBER : $6 . j 4$

7010 COLOR PRINTER HAS 300 DOTS FER INCH, 35 RESIDENT FONTS, A BUILI IN 20 MB HARD D RIVE, WILL PRINT TO THERMAL TRANSFER DAPER, 12OVAC, $60 \mathrm{HZ}$. MITS UBISHI, G650105, SER. M10444.

$600001 E A$

(

LINE ITEM NUMBER: 0043
COST/UNIT:
TOTAL COST:
$\$ 18,499.00$

$\$ 18,499.00$
FOR FURTHER INFORMATION

ELOY N. GIRON

ALBUOUERQUE

(505) 844.2746

NM 87185

IO FREEZE EQUIPMENT:

ELOY N. GIRON

ALBUOUEROUE

(505) $844-2746$

NM 87185

LOCATION OF EOUIPMENT:

SANDIA NATIONAL LABORATORIES

PROPERTY REAPPLICATION DIVISION 7617

ALBUQUERQUE NM 87123 
EFFECTIVE DATE: 07/01/94 EXPIRATION DATE: $07 / 31 / 94$

\section{FSC \\ COOE EXCESS PROPERTY DESCRIPTION}

REFERENCE NUMBER : 605

7010 COMP ACC XPANDR IBM EXPANSION UNIT THAT HAS A 20 MB HARD DRIVE.

$120 \mathrm{VAC}, 60 \mathrm{HZ}$.: BM, 5161, SER. 5053164.

REPORT NUMBER: $898355 \cdot 4097$

LINE ITEM NUMBER: 0004

REFERENCE NUMBER : 606

7010 COMPUTER 1986 APPLE MACINTOSH PLUS S/N F651434M0001A
No of

COND UNITS UNIT

600001 EA
ACQUISITION PRICE

COSTIUNIT:

(n)

REPORT NUMBER: $898277-4090$

LINE ITEM NUMBER: 0002

\section{$\$ 2,373.00$}

$\$ 2,373.00$

ALY N GIRONFORMATION:

ALBUOUERQUE

(505) $844-2746$

NM 87185

TO FREEZE EQUIPMENT

ELOY N. GIRON

ALBUOUEROUE

(505) $844-2746$

NM 87185

LOCATION OF EQUIPMENT:

SAMDIA MATIONAL LABORATORIES

PROPERTY REAPPLICATION DIV. 7617

PROPERTY REAPPLICATION DIV. 7617
ALBUOUEROUE
NM 87123
FOR FURTHER INFORMATION:

TED TORREZ

LOVELAND

(303) $490-7212$

CO 80539-3003

TO FREEZE EQUIPMENT:

TED TORREZ
LOVELAND

(303) $490-7212$

CO 80539-3003

LOCATION OF EOUIPMENT:

WESIERN AREA POWER ADMINISTRATION

5555 EAST CROSSROADS BLVO

5555 EAST CROSSROADS BLVD

LOVELAND

CO $80538-8986$ 
fFFECTIVE DATE: OT/01/04 EXPIRATION DATE: $0 i / 31 / 94$

\section{$\triangle S C$ \\ COOE EXCESS PROPERIY DESCRIPIION}

REFERENCE NUMBER : O07

T010 COMPUTER 1987 APFLE MUDEL:MACINTOSH SE S/N F7174FMMSO10

REPORT NUMEES: $99827 \% \cdot .90$

LINE ITEM NUMBER: 0001

REFERENCE NUMBER : 008

7010 COMPUTER 1987 IEM MOOEL: 5170,339 . DSCAT S/N 00267601

REPORT NUMBER: $898277 \cdot 40917$

$$
\text { NO OF }
$$

COND UNIIS UNIT

700001 EA

COST/UN1T:

TOTAL COST:

ACQUISITION PRICE

\section{$\$ 2,082.84$}

$\$ 2,082.84$

TED TORREZ

LOVELAND
(303) 4907212

Co $80539-3003$

TO FREEZE EOUIPMENT:

TED TORREZ

LOVELAND

(303) $490-7212$

CO $80539 \cdot 3003$

LOCATION OF EOUIFMENT:

WESTERN AREA POWER ADMINISIRATION

5555 EAST CROSSROADS BLVD

LOVELAND

CO $80538-8986$

\section{COST/UNIT: \\ TOTAL COST:}

$\$ 3,905.00$

$\$ 3,905.00$
FOR FURTHER INFORMATION:

TED TORREZ

LOVELAND

LOVELAND
(303) $490-7212$

CO $80539-3003$

TO FREEZE EOUIPMENT:

IED TORREZ

LOVELAND

(303) $490-7212$

CO $80539-3003$

LOCATION OF EOUIPMENT:

WESTERN AREA POWER ADMINISTRATION

5555 EAST CROSSROADS BLVD

LOVELAND

Co $80538-8986$ 
EFFECIIVF DATE: OI:01/94 EXFIRATION DATE: 07/31/94

$\mathrm{SC}$

CODE EXCESS PROPERIY DESCRIPIION

REFERENCE NUMBER : 000

7010 COMPUTER 1937 IANDON MODEL: TM7002 W/MONITOR S/N 70421
REFOR: NUMER: $908277 \cdot .000$

IINE ITEM NUMBER: 0004

REFERENCE NUMEER : 0 :

TO11 COMPUTER 9980 COMEAC MODEL: DESKPRO $2801405 / \mathrm{N}-912 A M 361404$

$4 \quad 00001$ EA

COST/UNIT:
TOTAL COST:

$\$ 4.220 .76$
$\$ 4.220 .76$

FOR FURTHER INFORMATION

TED TORREZ

OVELAND

(303) $490-7212$

Co $80539-3003$

TO FREEZE EQUIPMENT:

TED IORREZ

LOVELAND

(303) 490.7212

co $80539-3003$

LOCATION OF EQUIPMENT:

WESTERN AREA POWER ADMINISTRATION

S555 EAST CROSSROADS BLVO

LOVELAND

CO $80538-8986$ 
EFFECTIVE CAIE: OT/01/04 EXFIRATION DATE: OT/31/04

FST

COOE EXCESS PROPERIY DESCR:PIION

COND UNITS UNIT

ACOUISIIION PRICE

CONIACTIS)

7010 COMPUTER 280 COMPUIER WITH 3.1/2" DISK DRIVE, HARO DRIVE, PARALLEL PORT. 2 SERIAL FORIS AND VGA VIDEO FORT 1026 KB RAM. IZOVAC. IGM, MOC. 60.

ON001 EA

COST/UNIT: TOIAL COST:

\subsection{4 .00 $\$ .654 .00$}

.

LINE ITEM NUMBER: 0055

REFORT NUMEE: $398355-409$
RE EERENCE NUMGER:O12

$70 T$ COMFLIER 286 MACHINE, SUOKS FAM Z SERIAL POR:S. PARALLEL PORT AND VIDEO

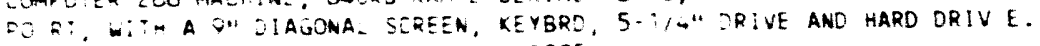

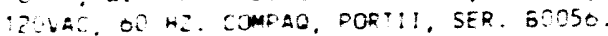

5 D0001 EA

COST/UNIT: TOTAL COST:
$\$ 3,150.00$ $\$ 3,150.00$

FOR FURTHER INFORMATION:

ELOY N. GIRON

AL BUDUEROUE

(505) 864.2746

TO FREEZE EQUIPMENT :

ELOY N. GIROW

ALBUOUEROUE

(505) $844-2746$

REPORT NUMGER: $898355 \cdot 609^{\circ}$
11NE ITEM NUMBER: 0046
LOCAIION OF EQUIPMENT

SAMDIA MATIONAL LABORATORIES

PROPERTY REAPPLICATION DIVISION T617

ALBUOUEROUE NM 87123 
FFFFTIIVE DAIF: 07/01/94 EXPIRATION DATE: $07 / 31 / 96$

ISC
COOE EXCESS PROPERTY OESCRIPIION

RE FERENCE NUMBER : 613

7010 COMPUIER 286 MACHINE 102- KB RAM 3-1/2" DISK DRIVE 73 M6 MARO DRIVE. PARAL LEL PORT. SERIAL FORT, AND VIDEO PORT. I2OVAC, SO HZ. IGM, MOO. PS260 vo of

COMD UMIIS UNII

ACOUISITION PRICE

$600001 E$ COST/UNIT:
TOTAL COST:
IINE ITEM NUMBER: 0054

REFERENCE NUMBER : 614

7010 COMPUTER 640KB RAM 5-1/4" DISK DRIVE, HARD DR:VE, 2 RS-232 PORIS, PARALLEL PORT AND VGA VIDEO PORT. $120 \mathrm{VAC}, 60 \mathrm{HZ}$. ATÓT, PCO30O. SER. 546328 !.

REPORT NUMBER: $898355-4091$
LINE ITEM MUMBER: D033
6 DOOOT EA COSTIUNIT:
TOTAL COST:

\section{$\$ 2.199 .00$} $\$ 2,149.00$
FOR FURTHER INFORMATION:

ELOY N. GIRON

ALBUOUEROUE

(505) $844-2746$

TO FREEZE EQUIPMENT:

ELOY N. GIROW

ALBUOUEROUE

(505) $846-2746$

MM 87185

LOCATION OF EOUIPMENT:

SANDIA MATIOMAL LABORATORIES PROPERTY REAPPLICATION DIVISION 7617 PROPERTY REAPPLICATION DIVISION 7617
ALBUOUEROUE 
COOE EXCESS PROPERIY DESCRIPIION

COND UNITS UNIT

i $00001 \mathrm{FA}$

COMFUTER OMA RAM ?; " $"$ OISK DRIVES, SERIAL PORT, PARALLEL PORT, AND

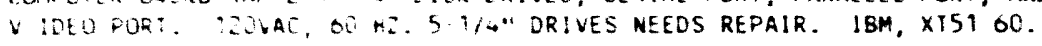

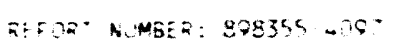

LINE ITEM NUMBER: DOO

DETEENCE NUMEE: : $=10$

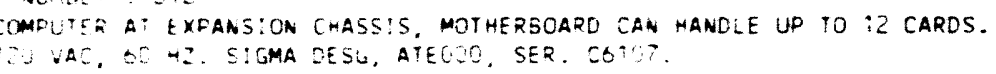

REPDRT NUMEER: $398355 \cdot-4097$

LINE ITEM NUMBER: 0066

S ONOOTEA
ACOUISITION PRICE

$\$ 2,247.00$

COST/UNIT:

TOTAL COST:

$52,247.00$

ELOY $N$. GIRON
ALBUOUEROUE

(505) $864 \cdot 2746$

IO FREEZE EOUIPMENT

ELOY N. GIRON

AL BUOUEROUE

(505) $844-2746$

LOCATION OF EOUIPMENT:

SANDIA NATIONAL LABORAIORIES

DROPERTY REAPPLICATION OIV. 7617

ALBUOUEROUE

Cost/unIt:

TOTAL COSI:

$\$ 1,449.00$

$\$ 1,469.00$

FOR FURTHER INFORMAIION

ELOY N. GIRON

ALBUQUERQUE

(505) $844-2746$

NM $87: 85$

TO FREEZE EQUIPMEN :

ELOY N. GIRON

ALBUQUEROUE

(505) $844-2746$

Mm 87185

LOCATION OF EOUIPMENT:

SAMD IA NATIOMAL LABORATORIES

PROPERTY REAPPLICATION DIV. 7617

AL BUOUEROUE 
EFFECIIVE DATE: $07 / 01 / 94$ EXPIRATION DATE: $07 / 31 / 94$

FSC

CODE EXCESS PROPERTY DESCRIPTION

REFERENCE MUMBER : 617

7010 COMPUTER MACINTOSH PLUS WITH 3-1/2" D!SK DRIVE AND 4096 KB RAM.

REPORT NUMBER: $898355-4097$

LINE ITEM NUMBER: 0030

REFERENCE MUMBER : 618

7010 COMPUTER, PERSOMAL 286 COMPUTER 6SOKB RAM. $120 \mathrm{VAC}, 60 \mathrm{HZ}$. IBM, 5170339,600001 EA 7072128.

Mo of

600002 EA
COMD UNITS UNII

ACQUISITION PRICE

COST/UNIT: TOTAL COSI:

$\$ 2,000.00$

54.000 .00

\author{
ELOY M. GIRON \\ ALBUOUEROUE \\ (505) $844-2746$ \\ 87185 \\ TO FREEZE EOUIPMENI: \\ ELOY N. GIRON \\ ALBUOUEROUE \\ (505) $846-2746$ \\ MM 87185 \\ LOCATION OF EOUIPMENT: \\ SAMDIA MATIOMAL LABORATORIES \\ PROPERIY REAPPLICAIION DIV. 7617 \\ ALBUOUEROUE \\ 87123
}

FOR FURTHER IMFORMATION:

$\cos /$ /UNIT

TOTAL COST:

$\$ 3,961.00$

$\$ 3.961 .00$

FOR FURTHER INFORMAIION:

ELOY N. GIROW

ALBUOUEROUE

(505) $864-2746$

m 87185

TO FREEZE EQUIPMENT

ELOY M. GIRON

ALBUOUEROUE

(505) $844-2746$

Mm 87185

LOCATION OF EQUIPMENT:

SAMDIA MATIONAL LABORATORIES

PROPERIY REAPPLICAIION DIVISION 7617

PROPERTY REAPPLICATION DIVISION 76 
EFFECIIVE DATE: OT/01/94 EXPIRAIION DATE: $07 / 31 / 94$

FSC

COOE EXCESS PROPERTY DESCRIPIION

$$
\text { ELIGIBLE EQUIPMENT }
$$

REFERENCE NUMGER : 019

7010 COMPUTER, PERSONAL 280 COMPUTER WITH 102- KB MEMORY, 2 5-1/4" DISK DOMIVES 2 SERIAL FORTS 2 PARALLEL PORTS AND VIDEO PORT 12OVAC 60 HZ ISM. 5170339 . SER. 7322677 .

REFORT NUMGER: $898355-409$

LINE ITEM NUMBER: 0002

REFERENCE NUMBER : OZO

COMPUTER, PEPSONAL A 386 COMPUTER, 4090 KG RAM 5-1/4" DISK DR!VE, $44 M B$ AARD DRIVE. ZOVAC, GC HZ. DATA GEN, DOTO046, SER. 849ACDOO.

COST/UNIT:
TOTAL COST:
ACOUISITION PRICE

COST/UNIT: IOTAL COST:

600001 EA

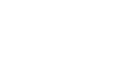

$\$ 33,033.00$
CONTACT(S)

FOR FURTHER INFORMATION

ALBUOUERQUE

(505) $844-2746$

NM 87185

IO FREEZE EOUIPMENT:

ELOY N. GIRON

ALBUOUEROUE

(505) $844-2746$

NM 87185

LOCATION OF EQUIPMENT

SANDIA NATIONAL LABORATORIES

PROPERTY REAPPLICATION DIVISION 7617

ALBUOUEROUE NM 87123

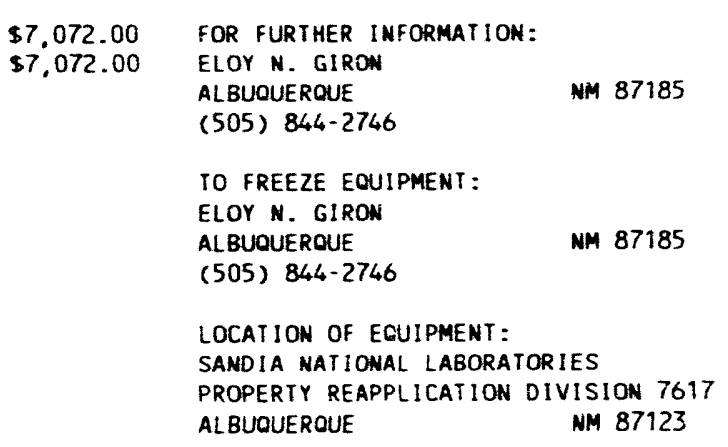


EFFECTIVE DATE: 07/01/94 EXPIRATION DATE: 07/31/94

COOE EXCESS PROPERTY DESCRIPIION

REFERENCE NUMBER : 621

7010 COMPUTER, PERSONAL OC WITH SLOKB RAM 2 5-1/4" DISK DRIVES, HARD DRIVE,

SERIAL PORT, VIDEO FORT AND PARALLEL PORT. 120VAC, 60 HZ. IBM, 5150174, SER. 1075215.

REPORT NUMGER: $898355-4091$

LINE ITEM NUMBER: 0005

REFERENCE NUMBER : 622

7010 COMPUTER, PERSONAL 640 KB RAM 5-1/4" DISK DR:VE, HARD DRIVE, PARALLEL PORT, SERIAL P ORT, AND VIDEO PORT. I2OVAC, SO HZ. IBM, 5150176, SER. 1126847.
NO OF

COND UNITS UNII

ACOUISITION PRICE

$600001 \mathrm{EA}$

COST/UNIT: TOTAL COST:

$\$ 1,843.00$ $\$ 1,843.00$

FOR FURTHER IMFORMAIION:

ELOY N. GIRON

ALBUOUERGUE

(505) $844-2746$

NM 87185

TO FREEZE EOUIPMENT:

ELOY N. GIRON

ALBUDUEROUE

(505) $844-2746$

NM 87185

LOCATION OF EQUIPMENT:

SANDIA NATIONAL LABORATORIES

PROPERIY REAPPLICATION DIVISION 7617

ALBUOUERQUE

NM 87123

600001 EA

COST/UNIT:

TOTAL COST:

$\$ 1.843 .00$

$\$ 1,843.00$

FOR FURTHER INFCRMATION:

ELOY N. GIRON

ALBUQUERQUE

(505) $844-2746$

NM 87185

TO FREEZE EOUIPMENT

ELOY N. GIRON

AL BUQUEROJE

(505) $844-2746$

NM 87185

LOCATION OF EOUIPMENT:

SAMDIA MATIONAL LABORATORIES

PROPERIY REAPPLICAIION OIVISION 7617

ALBUOUEROUE

NM 87123 
EFFECTIVE DATE: 07/01/94 EXPIRATION DATE: $07 / 31 / 94$

\section{FSC
COOE EXCESS PROPERTY DESCRIPIION}

REFERENCE NUMBER : 023

7010 OMPUTER PERSONAL PORTABLE COMPUTER WITH 8086 MICROPROCESSOR 272 KB

16 LINE , PER STEEN GY 80 COLUMNS AND STD KEYBRD. 120: $\therefore$, $60 \mathrm{HZ}$. HEW

PACK, HP 110 , SER. 2432 A208.

REPORT NUMBER: $898355 \cdot 4091$

LINE ITEM NUMBER: 0007

REFERENCE NUMBER : 624

作 COMPUTER, PERSONAL $640 \mathrm{~KB}$ RAM 5-1/4" DISK DRIVE, HARD DRIVE, SERIAL AND VIDEO PORT. 120VAC, 60 HZ. IBM, 5150176, SER. 1592695.
PORT, AN

$600001 \mathrm{EA}$ COST/UNIT:
TOTAL COST:

INE ITEM N'MBER: 0008

REPORT NUMBER: $898355-4091$
No of

ACQUISITION PRICE

\section{COST/UNIT:}

TOTAL COST:

\section{$\$ 2,097.00$ \\ $\$ 2,097.00$}

\section{FOR FURTHER INFORMATION: \\ ELOY $N$. GIRON \\ ALBUQUERQUE \\ (505) $844-2746$ \\ NM 87185}

TO FREEZE EQUIPMENT

ELOY N. GIRON

ALBUQUEROUE

(505) $844-2746$

NM 87185

LOCATION OF EQUIPMENT:

SANDIA NATIONAL LABORATORIES

PROPERTY REAPPLICATION DIVISION 7617

ALBUQUERQUE

NM 87123

FOR FURTHER INFORMATION:

ELOY N. GIRON

ALBUOUERQUE

(505) 844-2746

NM 87185

TO FREEZE EQUIPMENT :

ELOY N. GIRON

ALBUQUERQUE

(505) $844-2746$

NM 87185

LOCATION OF EOUIPMENT:

SANDIA NATIONAL LABORATORIES

PROPERTY REAPPLICATION DIVISION 7617

ALBUQUERQUE

NM 87123 
EFFECIIVE DATE: $07 / 01 / 94$ EXFIRATION DATE: $07 / 31 / 94$

FSC

\section{COOE EXCESS PROPERTY DESCRIPIION}

REFERENCE NUMBER : 025

7010 COMPUTER, PERSONAL XT MACHINE GQOK BASE MEMORY CGA VIDEO ADAPTER B800H VIDEO ADDRESS 2 5-1/4" FLOPPY DRIVES, 1 PARALLEL PORT, 1 SERIAL PORT. 12OVAC, $60 \mathrm{HZ}$. IBM, 5150176, SER. 1127454 .

REPORT NUMBER: $898355-4091$

LINE ITEM NUMBER: 0009

REFERENCE NUMBER : 626

7010 COMPUTER, PERSONAL XT COMPUTER WITH 640 KB RAM 5-1/4 INCH DISK DRIVE HARD DRIVE, 2 S ERIAL PORIS, PARALLEL PORT, AND VIDEO PORT. 120VAC, 60 HZ. IBM, 5160086, SER. 5697236

REPORT NUMBER: $898355-4091$

LINE ITEM NUMBER: 0010
NO OF

COND UNITS UNIT

ACOUISITION PRICE

$\$ 1,843.00$

$\$ 1,843.00$
COST/UNIT:
TOTAL COST:

00001 EA
$600001 E A$

COST/UNIT:
TOTAL COST:

$\$ 2.524 .00$ $\$ 2.524 .00$

FOR FURTHER INFORMATION

ELOY N. GIROM

ALBUQUERQUE

(505) 844-2746

NM 87185

TO FREEZE EQUIPMENT

ELOY N. GIRON

ALBUOUERQUE

(505) $844-2746$

NM 87185

LOCATION OF EQUIPMENT:

SANDIA NATIONAL LABORATORIES

PROPERTY REAPPLICATION DIVISION 7617

ALBUDUERQUE

NM 87123 
EFFECTIVE DATE: 07/01/04 EXPIRATION DATE: $07 / 31 / 94$

FSC
CODE EXCESS PROPERTY OESCRIPTION

REFERENCE NUMBER : 627

7010 COMPUTER PERSONAL 380 COMPUTER WITH 960 KB RAM $25-1 / 4 "$ DISK DRIVE, 20500001 EA MB HARD DRIVE. IZOVAC, 60 HZ. IBM, 5150174, SER. 787923.

REPORT NUMBER: $898355-2091$

INE ITEM NUMBER: 0011

REFERENCE NUMGER : 628

7010 COMPUTER PERSONAL XI COMPUTER HITH $640 \mathrm{~KB}$ RAM $25-1 / 4 "$ DISK DRIVE, HARD DRIVE, PARA LEL PORT AND SERIAL PORT. I20VAC, SO HZ. IBM, 5150176, SER. 1337841.

REPORT NUMBER: $898355-4091$

LINE ITEM NUMBER: 0012
NO OF

COND UNITS UNIT ACOUISITION PRICE

COST/UNIT:

TOTAL COST:
$\$ 1,843.00$
$\$ 1,843.00$

FOR FURTHER INFORMATION

ELOY N. GIRON

ALBUOUERQUE

(505) $844-2746$

NM 87185

TO FREEZE EQUIPMENT:

ELOY N. GIRON

ALBUQUERQUE

NM 87185

(505) $844-2746$

LOCATION OF EQUIPMENT:

SANDIA NATIONAL LABORATORIES

PROPERTY REAPPLICATION DIVISION 7617

PROPERTY REAPPLICATI

NM 87123

$\$ 1.843 .00$

$\$ 1,843.00$

- tOTAL COST:

FOR FURTHER INFORMATION:

ELOY N. GIRON

ALBUQUERQUE

(505) $844-2746$

NM 87185

TO FREEZE EQUIPMENT:

ELOY N. GIRON

ALBUQUEROUE

(505) $844-2746$

NM 87185

LOCATION OF EQUIPMENT:

SANDIA NATIONAL LABORATORIES

PROPERTY REAPPLICATION DIVISION 7617

PROPERTY REAPLICATION DI 
EFFECIIVE RATE: 07/01/04 EXPIRATION DATE: 07/31/94

\section{TOOE EXCESS PROPERTY DESCRIPTION}

REFERENCE NUMBER : 629 7010 COMPUIER, PERSONAL XT COMPUTER WITH 576 KB RAM, PARALLEL PORT, SERIAL
PORT, VIDEO PO RT, $25-1 / 4 "$ DISK DRIVES AND HARD DRIVE. 12OVAC, $60 \mathrm{HZ}$. IBM, 5150174, SER. 08597995

REPORT NUMBER: $898355-409$

INE ITEM NUMBER: 0013

REFERENCE NUMBER : 630

7010 COMPUTER, PERSONAL SYSTEM GOARD 8087, 640 KB MEMORY KEYGRD PARALLEL

PRINTER, VIDEO D ISPLAY UNIT, 2 DISKETIE DRIVES, ASYNC COMMUNICATIONS,

ALT ASYNC C OMMUNICATION. IZOVAC, $60 \mathrm{HZ}$. IBM.

REFORT NUMBER: $898355-409$

LINE ITEM NUMBER: 0014

$$
\text { NO OF }
$$

COND UNITS UNIT

ACQUISITION PRICE

$\$ 1,843.00$

COST/UNIT: TOTAL COST:

$\$ 1,843.00$

$00001 \mathrm{EA}$ TOTAL COST:

ELOY N. GIRON

ALBUQUERQUE

(505) $844-2746$

NM 87185

TO FREEZE EQUIPMENT

ELOY N. GIRON

ALBUOUERQUE

(505) $844-2746$

NM 87185

LOCATION OF EOUIPMENT:

SANDIA NATIONAL LABORATORIES

PROPERTY REAPPLICATION DIVISION 7617

ALBUOUEROUE

NM 87123

COST/UNIT:

TOTAL COST

$\$ 1,843.00$

$\$ 1,843.00$

OR FURTHER INFORMATION:

ELOY N. GIRON

ALBUQUEROUE

NM 87185

TO FREEZE EQUIPMENT:

ELOY N. GIRON

ALBUOUERQUE

(505) 844-2746

NM 87185

OCATION OF EQUIPMENT:

SANDIA NATIONAL LABORATORIES

PROPERTY REAPPLICATION DIVISION 7617

ALBUQUEROUE

NM 87123 
EFFCIIVE DATE: 0i/01/0. EXPIRATION DATE: 07/31/94

FSC

\section{COOE EXCESS PROPERTY DESCRIPIION}

REFERENCE NUMEER : O31

7010 COMPUTER, DERSONAL S4O KGM RAM 2 S-1/4" DISK DRIVE, SERIAL PORT, FARALIEI FORT AND $\checkmark$ IDEO PORT. IZOVAC, SO HZ. IBM.

REPOFT NUMEER - $398355 \cdot 409$

LINE ITEM NUMBER: 0015

REFERENCE NUMBER : 632

7010 COMPUTER, PERSONAL XI COMPUTER 640 KB RAM 5-1/4" DISK DRIVE, HARD DRIVE, 6 O0001 EA SERIAL PORT FARALIE: DORT AND VIDEO PORT. 12OVAC, $60 \mathrm{HZ}$. 1BM,

$5160080.507^{\prime} 59935$.

LINE ITEM NUMBER: 0016

$600001 \mathrm{EA}$
No of

COND UNITS UNIT

ACQUISITION PRICE

COST/UNIT:

TOTAL COST:

$\$ 1,843.00$

ELOY N. GIRON

ALBUQUERQUE

(505) $844-2746$

NM 87185

TO FREEZE EQUIPMENT:

ELOY N. GIRON

ALBUQUERQUE

(505) $844-2746$

NM 87185

LOCATION OF EOUIPMENT:

SANDIA NATIONAL LABORATORIES

PANOPERTY REAPPLICATION DIVISION 7617

ALBUOUEROUE

NM 87123

COST/UNIT:

TOTAL COST:

$\$ 2,524.00$

$\$ 2,524.00$

FOR FURTHER INFORMATION:

ELOY N. GIRON

ALBUOUERQUE

(505) $844-2746$

NM 87185

TO FREEZE EQUIPMENT :

ELOY N. GIRON

ALBUQUERQUE

(505) $844-2746$

NM 87185

LOCATION OF EQUIPMENT:

SANDIA NATIONAL LABORATORIES

PROPERTY REAPPLICATION DIVISION 7617

PROPERTY REAPPLICATION DIVISION 7617

NM 87123 
EFFECTIVE DATE: $07 / 01 / 94$ EXPIRATION DATE: $07 / 31 / 94$

FSC

\section{COOE EXCESS PROPERTY DESCRIPIION}

REFERENCE NUMBER : 633

7010 COMPUTER PERSONAL 64OKB MEMORY ONE 5-1/4" FLOPPY DRIVE SYSTEM BOARD 8087 KEYBRD, PA RALLEL PRINTER VIDEO DISPLAY UNIT. 120VAC, $60 \mathrm{HZ}$., IBM, 5150176, SER. 5411384 .

REPORT NUMBER: $898355-4091$

LINE ITEM NUMBER: 0018

REFERENCE NUMBER : 634

7010 COMPUTER, PERSONAL PC COMPUTER WITH 640 KB RAM SERIAL PORT, PARALLEL PORT, VIDEO POR T AND $51 / 4 "$ DISK DRIVE. I2OVAC, 60 HZ. IBM, 5160087, 5248491 .

REPORT NUMBER: $898355-4091$

LINE ITEM NUMBER: 0019
NO OF

COND UNITS UNIT

ACOUISITION PRICE

CONTACT(S)

600001 EA
COST/UNIT: TOTAL COST:

$\$ 1,843.00$

$\$ 1,843.00$

FOR FURTHER INFORMATION:

ELOY $N$. GIRON

ALBUQUERQUE

(505) $844-2746$

TO FREEZE EQUIPMENT :

ELOY N. GIRON

ALBUQUERQUE

(505) $844-2746$

LOCATION OF EQUIPMENT:

SANDIA NATIONAL LABORATORIES

PROPERTY REAPPLICATION DIVISION 7617

ALBUQUERQUE
NM 87185

87185
COST/UNIT:

TOTAL COST:
$\$ 2,524.00$ $\$ 2,524.00$
FOR FURTHER INFORMATION:

ELOY N. GIRON

ALBUQUERQUE

(505) $844-2746$

NM 87185

TO FREEZE EQUIPMENT :

ELOY N. GIRON

ALBUOUERQUE

(505) $844-2746$

MM 87185

LOCATION OF EQUIPMENT:

SANDIA NATIONAL LABORATORIES

PROPERTY REAPPLICATION DIVISION 7617

ALBUQUERQUE

NM 87123 
EFFECTIVE DATE: 07/01/94 EXPIRATION DATE: $07 / 31 / 94$

\section{CODE EXCESS PROPERTY DISCRIPTION}

REFERENCE NUMBER : 035

7010 COMPUTER, PERSONAL XT COMPUTER O60 KB RAM 25 -1/4" DISK DRIVE, SERIAL PORT PARALLEL PORT, VIDEO PORT AND IIMB HARD DRIVE. IZOVAC, $60 \mathrm{HZ}$.

REPORT NUMBER: $898355-4091$

LINE ITEM NUMBER: 0020

REFERENCE NUMEER : 630

7010 COMPUTER PERSONAL HAS AN 8" DIAGONAL SCREEN WITH TWO 5-1/4" DISK DRIVES

AN: A SO HA S A 2 OMB HARD D!SK, I2OVAC, 60 HZ. COMPAO, SER, 14220229.
$600001 E A$

COST/UNIT: TOTAL COST:

NO OF COND UNIIS UNIT COST/UNIT:
TOTAL COST:
$\$ 1,694.00$ $\$ 1.694 .00$

$00001 \mathrm{EA}$ TOTAL COST:

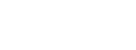

AL

ELOY N. GIRON
ALBUOUERQUE
(505) $844-2746$

\section{TO FREEZE EOUIPMENT:}

ELOY N. GIRON

ALBUOUEROUE

(505) $844-2746$

NM 87185

LOCATION OF EQUIPMENT.

SANDIA NATIONAL LABORATORIES

PROPERTY REAPPLICATION DIVISION 7617

ALBUOUEROUE

NM 87123

FOR FURTHER INFORMATION:

ELOY N. GIRON

ALBUQUERQUE

ALBUQUERQUE

NM 87185

TO FREEZE EQUIPMENT:

ELOY N. GIRON

ALBUQUEROUE

(505) $844-2746$

MM 87185

LOCATION OF EQUIPMENT:

SANDIA NATIONAL LABORATORIES

PROPERTY REAPPLICATION DIVISION 7617

ALBUQUERQUE

NM 87123 
EFFECTIVE DATE: 07/01/94 EXPIRATION DATE: 07/31/94

FSC

\section{COOE EXCESS PROPERTY DESCRIPTION}

ELIGIBLE EOUIPMENT

\section{REFERENCE NUMBER : 637}

7010 COMPUTER, PERSONAL SYSTEM BOARD $640 \mathrm{~KB}$ MEMORY KEYBRO PARALLEL PRINTER,

HZ. IB M, 5150174, SER. 838530 .

VIDEO DISPLAY UNIT, 2 DISKETTE DRIVES, 2 FIXED DISK DRIVES. 12OVAC, 60

No of

COND UNITS UNIT

$600001 \mathrm{EA}$

COST/UNIT:
TOTAL COST:

REPORT NUMGER: $898355-4091$

LINE ITEM NUMBER: 0022

\section{ACQUISITION PRICE}

OTAL COST:

\section{$\$ 1.843 .00$}

$\$ 1.843 .00$

FOR FURTHER INFORMATION:

ALBUOUEROUE

(505) $844-2746$

NM 87185

TO FREEZE EQUIPMENT:

ELOY N. GIRON

ALBUQUERQUE

(505) $844-2746$

NM 87185

LOCATION OF EOUIPMENT:

SANDIA MATIONAL LABORATORIES

PROPERTY REAPPLICAIION DIVISION 7617

ALBUOUEROUE

NM 87123

$\$ 2,362.00$

$\$ 2,342.00$

TOTAL COST:

FOR FURTHER INFORMATION:

ELOY N. GIRON

ALBUQUERQUE

(505) $844-2746$

NM 87185

TO FREEZE EOUIPMENT:

ELOY N. GIRON

ALBUQUEROUE

(505) $844-2746$

min 87185

LOCATION OF EQUIPMENT:

SANDIA MATIONAL LABORATORIES

PROPERTY REAPPLICATION DIVISION 7617

AL BUOUERQUE

NM 87123 
fst

\section{COOE EXCESS PROFERTY DESCRIPIION}

REFERENCE NUMBER : 030

T010 COMPUTER, PERSONAL 386AY MACHINE 387 MATH COPROCESSOR GLOK BASE MEMORY

$3200 \mathrm{~K}$ EXIENDE O MEMORY VGA VIDEO ADAPTER. I2OVAC, 60 HZ. IBM, 5170339

SER. 7213095 .
MO OF

COND UNITS UNIT $\quad$ ACOUISITION PRICE

$600001 E A$

COST/UN1T: TOTAL COST:

$\$ 3,932.00$

$\$ 3.932 .00$

(1)

FOR FURTHER INFORMATION:

ELOY $N$. GIRON

AL BUOUEROUE

(505) $84,4-2746$

m4 87185

TO FREEZE EOUIPMENT

ELOY N. GIROM

ALBUOUEROUE

(505) 846.2746

REPORT NUMEEF: $809355-409$

LINE ITEM NUMBER: 0026

FEFERENCE NUMEER : 040

COMPLIER, PERSONAL SYSTEM SOARD 808?, GLOKB MEMORY KEYBRD PARALLEL

ER:NTER, VIDEO 2 : SPLAV UN:T, 2 D:SKETTE DRIVES, ASYNC COMUNICATIONS,

$3 \quad 00001 E A$

COST/UNIT:

TOTAL COST:

$\$ 1.966 .00$

$\$ ? .964 .00$

LOCATION OF EOUIPMENT:

SAMDIA MarIONAL LABORATORIES

PROPERTY REAPPLICATION DIVISION 7617

ALBUOUEROUE NM 87123

A. ASYNE C OMMUNICATIONS I FIXED DISK DRIVE. IGM.

LINE ITEM MUMBER: 0027

\author{
FOR FURTHER INFORMATION: \\ ELOY N. GIRON \\ AL BUOUEROUE \\ (505) 846.2746 \\ ma 87185 \\ TO FREEZE EQUIPMENT: \\ ELOY N. GIRON \\ ALBUOUEROUE $864-2746$ \\ 87185 \\ LOCATION OF EOUIPMENT: \\ LOCATION OF EOUIPMENT: \\ PROPERTY REAPPLICATION DIVISION 7617 \\ PROPERTY REAPPLICATION DIVISION 7617
ALBUOUEROUE
}


EFFECTIVE DAIE: OT/01/04 EXFIRATION DATE: 07/31/96

FST

\section{COOE EXCESS PROPERIY DESCRIPTION}

REFERENCE NUMBER : 64

7010 COMPUTER. PERSONAL SYSTEM GOARD 8087 ONOKB MEMORY KEYGRD PARALLEL PRINTER VIDEO DISP LAY UNIT, 2 DISKETIE DRIVE. IGM, MOOEL 5160087, SER 4291832 .
No or

COND UNITS UNII

00001 EA

EA

cosinumit: TOTAL COST:

CONISIIION PRICE

$\$ 2.589 .00$ $\$ 2,589.00$

LINE ITEM NUMBER: 0028
CONTACT(S)

FOR FURTHER INFORMATION:

ELOY M. GIROM

AL BUOUEROUE

(505) $846-2746$

MM 87185

TO FREEZE EOUIPMENT:

ELOY W. GIRON
ALBUOUEROUE

(505) $844-2746$

MM $87 \div 85$

LOCATION OF EQUIPMENT:

SAMOIA MATIOUAL LABORATORIES

PROPERTY REAPPLICAIIOU OIVISION 7617

PROPERTY REAPPLICATION DIVISION ?617
ALQUOUFROUE

um 87123

\section{REFERENCE NUMBER : O4.}

7010 COMPUTER, PERSONAL 640 KG RAM, 2 FLOHPY DISK DRIVES PARALLEL POR 1,2

600001 EA SERIAL PORTS AN D CGA PORT. ZOVAC, $60 \mathrm{HZ}$. IGM, STIG2Z78, SER. 3013552.

REPORT NUMBER: $898355 \cdot 4091$

LINE IIEM NUMBER: 0029
TOTAL COST:

\section{$\$ 1,899.00$}

$\$ 1.399 .00$

\section{COST/UNIT:}

SOR FURTHER INFORMATION:

FiOY $M$. GIROA

AL BUQUEROLE

(505) $844-2746$

Nm 87185

TO FREEZE EQUIPMENT:

ELOY $N$. GIRON

ALBUOUEROUE

87185

(505) $844-2746$

LOCATION OF EOUIPMEN?

SAMOIA MATIONAL LABORATORIES

PROPERIY REAPPLICAIION OIVISION 7617

PROPERTY REAPPLICATION DIV

MM 87123 


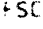 \\ COOE EXCESS PROEERTY OESCRIOTION}

REFERENCE NUMGER : OH

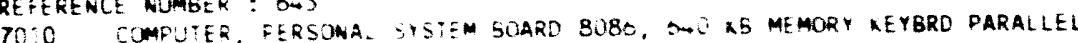
ERINIE V VDEC: O :SFLAY UNIT, Z DISAETIE DRIVE, ASYNC COMMUNICATIONS

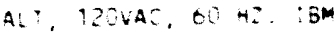

REFORT NLMEER: 809355 - NO

LIME ITEM MUMBER: C030

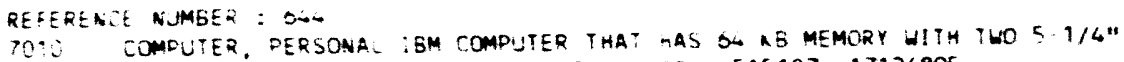

ilopor jisa za lves. izOVAC, oO Hz. :5m, 515107. "7126895.

6 O0001 EA

$\operatorname{cosi} / \sin 1 \mathrm{~T}$ :

TOTAL COST:

COND UNIIS UNII

- 0000: EA

COST/UNIT:

rotat $\cos 1$ :

REPORT NUMBER: $398355 \cdot 400^{\circ}$
LINE ITEM NUMBER : OU3

No of

ACOUISITION PRICE

S. .242 .00

5.242 .00

(2.0.00

ELOY N. GIRON

ALBUOUEROUE

(505) 84.2746

TO FREEZE EOUIPMEN:

ELOY N. GIRON

ALBUOUEROUE

(SOS) $864-2766$

LOCATION OF EOUI PMENT:

SAMOIA MATIOMAL LAGORATORIES PRCPERTY REAPPLICAIION DIVISION 7617

ALBUOUEROUF in 87123
59.694 .00 $\$ 1.694 .00$
FOR FURTHER INFORMATION

CLOY N. GIROM

ALBUDUEROUE

(505) $844-2746$

IO FREEZE EOUIPMENT

ELOY N. GIRCN

AL BUOUEROUE

(505) $8464-2746$

LOCATION OF EOUIPMENT:

SAMDIA MaTIOMAL LABORATORIES

PROPERTY REAPPLICATION CIVISION 7617

ALBUOUEROUE

im $37: 23$ 
EFFECTIVE DATE: 07/01/96 EXPIRATION DATE: 07/31/94

FSC

\section{COOE EXCESS PROPERTY OESCRIPIION}

REFEREMCE MUMBER : 645

7010 COMPUTER, PERSCMAL 6LOKB RAM, 5-1/4" DISK DRIVE HARD DRIVE, 2 SERIAL PORTS, PARALLEL PORT AND VIDEO PORT. I2OVAC, 60 HZ . 5-1/4" DISK DRIVE BAD. IBM, 5160086, SER. 54369795 .

REPOR: MUMBER: $898355-4001$

LINE ITEM MUMBER: 0032

REFEREMCE NUMBER : 646

7010 COMPUTER. PERSOMAL XY MACHINE $640 \mathrm{~K}$ BASE MEMORY CGA VIDEO ADAPTER B8OOH VIDEO ADDRES S A 5-1/4" FLOPPY, PARALLEL PORT, I SERIAL PORT. I2OVAC, $60 \mathrm{HZ}$. IBM. 5160,5463552 . wo of

Como units uni

ACOUISIIION PRICE

$700001 E A$ Cosifunit:
TOTAL COST:

\section{$\$ 2.183 .00$
$\$ 2,183.00$}

ELOY M. GIROW
ALBUDUEROUE

(505) $844-2746$

4 87185

TO FREEZE EQUIPMENT :

ELOY $N$. GIROW

ALBUOUEROUE

(S05) $844-2746$

87185

LOCATION OF ECUIPMEMT:

SAMDIA MATIOMAL LABORATORIES

PROPERTY REAPPLICATIOA DIVISION 7617

AL BUOUEROUE MT123
COST/UN 1T:

TOTAL COS: : $\$ 2,183.00$
$\$ 2.163 .00$
FOR FURTHER INFORMATION :

ELOY N. GIROM

AL BUOUEROUE

(505) $8646-2746$

87185

TO FREEZE EOUIPMENT:

ELOY N. GIROW

(505) $844-2746$

87185

LOCATIOM OF EOUIPMEMT :

SAMDIA MATIONAL LABORATORIES

PROPERTY REAPPLICATION DIVISION 7617

PROPERTY REAPPLICATION DIVISION 7617
ALBUOUERQUE 
EFFECTIVE DATE: 07;01/94 EXPIRATION DATE: 07/31/94

\section{CODE EXCESS PROPERTY DESCRIPTION}

REFERENCE NUMBER : 647

COMPUTER, PERSONAL SYSTEM GOARD 8087 G4OKB

PRINTER VIDEO DISP LAY UNIT I DISKETTE DRIVE ALT SYNCH COMMUNICATIONS,

FIXEO DISK DRIVE, 120VAC, $60 \mathrm{HZ}$. IBM, 5160

REPORT NUMGER: $898355-4091$

LINE IIEM NUMBER: 0036

\section{REFERENCE NUMBER : 348}

COMPUTER, PERSONAL AT WITH $2560 \mathrm{~K}$

DO $H Z$. IBM, 5170 , SER. 013 2815.
No of

COND UWITS UNIT

600001 EA

COSI/UNIT:
TOTAL COST:

COISITION PRICE

$\$ 2,183.00$

$\$ 2.183 .00$

ELOY N. GIRON

ALBUDUEROUE

(505) $844-2746$

TO FREEZE EQUIPAENT

ELOY N. GIROM

ALBUOUEROUE

(505) $844-2746$

LOCATION OF EQUIPMENT:

PROPERTY REAPPLICATION DIVISION 7617

ALBUOUEROUE

NM 87123

COST/UNIT:

YOTAL COST:

$\$ 2.529 .00$

\section{FOR FURTHER INFORMATION: \\ ELOY N. GIRON}

(505) $844-2746$

TO FREE2E EOUIPMENT:

ELOY N. GIROI

ALBUOUEROUE

MM 87185

REPORT NUMBER: $898355-4091$

LINE ITEM NUMBER: 0038
(505) $844-2746$

LOCATION OF EOUIPMENT:

TABOPATORIES PROPERTY REAPPLICATION DIVISION 7617 ALBUOUEROUE NM 87123 
EFFECTIVE DATE: 07/01/04 EXPIRATION DATF: 07/31/94

FSC

\section{COOE EXCESS PROPERTY DESCRIPTION}

REFERENCE NUMBER : 649

7010 COMPUTER, PERSONAL XI COMPUTER, 640KB RAM 2 5-1/4" CISX DRIVE, HARD DRIVE, SERIAL PO RT PARALLEL PORT, AND VIDEO PORT. $120 \mathrm{VAC}, 60 \mathrm{HZ}$. COMPAQ, DESKP RO, 45350516 .

REPORT NUMBER: $898355-409$

LINE ITEM NUMBER: 0039
No of

COND UNITS UNIT

$600001 \mathrm{EA}$

COST/UNIT: TOTAL COST:

\section{$\$ 2,882.00$ $2,882.00$}

ALBUOUERQUE

(505) $844-2746$

TO FREEZE EOUIPMENT:

ELOY $N$. GIRON

ALBUAUEROUE

(505) $844-2746$

LOCATION OF EOUIPMENT:

SANDIA NATIONAL LABORATORIES

PROPERTY REAPPLICATION DIVISION 7617

ALBUQUEROUE

NM 87123

REFERENCE NUMBER : 650

7010 COMPUTER, PERSONAL 1152 KB MEMORY TWO 5-1/4" FLOPPY DRIVES, TWO SERIAL PORT. TWO PAR ALLEL PORTS. 120VAC, 50 HZ. : BM, 5170, SFR. 0128332

$600001 \mathrm{EA}$

COST/UNIT:

TOTAL COST:

$\$ 3,466.00$

$\$ 3,466.00$

FOR FURTHER INFORMATIOM :

ELOY N. GIRON

ALBUQUEROUE

(505) $844-2746$

w4 87185

TO FREEZE EQUIPMENT:

ELOY N. GIRON

ALBUQUEROUE

(505) $844-2746$

4. 87185

LOCATION OF EQUIPMENT:

SANDIA NATIONAL LABORATORIES

PROPERTY REAPPLICATION DIVISION 7617

ALBUOUEROUE

NM $87 i 23$ 
EFFECTIVE DATE: O7/01/94 EXPIRAIION DATE: 07/31/94

FSC

\section{CODE EXCESS PROPERTY DESCRIPTION}

REFERENCE NUMBER : OS1 DRIVE, 2 SERIAL PORTS, PARALLEL PORT 120 VAC 60 HZ. NEEDS VIDEO BCARD. IBM, 5 170068, SER. 5123806

REPORT NUMBER: $898355-4091$

LINE ITEM NUMBER: 0041

REFERENCE NUMBER : 652

7010 COMPUTER PERSONAL SYSTEM BOARD 64OKB MEMORY KEYBRD PARALLEL PRINTER VIDEO DISPLAY U NIT 2 DISKETTE DRIVE, 120VAC, 60 HZ. IGM, 5160068, 6470762 .
NO OF

COND UNITS UNIT

ACQUISITION PRICE

700001 EA COST/UNIT:
TOIAL COST:

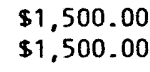

\section{ELOY N. GIRON \\ ALBUQUERQUE}

(505) $844-2746$

TO FREEZE EQUIPMENT

ELOY N. GIRON

ALBUQUERQUE

(505) $844-2746$

LOCATION OF EQUIPMENT:

SANDIA NATIONAL LABORATORIES

PROPERTY REAPPLICATION DIVISION 7617

ALBUOUEROUE

NM 87123

REPORT NUMGER: $898355-4091$

LINE ITEM NUMBER: 0042

\section{EA

\begin{abstract}
TOTAL COST:
\end{abstract}
$\$ 1.544 .00$
$\$ 1.544 .00$
FOR FURTHER INFORMATION:

ELOY N. GIRON

ALBUQUERQUE

(505) $844-2746$

NM 87185

TO FREEZE EQUIPMENT:

ELOY N. GIRON

ALBUQUERQUE

(505) $844-2746$

NM 87185

LOCATION OF EQUIPMENT

SANDIA NATIONAL LABORATORIES

PROPERTY REAPPLICATION DIVISION 7617

ALBUQUERQUE

NM 87123 
FFHCTIVE DATE: 07/01/94 EXFIRATION DATE: $07 / 31 / 94$

tse

\section{CODE EXCESS PROPERIY DESCRIPIION}

REFERENCE NUMGER : O53

7010 COMPUTER PERSONAL SYSTEM BOARD O4OKB MEMORY KEYBRD PARALLEL PRINTER,

VIDEO IDSPLAY UNIT 2 DISKETTE DRIVES ASYNC COMMUNICATIONS, 1 FIXED DISK DRIVE. I2OVAC, O0 HZ. 1BM, 5100, 6470096.

COND UNITS UNIT

ACQUISITION PRICE

$00001 \mathrm{EA}$ $\operatorname{COSI} / \mathrm{UNIT}$ TOTAL COST:

REPORT NUMEER : $898355 \cdot 4091$

LINE ITEM NUMBER: 0043

RE TERENCE NUMBER : 654

7010 COMPUTER PERSONAL SYSTEM SCARD GAOKB MEMORY KEYBRD PARALLEL PRINTER VIDEO DISPLAY U NIT Z DISKETTE DRIVE(S) ASYNC COMMUNICATIONS I F!XED OISK DRIVE(S). IZCVAC, SOI HZ. IEM.
REPORT NUMBER : $298355 \cdot-4091$

LINE ITEM NUMBER: 0044
$\$ 1,544.00$ $\$ 1,544.00$

$$
\text { ELOY N. GIRON }
$$

(505) $844-2746$

TO FREEZE EOUIPMENT:

ELOY N. GIRON

ALBUQUERQUE

(505) $844-2746$

NM 87185

LOCATION OF EOUIPMENT:

SANDIA NATIONAL LABORATORIES

SANDIA NATIONAL LABORATORIES

DROPERTY REAPPLICAY
ALBUQUEROUE

NM 87123

\author{
FOR FURTHER INFORMATION \\ ELOY N. GIRON \\ ALBUOUEROUE \\ (505) $844-2746$ \\ NM 87185 \\ TO FREEZE EQUIPMENT: \\ ELOY N. GIRON \\ ALBUOUEROUE \\ (505) $844-2746$ \\ NM 87185 \\ LOCATION OF EQUIPMENT: \\ SANDIA NAIIONAL LABORATORIES \\ PROPERTY REAPPLICATION DIVISION 7617 \\ PROPERTY REAPPLICATION DIVISION 7617 \\ AL BUQUERQUE \\ NM 87123
}


EFFECIIVE DATE: 07/01/94 EXPIRATION DAIE: $07 / 31 / 94$

\section{CODE EXCESS PROPERTY DESCRIFTION}

REFERENCE NUMBER : 055

RE 10 COMPUTER, PERSONAL SYSTEM EOARD $808 \%$ GLOK MEMORY KEYBRD PARALLEL

COMPUIER, PERSONAL SYSTEM BOARD $808 \%$, $440 \mathrm{~K}$ MEMORY KEYBRD PARALLEL
PRINTER VIDEO DISP LAY UNIT 2 DISKETTE DRIVES ASYNC COMMUNICATIONS.

'ZOVAC, OO HZ. IBM, 5100278, SER. 3023931.

REPORT NUMBER: $898355-4091$

LINE ITEM NUMGER: 0045

REFERENLE NUMBER : 650

C10 CONPUTER, PERSONAL 286A: MACHINE 287 MATH COPROCESSOR 640 KB GASE MEMORY 6 00001 EA 896K EXTEND ED MEMORY. 'ZOVAC, 60 HZ. IBM, 5170339, SER. 7143266

REPORT NUMBER: $898355-4091$

LINE ITEM NUMBER: 0047

COND UNITS UNII

NO OF

$\operatorname{COST} /$ UNIT:

TOTAL COST:

$\$ 2,247.00$

ELOY N. GIRON

ALBUQUERQUE

(505) $844-2746$

NM 87185

IO FREEZE EOUIPMENT:

ELOY N. GIRON

ALBUQUERQUE

(505) 844-2746

NM 87185

OCATION OF EQUIPMENT:

SANDIA MATIONAL LABORATORIES

PROPERTY REAPPLICATION DIVISION 7517

ALBUQUERQUE

NM 87123

\section{COST/UNIT:}

TOTAL COST:
$\$ 4.012 .00$
$\$ 4.012 .00$
FOR FURTHER INFORMATION:

ELOY N. GIRON

ALBUOUEROUE

505) $844-274$

NM 87185

TO FREEZE EQUIPMENT :

ELOY N. GIRON

(505) $844-2746$

NM 87185

LOCATION OF EOUI PMENT:

ANDIA MATIONAL LABORATORIES

PROPERTY REAPPLICATION DIVISION 7617

ALBUQUERQUE

(1) 87123 
EFFECTIVE DATE: $07 / 01 / 94$ EXPIRATION DATE: $07 / 31 / 94$

FSC

\section{COOE EXCESS PROPERTY DESCRIPTION}

REFERENCE NUMBER : 657

7010 COMPUTER, PERSONAL 386 COMPUTER HITH 8192KB RAM 5-1/4" DISK DRIVE, HARD DRIVE, SERIA L PORT VIDEO PORT. I2OVAC, 60 HZ. IBM, 5170339, 7170357.

LINE ITEM NUMBER: 0048

\section{REFERENCE NUMBER : 658}

7010 COMPUIER, PERSONAL G4OKB RAM 2 5-1/4" DISK DRIVES, PRINTER PORT, RS-232 7 00001 EA PORT AND VID EO PORT. 120VAC, 60 HZ. IBM, 5160278, SER. 3009621.
No of

COND UNITS UNIT

\section{ACQUISITION PRICE}

COST/UNIT:

TOTAL COST:

$\$ 3,020.00$ $\$ 3,020.00$

\section{$00001 \mathrm{EA}$}

A

TO FREEZE EQUIPMENT:

ELOY N. GIRON

(505) $844-2746$

NM 87185

LOCATION OF EQUIPMEMT:

SAMDIA NATIONAL LABORATORIES

PROPERTY REAPPLICATION DIVISION 7617

ALBUQUERQUE

\section{COST/UNII: TOTAL COST:}

$\$ 1.899 .00$

$\$ 1,899.00$

FOR FURTHER INFORMATION :

ELOY N. GIRON

ALBUDUEROUE

(505) $84.4-2746$

NM 87185

TO FREEZE EQUIPMENT:

ELOY N. GIRON

ALBUOUEROUE

(505) 844-2746

NM 87185

LOCATION OF EQUIPMENT:

SANDIA NATIOMAL LABORATORIES

PROPERTY REAPPLICATION DIVISION 7617

ALBUQUERQUE

NM 87123 
isC

\section{COOE EXCESS PROPERTY DESCRIPIION}

\section{REFERENCE NUMBER : 059}

7010 COMPUTER, PERSONAL 280 AT COMPUTER WITH PARALLEL PORT SERIAL PORT EGA VIDEO PORT, 512 KB RAM $25-1 / 4$ " DRIVES AND HARD DRIVE. 120VAC, $60 \mathrm{~Hz}$. IBM, 5170339.7153852 .

REFERENCE NUMBER : 660

7010 COMPUTER, PERSONAL 286 MICPO CHANNEL 287 MATH COPROCESSOR 384K EXTENDED MEMORY 64OK EASE MEMORY VGA. 120VAC, SO HZ. ISM, 8560041, SER. 8035749.

$600001 \mathrm{EA}$

No of

00001 EA
COND UNITS UNIT

ACOUISITION PRICE

COST/UNIT: TOTAL COST:

$\$ 3,932.00$ $\$ 3,932.00$

(5)

\section{TO FREEZE EQUIPMENT:}

ELOY $N$. GIRON

ALBUOUEROUE

(505) $844-2746$

LOCATION OF EOUIPMENT:

SANDIA NATIONAL LABORATORIES

PROPERTY REAPPLICATION DIVISION 7617

ALBUQUERQUE

NM 87123

COST/UNIT:
TOTAL COST:

$\$ 2,861.00$

$\$ 2,861.00$

FOR FURTHER INFORMATION:

ELOY N. GIRON

ALBUQUEROUE

(505) $844-2746$

NM 87185

TO FREEZE EQUIPMENT:

ELOY N. GIRON

ALBUQUEROUE

(505) $844-2746$

NM 87185

LOCATION OF EQUIPMENT:

SANDIA NATIONAL LABORATORIES

PROPERTY REAPPLICATION DIVISION 7617

ALBUQUERQUE

NM 87123 
EFFECIIVE DATE: 07/01/94 EXPIRATION DATE: 07/31/94

$\mathrm{FSC}$

COOE EXCESS PROPERTY DESCRIPTION

REFERENCE NUMBER : 661

7010 COMPUTER, PERSONAL 280XT MACHINE 640K BASE MEMORY L096K EXTENDED MEMORY EGA ADAPIER ADOOH VIDEO ADDRESS 43M HARD, ONE 3-1/4, ONE 5-1/4" FLOPPY. I2OV AC, $60 \mathrm{HZ}$. APCH401, SER. 71000037.

REPORT NUMBER: $899355-4001$

LINE ITEM NUMBER: 0053

REFERENCE NUMBER : 662

7010 COMPUTER, PERSONAL IBM 286 WITH 30 MB HARD DRIVE AND ALSO HAS A 3-1/4"

DISK DRIVE. 120VAC, $60 \mathrm{HZ}$. MOD. 8560071, SER. 9071396.

COSI/UNIT:

TOTAL COST:

LINE ITEM NUMBER: 0056

No of

COND UNITS UNIT

TOTAL COST :

REPORT NUMBER: $898355-4091$
ACQUISITION PRICE

$\$ 4,360.00$

$\$ 4,360.00$

ELOY N. GIRON

ALBUQUERQUE

(505) $844-2746$

NM 87185

TO FREEZE EQUIPMENT

ELOY N. GIRON

ALBUQUERQUE

(505) $844-2746$

MM 87185

LOCATION OF EQUIPMENT:

SANDIA NATIONAL LABORATORIES

PROPERTY REAPPLICATION DIVISION 7617

PROPERTY REAPPLICATION DIVISION 7617
$\$ 4.654 .00$

$\$ 4.654 .00$
NM 87185

(505) $844-2746$

TO FREEZE EQUIPMENT:

ELOY N. GIRON

ALBUQUEROUE

(505) $844-2746$

NM 87185

LOCATION OF EQUIPMENT:

SANDIA NATIONAL LABORATORIES

PROPERTY REAPPLICATION DIVISION 7617

PROPERTY REAPPLICATION DIVISION 7617 
EFFECTIVE DATE: 07/01/9. EXPIRAIION DATE: 07/31/94

\section{FSC
CODE EXCESS PROPERTY DESCRIPIION}

REFERENCE NUMBER : 003

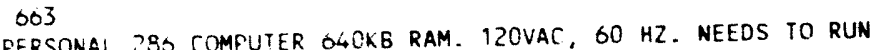
SET UP., EVER EX, 1800, SER. 53413.

REPORT NUMEER: $898355-4091$

LINE ITEM NUMBER: 0057

REFERENCE NUMBER : 664

: 286 AT COMPUTER BASE MEMORY 640 EXTENDED MEMORY 102 1 .

$120 V A C$, SO HZ. DELL COMPU, SYS2200, SER. SYS22001.

REPOR: NUMEER: $898355-4091$

LINE ITEM NUMBER: 0058
No of

ACOUISITION PRICE

$600001 \mathrm{EA}$ COST/UNIT:
TOTAL COST:

\section{$\$ 1,312.00$}

$\$ 1,312.00$

ELO N. GIRON

NM 87185

TO FREEZE EQUIPMENT

ELOY N. GIRON

ALBUOUEROUE

(505) $844-2746$

LOCATION OF EQUIPMENT:

SANDIA NATIONAL LABORATORIES

PROPERIY REAPPLICATION DIVISION 7617

ALBUQUEROUE

NM 87123

$\$ 1.544 .00$

$\$ 1,544.00$

TOTAL COST

$500001 \mathrm{EA}$

TOTAL COST:

FOR FURTHER INFORMATION:

ELOY N. GIRON

ALBUOUEROUE

NM 87185

TO FREEZE EOUIPMENT:

ELOY N. GIRON

ALBUOU

(505) $844-2746$

NM 87185

OCATION OF EQUIPMENT:

SANDIA NATIONAL LABORATORIES PROPERTY REAPPLICATION DIVISION 7617

AL BUOUEROUE

NM 87123 
EFFECTIVE DATE: 07/01/94 EXPIRATION DATE: 07/31/94

\section{COOE EXCESS PROPERTY DESCRIPIION}

REFERENCE NUMEER : 605

7010 COMPUIER, PERSONAL 286 MICRO CHANNEL 287 MATH COPROCESSOR O4OK BASE MEMORY 384 EXTEN DED MEMORY VGA VIDEO ADAPTER. 12OVAC, $60 \mathrm{HZ}$. IEM. 8550031, SER. 7223721

REPORT NUMGER: $898355-4091$

LINE ITEM NUMBER: 0059

REFERENCE NUMBER : 666

7010 COMPUTER, PERSONAL DIGITAL COMPUTER WITH A 5-1/4" DISK DRIVE, CAN ADD 3600001 EA HARD DRIVES. 120VAC, 60 HZ. DEC, BA123A2, WF 733052
MO OF
COND UNITS UNII

$600001 \mathrm{EA}$
ACQUISITION PRICE

COST/UNIT:

TOTAL COST

$\$ 3,327.00$

$\$ 3,327.00$

\section{CONTACT(S)}

FOR FURIHER INFORMATION:

ELOY N. GIRO

AL BUOUERQUE

(505) $844-2746$

in 87185

IO FREEZE EQUIPMENT:

ELOY N. GIRON

AL BUOUERQUE

(505) 844-2746

NM 87185

LOCATION OF EOUIPMENT

SANDIA MATIONAL LABORATORIES

PROPERTY REAPPLICATION DIVISION 7617

ALBUQUERQUE

Mi4 87123
COST/UNIT:

TOTAL COS.
$\$ 2.137 .00$

$\$ 2,137.00$

FOR FURTHER INFORMATION:

ELOY N. GIRON

AL BUQUEROUE

(505) $844-2746$

NM 87185

IO FREEZE EOUIPMENT :

ELOY N. GIRON

ALBUQUEROUE

(505) $844-2746$

MM 87185

LOCATION OF EOUIPMENT:

SAMDIA MATIONAL LABORATORIES

PROPERTY REAPPLICATION DIVISION 7617

ALBUOUERQUE

NM 87123 


\section{COOE EXCESS PROPERIY OESCRIPTION}

REFERENCE NUMBER : OB?

7010 COMPUIER, PLRSONAI 280 XI MACHINE, 287 MATH COPROCESSUR, G 6 OK BASE MEMORY, 38- EXTEN DEE MEMORY VGA VIDEO ADAPTER ANOH ADDRESS. 12OVAC, 00 HZ. IEM, 3 530EOI, SER. 7406010.

LINE ITEM NUMBER: 006 !

REFERENCE NUMBER : 608

7010 COMFUTER, PERSONAL 286 COMPUTER WITH $2048 \mathrm{KE}$ RAM 3-1/2" DISK DRIVE. SERIAL PORT, FAR ALEE POR AND VGA VIDEO PORT IZOVAC, GO HZ. IBM, $8530 E 01$ SER. T606152.
No of

COND UNITS UNII

ACQUISITION PRICE

- 00001 EA

COST/UNIT: TOTAL COST:

\section{$\$ 2,032.00$ $\$ 2.032 .00$}

(2.032.00

ELOY $N$. GIR

AL BUOUERQUE

(505) $846-2746$

NM 87185

TO FREEZE EQUIPMENT:

ELOY N. GIRON

ALBUOUER ROUE

(505) $844-2746$

47185

LOCATION OF EQUIPMENT:

SAMDIA MATIONAL LABORATORIES

PROPERTY REAPPLICATION DIVISION 7617

AL BUOUEROUE

NM 87123
6 O0001 EA COSTIUNIT: TOTAL COST: $\$ 2.550 .00$
$\$ 2,550.00$

FOR FURTHER INFORMATION:

ELOY W. GIRON

ALBUOUEROUE

(505) $844-2746$

MM 87185

IO FREEZE EOUIPMENT:

ELOY N. GIRON

ALBUOUEROUE

(505) $844-2746$

87185

LOCATION OF EDUIPMENT:

SANDIA MATIONAL LABORATORIES PROPERTY REAPPLICAIION DIVISION 7617 ALBUQUERQUE 
EFFECTIVE DATE: 07/01/94 EXPIRAIION DATE: $07 / 31 / 94$

COOE EXCESS PROPERTY DESCRIPTION

REFERENCE NUMBER : 669

7010 COMFUTER, PERSONAL 386 MICRO CHANNEL 640K BASE MEMORY 1280K EXTENDED MEMORY VGA VIDE O ADAPTER. 120VAC, 60 HZ. IBM, 8573061, SER. 0011518.

REPORT NUMBER: $898355 \cdot 4091$

REFERENCE NUMBER : 670

7010 COMPUTER, PERSONAL LAP TOP COMPUTER 8088, 640KB RAM, $23-1 / 2$ " DISK DRIVES, I1" ICD S CREEN AND STD KEYBRD. PONER FROM POWER SUPPLY. IMB, 5140 , SER, 0082465 .
REPORT NUMBER: $898355-4091$
NO of

COND UNITS UNIT

00001 EA
LINE ITEM MUMBER: 0063

ACQUISITION PRICE

\section{COST/UNIT:} TOTAL COST:

(n)

\section{$\$ 4.443 .00$ \\ $54,443.00$}

CONTACT(S)

FOR FURTHER IMFORMATION:

ELOY N. GIROM

ALBUOUEROUE

(505) $844-2746$

87185

TO FREEZE EOUIPMENT:

ELOY N. GIRON

ALBUOUEROUE

(505) $844-2746$

87185

LOCATION OF EOUI PMENT :

SANDIA MATIOMAL LABORATORIES

PROPERTY REAPPLICATION DIVISION 7617

ALBUOUEROUE
6 O0001 EA COST/UNIT: TOTAL COST: $\$ 1,297.00$
$\$ 1,297.00$
FOR FURTHER INFORMATION:

ELOY N. GIRON

AL BUOUERQUE

(505) $844-2746$

87185

TO FREEZE EQUIPMENT:

ELOY N. GIRON

ALBUOUEROUE

(505) $844-2746$

87185

LOCATION OF EQUIPMENT:

SANOIA MATIOMAL LABORATORIES

PROPERTY REAPPLICATION OIVISION 7617

\begin{tabular}{l} 
PROPERTY REAPPLICATION DIVISIOW 7617 \\
ALBUOUEROUE \\
\hline 47123
\end{tabular} 
EFFECTIVE DATE: OT/01/0. EXPIRATION DATE: 07/31/0\%

COOE EXCESS PROPERTY DESCRIPIION

REFERENCE NUMGER : OT1

7010 COMPUTER, PERSOMAL ATST 8086 CPU WITH 10MB HARE DRIVE, O40 RAM AND 5-1/4

FLOPPY DISK ORIVE. IZOVAC, $60 \mathrm{HZ}$

UNITS UNII

ACQUISITION PRICE

$\cos 1 /$ UNIT: TOTAL COST:

$\$ 1.735 .00$

$\$ 1.735 .00$

LINE ITEM NUMBER: 0005
REFERENCE NUMBER : 072

7010 COMPUTER PERSONA: IBM SYSTEM/2 PERSONA: COMPLTER, 286 CPU SPEED 1OMHZ WITH VGA VIDE O ADAPIER AND 3-1/2" 01SK DRIVE. ZOVAC, $30 \mathrm{HZ}$. BROKEN FACE PLA TE.
700001 EA

\section{COST/UNIT: TOTAL COST:}

\section{$\$ 2,363.00$}

$\$ 2,363.00$

FOR FURTHER IMFORMATION:

ELOY M. GIRON

ALBUOUEROUE

(505) $8464-2746$

m 87185

TO FREEZE ECUIPMENT:

ELOY N. GIRON

ELOY MUOUEROUE

(505) $844-2746$

$m 87185$ 


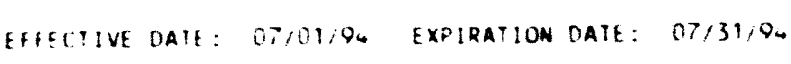

fsc

COOH EXIESS FROPERTY DESCR:PTIION

REFERENCE NUMBER : 603

7010 COMPUIER, PERSONAL XY MACHINE 808! MATH COPROCESSOR OWOK BASE MEMORY CGA VIDEO ADAPi ER. IZOVAC, O0 HZ. IBM, 4100080 , SER. 5609110.

D:YE :TTM NUMBER: 0006

REFERENCE NUMBER : Gi-

7010 COMPUTER, PERSONAL MAS $250 \mathrm{~KB}$ SYSTEM BOARO WITH A SIX PAX MEMORY BOARD

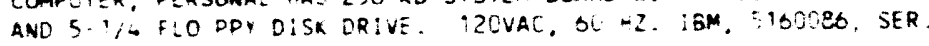

Sin 61806 .
No of

COND UNITS UMIT

ACOUISITION PRICE

$\$ 2,524.00$

Cost/UNIT:

TOTAL COST:

2.526 .00

\section{COST/UNIT:}

FOR FURTHER IMFORMAIIOM:

ELOY N. GIRON

AL BUCUEROUE

FOR FURTHER IMFORMATIOM:

ELOY $N$. GIRO

B7185

TO FREEZE FOUIPMENI:

ELOY N. GIRON

AL BUOUEROUE

(505) $844-2746$

87185

LOCATION OF EOUIPMENT

SAMOIA MAIIOMAL LABORATOR!ES PROPERIY REAPPLICATION DIVISION 7617

AL BUOUEROUE

4. 87123

\section{TO FREEZE EOUIPHENT}

ELOY N. GIRON

AL BUOUEROUE

(505) $844-2746$

LINE ITEM NUMBER: 0033

LOCATION OF EOUIPMENT

SAMDIA MATIOWAL LABORATORIES

PROPERIY REAPPLICATION DIV. 7617

AL BUOUER RUE 
FFECIIVE DAIE: OP/01/0 EXFIRATION DATE: O7/31/94

COOR EXCESS PROPERIY DESCRIFIION

REFERENCE NUMGER : STS

7010 COMPUTER PERSOMA 380 AT MACHINE OLOK BASE MEMORY 3392K EXTENDED MEMORY 0 O0001 EA VGA VIDEO A DAFTER ALIIOH VIDEO ADORESS. 120VAC, $00 \mathrm{HZ}$. IBM, 5160080 ,

VGA VIDEO

LNE :TEM NUMBER: 0034

REFERENCE AUMBER : OTO

\$10 COMFUTER, PERSONA: OLOKE AAM 5-1/4" UISK DRIVE, HARO DRIVE, SERIAL. PORT, CARALLEL DO RT, VIA VIOEO PORT. ZOVAC, SO HZ: :BM, 5.50170. 1137767.

0000: EA
No of

COND UNITS UNII

ACOUISITION PRICE

COSI/UNIT:

IOTAL COST:

\section{$\$ 2.524 .00$}

.

(c)

CONIACISS)

FOR FURTHER INFORMATION:

ELOY N. GIROW

ALBUOUEROUE

(505) $844 \cdot 2740$

MM 87185

TO FREEZE EOUIPMENT

ELOY N. GIRON

ALBUDUEROUE

mm 87185

LUCATION OF EQUIPMENT:

SANDIA MATIONAL LABORATORIES

PROPERTY PFAPPLICATION DIV. 7617

ALBUOUEROUE

NM 87123

COST/UNIT:

TOIAL COST:

\section{$\$ 1.843 .00$}

si.843.00
FOR FURTHER INFORMATION:
ELOY N. GIRON
ALBUOUEROUE
(505) $8.64-2746$

WM 87185

IO FREEZE EQUIPMENT:

ELOY N. GIRON

ALBUOUEROUE

(505) $8440-2746$

87185

LOCATION OF EQUIPMENT:

SAMDIA MATIOMAL LABORATORIES

PROPERTY REAPPLICATION DIV. 7617

ALBUOUEROUE 
EFFECTIVE DATE: 07/01/04 EXPIRATION DATE: 07/31/94

FSC

\section{COOE EXCESS PROPERIY DESCRIPTION}

REFERENCE NUMBER : OT7

7010 COMPUTER, PERSONAL RAINBON COMPUTER WITH A8088 PROCESSOR, 64KB MEMORY, REMUVABLE HAR D DISK AND RXSO DUAL DISK DRIVE. I20VAC, $60 \mathrm{HZ}$. DEC, PC10OB2, SE R. WFET203. no of

COND UNITS UNIT

ACQUISITION PRICE

600001 EA

COST/UNIT: TOTAL COST:

\section{$\$ 1.778 .00$ \\ $\$ 1.778 .00$}

(5)

REPORT NUMSER: $898355-4097$

LINE ITEM NUMBER: 0036
REFERENCE NUMBER : 678

7010 COMPUTER, PERSOMAL COMPUTER WITH 640KB RAM, SERIAL PORT, PARALLEL PORT. 600001 EA AND VIDEO PO RT. HAS 2 5-1/4" DISK DRIVES. 120VAC, $60 \mathrm{HZ} .5150174$. SER. 13492695
COST/UNIT: TOTAL COST:
$\$ 1.843 .00$ $\$ 1.843 .00$

FOR FURTHER INFORMATION:

ELOY N. GIRON

ALBUOUERQUE

(505) $844-2740$

NM 87185

TO FREEZE EQUIPMENT:

ELOY N. GIRON

ALBUQUEROUE

(505) $844-2746$

MM 87185

LOCATION OF EOUIPMENT

SANDIA MATIONAL LABORATORIES

PROPERTY REAPPLICATION DIV. 7617

PROPERTY REAPPLICATION DIV. 7617
ALBUQUERQUE 
DOE USED EMERGY RELAIED LABORATORY EQUIPMENT GRANT PROGRAM ELIGIBLE EOUIPMENT

EFFECIIVE DATE: 07/01/94 EXPIRAIION DATE: $07 / 31 / 94$

FSC
COOE EXCESS PROPERIY DESCRIPIION

REFERENCE NUMBER : 079

7010 COMPUTER, PERSONAL SYSTEM BOARO BLIVES ASYNC COMMUNICAIIONS 1 FIXED DISK VIDEO DISPLAY UNIT, 2 DISKETTE DRIVES ASYN
ORIVE. I2OVAC, 6O HZ. COMPAO. 14050210.

REPORT NUMBER: $898355-4097$

LINE ITEM NUMBER: 0038

No of

COND UNITS UNIT

ACOUISITION PRICE

$00001 \mathrm{EA}$

COST/UNIT:

TOTAL COST:

$\$ 1,839.00$
$\$ 1,839.00$

ELOY N. GIRON

(505) $844-2746$

TO FREEZE EQUIPMENT:

ELOY N. GIRON

ALBUQUEROUE

(505) $844-2746$

NM 87185

LOCATION OF EOUIPMENT:

SANDIA NATIONAL

PROPERTY REAPPLICATION DIV. 7617

ALBUOUERQUE

NM 87123

REFERENCE NUMBER : 580

SLOKB RAM 2 5-1/4" DISK DRIVES, HARD COMPUTER, PERSONAL COMPUTER WITH GLOKB RAM $25-1 / 4 "$ DISK ORIVES,

DRIVE174, SER. 0716293.

FOR FURTHER
ELOY N. GIRON
ALBUOUEROUE

ALBUQUERQUE $844-2746$

TO FREEZE EQUIPMENT

ELOY N. GIRON

ALBUQUEROUE

NM 87185

(505) $844-2746$

LOCATION OF EOUIPMENT:

IONAL LABORATORIES

PROPERTY REAPPLICATION DIV. 7617

ALBUDUUEROUE 
EFFECTIVE DATE: 07/01/94 EXPIRATION DATE: 07/31/94

FSC

\section{COOE EXCESS PROPERTY DESCRIPTION}

REFERENCE NUMBER : 681

7010 COMPUTER, PERSONAL HAS A 256K BYTE SYSTEM BOARD WITH CGA MONITOR BOARD AND A 5-1/4" FLOPPY DRIVE, 120VAC, 60 HZ. MISSING HARD DRIVE. IBM, 5160, 5248870

REPORT NUMBER: $898355-4097$

LINE ITEM NUMBER: 0040

REFERENCE NUMBER : 682

7010 COMPUTER, PERSONAL 640 KEYIES OF RAM 2 DISK DRIVES 1 PRINTER PORT. 1 RS- 700001 EA 232 PORT. BA D HARD DISK. IBM, 5150174, SER. 08596585.

No of

COND UNITS UNIT

$700001 \mathrm{EA}$
COST/UNIT: TOTAL COST:

REPORT NUMBER: $898355-4097$
LINE ITEM NUMBER: 0041

ACQUISITION PRICE

COST/UNIT: TOTAL COST: $\$ 2,524.00$

\section{ELOY N. GIRON
ALBUQUERQUE}

(505) $844-2746$

NM 87185

TO FREEZE EQUIPMENT :

ELOY N. GIRON

ALBUOUERQUE

(505) $844-2746$

NM 87185

LOCATION OF EQUIPMENT:

SANDIA NATIONAL LABORATORIES

SANDIA NATIONAL LABORATORIES
PROPERTY REAPPLICATION DIV. 7617

PROPERTY REAPPLICATION DIV. 7617
ALBUQUERQUE
NM 87123

$\$ 1,843.00$

$\$ 1,843.00$
FOR FURTHER INFORMATION:

ELOY N. GIRON

ALBUOUERQUE

(505) $844-2746$

NM 87185

TO FREEZE EQUIPMENT:

ELOY N. GIRON

ALBUOUEROUE

(505) $844-2746$

MM 87185

LOCATION OF EQUIPMENT

SANDIA NATIONAL LABORATORIES

PROPERTY REAPPLICATION DIV. 7617

ALBUQUERQUE 
EFFECTIVE DAIE: $07 / 01 / 94$ EXFIRATION DATE: $07 / 31 / 94$

$\mathrm{FSC}$

\section{CODE EXCESS PROPERTY DESCRIPTION}

REFERENCE NUMBER : 083

7010 COMPUTER PERSOMAL HAS A $250 \mathrm{~K}$ BYTE SYSTEM BOARD WITH A BASE MEMORY OF C40K GYTE, VI DEO ADAPTER CLA. AND 5-1/4" FLOPPY DRIVES, 12GVAC, $00 \mathrm{HZ}$ IBM, 5160080,5411351 .

REPORT NUMBER: $898355-4097$

LINE ITEM NUMBER: 0042

\section{REFERENCE NUMBER : $08 \%$}

TO10 COMPUTER PERSOMAL PORTASLE COMPUTER XI TYPE W1TH 256 KB RAM 2 5-1/4" DISK DRIVES F RINTER FORT, RS232 PORT, 9" DIAGONAL SCREEN/KEYBRD.

$700001 \mathrm{EA}$ IZOVAC, 60 H Z. IBM, 5155068, SER. 0041914.
No of

COND UNITS UNIT

ACQUISITION PRICE

COST/UNIT: TOTAL COST:

$\$ 2,524.00$ $\$ 2,524.00$

ELOY N. GIRON
ALBUQUERQUE

(505) $844-2746$

NM 87185

TO FREEZE EQUIPMENT :

ELOY N. GIRON

ALBUQUEROUE

(505) $844-2746$

NM 87185

LOCATION OF EQUIPMENT:

SANDIA NATIONAL LABORATORIES

PROPERTY REAPPLICATION DIV. 7617

$\begin{array}{ll}\text { PROPERTY REAPPLICATION DIV. } \\ \text { ALBUQUEROUE } & 87123\end{array}$

COST /UN IT:

TOTAL COST: $\$ 2.113 .00$

INFORMATION:

ELOY N. GIRON

ALBUQUERQUE

(505) $844-2746$

NM 87185

TO FREEZE EQUIPMENT:

ELOY N. GIRON

ALBUOUERQUE

(505) $844-2746$

MM 87185

LOCATION OF EQUIPMENT:

SAMDIA NATIONAL LABORATORIES PROPERTY REAPPLICATION DIV 7617 PROPERTY REAPPLICATION DIY 
FFFECTIVE DATE: 07/01/94 EXPIRATION DATE: 07/31/94

\section{FSC}

\section{CODE EXCESS PROPERTY DESCRIPIION}

\section{REFERENCE NUMBER : 685}

7010 COMPUTER PERSONAL HAS A $64 \mathrm{~KB}$ TO $256 \mathrm{~KB}$ CPUT WITH A 640 MB MEMORY CARD AND TWO 5-1/4" FLOPPY DISK DDRIVES. 120VAC, $60 \mathrm{HZ}$. IBM, 5150174, SER 677804.

\section{REFERENCE NUMBER : 686}

7010 COMPUTER PERSONAL HAS 4 OMBYTE HARD CARD PLUS $256 \mathrm{~K}$ BYTE SYSTEM BOARD AND

II ALSO HAS A 5-1/4" DISK DRIVE. I2OVAC, SO HZ. IBM, 5160087, 5184411.

REPORT NUMBER: $898355-4097$

LINE ITEM NUMBER: 0046

NO OF
COND UNITS UNIT

ACOUISITION PRICE

$\$ 1,843.00$ $\$ 1.843 .00$
$00001 \mathrm{EA}$

COST/UNIT:
TOIAL COST:

$600001 \mathrm{EA}$

COST/UNIT:

TOTAL COST:
$\$ 2.524 .00$ $\$ 2,524.00$
FOR FURTHER INFORMATION:

ELOY N. GIRON

ALBUOUEROUE

(505) $844-2746$

NM 87185

TO FREEZE EQUIPMENT :

ELOY N. GIRON

ALBUOUERQUE

(505) $844-2746$

NM 87185

LOCATION OF EQUIPMENT :

SANDIA NATIONAL LABORATORIES

PROPERTY REAPPLICATION DIV. 7617

ALBUQUERQUE 
EFFECTIVE DATE: 0i/01/96 EXPIRATION DATE: $07 / 31 / 94$

COOE EXCESS PROPERIY DESCRIPTION

REFERENCE NUMBER : 687

REFERENCE NUMBER : 687 COMPUTER PERSONAL HAS A $8 M H Z$ INTEL 3088 MICROPROCESSOR, 256KE RAM EXPANDABLE TO $640 \mathrm{KE}$. I2OVAC, $60 \mathrm{HZ}$. HEW PACK, HP150, SER. $2403 \mathrm{~A} 310$.

NO OF

COND UNITS UNI

ACQUISIIIION PRICE

$600001 \mathrm{EA}$

COST/UNIT:

TOTAL COST:

$\$ 1.560 .00$

$\$ 1,560.00$

REPORT NUMGER: $898355-409$

LINE ITEM NUMBER: 0047

REFERENCE NUMBER : 688

CONTEL 8088, 8MHZ MEMORY. 120VAC, 6 O0001 EA $60 \mathrm{HZ}$. HEW P ACK, HP150, $2403 \mathrm{~A} 333$.

REPORT NUMBER: $898355 \cdot 6097$

LINE ITEM NUMBER: 0048
COST/UNIT:

TOTAL COST:
$\$ 2.205 .00$

$\$ 2.205 .00$$$
\begin{aligned}
& \text { ELOY N. GIRON } \\
& \text { ALBUOUERQUE }
\end{aligned}
$$

ALBUOUEROUE

(505) $844-2746$

NM 87185

TO FREEZE EOUIPMENT:

ELOY N. GIRON
ALBUQUEROUE

(505) $844-2746$

LOCATION OF EQUIPMENT:

LOCATION OF EQUIPMENT:
SANDIA NATIONAL LABORATORIES

SANDIA NATIONAL LABORATORIES

PROPERTY REAPPLICATION DIV. 7617
ALBUDUERQUE 
EFFECIIVE DATE: $07 / 01 / 94$ EXPIRATION DATE: $07 / 31 / 94$

FSC

COOE EXCESS PROPERTY DESCRIPTION

REFERENCE NUMBER : 689

7010 COMPUTER, PERSONAL IBM PERSONAL COMPUTER AT HAS 512K SYSTEM BOARD, 30MB HARD DRIVE, AND TWO 5-1/4" DISK DRIVES. 120VAC, $60 \mathrm{HZ} .5170339$, 7197360.

REPORT NUMBER: $898355-4097$

LINE ITEM NUMBER: 0049

REFERENCE NUMBER : 690
7010 COMPUTER, PERSONAL 280 COMPUTER $25-1 / 4 "$ DISK DRIVES, HARD DRIVE PARALLEL PORT, 2 S ERIA! PORTS, AND VIDEO PORT. 120VAC, $60 \mathrm{HZ}$. IBM,

5170339. SER. 7217955.

INE ITEM NUMBER: 0050

개응 of

COND UNITS UNIT

ACOUISITION PRICE

$\$ 3,932.00$

$\$ 3.932 .00$

COST UNT:

$600001 \mathrm{EA}$

(2)

$$
\text { TO }
$$

ELOY FREZE EQUIPMEN

ELOY N. GIRON

ALBUQUEROUE
(505) $844-2746$

LOCATION OF EQUIPMENT:

SANDIA NATIONAL LABORATORIES

PROPERTY REAPPLICATION DIV. 7617

ALBUQUERQUE

$600001 E A$ COST/UNIT:
IOTAL COST:

\section{$\$ 4.557 .00$} $\$ 4,557.00$
FOR FURTHER INFORMATION:

ELOY N. GIRON

ALBUQUEROUE

(505) $844-2746$

NM 87185

TO FREEZE EQUIPMENT :

ELOY N. GIRON

ALBUQUERQUE

(505) $844-2746$

NM 87185

LOCATION OF EQUIPMENT:

LACAIIA NATIONAL LABORATORIES

SANDIA NATIONAL LABORATORIES
PROPERTY REAPPLICATION DIV. 7617

$\begin{array}{ll}\text { PROPERTY REAPPLICATION DIV. } & 7617 \\ \text { ALBUQUERQUE } & \text { NM } 87123\end{array}$ 
EFFECTIVE DATE: 07/01/94 EXPIRATION DATE: $07 / 31 / 94$

\begin{tabular}{|c|c|c|c|c|c|c|}
\hline $\begin{array}{l}\text { FSC } \\
\text { COOE }\end{array}$ & EXCESS PROPERTY DESCRIPTION & COND & $\begin{array}{l}\text { NO OF } \\
\text { UNITS UNIT }\end{array}$ & ACOUISITION & PRICE & CONTACT(S) \\
\hline \multirow[t]{3}{*}{$\begin{array}{l}\text { REFER } \\
7010\end{array}$} & $\begin{array}{l}\text { E NUMBER : } 691 \\
\text { COMPUTER, PERSONAL AT PERSONAL COMPUTER HAS S12K BYTE SYSTEM BOARD WITH } \\
\text { EXTENDED MEM ORY AND } 30 M \text { BYTE HARD DRIVE WITH ON } 5-1 / 4 " \text { DISK DRIVE. } \\
120 V A C, 60 \mathrm{HZ} \text {. IBM, } 5170339,7197006 \text {. }\end{array}$ & 6 & 00001 EA & $\begin{array}{l}\text { COST/UNIT: } \\
\text { TOTAL COST: }\end{array}$ & $\begin{array}{l}\$ 4,557.00 \\
\$ 4.557 .00\end{array}$ & $\begin{array}{l}\text { FOR FURTHER INFORMATION: } \\
\text { ELOY N. GIRON } \\
\text { ALBUQUERQUE } \\
\text { (505) } 844-2746\end{array}$ \\
\hline & LINE ITEM NUMBER: & 0051 & & & & $\begin{array}{l}\text { TO FREEZE EQUIPMENT : } \\
\text { ELOY } N \text {. GIRON } \\
\text { ALBUQUEROUE } \\
\text { (505) } 844-2746\end{array}$ \\
\hline & & & & & & $\begin{array}{l}\text { LOCATION OF EQUIPMENT: } \\
\text { SANDIA NATIONAL LABORATORIES } \\
\text { PROPERTY REAPPLICATION DIV. } 7617 \\
\begin{array}{ll}\text { ALBUQUERQUE } & \text { NM } 87123\end{array}\end{array}$ \\
\hline
\end{tabular}

REFERENCE NUMBER : 692 692 COMPUTER, PERSONAL WITH IO MH2, GOONA 60 HZ WITH MONITOR, HEW PACK.

COST/UNIT:

TOTAL COST: $98561 \times$ SER. $2650 A 159$.

REPORT NUMBER: $898355-4097$

LINE ITEM NUMBER: 0052
$\$ 2,314.00$

FOR FURTHER INFORMATION:

ELOY N. GIRON

ALBUQUEROUE

MM 87185

TO FREEZE EQUI PMENT:

ELOY N. GIRON

ALBUQUERQUE

(505) $844-2746$

MM 87185

LOCATION OF EQUIPMENT

SANDIA NATIONAL LABORATORIES

PROPERTY REAPPLICATION DIV. 7617

ALBUOUEROUE

NM 87123 
EFFECTIVE DATE: $07 / 01 / 94$ EXP!RATION DATE: $07 / 31 / 94$

\section{COOE EXCESS PROPERTY DESCRIPIION}

REFERENCE MUMBER : 693

7010 COMPUTER, PERSONAL HAS A 256K BYTE CFU AND TWO 5-1/4" DISK DRIVES. 120VAC,60 HZ. I BM, 5150166, SER. 1726757.

REPORT NUMBER: $898355-4097$

LINE ITEM NUMBER: 0053

REFERENCE NUMBER : 694

7010 COMPUTER, FERSONAL IBM PERSONAL COMPUTER WITH 256KB CPU AND TWO 5-1/4" FLOPPY DISK D RIVES AND CARD FOR CGA MONITOR, I2OVAC, 60 HZ. IBM, 5150 15424402.
NO OF

COND UNITS UNIT

600001 EA

COST/UNIT: TOTAL COST:

$\$ 1,694.00$

$\$ 1,694.00$

TOTAL cost:

TO FREEZE EQUIPMENT:

ELOY N. GIRON

ALBUQUERQUE
(505) $844-2746$

FOR FURTHER INFORMATION:

ELOY N. GIRON

ELOY N. GIRON

BUDUEROUE

NM 87185

LOCATION OF EOUIPMENT:

SAMDIA NATIONAL LABORATORIES

PROPERTY REAPPLICATION DIV. 7617

ALBUQUERQUE

NM 87123
600001 EA

COST/UNIT:
TOTAL COST:

$\$ 1.695 .00$

$\$ 1,695.00$

FOR FURTHER INFORMATION:

ELOY N. GIRON

ALBUOUEROUE

NM 87185

AL BUOUEROUE
(505) $844-2746$

TO FREEZE EQUIPMENT:

ELOY N. GIRON

(505) $844-2746$

M⿻ 87185

REPORT NUMBER: $898355-4097$

LINE ITEM NUMBER: 0054

LOCATION OF EQUIPMENT:

SAMDIA NATIONAL LABORATORIES

PROPERTY REAPPLICATION DIV. 7617

ALBUQUEROUE

NM 87123 
EFFECIIVE DATE: 07/01/94 EXPIRATION DATE: 07/31/94

TSC

\section{CODE EXCESS PROPERTY DESCRIPIION}

REFERENCE NUMBER : 695

7010 COMPUTER, PERSONAL HAS A 256 SYSTEM BOARD AND TWO 5-1/4" DISK DRIVE. 120VAC, 60 HZ. IBM, 5150176, SER. 1524450.

REPORT NUMBER: $898355-4097$

LINE ITEM NUMBER: 0055

\section{REFERENCE NLMBER : 696}

7010 COMPUTER, PERSONAL IEM FC HAS 256KE CPU WITH SIX PAX CARD AND ONE 5-1/4 FLOPFY DISK DRIVE II ALSO HAS CONNECTION FOR MONCHROME MONITOR. 12OVAC, $60 \mathrm{~Hz}$. 5150, 1520874, MISSING ONE FLOPPY DRIVE.

REPORT NUMBER: $898355-4097$

LINE ITEM NUMBER: 005t
NO OF

COND UNITS UNIT

$600001 \mathrm{EA}$

COST/UNIT: TOTA! COST:

ACQUISITION PRICE

$\$ 1,694.00$

$\$ 1,694.00$
$\$ 1,694.00$

(2)

ro

(505) $844-2746$

TO FREEZE EQUIPMENT:

ELOY $N$. GIRON

ALBUOUERQUE

(505) $844-2746$

CONTACISS)

LOCATION OF EQUIPMENT:

SAMDIA MATIONAL LABORATORIES

PROPERTY REAPPLICATION DIV. 7617

ALBUDUEROUE

NM 87123

COST/UNIT:

TOTAL COST:

$\$ 1,694.00$

$\$ 1,694.00$

$00001 E A$

$1,694.00$

AL

OR FURTHER INFORMATION:

ELOY N. GIRON

ALBUDERTE

NM 87185

TO FREEZE EQUIPMENT:

ELOY N. GIRON

ALBUQUEROUE

(505) $844-2746$

NM 87185

LOCATION OF EOUIPMENT:

SANDIA MAT IOMAL LABORATORIES

PROPERTY REAPPLICATION DIV. 7617

ALBUOUEROUE

NM 87123 
EFFECTIVE DATE: 07/01/94 EXPIRATION DATE: 07/31/94

FSC

\section{CODE EXCESS PROPERTY DESCRIPTION}

REFERENCE NUMBER : 507

HARO DRIVE SE RIAL PORT PARALLEL PORT AND VIDEO PORT. 12OVAC, $60 \mathrm{HZ}$.

FCO 300,0182526 .
7010 COMPUTER, PERSONAL XT TYPE COMPUTER WITH 640KB RAM 5-1/4" DISK DRIVE,

No of

COND UNITS UNIT

600001 EA COST/UNIT:
TOTAL COST:

ACQUISITION PRICE

$\$ 1,735.00$

$\$ 1.735 .00$

ELOY N. GIRON
ALBUOUEROUE
(505) $844-2746$

10 FREEZE EOUIPMENT:

ELOY N. GIRON

ALBUOUEROUE

(505) $844-2746$

MM 87185

LOCATION OF EOUIPMENT:

SANDIA NATIONAL LABORATORIES

PROPERTY REAPPLICATION DIV. 7617

\begin{tabular}{l} 
PROPERTY REAPPLICATION DIV. 7617 \\
ALLUDUERQUE \\
\hline
\end{tabular}

REFERENCE NUMBER : 693

7010 COMPUTER, PERSONAL SYSTEM BOARD 8087, S40 KB MEMORY, KEYGRD, PARALLEL,

PRINTER VIDEO DISPLAY UNIT I DISKETTE DRIVE ASYNC COMMUNICATIONS ALT

$0 \quad 00001$ EA

ASYNC COM MUNICATIONS. I2OVAC, 60 HZ. COMPAC, 101712.

INE ITEM NUMBER: 0058
COST/UNIT:
TOTAL COST:
$\$ 4.323 .00$
$\$ 4.323 .00$
FOR FURTHER INFORMATION:

ELOY N. GIRON

ALBUQUERQUE

(505) $844-2746$

NM 87185

TO FREEZE EQUIPMENT:

ELOY N. GIRON

ALBUOUERQUE

(505) $844-2746$

4.M 87185

LOCATION OF EOUIPMENT:

SANDIA NATIONAL LABORATORIES

PROPERTY REAPPLICATION DIV. 7617

AL BUDUEROUE

u4 87123 
EFFECTIVE DATE: 07/01/94 EXFIRATION DATE: 07/31/94

\section{CONE EXCESS PROPERTY DESCRIPTION}

REFERENCE NUMBER : 090

7010 COMPUTER FERSONAL XT COMPUTER WITH O4O KG RAM 2 5-1/4" DISK DRIVES, 2 SERIAL PORT, PARALLE: PORT AND VIDEO PORT, 120VAC, $60 \mathrm{HZ}$. IBM, 5160068, SER. OLZ2901S.

REPCRT NUMEER: $898355-0097$

LINE ITEM NUMBER: 0059

RE HERENCE NUMEE $₹$ : 700

OIAGONAL SCREEN, 1024KE MFMORY 3-1/2" DISK DRIVE, 7 00001 EA HAS POR:(S) IN BACK FOR MOUSE \& EXTERNAL HOOKUF. 12OVAC, $60 \mathrm{HZ}$. NEEDS MOUSE TEYBRD APFLE COMP, MOOO1A, FGUTOG?.

REPORT NUMEER: $898355-4097$

LINE ITEM NUMBER: 0060

NO of

COND UNITS UNIT

ACQUISITION PRICE

COST/UNIT:

TOTAL COST:

$\$ 1,544.00$

$\$ 1,544.00$

ELOY N. GIRON

ALBUOUEROUE

ALBUDUEROUE

87185

IO FREEZE EOUIPMENT:

ELOY N. GIRON

AL BUOTEROUE

(505) $844-2746$

87185

LOCATION OF EOUIPMENT.

SAMDIA MaTIONAL IABORATORIES

PROPERTY REAPPLICATION DIV. 7617

AL BUOUEROUE

COSI/UNIT:

TOTAL COST:
$\$ 1,934.00$

$\$ 1,934.00$
FOR FURTHER IMFORMATION:

ELOY N. GIRON

ALBUOUEROUE

(505) 844.2746

4M 87185

TO FREEZE EQUIPMENT :

ELOY N. GIRON

AL BUDUEROUE

(505) 846.2746

87185

LOCATION OF EOUIPMENT:

SANDIA MATIONAL LABORATORIES

SAMDIA MATIONAL LABORAIORIES

PROPERTY REAPPLICATION DIV. 7617
ALBUOUEROUE 
EFFECIIVE DATE: 07/01/94 EXPIRATION DATE: 07/31/94

FSC

\section{COOE EXCESS PROPERTY DESCRIPIION}

REFERENCE NUMBER : 701

7010 COMPUTER, PERSONAL IBM PERSONAL COMPUTER AT HAS A 64OKB SYSTEM GOARD.

$120 \mathrm{VAC}, 60 \mathrm{HZ}$. NEEDS WORK. IBM, 5170068, SER. 5200041.
LINE ITEM NUMBER: 0062

REPORT NUMBER: $398355-4097$
No of

COND UNITS UNI

$700001 \mathrm{EA}$
ACOUISITION PRICE

COST/UNit: TOTAL COST:

CINE ITEM NUBR: 0062

RETEREACE NUMBER : 702

7010 COMPUTER, PERSONAL 286 COMPUTER WITH $25-1 / 4 "$ DISK DRIVE, HARD DRIVE, SERIAL PORT P ARALLE DORT AMN VIDEO EGA PORT, I2OVAC, $60 \mathrm{HZ}, 5170068$. SER. 5206058 .

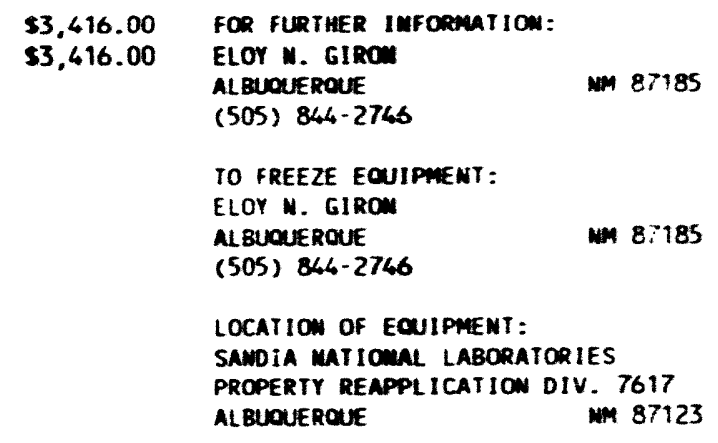

COST/UNIT: TOTAL COST:

600001 EA

$\$ 3.466 .00$ $\$ 3,466.00$
FOR FURTHER IMFORMATION:

ELOY N. GIRO

(505) $864-2746$

14. 87185

TO FREEZE EQUIPAENT:

ELOY N. GIROW

ALBUOUEROUE

(505) $844-2746$

m4 87185

LOCATION OF EOUIPMENT.

SAMOIA MATIOUAL LABORATORIES

PROPERTY REAPPL ICAIIOW DIV. 7617

PROPERIY REAPPLICAIIOW OIV. 7617
ALBUOUERQUE 
IfFC:IVE DATE: 07101/06 EXFIRATION DATE: 07/31/96

\section{COOE EXCESS PROPERTY DESCRIPTION}

REFERENCE MUMBER : 703

7010 COMPUTER, PERSONAL TOSHIEA PORTABLE COMPUTER WITH GLOK BYTE MEMORY, LCD SCREEN, THO 3-1/6" DISK DRIVES, AND IS IBM COMPATIBLE. OVDC. MISSINC POAER CORD. PA7OLZU. SER. 10624460.

PEPORT NUMEER: $898355-4097$

LINE ITEM NUMBER: 0065

REFERENCE N.JMEER : TOW COMPUTER, PERSONAL XI COMPUTER, 6LOO KB RAM 2 5-1/6" DISK DRIVES, HARD OR:VE, SERIA I PORTS, PARALLE PORT AND VIDEO PORT. $120 \mathrm{VAC}, 60 \mathrm{HZ}$. IBM, 516008 Q. SER. 3309851 .

$600001 \mathrm{EA}$

\section{COST/UNIT: \\ TOTAL COST:}

$\$ 2.517 .00$

$\$ 2.517 .00$

REPORT NUMBER: $898355 \cdot 4097$

LINE ITEM MUMBER: 0067
ACOUISIIION PRICE

$\$ 1.885 .00$

$\$ 1,885.00$

COST/UNIT:

TOTAL COST:

(2)

COMTACT(S)

FOR FURTHER IMFORMATION:

ELOY N. GIROW

ALBUOUEROUE

(505) $844-2746$

NM 87185

TO FREEZE EOUIPMENT

ELOY N. GIRO

(505) $844-2746$

87185

LOCATION OF EOUIPMENT:

SANDIA MATIOMAL LABORATORIES

PROPERTY REAPPLICATION OIV. TO:77

ALBUQUEROUE

im 87123

FOR FURTHER IMFORMATION:

ELOY M. GIROW

AL BUOUEROUE

(505) $844-2746$

4. 87185

TO FREEZE EOUIPMENT:

ELOY N. GIROW

ALBUOUEROUE

(505) $8466-2746$

LOCATION OF EQUIPMENT:

SAMDIA MATIOUAL LABORATORIES

SAMDIA MATIONAL LABORATORIES 7617

PROPERTY REAPPLICATION DIV. 7617
ALBUOUEROUE
87123 


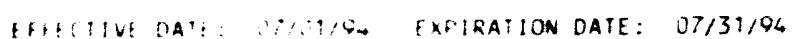

15t

\section{COOE EXCESS PROPERIY RESCRIPIION}

REFERENCE NUMBER : 705

7010 COMPUTER, PERSONAL FERSONAL SYSTEM/2 COMPUTER HITH 1024K GYYE MEMORY, 44 MBYTE HARD DISK AND ONE 3-1/4" DISKETTE DRIVE. 120VAC, OO HZ, 8560041. 3025800

REPCRT NUMBER: $993355-4097$

LINE ITEM NUMBER: 0068

REFE RE NLE NUMGER: TUS

PO1. COMPUTER, PERSONAL 386 SX COMPUTER, WITM GLOK GASE MEMORY, $5641 \mathrm{~K}$

EXTENDED MEMORY, 3. 1/2" DISK DRIVE. SERIAL PORT, PARALLEL PORT AND

VIDEO FORT. 8560 071, SER. 9006227 .

$700001 \mathrm{EA}$

COST/UNIT:

TOTAL COST:

$\$ 4,524.00$

FOR FURTHER INFORMATION

ELOY N. GIRON

AL BUOUEROUE

(505) $864 \cdot 2740$

NM 87185

TO FREEZE EQUIPMENT

CLOY N. SIRON

ALBUOUEROUE

(505) $844-2746$

Nm 87185

LOCATION OF EQUIPMENT.

SAMDIA NAIIONAL LABORATORIES

PROPERTY REAPPLICATION DIV. 7617

AL BUOUEROUE

im 87125 
EFFECTIVE DATE: 07/01/9. EXFIRATION DATE: DT/31/94

$\mathrm{FSC}$

CODE EXCESS PROPERTY DESCRIPTION

REFERENCE NUMBER : 707

7010 COMPUTER, PEPSONAL 236 COMPUTER 1024 KB RAM, 3-1/2" DISK DRIVE, 73 MB COMPUTER, PERSONAL 236 COMPUTER
HARD DRIVE, WI IH A PARALLEL DORT SERIAL PORT AND VIDEO PORT. 12OVAC, HARD DRIVE, HI IH A PARALLEL DORT
$60 \mathrm{HZ}$. 8560071 . SER. 9000094 .

REPOR T NUMGER: $898355-4097$

INE ITEM NUMBER: 0070

\section{REFERENCE NUMBER : 708}

7010 COMPUTER, PERSONA! 386 COMPUTER 9088KG RAM $31 / 2 "$ DISK DRIVE, SERIAL PRT. PARALLEL $P$ ORT AND VGA VIDEO PORT. IZGVAC, $60 \mathrm{HZ}$.

LINE ITEM NUMBER: 007

REPORT NUMBER: $898355-4097$
NO OF

COND UNITS UNIT

ACQUISIIION PRICE

$600001 \mathrm{EA}$

COST/UNIT:

TOTAL COST:

$\$ 3,582.00$

$\$ 3,582.00$

ELOY N. GIRON
ALBUQUERQUE

(505) $844-2746$

NM 87185

TO FREEZE EQUIPMENT:

ELOY N. GIRON

ALBUQUERQUE

(505) $844-2746$

LOCATION OF EQUIPMENT:

SANDIA NATIONAL LABORATORIES

PROPERIY REAPPLICATION DIV. 7617

ALBUQUERQUE

NM 87123
$700001 \mathrm{EA}$

COST/UNIT:

TOTAL COST:
$\$ 3,582.00$

$\$ 3,582.00$
FOR FURTHER INFORMATION:

ELOY N. GIRON

ALBUQUERQUE

(505) $844-2746$

NM 87185

TO FREEZE EQUIPMENT:

ELOY N. GIRON

ALBUOUERQUE

(505) $844-2746$

NM 87185

LOCATION OF EQUIPMENT

SANDIA NATIONAL LABORATORIES

PROPERTY REAPPLICATION DIV. 7617

ALBUOUEROUE

NM 87123 
FSC

\section{COOE EXCESS PROPERTY DESCRIPIION}

\section{REFERENCE NUMBER : 709}

7010 COMPUTER, PERSONAL 286 COMPUTER WITH 2 5-1/4" DISK DRIVE, HARD DRIVE, SERIAL PORT, P ARALLEL FORT AND VIDEO PORT. 120VAC, $60 \mathrm{HZ}$.

REPORT NUMBER: $898355 \cdot 609 \%$

LINE ITEM NUMBER: 0072
NO OF

COND UNITS UNIT

$500001 \mathrm{EA}$ COST/UNIT:
TOTAL COST:

$\$ 4,144.00$ $\$ 4,144.00$

ELOY N. GIRON

ALBUQUERQUE

(505) $844-2746$

TO FREEZE EQUIPMENT

ELOY N. GIRON

ALBUOUEROUE

(505) $844-2746$

LOCATION OF EQUIPMENT:

SANDIA NATIONAL LABORATORIES

PROPERTY REAPPLICATION DIV. 7617

ALBUQUERQUE

NM 87123

REFERENCE NUMBER : 710

7010 COMPUTER, PERSONAL PERSONAL SYSTEM II HAS 286 MICROPROCESSOR ONE MEMORY

$1024 \mathrm{~K}$ BYTE A ND 3-1/4" 0!SK DR!VE. 1ZOVAC, 60 HZ. MISSING HARD DRIVE.

$700001 \mathrm{EA}$

1BM, 85 60071, SER. 9061143

REPORT NUMBER: $898355-4097$

LINE ITEM NUMBER: 0073

\section{COST/UNIT:}

TOTAL COST:
$\$ 4.999 .00$

FOR FURTHER INFORMATION:
NM 87185

87185
$\$ 4,999.00$

ELOY N. GIRON

ALBUQUERQUE

(505) $844-2746$

NM 87185

TO FREEZE EOUIPMENT:

ELOY N. GIRON

ALBLOUERQUE

(505) $844-2746$

LOCATION OF EQUIPMENT:

SANDIA NATIONAL LABORATORIES

PROPERTY REAPPLICATION DIV. 7617

ALBUOUERQUE 


\section{COOE EXCESS PROPERTY UEESCRIPTION}

REFERENCE NUMBER : 711

7010 COAPUTER, PERSONAL 280 LPU HIIH A 30 MBYTE HARD DRIVE AND THO $5.1 / 4$

FLOPPY D:SK DRI VES. 12OVAC, 60 HZ. IBM, 5170339, SER. 55023529.

REPORT NUMEER: $898355-4097$

LINE ITEM NUMBER: 0074

REFERENCE NUMBER : 712

7010 COMPUTER PERSONAL HAS 640K BYTE MEMORY, PROCESSOR 340286 AT 12 MHZ, PARALEL INTERF ACES ONE 1.44 MEGABYTE $3-1 / 2 "$ DISK DRIVE AND A 40 MBYTE FIXED DIS K. IZOVAC, OO HZ. COMPAQ.

$600001 \mathrm{EA}$

COST/UNIT:

TOTAL COST:

$\$ 4,040.00$

$\$ 4,040.00$

FOR FURTHER INFORMATION :

ELOY N. GIRON

ALBUDUERQUE

FURTHER INFORMATION:

ELOY N. GIRON

AL BUQUER $844-2746$

TO FREEZE EQUIPMENT

ELOY N. GIRON

ALBUQUERQUE
(505) $844-2746$

NM 87185

OCATION OF EQUIPMENT:

SANDIA NATIONAL LABORATORIES

PROPERTY REAPPLICATION DIV. 7617

ALBUQUEROUE

NM 87123

TO FREEZE EQUIPMENT:

ELOY N. GIRON

ALBUQUERQUE

(505) 844-2746

NM 87185

REPORT NUMBER: $898355-4097$

LINE ITEM NUMBER: 0077

OCATION OF EQUIPMENT:

SANDIA NAIIONAL LABORATORIES

PROPERTY REAPPLICATION DIV. 7617

ALBUQUEROUE

NM 8712 
EFFECTIVE DATE: 07/01/94 EXPIRATION DATE: 07/31/94

FSC

COOE EXCESS PROPERTY DESCRIPTION

REFERENCE NUMBER : 713

7010 COMPUTER, PERSONAL HAS 640 KBYTES BASE MEMORY, 1024 EXTENDED MEMORY, TWO 6

3-1/4" DISK DRIVES AND IS A 2OMHZ CPU. 120VAC, $60 \mathrm{HZ}$. DELL COMPU.

SYS220, S ER. 22001284.

REPUR I NUMBER: $898355-4091$

LINE IIEM NUMBER: 0078

REFERENCE NUMBER : 714

7010 COMPUTER, PERSONAL 64OKB RAM 2 5-1/4" DISK DRIVE, SERIAL PORT, PARALLEL PORT AND VID EO PORT. 12OVA, $60 \mathrm{HZ}$. IGM, 5150174, 08077465.

$600001 \mathrm{EA}$

COST/UNIT:

TOTAL COST:

$\$ 1,843.00$

$\$ 1,843.00$

ELOY N. GIRON

ALBUOUERQUE

TO FREEZE EQUIPMENT:

ELOY N. GIRON

ALBUQUERQUE

(505) $844-2746$

LOCATION OF EQUIPMENT:

SANDIA NATIONAL LABORATORIES

PROPERTY REAPPLICATION DIV. 7617

ALBUQUERQUE
(505) $844-2746$

NM 87185

NM 87185

OF EOUIPMENT:

NATIONAL LABORATORIES

PROPERTY REAPP

NM 87123

NM 87185 
EFFECTIVE DATE: $07 / 01 / 94$ EXP!RAIION DATE: $07 / 31 / 94$

SSC

COOE EXCESS PROPERTY DESCRIPIION

REFERENCE NUMBER : 71

7010 COMPUTER, PERSONAL 280 WITH 64OK BYTES MEMORY WITH 1.375 EXTENIED MEMORY AND 3-1/4" D!SK DRIVE. 120VAC, 60 HZ. IBM, 8560071, SER. 72912551. CPU FAI LED TEST.
LINE ITEM NUMBER: 0080
No of

COND UNITS UNI

00001 EA

COST IUNIT:
TOTAL COST:

OTAL COST:

REFORT NUMBER: $898355-4097$

7010 COMPUTER, PERSONAL IS A 38 Ć WITH A 3-1/4" DISK DRIVE. IT IS ALSO PORTABLE AND IS REC HARGEABLE. ZENITH RAD, ZTE3034, B49EDO00.

$600001 E A$

\section{COST/UNIT:}

TOTAL COST:
$\$ 5,855.00$
$\$ 5,855.00$

FOR FURTHER INFORMATION:

ELOY N. GIRON

ALBUQUERQUE

(505) $844-2746$

NM 87185

TO FREEZE EQUIPMENT:

ELOY N. GIRON

ALBUOUERQUE

(505) $844-2746$

NM 87185

LOCATION OF EQUIPMENT:

SANDIA NATIONAL LABORATORIES

PROPERIY REAPPLICATION DIV. 7617

PROPERIY REAPPLICATION DIV. 7617
ALBUQUEROUE
NM 87123 
EFFECIIVE DATE: 07/01/94 EXPIRATION DATE: 07/31/94

isC

\section{CODE EXCESS PROPERTY DESCRIPTION}

REFERENCE NUMBER : 717

7010 COMPUIER PERSONAL 280 COMPUTER HITH 2048 KB RAM. 5-1/4" DISK DRIVE, 2

COMPUTER, PERSONAL 280 COMPUTER WITH 2048 KB RAM. 5
SERIAL PORTS 2 PARALLEL PORTS AND VGA VIDEO PORT. 120VAC, $60 \mathrm{HZ}$. AST

SERIAL PORTS 2 PARALLEL POR
RESEAR CH ASI286. TWP20032.

REPORI NUMBER: $893355-4097$

LINE ITEM NUMBER: 0082

\section{REFERENCE NUMEER : ?18}

7010 COMPUTER PERSOMAL 38 COMEUTER HITH A 5-1/4" DISK DRIVE, HARD DRIVE,

COMPUTER, PERSONAL 385 COMFUTER HITH A 5-1/4" DISK ORIVE, HARD DRIVE,
SERIAL PORT, F ARAL EL PORI AND VIDEO PORT, HITH 2048KB RAM. 120VAC, 60 H2. NE EOS TO RLN SET UP. WY32160, $15 \cup 29800$.

REPORT NUMEER: $898355-4097$

LINE ITEM NUMBER: 0083
NO OF

COND UNITS UNIT

\section{ACOUISITION PRICE}

COST/UNIT:

TOTAL COST:

$\$ 1,926.00$

$\$ 1,926.00$

ELOY N. GIRON

(505) $844-2746$

NM 87185

TO FREEZE EOUIPMENT:

ELOY N. GIRON

ALBUQUERQUE

(505) $844-2746$

NM 87185

LOCATION OF EOUIPMENT:

SANDIA NATIONAL LABORATORIES

PROPERTY REAPPLICATION DIV 7617

ALBUDUEROUE

COST/UNIT:

TOTAL COST:

$\$ 12,844.00$

$\$ 12,844.00$

FOR FURTHER INFORMATION:

ELOY N. GIRON

ALBUOUEROUE

NM 87185

TO FREEZE EQUIPMENT :

ELOY N. GIRON

ALBUQUERQUE

(505) $844-2746$

NM 87185

LOCATION OF EQUIPMENT:

SANDIA NATIONAL LABORATORIES

PROPERTY REAPPLICATION DIV. 7617

ALBUQUERQUE 
EFFECTIVE DATE: $07 / 01 / 94$ EXPIRATION DATE: $07 / 31 / 94$

FSC

\section{COOE EXCESS PROPERTY DESCRIPTION}

PEFERENCE NUMBER : 710

7010 COMPUTER PERSOHAL IBM 280 WITH $70 M$ M HARD DRIVE AND ALSO HAS A 3-1/4" DISK DRIVE. I 20VAC, $60 \mathrm{HZ}$. 8560071 , SER. 9098084.

REPQRT NUMGER: $898355-4097$

LINE ITEM NUMBER: 0086

\section{REFERENCE NUMBER : 720}

7010 COMPUTER PERSONAL HAS 6LOK GYTE MEMORY, EXTENDED 1.375 MBYTE MEMORY, AND A 3.14" D ISK DRIVE. 120VAC,60 HZ. IBM, 8560071, SER. 9113259 .
No of

COND UNITS UNI

ACQUISITION PRICE

$600001 \mathrm{EA}$

COST/UNIT:

TOTAL COST:

$\$ 3,749.00$

$\$ 3,749.00$

ELOY N. GIRON

ALBUQUEROUE

(505) $844-2746$

TO FREEZE EQUIPMENT:

ELOY N. GIRON

ALBUDUERQUE

(505) $844-2746$

MM 87185

LOCATION OF EQUIPMENT:

SANDIA NATIONAL LABORATORIES

PROPERTY REAPPLICATION DIV. 7617

ALBUOUERQUE
600001 EA COST/UNIT:

TOTAL COST:
$\$ 3,702.00$

$\$ 3,702.00$
FOR FURTHER INFORMATION:

ELOY N. GIRON

ALBUOUERQUE

(505) $844-2746$

NM 87185

TO FREEZE EOUIPMENT :

ELOY N. GIRON

NM 87185

(505) 844-2746

LOCATION OF EQUIPMENT

SANDIA NATIONAL LABORATORIES

PROPERTY REAPPLICATION DIV. 7617

ALBUQUEROUE 
EFFECTIVE DATE: $07 / 01 / 94$ EXPIRATION DATE: $07 / 31 / 94$

\section{CODE EXCESS PROPERTY DESCRIPIION}

REFERENCE NUMBEF : 721

7010 COMPUTER PERSONAL SYSTEM BOARD 8087 64OKB MEMORY KEYBRD PARALLEL PRINTER, VIDEO DIS FLAY UNIT, 2 DISKETTE DRIVES, ASYNC COMMUNICATIONS. 120VAC, $60 \mathrm{H} \mathrm{Z}$, IBM, 5160086, 5410575.

REPORT NUMGER: $898355-409$

LINE ITEM NUMBER: 0017

\section{REFERENCE NUMBER : 722}

7010 CONCENTRATOR RETIMI FOR LINKING COMPUTER OR WORKSTATIONS TO A MAIN CONCENTRATOR, RETIMI FOR LINKING COMPUTER OR WORKSTATIONS TO A MU SYNOPTICS, 2500C, SER. 111137 .
No of

COND UNITS UNIT

\section{ACQUISITION PRICE}

$600001 \mathrm{EA}$ COST/UNIT: TOTAL COST:

\section{$\$ 2,524.00$}

FOR FURTHER INFORMATION:

ELOY N. GIRON

ALBUQUERQUE

NM 87185

TO FREEZE EQUIPMENT:

ELOY N. GIRON

ALBUQUEROUE

(505) $844-2746$

NM 87185

LOCATION OF EQUIPMENT

SAMDIA NATIONAL LABORATORIES

PARDIA NATIONAL REAPPLILAIION DIVISION 7617

ALBUQUERQUE NM 87123

700001 EA

COST/UNIT:

TOTAL COST:

$\$ 1,036.00$
$\$ 1,036.00$

ELOY N. GIRON
ALBUQUERQUE
$(505) 844.2746$

TO FREEZE EQUIPMENT :

ELOY N. GIRON

ALBUQUEROUE

(505) $844-2746$

NM 87185

LOCATION OF EOUIPMENT:

SANDIA NATIGNAL LABORATORIES

SANDIA NATIGNAL LABORATORIES

PROPERTY REAPPLICATION DIV. 7617 
EFFECTIVE DATE: 07/01/0. EXPIRATION DATE: $07 / 31 / 94$

FSC

\section{CODE EXCESS PROPERIY DESCRIPIION}

REFERENCE NUMBER : 723

7010 CONCENTRATOR, RETIMI FOR LINKING COMPUTERS OR WORK STATIONS TO A MAIN COMPUTER SYSIEM. POWER GY POWER SUPPLY. MISSING POWER SUPPLY.

SYNOFTICS, $2500 \mathrm{C}$, SER. 111184.

LINE ITEM NUMBER: 0091

REFERENCE NUMEER : 724

7010 CPU 286 COMPU'ER H:TT 2689KS RAM 2 5-1/4" DISK DRIVES, 32 MB HARD DRI VE ? SERIAL FORTS FARAL 5 L FORT AND EGA VIDEO PORT. 12OVAC, $60 \mathrm{HZ}$. IGM. $517033^{\circ}$, SER. 7216384

No of

COND UNITS UNI

$700001 \mathrm{EA}$

\section{ACQUISITION PRICE}

COST/UNIT: TOTAL COST:
$\$ 1,036.00$
$\$ 1,036.00$

\section{FOR FURTHER INFORMATION:}

ELOY N. GIRON

ALBUQUEROUE

(505) $844-2746$

NM 87185

TO FREEZE EOUIPMENT:

ELOY N. GIRON

ALBUQUERQUE

(505) $844-2746$

NM 87185

LOCATION OF EOUIPMENT

SAMDIA NATIONAL LABORATORIES PROPERTY REAPPLICATION DIV. 7617 PROPERTY REAPPLICATION DIV 87123

$600001 \mathrm{EA}$
$\$ 3,932.00$ $\$ 3,932.00$

\section{FOR FURTHER INFORMATION}

ELOY N. GIRON

ALBUOUERQUE

(505) $844-2746$

NM 87185

TO FREEZE EQUIPMENT:

ELOY N. GIRON

ALBUOUERQUE

(505) $844-2746$

NM 87185

LOCATION OF EOUIPMENT:

SANDIA NATIONAL LABORATORIES PROPERTY REAPPLICATION DIVISION 7617 PROPERTY REAPPLICATION DIVI NM 87123 
EFFECTIVE DATE: 07/01/94 EXPIRATION DATE: $07 / 31 / 94$

FSC

COND UNITS UNIT

ACQUISITION PRICE

CONTACTSS)

REFERENCE NUMBER : 725

7010 CPU SUN CPU, HAS PORTS IN BACK FOR KEYBRD, ETHERNET, SERIAL B, SERIAL A, 700001 EA SCSI AND RGB. I2OVAC, $60 \mathrm{HZ}$. SUN, $4110 \mathrm{CDB}, 911 \mathrm{~K} 0094$.

COST/UNIT: TOTAL COST:

REPORT NUMBER: $898355-4091$

LINE ITEM NUMBER: 0069

REFERENCE NUMBER : 726

7010 CPU SYSTEM BOARD 6LOKB MEMORY KEYBRD PARALLEL PRINTER, VIDEO DISPLAY UNIT, 1 DISKETTE DRIVE. 12OVAC, $60 \mathrm{HZ}$.

$600001 \mathrm{EA}$

COST/UNIT: TOTAL COST:

LINE ITEM NUMBER: 0070

REPORT NUMBER: $898355-4091$
$\$ 6,000.00$ $\$ 6,000.00$

ELOY N. GIRON
ALBUQUEROUE

(505) $844-2746$

TO FREEZE EQUIPMENT :

ELOY N. GIRON

ALBUQUERQUE

(505) $844-2746$

LOCATION OF EQUIPMENT

SANDIA MATIONAL LABORATORIES

PROPERTY REAPPLICATION DIVISION 7617

ALBUOUEROUE

NM 87123 
EFFECTIVE DAIE: 07/01/94 EXPIRAIION DATE: $07 / 31 / 94$

$\mathrm{FSC}$

\section{COOE EXCESS PROPERTY DESCRIFIION}

REFERENCE NUMBER : 727

7010 DISC DRIVE BERNOULLI GOX IS A 20+20 EXTERNAL SUBSYSTEM HHICH CONNECTS TO A C OMPUTER THROUGH ONE OF OMEGAS HOST INTERFACE BOARDS. 12OVAC, $60 \mathrm{HZ}$ IOMEGA, A2ZOH, SER. 03 P 42 .

REPORT NUMEER: $898355-4097$

LINE IIEM NUMBER: 0018
NO OF

COND UNITS UNIT

ACQUISIIIION PRICE

COST/UNIT: TOTAL COST:

\section{$\$ 2.682 .00$}

$\$ 2.682 .00$

00001 EA

120.000

(50

FOR FURTHER INFORMATION:
ELOY N. GIRON

(505) $844-2746$

MM 87185

TO FREEZE EOUIPMENT:

ELOY N. GIRON

ALBUQUEROUE

(505) $844-2746$

m. 87185

LOCATION OF EQUIPMENT

SANDIA MATIOMAL LABORATORIES

PROPERTY REAPPLICAIION DIV. 76

ALBUQUERQUE

NM 87123

REFERENCE NUMBER : 728

70 IO DISC DRIVE GERNOULLI GOK HAS TWO DISK DRIVES, PLUS IT ALSO HAS A HOST

INTERF ACE BOARDS WITH IT. I2OVAC, 60 HZ. AZZOH. SER. OLIPM.

$60001 \mathrm{EA}$

COST/UNIT:

TOTAL COST:

$\$ 2.682 .00$
$\$ 2.682 .00$

FOR FURTHER INFORMATION:

ELOY N. GIRON

ALBUQUEROUE

(505) $844-2746$

NM 87185

TO FREEZE EQUIPMENT:

ELOY N. GIRON

ALBUOUEROUE

MM 87185

REPORT NUMBER: $898355-4097$

LINE ITEM NUMBER: 0019

LOCATION OF EOUIPMENT:
SANDIA MATIONAL LABORATORIES
PROPERTY REAPPLICATION DIV. 7617
ALBUDUEROUE 
EFFECIIVE DATE: $07 / 01 / 0$. EXPIRATION DATE: 07/31/94

FSC OSF OF

COOE EXCESS PROPERTY DESCRIPTION

REFERENCE NUMBER : 720

7010 DISC DRIVE BERNOULLI BOX IS A EXTERNAL SUBSYSTEM WHICH CONNECTS IO A COMPUIE $R$ IHROULH ONE OF IOMEGAS HOST INTERFACE BOARDS. 12OVAC, $60 \mathrm{HZ}$.

1 OMEGA, AZ2RH. SER. OTFUU.

REPORT NUMEEF: BIS35S TINT

LINE I IEM NUMBER: 0024

RE!ERENCE NUMBER : i3:

7010 DISC ORIVE GERNOUILI BOX IS A EXTERNAL SUBSSYSTEM WHICH CONNECTS TO A COMPUTE $R$ IHROUGH ONE OF : OMEGAS HOST INTERFACE SOARDS. 120VAC, $60 \mathrm{~Hz}$. $6266 x .60600302$
COND UNITS UNIT

ACOUISIIION PRICE

$00001 E A$ COST/UNIT: TOTAL COST:

$\$ 1,930.00$ $\$ 1,930.00$

\section{ELOY $N$. GIRON \\ ALBUOUEROUE \\ (505) $844-2746$}

FOR FURTHER INFORMATION:

TO FREEZE EQUIPMENT:

ELOY $N$. GIRON

ALBUOUERQUE

(505) $8 \div 2746$

NM 87185

LOCATION OF EQUIPMENI:

SAMDIA MATIONAL LABORATORIES

PROPERTY REAPPLICATION DIV. 7617

PROPERTY REAPPLICATION DIV. 7617
ALBUOUERQUE

$$
\text { NM } 87123
$$

$600001 \mathrm{EA}$

COST/UNIT:

TOTAL COST:

$\$ 2.245 .00$
$\$ 2.245 .00$
FOR FURTHER INFORMAIION:
ELOY N. GIRON

ALBUQUEROUE

(505) $844-2746$

NM 87185

TO FREEZE EQUIPMENT:

ELOY N. GIRON

ALBUOUEROUE

(505) $844-2746$

NM 87185

LOCATION OF EOUIPMENT:

SAMDIA MATIOMAL LABORATORIES

PROPERTY PEAPPLICATION DIV. 7617

ALBUOUEROUE

Nim 87123 
EFFECIIVE DATE: 07/01/06 EXPIRATION DATE: 07/31/94

sSC

COOE EXCESS PROPERTY DESCRIFIION

REFERENCE NUMEER : 31

7010 DISC DRIVE THE BERNOUL:I BOX IS A EXTERNAL SUBSYSTEM WHICH CONNECTS TO A

COM PUTER TMROUGM ONE OF IOMEGAS HOST INTERFACE BOARDS. 12OVAC, OO H Z

IOMEGA, A2ZOH, SER. DLFKK.

REFOR: NUMGER: $898355 \cdot 4007$

LINE ITEM NUMBER: 2023

REFERENCE NUMBER : T3Z

7010 DISC ORIVE, FI GERNOULL: BOX SYSTEM, DLAL DRIVE SYSTEM EACH DRIVE CNA HOLO 20 ME ORIVE IS IBM COMPATIBLE, IZOVAC, OO HiZ. IOMEGA, B2ZOX.

SER. 01600700 .
No of

COND UNITS UNIT

ACOUISIIION PRICE

$\$ 1,855.00$

$\operatorname{COSITUNIT}$

TOTAL COST:

$\$ 1,855.00$

ELOY N. GIROM

ALBUPUEROUE

(505) 846.2746

IO FREEZE EOUIPMENT:

ELOY N. GIRON

AL BUOUEROUE

(505) $844-2746$

m 87185

LCATION OF EQUIPMENT:

SANDIA MAIIONAL LAROPAIDOIES

PROPERTY REAPPLICATION DIV. 7617

AL BUOUEROUE

87123
6 O000T EA

COST/UNIT:

TOTAL COST:
$\$ 1,838.00$

$\$ 1,838.0$

\section{FOR FURTHER INFORMATION: \\ ELOY GIRON}

ALBLOUEROUE

(505) $844-2746$

Mm 87185

TO FREEZE EQUIPMENT :

ELOY N. GIRON

ALBUOUERQUE

(505) $844-2746$

4M 87185

LOCAIION OF EOUIPMENT:

SAMDIA MATIONAL LABORATORIES

PROPERTY REAPPLICAIION DIVISION 7617

AL BUOUEROUE

im 871 
EFFECTIVE DATE: 07/01/94 EXPIRATION DATE: $07 / 31 / 94$

FSC

\section{COOE EXCESS PROPERTY DESCRIPTION}

REFERENCE MUMBER : 733

7010 DISK DRIVE MOD: A220H, IOMEGA

REPORT NUMBER: $897402-4017$

REFERENCE MUMBER : 73

7010 DISK DRIVE MOO: 4869 , IBM

REPORT NUMBER: $897402-4017$
No of

COND UNITS UWIT

ACOUISITION PRICE

500001 EA

$\cos T / U N I T:$

IOTAL COST:

LINE ITEM NUMBER: R004

$500001 E A$

COST/UNIT:

TOTAL COST:

LINE ITEM NUMBER : ROOS
$\$ 1.520 .00$

$\$ 1,520.00$

AMARILLO

(806) $477 \cdot 3870$

:O FREEZE EQUIPMENT:

EVOW DAVIS

EvON DAvis

(806) $477-3870$

LOCATION OF EOUIPMENT:

MASON \& HAMGER

HWY 60 AND 2373

AMARILLO

Ix 79177-0001

IX 79177-000

TX 79177-0001

$\$ 3,997.00$ FOR FURTHER IMFORMATION:

$\$ 3,997.00$ EVOW DAVIS

AMAR ILLO

(806) 477.3870

IX 79177-0001

TO FREEZE EOUIPMENT:

EVON DAVIS

AMAR ILLO

(806) $477-3870$

Ix 79177-0001

LOCATION OF EOUIPMENT:

MASON \& HAMGER

FuY 60 MNO 237

AMARILLO

$7 \times 79177-0001$ 

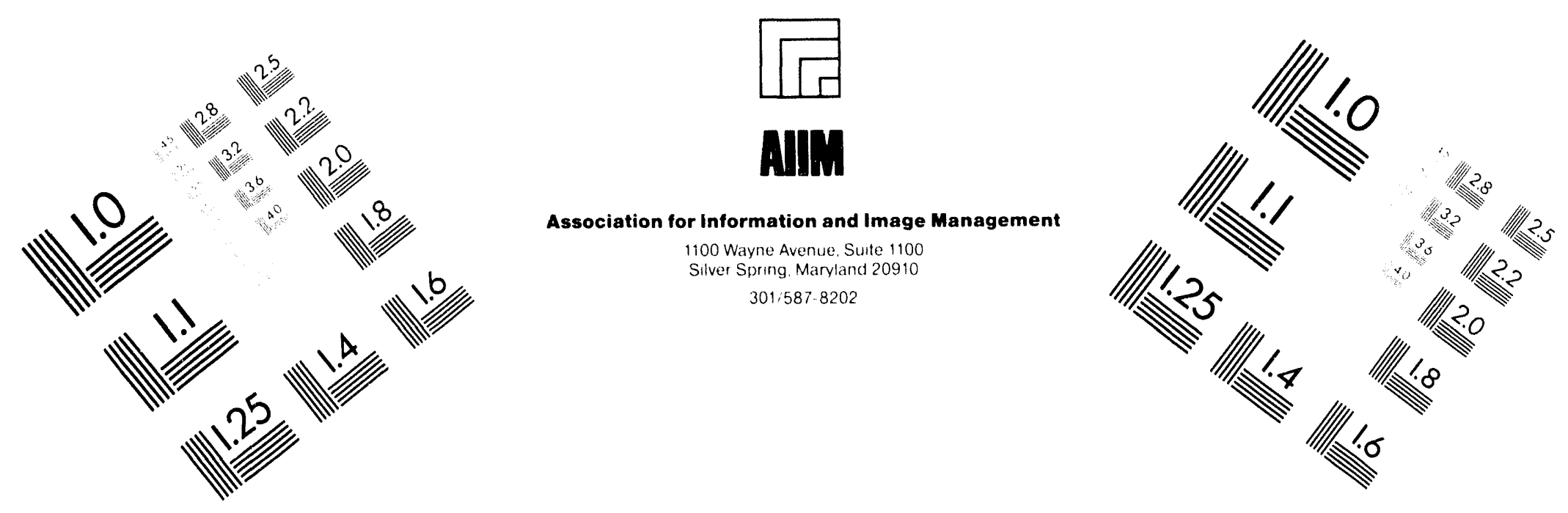

\section{Centimeter}

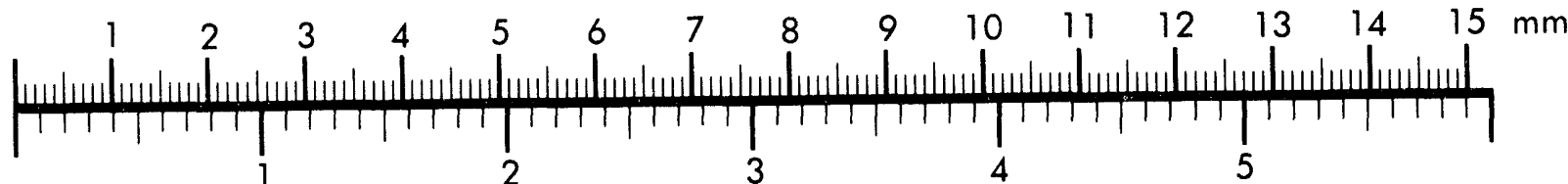
Inches
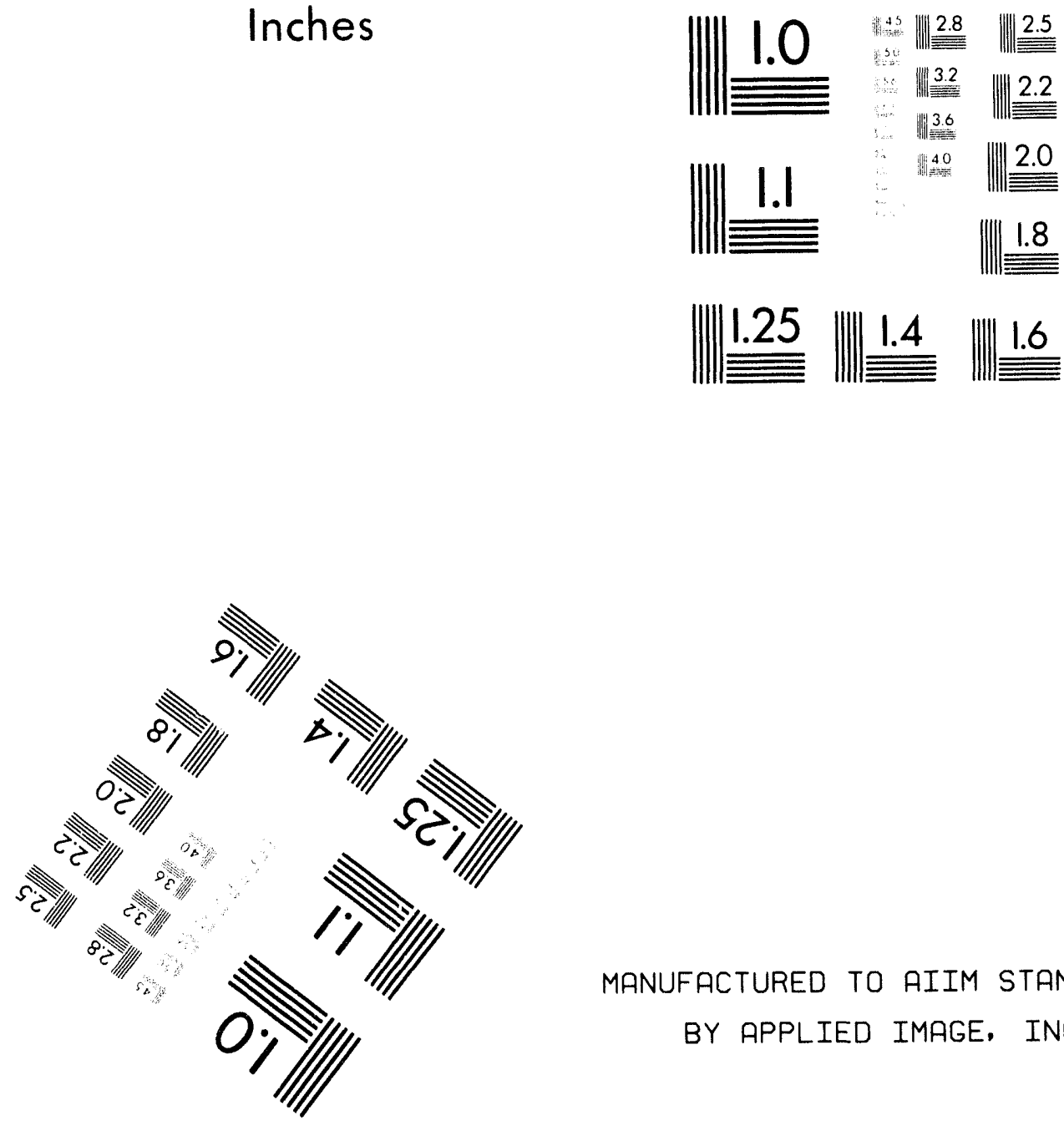

MANUFACTURED TO AIIM STANDARDS

BY APPLIED IMAGE, INC.

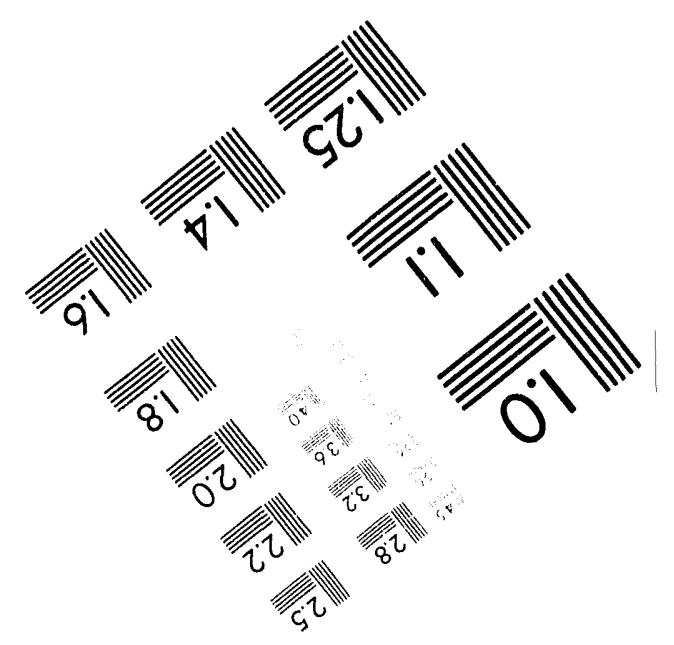



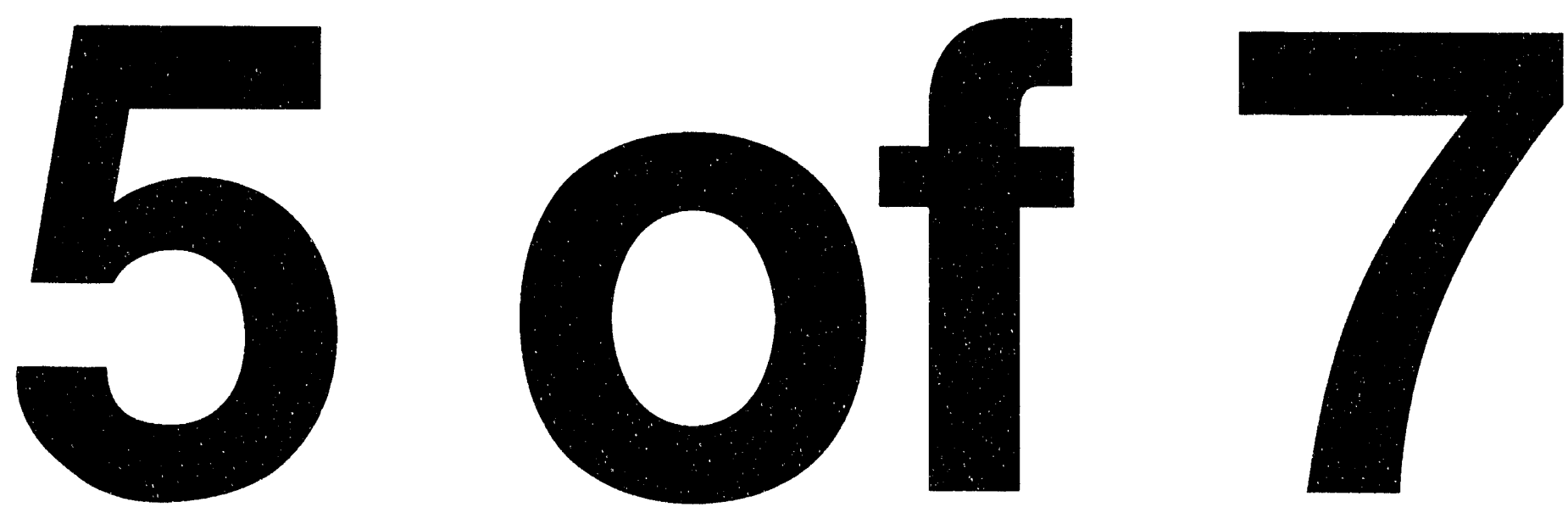
FSC

\section{CODE EXCESS PROPERIY DESCRIPIION}

REFERENCE NUMBER : 73 ;

7010 DISK DRIVE MOD: A22OH, IOMEGA

REPORT NUMSER: $897402-4018$

REFERENCE NUMBER : 730

7010 DISK DRIVE MOD: AZ2OH, IOMEGA

ELIGIBLE EOUIPMENT

PAGE 368

NO OF

COND UNITS UNIT

ACQUISETION PRICE

$400001 E A$

COST/UNIT:
TOTAL COST:

LINE ITEM NUMBER: RO04

$500001 E A$

COST/UNIT:
TOTAL COST:
LINE ITEM NUMBER: ROO5
REPORI NUMBER: $897402-6018$
CONTACT(S)

FOR FURTHER INFORMATION:

EVON DAVIS

AMARILLLO

(806) $477-3870$

TX 79177-0001

TO FREEZE EOUIPMENT:

EVON DAVIS

AMAR 1110

(806) $477-3870$

TX 79177-0001

LOCATION OF EOUIPMENT:

MASON \& HANGER

AMARILLO

IX 79177-0001

FOR FURTHER INFORMAIION

EVON DAVIS

AMARILLO

(806) $477-3870$

TX 79177-0001

TO FREEZE EQUIPMENT

EVON DAVIS

AMARILLO

(806) $477-3870$

TX 79177-0001

LOCATION OF EQUIPMENT :

MASON \& HANGER

HWY 60 AND 2373

AMARILLO

TX 79177-0001 
EFFECTIVE DAIE: 07/01/94 EXPIRATION DATE: $07 / 31 / 94$

FSC

COOE EXCESS PROPERTY DESCRIPIION

REFERENCE NUMBER : 737

7010 DISK DRIVE MOD: A220H, IOMEGA

REPORT NUMBER: $897402 \cdot 4018$

REFERENCE NUMBER : 738

7010 DISK DRIVE MOD: B220X, IOMEGA

REPORT HUMBER: $897402-4018$
NO

COND UNIIS UNIT

\section{ACQUISITION PRICE}

$400001 E A$

COST/UNIT:

LINE ITEM NUMBER: ROO6

\author{
400001 EA \\ COST/UNIT: \\ TOTAL COST:
}

LINE ITEM NUMBER: ROOT
CONTACT(S)

FOR FURTHER INFORMATION:

EVON DAVIS

AMARILLO

(806) $477-3870$

TX 79177-0001

TO FREEZE EQUIPMENT :

EVON DAVIS

AMARILLO

(806) $477-3870$

TX 79177-0001

LOCATION OF EQUIPMENT:

MASON \& HANGER

HWY 60 AND 2373

AMARILLLO

IX 79177-000
FOR FURTHER INFORMATION:

EVON DAVIS

AMARILLO

(806) $477-3870$

IX 79177-0001

TO FREEZE EQUIPMENT:

EVON DAVIS

AMAR

(806) $477-3870$

TX 79177-0001

LOCATION OF EQUIPMENT:

MASON \& HANGER

HWY 60 AND 2373

AMARILLO

TX 79177-0001 
EFFECTIVE DATE: 07/01/94 EXPIRATION DATE: 07/31/94

\section{CODE EXCESS PROPERTY DESCRIPTION}

REFERENCE NUMBER : 730

7010 DISK DRIVE MOD: B220X, IOMEGA

REPORT NUMGER: $897402-4018$

LINE ITEM NUMBER: R008

REFERENCE NUMBER : 740

SK DRIVE THE BERNOU:LI $60 X$ IS A EXTERNAL SUSSYSTEM WHICH CONNECTS TO A 600001 EA

COM PUTER THROUGH ONE OF IIMEGAS HOST INTERFACE GOARDS. 12OVAC, $60 \mathrm{~Hz}$.

OMEGA, AZZOH, SER. OBF3C.

REPORT NUMBER: $898355-4097$

LINE ITEM NUMBER: 0017

$400001 \mathrm{EA}$
NO OF

COND UNITS UNIT

ACQUISITION PRICE

COST/UNIT:

TOTAL COST:

$\$ 1,550.00$

$\$ 1,550.00$

ATER INFORMAIION :

VON DAVIS

AMARILLO

IX 79177-0001

TO FREEZE EQUIPMENT

EVON DAVIS

AMARILLLO

(806) $477-3870$

IX 79177-0001

LOCATION OF EQUIPMENT

MASON \& HANGER

HWY 60 AND 237

AMARILLO

TX 79177-000
COST/UNIT:

TOTAL COST:
$\$ 2,682.00$
FOR FURTHER INFORMATION:

ELOY N. GIRON

ALBUQUERQUE

(505) $844-2746$

NM 87185

TO FREEZE EQUIPMENT:

ELOY N. GIRON

ALBUQUERQUE

(505) 844-2746

NM 87185

LOCATION OF EOUIPMENT:

SANDIA NATIONAL LABORATORIES

PROPERTY REAPPLICATION DIV 7617

ALBUDUERQUE

NM 87123 
EFFECTIVE DATE: 07/01/94 EXPIRATION DATE: $07 / 31 / 94$

\section{NO OF}

CODE EXCESS PROPERTY DESCRIPTION

REFERENCE NUMBER : 741

7010 EXPANSION UNIT XT COMPUTER W/O4OKB RAM, SERIAL PORT, 2 5-1/4" DISK DRIVE, HARD D RIVE. IZOVAC, OO HZ. MISSING VIDEO CARD. IBM, 5161001, SER. 0147009

REPORT NUMBER: $898355-4091$

LINE ITEM NUMBER: 0076

REFERENCE NUMBER : 742

7010 EXPANSION UN!T HAS A 256 CPU AND A HARD DRIVE, II ALSO HAS A $5-1 / 4 "$ DISK

DRIVE. 12OVAC, 6O HZ. THIS UNIT IS NOT AN EXPANSION UNIT. IBM, 516100

$600001 \mathrm{EA}$ 1. 0147602
COND UNITS UNIT

7. $00001 \mathrm{EA}$

COST/UNIT:
IOTAL. COST:

UNIT:
COST:
COST/UNIT:
TOTAL COST:
$\$ 2,373.00$
$\$ 2,373.00$

$\$ 2,373.00$
$\$ 2,373.00$
$\$ 2,373.00$

\section{FOR FURTHER INFORMATION:}

ELOY N. GIRON

ALBUQUERQUE

(505) $844-2746$

NM 87185

TO FREEZE EQUIPMENT:

ELOY N. GIRON

ALBUQUEROUE

NM 87185

(505) $844-2746$

LOCATION OF EQUIPMENT:

SANDIA NATIONAL LABORATORIES

PROPERTY REAPPLICATION DIV. 7617

ALBUQUEROUE 
EFFETIVE DATE: OT/O1/Q4 EXPIRATION DATE: $07 / 31 / 94$

isc

COOE EXCESS PROPERTY DESCRIPTION

REFERENCE NIMMBER : $7 \% 3$

7010 FLOFPY DISC DRIVE MACINIOSH 2IMB HARD DRIVE, 120VAC, $60 \mathrm{HZ}$. APPLE COMF, MOi35, SER. FO120FO
NO OF

COND UNITS UNIT

$00001 \mathrm{EA}$

EA

TOTAL COST:

LINE ITEM NUMBER: 0073
REFORT NUMAER: $898355-\rightarrow 00$;
REFERENCE NUMBER : THM

7010 IMAGE HARDCOPY DEVICE PAPER SIZE IS $8.5 \times 11 "$. WARM UP TIME 5 Minutes, COPY TiME 18 SECON DS, IO SEC FOR THE NEXT COPY. PAPER STD 115VAC, 60 HZ. A ASER TECH, 3000405, SER. 89099847.
LINE ITEM NUMBER: 0078
FOR FURTHER INFORMATION:

ELOY N. GIRON

ALBUOUEROUE

(505) $844-2746$

NM 87185

IO FREEZE EQUIPMENT:

ELOY N. GIRON

ALBUOUEROUE

(505) $844-2746$

NM 87185

LOCATION OF EQUIPMENT:

SANDIA NATIONAL LABORATORIES

PROPERTY REAPPLICATION DIVISION 7617

ALBUQUEROUE NM 87123 
EFFECIIVE DATE: 07/01/94 EXPIRATION DATE: $07 / 31 / 94$

COOE EXCESS PROPERTY DESCRIPTION

REFERENCE NUMBER : 745

7010 MEMORY BOARD PLUG IN BOARD FOR COMPUTER, WITH A 25 PIN PORT IN BACK. FORCE CO MP. SRAM2ZB, SER. 633B300A.

REPORT NUMBER: $898355-4094$

LINE ITEM NUMBER: 0002
NOO OF

COND UNITS UNIT

ACQUISITION PRICE

$600001 \mathrm{EA}$

COST/UNIT:

TOTAL COST:

$\$ 2,490.00$

$\$ 2,490.00$
CONTACT(S)

OR FURTHER INFORMATION:

ALBUQUEROUE

505) $844-2746$

NM 87185

TO FREEZE EQUIPMENT:

ELOY N. GIRON

ALBUDUERQUE

(505) $844-2746$

NM 87185

LOCATION OF EOUIPMENT:

SANDIA NATIONAL LABORATORIES

PROPERTY REAPPLICATION DIVISION 7617

ALBUOUERQUE NM 87123

REFERENCE NUMBER : 746

\section{EA COST/UNIT:}

TOTAL COST:

$\$ 1.295 .00$
$\$ 1,295.00$

FOR FURTHER INFORMATION:

ELOY N. GIRON

ALBUQUERQUE

(505) 844-2746

NM 87185

TO FREEZE EQUIPMENT:

ELOY N. GIRON

ALBUOUERQUE

(505) $844-2746$

NM 87185

LOCATION OF EQUIPMENT :

SANDIA NATIONAL LABORATORIES

PROPERTY REAPPLICATION DIVISION 7617

PROPERIY REAPPLICATION DIVISION 7617
ALBUQUERQUE
NM 87123 
EFFECIIVE DATE: OT/01/94 EXFIRATION DATE: 07/31/94

FSC

REFERENCE NUMEER: 743

7010 MODULE MEMORY PLUG IN BOARD FOR COMPUTER. WITH A 25 PIN PORT IN BACK. FORCE CO MP, SRAMZES. IIBC3OOA.

REFOR: NUMEER: $998355-4094$

\section{CODE EXCESS PROPFRTY DESCRIPTION}

$$
\text { NO of }
$$

COND UNITS UNIT

ACQUISITION PRICE

$600001 \mathrm{EA}$

COST/UNIT: TOTAL COST:

\section{$\$ 1,650.00$}

$\$ 1,650.00$

ELOY N. GIRON

ALBUOUERQUE

(505) $844 \cdot 2746$

NM 87185

TO FREEZE EQUIPMENT

ELOY N. GIRON

ALBUOUEROUE

(505) $844-2746$

NM 87185

LOCATION OF EOUIPMENT:

SANDIA NATIONAL LABORATORIES

PROPERTY REAPPLICATION DIVISION 7617

ALBUQUERQUE

NM 87123

\section{REFERENCE NUMBER : 748}

O:- MOOULE MEMORY PLUE IN SOARO FOR COMPUTER, WITH A 25 PIN PORT IN BACK. FORCE CO MP. SRAMZZS, SER. TOCC3OJA.

$60001 \mathrm{EA}$

COST/UNIT: TOTAL COST:

$\$ 1,650.00$ $\$ 1,650.00$

ELOY N. GIRON

ALBUOUERQUE
(505) $844-2746$

NM 87185

TO FREEZE EOUIPMENT:

ELOY N. GIRON

ALBUQUERQUE

(505) $844-2746$

NM 87185

LOCATION OF EQUIPMENT

SANDIA NATIONAL LABORATORIES

PROPERTY REAPPLICATION DIV. 7617

ALBUQUERQUE

NM 87123 
DOE USED ENERGY RELATED LABORATORY EQUIPMENT GRANT PROGRAM ELIGIBLE EQUIPMENI

EFFECTIVE DATE: $07 / 01 / 94$ EXPIRATION DATE: $07 / 31 / 94$

\section{FSC}

CODE EXCESS PROPERTY DESCRIPIION

REFERENCE NUMBER : 749

7010 MONITOR COLOR MONITOR FOR SUN COMPUTER, HAS RGB CONNECTIONS, BRIGHINESS, CONTRAST DEGAUSS AND ON/OFF CONTROLS. 12OVAC, 60 HZ. SUN, HML119S, SER.

CONATOSZ3

REFERENCE NUMBER : 750

7010 MONITOR HIGH RESOLUTION MONOCHROME MONITOR. I2OVAC, $60 \mathrm{HZ}$. SIGMA DESIGN, LDM1019. SER. $581558 \mathrm{M}$.

REPORT NUMEER: $898355-4094$

LINE ITEM NUMBER: 0006

$$
\text { NO OF }
$$

COND UNITS UNIT

$60001 \mathrm{EA}$
COST/UNIT: TOTAL COST:

ACOUISITION PRICE

$\$ 5,000.00$ $\$ 5,000.00$
ELOY N. GIRON

ALBUOUERQUE
(505) $844-2746$

NM 87185

TO FREEZE EQUIPMENT

ELOY N. GIRON

ALBUQUERQUE

(505) $844-2746$

NM 87185

LOCATION OF EQUIPMENT:

SANDIA NATIONAL LABORATORIES

PROPERTY REAPPLICAIION DIVISION 7617

PROPERTY REAPPLICAIION DIVISION 7617
ALBUQUERQUE

$700001 \mathrm{EA}$

COST/UNIT:

TOTAL COST:

$\$ 1,560.00$

$\$ 1,560.00$

FOR FURTHER INFORMATION:

ELOY N. GIRON

AL BUQUERQUE

(505) $844-2746$

NM 87185

TO FREEZE EQUIPMENT:

ELOY N. GIRON

ALBUQUEROUE

(505) $844-2746$

NM 87185

LOCATION OF EQUIPMENT.

SANDIA NATIONAL LABORATORIES

FROPERTY REAPPLICATION DIVISION 7617

ALBUQUEROUE NM 8712 
EFFECTIVF DATE: OT/01/94 EXFIRATION DAIE: 07/31/94

FSC

COOE EXCESS PROPERTY DESCPIFIION

REFERENCE NUMBER : 75

7010 MONI:OR, PC 13" COLOR DISFLAY MONITOR HAS COLOR MODE SELECT GREEN, AMBER

AND WHITE ROVVAC, OO HZ. MITSUGISHI, EA3415A, SER. 6500812

REPOR: NUMGER: $898355-4094$

LINE ITEM NUMBER: 0010

REFERENCE NUMGER : 752

7010 MONITOR, FC 14" DIALONAL SCREEN, 256 COLORS FROM A PALETTE OF 4,006 $340 \times 480$ G RAPH!CS, 25 LINES GY 80 CHARACTERS. 120VAC, $60 \mathrm{HZ}$. IGM, 9514001 , SER. 1040701

no of

COND UNIIS UNIT

ACOUISITION PRICE

COST/UNIT: TOTAL COST:

$500001 E A$

$\$ 1,000.00$

ALB


EFFECIIVE DATE: 07/01/04 EXFIRATION DATE: $07 / 31 / 96$

COOE EXCESS PROPERTY DESCRIFIION

REFERENCE NUMBER : 753

7010 MONITOR, PC COLOR MONITOR WITH A 19" DIAGONAL SCREEN, BRIGHINESS,

CONTRAST AN D ON/OFF CONTROLS, WITH RGG CONNECTIONS, 12OVAC, $60 \mathrm{HZ}$.

HML11:S, SER. 913AROOZ.

REPORT NUMEER: $898355-4094$

LINE ITEM NUMBER: 0009

REFERENCF NUMBER : 754

7010 MONITOR, PC VGA COLOR MON!TOR WITH A 14" DIAGONAL SCREEN. 12OVAC, 60

HZ. IGM, 8514001 , SER. 1031254

$600001 E A$

COND UNITS UNIT

- 00001 EA

NU OF

COST/UNIT:

TOTAL. COST:

acouis

(n)

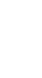


EFFECIIVE DAIS: OT/01/94 EXFIRATIL I DATE: 07/31/94

CODE EXCESS PROPERIY DESCRIPTION

REFERENCE NUMBER : 755

CONTRO, S, WITH A 15" DIALSONAL SCREEN. 120VAC, OO HZ. IBM, 8514001 ,

SER. 1130273
7010 MONITOR, PC VGA COLOR MONITOR WITH GRIGHTNESS CONTRAST AND ON/OFF

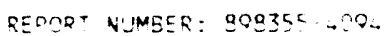

LINE :TEM NUMBER: 0008
COND UNITS UNIT

$500001 E A$

COST/UNIT:

TOTAL COST:

$\$ 1,266.00$

EL

TO FREEZE EQUIPMENT:
ELOY N. GIRON
ALBUQUERQUE

ELOY N. GIRON
ALBUDUERQUE

(505) $844-2746$

NM 87185

LOCATION OF EOUIPMENT:

SANDIA NATIONAL LAGORATORIES

PROPERTY REAPPLICATION DIVISION 7617

ALBUQUERQUE NM 87123

REFERENCE NUMBER : 750

7010 NODE PROCESSOR PLUG IN BOARD FOR NODE PROCESSOR, WITH A 15 PIN PORT IN GACK. CU MM MACHI, ETHERNET D10, SER. 20890.

$600001 E A$

COST/UNIT:

TOTAL COST:

$\$ 2,050.00$

$\$ 2,050.00$

FLR FURTHER INFORMATION:

ELOY N. GIRON

ALBUQUERQUE

NM 87185

(505) $844-2746$

TO FREEZE EOUIPMENT:

ELOY N. GIRON

ALBUOUEROUE

(505) 844-2746

NM 87185

REPORT NUMBER: $898355 \cdot 6094$

LINE IIEM NUMBER: 0011
LOCATION OF EOUIPMENT:

SANDIA NATIONAL LABORATORIES

PROPERTY REAPPLICAIION DIVISION 7617 ALBUQUEROUE 
FSC

\section{CODE EXCESS PROPERTY DESCRIPTION}

REFERENCE NUMBER : 757

7010 OSCILLOSCOPE, DIGITAL DUAL 200 MEGA SAMPLE/SECOND DIGITIZER, 50 MHZ BANDHIDTH PRE-TRIGÓ ER DISPLAY. HEW PACK, 54200D, SER. 2601 AO005.

REPORT NUMGER: $898355-4094$

LINE ITEM NUMBER: 0067

REFERENCE NUMBER : 758

7010 PC XT 64OKB RAM, 5-1/L" OISK DRIVE, HARD DRIVE, SERIAL PORT, PARALLEL $P \quad 700001$ EA ORT AND VIDEO PORT. I2OVAC, $60 \mathrm{HZ}$. DISK DRIVE NEEDS REPAIR. IBM. 5160,60725995 .

REPORT NUMBER: $898355-4091$

LINE ITEM NUMBER: 0037

NO OF

$500001 \mathrm{EA}$
COND UNITS UNII

ACQUISITION PRICE

COST/UNIT:
TOTAL COST:

$\$ 8,869.00$

\section{ALBUQUERQUE}

ELOY N. GIRON

(505) $844-2746$

NM 87185

TO FREEZE EQUIPMENT:

ELOY N. GIRON

ALBUDUERQUE

(505) $844-2746$

NM 87185

LOCATION OF EQUIPMENT:

SANDIA NATIONAL LABORATORIES

PROPERTY REAPPLICATION DIVISION 7617

PROPERTY REAPPLICATION DIVISION 7617
ALBUQUEROUE NM 87123
COST/UNIT:
TOTAL COST:

TOTAL COST:
$\$ 1,544.00$
$\$ 1,544.00$
FOR FURTHER INFORMATION:

ELOY N. GIRON

ALBUQUERQUE

(505) $844-2746$

NM 87185

TO FREEZE EQUIPMENT:

ELOY N. GIRON

ALBUQUERQUE

(505) 844-2746

NM 87185

LOCATION OF EOUIPMENT:

SANDIA NATIONAL LABORATORIES

PROPERTY REAPPLICATION DIVISION 7617

PROPERTY REAPPLICATION DIVISION 7617
ALBUQUEROUE 
EFFECTIVE DATE: OT/O1/94 EXPIRATION DATE: OT/31/94

ISC

\section{CODE EXCESS PROPERTY DESCRIPIION}

REFERENCE NUMBER : 759

7010 PERSONAL COMPIJTER 640 KE RAM, 5-1/4" DISK DRIVE, HARD DRIVE, SERIAL PERSONAL COMPIJTER 640 KE RAM, 5-1/4" DISK DRIVE, HARD DRIVE, SERIAL
PORT, PARALLEL PORT AND VIDEO PORT. 120VAC, 60 HZ. IBM XT 5160. 51844485 .

REPORT NUMBER: $898355-6091$

LINE ITEM NUMBER: 0004

\section{REFERENCE NUMBE?: 760}

PERSONAL COMPUTER 286 COMFUTER $1: 52$ KE RAM $25.1 / 4 "$ DISK DRIVE, HARD

DRIVE, 2 SERIA L FORIS, 2 PARALLEL DORTS ANS VIDEO PORT 12OVAC, $60 \mathrm{HZ}$

IBM, MR 5020

REPORT NUMBER: $898355-4097$

LINE ITEM NUMBER: 003
NO OF

COND UNITS UNIT

ACOUISITION PRICE

$600001 \mathrm{EA}$

COST/UNIT: IOTAL COST:

$\$ 2,524.00$ $\$ 2,524.00$

FOR FURTHER INFORMATION:

(505) $844-2746$

NM 87185

TO FREEZE EQUIPMENT

ELOY N. GIRON

ALBUDUEROUE

(505) $844-2746$

NM 87185

LOCATION OF EQUIPMENT:

SANDIA NATIONAL LABORATORIES

PROPERTY REAPPLICATION DIVISION 7617

ALBUOUEROUE

NM 87123

COST/UNIT:

TOTAL COST:

$\$ 3,581.00$

$\$ 3,581.00$

FOR FURTHER INFORMATION:

ELOY N. GIRON

ALBUQUERQUE
(505) $844-2746$

NM 87185

TO FREEZE EQUIPMENT:

ELOY N. GIRON

ALBUQUEROUE

(505) $844-2746$

NM 87185

LOCATION OF EQUIPMENT:

SANDIA NATIONAL LABORATORIES

PROPERTY REAPPLICATION DIV. 7617

ALBUOUEROUE

NM 87123 
EFFECTIVE DATE: 07/01/94 EXPIRATION DATE: $07 / 31 / 94$

FSt

CODE EXCESS PROPERTY DESCRIPTION

REFERENCE NUMBER : 761

7010 PRINTER 9 PIN DOT MATRIX PRINTER, PRINTS BIDIRECTIONAL, GUFFER CAPACITY $2 \mathrm{~K}$, PRINT SPEED 240 CPS IN DRAFT 40 CPS IN NLQ. I2OVAC, OO HZ. DE C, $2 K$, PRINT SPEED 240
LAL 10 , SER. TAGOQOTIA.

REPORT NUMGER: $898355-4094$

LINE ITEM NUMBER: 0042

REFERENCE NUMEER : 762

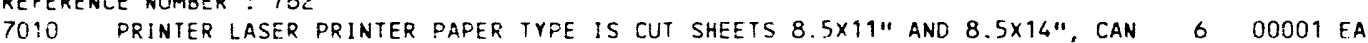
A LSO PRINT ON ENVELOPES. IZOVAC, 6O HZ.

REPORT NUMBER : $898355-4094$

LINE 1 IEM NUMBER: 0046

NO OF

$00001 E A$
COND UNITS UNIT

ACQUISITION PRICE

$\$ 1,005.00$

COST/UNIT: TOTAL COST:

$\$ 1,005.00$

$\$ 1,005.00$

ELOY N. GIRON

ALBUQUERQUE

(505) $844-2746$

NM 87185

TO FREEZE EOUIPMENT:

ELOY N. GIRON

ALBUQUERQUE

(505) $844-2746$

NM 87185

LOCATION OF EQUIPMENT:

SANDIA NATIONAL LABORATORIES

PROPERTY REAPPLICAIION DIVISION 7617

AL BUQUERQUE

NM 87123
COSTIUNIT: IOTAL COST:

$\$ 2,342.00$ $\$ 2,342.00$
FOR FURTHER INFORMATION:

ELOY N. GIRON

ALBUQUERQUE

(505) 844-2746

NM 87185

TO FREEZE EOUIPMENT :

ELOY N. GIRON

ALBUQUERQUE

(505) $844-2746$

NM 87185

LOCATION OF EQUIPMENT:

SANDIA NATIONAL LABORATORIES

PROPERTY REAPPLICATION DIVISION 7617

ALBUQUERQUE

UM 87123 
EFFECTIVE DATE: 07/01/94 EXPIRATION DAIE: 07/31/94

CODE EXCESS PROPERTY OESCRIPIION

REFERENCE NUMBER : 703

7010 PRINTER, LASER CAN PRINT LETTER OUALITY TEXT AND GRAPHICS, USES CASSETTES FOR I 1/2"X11" AND 8-1/2"X14" PAFER. 120VAC, 60 HZ. OUTPUT TRAY BROKEN.

REFERENCE NUMBER : 704

PRINTER, LINE OFFERS GOTH REAR AND BOTTON PAFER FEED. 115/230V, 50-60 7 00001 EA HZ. TEXA S INST, 810, SER. 2281500 .

LINE ITEM NUMBER: 0051

$$
\text { No of }
$$

COND UNITS UNII

7. 00001 EA
COST/UNIT: IOTAL COST:

$\$ 6,880.00$ $\$ 6,880.00$
ACQUISITION PRICE

\section{FOR FURTHER INFORMATION: \\ ELOY N. GIRON \\ ALBUQUERQUE \\ NM 87185}

(505) $844-2746$

TO FREEZE EOUIPMENT:

ELOY N. GIRON

ALBUQUERQUE

NM 87185

(505) $844-2746$

LOCATION OF EOUIPMENT :

SANDIA NATIONAL LABORATORIES

PROPERTY REAPPLICATION DIVISION 7617

ALBUOUERQUE
COST/UNIT:

TOTAL COST:
$\$ 1,579.00$ $\$ 1,579.00$

FOR FURTHER INFORMATION

ELOY N. GIRON

ALBUQUERQUE

(505) 844-2746

NM 87185

TO FREEZE EQUIPMENT

ELOY N. GIRON

ALBUQUEROUE

(505) $844-2746$

NM 87185

LOCATION OF EQUIPMENT:

SANDIA NATIONAL LABORATORIES

PROPERTY REAPPLICATION DIVISION 7617

ALBUQUEROUE 
EFFECTIVE DATE: 07/01/94 EXPIRATION DATE: 07/31/94

$\operatorname{cose}$

REFERENCE NUMEER : 765

7010 PRINTER, PC DIGITAL SCRIPTWRITER IS A STO LASER GRAPHICS PRINTER THAN PROVIDE S CHARACTER FONT FLEXABILITYY AND QUALITY TEXT. 12OVAC, $60 \mathrm{HZ}$ LN O3A, SER. HY02013.
NO OF

COND UNITS UNIT

$00001 \mathrm{EA}$

TOTAL COST:

LINE ITEM NUMBER: 0047
REPORT NUMEER: $898355 \div 094$
REFERENCE NUMBER : 766

7010 PRINTER, PC DIGITAL PRINTER CAN USE THE FONT CARTRIDGES AND $8-1 / 2 \times 11 "$ PAPER C ASSETTE. 120VAC, $60 \mathrm{HZ}$. NEEDS SERVICING BECAUSE PAPER JAMS.

LNO 3AAZ, SER. HYSO972.
LINE I IEM NUMBER: 0049

\section{$700001 \mathrm{EA}$} TOTAL COST:
$\$ 3,245.00$
$\$ 3,245.00$ $\$ 3,245.00$

FOR FURTHER INFORMATION:

ELOY N. GIRON

ALBUOUERQUE

(505) $844-2746$

NM 87185

TO FREEZE EQUIPMENT:

ELOY N. GIRON

(505) $844-2746$

NM 87185

LOCATION OF EOUIPMENT :

SANDIA NATIONAL LABORATORIES

PROPERTY REAPPL!CATION DIVISION 7617 ALBUQUEROUE NM 87123 
EFFECTIVE DATE: BT/OY:O4 EXFIRAIION DATE: 07/31/96

\section{COOE EXCESS PROPERTY DESCRIFIION}

REFERENCE NUMBER : 70

7010 PRINTER, FC DOI MATRIX PRINTER THAT HAS 130 COLUMN PRINTINGS WIOTH, DRAFT NLC FRINT WITH CONINUOUS PAPER FEED. ?ZOVAC, OO HZ. MOD. 479 , SER. 191345 .

REPORT NUMBER: $898355 \cdot .096$

LINE ITEM NUMGER: 0036

REFERENCE NUMEER : iBS

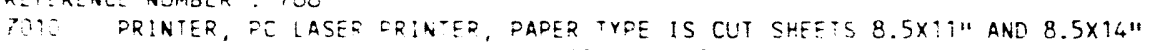
CAN A LSO PRIN ON ENVELOPES. 12UVAC, $60 \mathrm{HZ}$. HEW FACK., 2080A, SER. 26 $02 J 655$
NO OF

COND UNIIS UNIT

ACOUISITION PRICE

$600001 E A$

COST/UNIT:

TOTAL COST:

$\$ 1,159.00$

$\$ 1.159 .00$

\section{FOR FURTHER INFORMATION:}

ELOY N. GIRON

ALBUOUERQUE

(505) $844 \cdot 2746$

NM 87185

TO FREEZE EQUIPMENT

ELOY N. GIRON

ALBUQUERQUE

(505) $844-2746$

NM 87185

LOCATION OF EOUIPMENT:

SANDIA NATIONAL LABORATORIES

PROPERTY REAPPLICATION DIVISION 7617

ALBUQUEROUE

FOR FURTHER INFORMATION:

ELOY N. GIRON

ALBUOUERQUE

NM 87185

(505) $844-2746$

TO FREEZE EOUIPMENT:

ELOY N. GIRON

ALBUQUERQUE

(505) $844-2746$

NM 87185

LOCATION OF EQUIPMENT:

SANDIA NATIONAL LABORATORIES

PANDIA NATIONAL LABORATORIES

PROPERTY REAPPLICATION DIVISION 7617
ALBUQUERQUE
NM 87123 
EFFECTIVE DATE: $07 / 01 / 0_{4}$ EXPIRATION DATE: $07 / 31 / 94$

FSC

CODE EXCESS PROPERTY DESCRIPTION

REFERENCE NUMBER : 760

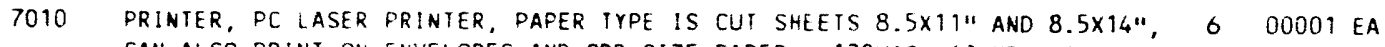
CAN ALSO PRINT ON ENVELOPES AND DOD SIZE PAPER. 120VAC, $60 \mathrm{HZ}$. HEW P $A C K, 2686 A, 2602 J 373$

REPORI NUMBER: $898355 \cdot 6094$

LINE ITEM NUMBER: 0037

REFERENCE NUMBER : 770

7010 PRINTER, FC LASER PR!NIER, PAPER TYPE IS CUT SHEETS $8.5 \times 11$ " AND 8.5 $514 ", 600001$ EA

CAN PRINT ENVELOPES AND ODD SIZE PAPER. I2OVAC, OD HZ. HEW PACK, 268

6A, 2531J277

REPORT NUMBER: $898355-4096$

INE IIEM NUMBER: 0038

No of

COND UNITS UNII

ACQUISITION PRICE

$\$ 2,677.00$

COST/UNIT:

TOTAL COST:

$\$ 2,677.00$

INFORMATION:

ELOY N. GIRON

ALBUQUERQUE

(505) $844-2746$

NM 87185

TO FREEZE EQUIPMENT :

ELOY N. GIRON

ALBUOUEROUE

(505) $844-2746$

NM 87185

LOCATION OF EQUIPMENT:

SANDIA NATIONAL LABORATORIES

PROPERTY REAPPLICATION DIVISION 761

AL BUQUERQUE

NM 87123

COST/UNIT:

TOTAL COST:
FOR FURTHER INFORMATION:

ELOY N. GIRON

ALBUQUERQUE

(505) $844-2746$

NM 87185

TO FREEZE EQUIPMENT:

ELOY N. GIRON

ALBUDUEROUE

(505) $844-2746$

NM 87185

LOCATION OF EQUIPMENT:

SANDIA NATIONAL LABORATORIES

PROPERTY READPLICATION DIVISION 7617

ALBUQUERQUE

NM 87123 
EFFECTIVE DATF: 07/01/04 EXPIRATION DATE: 07/31/94

\section{CODE EXCESS PROPERTY OESCRIPIION}

REFERENCE NUMBER : $P 71$

7010 PRINTER FC LASER PRINTER, PAPER TYPE IS CUT SHEETS, 8.5 $\times 11 "$ AND

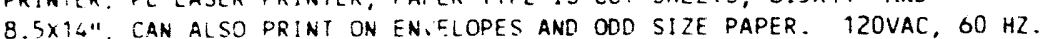

HEW PACK, $2080 \mathrm{~A}, 2424 \mathrm{JOB3}$

REPORT NUMGER: $808355-6096$

LINE ITEM NUMBER: 0039

REFERENCE NUMBER : 772

DOSER PRINTER PAPER TYPE IS CUT SMEETS $8.5 \times 11 "$ AND $8.5 \times 14 "$ FAN A ISO PRINT ON ENVELODES, ZOVAC, GO HZ. HEW PACK, 2086A, SER. 260 23.30
NO OF

COND UNITS UNII ACOUISITION PRICE

$600001 \mathrm{EA}$

COST/UNIT:

TOTAL COST:

$\$ 2,481.00$

$\$ 2,481.00$

ELOY N. GIRON

(505) $844-2746$

NM 87185

TO FREEZE EQUIPMENT:

ELOY N. GIRON

ALBUDUEROUE

(505) $844-2746$

NM 87185

LOCATION OF EQUIPMENT:

SANDIA NAIIONAL LABORATORIES

PROPERTY REAPPLICATION DIVISION 7617

ALBUQUERQL'E

NM 87123

COST/UNIT:
TOTAL COST:

$\$ 2,677.00$

FOR FURTHER INFORMATION:

ALBUQUEROUE

(505) $844-27$

NM 87185

TO FREEZE EQUIPMENT :

ELOY N. GIRON

ALBUDUERQUE

NM 87185

(505) $844-2746$

LOCATION OF EQUIPMENT:

SANDIA NATIONAL LABORATGRIES

PROPERTY REAPPLICATION DIVISION 7617

ALBUOUEROLE

NM 87123 
EFFECIIVE DATE: O:/01/94 EXPIRATION DATE: 07/31/94

FSC

\section{CODE EXCESS PROPERTY DESCRIPIION}

REFERENCE NUMBER : 773 WORK ST ATION WITH CHARACTER FONTS FLEXIBILIITY. 12OVAC, 60 HZ. NEEDS VING. RICOH AMER, LASER60, 13059

INE ITEM NUMBER: 0045
7010 PRINTER, PC LASER JET PRINTER THAT PROVIDES LETIER QUALITY TEXT FOR A

NO OF

COND UNIIS UNIT

7. $00001 \mathrm{EA}$ TOTAL COST: ACQUISITION PRICE

$\$ 2.533 .00$ $\$ 2.533 .00$

ELOY N. GIRON
ALBUOUEROUE
(505) $844-2746$

NM 87185

TO FREEZE EQUIPMENT

ELOY N. GIRON

ALBUOUEROUE

(505) $844-2746$

NM 87185

LOCATION OF EQUIPMENT:

SANDIA NATIONAL LABORATORIES

SANDIA NATIONAL LABORATORIES

AL BUQUERQUE

RE+ERENCE NUMBER : 774

PRINTER, PC LASER PRINTER PAPER TYPE IS CUT SHEEIS 8.5 111 " AND $8.5 \times 14 "$

CAN A LSO PR!NT ENVELODES, PRINTER CAN USE FONT CARTRIDGES. 120VAC, 6

$600001 E A$

H2. HEW PACK, 2680A, SER. A2550J22.
COST/UNIT:
TCTAL COST:

$\$ 2,677.00$
ELOT N. GIRON
ALBUCUEROUE

(505) $844-2746$

NM 87185

TO FREEZE EOUIPMENI

ELOY N. GIRON

ALBUQUERQUE

(505) $844-2746$

NM 87185

LOCATION OF EQUIPMENT

SANDIA NATIONAL LABORATORIES

PROPERTY REAPPLICATION DIVISION 7617

PROPERTY REAPPLICATION DIVISION 7617
ALBUOUERQUE NM 87123 
EFFECIVE DATE: OP/B1/84 EXF!RATION DATE: 07/31/94

\section{isi \\ CONE EXEESS RRUPERV OESCRIEZOON \\ NO OF}

PEFERENCE NUMBER: T:S

7010 DRINTER, DC ASER IE: RAINISR IS A PERSONAL LASER PRINTER THAT PROVIDES IETTE R OUA.: 'Y 'EX: AN GRAFH:CS :OR A WORX STATION WITH CHARACTER FON GEXIGIL: ANE :S IDEAL FOR WORK PROCESSING, IOOVAC, DO

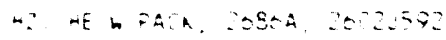

REFOR: NMSER: $898355-439$

LINE ITEM NUMGER: OO50

REFERENCE NUMBER: TOO

FRINTER, SERIAL NEC FR:NTER IS A SFINHRITER SERIAL PRINTER THAT CAN

ORIN IETTER QUA:: Y IEST AND IT ALSO HAS A S!NGLE BIN CUT SHEET FEEDER.

izOV AE, s? +2 .

REPORT NUMBER: $898355-4094$

LINE ITEM NUMBER: 0044
COND UNITS UNII

ACQUISIIION PRICE

CONTACTSS)

00001 EA

COST/UNIT:
TOTAL COST:

$\$ 2,677.00$

$\$ 2,677.00$

FOR FURTHER INFORMATION:

ELOY N. GIRON

AL BUOUEROUE

(505) $844-2746$

NM 87185

TO FREEZE EOUIPMENT:

ELOY N. GIRON

ALBUQUERQUE

NM 87185

(505) $844-2746$

LOCATION OF EQUIPMENT:

SANDIA NAYIONAL LABORATORIES

PROPERTY REAPPLIICATION OIVISION 7617

PROPERTY REAPPLICAIION OIVISION 7617
ALBUQUERQUE
NM 87123
$00001 E A$

COST/UNIT:

TOTAL COST:

$\$ 1.529 .00$
$\$ 1,529.00$

FOR FURTHER INFORMATION:
ELOY N. GIRON
ALBUOUEROUE

(505) $844-2746$

NM 87185

TO FREEZE EQUIPMENT:

ELOY N. GIRON

ALBUQUEROUE

(505) $844-2746$

NM 87185

LOCATION OF EQUIPMENT

SANDIA NATIONAL LABORATORIES

PROPERTY REAPPLICATION DIVISION 7617

PROPERTY REAPPLICATION DIVISION 7617
ALBUQUEROUE 
EFFECTIVE DAIE: 07/01/94 EXFIRAIION DATE: 07/31/94

COOE EXCESS PROPERIY DESCRIPIION

REFERENCE NUMBER : 777

7010 PS2 COMPUTER 1026 KG RAM 3-1/2" DISK DRIVE, SERIAL PORT, PARALLEL PORT AND VGA VIDEO PORT. I2OVAC, 6OHZ. 8560071, SER. 9102734.
No of

COND UNITS UNIT

$700001 \mathrm{EA}$

TOTAL COST:

LINE ITEM NUMBER: 0084
REPORT NUMBER: $898355-4097$

REFERENCE NUMBER : 778

70:0 READ-PRINT MICROFICHE USE FOR READING AND PR!NTING MICROFICHE. IZOVAC, 5 D0001 EA $60 \mathrm{HZ}$., 3M CO., 800F, SER. 147062.

REPORT NUMBER: $898355-4094$

LINE IIEM NUMBER: 0056
COST/UNIT:

TOTAL COST:
ACOUISITION PRICE

$\$ 4.591 .00$ $\$ 4.591 .00$

CONTACT(S)

FOR FURTHER INFORMATION:

ELOY N. GIRON

ALBUQUERQUE

505) $844-2746$

NM 87185

TO FREEZE EQUIPMENT:

ELOY N. GIRON

505) $844-2746$

NM 87185

LOCATION OF EOUIPMENT:

SANOIA NATIONAL LABORATORIES

PROPERTY REAPPLICATION DIV. 7617

ALBUOUEROUE

NM 87123

$\begin{array}{ll}\$ 1,547.00 & \text { FOR FURTHER INFORMATION: } \\ \$ 1,547.00 & \text { ELOY N. G. ON } \\ & \text { ALBUQUERQUE } \\ & \text { (505) } 844-2746 \\ & \\ & \text { TO FREEZE EQUIPMENT: } \\ & \text { ELOY N. GIRON } \\ & \text { ALBUQUEROUE } \\ & \text { (505) } 844-2746 \\ & \text { LOCATION OF EOUIPMENT: } \\ & \text { SANDIA NATIONAL LABORATORIES } \\ & \text { PROPERTY REAPPLICATION DIVISION } 7617 \\ & \text { ALBUQUEROUE } \\ & \text { NM } 87123\end{array}$


FSC

\section{CODE EXCESS PROPERTY DESCRIPIION}

REFFRENCE NUMBER : 770

7010 REFLECTOMEIER 5MHZ PLUG IN UNIT WITH 10 S 10 8ONS PULSER THAT CAN BE USED WITH A TEKTRONIX SCOPE. POWER FROM SCOPE. ORIONICS. OTDRI03, SER. O O7909.
NO) OF

COND UNIIS UNIT

$600001 \mathrm{EA}$

\section{ACQUISITION PRICE}

$\operatorname{COST/UNIT}$ TOTAL COST:

\section{$\$ 6,800.00$} $25,800.00$

\section{CONTACT(S)}

\section{OR FURTHER INFORMATION: \\ ELOY N. GIRON \\ ALBUDUEROUE \\ NM 87185}

IO FREEZE EQUI PMENT:

ELOY N. GIRON

ALBUQUERQUE

(505) $844-2746$

NM 87185

LINE ITEM NUMBER: 0055

OCATION OF EQUIPMENT:

SANDIA NATIONAL LABORATORIES PROPERTY REAPPLICATION DIVISION 7617

ALBUOUERQUE NH 87123

\section{REFERENCE NUMEER : 780}

7010 SCOPE, GENERAL DURE TEK DORTABLE OSCILLOSCOPE W:TH 200MHZ AT 2MV/DIV,

INS/DIV SWEEP R AGE, 8XIO CM DISPLAY AND DMG4. 12OVAC, $60 \mathrm{HZ}$
LINE ITEM NUMBER: 0066
60001 EA

$\operatorname{COST/UNIT}$

TOTAL COST:

$\$ 5,211.00$
FOR FURTHER INFORMATION
ELOY N. GIRON
ALBUOUERQUE
(505) $844-2746$
NM 87185
TO FREEZE EQUIPMENT:
ELOY N. GIRON
ALBUOUERQUE
NM 87185
(505) $844-2746$
LOCATION OF EOUIPMENT:
SANDIA NATIONAL LABORATORIES
PROPERTY REAPPLICATION DIVISION 7617
ALBUOUERQUE 
EFFECIIVE DATE: $07 / 01 / 94$ EXPIRATION DATE: $07 / 31 / 94$

isC

\section{COOE EXCESS PROPERTY DESCRIPIION}

\section{REFERENCE NUMBER : 781}

7010 SCOPE, GENERAL PURP SCOPE HAS 5OOMHZ AT $10 \mathrm{MV} / \mathrm{DIV}$ AND IS 500PS/DIV. FASTEST, IT ALSO HAS CAL IBRATES SWEEF RATE AND GREATER THAN $15 \mathrm{CM} / \mathrm{NS}$ ENHANCED CRT READOUT. 12OVAC, $00 \mathrm{HZ}$. TEKIRONIX.
NO OF

COND UNITS UNI

00001 EA TOTAL COST

ACQUISITION PRICE

$\$ 3.162 .00$ $\$ 3.162 .00$

REPORT NUMBER : $898355-4094$

LINE ITEM NUMBER: 0071

\section{REFERENCE NUMBER : ?8?}

7010 SCOPE, GENERAL PURP TEKTRONIX SCOPE HAS 50OMHZ AY OOMV/DIV WITH 1GHZ DIRECT ACCESS UN IT, SOOPS/DIV FASTEST CALISRATED SWEEP RATE AND IS GREATER THAN 1 SCM/AS. 12OVAC, OO HZ.

REPORT NUMBER: $898355-4094$

LINE ITEM NUMBER: D074
600001 EA
TOTAL COST:
$\$ 4,354.00$
$\operatorname{CONTACT(S)}$

FOR FURTHER INFORMATION :

ELOY N. GIRON

ALBUQUEROUE

NM 87185

(505) $844-2746$

TO FREEZE EQUIPMENT:

ELOY N. GIRON

ALBUQUERQUE

(505) $844-2746$

NM 87185

LOCATION OF EOUIPMENT:

SANDIA NATIONAL LABORATORIES

PROPERTY REAPPLICAIION DIVISION 7617

ALBUQUERQUE
FOR FURTHER IHFORMATION:

ELOY N. GIRON

ALBUOUEROUE

(505) 844.2746

NM 87185

TO FREEZE EQUIPMENT:

ELOY N. GIRON

(505) $844-2746$

NM 87185

LOCATION OF EQUIPMENT:

SANDIA NAT! ONAL LABORATORIES

PROPERTY REAPPLICATION DIVISION 7617

ALRUOUERQUE

NM 87123 
EFFETIVE DATE: OT/O1/N EXFIRATION DATE: OT/31/94

FSC

\section{COOE EXCESS PROPERTY DESCRIPTION}

\section{REFERENCE NUMBER : 783}

7010 SCOPE, GENERAL 500MHZ SCOPE HAS TWO LEFTHAND P-I COMPARTMENTS FOR VERTICAL AND I S COMPATIBLE WITH ALL 7000 SERIES P-1, 120VAC, $60 \mathrm{hZ}$.

TEKTRONIX, 700\%, SER. B09209.

REPOE: NUMEEF. $\quad 373355-20$

LINE IIEM NUMBER: 0075

REFERENCE NUMBER : 78 ;

7010 SCOPE, GENERAL PURF HIS :NSTEUMENT IS A K OHM AC-DC SCOPE EQUIPPED WITH 500001 EA MODEL 975 OM M-COUNTER WUTH A RANGE OF TOKHY. IZOVAC, $60 \mathrm{HZ}$. VU-DATA, DSO5OB, SER 3021.
No of

COND UNITS UNIT

$600001 \mathrm{EA}$ COST/UNIT: TOTAL. COST:

ACQUIS:

COST/UNIT:

IOTAL COSI:
$\$ 2,484.00$

$\$ 2,484.00$

FOR FURTHER INFGRMATION:

ELOY N. GIRON

ALBUQUERQUE

(505) $844-2746$

NM 87185

IO FREEZE EQUI PMENT:

ELOY N. GIRON

ALBUOUERQUE

(505) $844-2746$

NM 87185

LOCATION OF EQUIPMENT:

SANDIA NATIONAL LABORATORIES

PROPERTY REAPPLICATION DIVISION 7617

PLBUUQUEROUE

NM 87123 
EFFECTIVE DATE: $07 / 01 / 94$ EXPIRATION DATE: $07 / 31 / 94$

CODE EXCESS PROPERTY DESCRIPTION

REFERENCE NUMBER : 785

7010 SCOPE, STORAGE HAS 1000 CM/US STORED WRITING SPEED, LONG VIEW IIME, MULTIMODE ST ORAGE AND DC TO 1000 MHZ BANDWIDTH I2OVAC, $60 \mathrm{~Hz}$.

MUL TIMODE ST ORAGE AND DC TO
TEKTRONIX 7633 , SER. 227509.
NO OF

COND UNIIS UNIT

$7 \quad 00001 \mathrm{EA}$

TOTAL COST:

ACOUISITION PRICE

\section{$\$ 6,873.00$} $\$ 6,873.00$
REPORT NUMBER: $898355-4094$

LINE ITEM NUMBER: 0065

REFERENCE NUMBER : 786

7010 SCOPE, STORAGE HAS $1000 \mathrm{CM} / \mathrm{US}$ STORED WRITING SPEED, LONG VIEW TIME, MULTIMODE ST ORAGE AND DC TO $100 \mathrm{MHZ}$ BANDWIDTH. I2OVAC, $60 \mathrm{HZ}$.

$500001 \mathrm{EA}$

COST/UNIT: MUTTHON 7633 , SER, 8172712 .

FOR FURTHER INFORMATION:

ELOY N. GIRON

ALBUOUEROUE
(505) $844-2746$

NM 87185

TO FREEZE EQUIPMENT:

ELOY N. GIRON

ALBUQUEROUE

(505) $844-2746$

NM 87185

REPORT NUMBER: $898355-4094$

LINE ITEM NUMBER: 0068

LOCATION OF EQUIPMENT:

SANDIA NATIONAL LABORATORIES

PROPERTY REAPPLICATION DIVISION 7617

PROPERTY REAPPLICATION DIVISION 7617
ALBUQUEROUE 
EFFECIIVE DATE: 07/01/04 EXPIRATION DATE: 07/31/94

CODE EXCESS PROPERIY DESCRIPIION

REFERENCE NUMBER : 787

7010 SCOPE, STORAGE HAS NORMAL, WRITING MODES, 1801A DUAL CHANNEL 50 MHZ VERTICAL MP AND $1920 \mathrm{C}$ TIME GASE. $120 \mathrm{VAC}, 60 \mathrm{HZ}$. HEW PACK, 184A, SER. $35 A 0 C 923$.
NO OF

COND UNITS UNIT

$00001 \mathrm{EA}$

COST/UNIT:

TOTAL COST:

REPORT NUMBER: $898355-4004$

REFERENCE NUMBER : 788

7010 SEALING MACHINE 1986 MODEL, MOD: HSC 950324 , DOBOY

REPORT NUMBER: $897402-4019$

\section{$200001 E A$}

LINE ITEM NUMBER: ROOI
ACQUISITION PRICE

$\$ 3,721.00$

$\$ 3,721.00$
CONTACT(S)

FOR FURTHER INFORMATION:

ELOY N. GIRON

ALBUQUEROUE

(505) $844-2746$

NM 87185

TO FREEZE EQUIPMENT

ELOY N. GIRON

ALBUOUERQUE
(505) $844-2746$

NM 87185

LOCATION OF EQUIPMENT:

SANDIA NATIONAL LABORATORIES

PROPERTY REAPPLICATION DIVISION 7617

ALBUQUERQUE NM 87123
$\$ 1,225.00$
$\$ 1,225.00$

FOR FURTHER INFORMATION :

EVON DAVIS

AMARILIO

(806) $477-3870$

TX 79177-0001

TO FREEZE EOUIPMENT :

EVON DAVIS

AMARILLO

TX 79177-0001

(806) $477-3870$

LOCATION OF EQUIPMENT

MASON \& HANGER

HWY 60 AND 2373

AMARILLO

TX 79177-0001 
EFFECTIVE DATE: 07/01/94 EXPIRATION DATE: $07 / 31 / 94$

FSC

COOE EXCESS PROPERTY DESCRIPIION

REFERENCE NUMBER : 789

7010 SYSTEM UNIT MOD: 386/20E, COMPAO
NO OF

COND UNIIS UNI

ACOUISITION PRICE

$4 \quad 00001 \mathrm{EA}$ COST/UNIT:
TOTAL COST:
LINE ITEM NUMBER: R001
REPORT NUMBER: $897602-4018$

\section{$\$ 6.955 .00$} $\$ 6,955.00$

FOR FURTHER INFORMATION:

EVON DAVIS

(806) $477-3870$

ix 79177-0001

TO FREEZE EQUIPMENT:

EVON DAVIS

AMARILLO

(806) $477-3870$

IX 79177-0001

LOCATION OF EQUIPMENT:

MASON \& HANGER

HUY 60 AND 2373

AMARILLO

TX 79177-0001

REFERENCE NUMBER : 790 7010 WORK STATION GRAPHICS WORK STATION WITH 8" DIAGONAL MONITOR AND KEYBRD,
IT ALS O HAS 4 MG MEMORY. 12OVAC, 60 HZ. HAS TWO PARTS. SILICON, W2400C , SER. 03263.
$600001 \mathrm{EA}$ TOTAL COST:
$\$ 3,700.00$

FOR FURTHER INFORMATION

ELOY N. GIRON

ALBUQUEROUE

(505) $844-2746$

NM 87185

TO FREEZE EQUIPMENT:

ELOY N. GIRON

ALBUOUERQUE

(505) $844-2746$

NM 87185

REPORT NUMBER : $898355 \cdot 4094$

LINE ITEM NUMBER: 0079

LOCATION OF EQUIPMENT:

SANDIA NATIONAL LABORATORIES PROPERTY REAPPLICATION DIVISION 7617 AROPERTY REAPPLICATIO 87123 
EFFECIIVE DAIE: 07/01/94 EXPIRATION DATE: 07/31/94

$F S C$

\section{CODE EXCESS PROPERIY DESCRIPTION}

REFERENCE NUMBER : 791

7020 COMPUTER MFR. $16 \mathrm{M}$, MODEL 5170 PROPERTY $\# X-17553$

REPORT NUMBER: $898264-4129$

LINE ITEM NUMBER: 0050

$$
\text { NO OF }
$$$$
\text { COND UNITS UNIT }
$$

$500001 \mathrm{EA}$
ACQUISITION PRICE

$\begin{array}{rr}\text { COST/UNIT: } & \$ 7,755.00 \\ \text { TOTAL COST: } & \$ 7,755.00\end{array}$

$\$ 7.755 .00$
REFERENCE NUMBER : 792

7020 COMPUIER MODE. ZENITH, ZF110-22/468 PROPERTY $\# \times .10457$

REPORT NUMBER: $898264-4129$
LINE ITEM NUMBER: 0042

\section{$400001 E A$}

$\$ 4,325.00$
$\$ 4,325.00$

FOR FURTHER INFORMATION:

REBECCA FINCH

GRAND JUNCTION

(303) $248-6623$

CO $81503-5504$

TO FREEZE EQUIPMENT:

REBECCA FINCH

GAND JUNCTION

(303) 2486623

CO 81503-5504

LOCATION OF EQUIPMENT :

RUST GEOTECH

2597 B $3 / 4$ ROAD

GRAND JUNCTION

CO 81503-5504 
EFFECIIVE DATE: 07/01/9. EXPIRATION DATE: $07 / 31 / 94$

ISC

\section{CODE EXCESS PROPERTY DESCRIPIION}

REFERENCE NUMBER : 793

7020 COMPUTER MODEL ZENITH ZF $110-22 / 192$ PROPERTY \# $X-1646$

REPORT NLMBER: $39826 \div-4129$

INE ITEM NUMBER: 0043

REFERENCE NUMBER : 796

7020 COMPUIER MODEL ZENITH ZF $151-52$ PROPERTY \#X-16698

REPORT NUMBER: $898264-4129$ mo or

COND UNITS UNIT

ACQUISITION PRICE

$4 \quad 00001 \mathrm{EA}$

COST/UNIT:

TOTAL COST:

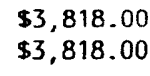

REBECCA FINCH

GRAND JUNCTION

(303) 248-6623

CO $81503-5504$

TO FREEZE EQUIPMENT :

REBECCA FINCH

GRAND JUNCTION

(303) $248-6623$

CO 81503-5504

LOCATION OF EQUIPMENT -

RUST GEOTECH
2597 B $3 / 4$ ROAD

GRAND JUNCIION

CO 81503-5504
$700001 \mathrm{EA}$

COST/UNIT:

IOTAL COST:

$\$ 2,308.00$

$\$ 2,308.00$
FOR FURTHER INFORMATION

REBECCA FINCH

GRAND JUNCTION

CO 81503-5504

(303) 248-6623

TO FREEZE EOUIPMENT:

REBECCA FINCH

GRAND JUNCTION

(303) 248-6623

LOCATION OF EOUIPMENT:

RUST GEOTECH

2597 B $3 / 4$ ROAD

GRAND JUNCTION

CO 81503-5504 
EFFECTIUE DaTE: 07/01/04 EXFIRATION DATE: O7/31/94

$+\mathrm{SC}$

\section{COOE EXCESS PROPERTY DESCRIFIION}

REFERENCE NUMGER : 705

7020 COMPUTER MODEL 2EVI:H 2F $1111.32,192$ PROPERTY $\# \times-16700$

REPOR: NUMBER: $898264-6120$

LINE ITEM NUMBER: 0045

REFERENCE NUMBER : 790

7020 COMPUTER MOOEL ZENITH ZW158-43 PROPERTY \# X-13780

REPORT NUMBER: $898264-4129$

No of

COND UNITS UNII

ACQUISITION PRICE

$400001 \mathrm{EA}$

COST/UNIT: TOTAL COST:

\section{$\$ 2.915 .00$ \\ $\$ 2,915.00$}

REBECCA FINCH
GRAND JUNCTION

(303) $248-6623$

CO $81503-5504$

TO FREEZE EOUIPMENT:

REBECCA FINCH

GRAND JUNCTION

(303) $248-6623$

CO $81503-5504$

LOCATION OF EQUIPMENT:

RUST GEOTECH

2597 B 3/4 ROAD

GRAND JUNCTION

CO $81503-5504$

\section{$500001 \mathrm{EA}$ COST/UNIT:}

TOIAL COST:

LINE ITEM NUMBER: 0046
$\$ 1,539.00$

REBECCA FINCH

GRAND JUNCTION

(303) $248-6623$

TO FREEZE EQUIPMENT:

REBECCA FINCH

GRAND JUNCTION

(303) 248-6623

CO 81503-5504

LOCATION OF EQUIPMENT

RUST GEOTECH

2597 B $3 / 4$ ROAD

GRAND JUNCTION

CO 81503-5504 
EFFECTIVE DATE: 07/01/94 EXPIRATION DATE: $07 / 31 / 94$

FSC

\section{COOE EXCESS PROPERTY DESCRIPTION}

\section{REFERENCE NUMBER : 707}

7020 COMPUTER MODEL ZENITH ZW158-42 PROPERTY \#X-10780

REPORT NUMBER: $898204-4129$

REFERENCE NUMBER : 798

7020 COMPUTER MODEL ZENITH ZW158-42 PROPERTY $\# x-10701$

REPORT NUMBER: $898256-6120$
No of

COND UNITS UNIT

ACQUISITION PRICE

$400001 \mathrm{EA}$

COST/UNIT:

TOTAL COST:

$\$ 2,514.00$

$\$ 2.514 .00$

LINE ITEM NUMBER: 0047

$800001 E A$

COST/UNIT:

IOTAL COST:

$\$ 2,560.00$

LINE ITEM NUMBER: 0048
FOR FURTHER INFORMATION:

REBECCA FINCH

GRANC JUNCTION

(303) $248-6623$

CO 81503-5504

IO FREEZE EQUIPMENT:

REBECCA FINCH

GRAND JUNCTIION

(303) $248-6623$

Co 81503-5504

LOCATION OF EQUIPMENT:

RUST GEOTECH

2597 B $3 / 4$ ROAD

GRAND JUNCTION

CO $81503-5504$ 
DOE USED ENERGY RELATED LABORATORY EOUIPMENT GRANT PROGRAM

EFFECTIVE DATE: G7/01/94 EXFIRATION DATE: 07/31/96 ELIGIBLE EOUIPMENT

\section{FSC
CODE EXCESS PROPERIY DESCRIPTION}

REFERENCE NUMBER : 700

7020 COMPUTER MOOEL ZENITH ZW111-321648 PROPERTY \# X-1079

REPORI NUMGER: $89820 \omega-+120$

REFERENCE NUMBER : 800

7020 COMPUTER MODEL ZENITH 2W15, 52 PROPERTY \#X-17555

REPORT NUMEER: $898264-4129$

LINE ITEM NUMBER: 005
INE ITEM NUMBER: 0049

No of

COND UNITS UNIT

ACOUISIIIION PRICE

400001 EA

COST/UNIT:

TOIAL COST:

$\$ 4,748.00$

$\$ 4.748 .00$

4 O0001 EA

COST/UNIT:

TOTAL COST:

$\$ 5,507.00$
$\$ 5.507 .00$
CONTACT(S)

FOR FURTHER INFORMATION:

REBECCA FINCH

GRAND JUNCTION

(303) 248-6623

TO FREEZE EQUIPMENT:

REBECCA FINCH

GRAND JUNCTION

(303) $248-6623$

co 81503-5504

LOCATION OF EQUIPMENT:

RUST GEOTECH

2597 B 3/4 ROAD

GRAND JUNCTION

CO 81503.5504

FOR FURTHER INFORMATION :

REBECCA FINCH

GRAND JUNCTIION

Co 81503.5504

TO FREEZE EQUIPMENT:

REBECCA FINCH

GRAND JUNCTION

GRAND JUNCT1ON $248-6623$

LOCATION OF EQUIPMENT:

RUST GEOTECH

2597 B 3/4 ROAD

GRAND JUNCTION

Co $81503-550$ 
EFFECIIVE DAIE: $07 / 01 / 9$, EXPIRATION DATE: $07 / 31 / 94$

FSC

\section{COOE EXCESS PROPERTY DESCRIPIION}

REFERENCE NUMBER : 801
7020 COMPUTER MODEL ZENITH $2 F 110-22 / 192$ PROPERTY $\# \times-17550$
Mo of

COND UMITS UNIT

ACOUISITION PRICE

$4 \quad 50001 E A$

TOTAL COST

\section{$56,602.00$}

56.602 .00

INE ITEM NUMBER: 0052

$4 \quad 00001 E A$

$\$ 5.019 .00$

$\$ 5.019 .00$
REFERENCE NUMBER : 802
7020 COMPUTER MODEL ZENITH ZWG121-32 PROPERTY $\# X-1755 ?$

REPORT NUMBER: $898264-4129$
LINE ITEM NUMBER: 0053

\section{TOTAL COST:}

\section{rowTACTSS)}

FOR FURTHER INFORMATION :

REBECCA FINCH

GRAND JUMCTION

CO 81503.5504

TO FREEZE EQUIPMENT:

REBECCA FINCH

GRAND JUNCTION

Co $81503-5504$

OCAIION OF EOUIPMENT:

RUST GEOIECH
2597 B $3 / 4$ ROAD

GRAND JUNCTION

CO $81503 \cdot 5504$

FOR FURTHER INFORMAIION:

REBECCA FINCH

GRAND JUNCTION

CO $81503 \cdot 5504$

TO FREEZE EQUIPMENT:

REBECCA FINCH

GRAND JUMCTION

(303) 248-6623

CO 81503.5504

LOCATION OF EOUIPMENT:

RUSI GEOTECH

2597 B 3/4 ROAD

GRAND JUNCTION

CO $81503-5504$ 
EFFECTIVE DATE: 0i/01/9. EXPIRATION DATE: $0 i / 31 / 04$

\section{COOE EXCESS PROPERTY DESCRIPTION}

PEFERENCE MUMBER : 803

7020 COAPUTER MOCEL ZW $111.32 / 192$ PROPERTV $\$$ X -17558

REPORT NUMEER: $89820-1.120$

LINE IIEM NUMBER: 005

No

OND UNITS UNIT

ACOUISITION PRICE

$400001 \mathrm{EA}$

COST/UNIT:

TOTAL COST:

$\$ 5.606 .00$
COST/UNIT:

TOTAL COST:
$\$ 3,838.00$

$\$ 3,838.00$

FOR FURTHER INFORMATION:

REBECCA FINCH

GRAND JUNCTION

(303) $248-6623$

CO $81503-5504$

TO FREEZE EOUIPMENT

REBECCA FINCH

GRAND JUNCTION

(303) 248-6623

Co $81503-5504$

LOCATION OF EOUIPMENT

RUST GEOTECH

2597 B 3/4 ROAD

GRAND JUNCTION

CO $81503-5504$ 
EFFECTIVE DATE: 07/01/94 EXFIRATION DATE: 07/31/96

FST

\section{COOE EXCESS PROPERTY DESCRIPIION}

REFERENCE NUMGER : 805

7020 COMPUTER MOOEL ZENITH ZW118-42 PROPERTY $\#$ X-17571

REPORT NUMBER: $898206-412$

REFERENCE NUMBER : 806

7020 COMPUTER MODEL 2ENITH ZW118 42 PROPERTY \#X 97573

REPORT NUMBER: $898264-4129$
LIME IIEM NUMBER: 0058

\section{Mo of}

COND UNIIS UNIT

ACQUISITION PRICE

$5 \quad 00001 \mathrm{EA}$

COST/UNIT: IOIAL COST:

$\$ 5.116 .00$ $\$ 5.116 .00$

400001 EA

COST/UNIT:

TOTAL COST:

IINE ITEM NUMBER: 0059
CONTACT(S)

FOR FURTHER INFORMATIOW:

REBECCA FINCH

GRAND JUNCTION

(303) $248-6623$

CO $81503-5504$

TO FREEZE EOUIPMENT:

REBECCA FINCH

GRAND JUNCTION

CO $81503-5504$

(303) 248-6623

LOCATION OF EOUIPMENT:

RUST GEOTECH

2597 B 3/4 ROAD

GRANO JUNCTION

CO 81503-5504
$\$ 5.507 .00$

$\$ 5.507 .00$
FOR FURTHER INFORMATION:

REBECCA FINCH

GRAND JUNCIION

(303) 248-6623

CO 81503.5504

TO FREEZE EOUIPMENT

REBECCA FINCH

GRAND JUHCTION

(303) 248-6623

CO $81503-5504$

LOCATION OF EOUIPMENT :

RUST GEOTECH

2597 B 3/4 ROAD

GRAND JUNCIION

CO 81503-5504 
EFFECTIVE DATE: OT/0:/9. EXPIRATION DATE: OT/31/94

$\operatorname{sc}$

\section{COOE EXCESS PROPERTY DESCRIPIION}

REFERENCE NUMBER : 807
7020 COMPUIER MODEL $2 E N I T H 2 F \quad 110 \cdot 22 / 192$ PROPERTY $\approx x \cdot 17045$

ELIGIBLE EQUIPMENT

NO of

COND UNITS UNIT

ACQUISITION PRICE

$4 \quad 00001$ EA

COST/UNIT:
TOTAL COST:

LINE ITEM NUMBER: 0060
REFERENCE NUMBER : 808

7020 COMPUTER MODEL ZENITH ZW'10-32/192 DROPERIY EX-1765?

REPORT NUMGER: $898204-4129$
$500001 \mathrm{EA}$

COST/UNIT:

TOTAL COSi:

$\$ \$, 246.00$

FOR FURTHER INFORMATION:

REBECCA FINCH

GRAND JUNCTION

CO 81503.5504

TO FREEZE EQUIPMENT:

REBECCA FINCH

GRAND JUNCTION

(303) 248-6023

CO $81503-5504$

LOCATION OF EOUIPMENT:

RUST GEOTECH

2597 B 3/4 ROAD

GRAND JUNCTION

Co 81503-5504 
EFFFCIIVE DATE: 07/01/94 EXFIRATION DATE: 07/31/94

$\mathrm{FSC}$

COOE EXCESS PROPERTY DESCRIPIION

REFERENCE NUMBER : 809

7020 COMPUTER MODEL ZENITH 2W151-152 PROPERTY \#X-17670

REPOR: NUMBER: $898264-4129$

LINE ITEM NUMBER: 0063

\begin{tabular}{ccc}
$\begin{array}{c}\text { NO OF } \\
\text { COND UNITS UNII }\end{array}$ & \multicolumn{3}{c}{ ACOUISIIIION PRICE } \\
400001 EA & $\begin{array}{r}\text { COST/UNIT: } \\
\text { TOTAL COSI: }\end{array}$ & $\$ 5,357.00$ \\
& & $\$ 5,357.00$
\end{tabular}

REFERENCE NUMGER : 810

7020 COMPUTER MODEL ZENITH ZW110-32/192 PROPERTY \#X-17791

REPORT NUMBER : $898264-4129$

LINE ITEM NUMBER: 006
CONTACI (S)

FOR FURTHER INFORMATION:

REBECCA FINCH

$$
\text { GRAND JUNCTION }
$$

CO 8i503-5504

TO FREEZE EQUIPMENT:

REBECCA FINCH

GRAND JUNCTION

(303) 248.6623

CO 81503-5504

LOCATION OF EOUIPMENT:

RUST GEOTECH

2597 B 3/4 ROAD

GRAND JUNCTION

Co 81503-550
$400001 E A$

COSI/UNIT:

TOTAL COST:

$\$ 5,644.00$

$\$ 5.444 .00$
FOR FURTHER INFORMATION:

REBECCA FINCH

GRAND JUNCTION

CO $81503-5504$

(303) 248.6623

TO FREEZE EQUIPMENT :

REBECCA FINCH

GRAND JUNCTION

(303) 248-6623

Co 81503-5504

LOCATION OF EOUIPMENT.

RUST GEOTECH

2597 B $3 / 4$ ROAD

GRAND JUNCTION

CO 81503-5504 
EFFECTIVE DATE: 07/01/94 EXPIRATION DATE: 07/31/94

FSC NO OF

\section{COOE EXCESS PROPERTY DESCRIPTION}

REFERENCE NUMBER : 81

7020 COMPUTER MOOEL, ZENITH $2 F 110-22 / 648$ PROPERTY $\# X-17569$

REPORT NUMBER: $898264-4129$

LINE ITEM NUMBER: 0057
ACQUISIIIION PRICE

COSTIUNIT: TOTAL COST:

$4 \quad 00001 \mathrm{EA}$

\section{$\$ 8,736.00$} $\$ 8,736.00$

\section{FOR FURTHER INFORMATION:}

REBECCA FINCH

GRAND JUNCTION

(303) $248-6623$

CO 81503-5504

TO FREEZE EQUIPMENT:

REBECCA FINCH

GRAND JUNCTION

(303) $248 \cdot 6623$

LOCATION OF EQUIPMENT:

RUST GEOTECH

2597 B 3/4 ROAD

GRAND JUNCTION

CO 81503-5504

$81503-5504$

REFERENCE NUMBER : 812

7020 COMPUTER TEXAS INSTRUMENTS MODEL 10019 PROPERTY \#C4394
$600001 \mathrm{EA}$

COST/UNIT: IOTAL COST:
$\$ 21.101 .00$ $\$ 21,101.00$

LINE ITEM NUMBER: OCO2
REPORT NUMBER: $898264-4129$
FOR FURTHER INFORMATION:

REBECCA FINCH

GRAND JUNCTION

(303) 248.6623

co $81503-5504$

TO FREEZE EOUIPMENT:

REBECCA FINCH

GRAND JUNCTION

(303) 248-6623

CO 81503-5504

LOCATION OF EQUIPMENT:

RUSI GEOTECH

2597 B 3/4 ROAD

GRAND JUNCTIION

CO $81503-550$ 
EFFECTIVE DAIE: 07/01/94 EXPIRATION DATE: $07 / 31 / 94$

FSC

\section{COOE EXCESS PROPERTY DESCRIPTION}

REFERENCE NUMBER : 813

7020 COMPUTER ZENITH MODEL 2 F $110-22$ PROPERTY \#C-3820

REPORT NUMBER : $898264-4129$

\section{REFERENCE NUMBER : 814}

7020 COMPUTER ZENITH MODEL ZW110-32/192 PROPERTY $\# \times 3799$

REPORT NUMBER: $898264-4129$
LINE ITEM NUMBER: 0001

NO OF

COND UNITS UNIT

ACQUISITION PRICE

$60001 \mathrm{EA}$

COST/UNIT:
TOTAL COST:

\section{$\$ 3,838.00$}

$\$ 3,838.00$

$700001 \mathrm{EA}$

COST/UNIT:

TOTAL COST:

$\$ 5,246.00$

$\$ 5,246.00$

LINE ITEM NUMBER: 0004
CONTACT(S)

OR FURTHER INFORMATION:

REBECCA FINCH

GRAND JUNCTION

CO $81503-5504$

TO FREEZE EOUIPMENT:

REBECCA FINCH

GRAND JUNCTION

(303) 248-6623

co 81503-5504

LOCATION OF EQUIPMENT:

RUST GEOTECH

2597 B $3 / 4$ ROAD

GRAND JUNCTION

CO 81503-5504

FOR FURTHER INFORMATION :

REBECCA FINCH

GRAND JUNCTION

CO 81503-5504

TO FREEZE EQUIPMENT:

REBECCA FINCH

GRAND JUNCTION

(303) 248-6623

CO 81503-5504

LOCATION OF EQUIPMENT:

RUST GEOTECH

2597 B $3 / 4$ ROAD

GRAND JUNCTION

CO 81503-5504 
EFFECTIVE DATE : $07 / 01 / 94$ EXPIRATION DATE: $07 / 31 / 94$
FSC

\section{COOE EXCESS PROPERTY DESCRIPIION}

RE FERENCE NUMBER : 815

7020 COMPUTER ZENITH MODEL ZW111-32/448 PROPERTY \#X3877

REPORT NUMBER: $898264-4129$
ELIGIBLE EQUIPMENT
NO OF

COND UNITS UNIT

$4 \quad 00001 \mathrm{EA}$

$\operatorname{COST/UNIT}$

TOTAL COST:

LINE ITEM NUMBER: 0005 COST/UNIT:
TOTAL COST:
$\$ 2,915.00$ $\$ 2,915.00$
$\$ 8,014.00$
FOR FURTHER

GRAND JUNCTION

(303) $248-6623$

Co $81503-5504$

TO FREEZE EQUIPMENT:

REBECCA FINCH

GRAND JUNCTION

CO $81503-5504$

(303) 248-6623

LOCATION OF EQUIPMENT :

RUST GEOTECH

2597 B 3/4 ROAD

GRAND JUNCTION

CO 81503-5504
REFERENCE NUMBER : 816
7020 COMPUTER ZENITH, $2 F 111132 / 192$ PROPERTY $\$ \mathrm{X}-3974$

PEPPOR N NUMBER: $898264-4129$
LINE ITEM NUMBER: 0006

\author{
FOR FURTHER INFORMATION : \\ REBECCA FINCH \\ GRAND JUNCTION \\ (303) $248-6623$ \\ CO $81503-5504$ \\ TO FREEZE EQUIPMENT : \\ REBECCA FINCH \\ GRAND JUNCTION \\ (303) $248-6623$ \\ CO 81503-5504 \\ LOCATION OF EQUIPMENT: \\ RUST GEOTECH \\ 2597 B $3 / 4$ ROAD
}

CO 81503-5504 
EFFECTIVE DATE: 07/01/94 EXPIRATION DAIE: $07 / 31 / 94$ FSC

\section{CODE EXCESS PROPERIY DESCRIPIION}

REFERENCE NUMBER : 897

7020 COMPUTER ZENITH MODEL ZWG121-32 PROPERTY \#X-3981

REPORT NUMBER: $898264-4129$

\section{REFERENCE NUMBER : 818}

7020 COMPUTER ZENITH MODEL ZWG121-32 PROPERTY \#X-3983

REPORT NUMBER: $898264-4129$
No of

COND UNITS UNIT

ACQUISITION PRICE

$400001 E A$

COST/UNIT:

TOTAL COST:

$5,019.00$

LINE ITEM NUMBER: 0007

$600001 \mathrm{EA}$ COST/UNIT:
TOTAL COST:
LINE ITEM NUMBER: 0008
CONTACT(S)

FOR FURTHER INFORMATION:

REBECCA FINCH

GRAND JUNCTION

CO $81503-5504$

TO FREEZE EQUIPMENT :

REBECCA FINCH

GRAMD JUMCIION

(303) 248-6623

Co $81503-5504$

LOCATION OF EQUIPMENT

RUST GEOTECH

2597 B 3/4 ROAD

GRAND JUNCTION

CO $81503-5504$

FOR FURTHER INFORMATION:

REBECCA FINCH

GRAND JUNCTION

(303) 248-6623

CO $81503-5504$

TO FREEZE EQUIPMENT :

REBECCA FINCH

RRAND JUNCTION

CO 81503-5504

OCATION OF EOUIPMENT :

RUST GEOTECH

2597 B 3/4 ROAD

GRAND JUNCTION

CO 81503-5504 
EFFECTIVE DATE: $07 / 01 / 94$ EXPIRATION DATE: $07 / 31 / 94$

FSC

\section{CODE EXCESS PROPERTY DESCRIPTION}

REFERENCE NUMGER : 819

7020 COMPUTER ZENITH, 2G111-32/448 PROPERTY \#X-3985

REFORT NUMBER: $898264 \cdot 4129$

REFERENCE NUMBER : 820

7020 COMPUTER ZENITH MODEL ZW158-42

REPORT NUMBER: $898264-4129$

NO OF

COND UNITS UNI

ACQUISITION PRICE

$\$ 3.770 .00$

$700001 \mathrm{EA}$

COST/UNIT:

TOTAL COST:

$\$ 3,770.00$

REBECCA FINCH

(303) $248-6623$

CO 81503-5504

TO FREEZE EOUIPMENT :

REBECCA FINCH

GRAND JUNCTION

(303) $248-6623$

Co $81503-5504$

LOCATION OF EOUIPMENT

RUST GEOTECH

2597 B 3/4 ROAD

GRAND JUNCTION

CO 81503-5504

$500001 \mathrm{EA}$

COST UNIT:
TOTAL COST:

LINE ITEM NUMBER: 0010 $\$ 5,566.00$

FOR FURTHER INFORMATION :

REBECCA FINCH

GRAND JUNCTION

Co 81503-5504

TO FREEZE EOUIPMENT

REBECCA FINCH

GRAND JUNCTION

(303) $248-6623$

CO $81503-5504$

LOCATION OF EQUIPMENT:

RUST GEOTECH

2597 B 3/4 ROAD

GRAND JUNCTION

CO 81503-5504 
EFFECIIVE DATE: 07/01/94 EXPIRATION DATE: $07 / 31 / 96$

FSC

\section{COOE EXCESS PROPERIY DESCRIPIION}

REFERENCE NUMBER : 821

7020 COMPUTER ZENITH ZW158-43 PROPERTY \#X-12945

REPORT NUMBER: $898264-4129$

LINE ITEM NUMBER: 0012

NO of

COND UNITS UNIT

ACQUISITION PRICE

$400001 \mathrm{EA}$

COST/UNIT:

TOTAL COST:

$\$ 1,747.00$
COST/UNIT:
TOTAL COST:
$\$ 3.163 .00$

$\$ 3,163.00$

FOR FURTHER INFORMATION:

REBECCA FINCH

GRAND JUNCTION

CO $81503-5504$

TO FREEZE EQUIPMENT

REBECCA FINCH

GRAND JUNCTION

(303) 248-6623

Co 81503-5504

LOCATION OF EQUIPMENT :

RUST GEOTECH

2597 B 3/4 ROAD

GRAND JUNCTION

Co $81503-15504$ 
EFFECIIVE DATF: OT/01/94 EXFIRATION DATE: $07 / 31 / 94$

FSC

COOE EXCESS PROPERIY DESCRIFIION

REFERENCE NUMBER : 823

7020 COMPUTER ZENITH ZW168-42 MODEL PROPERTY \#X-13068

REPORT NUMEER: $898264-4129$

AEFERENTE NUMBER : 824

7020 COMPUIER ZENITH MODEL ZW159-3 PROPERTY $\# X-13103$

REPORT NUMBER: $898264-4129$
INE I TEM NUMBER: 0014

No of

COND UNITS UNIT

ACQUISITION PRICE

400001 EA

COST/UNIT: TOTAL COST:

\section{$\$ 1,389.00$} $\$ 1,389.00$

\section{REBECCA FINCH}

FOR FURTHER INFORMATION:

GRAND JUNCTION

CO 81503-5504

TO FREEZE EOUIPMENT:

REBECCA FINCH

GRAND JUNCTION

(303) $248-6623$

Co $81503-5504$

LOCATION OF EQUIPMENT :

RUST GEOTECH

2597 B $3 / 4$ ROAD

GRAND JUNCTION

CO 81503-5504
400001 EA COST/UNIT: TOTAL COST:

LINE ITEM NUMBER: 0015
$\$ 1,774.00$ $\$ 1,774.00$
FOR FURTHER INFORMATION:

REBECCA FINCH

GRAND JUNCTION

(303) 248-6623

Co 81503-5504

TO FREEZE EQUIPMENT:

REBECCA FINCH

GRAND JUNCTION

(303) $248-6623$

CO $81503-5504$

LOCATION OF EQUIPMENT :

RUST GEOTECH

2597 B 3/4 ROAD

GRAND JUNCTION 
EFFECTIVE DATE: 07/01/94 EXPIRATION DATE: $07 / 31 / 94$

FSC

\section{CODE EXCESS PROPERIY DESCRIPIION}

REFERENCE NUMBER : 825

7020 COMPUTER ZENITH MOOEL ZW159-3 PROPERTY \#X-13172

REPORT NUMBER : $898264-4129$

REFERENCE NUMBER : 826

7020 COMPUTER ZENITH MODEL ZW159-3 PROPERTY \#X-13215

REPORT NUMBER: $898264-4129$
NO OF

COND UNITS UNIT

ACQUISITION PRICE

$400001 \mathrm{EA}$

COST/UNIT:
TOTAL COST:

$\$ 1,450.00$

LINE ITEM NUMBER: 0016

$400001 \mathrm{EA}$

COST/UNIT:
TOTAL COST:

LINE ITEM NUMBER: 0019
CONTACTSS

FOR FURTHER INFORMATION

REBECCA FINCH

GRAND JUNCTION

CO 81503-5504

TO FREEZE EOUIPMENT:

REBECCA FINCH

GRAND JUNCTION

(303) $248-6623$

Co 81503-5504

LOCATION OF EQUIPMENT :

RUST GEOTECH

2597 B $3 / 4$ ROAD

GRAND JUNCTION

CO 81503-5504

FOR FURTHER INFORMATION:

REBECCA FINCH

GRAND JUNCTION

CO 81503-5504

(303) 248.6623

TO FREEZE EQUIPMENT

REBECCA FINCH

GRAND JUNCTION

CO $81503-5504$

OCATION OF EQUIPMENT

RUST GEOTECH

2597 B $3 / 4$ ROAD

GRAND JUMCTIOM

CO 81503-5504 


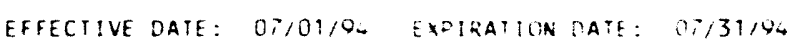

FSC

CODE EXCESS PROPERTY DESCRIPTION

REFERENCE NUMBER : 827

7020 COMPUIER ZENITH MOOEL $2 F 148 \cdot 41$ FROPERTY \#X-13328

REPORT NUMBER: $39820 \cdot-4120$

LINE ITEM NUMBER: 0020

REFERENCE NUMGER : 828

7020 COMPUTER ZENITH MOCEL ZW159-3 PROPERTY \#X-13302

REPORT NUMBER: $898264-4129$

NO OF

COND UNITS UNIT

ACQUISITION PRICE

$500001 \mathrm{EA}$

COST/UNIT:

TOTAL COST:

139.00

$$
\text { REBECCA FINCH }
$$

INFORMATION :

GRAND JUNCTION

CO $81503-5504$

IO FREEZE EOUIPMENT:

REBECCA FINCH

GRAND JUNCIION

(303) 248-6623

c0 81503-5504

LOCATION OF EQUIPMENT

RUST GEOTECH

2597 B $3 / 4$ ROAD

GRAND JUNCTION

co $81503-5504$

$500001 \mathrm{EA}$
COSTIUN!T:
IOTAL COST:
LINE ITEM NUMBER: 0021
FOR FURTHER INFORMATION:

REBECCA FINCH

GRAND JUNCTION

CO 81503-5504

(303) $248-66$

TO FREEZE EOUIPMENT:

REBECCA FINCH

GRAND JUMCTION

CO 81503-5504

LOCATION OF EOUIPMENT

RUST GEOTECH

2597 B 3/4 ROAD

GRAND JUNCTION

Co 81503-5504 
EFFECIIVE DATE: 07/01/96 EXPIRATION DATE: 07/31/94

FSC

\section{CODE EXCESS PROPERTY DESCRIPTION}

REFERENCE NUMBER : 829

7020 COMPUTER ZENITH 2F148-42 PROPERTY *X-13402

REPORT NUMBER: $898264-4129$

REFERENCE NUMBER : 830

7020 COMPUTER ZENITH 2W159-3 PROPERTY \#X-13406

REPORT NUMBER: $898264-4129$

\begin{tabular}{lll} 
NO OF & \multicolumn{2}{c}{ ACOUISITION PRICE } \\
COMD UMITS UNIT & & $\$ 1,103.00$ \\
400001 EA & $\begin{array}{r}\text { COSTIUNIT: } \\
\text { TOTAL COST: }\end{array}$ & $\$ 1,103.00$
\end{tabular}

LINE ITEM MUMBER: 0024

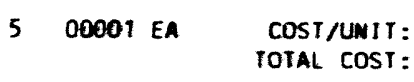

LIME ITEM NUMBER: 0025
TOTAL COST:

$\$ 1,851.00$

$\operatorname{COMTACT}(S)$

FOR FUR IHER IMFORMAIION :

REBECCA FIMCH

GRAND JUMCTION

(303) 248-6623

CO 81503-55.04

TO FREEZE EOUIPMEMT:

REBECCA FINCH

GRAMD JUNCTIOW

(303) 248-6623

Co 81503-5504

LOCATION OF EQUIPNENT:

RUST GEOTECH

2597 B $3 /$

GRAND JUMCTION

CO $81503-550$

FOR FURTHER IMFORMATION

REBECCA FINCH

GRAND JUNCTION

CO $81503-5504$

TO FREEZE EOUIPMENT:

REBECCA FIMCH

GRAMD JUNCTIO

(303) 248-6623

CO 81503-5504

LOCATION OF EOUIPMENT

RUST GEOTECH

2597 B $3 / 4$ ROAD

GRAND JUNCTION 
DOE USED EMERGY RELATED LABORATORY EQUIPMENT GRANT PROGRAM

EFFECTIVE DATE: D7/01/9- EXFIRATION DATE: 07/31/94

FSC

COOE EXCESS PROFERTY OESCRIPIION

REFERENCE NUMGER : 83

7020 COMPUTER ZENITH $2 W 150-1$ PROPERTY $\# X-13411$

REPOR: NUMBER: $898264-4120$

REFERENCE NUMGER : 832

TO2O COMPUTER ZENITH 25248-8: PROPERTY $\$ X-13412$

REPORT MUMBER: $898264-4129$

ELIGIBLE EOUIPMENT

$$
\text { No of }
$$

COND UNITS UNIT

ACQUISITION PRICE

5 O0001 EA

COST/UNIT: TOTAL COST:

\section{$\$ 1.250 .00$}

$\$ 1,250.00$

LINE ITEM MUMBER: 0026

50000 : EA

COST/UNIT:

TOTAL COST:
$\$ 2.169 .00$
$\$ 2.169 .00$

LIME ITEM MUMBER: 0027
CONTACI(S)

FOR FURIHER IMFORMATION:

REBECCA FINCH

GRAMD JUMCTION
(303) $248-6623$

CO 81503-5506

TO FREEZE EOUIPMEMT :

REBECCA FIMCH

GRAMD JUWCTION

(303) 248-6623

Co 81503-5504

LOCATION OF EQUIPMENT:

RUST GEOTECH

2597 B $3 / 4$ ROAD

GRAMD JUNCTION

CO $81503-5506$

FOR FURTHER INFORMATION:

REBECCA FINCH

GRAND JUNCIION

Co $81503-5506$

(303) $248-6623$

TO FREEZE EOUI PMENT

REBECCA FIMCH

GRAND JUWCTIOO

(303) 248-6623

CO 81503-5504

LOCAIION OF EOUIPMENT:

RUST GEOTECH

2597 B $3 / 4$ ROAD

GRAMD JUNCTION

CO $81503-5504$ 
EFFECTIVE DATE: $07 / 01 / 94$ EXPIRATION DATE: $07 / 31 / 94$

FSC

\section{COOE EXCESS PROPERTY DESCRIPIION}

REFERENCE MUMBER : 833

7020 COMPUTER ZENITH MOOEL ZWI59-2 PROPERTY $\#$ X-13636

REPORT NUMEER: $89820 \%-\$ 129$

REFERENCE MUMBER : 83

7020 COMPUTER ZENITH MOOEL ZW159-3 PROPERTY EX-:3489

REPORT NUMBER: $898264-4129$

ELIGIBLE EOUIPMEMT

No of

COMD UNITS UNII

$5 \quad 00001 \mathrm{EA}$

COSTIUMIT: TOTAL COST:

LINE ITEM NUMBER: 0028

$5 \quad 00001$ EA

COST/UNIT: TOTAL COST:

$\$ 1.403 .00$

IINE ITEM NUMBER: 0029 $\$ 1,403.00$

$\$ 2,760.00$ 2.760 .00

REBECT THER IMFT

REBECCA FIMCH

GRARD JUACHON

Co $81503-5504$

TO FREEZE EQUIPMENT:

REBECCA FIMCH

GRAND JUWCTIOM

(303) 248-6623

co $81503-550<$

LOCATION OF EOUIPMENT:

RUST GEOIECH

2597 B $3 / 4$ ROAD

GRAKD JUACTION

CO 81503-5504

FOR FURTHER INFORMATION:

REBECCA FIMCH

GRAND JUNCTIOA

(303) 248-6623

Cu 81503-5504

TO FREEZE EQUIPMENT:

REBECCA FIMCH

GRAMD JUWCTIOW

(303) 248-6623

CO 81503.5504

LOCATION OF EOUIPMENT:

RUST GEOTECH

2597 B $3 / 4$ ROND

GRAND JUWCTION 
EFFECTIVE DAIE: 07/01/94 EXPIRATION DATE: $07 / 31 / 96$

\section{COOE EXCESS PROPERTY DESCRIPIION}

REFERENCE NUMBER : 835
7020 COMPUTER ZENITH, MOOEL $2 F 280$ PROPERTY \# X-13736

REPORT NUMGER: $39826-\div 129$

REFERENCE NUMBER : 836
7020 COMPUTER ZEMITH MODEL ZWL 183.92 PROPERTY \#X-1373.

REPORT NUMBER: $898264-4129$ no of

COMD UNITS UNIT

ACOUISITION PRICE

$700001 \mathrm{EA}$

COST/UNIT:

TOTAL COST:

LINE ITEM NUMBER: 0030

4 O0001 EA COST/UNIT:

TOTAL COST:

LINE ITEM NUMBER: 0031
400001 EA COST/UNIT:
$\$ 1,949.00$
$\$ 1,949.00$

Gra

FOR FURTHER INFORMATION:

REBECCA FIMCH

GRAND JUWCTION

co $81505-5506$

TO FREEZE EOUIPMENT:

REBECCA FINCH
GRAND JUMCTION

GRAND JUMCTION

Co 81503-5504

LOCATION OF EOUIPMENT:

RUST GEOTECH

2597 B $3 / 4$ ROAD

GRAND JUNCTION

Co $81503-5504$

FOR FURTHER INFORMATION:

REBECCA FINCH

GRAND JUNCTION

CO $81503-5504$

(303) 248-6623

IO FREEZE EQUIPMENT:

REBECCA FINCH
GRAND JUMCTION

(303) 248-6623

CO 81503-5504

LOCATION OF EQUIPMENT:

RUST GEOTECH

2597 B $3 / 4$ ROAD

GRAND JUNCTION

Co 81503-5506 
EFFECIIVE DATE: 07/01/94 EXPIRATION DATE: $07 / 31 / 94$

COOE EXCESS PROPERIY DESCRIPIION

REFERENCE MUMBER : 837

TO20 COMPUTER ZENITH, 2286-25 PROPERTY \# X-13855

REPORT NUMEER: $898264-4129$

\section{No of}

LINE ITEM NUMBER: 0032

COND UNIIS UNIT

600001 EA

\section{COST/UNIT: \\ TOTAL COST:}

$700001 \mathrm{EA}$

REFERENCE MUMBER : 838

7020 COMPUTER 2ENITH, MODEL ZWL 18393 PROPERTY \# X-13919

REPORT NUMBER: $898264-4129$
LINE ITEM NUMBER: 0033

\section{CQUISITION PRICE}

\section{$\$ 1,862.00$}

$\$ 1,862.00$

GRAND JUNCTIO

(303) $248-6623$

TO FREEZE EOUIPMENT :

REBECCA FINCH

GRAMD JUMCTION

(303) 248-6623

LOCAIION OF EQUIPMENT

RUST GEOTECH
2597 B $3 / 4$ ROAD

GRAND JUNCTION
Co $81503-5504$

CO $81503-550$

Co $81503-5504$
$\$ 2,362.00$

COST/UNIT:
TOTAL COST:

2.362 .00
FOR FURTHER INFORMATION

REBECCA FINCH

GRAND JUNCTIO

(303) 248 -6623

CO 81503-5504

TO FREEZE EOUIPMENT:

REBECCA FINCH

GRAMD JUNCTION

(303) $248-6623$

CO $81503-5564$

LOCATION OF EOUIPMENT:

RUST GEOTECH

2597 B $3 / 4$ ROAD

GRAND JUNCTION

CO 81503-5504 
EFFECTIVE DATE: 07/01/94 EXPIRATION DATE: 07/31/94

\section{FODE EXCESS PROPERTY DESCRIPTION}

$$
\text { No of }
$$

LINE ITEM NUMBER: 0034

COND UNIIS UNIT

$600001 \mathrm{EA}$

COST/UNIT: IOTAL COST:

$\$ 1.404 .00$

$\$ 1,404.00$

$7 \quad 00001$ EA

COST/UNIT:

TOTAL COST:

LINE ITEM NUMBER: 0035

ACOUISITION PRICE
REPORT NUMBER : $898264-4129$
REFERENCE NUMBER : 839

TO2O COMPUTER ZENITH, MODEL $2159 / 13$ PROPERTY \# X-14022

REPORT NUMBER : $898264-4129$

REFERENCE NUMGER : 840
CONTACT(S)

FOR FURTHER INFORMATION:

REBECCA FINCH

GRAND JUNCTION

(303) $248-6623$

CO $81503-5504$

IO FREEZE EOUIPMENT:

REBECCA FINCH

GRAND JUNCTION

CO 81503-5504

(303) 248-6623

LOCATION OF EQUIPMENT :

RUST GEOTECH

GRAND JUNCTION

CO 81503-5504

FOR FURTHER INFORMATION:

REBECCA FINCH

GRAND JUNCTION

(303) 248-6623

CO $81503-5504$

TO FREEZE EOUIPMENT:

REBECCA FINCH

GRAND JUNCTION

(303) $248-6623$

CO 81503-5504

LOCATION OF EQUIPMENT:

RUST GEOTECH

2597 B 3/4 ROAD

GRAND JUNCIION

CO $81503-5504$ 
EFFECIIVE DATE: $07 / 01 / 94$ EXPIRATION DATE: $07 / 31 / 94$

\section{CODE EXCESS PROPERTY DESCRIPIION}

REFERENCE NUMBER : 841

7020 COMPUTER ZENITH MODEL 2386-20/70 PROPERTY \# X-15485

REPORT NUMBER: $898264-4129$

REFERENCE NUMBER : 842

7020 COMPUTER ZENITH MODEL ZW158-42 PROPERTY \# X-16377

REPORT NUMBER: $898264-4129$

$$
\text { No of }
$$

COND UNITS UNIT

$800001 \mathrm{EA}$

COST/UNIT: TOTAL COST:

LINE ITEM NUMBER: 0036

$500001 \mathrm{EA}$

COST/UNIT:

TOTAL COST:

LINE ITEM NUMBER: 0038
CQUISITION PRICE

$\$ 3,338.00$ $\$ 3,338.00$

$\$ 2,510.00$ $\$ 2,510.00$

REBECCA FINCH

REBECCA FINCH

GRAND JUNCTION
(303) 248-6623

TO FREEZE EQUIPMENT

REBECCA FINCH

GRAND JUNCTION

(303) 248-6623

LOCATION OF EQUIPMENT:

RUST GEOTECH

2597 B 3/4 ROAD

GRAND JUNCTION

CO $81503-5504$ 
EFFECTIVE DATE: 07/01/94 EXPIRATION DATE: $07 / 31 / 94$

FSC

\section{COOE EXCESS PROPERTY DESCRIPTION}

REFERENCE NUMBER : 843

7020 COMPUTER ZENITH MOOEL ZW110-22/192 PROPERTY \#X-16405
COND UNITS UNIT

$400001 E A$

\section{COST/UNIT:} TOTAL COST:

\section{ACQUISITION PRICE}

REPORT NUMBER: $898264-4129$

LINE ITEM NUMBER: 0039

\author{
$500001 \mathrm{EA}$
}

REFERENCE NUMBER : 844
$7020 \quad$ COMPUTER ZENITH MOOEL ZW158-43 PROPERTY $\sharp \mathrm{X}-16417$

REPORT NUMBER: $898264-4129$
TOTAL COST:

LINE ITEM NUMBER: 0040
$\$ 3,818.00$
OR FURTHER INFORMATION:

REBECCA FINCH

GRAND JUNCTION

(303) 248-6623

TO FREEZE EQUIPMENT:

REBECCA FINCH

GRAND JUNCTION

(303) $248-6623$

LOCATION OF EQUIPMENT:

RUST GEOTECH

2597 B 3/4 ROAD

GRAND JUNCTION

CO $81503-5504$
CO $81503-5504$

CO 81503-5504
FOR FURTHER INFORMATION:

REBECCA FINCH

GRAND JUNCTION

Co $81503-5504$

TO FREEZE EQUIPMENT :

REBECCA FINCH

GRAND JUNCTION

CO 81503-5504

303) 248-6623

LOCATION OF EQUIPMENT :

RUST GEOTECH

2597 B $3 / 4$ ROAD

GRAND JUNCTION

CO 81503-5504 
EFFECTIVE DATE: $07 / 01 / 94$ EXPIRATION DATE: $07 / 31 / 94$

FSC

\section{COOE EXCESS PROPERTY DESCRIPIIOA}

REFERENCE NUMBER : 845

7020 COMPUTERS ZENITH ZW158-43 PROPERTY $\# X-12770$ AND X-12771 no of

COND UNITS UNIT

$400002 \mathrm{EA}$

REPORT NUMBER: $898264-4129$

LINE ITEM NUMBER: 0011

\section{ACQUISITION PRICE}

\section{COST/UNIT:} TOTAL COST:

\section{$\$ 1.539 .00$ $\$ 3,078.00$}

CONTACT(S)

FOR FURTHER INFORMATION:

REBECCA FINCH

GRAND JUNCTION

CO 81503-5504

(303) 248-6623

TO FREEZE EQUI PMENT :

REBECCA FINCH

GRAND IUNCTION

(303) 248-6623

LOCATION OF EQUIPMENT

RUST GEOTECH

2597 B $3 / 4$ ROAD

GRAND JUNCTION

Co $81503-5504$
COST/UNIT: TOTAL COST:
$\$ 1,635.00$ $\$ 11,445.00$
FOR FURTHER INFORMATION:

REBECCA FINCH

GRAND JUNCTION

CO 81503-5504

(303) $248-6623$

TO FREEZE EQUIPMENT:

REBECCA FINCH

GRAMD JUNCTION

(303) 248-6623

CO 81503-5504

LOCATION OF EQUIPMENT

RUST GEOTECH

2597 B 3/4 ROAD

GRAND JUNCTION

CO 81503-5504 
EFFECTIVE DATE: 07/01/96 EXPIRATION DATE: 07/31/94

\section{FSC
COOE EXCESS PROPERTY DESCRIPTION}

REFERENCE NUMBER : 847

7020 COMPUTERS ZENITH MODEL $2 W 159-3$ PROPERTY \#'S X-13207 AND X-13210

$$
\text { NO OF }
$$

COND UNITS UNIT

500002 EA

COST/UNIT: TOTAL COST:
LINE ITEM NUMBER: 0018

REPORT NUMBER: $898264-4120$
REFERENCE NUMBER : 848
7020 COMPUTERS ZEN1TH MODEL ZW159-3 PROPERTY $=$ 'S $x-13367$ AND X-13368
500002 EA
COST/UNIT: TOTAL COST:
$\$ 1,475.00$

$\$ 2,950.00$

FOR FURTHER INFORMATION:

REBECCA FINCH

GRAND JUNCTION

(303) 248-6623

CO $81503-5504$

TO FREEZE EQUIPMENT

REBECCA FINCH

GRAND JUNCTION

(303) 248-6623

Co $81503-5504$

LOCATION OF EOUIPMENT:

RUST GEOTECH

2597 B 3/4 ROAD

GRAND JUNCTION 
EFFECTIVE DATE: 07/01/94 EXPIRATION DATE: 07/31/94

\section{FSC
CODE EXCESS PROPERTY DESCRIPIION}

REFERENCE NUMBER : 849

7020 COMPUTERS ZENITH ZW159-12 PROPERTY \#'S X-13397 AND X-13434

REPORT NUMBER: $898264-4129$

LINE ITEM NUMBER: 0023

REFERENCE NUMBER : 850

7020 PCOMPUTER MODEL ZENITH ZWG121-32 PROPERTY \# X-16456

REPORT NUMBER : $898264-4129$

LINE ITEM NUMBER: 0041
NO OF

COND UNITS UNIT

ACQUISITION PRICE

$500002 \mathrm{EA}$

COST/UNIT: TOTAL COST:

$\$ 1,319.00$ $\$ 2,638.00$

\section{FOR FURTHER INFORMATION:}

REBECCA FINCH

GRAND JUNCTION

(303) $248-6623$

CO $81503-5504$

TO FREEZE EQUIPMENT:

REBECCA FINCH

GRAND JUNCTION

(303) $248-6623$

CO 81503-5504

LOCATION OF EQUIPMENT:

RUST GEOTECH

2597 B $3 / 4$ ROAD

GRAND JUNCTION

Co 81503-5504
$400001 \mathrm{EA}$ COST/UNIT:
TOTAL COST:

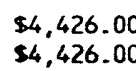

$\$ 4,426.00$
FOR FURTHER INFORMATION:

REBECCA FINCH

GRAND JUNCTION

(303) 248-6623

CO 81503-5504

TO FREEZE EQUIPMENT:

REBECCA FINCH

GRAND JUNCTION

(303) 248-6623

CO 81503-5504

LOCATION OF EOUIPMENT:

RUST GEOTECH

2597 B 3/4 ROAD

GRAND JUNCTION

CO 81503-5504 
EFFECTIVE DATE: $07 / 01 / 94$ EXPIRATION DATE: $07 / 31 / 94$

\section{CODE EXCESS PROPERTY DESCRIPIION}

REFERENCE NUMBER : 851

X94-04-019 MANUF. HEHLET

\# N/A SERIAL \# 2641V45192

BAR CODE 85002:

REPORT NUMEER : $895529-4109$

LINE ITEM NUMBER: 0018

REFERENCE NUMBER : $85 ?$

TO21 COMPUTER MFG WIN LAES 280 AT ACO DATE: $\rightarrow / 90$ PEF NO: 29750.03

$500001 E A$

REPORT NUMGER: $890531-\div 103$

LINE ITEM NUMBER: 0040
COST/UNIT:

TOTAL COST:

$\$ 1,510.00$
$\$ 1,510.00$

NO OF

COND UNITS UNIT

ACQUISITION PRICE

COST/UNIT: TOTAL COST:

$\$ 1,375.00$

HILLIS J. MILLER

CINCINNATI

(513) $738-6630$

TO FREEZE EOUIPMENT:

WILLIS J. MILLER

CINCINNAT

(513) $738-6630$

OH 45239-8704

LOCATION OF EQUIPMENT:

U.S. DOE / FERMCO

7400 WILLEY ROAD

FERNALD

$\mathrm{OH} 45030$

FDR FURTHER INFORMATION:

NANCY J. SITTON

RICHLAND

(509) $376-5108$

WA 99352

TO FREEZE EQUIPMENT:

NANCY J. SITTON

RICHLAND

WA 99352

(509) 376-5108

LOCATION OF EQUIPMENT:

WESTINGHOUSE HANFORD COMPANY

ESS\&S, 1167A BLDG/1100 AREA

RICHLAND 
EFFECTIVE DATE: $07 / 01 / 94$ EXPIRATION DATE: $07 / 31 / 94$

COOE EXCESS PROPERTY DESCRIPTION

No of

COND UNITS UNIT

ACOUISITION PRICE

REFERENCE NUMBER : 853

7021 COMPUTER MFG: IBM 5160 XT ACO DATE: 12/86 REF NO: 29813-01

$500001 \mathrm{EA}$

COSTIUNIT: TOTAL COST:

$\$ 2,550.00$ $\$ 2,550.00$

REPORT NUMBER: $890531-4.103$

LINE ITEM NUMBER: 0068

REFERENCE NUMBER : 854

COMPUTER MFG: APPLE MACINTOSH MODEL: M5251 ACO DATE: 9/88 REF NO: $29777-01$

REPORT NUMBER: $890531-4103$

LINE ITEM NUMBER: 0045
$500001 \mathrm{EA}$

COST/UNIT:
TOTAL COST:

$\$ 2,445.00$

$\$ 2,445.00$
CONTACT(S)

FOR FURTHER INFORMATION:

NANCY J. SITTON

RICHLAND

(509) 376-5108

WA 99352

TO FREEZE EQUIPMENT:

NANCY J. SITTON

RICHLAND

(509) $376-5108$

WA 99352

LOCATION OF EOUIPMENT :

WESTINGHOUSE HANFORD COMPAN ESS\&S, 1167A BLDG/1100 AREA RICHLAND
FOR FURTHER INFORMATION:

RICHLANO

WA 99352

509) $376-5108$

TO FREEZE EOUIPHENT :

NANCY J. SITTON

RICHLAND

WA 99352

(509) $376-5108$

LOCATION OF EOUIPMENT:

WESTINGHOUSE HANFORD COMPANY

ESS\&S, 1167A BLDG/1100 AREA

RICHLAND 
EFFECTIVE DATE: 07/01/94 EXPIRATION DATE: $07 / 31 / 94$

FSC

\section{COOE EXCESS PROPERTY DESCRIPTION}

REFERENCE NUMBER : 855

REFERENCE NUMBER : 855
7021 COMPUTER MFG: COMPAO 286 ACO DATE: 4/91 REF NO: 29402-05
REPORT NUMBER: $890531-4103$

LINE ITEM NUMBER: 0015

REFERENCE NUMBER : 856

7021 COMPUTER MFG: COMPAO PORTABLE 286 MODEL: 40 ACO DATE: $8 / 88$ REF NO: 29784.01

REPORT NLMBER: $890531-4103$
No of COND UNITS UNIT

ACOUISITION PRICE

$600001 \mathrm{EA}$

COST/UNIT: TOTAL COST:

$\$ 3.681 .00$ $\$ 3,681.00$

FOR FURTHER INFORMAIION: NANCY J. SITTOM

RICHLAND

WA 99352

509) $376-5108$

TO FREEZE EQUIPMENT

NANCY J. SITTOW

RICHLAND

(509) $376-5108$

WA 99352

LCATION OF EQUIPMENT:

WESTINGHOUSE HANFORD COMPANY ESS\&S, 1167A BLDG/1100 AREA

RICHLAND
$600001 \mathrm{EA}$ COST/UNIT: TOTAL COST:

$\$ 9,320.00$ $\$ 9,320.00$
FOR FURTHER INFORMATION

NANCY J. SITTON

RICHLAND

HA 99352

(509) 376-5108

TO FREEZE EQUIPMENT :

NANCY $J$. SITTON

RICHLAND

(509) $376-5108$

WA 99352

LOCATION OF EQUIPMENT:

WESTINGHOUSE HANFORD COMPANY ESS8S, 1167A BLDG/1100 AREA

RICHLAND

WA 99352 
EFFECTIVE DATE: 07/01/94 EXPIRATION DATE: 07/31/94

FSC

\section{COOE EXCESS PROPERTY DESCRIPIION}

REFERENCE NUMBER : 857

7021 COMPUTER MFG: COMPAQ PORTABLE 286 MODEL: 40 ACO DATE: 8/88 REF NO: $29784-02$
No of

COND UNITS UNIT

ACQUISITION PRICE

600001 EA

COST/UNIT: TOTAL COST:

LINE ITEM MUMBER: 0049
REFERENCE NUMBER : 858

7021 COMPUTER MFG: COMPAO 286 ACO DATE: 4/88 REF NO: 29845-01
COST/UNIT:
TOTAL COST:
$800001 E$
LINE ITEM NUMBER: 0075
REPORT NUMBER: $890531-4103$

\section{$\$ 9,319.00$
$\$ 9.319 .00$}

MANCY J. SITIOW
RICHLANO

(509) $376-5108$

TO FREEZE EOUIPAENT:

MANCY J. SITTON

RICHLAND

(509) $376-5108$

LOCATION OF EQUIPMENT

WESTINGHOUSE HAMFORD COMPANY

ESS\&S, 1167A BLDG/1100 AREA

RICHLAND

WA 99352
WA 99352

WA 99352
$\$ 1.724 .00$ $\$ 1.724 .00$

FOR FURTHER INFORMATIOW:

MANCY J. SITTON

RICHLAND

(509) $376-5108$

WA 99352

TO FREEZE EOUIPMENT :

MANCY J. SITTON

RICHLAND

(509) $376-5108$

WA 99352

LOCATION OF EOUIPMENT:

WESTINGHOUSE HANFORD COMPANY

ESS\&S, 1167A BLDG/1100 AREA

ESSES, 1167A BLDG/1100 AREA
RICHLAND 
EFFECTIVE DATE: $07 / 01 / 94$ EXPIRATION DATE: $07 / 31 / 94$

\section{isC \\ CODE EXCESS PROPERTY DESCR!PIIION}

7021 COMPUTER MFG: COMPAO ACO DATE: 8/88 REF NO: 29853-01

ELIGIBLE EQUIPMENT

No of

COND UNITS UNI

ACOUISITION PRICE

$500001 \mathrm{EA}$
REFERENCE NUMBER : 860

7021 COMPUTER MFG: COMPAO PORTABLE ACE DATE: 12/87 REF NO: 29853-02
TOIAL COST:
$\$ 9,927.00$
$\$ 9,927.00$
REPORT NUMBER: $890531-4103$
LINE ITEM NUMBER: 0083
$500001 \mathrm{EA}$
COSTIUNIT:
TOTAL COST:
LINE ITEM NUMBER: QOOS

\section{CONTACT(S)}

\section{OOR FURTHER IMFORMATIOM:}

NANCY i. SITHON

RICHLAND

(509) $376-5108$

WA 99352

TO FREEZE EOUIPMEMT

NANCY J. SITTON

RICHLAND

(509) 376.5108

WA 99352

LOCATION OF EOUIPMENT:

UESIINGHOUSE HAMFORD COMPANY

ESS8S, 1167A BLDG/1100 AREA

RICHLAMO

REPORT NUMEER: 890531-4:03
$\$ 3.800 .00$

$\$ 3,800.00$

\section{FOR FURTHER INFORMATION :}

MANCY J. SIITON

RICHLAND

WA 99352

(509) $376-5108$

TO FREEZE EQUIPMENT:

MANCY J. SITTOW

RICHLAND

(509) $376-5108$

WA 99352

LOCATION OF EOUIPMENT

WESTIMGHOUSE HAMFORD COMPANY

ESS\&S, 1167A BLDG/1100 AREA

ESS\&S, 1167A GLDG/1100 AREA 99352 
EFFECTIVE DATE: 07/01/94 EXPIRATION DATE: 07/31/96

FSC

\section{CCEE EXCESS PROPERTY DESCR:PTION}

REFERENCE MUMBEP : 861
7021 COMPUTER MFG: COMPAO PORTABLE ACO DATE: 12/87 REF NO: $29853-03$

ELIGIBLE EOUIPMENT

REPORT NUMBER: $890531-4103$

LINE ITEM MUMBER: IESS wo of

COND UNITS UNIT

5 O0001 EA

\section{ACOUISITIOU PRICE}

COST/UNIT:
TOTAL COST: AL COST:

\section{$\$ 3.800 .00$ $\$ 3,800.00$}

\section{COMitaci(S)}

FOR FURTHER INFORMATION: MANCY J. SITTOW

RICHLAN (509) $376-5108$

wh 99352

IO FREEZE EOUIPMEWT:

MANCY J. SITTO

$$
\text { RICHLANO }
$$

(509) $376-5108$

UA 99352

LOCATION OF EQUIPMEN :

LESIIMGHOUSE MAMFORD COMPAMY

LESTIMGHOUSE MANFORD COAPAMY

RICHLANO

WA 99352

REFERENCE NUMBER : 862

7021 COMPUTER MFG: GEMESIS 111 ACO DATE: 2/89 REF NO: 29568.02

5 O0001 EA

COST/UNIT:
TOTAL COSI:

$\$ 1.646 .00$

FOR FURTHER IMFCRMATION:

MAMCY J. SITTON

RICHLAN

(509) 376.5103

UA 99352

TO FREEZE EOUIPMENT:

MANCY J. SITTOW

RICHLAND

(509) $376-5108$

wa 99352

LOCATION OF EQUIPMENT:

LESTIMGHOUSE HAMFORD COMPAMY

ESSES, 1167A BLDG/1100 AREA

RICHLAND 
EFFECTIVE DAIE: 07/01/9. EXPIRATION DATE: 07/31/96

isc

COOE EXCESS PROPERIY DESCRIDIION

REFERENCE MUMBER : 863

7021 COMPLIER MFG: GENESIS III ACO DAIE: 1/89 REF NO: $29605-02$

\section{Mo of}

COND UNITS UNIT

6 O000I EA
ACOUISITIOM PRICE

cosi/unit: TOIAt cosi:
LINE ITEM MUMBER: 0033

REPORT NUMGER: $890531-4103$
REFERENCE NUMBER : 804

7021 COMPUTER MFG: GENES1S III ACO DATE: 8/88 REF NC: 29723-01
600001 EA
COST/UNIT:
IOTAL COSI:
$\$ 1.560 .00$ $\$ 1,560.00$

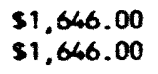

FOR FURTHER IMFORMAIION:

WAMCY J. SIITOM

RICHLAMD

un 99352

(509) $376-5108$

IL TREEZE EOUIPMENT

MANCY J. SITTOW

RICHLAND

WA 99352

LOCATION OF EOUIPMENT:

UESTIMGHOUSE HAMFORD COMPANY

ESSSS, 1167A BLDG/1100 AREA

RICHLAMO 99352

REPORT NUMBER: $890531-4103$

LINE ITEM MUMBER: 003

FOR FURTHER INFORMAIION:

MANCY J. SITTOW

RICHLAND

WA 99352

TO FREEZE EQUIPMENT:

MANCY J. SITTOW

RICHLANO

(509) $376-5108$

WA 99352

LOCATION OF EOUIPMENT:

HESTIMGHOUSE HAMFORD COMPAMY

ESS\&S, 1167A BLDG/1100 AREA

RICHLAND

WA 99352 
EFFECTIVE DATE: $07 / 01 / 94$ EXPIRATION DATE: $07 / 31 / 94$

FSC

COOE EXCESS PROPERTY DESCRIPTION

REFERENCE MUMBER : 865

7021 COMPUTER MFG: GEMES1S III ACO DATE: 29723-02 REF MO: 29723.02
REPORT NUMBER: $890531-4103$

LIME ITEM MUMBER: Q035
No of

COND UNITS unI

400001 EA

\section{ACQUISIIIION PRICE}

COST/UNIT: IOTAL COST:
$\$ 1,560.00$

$\$ 1,560.00$

\section{$\operatorname{com} \operatorname{sact}(S)$}

FOR FURTHER INFORMAIION:

MaNCY J. SITTOM

$$
\text { RICHLAMD }
$$

(509) $376-5108$

99352

IO FREEZE EOUIPMENT :

MANCY J. SITTOW

RICHLAM

WA 99352

(509) $376-5108$

LOCATION OF EQUIPMENT

LESTIMGHOUSE HAMFORO COMPAMY

ESS\&S, 1167A BLDG/1100 AREA

RICHLAMD

WA 99352

\section{REFERENCE MUMBER : 806}

7021 COMPUTER MFG: GENESIS III ACO DATE: UNKNOW REF NO: 29778-01

800001 EA

COST/UNIT:

TOTAL COST:

$\$ 1.646 .00$

1.646 .00

FOR FURTHER IMFORMATION

MANCY J. SITTON

RICHLAND

(509) $376-5108$

WA 99352

TO FREEZE EOUIPMENT

MANCY J. SITTON

RICHLAND

WA 99352

(509) $376-5108$

LOCATION OF EQUIPMENT:

WESTINGHOUSE HAMFORD COMPANY

ESS\&S, 1167A BLDG/1100 AREA

RICHLAND

WA 99352 
EFFECTIVE DATE: CI/01/94 EXPIRATION DATE: 07/31/94

\section{CODE EXCESS PROPERTY DESCRIPTION \\ REFERENCE NUMBER : 807}

7021 COMPUTER MFG: GENEIS 111 ACO DATE: 5/88 REF NO: 29790-04
No of

COND UNIIS UNIT

$500001 E A$
ACOUISITION PRICE

COSI/UNIT: TOTAL COST:

$\$ 1,560.00$ $\$ 1.560 .00$
LINE ITEM NUMBER: 0054
REPORT NUMBER: $890531-4103$
REFERENCE NUMEER : 868
7021 COMPUTER MFG: GENESIS III ACO DATE: $4 / 88$ REF NO: $29791-01$
900001 EA
COST/UNIT: TOTAL COST:
$\$ 1,560.00$ $\$ 1.560 .00$
REPORT NUMBER: $890531-4103$
LINE ITEM NUMBER: 0057
FOR FURTHER INFGRMATION:

NANCY J. SITTOW

RICHLAND

WA 99352

(509) $376-5108$

TO FREEZE EQUIPMENT:

NANCY J. SITTOO

RICHLAND

(509) $376-5108$

WA 99352

LOCATION OF EOLIPMENT:

WESTINGHOUSE HANFORD COMPANY ESS\&S, 1167A BLDG/1100 AREA

RICHLAND

WA 99352 
EFFECTIVE DATE: $07 / 01 / 94$ EXPIRATION DATE: $07 / 31 / 94$

COOE EXCESS PROPERTY DESCRIPTION

REFERENCE NUMBER : 869

7021 COMPUTER MFG: GENESIS 111286 ACO DATE: $1 / 89$ REF NO: 29793-01

REPORT NUMBER: $890531-4103$

LINE ITEM NUMBER: 0058

REFERENCE NUMBER : 870

7021 COMPUTER MFG: GENESIS 111286 ACO DATE: 10/88 REF NO: 29793-02

REPORT NUMBER: $890531-4103$

LINE ITEM NUMBER: 0059
NO OF

COND UNITS UNII

ACQUISITION PRICE

$500001 E A$

COST/UNIT:

$\$ 1,646.00$

$\$ 1,646.00$
$\$ 1,646.00$

TO

TO FREEZE EQUIPMENT:

NANCY J. SITTON

RICHLAND

(509) $376-5108$

LOCATION OF EQUIPMENT:

WESTINGHOUSE HANFORD COMPANY

ESS\&S, 1167A BLDG/1100 AREA

RICHLAND

HA 99352

WA 99352

HA 99352

\section{EA \\ COST/UNIT:
TOTAL COST:}

$\$ 1,560.00$

$\$ 1,560.00$

FOR FURTHER INFORMATION:

NANCY J. SITTON

RICHLAND

WA 99352

TO FREEZE EQUIPMENT

NANCY $J$. SITTON

RICHLAND

(509) $376-5108$

WA 99352

LOCATION OF EQUIPMENT:

WESTINGHOUSE HANFORD COMPANY

ESS\&S, 1167A BLDG $/ 1100$ AREA

RICHLAND

WA 99352 
EFFECTIVE DATE: 0i/01/94 EXPIRATION DATE: 07/31/94

\section{CODE EXCESS PROPERTY DESCRIPIION}

REFERENCE NUMBER : 871

7021 COMPUTER MFG: GENES1S III ACO DATE: 1/89 REF NO: 29810-03

ELIGIBLE EOUIPMENT

NO OF

COND UNIIS UNIT

ACQUISITION PRICE

$400001 E A$

COST/UNIT:

TOTAL COST:

$\$ 1,646.00$
LINE ITEM NUMBER: 0067

REPORT NUMEER: $890531 \div 103$

\section{REFERENCE NUMBER : 872}

7021 COMPUTER MEG: GENESIS 111 ACO DATE: $11 / 88$ REF NO: $29835-02$
$400001 E A$
$\$ 1,560.00$ $\$ 1,560.00$

FOR FURTHER INFORMATION:

NANCY J. SITTON

RICHLAND

(509) 376.5108

WA 99352

TO FREEZE EOUIPMENT:

NANCY $J$. SITTON

RICHLAND

(509) $376-5108$

WA 99352

LOCATION OF EQUIPMENT :

WESTINGHOUSE HANFORD COMPANY

ESS\&S, 1167A BLDG/1100 AREA

ESS\&S, 1167A BLDG/1100 AREA
RICHLAND 
EFFECIIVE DATE: 07/01/94 EXPIRATION DATE: $07 / 31 / 94$

ELIGIBLE EOUIPMENT
FSC

CODE EXCESS PROPERTY DESCRIPIION

\section{REFERENCE NUMBER : 873}

7021 COMPUTER MFG: GENESIS III ACO DATE: 1/89 REF NO: 29838-01
REPORT NUMBER: $890531-4103$

LINE ITEM NUMBER: 0073
NO OF

$500001 \mathrm{EA}$
COND UNITS UNIT

\section{ACQUISITION PRICE}

COST/UNIT: TOTAL COST:

\section{$\$ 1,646.00$ \\ $\$ 1,646.00$}

\section{CONTACT(S)}
FOR FURTHER INFORMATION:
NANCY J. SITTON
(509) $376-5108$
WA 99352

TO FREEZE EOUIPMENT:

NANCY J. SITTON

RICHLAND

WA 99352

(509) $376-5108$

LOCATION OF EOUIPMENT:

WESTINGHOUSE HANFORD COMPANY

ESS\&S, 1167A BLDG/1100 AREA

ESS\&S, 1167A BLDG/1100 AREA
RICHLAND
HA 99352

REFERENCE NUMBER : 874

7021 COMPUTER MFG: GENESIS III ACO DATE: 3/88 REF NO: 29892-03

$500001 \mathrm{EA}$

COST/UNIT:

TOTAL COST:

$\$ 1,560.00$

$\$ 1,560.00$

FOR FURTHER INFORMATION:

NANCY J. SITTON

RICHLAND

(509) 376.5108

WA 99352

TO FREEZE EOUIPMENT:

NANCY J. SITTON

RICHLAND

WA 99352

(509) $376-5108$

LOCATION OF EQUIPMENT :

WESTINGHOUSE HANFORD COMPANY

ESS\&S, 1167A BLDG/1100 AREA

RICHLAND

WA 99352 
EFFECTIVE DATE: $07 / 01 / 94$ EXPIRATION DATE: $07 / 31 / 94$

FSC

COOE EXCESS PROPERTY DESCRIPTION

REFERENCE NUMBER : 875

7021 COMPUTER MFG: GENESIS III ACO DATE: 5/89 REF NO: 29984-02

REPORT NUMBER: $890531-4103$

LINE ITEM NUMBER: 0116

REFERENCE NUMBER : 870

7021 COMPUTER MFG: : BM AT 5170 ACO DATE: 2.87 REF NO: 29503-01

LINE ITEM NUMBER: 0020

REPORT NUMBER: $890531-4103$
No of

COND UNIIS UNIT

ACQUISITION PRICE

$500001 E A$

COST/UNIT:

TOTAL COST:

$\$ 1,646.00$

$\$ 1,646.00$

FOR FURTHER INFORMATION:

NANCY J. SITTON

RICHLAND

(509) $376-5108$

WA 99352

TO FREEZE EQUIPMENT:

NANCY J. SITTON

RICHLAND

WA 99352

(509) $376-5108$

LOCATION OF EQUIPMENT

UESTINGHOUSE YAMFORD COMPANY

ESS\&S, 1167A BLDG/1100 AREA

RICHLAND

WA 99352
$500001 \mathrm{EA}$ COST/UNIT:

TOTAL COST:

$\$ 3,545.00$

$\$ 3,545.00$
FOR FURTHER INFORMATION :

NANCY J. SITTON

RICHLAND
(509) $376-5108$

WA 99352

TO FREEZE EQUIPMENT:

NANCY J. SITTON

RICHLAND

WA 99352

(509) 376-5108

LOCATION OF EQUIPMENT:

WESTINGHOUSE HANFORD COMPANY

ESS\&S, 1167A BLDG/1100 AREA

RICHLAND

WA 9935 
EFFECTIVE DATE: 07/01/94 EXPIRATION DATE: 07/31/94

FSC

\section{CODE EXCESS PROPERTY DESCRIPTION}

REFERENCE NUMBER : 877

7021 COMPUTER MFG: IBM XT 5160 ACO DATE: UNKNOWN REF NO: 29503-02
No of

COND UNITS UNI

ACQUISITION PRICE

$500001 \mathrm{EA}$ COSTIUNIT
TOTAL COST

$\$ 1,665.00$

$$
\$ 1,665.00
$$

LINE ITEM NUMBER: 0021
COST/UNIT: TOTAL COST:
LINE ITEM NUMBER: 0022
$800001 \mathrm{EA}$

\section{COMPUTER MFG:}

REF NO: 29525-01
CONTACT(S)

FOR FURTHER INFORMATION:

NANCY J. SITTON

RICHLAND

(509) $376-5108$

WA 99352

TO FREEZE EQUIPMENT:

NAMCY J. SITTON

RICHLAND

WA 99352

(509) 376.5108

LOCATION OF EQUIPMENT:

WESTINGHOUSE HANFORD COMPANY

ESS\&S, 1167A BLDG/1100 AREA

RICHLAND

WA 99352

REPORT NUMBER: $890531-4103$

$\$ 4.077 .00$

FOR FURTHER INFORMATION:

NANCY J. SITTON

RICHLAND

(509) $376-51118$

WA 99352

TO FREEZE EOUIPMENT

NANCY J. SITTON

RICHLAND

WA 99352

(509) 376-5108

LOCATION OF ECUIPMENT:

WESTINGHOUSE HANFORD COMPANY

ESS\&S, 1167A BLDG/1100 AREA

RICHLAND

HA 99352 
EFFECTIVE DATE: 07/01/94 EXPIRATION DATE: $07 / 31 / 94$

\section{COOE EXCESS PROPERTY DESCRIPTION \\ REFERENCE NUMBER : 879}

7021 COMPUTER MFG: IBM AT 5170 ACQ DATE: 8/87 REF NO: 29568-03
NO OF
$500001 E A$

\section{COST/UNIT:}

REPORT NUMGER: $890531-4103$
LINE ITEM NUMBER: 0026

\section{REFERENCE NUMBER : 88}

7021 COMPUTER MFG: IBM T 5150 ACO DATE: UNKNOWN REF NO: 29568-04
$5 \quad 00001 \mathrm{EA}$
COST/UNIT: TOTAL COST:
$\$ 2,197.00$ $\$ 2,197.00$
REPORT NUMEER: $890531-4103$
INE ITEM NUMEER: 0027
$\$ 2,174.00$ $\$ 2,174.00$
FOR FURTHER INFORMATION: NANCY J. SITTON

RICHLAND

WA 99352

(509) $376-5108$

TO FREEZE EQUIPMENT :

NANCY J. S!TTON

(509) $376-5108$

WA 99352

LOCATION OF EQUIPMENT

WESTINGHOUSE HANFORD COMPANY ESS\&S, 1167A BLDG/1100 AREA

RICHLAND

FOR FURTHER INFORMATION: NANCY J. SITTON

RICHLAND

(509) $376-5108$

TO FREEZE EQUIPMENT:

NANCY J. SITTON

RICHLAND

WA 99352

(509) $376-5108$

LOCATION OF EQUIPMENT :

WESTINGHOUSE HANFORD COMPANY ESS\&S, 1167A BLDG/1100 AREA

RICHLAND 
EFFECTIVE DATE: $07 / 01 / 94$ EXPIRATION DATE: $07 / 31 / 94$

FSC

\section{COOE EXCESS PROPERTY DESCRIPTION}

REFERENCE NUMBER : 88

7021 COMPUTER MFG: 1 BM 5170 ACO DATE: 11/87 REF NO: 29571-01
NO OF

COND UNITS UNLT

\section{ACQUISITION PRICE}

$500001 \mathrm{EA}$ COST/UNIT:
TOTAL COST:

$\$ 3,500.00$ $\$ 3,500.00$

LINE ITEM NUMBER: 0028

REFERENCE NUMBER : 882

7021 COMPUTER MFG: 1BM PS2 ACO DATE: 10/90 REF NO: 29582-01

REPORT NUMBER: $890531-4103$

LINE ITEM NUMBER: Q029

$800001 \mathrm{EA}$

COST/UNIT:
TOTAL COST:

$\$ 3.119 .00$

$\$ 3,119.00$

RICHLAND

(509) $376-5108$

NA 99352

TO FREEZE EOUIPMENT :

MANCY J. SITTON

RICHLAND

(509) $376-5108$

LOCATION OF EQUIPMENT:

WESTINGHOUSE HANFORD COMPANY

ESS\&S, 1167A BLDG/1100 AREA

RICHLAND

WA 99352 
EFFECIIVE DATE: $07 / 01 / 94$ EXPIRATION DATE: $07 / 31 / 94$

\section{COOE EXCESS PROPERTY DESCRIPIION}

REFERENCE NUMBER : 883
7021 COMPUTER MFG: IBM PS2 ACO DATE: 8/89 REF NO: $29665-01$

REPORT NUMBER: $890531-4103$

LINE ITEM NUMBER: 0032

REFERENCE NUMBER : 884

7021 COMPUTER MFG: IBM 5170 ACQ DATE: 7/87 REF NO: 29750-01

$600001 \mathrm{EA}$

COST/UNIT:

TOTAL COST:

LINE ITEM NUMBER: Q037

COND UNITS UNIT

$00001 \mathrm{EA}$

COST/UNIT: TOTAL COST:

REPORT NUMBER: $890531-4103$
NO OF

\section{$\$ 4.734 .00$}

$\$ 4,734.00$

NANCY J. SITTON

WA 99352

TO FREEZE EQUIPMENT

NANCY J. SITTON

RICHLAND

(509) $376-5108$

LOCATION OF EQUIPMENT:

WESTINGHOUSE HANFORD COMPANY

ESS\&S, 1167A BLDG/1100 AREA

RICHLAND

WA 99352
$\$ 3.545 .00$ $\$ 3,545.00$
FOR FURTHER INFORMATION:

NANCY J. SITTON

WA 99352

TO FREEZE EQUIPMENT:

NANCY J. SIITON

RICHLAND

(509) $376-5108$

LOCATION OF EQUIPMENT:

WESTINGHOUSE HANFORD COMPANY

ESS\&S, 1167A BLDG/1100 AREA

RICHLAND

WA 99352 
EFFECTIVE DATE: 07/01/94 EXPIRATION DATE: 07/31/94

FSC

COOE EXCESS PROPERTY DESCRIPIION

REFERENCE NUMBER : 885

7021 COMPUTER MFG: 1 BM 5170 ACO DATE: UNKNOWN REF NO: 29756-01

REPORT NLMBER: $890531-4103$

LINE ITEM NUMBER: 0038

\section{REFERENCE NUMBER : 880}

7021 COMPUTER MFG: IBM 5170 ACO DATE: 2/87 REF NO 29790- 02
NO OF

COND UNITS UNIT

ACQUISITION PRICE

50001 EA

COST/UNIT:

TOTAL COST:

$\$ 3,000.00$

$\$ 3,000.00$

NANCY J. SITTON

(509) $376-5108$

(509) 376-5108

TO FREEZE EOUIPMENT:

NANCY J. SITTON

RICHLAND

509) $376-5108$

LOCATION OF EQUIPMENT:

WESTINGHOUSE HANFORD COMPANY

ESS\&S, 1167A BLDG/1100 AREA

RICHLAND

REPORT NUMBER: $890531-4103$

LINE ITEM NUMBER: Q052
$500001 \mathrm{EA}$ COSI/UNII:

TOTAL COST:

$\$ 3,545.00$

$\$ 3,545.00$

FOR FURTHER INFORMAIION:

NANCY J. SITTON

RICHLAND
(509) $376-5108$

TO FREEZE EOUIPMENT :

MANCY J. SITTON

RICHLAND

WA 99352

(509) $376-5108$

LOCATION OF EQUIPMENT:

WESTINGHOUSE HANFORD COMPANY

ESS\&S, 1167A BLDG/1100 AREA

RICHLAND

WA 99352 
EFFECTIVE DATE: $07 / 01 / 94$ EXPIRATION DATE: $07 / 31 / 94$

FSC

CODE EXCESS PROPERTY DESCRIPTION

REFERENCE NUMBER : 887

7021 COMPUTER MFG: IBM 5170 ACQ DATE: 11/87 REF NO: 29796-01
No of

COND UNITS UNIT

ACQUISITION PRICE

$4 \quad 00001$ EA
TOTAL COST:
LINE ITEM NUMBER: 0061

REPORT NUMBER: $89053:-4103$
REFERENCE NUMBER : 888

7021 COMPUTER MFG: IBM PS2 ACO DAIE: 8/91 REF NO: 29799-011
$800001 E A$ COST/UNIT:
TOTAL COST:
LINE ITEM NUMBER: 0062

\section{$\$ 3,500.00$}

$\$ 3,500.00$$$
\text { RICH }
$$

TO FREEZE EQUIPMENT:

NAMCY J. SITTON

RICHLAND
(509) $376-5108$

LOCATION OF EQUIPMENT

WESTINGHOUSE MANFORD COMPAMY

WESTINGHOUSE HANFORD COMPANY

ESS\&S, 1167A BLDG/1100 AREA
RICHLAND
WA 99352

WA 99352

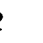


EFFECTIVE DATE: 07/01/94 EXPIRATION DATE: $07 / 31 / 94$

\section{REFEREMCE MUMBER : 88}

7021 COMPUTER MFG: IBM 5170 ACO DATE: 11/87 REF NO: 29808-01
REPORT NUMBER: $890531-6103$

\section{REFERENCE NUMBER : 890}

7021 COMPUTER MFG: IBM 5170 ACO DATE: 6/87 REF NO: 29846-01
No o

COND UNITS UNIT

$500001 \mathrm{EA}$
LINE ITEM MUMBER: 0063

ACQUISITION PRICE

COST/UNIT:

TOTAL COST:
LINE ITEM MUMBER: 0076
$800001 \mathrm{EA}$

COST/UNIT:

TOTAL COST:

$\$ 3.545 .00$

$\$ 3,545.00$
CONTACT(S)

$\$ 3,545.00$ $\$ 3,545.00$

FOR FURTHER IMFORMATIOW:

MANCY J. SITTOW

RICHLAND

(509) $376-5108$

un 99352

TO FREEZE EOUIPMEMT:

MANCY J. SITTO

RICHLANO

wa 99352

(509) 376.5108

LOCATION OF EQUIPHEWT:

WESTIMGHOUSE MANFORD COMPANY

ESSES, 1167A BLDG/1100 AREA

RICHLAMD

UA 99352

REPORT NUMBER: $890531-4103$

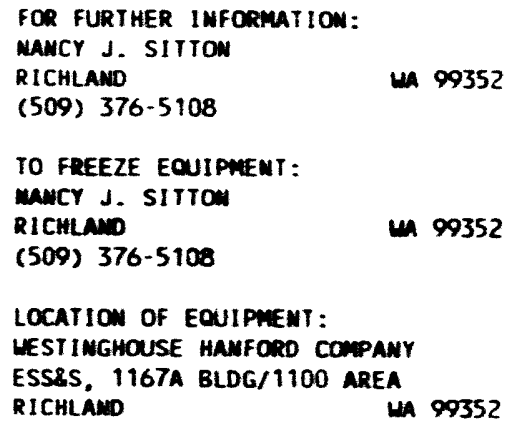


EFFECTIVE DATE: $07 / 01 / 94$ EXPIRATION DATE: $07 / 31 / 94$

\section{COOE EXCESS PROPERTY ZESCRIPIION \\ REFERENCE MUMGER : 891}

7021 COMPUTER MFG. IBM 5170 ACO DATE: 7/86 REF NO: 29848-01

REPORT NUMBER : $890531-4103$

LINE ITEM MUMBER: 0078

REFERENCE NUMBER : 892

7021 COMPUTER MFG: IBM 5170 ACO DATE: 12.87 REF NO: 29848.02
No of

COND UNITS UNIT

ACOUISITION PRICE

$5 \quad 00001 E A$

\section{COST/UNIT:
TOTAL COST:}

54.523 .00

54.523 .00

\section{MANCY J. SITTO \\ RICHLANO}

FOR FURTHER IMFORMATION:

un 99352

IO FREEZE EQUIPMENT:

MaMcr J. SIITOW

RICHLNOO

(S09) 376.5108

wa 99352

LOCATION OF EOUIPMENT:

LESTIMGHOUSE MAMFORO COMPANY

ESSSS, 1167A BLDG/1100 AREA

RICHLANO

man 99352

$\begin{array}{rrr} & \\ \text { COSTIUN1T: } & \$ 3.500 .00 \\ \text { TOTAL COST: } & \$ 3.500 .00\end{array}$

FOR FURTHER INFORMATION:
MAMCY $J$. SITION

RICHLAKD

(509) $376-5108$

ua 99352

TO FREEZE EQUIPMENT:

MANCY J. SITTOW

RICHLAMD

uA 99352

(509) $376-5108$

LOCATIOW OF EOUIPMENT:

LESTIMGHOUSE HAMFORD COMPANY ESS\&S, 1167A BLDG/1100 AREA

RICHLAMD 
EFFECTIVE DATF: $07 / 01 / 04$ EXPIRATION DATE: $07 / 31 / 96$

\section{COOE EXCESS PROPERTY DESCRIPIION}

REFERENCE NUMBER : 893

7021 COMPUTER MFG: IBM 5170 ACO DATE: 11/87 REF NO: 29869-01

REPORT NUMBER: $890531-4103$

LINE ITEM NUMBER: 0081

\section{REFERENCE NLMBER : 89}

7021 COMPUTER MFG: IBM 'S2 ACO DATE: 2/90 REF NO: 29992.04

REPORT NUMBER: $890531-4103$

LINE ITEM MUMBER: 0104

$$
\text { no of }
$$

COND UNIIS UMIT

ACQUISITION PRICE

800001 EA

COST/UNIT: TOTAL COST:

$\$ 3.500 .00$ $\$ 3.500 .00$
FOR FURTHER INFORMAT: IOW:
mancy I. SITrow
RICHLAND
(509) $376-5108$
ma 99352

TO FREEZE EOUIPMENT

MANCY $J$. SITTON

RICHLAND

wa 99352

LCATION OF EQUIPMENT:

HESTIMGHOUSE MANFORD COMPANY

ESS\&S. 1167A BLDG/1100 AREA

RICHLAND

un 99352

5 O0001 EA

COST/UNIT:

toral cost:

$\$ 3.796 .00$
$\$ 3.796 .00$

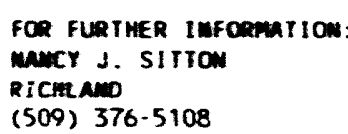

WA 99352

IO FREEZE EQUIPMENT:

MANCY J. SITTON

RICHLAND

(509) 376.5108

LOCATION OF EOUIPMENT:

VESIIMGHOUSE HAMFOOD COMPAMY

ESS\&S, 1167A BLDG/1100 AREA

RICHLAND

WA 99352 
EFFECTIVE DAIE: 07/01/94 EXPIRATION DATE: 07/31/94

$\mathrm{SC}$

COOE EXCESS PROPERTY DESCRIPTION

REFERENCE NUMBER : 895

$7021^{\circ}$ COMPUTER MFG: IBM 5170 ACO DATE: 12.87 REF NO: 29902-02

REPORT NUMBER: $890531-4103$

LINE ITEM MUMBER: 0105

REFERENCE NUMBER : 890

7021 COMPUTER MFG: IEM SSZ ACO DATE: 5/90 REF NO: 29906-01
NO of

COND UNITS UNIT

ACQUISITION PRICE

60001 EA

COST/UNIT:

IOTAL COST:

$\$ 3.500 .00$

$\$ 3,500.00$

REPORT NUMBER : $890531-4103$

LINE ITEM MUMBER: 0108
$4 \quad 00001 E A$

$$
\begin{aligned}
& \text { COST/UNIT: } \\
& \text { TOIAL COST: }
\end{aligned}
$$

$\$ 2.706 .00$

$\$ 2.706 .00$
CONTACI(S)

FOR FURTHER INFORMAIION:

NAMCY J. SITTON

$$
\text { RICHLAND }
$$

(509) $376-5108$

WA 99352

TO FREEZE EOUIPMENT:

MANCY J. S!TTON

RICHLAND

WA 99352

509) $376-5108$

OCATION OF EOUIPMENT:

WESTINGHOUSE HANFORD COMPANY

ESS8S, 1167A BLDG/1100 AREA

RICHLAND
FOR FURTHER INFORMATION:

NANCY J. SITION

$$
\text { RICHLAND }
$$

(509) $376-5108$

TO FREEZE EQUIPMENT :

MAMCY J. SITTON

RICHLAND

WA 99352

(509) $376-5108$

LOCATION OF EQUIPMENT

LESTINGHOUSE HANFORD COMPANY

ESS\&S, 1167A BLDG/1100 AREA

RICHLAND 
EFFECTIVE DATE: 07/01/94 EXPIRATION DATE: $07 / 31 / 94$

ELIGIBLE EQUIPMENT

FSC

COOE EXCESS PROPERTY DESCRIPTION

REFERENCE NUMBER : 897

7021 COMPUTER MFG: IBM 5170 ACQ DATE: 8//87 REF NO: 29920-01
NO OF COND UNITS UNIT ACQUISITION PRICE

$500001 \mathrm{EA}$ $\begin{aligned} \text { COST/UNIT: } & \$ 3,500.00 \\ \text { TOTAL COST: } & \$ 3,500.00\end{aligned}$

REPORT NUMBER: $890531-4103$

LINE ITEM NUMBER: 0109

REFERENCE NUMBER : 898

7021 COMPUTER MFG: IBM PCAT ACO DATE: 4/86 REF NO: 29932-01

$4 \quad 00001 \mathrm{EA}$

COST/UNIT: TOTAL COST:

$\$ 4,040.00$

$\$ 4,040.00$

\section{CONTACT(S)}

FOR FURTHER INFORMATION: NANCY J. SITTON

RICHLAND

(509) $376-5108$

WA 99352

TO FREEZE EQUIPMENT :

MANCY J. SITTON

$$
\text { RICHLAND }
$$

(509) $376-5108$

WA 99352

LOCATION OF EQUIPMENT:

WESTINGHOUSE HANFORD COMPANY

ESS\&S, 1167A BLDG/1100 AREA

RICHLAND WA 99352

REPORT NUMBER: $890531-4103$

LINE ITEM NUMBER: 0110
FOR FURTHER INFORMATION:

NANCY J. SITTON

RICHLAND

(509) $376-5108$

WA 99352

TO FREEZE EQUIPMENT :

NANCY J. SITTON

RICHLAND

(509) 376-5108

WA 99352

LOCATION OF EOUIPMENT:

WESTINGHOUSE HANFORD COMPANY

ESS\&S, 1167A BLDG $/ 1100$ AREA

RICHLAND

WA 99352 
EFFECTIVE DATE: $07 / 01 / 94$ EXPIRATION DATE: $07 / 31 / 94$

FSC

COOE EXCESS PROPERTY DESCRIPIION

REFERENCE NUMBER : 899

TO21 COMPUTER MFG: TSI MICROELECTRON MOOEL: T386-80 TYPE: SYSTEM 2

ACO DATE: $9 / 87$ REF NO: $29562 \cdot 01$

REPORT NUMBER: $890531-4103$

LINE ITEM NUMBER: 0023

REFERENCE NUMBER : 900

1021 COMPUTER MFG: WIN LABS MOOEL: 386 SX ACO DATE: 1/91 REF NO: $16350-01$

REPORT NUMBER: $890531 \cdot 4103$

LINE ITEM NUMBER: 001
No of

COND UNITS UNIT

ACQUISITION PRICE

CONTACT(S)

$900001 \mathrm{EA}$

COST/UNIT: TOTAL COST:

$\$ 4,495.00$ $\$ 4,495.00$

FOR FURTHER INFORMATION:

NANCY J. SITTON

RICHLAND

WA 99352

TO FREEZE EQUIPMENT :

NANCY J. SITTON

RICHLAND

HA 99352

(509) $376-5108$

LOCATION OF EQUIPMENT:

WEST INGHOUSE HANFORD COMPANY

ESS\&S, 1167A BLDG/1100 AREA

RICHLAND

WA 99352

900001 EA

COST/UNIT: TOTAL COST:

$\$ 2,010.00$ $\$ 2,010.00$

FOR FURTHER INFORMATION:

NANCY J. SITTON

RICHLAND

WA 99352

TO FREEZE EQUIPMENT :

MANCY J. SITTON

RICHLAND

(509) $376-5108$

WA 99352

LOCATION OF EQUIPMENT

WESIINGHOUSE HANFORD COMPANY

ESS\&S, 1167A BLDG/1100 AREA

RICHLAND WA 99352 
EFFECIIVE DATE: 07/01/94 EXPIRATION DATE: $07 / 31 / 94$

\section{FSC \\ COOE EXCESS PROPERIY DESCRIPTION}

REFERENCE NUMBER : 901

7021 COMPUTER MFG: WIN LABS TUBO AT MODEL: 8028710 ACO DATE: $6 / 89$ REF NO: $28952-01$
REFERENCE NUMBER : 902

7021 COMPUTER MFG: WIN LABS TURRBO AT ACO DATE: 1/90 REF NO: 29402.02
No of

COND UNITS UNIT

ACQUISITION PRICE

$400001 E A$

COST/UNIT: TOTAL COST:
LINE ITEM NUMBER: 0012

REPORT NUMBER: $890531-4103$
$500001 \mathrm{EA}$
LINE ITEM NUMBER: 0013

CONTACT(S)

$\$ 1,610.00$

$\$ 1,610.00$

INFORMATION :

NANCY J. SITTON

RICHLAND

HA 99352

(509) $376-5108$

TO FREEZE EQUIPMENT:

NANCY J. SITTON

RICHLAND

WA 99352

(509) $376-5108$

LOCATION OF EQUIPMENT:

WESTINGHOUSE HANFORD COMPANY

ESS\&S, 1167A BLDG/1100 AREA

RICHLAND

WA 99352

REPORT NUMBER: $890531-4103$
COST/UNIT: TOTAL COST:

$\$ 1,610.00$ $\$ 1,610.00$
FOR FURTHER INFORMATION:

NANCY J. SITTON

RICHLAND

(509) $376-5108$

WA 99352

TO FREEZE EQUIPMENT:

NANCY J. SITTON

RICHLAND

WA 99352

(509) $376-5108$

LOCATION OF EQUIPMENT:

WESTINGHOUSE HANFORD COMPANY

ESS\&S, 1167A BLDG/1100 AREA

RICHLAND

WA 99352 
EFFECIIVE DATE: 07/01/94 EXPIRATION DATE: $07 / 3: / 94$

FSC

\section{CODE EXCESS PROPERTY DESCRIPTION}

REFERENCE NUMBER : 903

7021 COMPUTER MFG: WIN LABS TURBO AT ACO DATE: 3.90 REF NO: 29402-04

REPORT NUMBER: $890531 \cdot 4103$

LINE ITEM NUMEER: 0014

REFERENCE NUMBER : 904

7021 COMPUTER MFG: WIN LAGS PERSONAL AT 286 ACO DATE: 5/90 REF NO: 29403-01

$400001 \mathrm{EA}$

$\operatorname{COST/UNIT}$

TOTAL COST:

$\$ 1,510.00$

FOR FURTHER INFORMATION:

NANCY J. SITTON

RICHLAND

(509) $376-5108$

WA 99352

TO FREEZE EOUIPMENT:

NANCY J. SITTON

REPORT NUMBER: $890531 \cdot 4103$

LINE ITEM NUMBER: 0016

RICHLAND

(509) $376-5108$

WA 99352

LOCAIION OF EQUIPMENT:

WESTINGHOUSE HANFORD COMPANY ESS\&S, 1167A BLDG/1100 AREA

ESS\&S, 1167A BLDG/1100 AREA 9935 
EFFECTIVE DATE: 07/01/94 EXPIRATION DATE: $07 / 31 / 94$

COOE EXCESS PROPERTY DESCRIPTION

REFERENCE NUMBER : 905

7021 COMPUTER MFG: WIN LABS AT MOOEL: 8028710 ACO DATE: 6/89 REF NO: $29427 \cdot 01$

REPORT NUMBER: $890531-4103$

LINE ITEM NUMBER: 0017

\section{REFERENCE NIMBER : 906}

7021 COMPUTER MFG: WIN LABS 3869: ACO DATE: 11/90 REF NO: 29435.01

REPORT NUMBER: $890531-4103$

LINE ITEM NUMBER: 0018
No O

COND UNITS UNI

$500001 \mathrm{EA}$

COST/UNIT: TOTAL COST:

$\$ 1,610.00$ $\$ 1,610.00$

NANCY J. SITTON

RICHLAND

WA 99352

(509) $376-5108$

TO FREEZE EQUIPMENT:

NANCY J. SITTON

RICHLAND

WA 99352

509) $376-5108$

LOCATION OF EQUIPMENT:

WESTINGHOUSE HANFORD COMPANY

SS\&S, 1167A BLDG/1100 AREA

RICHLAND

WA 99352

$400001 \mathrm{EA}$

COST/UNIT:

TOTAL COST:

$\$ 1,510.00$

$\$ 1,510.00$

FOR FURTHER INFORMATION:

NANCY J. SITTON

RICHLAND

509) $376-5108$

WA 99352

TO FREEZE EQUIPMENT:

NANCY J. SITTOH

RICHLAND

WA 99352

(509) $376-5108$

LOCATION OF EQUIPMENT:

WESTINGHOUSE HANFORD COMPANY

ESS\&S, 1167A BLDG/1100 AREA

RICHLAND

WA 99352 
EFFECTIVE DATE: $07 / 01 / 94$ EXPIRATION DATE: $07 / 3 \mathrm{i} / 94$

FSC

\section{CODE EXCESS PROPERTY DESCRIPIION}

REFERENCE NUMBER : 907

7021 COMPUTER MFG: HIN LABS TURBO AT ACO DATE 7/89 REF NO: 29568-01

REPORT NUMBER: $890531-4: 103$

INE ITEM NUMBER: 0024

REFERENCE NUMBER : 908

7021 COMPUTER MFG: WIN LAES TURBO AT ACO DATE: 10/89 REF NO: 29615-01

$500001 \mathrm{EA}$

COST/UNIT:

TOTAL COST:

LINE ITEM NUMBER: 0030

NO OF

COND UNITS UNIT

$500001 \mathrm{EA}$

COST/UNIT:

TOTAL COST:

REPORT NUMBER: $890531-4103$
ACOUISITION PRICE

$\$ 1,610.00$

$\$ 1,610.00$

NANCY J. SITTON
RICHLAND

(509) $376-5108$

TO FREEZE EQUIPMENT:

NANCY J. SITTON

RICHLAND

WA 99352

(509) $376-5108$

LOCATION OF EQUIPMENT

WESTINGHOUSE HANFORD COMPANY

ESS\&S, 1167A BLDG/1100 AREA

RICHLAND

WA 99352
$\$ 1,610.00$

$\$ 1,610.00$
FOR FURTHER INFORMATION :

NANCY J. SITION

RICHLAND

(509) $376-5108$

WA 99352

TO FREEZE EOUIPMENT :

NANCY J. SITTON

RICHLAND

WA 99352

(509) $376-5108$

LOCATION OF EQUIPMENT

WESTINGHOUSE HANFORD COMPANY

ESS\&S, 1167A BLDG/1100 AREA

RICHLAND 
DOE USED EMERGY RELATED LABORATORY EQUIPMENT GRANT PROGRAM

ELIGIBLE EOUIPMENT

EFFECTIVE DATE: 07/01/94 EXPIRATION DATE: 07/31/94

\section{COOE EXCESS PROPERTY DESCRIPTION}

REFERENCE NUMBER : 909

7021 COMPUTER MFG: WIN LABS 386SX ACO DATE: 5/91 REF NO: $29660-01$
No OF

COND UNITS UNIT

ACQUISITION PRICE

$400001 \mathrm{EA}$

COST/UN1T:

TOTAL COST:

$\$ 1,827.00$

$\$ 1,827.00$

REPORT NUMBER: $890531-4103$

LINE ITEM NUMBER: 003

REFERENCE NUMBER : 910

7021 COMPUTER MFG: WIN LABS AT 286 REF NO: $29723-03$ ACO DATE: $1 / 90$

$4 \quad 00001 \mathrm{EA}$

COSINUNIT:

TOTAL COST:

$\$ 1.610 .00$
$\$ 1,610.00$

REPORT NUMBER: $890531-4103$

LINE ITEM NUMBER: 0036

\section{CONTACTSS)}

FOR FURTHER INFORMATION:

NANCY J. SITTON

RICHLAND

(509) $376-5108$

WA '19352

TO FREEZE EQUIPMENT :

NANCY I. SITION

RICHLAND

(509) 376-5108

WA 99352

LOCATION OF EQUIPMENT:

WESTINGHOUSE HANFORD COMPANY

WESTINGHOUSE HANFORD COMPANY
ESS\&S, 1167A BLDG/1100 AREA

ESS\&S, 1167A BLDG/1100 AREA
RICHLAND
WA 99352

FOR FURTHER INFORMATION:

MANCY J. SITTON

(509) $376-5108$

WA 99352

TO FREEZE EQUIPMENT

NANCY J. SITTON

RICHLAND

(509) $376-5108$

WA 99352

LOCATION OF EQUIPMENT :

WESTINGHOUSE HANFORD COMPANY

ESS\&S, 1167A BLDG/1100 AREA

ESS\&S, 1167A BLDG/1100 AREA
RICHLAND 
EFFECTIVE DATE: 07/01/04 EXFIRATION DATE: $07 / 31 / 94$

FSC
CODE EXCESS PROPERTY DESCRIPTION

REFERENCE NUMBER : 911

7021 COMPUTER MFG: WIN LABS TURBO AT ACO DATE: 9/89 REF NO: 29756-02
NO OF

COND UNITS UNIT

ACQUISITION PRICE

500001 EA

COST/UNIT: TOTAL COST:

$\$ 1.610 .00$

$\$ 1,610.00$
LINE ITEM NUMBER: Q039
REPORT NUMBER: $890531-4103$ 
EFFECIIVE DATE: 07/01/94 EXPIRATION DATE: 07/31/94

\section{FSC \\ COOE EXCESS PROPERTY DESCRIPIION}

REFERENCE NUMBER : 913

7021 COMPUTER MFG: WIN LABS 286 ACO DATE: 7/90 REF NO: 29756-05
NO OF COND UNITS UNIT

$500001 \mathrm{EA}$ COST $/ U N I T:$
TOTAL COST: TOTAL COST:
LINE ITEM NUMBER: 0042

\section{REFERENCE NUMBER * 916}

\section{COMPUTER MFG: WIN LABS AT 286 ACO DATE: 3/90 REF NO: 29756-06}

$500001 \mathrm{EA}$

\section{COST/UNIT: TOTAL COST:}

$\$ 1,510.00$
$\$ 1,510.00$

FOR FURTHER INFORMATION:

NANCY J. SITTON

RICHLAND (509) $376-5108$

WA 99352

10 FREEZE EQUIPMENT:

MANCY J. SITTON

RICHLAND

(509) 376-5108

WR 99352

LOCATION OF EQUIPMENT:

WESTINGHOUSE HANFORD COMPANY

ESS\&S, 1167A BLDG/1100 AREA

RICHLAND

WA 99352

HA 99352

WA 99352

OREA

WA 99352 
EFFECTIVE DATE: $07 / 01 / 94$ EXPIRATION DATE: $07 / 31 / 94$

FSC

\section{COOE EXCESS PROPERIY DESCRIPIION}

REFERENCE NUMBER : 015

7021 COMPUTER MFG: WIN LABS TURBO AT ACQ DATE UNKNOWN REF NO: 29763-01

REPORT NUMGER: $890531-4103$

LINE ITEM NUMBER: 0044

REFERENCE NUMBER : 916

7021 COMPUTER MFG: WIN LABS TURBO AT ACO DATE: UNKNOWN REF NO: 29781-0:

$500001 \mathrm{EA}$
COST/UNIT:

TOTAL COST:

ACOUISITION PRICE

COST/UNIT:

TOTAL COST:

$\$ 1,610.00$

$\$ 1,610.00$

TOTAL COST:

$\$ 1,610.00$

$\$ 1,610.00$

FOR FURTHER INFORMATION:

NANCY J. SITTON

RICHLAND

OR FURTHER INFORMATION:

NANCY J. SITTON

RICHLAMD

WA 99352

TO FREEZE EQUIPMENT:

NANCY J. SITTON

RICHLANO

(509) $376-5108$

LOCATION OF EQUIPMENT:

WESTINGHOUSE HANFORD COMPANY

ESS\&S, 1167A BLDG/1100 AREA

RICHLAND

TO FREEZE EOUIPMENT:

HANCY J. SITTON

RICHLAND

(509) $376-5108$

LOCATION OF EOUIPMENT:

WESTINGHOUSE HANFORD COMPANY

ESS\&S, 1167A BLDG/1100 AREA

RICHLAND 
EFFECTIVE DATE: 07/01/94 EXPIRATION DATE: 07/31/94

FSC
CODE EXCESS PROPERTY OESCRIPTION

REFERENCE NUMBER : 917

7021 COMPUTER MFG: WIN LABS 286 AT ACO DATE: 9/90 REF NO: 29784-04

REPORT NUMBER: $890531-4103$

LINE ITEM NUMBER: 0050

REFERENCE NUMBER : 918

7021 COMPUTER MFG: WIN LABS TURBO AT 286 ACO DATE: 9/89 REF NO: 29790-01

$500001 \mathrm{EA}$

COST/UNIT:

TOTAL COST:

$\$ 1.610 .00$

$\$ 1,610.00$
CONTACTSS)

FOR FURTHER IMFORMATION:

NANCY J. SITTON

RICHLAND

WA 99352

(509) $376-5108$

TO FREEZE EQUIPMENT:

NANCY $J$. SITTON

RICHLAMD

WA 99352

(509) $376-5108$

LOCATION OF EQUIPMENT:

WESTIMU.LOUSE HAMFORD COMPANY

ESS\&S, 1167A BLDG/1100 AREA

RICHLAND

WA 99352

REPORT NUMBER: $890531-4103$

LINE ITEM NUMBER: 0051

\author{
MANCY J. SITTON \\ RICHLAND \\ WA 99352 \\ (509) $376-5108$ \\ TO FREEZE EQUIPMENT: \\ MANCY J. SITION \\ RICHLAND \\ WA 99352 \\ (509) $376-5108$ \\ LOCATIOM OF EQUIPMENT: \\ WESIINGHOUSE HAMFORD COMPANY \\ ESS\&S, 1167A BLDG/1100 AREA \\ RICHLAND

INORMATION: 
EFFECTIVE DATE: 07/01/94 EXPIRATION DATE: 07/31/94

FSC

REFERENCE MUMBER : 919

702! COMPUTER MFG: WIN LABS TURBO AT ACO DATE: 6/89 REF NO:29790-03
COOE EXCESS PROPERTY DESCRIPIION
REPORT NUMBER: $890531-4103$

LINE ITEM NUMBER: 0053
No of

COND UNIIS UNIT

500001 EA

\section{ACQUISITION PRICE}

COST/UNIT: TOTAL COST:
$\$ 1,610.00$

$\$ 1,610.00$

COMTACT(S)

FOR FURTHER IMFORMATION:

MANCY J. SITTON

RICHLAND

HA 99352

(599) $376-5108$

TO FREEZE EOUIPMENT:

MANCY J. SITTOW

RICHLAND

(509) 376.5108

LOCATION OF EOLIPMENT

WESTINGHOUSE HANFORD COMPANY

ESS\&S, 1167A BLOG/1100 AREA

RICHLAND

REFERENCE NUMBER : 920

7021 COMPUTER MFG: WIN LABS 286 ACO DATE: 9/90 REF ND: 29790-05

COST/UNIT:

TOTAL COST:
LINE ITEM NUMBER: 0055

REPORT NUMBER: $890531-6103$
$\$ 1.510 .00$

FOR FURTHER INFORMATION:

MANCY J. SITTON

RICHLAND

WA 99352

TO FREEZE EQUIPMENT:

MANCY J. SITTON

RICHLAND

(509) $376-5108$

UA 99352

LOCATION OF EQUIPMENT

UESTING'TOUSE HANFORD COMPANY

ESS8S, 1167A BLDG/1100 AREA

RICHLAND

WA 99352 
EFFECTIVE DATE: 07/01/94 EXPIRATION DATE: $07 / 31 / 94$

COOE EXCESS PROPERTY DESCRIPIION

REIERENCE MUMBER : 921

7021 COMPUTER MFG: WIN LABS 386SX ACO DATE: 5/91 REF NO: $29790-08$
No of

COND UNITS UNIT

ACQUISITION PRICE

$500001 \mathrm{EA}$

COST/UNIT: TOTAL COST:
LINE ITEM MUMBER: 0056

\section{REFEREMCE MUMBER : 922}

7021 COMPUTER MFG: WIN LAB 286 ACO DATE: 7/90 REF NO: 29793-03
LINE ITEM MUMBER: D060

REPORT MUMBER: $890531-4103$

$\$ 1,827.00$
$\$ 1.827 .00$

FOR FURTHER INFORMATION:

MAMCY J. SITtTo

RICHLAND

un 99352

(509) $376-5108$

TO FREEZE EQUIPNENT:

WAWCY J. SITTOW

RICHLAND

wa 99352

(509) $376-5108$

LOCATIOW OF EQUIPMENT:

UESTINGHOUSE HAMFORD COMPAMY

ESS\&S, 1167A BLDG/1100 AREA

RICHLAND 
EFFFCTIVE DATE: 0,/UI/94 EXPIRATION DATE: $07 / 31 / 94$

COOE EXCESS PROPERTY DESCRIPTION

REFERENCE NUMBER : 923

7021 COMPUTER MFG: WIN LABS 286 IURBC AT ACO DATE: 12/89 REF MO: 29808-02

REPORT NUMBER: $890531-4103$

LINE ITEM NUMBER: 0064

REFERENCE NUMBER : 924

7021 COMPUTER MFG: WIN LABS AT 286 ACO DATE: $4 / 90$ REF NO: $29810-01$

LINE ITEM MUMBER: 0065

REPORT NUMBER: 8905314103
No of

COND UNITS UNIT

ACOUISITION PRICE

500001 EA

COST/UNIT:

TOTAL COST:

$\$ 1.610 .00$

$\$ 1,610.00$

MANCY 1. SITTON
RICHLAND

(509) $376-5108$

HA 99352

iO FREEZE EQUIPMENT:

MANCY J. SITTOU

RICHLAND

WA 99352

(509) 376-5108

LOCATION OF EQUIPMENT:

UESTINGHOUSE HAMFORD COMPANY

ESS\&S, 1167A BLDG/1100 AREA

RICHLAMD

WA 99352
400001 EA

$\operatorname{cosT/UNIT:}$

TOTAL COST:
51.510 .00

$\$ 1.510 .00$
FOR FURTHER IMFORMATION:

MANCY J. SITTON$$
\text { RICHLAND }
$$

(509) $376-5108$

TO FREEZE EQUIPMENT :

mancY J. SITtOo

RICHLAMD

UA 99352

(509) $376-5108$

LOCATIOM OF EQUIPMENT:

UESTIMGHOUSE HAMFORD COMPAMY

ESS\&S, 1167A BLDG/1100 AREA

RICHLAMD 

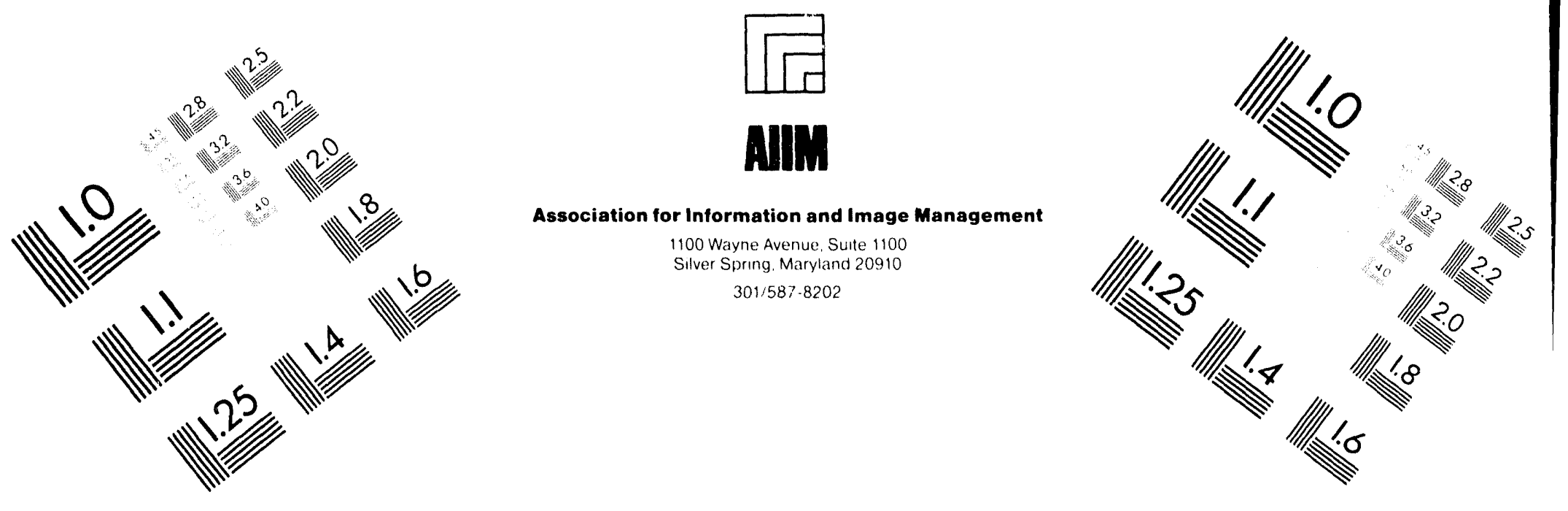

\section{Centimeter}

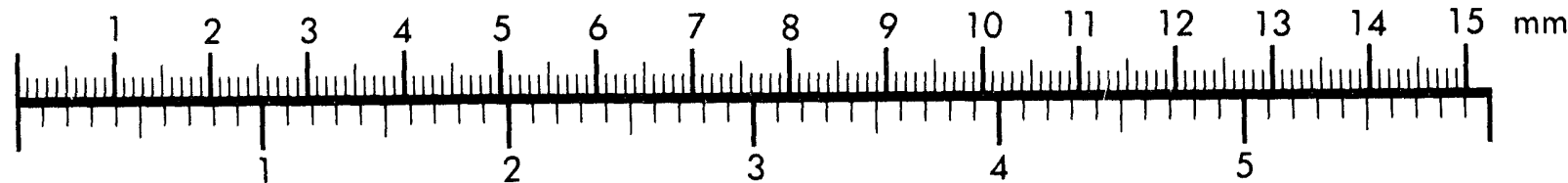

Inches
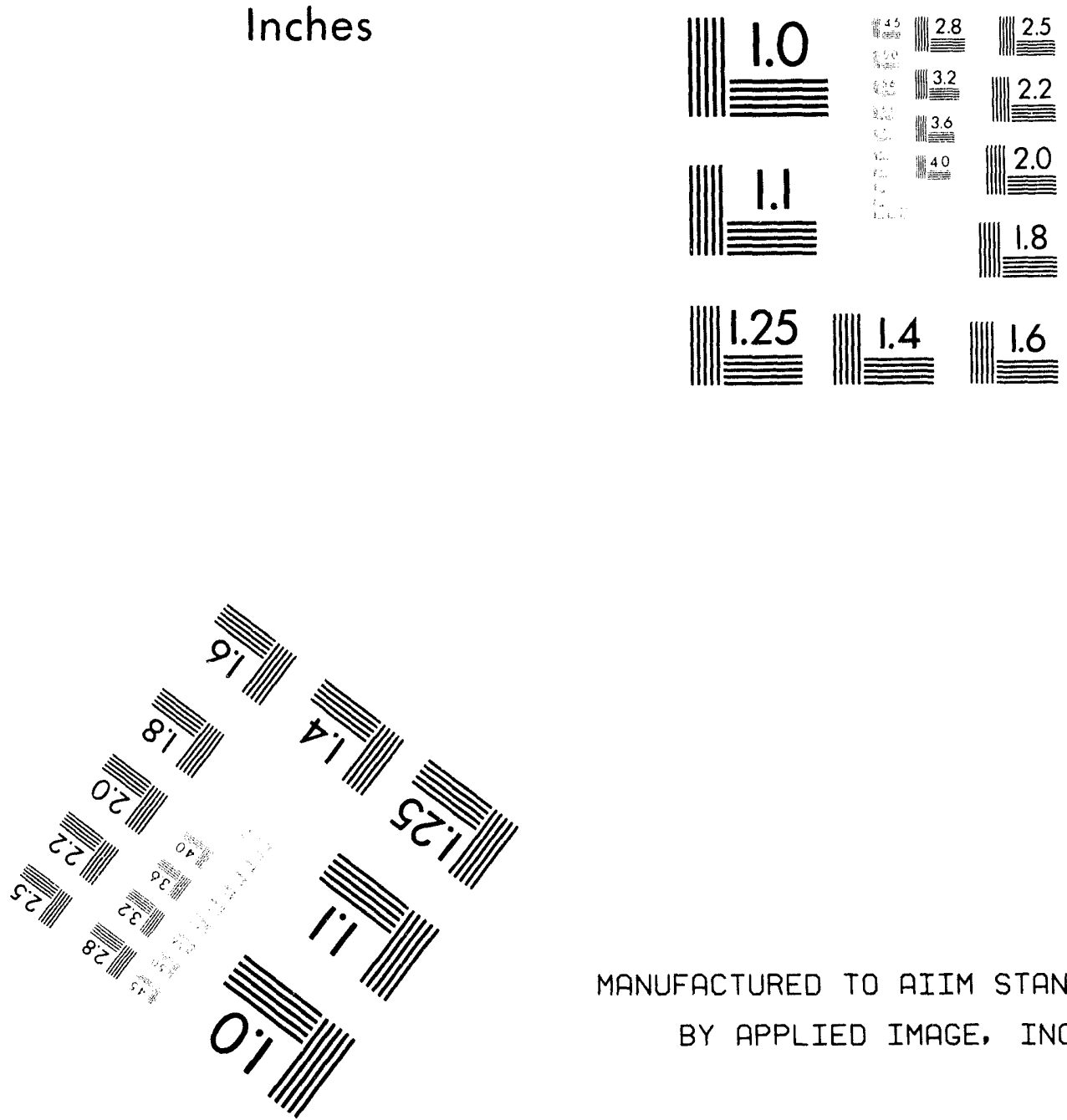

MANUFACTURED TO AIIM STANDARDS

BY APPLIED IMAGE, INC.

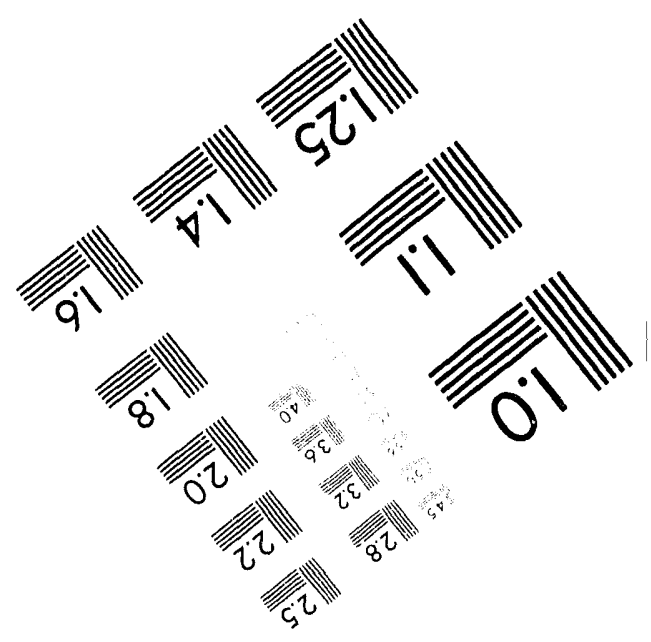



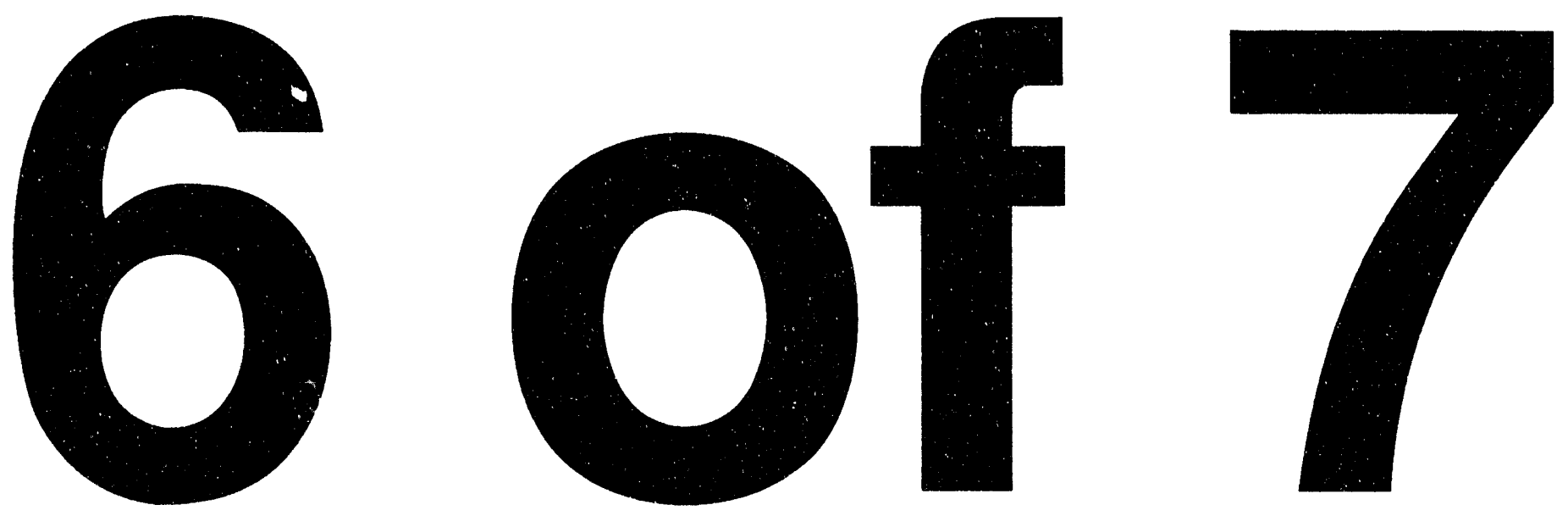
EFFECIIVE DATE: 07/01/94 EXPIRATION DATE: $07 / 31 / 94$

FSC

\section{CODE EXCESS PROPERTY OESCRIPTION}

REFERENCE NUMBER : 925

7021 COMPUTER MFG: WIN LABS AT 286 ACO DATE: $4 / 90$ REF NO: $29810-02$
NO OF

COND UNITS UNI

ACQUISITION PRICE

400001 EA COST UNIT:
TOTAL COST:

$\$ 1,510.00$ $\$ 1,510.00$

LINE ITEM NUMBER: 0066
REPORT NUMBER: $890531-4103$
REFERENCE NUMBER : 926

7021 COMPUTER MFG: WIN LABS TURBO AT 286 ACO DATE: 9/89 REF NO: $29835-01$
400001 EA COST /UNIT:
TOTAL COST:

FOR FURTHER INFORMATION: NANCY J. SITTON RICHLAND

(509) $376-5108$

WA 99352

TO FREEZE EOUIPMENT:

NANCY J. SITTON

RICHLAND

WA 99352

(509) $376-5108$

LOCATION OF EQUIPMENT:

WESTINGHOUSE HANFORD COMPANY ESS\&S, 1167A BLDG/1100 AREA

RICHLAND 
EFFECTIVE DATE: 07/01/94 EXPIRATION DATE: 07/31/94

COOE EXCESS PROPERIY DESCRIPIION

REFERENCE NUMBER : 927

7021 COMPUTER MFG: WIN LABS TURBO AT ACQ DAIE: 9/39 REF NO: 29835-04
No of

COND UNIIS UNIT

$400001 E A$

COST/UNIT:

TOTAL COST:

CQUISITION PRICE

$\$ 1,610.00$

$\$ 1,610.00$

LINE ITEM NUMBER: 0072

REFERENCE NUMBER : 928

7021 COMPUTER MFG: WIN LABS AT 286 ACO DATE: 3/90 REF NO: 29838-03

$500001 E A$

COST/UNIT:

TOTAL COST:

$\$ 1,510.00$
$\$ 1,510.00$

LINE ITEM NUMBER: 0074

REPORT NUMBER : $890531-4103$
CONTACT(S)

FOR FURTHER INFORMATION:

NANCY J. SITTON

RICHLAND

WA 99352

TO FREEZE EOUIPMENT:

NANCY J. SITTON

RICHLAND

(509) $376-5108$

LOCATION OF EQUIPMENT :

WESTINGHOUSE HANFORD COMPANY

ESS\&S, 1167A BLDG/1100 AREA

RICHLAND

FOR FURTHER INFORMAIION:

NANCY J. SITTON

RICHLAND

WA 99352

509) $376-5108$

TO FREEZE EQUIPMENT:

NANCY J. SITTON

RICHLAND

(509) $376-5108$

LOCATION OF EQUIPMENT:

WESTINGHOUSE HANFORD COMPANY

ESS\&S, 1167A BLDG/1100 AREA

RICHLAND 
EFFECTIVE DATE: $07 / 01 / 94$ EXPIRATION DATE: $07 / 31 / 94$

$$
\text { ELIGIBLE EQUIPMENT }
$$

\section{FSC}

COOE EXCESS PROPERTY DESCRIPTION

REFERENCE NUMBER : 929

7021 COMPUTER MFG: WIN LABS TURBO AT ACO DATE: 6/89 REF NO: 29848-03
No of
COND UNITS UNIY

$500001 \mathrm{EA}$

\section{ACQUISITION PRICE}

\section{$\operatorname{COST/UNIT:}$} TOTAL COST:

\section{$\$ 1,610.00$ \\ $\$ 1,610.00$}

REPORT NUMBER: $890531-4103$

LINE ITEM NUMBER: 0080
REFERENCE NUMBER : 930

7021 COMPUTER MFG: WIN LABS TURBO AT 286 ACO DATE: 12/89 REF NO: 29850-01
$500001 \mathrm{EA}$
COST/UNIT:
TOTAL COST:
$\$ 1,610.00$

$\$ 1,610.00$
FOR FURTHER INFORMATION:

NANCY J. SITTON

RICHLAND

(509) $376-5108$

WA 99352

TO FREEZE EQUIPMENT :

NANCY J. SITTON

RICHLAND

WA 99352

(509) $376-5108$

LOCATION OF EQUIPMENT :

WESTINGHOUSE HANFORD COMPANY

ESS\&S, 1167A BLDG/1100 AREA

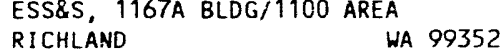


EFFECTIVE DATE: 07/01/94 EXPIRATION DATE: $07 / 31 / 94$

FSC

COOE EXCESS PROPERTY DESCRIPTION

REFERENCE NUMBER : 931

7021 COMPUTER MFG: WIN LABS TURBO AT ACO DATE: 10/88 REF NO: 29853-04

REPORT NUMBER: $890531 \cdot 4103$

INE ITEM NUMBER: 0086

REFERENCE NUMBER : 932

7 GP1 COMPUTER MFG: WIN LABS TUREO AT ACO DATE: 10/88 REF NO: 29853-05

$500001 \mathrm{EA}$

COST/UNIT:

TOTAL COST:

$\$ 1,000.00$

$\$ 1,000.00$

REPORT NUMBER: 890531.4103

LINE ITEM NUMBER: 0087
FOR FURTHER INFORMATION:
NANCY J. SITTON
RICHLAND

(509) $376-5108$

TO FREEZE EQUIPMENT:

NANCY J. SITTON

RICHLAND

(509) $376-5108$

WA 99352

WA 99352

LOCATION OF EQUIPMENT:

WESTINGHOUSE HANFORD COMPANY

ESSES, 1167A BLDG/1100 AREA

RICHLAND 
EFFECTIVE DATE: 07/01/94 EXPIRATION DATE: $07 / 31 / 94$

FSC

\section{COOE EXCESS PROPERTY DESCRIPTION}

REFERENCE NUMBER : 933

7021 COMPUTER MFG: WIN LABS TURBO AT ACQ DATE: 10/88 REF NO: 29853-06
No of

COND UNITS UNIT

ACQUISITION PRICE

$500001 \mathrm{EA}$

COST/UNIT:

TOTAL COST:

$\$ 1,000.00$

$\$ 1,000.00$

REPORT NUMBER: $890531-4103$

LINE ITEM NUMBER: Q088

REFERENCE NUMBER : 934

7021 COMPUTER MFG: WIN LABS TURBO AT ACO DATE: 10/88 REF NO:29853-07

\section{COSI/UNIT:}

$\$ 1,000.00$

$\$ 1,000.00$
$\underline{\operatorname{CONTACT}(S)}$

FOR FURTHER INFORMATION:

NANCY J. SITTON

RICHLAND

(509) $376-5108$

WA 99352

IO FREEZE EQUIPMENT:

NANCY J. SITTON

RICHLAND

(509) $376-5108$

LOCATION OF EQUIPMENT:

WESTINGHOUSE HANFORD COMPANY

ESS\&S, 1167A BLDG/1100 AREA

RICHLAND

WA 99352

REPORT NUMBER: 890531.4103

LINE ITEM NUMBER: 0089
FOR FURTHER INFORMATION:

NANCY J. SITTON

RICHLAND

(509) $376-5108$

WA 99352

TO FREEZE EQUIPMENT:

NANCY J. SITTON

RICHLAND

(509) $376-5108$

WA 99352

LOCATION OF EQUIPMENT:

WESTINGHOUSE HANFORD COMPANY ESS\&S, 1167A BLDG/1100 AREA

RICHLAND 
EFFECTIVE DATE: $07 / 01 / 94$ EXPIRATION DATE: $07 / 31 / 94$

COOE EXCESS PROPERTY OESCRIPTION

REFERENCE NUMBER : 935

7021 COMPUTER MFG: WIN LABS TUBO AT ACQ DATE: 10/88 REF NO: 29853-08

REPORT NUMEER: $890531-4103$

INE ITEM NUMBER: Q090

REFERENCE NUMBER : 930

7021 COMPUTER MFG: HIN LAES TURBO AT ACO DATE: 10/88 REF NO: 29853.09

REPORT NUMBER: $890531-4103$

LINE ITEM NUMBER: 0091
NO OF

COND UNITS UNIT

ACQUISITION PRICE

$500001 \mathrm{EA}$

COSTIUNIT:
TOTAL COST:

$\$ 1,000.00$

$\$ 1,000.00$

$\$ 1,000.00$

NANCY J. SITTON
RICHLAND

(509) $376-5108$

WA 99352

TO FREEZE EQUIPMENT:

NANCY $J$. SITTON

RICHLAND

(509) $376-5108$

WA 99352

LOCATION OF EOUIPMENT:

WESTINGHOUSE HANFORD COMPANY

ESS\&S, 1107A BLDG/1100 AREA

RICHLAND

WA 99352

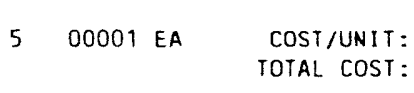

$\$ 1.000 .00$

$\$ 1,000.00$

FOR FURTHER INFORMAIION:

NANCY J. SITTON

RICHLAND

WA 99352

(509) $376-5108$

TO FREEZE EQUIPMENT:

NANCY $J$. SITTON

RICHLAND

WA 99352

(5C9) $376-5108$

LOCATION OF EOUIPMENT:

WESTINGHOUSE HANFORD COMPANY

ESS\&S, 1167A BLDG/1100 AREA

RICHLAND

WA 99352 
EFFECTIVE DATE: $07 / 01 / 94$ EXPIRATION DATE: $07 / 31 / 94$

FSC

CODE EXCESS PROPERTY DESCRIPIION

REFERENCE NUMBER : 93

7021 COMPUTER MFG: WIN LABS TURBO AT ACQ DATE: 2/89 REF NO: $29853-10$

REPORT NUMBER: $890531-4103$

LINE ITEM NUMBER: 0092

REFERENCE NUMBER : 938

7021 COMPUTER MFG: WIN LABS TURBO AT ACO DATE: 6/89 REF NO: 29853-11

$500001 E A$

COST/UNIT:
TOTAL COST:

$\$ 2,623.00$

FOR FURTHER INFORMATION:

NANCY A. SITTON

RICHLAND

(509) $376-5108$

WA 99352

TO FREEZE EQUIPMENT:

NANCY J. SITTON

RICHLAND

(509) $376-5108$

WA 99352

LOCATION OF EQUIPMENT:

WESTINGHOUSE HANFORD COMPANY

ESS\&S, 1167A BLDG/1100 AREA

RICHLAND

WA 99352 
EFFECTIVE DATE: 07/01/94 EXPIRATION DATE: $07 / 31 / 94$

COOE EXCESS PROPERTY DESCRIPTION

REFERENCE NUMBER : 930

7021 COMPUTER MFG: WIN LAES TURBO AT ACO DATE: 8/89 REF NO: 29853-12

REPOR: NUMEER: $890531-\rightarrow 103$

LINE ITEM NUMBER: 0094

REFERENCE NUMBER : 940

7021 COMPLIER MFG: WIN LAES TURBC AT ACO DATE: 8/89 REF NO: 29853-13

REPORT NUMBER: $890531-4103$

LINE ITEM NUMBER: 0095
NO OF

COND UNITS UNIT

ACQUISITION PRICE

$500001 E A$

COST/UNIT:

TOTAL COST:

$\$ 2.571 .00$

$\$ 2.571 .00$

RICHLAND

(509) $376-5108$

WA 99352

TO FREEZE EQUIPMENT:

NANCY J. SITTON

RICHLAND

WA 99352

(509) $376-5108$

LOCATION OF EQUIPMENT

UESTINGHOUSE HANFORD COMPANY

ESS\&S, 1167A BLDG/1100 AREA

RICHLAND

WA 99352

TOTAL COST:

$\$ 2,571.00$

$\$ 2,571.00$
FOR FURTHER INFORMATION:

NANCY J. SITTON

RICHLAND
(509) $376-5108$

WA 99352

TO FREEZE EQUIPMENT:

NANCY J. SITTON

RICHLAND

WA 99352

(509) $376-5108$

LOCATION OF EQUIPMENT:

WESTINGHOUSE HANFORD COMPAN

ESS\&S, 1167A BLDG/1100 AREA

RICHLAND 
EFFECTIVE DATE: $07 / 01 / 94$ EXPIRATION DATE: $07 / 31 / 94$

FSC

COOE EXCESS PROPERTY DESCRIPIION

REFERENCE NUMBER : 94

7021 COMPUTER MFG: WIN LABS TURBO AT ACO DATE: 8/89 REF NO: 29853-14
NO

COND UNITS UNIT

ACQUISITION PRICE

$500001 \mathrm{EA}$ COST/UNIT: TOTAL COST:
LINE ITEM NUMBER - 0096

REFERENCE NUMBER : 942

7021 COMPUTER MFG: WIN LABS 386 SX ACO DATE: 1/91 REF NO: 29867-01

$500001 \mathrm{EA}$
COST /UNIT:
IOTAL COST:

TOTAL COST:
REPORT NUMBER: $890531-4103$
LINE ITEM NUMBER: 0097

\section{$\$ 2,571.00$ $\$ 2,571.00$}

CONTACT(S)

FOR FURTHER INFORMATION: NANCY J. SITTON (509) 376.5108

WA 99352

TO FREEZE EQUIPMENT:

NANCY J. SITTON

RICHLAND

(509) $376-5108$

WA 99352

LOCATION OF EQUIPMENT:

WESIINGHOUSE HANFORD COMPANY ESS\&S, 1167A BLDG/1100 AREA ESS\&S, 1167A BLDG/1100 AREA
RICHLAND

FOR FURTHER INFORMATION: NANCY J. SITTON RICHLAND

(509) $376-5108$

TO FREEZE EQUIPMENT:

NANCY J. SITTON

RICHLAND

(509) $376-5108$

WA 99352

LOCATION OF EQUIPMENT:

WESTINGHOUSE HANFORD COMPANY ESS\&S, 1167A BLDG/1100 AREA ESS\&S, 1167A BLDG/1100 AREA 99352 
EFFECTIVE DATE: 07/01/96 EXFIRATION DATE: OT/31/94

\section{FSC EXCESS PROPERTY DESCRIPIION}

\section{REFERENCE NUMBER : 943}

: 943 WIN LABS IURBO AT ACO DAIE: 5/89 REF NO: 29892-01

ELIGIBLE EOUIPMENT

REFERENCE NUMBER : 944

7021 COMPUTER MFG: WIN LABS 236 ACO DATE: 8/90 REF NO: 29904-01
No O

$500001 \mathrm{EA}$
COND UNITS UNIT

\section{ACQUISITION PRICE}

\section{COST/UNIT:
TOTAL COST:}

$\$ 1,610.00$
REPORT NUMBER: $890531-4103$

LINE ITEM NUMBER: 0101
$500001 E A$
COST IUNIT:
TOTAL COST
INE ITEM NUMBER: 0106

CONIACT(S)

FOR FURTHER INFORMATION:

NANCY J. SITTON

RICHLAND

(509) $376-5108$

WA 99352

TO FREEZE EQUIPMENT:

NANCY J. SITTON

RICHLAND

WA 99352

(509) 376.5108

LOCATION OF EQUIPMENT:

HESTINGHOUSE HANFORD COMPANY

ESS\&S, 1167A BLDG/1100 AREA

RICHIAND HA 9935

WA 99352

REPORT NUMBER: $890531-4103$
$\$ 1,610.00$

$\$ 1,610.00$
OR FURTHER INFORMATION:

NANCY J. SITTON

ICHLAND

(509) $376-5108$

WA 99352

YO FREEZE EOUIPMENT:

NANCY J. SITTON

RICHLAND

WA 99352

(509) $376-5108$

LOCATION OF EQUIPMENT:

WESTINGHOUSE HANFORD COMPANY

ESS\&S, 1167A BLDG/1100 AREA

RICHLAND WA 99352 
EFFECTIVE DATE: $07 / 01 / 94$ EXPIRATION DATE: $07 / 31 / 94$

FSC

\section{COOE EXCESS PROPERTY OESCRIPTION}

REFERENCE NUMBER : 945
7021 COMPUTER MFG: WIN LABS 286 ACO DATE: $12 / 89$ REF NO: $29904-02$

ELIGIBLE EQUIPMENT

No of

COND UNITS UNIT

$500001 \mathrm{EA}$

\section{ACOUISITION PRICE}

COSTIUNIT: TOTAL COST:

LINE ITEM NUMBER: 0107
REPORT NUMEER : $890531-4103$
REFERENCE NUMBER : 946

7021 COMPUTER MFG: WIN LABS TURBO AT ACO DATE: 11/89 REF NO: 29942-01
$800001 E A$
COST/UNIT:
TOTAL COST:

LINE ITEM NUMBER: 0111

\section{$\$ 1.610 .00$ \\ $\$ 1,610.00$}

CONTACT(S)

FOR FURTHER INFORMATION:

MANCY J. SITTON

RICHLAND

(509) $376-5108$

WA 99352

TO FREEZE EQUIPMENT:

NANCY J. SITTON

RICHLAND

(509) 376.5108

WA 99352

LOCATION OF EQUIPMENT

WESTINGHOUSE HANFORD COMPANY

WESTINGHOUSE HANFORD COMPANY

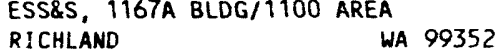

REPORT NUMBER: $890531-4103$

FOR FURTHER INFORMATION:

NANCY J. SITTON

PICHLAND

(509) $376-5108$

WA 99352

TO FREEZE EQUIPAENT :

NANCY J. SITTON

RICHLAND

(509) $376-5108$

WA 99352

LOCATION OF EQUIPMENT:

UESTINGHOUSE HANFORD COMPANY

ESS\&S, 1167A BLDG/1100 AREA

RICHLAND

WA 99352 
EFFECTIVE DATE: 07/01/94 EXPIRAIION DATE: $07 / 31 / 94$

FSC

REFERENCE NUMBER : 947

7021 COMPUTER MFG: WIN LABS 386SX ACO DATE:11/90 REF NO: 29955-01

\section{COOE EXCESS PROPERTY DESCRIPIION}

NO OF COND UNITS UNIT. ACQUISITION PRICE

$400001 \mathrm{EA}$ COST/UNIT:
TOTAL COST:
$\$ 2,010.00$
$\$ 2,010.00$

REPORT NUMBER: $890531-4103$

LINE ITEM NUMBER: 0112

REFERENCE NUMBER : 948

7021 COMPUTER MFG: HIN LABS 3865X ACO DATE: 29955-02

REPORT NUMBER: $890531-4103$

LINE ITEM NUMBER: 0113
$400001 \mathrm{EA}$

COST/UNIT:
TOTAL COST:

$\$ 1,827.00$

$1,827.00$

\section{CONTACISS}

FOR FURTHER INFORMATION: NANCY J. SITTON

RICHLAND

WA 99352

TO FREEZE EQUIPMENT:

NANCY J. SITTON

RICHLAND

(509) $376-5108$

WA 99352

LOCATION OF EQUIPMENT:

WESTINGHOUSE HANFORD COMPANY ESS\&S, 1167A BLDG/1100 AREA

RICHLAND

FOR FURTHER INFORMATION:

NANCY J. SITTON

RICHLAND

WA 99352

TO FREEZE EQUIPMENT:

NANCY J. SITTON

RICHLAND

(509) $376-5108$

WA 99352

LOCATION OF EGUIPMENT:

WESTINGHOUSE HANFORD COMPANY

ESS\&S, 1167A BLDG/1100 AREA

RICHLAND 
EFFECTIVE DATE: $07 / 01 / 94$ EXPIRATION DATE: $07 / 31 / 94$

FSC

\section{COOE EXCESS PROPERTY DESCRIPIION}

REFERENCE NUMBER : 949

7021 COMPUTER MFG: WIN LABS 386SX ACQ DATE: 9/91 REF NO: 29955-03

REPORT NUMBER: $890531-4103$

LINE ITEM NUMBER: 0114

REFERENCE NUMBER : 950

7021 COMPUTER MFG: WIN LABS 386SX ACO DATE: 1/91 REF NO: 29984-01

\section{EA COST/UNIT: \\ TOTAL COST:}

REPORT NUMBER: $890531-4103$

LINE ITEM NUMBER: 0115

NO OF

COND UNITS UNIT

400001 EA COST/UNIT: TOTAL COST:
ACOUISITION PRICE

$\$ 1,591.00$ $\$ 1,591.00$

NANCY J. SITTON

RICHLAND

RICHLAND
(509) $376-5108$

TO FREEZE EQUIPMENT

NANCY J. SIITON

RICHLAND

(509) $376-5108$

LOCATION OF EQUIPMENT:

WESTINGHOUSE HANFORD COMPANY

ESS\&S, 1167A BLDG/1100 AREA

RICHLAND

WA 99352


EFFECTIVE DATE: 07/01/94 EXPIRATION DATE: $07 / 31 / 94$

FSC

CODE EXCESS PROPERTY DESCRIPTION

REFERENCE NLMBER : 951

7021 COMPUTER MFG: WIN LABS 286 AT ACO DATE: $4 / 90$ REF NO: 29984-03
NO of

COND UNITS UNIT

$500001 \mathrm{EA}$
ACOUISITION PRICE

COST/UNIT:

TOTAL COST:

LINE ITEM NUMBER: 0117

REFERENCE NUMBER : 952

7021 COMPUTER MFT: WIN LABS TURBO AT 286 ACO DATE: 9/90 REF NO: 29815-01

$500001 \mathrm{EA}$
COST/UNIT: TOTAL COST:

\section{$\$ 1,510.00$} $\$ 1,510.00$

FOR FURTHER INFORMATION: NANCY J. SITTON

CONTACTSS)

FOR FURTHER INFORMAIION:

NANCY J. SITTON

RICHLAND

WA 99352

(509) $376-5108$

TO FREEZE EQUIPMENT :

NANCY J. SITTON

RICHLAND

WA 99352

LOCATION OF EQUIPMENT:

WEST INGHOUSE HANFORD COMPANY

ESS\&S, 1167A BLDG/1100 AREA

RICHLAND

WA 99352

REPORT NUMBER: $890531-6103$

LINE ITEM NUMBER: 0069 RICHLAND

(509) $376-5108$

WA 99352

TO FREEZE EQUIPMENT:

NANCY J. SITTON

RICHLAND

(509) $376-5108$

WA 99352

LOCATION OF EQUIPMENT:

WESTINGHOUSE HANFORD COMPANY ESS\&S, 1167A BLDG/1100 AREA

RICHLAND 
EFFECTIVE DATE: $07 / 01 / 94$ EXPIRATION DATE: $07 / 31 / 94$

FSC

\section{COOE EXCESS PROPERTY DESCRIPIION}

REFERENCE NUMBER : 953

7021 COMPUTER-PC, MFR. IBM, MODEL 5170, ACO. YR. 86, PROP. NO. 4575175
No of

COND UNITS UNI

ACQUISITION PRICE

$7 \quad 00001 E$

COST/UNIT:

TOTAL COST:

$\$ 2,139.14$

$\$ 2,139.14$
REPORT NUMBER: 899102-4173 - LINE ITEM NUMBER: 0001

REFERENCE NUMBER : 954

7021 COMPUTER-PC, MFR. APPLE, MODEL MOO01A, ACQ. YR. 87, PROP. NO. 4691967
COST/UNIT:

TOTAL COST:
$\$ 1,249.00$

$\$ 1,249.00$
$\operatorname{CONTACT}(S)$

FOR FURTHER INFORMATION:

JOANNE BREZNIK

LIVERMORE

CA 94550

(510) $424-4101$

TO FREEZE EQUIPMENT:

JOANNE BREZNIK

(510) $424-4101$

LOCATION OF EQUIPMENT

LAWRENCE LIVERMORE NATIONAL LAB

EXCESS SECTION, L-696

LIVERMORE

CA 94550

FOR FURTHER INFORMATION

JOANNE BREZNIK

LIVERMORE

CA 94550

(510) $424-4101$

TO FREEZE EQUIPMENT:

JOANNE BREZNIK

IVERMORE

CA 94550

(510) $424-4101$

LOCATION OF EOUIPMENT:

LAURENCE LIVERMORE NATIONAL LAB

EXCESS SECTION, L-696

LIVERMORE

CA 94550 
EFFECTIVE DATE: 07/01/94 EXPIRATION DATE: 07/31/94

\section{CODE EXCESS PROPERTY DESCRIPTION}

\section{REFERENCE NUMBER : 955}

7021 COMPUTER-PC, MFR. IBM, MODEL 5160, ACQ. YR. 86, PROP. NC. 4425081

REPORT NUMBER: $899102 \cdot 4173$

LINE ITEM NUMBER: 0003
NO OF

$600001 E A$ COST NUNIT:
TOTAL COST:

\section{$\$ 1,490.44$ $\$ 1,490.44$}

COND UNITS UNIT

CONTACT(S)

FOR FURTHER INFORMATION:

JOANNE BREZNIK

IVERMORE

CA 94550

(510) $424-4101$

TO FREEZE EOUIPMENT:

JOANNE BREZNIK

LIVERMORE

(510) $424-4101$

CA 94550

LOCATION OF EQUIPMENT:

LAWRENCE LIVERMORE NATIONAL LAB

EXCESS SECTION, L-696

LIVERMORE

CA 94550

REFERENCE NUMBER : 956

7021 COMPUTER-PC, MFR, IBM, MODEL 5160, ACO. YR. 86, PROP. NO. 4425111

600001 EA

COST/UNIT:

TOTAL COST:

$\$ 1,490.44$

$\$ 1,490.44$

FOR FURTHER INFORMATION

JOANNE BREZNIK

LIVERMORE

CA 94550

TO FREEZE EQUIPMENT:

JOANNE BREZNIK

LIVERMORE

(510) $424-410$

CA 94550

LOCATION OF EQUIPMENT:

LAURENCE LIVERMORE NATIONAL LAB

EXCESS SECTION, L-696

LIVERMORE

CA 94550 
EFFECTIVE DATE: 07/01/94 EXPIRATION DATE: $07 / 31 / 94$

FSC

\section{COOE EXCESS PROPERTY DESCRIPTION}

REFERENCE NUMBER : 957

TO21 COMPUTER-PC, MFR. IBM, MOOEL 5170, ACQ. YR. 86, PROP. NO. 4554958

REPORT NUMBER: $899102-4173$

LINE ITEM NUMBER: 0005

REFERENCE MUMBER : 958

7021 COMPUIER-PC, MFR. IBM, MODEL 5160, ACO YR. 86, PROP. NO. 4556303

$600001 \mathrm{EA}$

COST/UNIT:

TOTAL COST:

$\$ 1,394.00$

LINE ITEM NUMBER: 0006

NO OF

COND UNITS UNIT

ACQUISITION PRICE

$\$ 2,390.42$

REPORT NUMBER: $899102-4173$
$\$ 2,390.42$

FURTHER INFORMATION:

JOANKE BREZNIK

LIVERMORE

(510) $424-4101$

CA 94550

TO FREEZE EQUIPMENT

JOANNE BREZNIK

LIVERMORE

CA 94550

$424-4101$

LOCATION OF EQUIPMENT:

LAWRENCE LIVERMORE NATIONAL LAB

EXCESS SECTION, L-696

LIVERMORE

CA 94550
FOR FURTHER INFORMATION:

JOANNE BREZNIK

LIVERMORE

(510) $424-4101$

TO FREEZE EQUIPMENT:

JOANME BREZNIK

CA 94550

(510) $424-4101$

LOCATION OF EOUIPMENT

LAWRENCE LIVERMORE NATIONAL LAB

EXCESS SECTION, L-696

LIVERMORE

CA 94550 
EFFECTIVE DATE: 07/01/94 EXPIRATION DATE: 07/31/94

\section{FSC \\ COOE EXCESS PROPERTY DESCRIPTION}

REFERENCE NUMBER : 959

7021 COMPUIER.PC, MFR. IBM, MODEL 5160, ACQ. YR 87, PROP. NO. 3336739
NO OF

COND UNITS UNIT

ACQUISITION PRICE
$\$ 2.948 .00$ $\$ 2,948.00$
COST/UNIT:

LINE ITEM NUMBER: 0008
REPORT NUMBER: $899102-4173$
REFERENCE NUMBER : 960
7021 COMPUTER-PC, MFR. IBM, MODEL 5160, ACO YR. 86, PROP. NO. 4418519
600001 EA
COST/UNIT:
$\$ 1,490.44$

FOR FURTHER INFORMATION:

JOANNE BREZNIK

LIVERMORE

FOR FURTHER INFORMATION:

JOANNE BREZNIK

IVERMORE

CA 94550

TO FREEZE EQUIPMENT:

JOANNE BREZNIK

IVERMORE

(510) $424-4101$

CA 94550

LOCATION OF EQUIPMENT:

LAURENCE LIVERMORE NATIONAL LAB

EXCESS SECTION, L- 696

LIVERMORE

CA 94550

(510) $424-4191$

CA 94550

TO FREEZE EQUIPMENT:

JOANNE BREZNIK

LIVERMORE

CA 94550

REPORT NUMBER: $899102-4167$

LINE ITEM NUMBER: 0001

(510) $424-4191$

LOCATION OF EQUIPMENT:

LAURENCE LIVERMORE NATIONAL LAB

EXCESS PROPERTY SECTION, L-696

LIVERMORE

CA 94550 
EFFECTIVE DATE: $07 / 01 / 94$ EXPIRATION DATE: $07 / 31 / 94$

FSC

COOE EXCESS PROPERTY DESCRIPTION

\section{REFERENCE NUMBER : 961}

7021 COMPUTER-PC, MFR. APPLE, MOOEL MO001A, ACQ. YR 87, PROP. NO. 4648732

ELIGIBLE EOUIPMENT

NO OF

COND UNITS UNIT

$600001 \mathrm{EA}$

\section{ACOUISITION PRICE} COST/UNIT:
TOIAL COST:

\section{$\$ 1,249.00$}

$\$ 1,249.00$

CONTACT(S)

FOR FURTHER INFORMATION:

JOANNE RREZNIK

LIVERMORE

(510) $424-4191$

CA 94550

TO FREEZE EQUIPMENT:

JOANNE BREZNIK

LIVERMORE

CA 94550

(510) $424-4191$

LOCATION OF EQUIPMENI:

LAURENCE LIVERMORE NATIONAL LAB

EXCESS PROPERTY SECTION, L-696

EXCESS PROPERTY SECTION, L-696
LIVERMORE
CA 94550

REFERENCE NUMBER : 962

7021 COMPUTER-PC, MFR. IBM, MODEL 5160, ACO. YR. 86, PROP. NO. 4639921

$500001 E A$

COST/UNIT:

TOTAL COST:

$\$ 2,089.00$

$\$ 2,089.00$

FOR FURTHER INFORMATION:

JOANNE BREZNIK

LIVERMORE

CA 94550

TO FREEZE EOUIPMENT:

JOANNE BREZNIK

LIVERMORE

(510) $424-4191$

LOCATION OF EQUIPMENT:

LAURENCE LIVERMORE NATIONAL LAB

EXCESS PROPERTY SECTION, L-696

LIVERMORE

CA 94550 
EFFECTIVE DATE: OT/O1/04 EXPIRATION DATE: O7/31/94

FS:

\section{CODE EXCESS PROPERTY DESCRIPTION}

REFERENCE NUMGER : 903

7021 COMPUTER-PC, MFR. IBM, MOOEL 5100, ACO. YR. 86, PROP. NO. 4351335

REPORT NUMBER: $899102 \cdot 10$ ?

LINE ITEM NUMBER: 0004

REFERENCE NUMBER : QOC

TOZ: COMPUTER-PC, MFR. I GM, MONEL 5170, ACO. YR. 86, PROP. NO. 4554729

$500001 E A$

REPORT NUMBER: $899102-4167$

LINE ITEM NUMBER: 0005
COST/UNIT:

TOTAL COST:

$\$ 2.390 .42$

$\$ 2,390.42$

NO OF

COND UNITS UNIT

COST/LNIT: TOTAL COST:

$\$ 1,500.00$ $\$ 1,500.00$

IHER INFRMATION:

JOANNE BREZNIK

LIVERMORE

(510) $424-4191$

TO FREEZE EOUIPMENT

JOANNE BREZNIK

LIVERMORE

(510) $424-4191$

LOCATION OF EQUIPMENT

LAWRENCE LIVERMORE NATIONAL LAB

EXCESS PROPERTY SECTION, L-696

LIVERMORE

CA 94550

FOR FURTHER INFORMATION:

JOANNE BREZNIK

LIVERMORE

(510) $424-4191$

CA 94550

TO FREEZE EOUIPMENT:

JOANNE BRE2NI

CA 94550

(510) $424-4191$

LOCATION OF EQUIPMENT

LAWRENCE LIVERMORE NATIONAL LAB

EXCESS PROPERTY SECTION, L-696

LIVERMORE

CA 94550 
EFFECTIVE DATE: $07 / 01 / 94$ EXPIRATION DATE: $07 / 31 / 94$

FSC

\section{COOE EXCESS PROPERTY DESCRIPIION}

REFERENCE NUMBER : 965

7021 COMPUTER-PC, MFR. IGM, MODEL 5160, ACO, YR, 86, PROP. NO. 4555054

REPORT NUMBER : $899102-4167$

LINE ITEM NUMBER: 0006

REFERENCE HUMBER : 966

7021 COMPUTER-PC, MFR. MEWLETT FACKARD, MOOEL 45711B, ACQ. YR. 86 P ROP. NO. 5 O0001 EA 4577541

NO OF

$500001 \mathrm{EA}$
COND UNITS UNIT

ACQUISITION PRICE

COST/UNIT:

TOTAL COST:

$\$ 1,394.00$

$\$ 1,394.00$

JOANNE BREZNIK

(510) $424-4191$

CA 94550

TO FREEZE EQUIPMENT

JOANNE BREZNIK

LIVERMORE

CA 94550

(510) $424-419$

LOCATION OF EQUIPMENT

LAURENCE LIVERMORE NATIONAL LAB

EXCESS PROPERTY SECTION, L-696

LIVERMORE
COST/UNIT:

TOTAL COST:
$\$ 1,869.30$

$\$ 1,869.30$
FOR FURTHER INFORMATION:

JOANNE BREZNIK

LIVERMORE

(510) $424-4191$

CA 94550

TO FREEZE EQUIPMENT:

JOANME BREZNIK

LIVERMORE

CA 94550

REPORT NUMBER: $899 \div 02-4167$

LINE ITEM NUMBER: 0008
(510) $424-4191$

LOCATION OF EQUIPMENT

LAURENCE LIVERMORE NATIONAL LAB

EXCESS PROPERTY SECTION, L-696

LIVERMORE 
EFFECTIVE OATE: 07/01/96 EXPIRATION DATE: 07/31/94

NO Of

COOE EXCESS PROPERTY OESCRIPIION

COND UNITS UNIT

$600001 E A$

: 967

7021 COMPUTER-PC, MFR. APPLE, MOOEL MOOTA, ACO. YR. 86, PROP. NO. 4618094

REPORT NUMBER: $399102-4167$

LINE IIEM NUMBER: 0009

REFERENCE NUMBER : 958

7021 COMPUTER-DC, MFR. IBM, MODEL 5160, ACQU. YR. 86. PROP. NO 4617547

500001 EA

COST/UNIT: TOTAL COST:

REPORT NUMBER: $899102-4167$

LINE ITEM NUMBER: 0010
ACOUISITION PRICE

COST/UNIT: TOTAL COST:

\section{$\$ 1,249.00$}

$\$ 1.249 .00$

JOANNE BREZNIK
LIVERMORE

(510) $424-4191$

TO FREEZE EQUIPMENT :

JOANNE BREZNIK

LIVERMORE

(510) $424-4191$

LOCATION OF EOUIPMENT:

LAWRENCE LIVERMORE NATIONAL LAB

EXCESS PROPERTY SECTION , L-696

IIVERMORE
$\$ 1,394.00$

$\$ 1,394.00$
FOR FURTHER INFORMATION:

JOANNE BREZNIK

LIVERMORE

(510) $424-4191$

TO FREEZE EQUIPMENT:

JOANNE BREZNIK

IVERMORE

CA 94550

(510) $424-4191$

LOCATION OF EOUIPMENT:

LALRENCE LIVERMORE NATIONAL LAB

EXCESS PROPERTY SECIION, L-O96

LIVERMORE

CA 94550 
EFFECTIVE DATE: 07/01/94 EXPIRATION DATE: 07/31/94

FSC

\section{COOE EXCESS PROPERTY DESCRIPIION}

REFERENCE MUMBER : 969

7021 COMPUTER-PC, MFR. IBM, MODEL 5160, ACO. YR. 86, PROP. NO. 4576462
Mo of

COND UWITS UMIT

\section{ACQUISITION PRICE}

$5 \quad 00001 E A$
$\$ 1,394.00$

$\$ 1,394.00$

REPORT MUMBER: $899102-4167$

LINE ITEM NUMBER: 0011

REFERENCE MUMBER : 970

7021 COMPUTER-PC, MFR. IBM, MODEL 5160, AO. YR. 86, PROP. NO. 4556464

$500001 \mathrm{EA}$

COST/UNIT:

TOTAL COST:

$\$ 1.380 .11$

$\$ 1,380$.

JOANNE BREZNIK

LIVERMORE

(510) $424-4191$

CA 94550

TO FREEZE EQUIPMENT:

JOANME BREZNIK

LIVERMORE

(510) $424-4191$

CA 94550

LOCATION OF EQUIPMENT:

LOCATION OF EQUIPMENT:

EAWRENCE LIVERMORE NATIONAL LAB

EXCESS PROPERTY SECTION, L-696

LIVERMORE 
DOE USED ENERGY RELATED LABORATORY EOUIPMENT GRANT PROGRAM ELIGIBLE EOUIPMENT

EFFECTIVE DAIE: 07/01/94 EXPIRAIION DATE: $07 / 31 / 94$

CODE EXCESS PROPERTY DESCRIPTION

REFERENCE NUMBER : 971

7021 COMPUTER-PC, MFR. IBM, MODEL 5160, ACQ. YR. 86, PROP. NO. 4556389

REPORT NUMBER: $899102 \cdot 4167$

LINE ITEM NUMBER: 0013

REFERENCE NUMBER : 972

7021 COMPUTER-PC, MFR. IBM, MOOEL 8560, ACO. YR. 87, PROP. NO. 4758554

$500001 E A$

COST/UNIT:

TOTAL COST:

$\$ 3.336 .00$ $\$ 3,336.00$

LINE ITEM NUMBER: 0014

COND UNITS UNIT

ACQUISITION PRICE

REPORT NUMBER: $899102-4167$
NO OF

CONTACT(S)

FOR FURTHER INFORMATION:

JOANME BREZNIK

LIVERMORE

CA 94550

TO FREEZE EQUIPMENT

JOANNE BREZNIK

LIVERMORE

(510) $424-4191$

CA 94550

LOCATION OF EOUIPMENT:

LAWRENCE LIVERMORE NATIONAL LAB

EXCESS PROPERTY SECTION, L-696

LIVERMORE CA 94550
FOR FURTHER INFORMATION:

JOANNE BREZNIK

LIVERMORE

CA 94550

TO FREEZE EQUIPMENT:

JOANNE BREZNIK

IIVERMORE

(510) $424-4191$

CA 94550

LOCATION OF EQUIPMENT:

LAURENCE LIVERMORE NATIONAL LAB

EXCESS PROPERTY SECTION, L-696

LIVERMORE CA 94550 
EFFECTIVE DATE: 07/01/96 EXPIRATION DATE: 07/31/94

FSC

COOE EXCESS PROPERTY OESCRIPTION

REFERENCE NUMBER : 973
7021 COMPUTER-PC, MFR. TOSHIBA, MODEL T1100, ACO. YR. 87, PROP. HO. 4774622

COND UNITS UNIT

ACQUISITION PRICE

$600001 \mathrm{EA}$

COST/UNIT:
TOTAL COST:

$\$ 1,795.00$
$\$ 1,795.00$

REPORT NUMBER: $899102-6167$

LINE 1 TEM NUMBER: 0015

REFERENCE NUMBER : 974

7021 COMPUTER-PC, MFR. ZENITH, MODEL ZWL 18392, ACO. YR. 87, PROP. N 0.

$600001 \mathrm{EA}$ 4739140
COST/UNIT:

TOTAL COST:
$\$ 2,475.00$
$\$ 2,475.00$

REPORT NUMBER: $899102-4167$

LINE ITEM NUMBER: 0016
CONTACT(S)

FOR FURTHER INFORMATION

JOANNE BREZNIK

LIVERMORE

CA 94550

TO FREEZE EQUIPMENT:

JOANNE BREZNIK

LIVERMORE

(510) $424-4191$

CA 94550

LOCATION OF EQUIPMENT:

LAURENCE LIVERMORE NATIONAL LAB

EXCESS PROPERTY SECTION, L- 696

LIVERMORE

FOR FURTHER INFORMATION:

JOANNE BREZNIK

LIVERMORE

(510) $424-419$

TO FREEZE EQUIPMENT:

JOANNE BREZNIK

LIVERMORE

(510) $424-4191$

CA 94550

LOCATION OF EQUIPMENT:

LALRENCE LIVERMORE MATIONAL LAB

EXCESS PROPERTY SECTION L-696

EXCESS PROPER!Y SECTION, L-696
LIVERMORE
CA 94550 
EFFECIIVE DATE: $07 / 01 / 94$ EXPIRATION DATE: $07 / 31 / 94$

\section{FSC \\ COOE EXCESS PROPERTY DESCRIPTION}

REFERENCE NUMBER : 975

7021 COMPUTER-PC, MFR. TOSHIBA, MODEL T1100, ACQ. YR. 88, PROP. NO 4788674
LINE ITEM NUMBER: 0017

$$
\text { NO OF }
$$

COND UNITS UNIT

600001 EA
ACQUISITION PRICE

COST/UNIT: TOTAL COST:

$\$ 1,403.00$ $\$ 1,403.00$
REPORT NUMBER: $899102-4167$

\section{REFERENCE NUMBER : 976}

7021 COMPUTER-PC, MFR. IBM, MODEL 8550, ACO. YR. 88, PROP. NO. 4911423
$500001 \mathrm{EA}$

\section{COST/UNIT: TOTAL COST:}

$\$ 2,516.00$ $\$ 2,516.00$
REPORT NUMBER: $899102-4167$
LINE ITEM NUMBER: 0018

\author{
FOR FURTHER INFORMATION: \\ JOANNE BREZNIK \\ LIVERMORE \\ CA 94550 \\ TO FREEZE EQUIPMENT: \\ JOANNE BREZNIK \\ LIVERMORE \\ (510) 424-4191 \\ CA 94550 \\ LOCATION OF EQUIPMENT : \\ LAWRENCE LIVERMORE NATIONAL LAB \\ EXCESS PROPERTY SECTION, L-696 \\ LIVERMORE$$
696
$$

CA 94550

CA 94550

EQUIPMENT:

LIVERMORE NATIONAL LAB

EXCESS PROPERTY SECTION, L-696

CA 94550 
EFFECTIVE DATE: 07/01/94 EXPIRATION DATE: $07 / 31 / 94$

FSC

\section{COOE EXCESS PROPERTY DESCRIPIION}

REFERENCE NUMBER : 977

7021 COMPUTER-PC, MFR. IBM, MODEL 5160, ACQ. YR. 86, PROP. NO. 4425746
No of

COND UNITS UNIT

ACOUISITION PRICE

$600001 E A$

COST/UNIT:

TOTAL COST:

LINE ITEM NUMBER: 0019
REPORT NUMBER: $899102-4167$

REFERENCE MUMBER : 978

7021 COMPUIER-PC, MFR. APPLE, MODEL MOO1A, ACO. YR. 86, PROP. NO 4617318

$600001 \mathrm{EA}$
COST/UNIT:

REPORT NUMBER: $899102-4167$
LINE ITEM NUMBER: 0020
$\$ 1,394.00$

$\$ 1,394.00$
$\operatorname{CONTACT(S)}$

FOR FURTHER INFORMATION:

JOANNE BREZNIK

LIVERMORE

CA 94550

(510)

TO FREEZE EQUIPMENT:

JOANNE BREZNIK

CA 94550

(510) 424-4191

LOCATION OF EQUIPMENT:

LAURENCE LIVERMORE NATIONAL LAB

EXCESS PROPERTY SECTION, L-696

LIVERMORE

CA 94550

$1,249.00$

$\$ 1,249.00$

FOR FURTHER INFORMATION :

JOANNE BREZNIK

LIVERMORE

CA 94550

(510) $424-4191$

TO FREEZE EOUIPMENT:

JOANME BREZNIK

LIVERMORE

CA 94550

(510) $424-4191$

LOCATION OF EQUIPMENT

LAWRENCE LIVERMORE NATIONAL LAB

EXCESS PROPERTY SECTION, L-696

LIVERMORE

CA 94550 
EFFECIIVE DATE: $07 / 01 / 94$ EXPIRATION DATE: $07 / 31,94$

\section{FSC \\ COOE EXCESS PROPERTY DESCRIPTION \\ REFERENCE NUMBER : 979}

7021 COMPUTER-PC, MFR. APPLE, MOOEL MO001, ACQ. YR. 86, PROP. NO. 4417482

REPORT NUMBER: $899102-4167$

LINE ITEM NUMBER: 0021

REFERENCE NUMBER : 980

7021 COMPUTER-PC, MRF. IBM, MODEL 5160, ACO. YR. 86, PROP. NO. 4425234

$500001 \mathrm{EA}$

REPORT NUMBER: $899102-4167$

LINE ITEM NUMBER: 0007
COST/UNIT:

TOTAL COST:

$\$ 1,490.44$

$\$ 1,490.44$

NO OF

UNITS UNIT

ACQUISITION PRICE

$\$ 2,152.20$ $\$ 2,152.20$

COST/UNIT:
TOTAL COST (

JOANNE BREZN IK

IVERMORE

CA 94550

(510) $424-4191$

TO FREEZE EQUIPMENT:

JOANNE BREZNIK

LIVERMORE

(510) $424-4191$

CA 94550

LOCATION OF EQUIPMENT:

LAURENCE LIVERMORE NATIONAL LAB

EXCESS PROPERTY SECTION L-696

LIVERMORE

CA 94550

FOR FURTHER INFORMATION:

JOANNE BREZNIK

LIVERMORE

CA 94550

TO FREEZE EOUIPMENT:

JOANNE BREZNIK

LIVERMORE

(510) $424-4191$

CA 94550

LOCATION OF EQUIPMENT:

LAWRENCE LIVERMORE NATIONAL LAB EXCESS PROPERTY SECTION, L-696

LIVERMORE

A 94550 
DOE USED ENERGY RELATED LABORATORY EOUIPMENT GRANT PROGRAM

EFFECTIVE DATE: 07/01/94 EXPIRATION DATE: 07/31/94

FSC

COOE EXCESS PROPERTY DESCRIPTION

REFERENCE NUMBER : 981

7025 BACK EVEREX TAPE, SN-AT220011039

REPORT NUMBER: $894609-9090$

\section{REFERENCE NUMBER : 982}

7025 BACKUP EVEREX TAPE AAT20004880

REPORT NUMBER: $894609-9090$

ELIGIBLE EQUIPMENT

No OF

COND UNIIS UNIT

$600001 \mathrm{EA}$

COST/UNIT: TOTAL COST:

LINE ITEM NUMBER: 43
$600001 \mathrm{EA}$

COST/UNIT: TOTAL COST:

LINE ITEM NUMBER: 41
ACQUISITION PRICE

$\$ 1,124.00$ $\$ 1,124.00$

$\$ 1,124.00$ $\$ 1,124.00$
FOR FURTHER INFORMATION : DAVID MITCHELL

AIKEN

SC 29802

(803) $725-9540$

TO FREEZE EOUIPMENT:

DAVID MITCHELL

AIKEN

(803) $725-9540$

SC 29802

LOCATION OF EQUIPMENT:

WESTINGHOUSE

SAVANNAH RIVER SITE

AIKEN

SC 29802 
EFFECTIVE DATE: $07 / 01 / 94$ EXPIRATION DATE: $07 / 31 / 96$

FSC
COOE EXCESS PROPERTY DESCRIPIION

REFERENCE NUMBER : 983

7025 BACKUP EVEREX TAPE, SN-AT20011038

REPORT NUMBER: $894609-9090$

REFERENCE NUMBER : 984

7025 BACKUP EVEREX TAPE, SN-DX11397

REPORT NUMBER: $894609-9090$
ELIGIBLE EOUIPMENT
No of

COND UNITS UNIT

COST/UNIT:
TOTAL COST:

LINE ITEM NUMBER: 42
$600001 \mathrm{EA}$
COST/UNIT:
TOTAL COST:
LINE ITEM NUMBER: 48
$600001 \mathrm{EA}$
$\$ 1,235.00$
$\$ 1,235.00$

FOR FURTHER INFORMATION:

DAVID MITCHELL

AIKEN

SC 29802

803) $725-9540$

TO FREEZE EQUIPMENT:

DAVID MITCHELL

AIKEN

(803) $725-9540$

SC 29802

LOCATION OF EQUIPMENT:

WEST INGHOUSE

SAVANNAH RIVER SITE

AIKEN

SC 29802 
EFFECTIVE DATE: $07 / 01 / 94$ EXPIRATION DATE: $07 / 31 / 94$

FSC

COOE EXCESS PROPERTY DESCRIPTION

REFERENCE NUMBER : 985

7025 BERNOULLI DISK DRIVE, SN-03K3E

REPORT NUMBER: $894609-9091$
NO OF

COND UNITS UNIT

$500001 E A$
LINE ITEM NUMBER: 31

ACQUISITION PRICE

\section{COST/UNIT:
TOTAL COST:}

TOTAL COST:

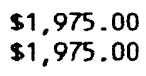

\section{FOR FURTHER INFORMATION:}

DAVID MITCHELL

AIKEN

(803) $725-9540$

SC 29802

TO FREEZE EQUIPMENT

DAVID MITCHELL$$
\text { DAVID }
$$

(803) $725-9540$

SC 29802

LOCAIION OF EOUIPMENT:

WESTINGHOUSE

SESTINGHOUSE

SAVANMAY
COST/UNIT: TOTAL COST:

REFERENCE NUMBER : 986

BERNOULLI DRIVE SYSTEM DUAL DRIVE SYSTEM EACH DISK CAN HOLD 44 MB, DRIVE 600001 EA

IS IBM COMPATIB LE. 120VAC, $60 \mathrm{HZ}$. IOMEGA, B244X, SER. 40193700

REPORT NUMBER: $898355-4095$

LINE ITEM NUMBER: 0018

FOR FURTHER INFORMATION :

ELOY N. GIRON

ALBUQUERQUE

(505) $844-2746$

NM 87185

TO FREEZE EOUIPMENT:

ELOY N. GIRON

ALBUOUERQUE

(505) $844-2746$

NM 87185

LOCATION OF EOUIPMENT:

SANDIA NATIONAL LABORATORIES

PROPERTY REAPPLICATION DIVISION 7617

PROPERTY REAPPLICATION DIVISION 7617
ALBUQUEROUE
NM 87123 
FSC

\section{COOE EXCESS PROPERTY DESCRIPTION}

REFERENCE NUMBER : 987

7025 BERNOULLI MFG. OMEGA MOOEL \# B264X SERIAL \# 4040190001 EGŚ ID 008001-00 ONE OF THE DRIVES IS DEFECTIVE

YEAR ACO 1990 DOE $\# 00045915-00$

REPORT NUMBER: $898289-4049$

LINE ITEM NUMBER: 101R

REFERENCE NUMBER : 988

7025 CADDSIATION MFR: SUN MICROSYSTEM, MODEL 247-EEC, S/N 820F1406, ACO. DATE: $8 / 88$, APPROX. HT: 100 LBS., 21 "W $\times 20 " \mathrm{~L} \times 20 \mathrm{H}$.
NO OF

COND UNITS UNIT

$900001 \mathrm{EA}$

COST/UNIT:

TOTAL COST:

ACQUISITION PRICE

$\$ 1,869.00$

$\$ 1,869.00$

FOR FURTHER INFORMATION:

G.A. ELVE

(303) $465-3765$

CO 80402-0464

TO FREEZE EQUIPMENT:

G.A. ELVEY

GOLDEN

(303: $465-3765$

CO 80402-0464

LOCATION OF EQUIPMENT:

EGE ROCKY FLATS, INC.

BUILDING 020-BROOMFIELD FACILITY

BROOMFIELD

CO 80020
400001 EA

COST/UNIT:

TOTAL COST:
$\$ 15,472.00$ $\$ 15,472.00$

OR FURTHER INFORMATION:

JANE T F. BROOKS

LIVERMORE

LIVERMORE
$(510) 294-2186$

CA $94550-0969$

TO FREEZE EQUIPMENT:

JANET $F$. BROOKS

IVERMORE

(510) $294-2186$

CA $94550-0969$

LOCATION OF EOUIPMENT:

SANDIA NATIONAL LABORATORIES

PROPERTY REAPPLICATION DEPT. 8532-1

LIVERMORE

CA $94550-0969$ 
EFFECTIVE DATE: 07/01/94 EXPIRATION DATE: $07 / 31 / 94$

FSC

COOE EXCESS PROPERTY DESCRIPTION

REFERENCE MUMBER : 989

7025 COMPUTER COMPAO PORTABLE SN-1406020788

REPORT MUMBER: $894609-9090$

REFERENCE NUMBER : 990

7025 COMPUTER EPSON EQUITY 111 , SN-A010642

REPORT MUMBER: $894609-9090$
MO OF

COND UNITS UNIT

\section{ACOUISITION PRICE}

$5 \quad 00001 \mathrm{EA}$

COST/UNIT:
TOTAL COST:

LINE ITEM NUMBER: 64

$600001 \mathrm{EA}$

COST/NAIT:
OTAL COST:

$\$ 33,321.00$

LIME IPEM MUNBER: 62

\section{CONTACT(S)}

FOR FURTHER INFORMATION:

DAVID MITCHELL

AIKEN

(803) $725-9540$

TO FREEZE EOUIPMENT:

DAVID MITCHELL

AIKEN

(803) $725-9540$

LOCATION OF EQUIPMENT:

WESTINGHOUSE

SAVAMNAH RIVER SITE

AIKEN

SC 29802

FOR FURTHER INFORMATION:

DAVIO MITCHELL

AIKEN

SC 29802

(803) $725-9540$

TO FREEZE EOUIPMENT:

DANTD MITCHELL

AIKEM

SC 29802

(803) $725-9540$

LOCATION OF EQUIPMENT:

WESTINGHOUSE

SAVANNAH RIVER SITE

AIKEN

SC 29802 
EFFECTIVE DATE: 07/01/94 EXPIRATION DATE: $07 / 31 / 94$

\section{fSC}

COOE EXCESS PROPERTY DESCRIPIION

\section{REFERENCE NUMBER : 99}

7025 COMPUTER MFG. IBM MOOEL \# 5160 SERIAL \# 53703065160 EG\&G $10 \quad 010548-00$

YEAR ACO 1986 DOE $\# 00024794-00$

REPORT NUMBER: $898289 \cdot 4049$

LINE ITEM NUMBER: $103 R$

REFERENCE NUMBER : 992

7025 COMPUTER MFG. IBM MOOEL \#5160 SERIAL = 54570405100

EGAG DO 010551.00

YEAR ACQ 1985 DOE $=00041357-00$
NO OF
COND UNITS UNIT

B $00001 \mathrm{EA}$
ACOUISITION PRICE

COST /UNIT: TOTAL COST:

\section{$\$ 7.250 .53$ $\$ 7,250.53$}

CONTACT(S)

FOR FURTHER INFORMATION:

G.A. ELVEY

CO $80402-0464$

$465-3765$

TO FREEZE EOUIPMENT:

G.A. ELVEY

GOLDEN

c0 $80402-046$

(303) $465-3765$

LOCATION OF EQUIPMENT

EGEG ROCKY FLATS, INC.

BUILDING 020-BROOMFIELD FACILITY

BROOMFIELD

CO 80020

$8 \quad 00001$ EA

COST/UNIT:

TOTAL COST

$\$ 2.649 .00$

$\$ 2,649.00$

FOR FURTHER INFORMATION:

G.A. ELVEY

GOLDEN

CO 80402-0466

3) $465-3765$

TO FREEZE EOUIPMENT:

G.A. ELVEY

GOLDEN

(303) $465-3765$

CO $80402-0464$

LOCATION OF EOUIPMENT:

EGEG ROCKY FLATS, INC.

BUILOIMG O20-BROOMFIELD FACHUITY

BROOMF IELD 
EFFECTIVE DATE: 07/01/94 EXPIRATION DATE: 07/31/94

FSC

COOE EXCESS PROPERTY DESCRIPIION

REFERENCE NUMBER : 993

7025 COMPUTER MFG. IBM MOOEL \# 5161 SERIAL \#01552875161

EG\&G ID $010552-00$

YEAR ACO 1985 DOE $\# 00041358-00$

REPORT NUMBER: $898289-4049$

LINE ITEM NUMBER: $106 R$

\section{No o}

COND UNITS UNIT

ACOUISITION PRICE

$800001 E A$

COST/UNIT:

TOTAL COST:

$\$ 1,421.00$

$\$ 1,421.00$

EG\&G ID 010921-00

YEAR ACO 1986 DOE \#00024347-00

REPORT MUMBER : $898289-4049$

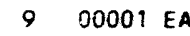 \\ COSI/UNII:
TOTAL COSI: \\ $900001 \mathrm{EA}$}

LINE ITEM MUMBER: 1158
CONTACISS)

FOR FURTHER INFORMAI!ON:

G.A. ELVEY

GOLDEN

CO $80402 \cdot 0464$

(303) $465-3765$

TO FREEZE EOUIPMENT:

G.A. ELVEY

co 80402-0464

(303) $465 \cdot 3765$

LOCATION OF EOUIPMENT:

EG\&G ROCKY FLAIS, INC.

BUILDING 020-BROOMFIELD FACILITY

BROOMFIELD

CO 80020

FOR FURTHER INFORMAIION:

G.A. ELVEY

GOLDEN

Co $80402-0464$

(303) 465-3765

TO FREEZE EQUIPMENT:

G.A. EIVEY

CO 80402-0464

(303) $465-3765$

LOCATION OF EOUIPMENT

EGQG ROCKY FLATS, INC.

BUILDING 020-BROOMFIELD FACILITY

BROOMF IELD

CO 80020 
EFFECTIVE DATE: $07 / 01 / 94$ EXPIRATION DATE: $07 / 31 / 94$

FSC
COOE EXCESS PROPERTY DESCRIPTION

\section{No of COND UNITS UNIT}

900001 EA
REFERENCE MUMBER : 995

7025 COMPUTER MFG. 1 BM MOOEL \# 5150 SERIAL \# 0630181 EGQG ID $010922 \cdot 00$

YEAR ACO 1984 DOE \# 00004628-00

REPORT NUMBER: $898289-4049$

LINE ITEM MUMBER: 11T6R

COST/UNIT: TOTAL COST:

UISITION PRICE

$\$ 3.497 .00$ $\$ 3,497.00$

\begin{tabular}{|c|c|c|}
\hline 5 & 00001 EA & $\begin{array}{l}\text { COST/UNIT } \\
\text { TOTAL COST }\end{array}$ \\
\hline
\end{tabular}

REFERENCE NUMGER : 996

7025 COMPUTER MFG. IBM MODEL \# 5150 SERIAL \# 0630178 EGAS ID $010926-00$

VEAR ACO 1984 DOE $\# 00004647-00$

REPORT NUMBER: $898289-4049$
LINE ITEM NUMBER: 117R $\$ 1,843.00$
$\$ 1,843.00$
FOR FURTHER INFORMATION

G.A. ELVEY

GOLDEN

CO 80402-0464

(303) 465-3765

CO 80402-0464

GOLDEN

$0080402-0464$

TO FREEZE EQUIPMENT:

G.A. ELVEY

GOLDEN

CO 80402-0464

LOCAIION OF EOU:FMENT:

EGSG ROCKY FLATS, INC.

BUILDING CZR-BROOMFIELD FACILITY

BROOMFIELD

CO 80020

TO FREEZE EOUIPMENT:

G.A. ELVEY

GOLDEN

(303) $465-3765$

co $80402-0464$

LOCATION OF EQUIPMENT

EG\&G ROCKY FLATS, INC.

BUILDING 020-BROOMFIELD FACILITY

BROOMFIELD

CO 80020 
EFFECTIVE DATE: 07/01/94 EXPIRATION DATE: $07 / 31 / 94$

\section{FSC \\ no of}

COOE EXCESS PROPERTY DESCRIPIION

REFERENCE NUMBER : 997

7025 COMPUTER MFR: APPLE, MOOEL M5010, S/N F73611PMS010, ACO. DATE: 10/87. AP PROX. WT: 20 LBS. , 10"W $\times 11^{\prime \prime L} \times 13^{\prime \prime H}$.

REPORT NUMBER: $899105-4083$

LINE ITEM MUMBER: 0001

\section{REFERENCE NUMBER : 998}

7025 COMPUTER MFR: APPLE, MOOEL M0001A, S/N F4466RLM0001W, ACO. DATE:

12/84, APPROX WI: 25 LBS. . 10"W $\times 19$ "L $\times 14^{\prime \prime H}$

600001 EA

COST/UNIT:
TOTAL COST:

LINE ITEM MUMBER: 0002

COND UNITS UNIT
ACOUISIIION PRICE

COST/UNIT:

TOTAL COST:

$\$ 2,150.00$
$\$ 2,150.00$

FOR FURTHER INFORM
JAMET F. BROOKS

LIVERMORE

(510) $294-2186$

CA $94550-0969$

TO FREEZE EOUIPMENT :

JANET $F$. BROOKS

LIVERMORE

CA $94550-0969$

(510) $294-2186$

LOCATION OF EQUIPMENT:

SAMDIA MATIOMAL LABORATORIES

PROPERTYY REAPPLICATION DEPT 8532-1

LIVERMORE

CA $94550-0969$
$\$ 1.657 .00$

$\$ 1,657.00$
FOR FURTHER INFORMATION

JANET F. BROOKS

LIVERMORE

(510) $294-2186$

CA $94550-0969$

TO FREEZE EOUIPMENT:

JANET F. BROOKS

LIVERMORE

(510) $294-2186$

CA $94550-0969$

LOCATION OF EQUIPMENT :

SAMDIA MATIONAL LABORATORIES

PROPERTY REAPPLICATION OEPT. 8532-1

PROPERTY REAPPLICATION DEPT. 8532-1 
EFFECTIVE DATE: $07 / 01 / 94$ EXPIRATION DATE: $07 / 31 / 94$

FSC

COOE EXCESS PROPERTY DESCRIPTION

REFERENCE NUMBER :

7025 COMPUTER MFR: APPLE, MO001A, S/N F7072JXM0001A, ACQ. DATE: $3 / 87$, APPROX. WT: 30 LBS., 10"W $\times 11^{\prime \prime L} \times 13^{\prime \prime H}$.

REPORT NUMEER: $899105-4083$

LINE ITEM NUMBER: 0004

REFERENCE NUMBER : 1000

7025 COMPUTER MFR: HETRA, MODEL KT-7/PC, S/N H86120516, ACO. DATE: $12 / 88$, APP ROX. WT: 35 LBS, $20^{\prime \prime W} \times 17^{\prime \prime L} \times 18^{\prime \prime H}$
No of

COND UN!TS UNIT

ACOUISITION PRICE

$500001 E A$

$\operatorname{COST/UN1T}$

TOTAL COST:

$\$ 2,100.00$

$\$ 2,100.00$

JANET F. BROOKS

(510) $294-2186$

CA $94550-0969$

TO FREEZE EQUIPMENT :

JANET $F$. BROOKS

LIVERMORE

(510) $294-2186$

CA $94550-0969$

LOCATION OF EOUIPMENT:

SAMDIA NAIIONAL LABORATORIES

PROPERTY REAPPLICATION DEPT. 8532-

LIVERMORE

CA $94550-0969$

REPORT NUMBER: $899105-4083$

LINE ITEM NUMBER: 0006
COST/UNIT:

TOTAL COST:
$\$ 3.633 .00$

$\$ 3,633.00$
FOR FURTHER INFORMATION:

JAKET $F$. BROOKS

$$
\text { LIVERMORE }
$$

(510) $294-2186$

CA $94550-0969$

TO FREEZE EOUIPMENT :

JANET $F$. BROOKS

LIVERMORE

CA $94550-0969$

(510) $294-2186$

LOCATION OF EOUIPMENT:

SANDIA NATIONA: LABORATORIES

PROPERTY REAPPLICATION DEPT. 8532 -

LIVERMORE

CA $94550-0969$ 
EFFECTIVE DATE: 07/01/94 EXPIRATION DATE: 07/31/94

FSC

\section{COOE EXCESS PROPERTY DESCRIPIION}

REFERENCE NUMBER : 1001

7025 COMPUTER PORTABLE PLUS, SH-152200680061 COMPAO

REPORT NUMBER: $894609-9090$

LIME ITEM NUMBER: 63

No of

COMD UNITS UNIT

ACQUISITION PRICE

$600001 \mathrm{EA}$

COST/UNIT: TOTAL COST:

\section{$\$ 2,864.00$} $\$ 2,864.00$

REFERENCE NUMBER : 1002

7025 COMPUTER, COMPAO 286, MDL 2551, S/N 4744AM1B0B36, W/MONITOR, THOMPSON,

MDL 4375 M, S/N 000044, COLOR AND XEYBOARD
LINE ITEM NUMBER: 0013
$400001 \mathrm{EA}$

$\cos T / U \operatorname{Lit}$

TOTAL COST:
$\$ 2,000.00$ $\$ 2,000.00$
REPORT MUMBER: $892401-4080$ 
EFFECIIVE DATE: $07 / 01 / 94$ EXPIRATION DAIE: $07 / 31 / 94$

FSC

\section{COOE EXCESS PROPERTY DESCRIPIION}

\section{REFERENCE NUMBER : 1003}

7025 COMPUTER, COMPAO 286, MDL 2551, S/N 4744AMIB0980, :!'MONITOR, NEC NTERNAL REMOVABLE MEDIA BASED HOUSING W/CONTROLLER CARD IS INSTALLE $D$ - ME DIA IS REMOVED, TO BE OPERATIONAL NEED DOS SOFTWARE AND HARD DISK MEDIA W/CONTROLLER CARD

LINE ITEM NUMBER: 0014

\section{REFERENCE NUMBER : 1004}

7025 COMPUTER, COMPAO 386-16, MDL 2570, S/N 4711AJ3B0309, W/KEYBOARD, INTERNAL REMOVABLE MEDIA BASE CONTROLLER CARD \& HOUSING INSTALLED NAL HA RD DISK MEDIA H/CONTROLLER CARD MULTISYNC, MDL JC-1402HMA, S/N 7YD61679M, COLOR, AND KEYBOARD, AN MEDIA IS REMOVED, TO BE OPERATIONAL NEEDS DOS SOFTHARE AND INTER

$$
\text { NO OF }
$$

COND UNITS UNIT

$800001 \mathrm{EA}$
COSTIUNIT: TOTAL COST: IT: $\$ 4,625.00$
FOR FURTHER INFORMATION:

JENNI FER IMHOFF

WEST MIFFLIN

PA 15122-0109

TO FREEZE EQUIPMENT :

JENN I FER IMHOFF

WEST MIFFLIN

(412) $476-5328$

PA 15122-0079

LOCATION OF EQUIPMENT:

BETTIS ATOMIC POWER LABORATORY

814 PITTSBURGH/MCKEESPORT BLVD

WESI MIFFLIN

PA 15122-0079 
EFFECTIVE DATE: $07 / 01 / 94$ EXPIRATION DATE: $07 / 31 / 94$

FSC

\section{CODE EXCESS PROPERTY DESCRIPTION}

REFERENCE NUMBER : 1005

7025 COMPUTER, COMPAO 286, MDL 2551, S/N 4744AMIBIB1347, REC 1988, W/MONITOR,

MU LTISYNC 11, MDL JC-1402HMA, S/N 79002776A AND KEYBOARD, AN INTERN AL REMOVABLE MEDIA BASED HOUSING W/CONTROLLER CARD IS INSTA

LLED - MEDIA IS REMOVED, TO BE OPERATIONAL NEED DOS SOFTHARE AND INTER

NAL HARO ORIVE MEIDA W/CONTROLLER CARD

REPORT NUMBER: $892401-4080$

LINE ITEM NUMBER: 0058

REFERENCE NUMBER : 1006

7025 COMPUTER, COMPAO 286, MDL 2551, S/N 4745AMIBI269, REC 1988, W/KEYBOARD,

AN INTERNAL REMOVABLE MEDIA BASED HOUSING W/CONTROLLER CARD IS INSTA

LLED - MEDIA IS REMOVED. TO BE OPERATIONAL NEED DOS SOFTWAR

E AND INTERNAL HARD DRIVE MEDIA W/CONTROLLER CARD

\section{No OF}

COND UNITS UNIT

ACOUISITION PRICE

800001 EA

COST/UNIT:

TOTAL COST:

$\$ 4.975 .00$ $\$ 4,975.00$

\section{JENNI IFER IMHOFF
WEST MIFFLIN}

(412) $476-5328$

TO FREEZE EOUIPMENT:

JENNI FER IMHOF

WEST MIFFLIN
(412) $476-5328$

PA 15122-0079

LOCATION OF EQUIPMENT:

BETTIS ATOMIC POWER LABORATORY

814 PITTSBURGH/MCKEESPORT BLVD

WEST MIFFLIN

PA 15122-0079
COST/UNIT: IOTAL COST:

$\$ 4.175 .00$ $\$ 6.175 .00$
FOR FURTHER INFORMATION:

JENNI FER IMHOFF

HEST MIFFLIN

(412) $476-5328$

PA 15122-0109

TO FREEZE EQUIPMENT:

JENNI FER IMHOF

(412) $476-5328$

PA 15122-0079

LOCATION OF EQUIPMENT:

BETTIS ATOMIC POWER LABORATORY

814 PITTSBURGH/MCKEESPORT BLVD

WEST MIFFLIN

PA 15122-0079 
EFFECTIVE DATE: $07 / 01 / 94$ EXPIRATION DATE: $07 / 31 / 94$

\section{COOE EXCESS PROPERTY DESCRIPTION}

\section{PEFERENCE NUMBER : 1007}

7025 COMPUTER IBM XT, MDL 5170, S/N 5170-7148671, W/MONITOR, PRINCETON COMPUTER, IBM XT, MDL S1T TYZ9131 AND KEYBOARD AN INTERNAL REMOVABLE GRAPHICS MDL HXI2E, S/N TY 2913 IN AND IS INSTALLED - MEIDA IS REMO VED IA BASE HOUSING WIONAL NEED DOS SOFTWARE AND INTERNAL HARD DISK MEDIA H VED, T O BE OPERATI

7025 COMPUTER, IBM XT, MDL 5160, S/N 56268175160, W/MONITOR, NEC MULTISYNC

作

MEDIA BASE HOUSING U/CONTROLLER CARD IS INSTALLED - MEDIA

MEDIA BASE HOUSING W/CONTROLLER CARD IS INSTALARE AND INTERNAL HARD DISK

MEDIA W/CONTROLLER CARD

INE ITEM NUMBER: 0005
No 0
COST/UNIT: TOTAL COST:

$\$ 6,249.00$ $\$ 6,249.00$

$00001 \mathrm{EA}$

COST/UNIT:

TOTAL COST:

$\$ 5,000.00$

$\$ 5,000.00$
FOR FURTHER INFORMATION:

JENNI FER IMHOF

WEST MIFFLIN

(412) $476-5328$

PA $15122-0109$

TO FREEZE EQUIPMENT

JENNI FER IMHOFF

WEST MIFFLIN

(412) $476-5328$

PA 15122-0079

LOCATION OF EQUIPMENT

BETIIS ATOMIC POWER LABORATORY

814 PITTSBURGH/MCKEESPORT BLVD

HEST MIFFLIN

PA 15122-0079 
EFFECTIVE DATE: $07 / 01 / 94$ EXPIRATION DATE: $07 / 31 / 94$

\section{FSC}

COOE

\section{EXCESS PROPERTY DESCRIPTION}

7025 COMPUTER, IBM XT, MDL 5170, S/N 01379175170, W/MONITOR, IBM, MDL 5154 S/N 0096803 . ENHANCE COLOR DISPLAY, \& KEYBOARD, AN INTERNAL REMOVABLE MEDIA BASE HOUSING W/CONTROLLER CARD IS INSTALLED - MEDIA

IS REM OVED, TO BE OPERATIONAL NEED DOS SOFTWARE AND INTERNAL HARD DISK MEDIA W/CONTROLLER CARD

REPORT NUMBER: $892401-4080$

LINE" ITEM NUMBER: 0007

REFERENCE NUMBER : 1010

7025 COMPUTER, IBM XT, MDL 5160, S/N 56279615160, W/KEYBOARD, AN INTERNAL REMOVA BLE BASE HOUSING H/CONTROLLER CARD IS INSTALLED - MEDIA IS REMOVE $D$, TO BE OPERATIONAL NEED DOS SOFTWARE AND INTERNAL HARD DI

SK MED IA W/CONTROLLER CARD

\author{
REPORT NUMBER: $892401-4080$
}

LINE ITEM NUMBER: 0009
NO OF

COND UNITS UNIT

ACQUISITION PRICE

800001 EA

COST/UNIT:
TOTAL COST:

$\$ 6,200.00$

$\$ 6,200.00$
CONTACT(S)

FOR FURTHER INFORMATION:

JENNIFER IMHOFF

WEST MIFFLIN

(412) $476-5328$

PA 15122-0109

TO FREEZE EQUIPMENT:

JENNIFER IMHOFF

WEST MIFFLIN
(412) $476-5328$

PA 15122-0079

LOCATION OF EQUIPMENT:

BETTIS ATOMIC POWER LABORATORY

BETTIS ATOMIC POWER LABORATORY

WEST MIFFLIN
COST/UNIT:

TOTAL COST:

$\$ 4,200.00$
$\$ 4,200.00$

FOR FURTHER INFORMATION:

JENNIFER IMHOFF

(412) $476-5328$

PA 15122-0109

TO FREEZE EQUIPMENT:

JENNIFER IMHOFF

WEST MIFFLIN

PA 15122-0079

(412) $476-5328$

LOCATION OF EQUIPMENT:

BETTIS ATOMIC POWER LABORATORY

B14 PITISBURGH/MCKEESPORT BLVD

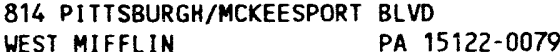


EFFECIIVE DATE: $07 / 01 / 94$ EXPIRATION DATE: $07 / 31 / 94$

COOE EXCESS PROPERTY DESCRIPTION

REFERENCE NUMBER : 1011

7025 COMPUTER, IBM XT, MDL 5160, S/N 55844825160, W/MONITOR, MULTISYNC II, MDL I 403 HMA, S/N 93K08135P. AND KEYBOARD, AN INTERNAL REMOVABLE MEDIA MDL 1403 HASE, S/N $93 K 08135$ P , AND KEYBOARD, AN INTERNAL REMOVAB

BASE HOUSING W/CONTROLLER CARD IS INSTALLED - MEDIA IS REMO
VED I O BE OPERATIONAL NEED DOS SOFTWARE AND INTERNAL HARD DISK MEDIA VED, T O BE OPERATI
/CONTROLLER CARD

REFORT NUMSER: $892401-4080$

LINE ITEM NUMBER: 0010

REFERENCE NUMBER : 1012

COMPUTER, IBM XT, MDL 5160, S/N 62185335160, W/MONITOR, MULTISYNC 2A,

MDL 1 403HMA, S/N 99L120462, AND KEYBOARD, AN INTERNAL REMOVABLE MEDIA

BASE HOUSING W/CONTROLLER CARD IS INSTALLED - MEDIA IS REMO

VED, T O BE OPERATIONAL NEED DOS SOFTWARE AND INTERNAL HARD DISK MEDIA $W$

ICONTROLLER CARD

LINE ITEM NUMBER: 0011
NO OF

COND UNITS UNI

ACOUISITION PRICE

$800001 E A$ COST/UNIT:
TOTAL COST:

$\$ 5,000.00$ TOIAL COST: $\$ \$ 5,000.00$

JE

\section{WEN}
TO FREEZE EOUIPMENT:
JENNIFER IMHOFF

WEST MIFFLIN

(412) $476-5328$

PA 15122-0079

LOCATION OF EQUIPMENT:

BETTIS ATOMIC POWER LABORATORY 814 PITTSBURGH/MCKEESPORT BLVD

WEST MIFFLIN

PA 15122-0079
$800001 E A$
COST/UNIT:
TOTAL COST:
$\$ 5,000.00$
$\$ 5,000.00$
FOR FURTHER INFORMATION:

JENNIFER IMHOFF

WEST MIFFLIN

(412) $476-5328$

PA $15122-0109$

TO FREEZE EQUIPMENT:

JENNIFER IMHOFF

WEST MIFFLIN

(412) $476-5328$

PA 15122-0079

LOCATION OF EQUIPMENT:

BETIIS ATOMIC POWER LABORATORY

814 PITTSBURGH/MCKEESPORT BLVD

WESI MIFFLIN

PA 15122-0079 
EFFECTIVE DATE: 07/01/94 EXPIRATION DATE: 07/31/94

FSC

\section{COOE EXCESS PROPERTY DESCRIPIION}

REFERENCE NUMBER : 1013

7025 COMPUTER, IBM XT-386, MDL 5160, S/N 55830075160, UPGRADED, W/KEYBOARD

$30 L B$ AN INTERNAL REMOVABLE MEDIA BASED HOUSIHG H/CONTROLLER CARD IS

INSTALLLED - MEDIA REMOVED, TO OPERATE NEED DOS SOFTWARE AND

INTER NAL HARD DRIVE MEDIA W/CONTROLLER CARD

REPORT NUMBER: $892401-4080$

LINE ITEM NUMBER: 0023
No of

COND UNITS UNIT

ACQUISITION PRICE

800001 EA

COST/UNIT

TOTAL COST

$\$ 2.500 .00$

$\$ 2,500.00$

Th

(412) $476-5328$

TO FREEZE EQUIPMENT:

JENNIFER IMHOFF

WEST MIFFLIN

(412) $476-5328$

PA 15122-0079

LOCATION OF EQUIPMENT:

BETTIS ATOMIC POWER LABORATORY

814 PIIT SBURGH/MCKEESPORT BLVD

WEST MIFFLIN

PA 15122-0079

REFERENCE NUMBER : 1014

COMPUTER, JAMECO 386-16, MOL JE1019, S/N NONE, W/KEYBOARD, INTERNAL

REMOVAB LE MEDIA BASE CONTROLLER CARD \& HOUSING INSTALLED - MEDIA IS

REMO VED, TO BE OPERATIONAL NEEDS DOS SOFTWARE AND INTERMAL HARD

$800001 \mathrm{EA}$

COST/UNIT:

TOTAL COST:

$\$ 4,200.00$

$\$ 4,200.00$

DISK MEDIA W/CONTROLLER CARD
LIME ITEM NUMBER: 0044
REPORT NUMBER: $892401-4080$

\section{FOR FURTHER INFORMATION: \\ JENNI FER IMHOFF \\ WEST MIFFLIN \\ (412) $476-5328$}

PA 15122-0109

TO FREEZE EQUIPMENT:

JENNI FER IMHOFF

WEST MIFFLIN

PA 15122-0079

(412) $476-5328$

LOCATION OF EOUIPMENT:

BETTIS ATOMIC POWER LABORATORY

814 PITISBURGH/MCKEESPORT BLVD

WEST MIFFLIN

PA 15122-0079 


\section{EFFECTIVE DATE: 07/01/94 EXPIRATION DATE: $07 / 31 / 94$}

FSC

\section{COOE EXCESS PROPERTY DESCRIPTION}

REFERENCE NUMBER : 1015

7025 COMPUTER, KAYPRO 286, S/N 373253, REC 1987, W/MONITOR, PRINCETON GRAPHICS, MDL HX12E, S/N TY 49895 AND KEYBOARD, AN INTERNAL REMOVABLE MEDIA BASE HOUSING W/CONTROLLER CARD IS INSTALLED - MEIDA IS REMO VED, T O BE OPERATIONAL NEED DOS SOFTWARE AND INTERNAL HARD DISK MEDIA W ICONTROLLER CARD.
REPORT NUMBER: $892401-4080$
LINE ITEM NUMBER: 0003
No of

800001 EA
COND UNITS UNIT
ACQUISITION PRICE

COST/UNIT: TOTAL COST:
$\$ 3,356.00$

$\$ 3,356.00$

\section{REFERENCE NUMBER : 1016}

7025 COMPUTER, KAYPRO 286I, S/N 347821, W/MONI TOR, PRINCETON GRAPHICS, MDL HX12E, S/N TY29669, AND KEYBOARD, AN INTERNAL REMOVABLE MEDIA BASE HOU SING W/CONTROLLER CARD IS INSTALLED - MEDIA IS REMOVED, TO BE OPE RATIONAL NEED DOS SOFTWARE AND INTERNAL HARD DISK MEDIA W/CONTROL LER CARD
$800001 \mathrm{EA}$ TOTAL COST: $\$ 3,356.00$

FOR FURTHER INFORMATION:

JENNIFER IMHOFF

WEST MIFFLIN (412) $476-5328$

PA $15122-0109$

TO FREEZE EQUIPMENT:

JENN I FER IMHOFF

$$
\text { WEST MIFFLIN }
$$

(412) $476-5328$

PA 15122-0079

LOCATION OF EQUIPMENT:

BETIIS ATOMIC POWER LABORATORY 814 PITTSBURGH/MCKEESPORT BLVD WEST MIFFLIN 
EFFECTIVE DATE: $07 / 01 / 94$ EXPIRATION DATE: $07 / 31 / 94$

FSC

\section{COOE EXCESS PROPERTY DESCRIPTION}

REFERENCE NUMBER : 1017

7025 COMPUIER, XAYPRO 2861, S/N 353802, W/MONITOR, SONY TRINITRON SUPER FINE PIT CH, MDL CPD-1302, S/N S021886, AND KEYBOARD, AN INTERNAL REMOVABL E MEDIA BASE HOUSING W/CONTROLLER CARD IS INSTALLED - MEDIA

IS RE MOVED, TO BE OPERATIONAL NEED DOS SOF TWARE AND INTERNAL HARD DISK MEDIA W/CONTROLLER CARD

\section{REFERENCE NUMBER : 1018}

7025 COMPUTER, KAYPRO 2861, S/N 371161, H/MONITOR, PRINCETON HX12E, S/N

KWI 1934, AND KEYBOARD, AN INTERNAL REMOVABLE MEDIA BASE HOUSING

W/CONTROL LER CARD IS INSTALLED - MEDIA IS REMOVED TO BE OPERATIONAL

NEED DOS SOFTHARE AND INTERNAL HARD DISK MEDIA W/CONTROLLER CARD.
COST/UNIT:

TOIAL COST:
ACQUISITION PRICE

COST/UNIT:

$\$ 3,625.00$

$$
\begin{aligned}
& \text { JENNIFER IMHOFF } \\
& \text { WEST MIFFLIN }
\end{aligned}
$$

(412) $476-5328$

PA $15122-0109$

TO FREEZE EQUIPMENT

JENNI FER IMHOFF

WEST MIFFLIN

PA 15122-0079
LOCATION OF EQUIPMENT:

BETTIS ATOMIC POWER LABORATORY 814 PITTSBURGH/MCKEESPORT BLVD

WEST MIFFLIN
$\$ 3,625.00$

$\$ 3,625.00$
FOR FURTHER INFORMATION:

JENNIFER IMHOF F

WEST MIFFLIN

PA 15122-0109

(412) $476-5328$

TO FREEZE EOUIPMENT

JENN I FER IMHOFF

WEST MIFFLIN

PA 15122-0079

(412) $476-5328$

LOCATION OF EQUIPMENT:

BETTIS ATOMIC POWER LABORATORY

814 PITISBURGH/MCKEESPORT BLV

WEST MIFFLIN

PA 15122-0079 
EFFECTIVE DATE: 07/01/94 EXPIRATION DATE: 07/31/96

FSC

\section{COOE EXCESS PROPERIY OESCRIPTION}

REFERENCE NUMBER : 1019

TO25 COMPUTER, KAYPRO $2961, S / N 368208$, UPGRADED TO 386, REC $12 / 86$

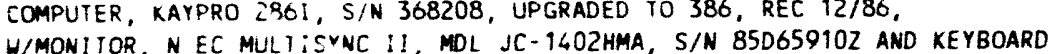

W/MONITOR, N EC MULIIS SNC II, MDL JC- 1402 HMA, S/N 85D65910Z AND KEYE

CARD IS INSTALLED. MEIDA IS REMOVED, TO BE OPERATIONAL MEED DOS SOFTW

ARE AND INTERNAL HARD DRIVE MEDIA W/CONTROLLER CARD.

REPORT NUMBER: $892401-4080$

LINE ITEM NUMBER: 0024

\section{NO O}

COND UNITS UNIT

ACQUISITION PRICE

$800001 \mathrm{EA}$

COST/UNIT:
TOTAL COST:

$\$ 3,625.00$

$\$ 3.625 .00$

\section{(412) 476 -53}

JENMIFER IMHOF

PA 15122-0109

TO FREEZE EOUIPMENT:

EENMIFER IMHOF

NEST MIFFLIN

(412) $476-5328$

PA $15122-0079$

LOCATION OF EQUIPMENT:

BETTIS ATOMIC PONER LABORATORY

B14 PITTSBURGH/MCKEESPORT BLV

UEST MIFFLIN

PA $15122-0079$

\section{REFERENCE NUMBER : 1020}

COMPUTER, KAYPRO 2861, S/N 368222, UPGRADED TO 386, REC $1 / 87$

12E, COLOR GRAPHIC, S/N TY32544, AND KEYBOARD,

ROLLER CARD IS IN

STALLE D - MEDIA IS REMOVED, TO BE OPERATIONAL NEED DOS SOFTWARE AND IN

ERNAL HARD DRIVE MEDIA W/CONTROLLER CARD
800001 EA

COST $/ U N 1 T:$
TOTAL COST:

$\$ 2.950 .00$
$\$ 2.950 .00$
FOR FURTHER INFORMATION:

JENNIFER IMHOF

NEST MIFFLIN

PA $15122-0109$

412) $476-5328$

TO FREEZE EQUIPMENT:

JEMNIFER IMHOF

WEST MIFFLIN

PA 15122-0079

REPORT NUMBER: $892401-4080$

LINE ITEM MUMBER: 0025

OCATION OF EOUIPMENT:

BETTIS ATOMIC POUER LABORATORY

814 PITTSBURGH/MCKEESPORT BLVD

UEST MIFFLIN

PA 15122-0079 
EFFECTIVE DATE: 07/01/96 EXPIRATION DATE: $07 / 31 / 96$

\section{FSC \\ NO of}

COOE EXCESS PROPERTY DESCRIPTION

REFERENCE MUMBER : 102

7025 COMPUTER, LAP TOP MFR: NEC, MOOEL ULTRALITE, S/N 9100355HB, ACQ. DATE: COMPUTER, LAP TOP MFR: NEC, MOOEL ULTRALITE, S
4/89, APPR OX. WT: IS LABS., 12"W $\times 8^{\prime \prime L} \times 2^{\prime \prime H}$.

REPORT NUMBER: $899105-4083$

LINE ITEM MUMBER: 0010

REFERENCE NUMBER : 1022

7025 COMPUTER, PERSONAL HARD DRIVE AND TAPE DRIVE UNIT FOR A SUN COMPUTER SYSTEM. 120VAC, $60 \mathrm{HZ}$. SUN MICROS, 511, SER. 828F0432.

$600001 \mathrm{EA}$

COST/UNIT:

TOTAL COST:

\section{$\$ 7.576 .00$ $\$ 7.576 .00$}

LINE ITEM NUMBER: 0005

COND UNITS UNIT ACOUISIIIION PRICE

COST/UNIT:

TOTAL COST:

$\$ 2,589.00$
$\$ 2,589.00$

REPORT MUMBER: $898355-6095$
JAMET F. BR IMFOR

JANET F. BROOKS

CA $94550-0969$

IO FREEZE EQUIPMENT:

JANET F. BROOKS

LIVERMORE

(510) $294-2186$

CA $94550-0969$

LOCATIOW OF EQUIPMENT:

SAMDIA MATIOMAL LABORRTORIES

PROPERIY REAPPLICATION DEPT. 8532-1

LIVERMORE

CA $94550-0969$
FOR FURTHER INFORMATION:

ELOY $N$. GIRO

ALBUOUERQUE

(505) $844-2746$

m 87185

TO FREEZE EOUIPMENT:

ELOY N. GIRON

ALBUOUEROUE

(505) $844-2746$

47185

LOCATION OF EQUIPMENT:

SAMDIA MATIONAL LABORATORIES

PROPERTY REAPPLICATION DIVISION 7617

ALBUQUEROUE

in 37123 
EFFECTIVE DATE: 07/01/94 EXPIRATION DATE: 07/31/94

\section{FSC \\ COOE EXCESS PROPERTY DESCRIPTION}

REFERENCE MUMBER : 1023

7025 COMPUTER, PERSONAL PART OF THE COLOR GRAPHICS WORKSTATION HAS A 5-1/4" DISK DRIVE AN D TWO SCSI PORTS. 12OVAC, GO HZ. TEKTROWIX, 4317, SER. B040435.

REPORT NUMBER: $898355-4095$
NO OF

COND UNITS UNIT

ACOUISITION PRICE

$600001 E A$

COST/UNIT:

TOTAL COST:

$\$ 5.076 .00$
$\$ 5.076 .00$

FOR FURTHER INFORMATIOA:

ELOY M. GIRO

ALBUOUEROUE

(SOS) $844-2746$

87185

TO FREEZE EQUIPMEMT:

ELOY N. GIROW

ALBUOUEROUE

(505) $844-2746$

87185

LOCATION OF EOUIPMENT:

SAMDIA MATIOUAL LABORATORIES

PROPERTY REAPPLICATIOI DIVISIOU 7617

PROPERTY REAPPLICATION DIVISIOW

min 87123

REFERENCE MUMBER : 102

7025 COMPUTER, PERSONAL, COMPAO 286, MDL 2551, S/N 4764AM1B0398, H/MONITOR NEC MULTISYNC 11. MOL JC1402HMA, S/N TYD62379M. AND KEYBOARD. AN INTE RMAL REMOVABLE MEDIA BASE HOUSING H/CONTROLLER CARD IS INST

ALLED - MEDIA IS REMOVED, TO BE OPERATIONAL NEEO DOS SOFTHARE AND INTER

800001 EA

COSTIUNIT:

TOTAL COST:

$\$ 4.000 .00$

$\$ 4,000.00$

FOR FURTHER INFORMATION :

JEMMIFER IMHOF $F$

LEST MIFFLIM

(412) $476-5328$

PA 15122-0109

TO FREEZE EOUIPMENT:

JEMNI IER IMHOFF

LEST MIFFLIN

(412) $476-5328$

PA $15122-0079$

REPORT NUMBER: $892401-4080$

LINE ITEM MUMBER: 0001

LOCATIOW OF EQUIPMENT:

BETTIS ATOAIC POAER LABORATORY

814 PITTSQUPGH/MCKEESPORT BLID

IEST MIFFLIN

PA 15122-0079 
EFFECTIVE DATE: 07/01/94 EXPIRATION DATE: 07/31/94

\section{COOE EXCESS PROPERTY OESCRIPTION}

REFERENCE MUMBER : 1025

T025 COMPUTER, PERSOMAL, COMPAO 286, MOL 2551, S/N 4744AM181139, W/MONITOR,

WUIISYMC II, MOL JC1602HMA, S/M 7XD03438A, AMO KEYBOARD. AN INTE RMAL

REMOVABLE MEDIA BASE HOUSIMG W/CONTROLLER CARD IS INST

ALIED - MEDIA IS REMOVED. TO BE OPERATIONAL MEED DOS SOFTMARE AMD INTER

MAL HARD DISK MEDIA H/CONTROLLER CARD.

REPORT MUMBER: $892401-4080$

LINE ITEM MUMBER: 0002

\section{REFERENCE MUMBER : 1026}

7025 COMPLIER, PERSOMAL, IBM-XT, MOL 5160, S/M 55837555160, W/KEYBOARD,

IMTERMAL REMOVABLE MEDIA BASE CONTROLLER CARD \& HOUSING INSTALLED

MEDIA IS REMOVED, TO BE OPERATIONAL MEEDS DOS SOFTMARE AND INTER

MAL HA RD DISK MEDIA W/CONTROLLER CARD

$\operatorname{COST/UNIT:}$

TOTAL COST:

$\$ 24.200 .00$

FOR FURTHER IMFORMATION:

JEMMI IFER IMHOFF

KEST MIFFLIN

(412) $476-5328$

PA 15122-0109

TO FREEZE EQUIPMENT:

JEMMIFER IMHOFF

EST MIFFLIM

(412) 476-5328

PA 15122-0079

REPORT MUMBER : $892401-4030$

LINE ITEM MIMBER: 0040
OCATION OF EOUIPAEMT:

BEITIS ATOMIC PONER LABORATORY

814 PIITSBURGH/MCKEESPORT BLVD

UEST MIFFLIN

PA 15122-0079 
EFFECIIVE DATE: 07/01/94 EXPIRATION DATE: 07/31/94

FSC

\section{COOE EXCESS PROPERTY DESCRIPTION}

7025 COMPUTER, PERSONAL, IBM-XT, MDL 5160, S/N 56032175160, H/KEYBOARD, COMPUTER, PERSONAL, IBM-XT, MOL S160, S/N S6032175160, W/KEYBOARD, INTERNAL REMOVABLE MEDIA BASE CONIROLLER CARD \& HOUSING INSTALLED MEDIA IS REMOVED, TO GE OPERATIONAL ME

REPORT NUMBER: $892401-4080$

LINE ITEM NUMBER: 004
REFEREMCE MUMBER : 1027

MO of

COND UNITS UNIT

ACOUISIIIION PRICE

$800001 E A$

COST/UNIT:
TOTAL COST:

\section{$\$ 5,050.00$}

$\$ 5,050.00$

JEMMI FER IMHOF

(412) $476-5328$

PA 15122-0109

TO FREEZE EQUIPMENT:

JEMNI FER IMHOF

WEST MIFFLIN

(412) $476-5328$

PA 15122-0079

LOCATION OF EOUIPMENT:

BETTIS ATOMIC PONER LABORATORY

814 PITTSBURGH/MCKEESPORT BLVD

WEST MIFFLIN

REFERENCE NUMBER : 1028

7025 COMPUTER, PERSOHAL, IBM-XT, MDL 5160, S/N 57122385160, W/KEYBOARD,

INTERNAL REMOVABLE MEDIA BASE CONTROLLER CARD \& HOUSING INSTALLED -

$800001 E A$

COST/UNIT:

MEDIA IS REMOVED, TO BE OPERATIONAL NEEDS DOS SOFTUARE AND INTER

COST UNIT:
TOTAL COST:

NAL HA RD DISK MEDIA W/CONTROLLER CARD

REPORT NUMBER: $892401-4080$

LINE ITEM NUMBER: 0042

FOR FURTHER INFORMATION:
$\$ 4.200 .00$

$\$ 4,200.00$

JEMNI FER IMHOF

(412) $476-5328$

PA 15122-0109

TO FREEZE EOUIPMENT:

JEMNIFER IMHOFF

WEST MIFFLIN

(412) $476-5328$

PA 15122-0079

LOCATION OF EOUIPMENT:

BETTIS ATOMIC PONER LABORATORY

814 PITTSBURGH/MCKEESPORT BLVD

WEST MIFFLIN

PA 15122-0079 


\section{EFFECTIVE DATE: 07/01/94 EXPIRAIION DATE: $07 / 31 / 94$}

FSC

\section{COOE EXCESS PROPERIY DESCRIPTION}

REFERENCF. NUMBER : 1029

7025 COMPUTER, PERSONAL, IBM XT, MDL 5160, S/N 577123285160, W/MONITOR, NEC MULT ISYNC IIA, MDL JC-1403HMA, S/N 92KOT949T, AND KEYBOARD, AN INTERN AL REMOVABLE MEDIA BASE HOUSING W/CONTROLLER CARD IS INSTAL

LED - MEDIA IS REMOVED, TO BE OPERATIONAL MEEDS DOS SOFTHARE AND INTERN

AL HARD DISK MEDIA $W /$ CONTROLLER CARD

REPORT NUMBER: $892401-4080$

LINE ITEM NUMBER: 0049

\section{REFERENCE NUMBER : 1030}

7025 COMPUTER, PERSONAL, COMPAO 286, MDL 2551, S/N 4852AM2B0695, W/MONITOR IBM, MDL 8513001, AND KEYBOARD, AN INTERNAL REMOVABLE MEDIA BASE HOUS

ING W/CONTROLLER CARD IS INSTALLED - MEIDA IS REMOVED, TO B

E OPER ATIONAL MEED DOS SOFTWARE AND INTERMAL HARD DISK MEIDA W/CONTEROL LER CARD
No of

COND UNITS UNIT

$800001 \mathrm{EA}$

COST/UNIT:
TOTAL COST:

CQUISITION PRICE

$\$ 4.000 .00$

$\$ 4,000.00$

PA 15122-0109

FOR FURTHER INFORMATION:

412) $476-5328$

TO FREEZE EQUIPMENT

JEMNI FER IMHOF F

WEST MIFFLIN

PA 15122-0079

(412) $476-$

LOCATION OF EQUIPMENT:

BETTIS ATOMIC POWER LABORATORY
814 PITTSBURGH/MCKEESPORT BLVD

WEST MIFFLIN

PA $15122-0079$

$800001 \mathrm{EA}$

COST/UNIT:

TOTAL COST:

$\$ 4,000.00$
$\$ 4,000.00$

FOR FURTHER INFORMATION:

JENNIFER IMHOFF

WEST MIFFLIN

(412) $476-5328$

PA 15122-0109

TO FREEZE EOUIPMENT:

JEMNIFER IMHOFF

UEST MIFFLIN

PA 15122-0079

(412) $476-5$

LOCATION OF EOUIPMENT:

BETTIS ATOMIC PONER LABORATORY

BETT AT ATOMIC PONER LABORATORY

814 PITTSBURGH/MCKEESF

WEST MIFFLIN

PA 15122-0079 
EFFECTIVE DATE: 07/01/94 EXPIRATION DATE: $07 / 31 / 94$

FSC

\section{COOE EXCESS PROPERTY DESCRIPIION}

REFERENCE NUMBER : 1031

7025 COMPUTER, PORTABLE MFR: APPLE, MODEL M5120, S/N F3034N8HM59, ACQ. DATE: 10/90, APP ROX. WT: 20 LBS., 15" $W \times 141 \mathrm{~L} \times 4 " \mathrm{H}$

LINE ITEM NUMBER: 0003
REPORT NUMBER: $899105-4083$
NO OF

COND UNITS UNIT

$600001 \mathrm{EA}$
ACQUISIIIION PRICE

COST/UNIT: TOTAL COST:

\section{$\$ 3,699.00$ $\$ 3,699.00$}

REFERENCE NUMBER : 1032

7025 COMPUTER, PORTABLE MFR: HP, MODEL D1001A, S/N 2741A16281, ACO. DATE: 2/87. APPROX. WT: 30 LBS., $16^{\prime \prime W} \times 14^{\prime \prime L} \times 4^{\prime \prime H}$, INSIDE CASE.
$400001 \mathrm{EA}$ COST/UNIT:
TOTAL COST:
$\$ 2,750.00$ $\$ 2,750.00$

FOR FURTHER INFORMATION:

JANET F. BROOKS

LIVERMORE

(510) 294-2186

CA $94550-0969$

TO FREEZE EQUIPMENT:

JANET F. BROOKS

LIVERMORE

(510) $294-2186$

LOCATION OF EQUIPMENT:

SANDIA NATIONAL LABORATORIES

PROPERTY REAPPLICATION DEPT. $8532-1$

LIVERMORE

CA $94550-0969$ 
EFFECTIVE DATE: $07 / 01 / 94$ EXPIRATION DATE: $07 / 31 / 94$

FSC

\section{CODE EXCESS PROPERTY DESCRIPIION}

REFERENCE NUMBER : 1033

7025 COMPUTER-WORKSTATION, MFR. SUN MICROSYS, MOOEL 350M4, ACO. YR. 86, PROP. 600001 EA NO. 4614409

REPORT NUMBER: $899102-4168$

LINE ITEM NUMBER: 0001

REFERENCE NUMBER : 1034

7025 COMPUTER-HORKSTAIION, MFR. NEXI INC., MOOEL N1000, ACO. YA. 19 89, PROP. 900001 EA NO. 5030239
No of

COND UNITS UNIT

ACQUISITION PRICE

COST/UNIT: TOTAL COST:

COST/UNIT: TOTAL COST:
LINE ITEM NUMBER: 0006

$\$ 5,530.00$

$\$ 5,530.00$

JOANME BREZNIK
LIVERMORE

(510) $424-4101$

CA 94550

TO FREEZE EQUIPMENT

JOANNE BREZNIK

JOANNE BREZ

(510) $424-4101$

CA 94550

LOCATION OF EQUIPMENT:

LAURENCE LIVERMORE NATIONAL LAB

EXCESS PROPERTY SECTION, L-696

LIVERMORE
FOR FURTHER INFORMATION:

JOANNE BREZNIK

LIVERMORE

(510) $424-4101$

CA 94550

TO FREEZE EOUIPMENT:

JOANNE BREZNIK

JOANNE BRE

CA 94550

LOCATION OF EQUIPMENT:

LAWRENCE LIVERMORE NATIONAL LAB

EXCESS PROPERTY SECTION, L-696

LIVERMORE 
DOE USED ENERGY RELATED LABORATORY EQUIPMENT GRANT PROGRAM

EFFECTIVE DATE: $07 / 01 / 94$ EXPIRATION DATE: $07 / 31 / 94$

FSC

REFERENCE NUMBER : 1035

7025 CPU COMPUCOM DESKPRO, SN-4916H23H0757

REPORT NUMBER: $894609-9090$

REFERENCE NUMBER : 1036

7025 CPU IBM XT, SN -519044375160

PEPORT NUMBER: $894609-9090$

ELIGIBLE EQUIPMENT

No of COND UNITS UNIT ACOUISITION PRICE

$400001 \mathrm{EA}$

COST/UNIT: TOTAL COST:

LINE ITEM NUMBER: 29

$600001 \mathrm{EA}$

COST/UNIT:
TOTAL COST :

LINE ITEM NUMBER: 28
$\$ 2,206.00$ $\$ 2,206.00$

CONTACT(S)

FOR FURTHER INFORMATION:

DAVID MITCHELL

AIKEN

SC 29802

(803) $725-9540$

TO FREEZE EQUIPMENT:

DAVID MITCHELL

AIKEN

(803) $725-9540$

SC 29802

LOCATION OF EQUIPMENT:

WESTINGHOUSE

SAVANMAH RIVER SITE

AIKEN

SC 29802
$\$ 6,351.00$

$\$ 6,351.00$
FOR FURTHER INFORMATION: DAVID MITCHELL

AIKEN

SC 29802

TO FREEZE EQUIPMENT:

DAVID MITCHELL

AIKEN

(803) $725-9540$

LOCATION OF EQUIPMENT:

WESTINGHOUSE

SAVANMAH RIVER SIIE

AIKEN

SC 29802 
EFFECTIVE DATE: 07/01/94 EXPIRATION DATE: 07/31/94

FSC

\section{COOE EXCESS PROPERTY DESCRIPTION}

REFERENCE NUMBER : 1037

7025 CPU IBM XT, SN-63582375160

REPORT MUMBER: $894609-9090$

LINE ITEM NUMBER: 57

No of

COND UNITS UNIT

ACOUISITION PRICE

$600001 \mathrm{EA}$ COST/UNIT:
TOTAL COST:

$\$ 2.479 .00$ $\$ 2,479.00$
CPU MFG. IBM MODEL \# 5150 SERIAL

YEAR ACQ 1985 DOE \# 00029262-00

REPORT MUMBER: $898289-4049$

\section{EA COST/UNIT: \\ TOTAL COST:}
$\$ 1,843.00$
$\$ 1,843.00$

FOR FURTHER INFORMATION

G.A. ELVEY

GOLDEN

ORTHER IMFORMATION:

DAVID MITCHELL

TO FREEZE EOUIPMENT:

DAVID MITCHELL

(803) $725-9540$

LOCATION OF EQUIPMENT:

WESTINGHOUSE

SAVANNAH RIVER SITE

AIKEN

SC 29802

(303) $465-3765$

CO $80402-0464$

TO FREEZE EQUIPMENT :

G.A. ELVEY

GOLDEN

(303) 465-3765

CO $80402-0464$

LOCATION OF EOUIPMENT:

EG\&G ROCKY FLATS, INC.

BUILDING 020-BROOMFIELD FACILITY

BROOMFIELD

Co 80020 
EFFECTIVE DATE: $07 / 01 / 94$ EXPIRATION DATE: 07/31/94
CODE EXCESS PROPERTY DESCRIPTION

REFERENCE NUMBER : 1039

7025 CPU PREMIUM AST 286, SN-US109728

REPORT NUMBER: $894609-9091$

REFERENCE NUMBER : 1040

7025 CPU PREMIUM AST 286, SN-US109721

REPORT NUMBER: $894609-9091$
NO OF

COND UNITS UNIT

ACQUISITION PRICE

$600001 \mathrm{EA}$

COST/UNIT:
TOTAL COST:

LINE ITEM NUMBER: 6

$600001 E A \quad$ COST/UNIT:
TOTAL COST:

LINE ITEM NUMBER: 7
CONTACT(S)

FOR FURTHER INFORMATION:

DAVID MITCHELL

AIKEN

(803) $725-9540$

TO FREEZE EOUIPMENT :

DAVID MITCHELL

AIKEN

SC 29802

(803) $725-9540$

LOCATION OF EQUIPMENT:

MESTINGHOUSE

SAVAMNAH RIVER SITE

AIKEN

SC 29802
$\$ 2,787.00$
FOR FURTHER INFORMATION:

OAVID MITCHELL

AIKEN

SC 29802

(803) $725-9540$

TO FREEZE EQUIPMENT:

DAVID MITCHELL

AIKEN

SC 29802

(803) $725-9540$

LOCATION OF EQUIPMENT:

WESTINGHOUSE

SAVANMAH RIVER SITE

AIKEN 
EFFECTIVE DATE: 07/01/94 EXPIRAIION DATE: 07/31/94

FSC

COOE EXCESS PROPERTY DESCRIPTION

REFERENCE NUMBER : 104

7025 CPU PREMIUM AST 286, SN-US135184

REPORT NUMBER: $894609-9091$

REFERENCE NUMBER : 1042

7025 CPU PREMIUM AST 286, SN-US 109693

REPORT NUMBER: $894609-9091$ no of

COND UNITS UNIT

ACQUISITION PRICE

$600001 \mathrm{EA}$

COST/UNIT:
TOTAL COST:

LINE ITEM NUMBER: 8

$600001 \mathrm{EA}$

COST/UNIT:

TOTAL COST:

LINE ITEM NUMBER: 9

\section{$\$ 2.695 .00$}

$\$ 2,695.00$

$\$ 2,787.00$

FOR FURTHER INFORMATION

DAVID MITCHELL

AIKEN $255-9540$

SC 29802

TO FREEZE EQUIPMENT

DAVID MITCHELL

AIKEN $725-9540$

SC 29802

LOCATION OF EQUIPMENT:

WESTINGHOUSE

SAVANNAH RIVER SITE

AIKEN 
EFFECTIVE DATE: $07 / 01 / 94$ EXPIRATION DATE: $07 / 31 / 94$

FSC

COOE EXCESS PROPERTY DESCRIPTION

REFERENCE NUMBER : 1043

7025 DERHOULLIE IOMEGA DISK DRIVE, SN-OTSDS

REPORT NUMBER: $894609-9091$

INE ITEM NUMBER: 29

7025 DISC DRIVE BERNOULLI BOX SYSTEM, DUAL DRIVE SYSTEM EACH DISK CAN HOLD 10600001 EA

MB, DRIVE IS IBM COMPATIBLE. 120VAC, 60 HZ. A210H, SER. 0267A.

COST/UNIT:

TOTAL COST:

COND UNITS UNI

$600001 E A$

TOTAL COST:
NO OF

ACOUISITION PRICE

$\$ 1,750.00$

$\$ 1,750.00$$$
\text { DAV }
$$

(803) $725-9540$

SC 29802

TO FREEZE EQUIPMENT:

DAVID MITCHELL

AIKEN

(803) $725-9540$

SC 29802

LOCATION OF EQUIPMENT :

WESTINGHOUSE

SAVAMMAH RIVER SITE

AIKEN

SC 29802

$\$ 2,215.00$

FOR FURTHER INFORMATION :

ELOY N. GIRON

ALBUQUERQUE

(505) $844-2746$

NM 87185

TO FREEZE EOUIPMENT:

ELOY N. GIRO

(505) $844-2746$

LOCATION OF EOUIPMENT.

SANDIA NATIONAL LABORATORIES

SANDIA NATIONAL LABORATORIES

PROPERTY REAPPLICATION DIVISION 7617
ALBUOUEROUE 
EFFECTIVE DATE: $07 / 01 / 94$ EXPIRAIION DATE: $07 / 31 / 94$

FSC

\section{CODE EXCESS PROPERTY DESCRIPTION}

REFERENCE MUMBER : 1045

7025 DISC DRIVE OISK DRIVE SYSTEM USES 5-1/4" DISK, EACH DRIVE HOLDS 20

712571 .

REPORT NUMBER: $898355-4095$

LINE ITEM NUMBER: 0016

REFERENCE MUMBER : 1046

7025 DISC DRIVE, PC STORAGE UNIT HAS A REMOVEABLE STEAMER TAPE. 120VAC, 60 HZ TEKT RONIX, 4944118, B020703.

LINE ITEM NUMBC̈R: 0012
No of

COND UNITS UNIT

ACQUISITION PRICE

600001 EA

COST/UNIT:

TOTAL COST:

$\$ 3,500.00$

$\$ 3,500.00$

\section{ALBUOUEROUE}

INFORMATION

(505) $844-2746$

NM 87185

TO FREEZE EQUIPMENT:

ELOY N. GIRON

ALBUQUEROUE

(505) $844-2746$

NM 87185

LOCATION OF EQUIPMENT:

SANDIA MATIOMAL LABORATORIES

PROPERTY REAPPLICATION DIVISION 7617

ALBUQUERQUE

NM 87123

TOTAL COST:

$\$ 1,000.00$

FOR FURTHER INFORMATION:

ELOY N. GIRON

ALBUOUERQUE

(505) $844-2746$

NM 87185

TO FREEZE EQUIPMENT:

ELOY N. GIRON

(505) $844-2746$

NM 87185

LOCATION OF EQUIPMENT:

SAMDIA NATIONAL LABORATORIES

PROPERTY REAPPLICATION DIVISION 7617

PROPERTY REAPPLICATION DIVISION 7617
ALBUQUERQUE
NM 87123 
EFFECTIVE DATE: 07/01/94 EXPIRATION DATE: 07/31/94

\section{FSC
COOE EXCESS PROPERTY DESCRIPTION}

REFERENCE MUMBER : 1047

7025 DISC DRIVE, PC USES THE 3-1/2" DISK DUAL DISK DRIVE SYSTEM EACH DRIVE IS DOUBLE SIDED THE DISK DRIVE HAS A TOTAL CAPACITY OF 1420K BYTES, 120VAC GO HZ. HEW PACK, 9122C, SER. 2804A245.

REFERENCE HUMBER : 1048

7025 DISC DRIVE, PC DISK DRIVE WITH 2 SCSI PORT IN BACK FOR COMPUTER, HAS OISC DRIVE, PC DISK DRIVET ROLS. 12OVAC, 60 HZ. MICRONET T, SB1T3PC, SER. 0514130.
LINE ITEM NUMBER: 0020
600001 EA

$\operatorname{COSI} /$ UN $1 \mathrm{~T}$ TOTAL COST:
ACQUISITION PRICE

COST/UNIT: TOTAL COST:

$60001 \mathrm{EA}$ REPORT NUMBER: $898355-4096$
LINE ITEM NUMBER: 0010

\section{$\$ 1,098.00$} $\$ 1,098.00$

\section{ELOY N. GIRON \\ ALBUOUEROUE}

(505) $844-2746$

TO FREEZE EQUIPMENT:

ELOY N. GIROW

ALBUOUEROUE

(505) $844-2746$

LOCATION OF EOUIPMENT:

SAMDIA MATIONAL LABORATORIES SANDIA WATIONAL LABORATORIES ALBUDUEROUE MM 87123
$\$ 1,494.00$ $\$ 1,494.00$
FOR FURTHER INFORMATIOA:

ELOY N. GIRON

ALBUQUEROUE

(505) $844-2746$

NM 87185

TO FREEZE EQUIPMEMT :

ELOY N. GIRON

ALBUOUEROUE

(505) $844-2746$

MM 87185

LOCATION OF EOUIPMENT:

ELOY N. GIRON

PROPERIY REAPPLICATION DIV. 7617

PROPERTY REAPPLICATION DIV 7617

im 87123 
EFFECTIVE DATE: 07/01/96 EXPIRATION DATE: 07/31/94

\section{COOE EXCESS PROPERTY DESCRIPTION}

\section{REFERENCE MUMBER : 1049}

7025 DISK COPIER, DESKTOP, MFG DATA PATH, MOL AL525D, S/W 5040
Mo of

COND UNITS UNIT

ACQUISITION PRICE

600001 EA COST/UNIT:
TOTAL COST:

$\$ 1,900.00$ $\$ 1,900.00$

FOR FU:THER IMFOBCATIOO:

JEMMIFER IMHOF

NEST MIFFLIN

PA 15122-0109

TO FREEZE EQUIPHENT:

JEMUIFER IMHOFF

JEST MIFFLIN

(412) $476-5328$

PA 15122-0079

LOCATION OF EOUIPMEMT:

BETIIS ATOMIC POUER LABORATORY

\$14 PITISBURGH/MCKEESPORT BLVO

LEST MIFFLIN

PA 15122-0079

REFERENCE MUMBER : 1050

7025 DISK DRIVE

* w/a Serial \# n/a

X94-04-010 MANUF. UNKNOWN MOOEL

00001 EA

COST/UNIT:

TOTAL COST:

$\$ 1,470.00$
$\$ 1,470.00$

FOR FURTHER IMFORMATION:

HILLIS J. MILLER

CINCImart!

(513) $738-6630$

OH $45239-8704$

TO FREEZE EOUIPMENT :

WILLIS J. MHILER

OH $45239-8704$

REPORT MUMBER: $895529-4109$

LINE ITEM MUMBER: 0012

(513) $738-6630$

LOCATIOM OF EOUIPMENT:

U.S. DOE/FERACO

7400 WILLEY ROAD

FERMALD

OH 45030 
EFFECTIVE DAIE: $07 / 01 / 04$ EXPIRATION DATE: $07 / 31 / 94$

\section{FSC \\ COOE EXCESS PROPERTY DESCRIPTION}

7025 DISK DRIVE 69196, MANUF: IOMEGA MOOEL A210H S/N O3NRC

1 EACH, $2 \times 10$ MEG. SIZE: $12^{\mu W} \times 14^{\mu F} / R \times 8^{4} \mathrm{H}$ EST WT: 30 LBS MFGR YR:

1987

REPORT NUMBER: $896404-4120$

LINE ITEM MUMBER: 0015
REFERENCE MUMBER : 1051

Mo of

COND UNITS UNII ACOUISITION PRICE

$5 \quad 00001 \mathrm{EA}$

$\cos T / \lim _{1} \mathrm{~T}$ :

TOTAL COST:

$\$ 1.466 .00$
$\$ 1,486.00$

BURBARA HOLMES, REP.

KAMSAS CITY

(816) $997-2976$

TO FREEZE EOUIPIENT :

BARBARA HOLMES, REP.

KaWSAS CITY

(816) $997-2976$

LOCATION OF EOUIPMENT

ALLIEDSIGUAL. INC

2000 EAST 95TH STREET

KAMSAS CITY

110 $64131-3095$
$\$ 1.466 .00$
REFEREMCE MUMBER : 1052

7025 DISK DRIVE 69198, MANUF: IOMEGA MOOEL A210H S/N O3NPZ

1 EACH, $2 \times 10$ MEG. SIZE: $12^{" W} \times 14^{\prime \prime F} / R \times 8^{\prime \prime H}$ EST WT: 30 LBS MFGR YR:

5 O0001 EA

COST/UNIT:

1987

REPORT MUMBER: 896404-4126

LINE ITEM MUMBER: 0017
MO $64141-6159$

10 64141-6159
$\$ 1,466.00$

TOIAL COST:

BARBARA HOLMES, REP.

KaWSAS CIIY

(816) $997-2976$

TO FREEZE EOUIPMEWT:

BARBARA MOLMES, REP

Kausas CITY

(816) $997-2976$

LOCATION OF EQUIPMEMT

ALLIEDSIGMAL. INC.

2000 EAST 95TH STREET

KAMSAS CITY 


\section{EFFECTIVE DATE: 07/01/96 EXPIRATION DATE: 07/31/96}

FSC

\section{COOE EXCESS PROFERTY DESCRIPIION}

\section{REFEREWCE MUMBER : 1053}

7025 DISK DRIVE 71065 , MANUF: IOMEGA MOOEL A210H S/N O7AAS 1 EACH, $2 \times 10$ MEG. SIZE: $12^{" W} \times 14^{4 F / R} \times 8^{\prime \prime H}$ EST WT: 30 LBS MFGR YR 1988

REPORT MUMBER: $896404-4126$

LINE ITEM MUMBER: 0049

REFERENCE MLMABER : 1054

7025 DISK DRIVE 71669, MANUF: 1OMEGA MOOEL A210H S/N O7AEL 1 EACH, $2 \times 10$ MEG. S12E: $12^{\prime \prime W} \times 14^{\prime \prime F / R ~} \times 8^{\prime \prime H}$ EST WT: 30 LBS MFGR YR 1988 wo of

COND UAITS UNIT

$500001 E A$

COST/UWIT:
TOTAL COST:

\section{ACOUISITIOO PRICE}

\section{$51,620.00$}

$\$ 1,620.00$
COMTACI(S)

FOR FURTMER IMFORMAIIOW:

BARBARA MOLINES. REP.

KANSAS CITY

(816) $997-2976$

mo $64141-6159$

TO FREEZE EOUIPWENT:

BARBARA MOLMES, REP.

KAMSAS CITY

(816) $997-2976$

LOCATIOM OF EQUIPMENT

ALLIEDSIGMAL, IMC.

KAMSAS CITY

mo $64131-3095$
COST/UNIT:

TOTAL COST:
$\$ 1.620 .00$

$\$ 1,620.00$

FOR FURTHER INFORMATION:

BARBARA HOLMES, REP.

KAMSAS CITY

(816) $997-2976$

Mo $64141-6159$

TO FREEZE EQUIPMEMT:

BARBARA HOLMES, REP.

KANSAS CITY

$1064141-6159$

REPORT MUMBER: $396404-4126$

LINE ITEM NUMBER: 0052

(816) $997-2976$

LOCATION OF EQUIPMENT

ALLIEDSIGUAL, INC.

2000 EAST OSTH STREET

KANSAS CITY 
EFFECTIVE DATE: 07/01/94 EXPIRATIOW DATE: 07/31/94

\section{COOE EXCESS PROPERIY DESCRIPIION}

REFERENCE MUMBER : 1055

7025 DISX DRIVE 73951 MANUF: 1 OMEGA MOOEL A210H S/N OA4AA

1 EACH. $2 \times 10$ MEG. SIZE: $12^{W W} \times 14 " \mathrm{WF} / \mathrm{R} \times 8^{\prime \prime H}$ EST WT: 30 LBS MFGR YR: ELIGIBLE EOUIPMENT

REPORT NUMBER: $896404-4126$

LINE ITEM NUMBER: 0016

REFERENCE MUMBER : 1056

7025 DISK DRIVE 77.11, MANUF: IOMEGA MODEL A210H S/N 1010210013

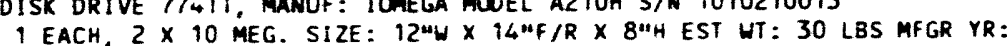

$\operatorname{cosT/UNIT:}$

TOTAL COST:
No of

COND UMITS UNIT

ACOUISITIOH PRICE

$500001 \mathrm{EA}$

COST/NMIT:

TOTAL COST:

$\$ 1.281 .00$ contacts

FOR FURTHER INFORMATION:

BARBARA HOLMES, REP.

KANSAS CITY

mo 64141-6159

TO FREEZE EOUIPMENT:

BarBarA HOLMES, REP.

$19064141-6159$

(816) $997-2976$

LOCATION OF EQUIPMENT

ALLIEDSIGMAL, IMC

2000 EAST 95TH STREET

KANSAS CITY

OR FURTHER INFORMATION

BARBARA HOLLES, REP.

KANSAS CITY

MO $64141-6159$

TO FREEZE EOUIPMEMT:

BARBARA HOLMES, REP.

KANSAS CITY

(816) $997-2976$

LOCATION OF EOUIPMENT

ALL IEDSI GNAL. INC.

2000 EAST 95TH STREET

KANSAS CITY

MO $64131-3095$ 
EFFECTIVE DATE: 07/01/94 EXPIRATION DATE: 07/31/94

\section{FS}

COOE EXCESS PROPERTY DESCRIPTION

REFERENCE MMER : 1057

7025 DISK DRIVE 78026, MAMUF: IOMEGA MOOEL A210H S/N 1010260004

1 EACH, $2 \times 10$ MEG. SIZE: $12^{M W} \times 14^{" 1 F / R} \times 8^{" 1 H}$ EST WT: 30 LBS MFGR YR: 1990

REPORT MUMBER: $896404-4126$

LINE ITEM MUMBER: 0050

REFERENCE MUMBER : 1058

TO25 DISK DRIVE 78231, MANUF: IOMEGA MODEL A210H S/N 1010180039

1 EACH, $2 \times 10$ MEG. SIZE: $12 " \mathrm{~W} \times 14$ "F/R $\times 8$ "H EST WT: 30 LBS MFGR YR:

1990
No of

COND Unirs uni

500001 EA

COST/UNIT: TOTAL COST:

COUISITION PRICE

$\$ 1.492 .00$ $\$ 1.492 .00$

(816) $997-2976$

TO FREEZE EOUIPMENT:

BARBARA HOLMES, REP.

KANSAS CITY

(816) $997-2976$

LOCATION OF EQUIPAENT:

ALLIEDSIGOAL, INC.

2000 EAST 95IH STREET

KaNSAS CITY

Mo $64131-3095$ 500001 EA COST/UNIT:
$\$ 1,492.00$ $\$ 1,492.00$
FOR FURTHER INFORMATION: BARBARA HOLMES, REP. KANSAS CITY (816) $997-2976$

Mo 64141-6159

TO FREEZE EOUIPMENT: BARBARA HOLMES, REP.

KANSAS CITY

(816) $997-2976$

$1064141-6159$

LOCATION OF EOUIPMENT: ALLIEDSIGMAL. INC. KANSAS CITY 
EFFECTIVE DATE: 07/01/94 EXPIRATION DATE: $07 / 31 / 94$

\section{FSC \\ NO OF}

COOE EXCESS PROPERTY DESCRIPTIOM

REFERENCE NUMBER : 1059

REFERENE NUMBER : 78230 MANUF: IOMEGA MOOEL A21OH S/N 1010340009

1 EACH $2 \times 10$, MANUF: IOMEGA MODEL A21OH S/N 1010340009 SIZE:

1990

REPORT NUMBER: $896404-4126$

LINE ITEM NUMBER: 0051

REFERENCE NLMBER : 1060

7025 DISK DRIVE 79282 MANUF: IOMEGA MODEL A210H S/N 1011110015

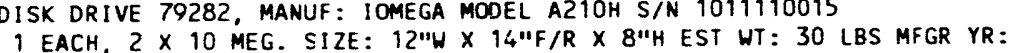
1991
COND UNITS UNIT

ACQUISITION PRICE

$500001 \mathrm{EA}$

COST/UNIT:

TOTAL COST:

$\$ 1,492.00$

$\$ 1,492.00$

作

(816) $997-2976$

TO FREEZE EQUIPMENT:

BARBARA HOLMES, REP.

KANSAS CITY

(816) $997-2976$

LOCATION OF EQUIPMENT :

ALLIEDSIGNAL, INC.

2000 EAST 95TH STREE

KANSAS CITY

Mo $64131-3095$

MO 64141-6159

$500001 \mathrm{EA}$

COST/UNIT:

TOTAL COST:

$\$ 1,388.00$
$\$ 1,388.00$
FOR FURTHER INFORMATION:

BARBARA HOLMES, REP.

KANSAS CITY

(816) $997-2976$

MO $64141-6159$

TO FREEZE EOUIPMENT:

BARBARA HOLMES, REP.

KANSAS CITY

(816) $997-2976$

LOCATION OF EQUIPMENT:

ALLIEDSIGNAL, INC.

2000 EAST 95TH STREE

KANSAS CITY

MO 64131-3095 
EFFECTIVE DATE: 07/01/94 EXPIRATION DATE: 07/31/94

FSC

COOE EXCESS PROPERTY DESCRIPTION

REFERENCE NUMBER : 106

7025 DISK ORIVE BERNOULLI BOX SYSTEM, DUAL DRIVE SYSTEM EACH DISK CAN HOLD 10600001 EA MV. DRIVE IS IBM COMPATIBLE. 120VAC, $60 \mathrm{HZ}$. BOUGHTON, P001253.

REPORT NUMBER: $898355-4096$

LINE ITEM NUMBER: 0004

\section{REFERENCE MUMBER : 1062}

7025 DISK ORIVE MFG: TALLGRASS MOOEL: TG5025 ACO DATE: 5/85 REF NO: $53550-03$
No of

COND UNITS UNIT

ACOUISITION PRICE

COST/UNIT:

TOTAL COST:

\section{$\$ 1,907.00$}

$\$ 1,907.00$

FOR FURTHER INFORMATION:

ELOY N. GIRON

ALBUOUERQUE

(505) $844-2746$

NM 87185

TO FREEZE EQUIPMENT:

ELOY N. GIRON

ALBUQUERQUE

(505) $844-2746$

NM 87185

LOCATION OF EQUIPMENT:

ELOY N. GIRON

ELOY N. GIRON

REAPPLICATION DIV. 7617

ALBUQUERQUE
$500001 \mathrm{EA}$ COST/UNIT:
TOTAL COST :

LINE ITEM NUMBER: 0126
REPORT NUMBER: $890531-4103$
$\$ 2,310.00$
FOR FURTHER INFORMATION:

NANCY J. SITTON

RICHLAND

(509) $376-5108$

WA 99352

TO FREEZE EQUIPMENT

NANCY J. SITTON

RICHLAND

(509) $376-5108$

WA 99352

LOCATION OF EQUIPMENT :

WESTINGHOUSE HANFORD COMPANY

ESS\&S, 1167A BLDG/1100 AREA

RICHLAND

WA 99352 
DOE USED ENERGY RELATED LABORATORY EOUIPMENT GRANT PROGRAM ELIGIBLE EQUIPMENT

EFFECTIVE DATE: 07/01/94 EXPIRATION DATE: $07 / 31 / 94$

FSC

COOE EXCESS PROPERIY DESCRIPIION

REFERENCE NUMBER : 1063

7025 DISK DRIVE MFG: IOMEGA MODEL: A220AH ACQ DATE: 6/86 REF NO: $53550-05$

REPORT NUMBER: $890531-4103$

LINE ITEM NUMBER: 0128

$$
\text { No of }
$$

COND UNITS UNIT

ACQUISITION PRICE

$500001 \mathrm{EA}$

COST/UNIT: TOTAL COST:

\section{$\$ 2,896.00$} $\$ 2,896.00$

FOR FURTHER INFORMATION:

NANCY 3 . SITTON

RICHLAND

WA 99352

TO FREEZE EQUIPMENT

NANCY J. SITTON

RICHLAMD

(509) $376-5108$

WA 99352

LOCATION OF EQUIPMENT:

WESTINGHOUSE HANFORD COMPANY

ESS\&S, 1167A BLDG/1100 AREA

ESS\&, IISTA BLDG/1100 AREA 99352

WA 99352

REFERENCE NUMBER : 1064

7025 DISK DRIVE MFG: IOMEGA MOOEL: A22OH ACO DATE: 6/86 REF NO: $53550-06$

REPORT NUMBER: $890531-4103$

LINE ITEM NUMBER: 0129
500001 EA COST/UNIT:

TOTAL COST:

$\$ 2,896.00$

FOR FURTHER INFORMATION:

NANCY $J$. SITTON

RICHLAND

(509) $376-5108$

WA 99352

TO FREEZE EOUIPMENT

NANCY J. SITTON

RICHLAND

(509) $376-5108$

WA 99352

LOCATION OF EQUIPMENT

WESTINGHOUSE HANFORD COMPANY

ESS\&S, 1167A BLDG/1100 AREA
RICHLAND 
EFFECTIVE DATE: $07 / 01 / 94$ EXPIRATION DATE: $07 / 31 / 94$

FSC

CODE EXCESS PROPERTY DESCRIPTION

REFERENCE NUMBER : 1065

7025 DISK SUBSYSTEM PART OF A SUM COMPUTER SYSTEM HITH A HARD DRIVE AND TAPE DRIVE, H AS 7 PI BOARDS AND CAN HOLD 5 MORE. 120VAC, $60 \mathrm{HZ}$. SUN MICROS, 1267 , SER. 643E0617.

REPORT NUMBER: $898355-4096$

LINE ITEM NUMBER: 0011

REFERENCE NUMBER : 1066

DISK/TAPE DRIVE EXTERNAL DRIVE FOR SUN COMPUTER HAS A TAPE DRIVE AND

HARD DRIVE, HAS TWO SCSI PORTS IN BACK. 12OVAC, 60 HZ. SUN, 511, SER.

$$
907 \text { OOS } 61 \text {. }
$$

$600001 \mathrm{EA}$
NO OF

COND UNITS UNIT

$600001 \mathrm{EA}$

REPORT NUMBER: $898355-4095$
LINE ITEM NUMBER: 0017

ACQUISITION PRICE COST/UNIT: TOTAL COST

$\$ 11,115.00$

ELOY N. GIRON
ALBUQUERQUE

(505) $844-2746$

NM 87185

TO FREEZE EOUIPMENT :

ELOY N. GIRON

ALBUOUEROUE

(505) $844-2746$

LOCATION OF EQUIPMENT:

ELOY N. GIRON

PROPERTY REAPPLICATION DIV. 7617

ALBUOUERQUE

NM 87123
$\$ 8,000.00$

$\$ 8,000.00$

COST/UNIT:

FOR FURTHER INFORMATION:

ELOY N. GIRON

ALBUQUERQUE

NM 87185

(505) $844-2746$

TO FREEZE EQUIPMENT

ELOY N. GIRON

ALBUQUEROUE

NM 87185

(505) $844-2746$

LOCATION OF EOUIPMENT:

SAMDIA MAIIONAL LABORATORIES

SANDIA NATIONAL LABORATORIES

ALBUQUERQUE NIS 87123 
EFFECTIVE DATE: $07 / 01 / 94$ EXPIRATION DATE: $07 / 31 / 94$

\section{FSC \\ COOE EXCESS PROPERTY DESCRIPTION}

REFERENCE NUMBER : 1067

7025 DRIVE IBM 5.25 DISK, SN-86-0373534

REPORT NUMBER: $894609-9091$
NO OF

COND UNITS UNIT

ACQUISITION PRICE

$500001 \mathrm{EA}$

COST /UNIT:

TOTAL COST:

$\$ 2,040.00$ $\$ 2,040.00$

LINE ITEM NUMBER: 28

\section{REFERENCE NUMBER : 1068}

7025 FLOPPY DISC DRIVE 20 MEGA BYTE HARD DRIVE FOR MAINTOSH COMPUTER,

120VAC, 60 HZ. AP PLE COMP, MO135. SER. F69132.

REPORT NUMBER: 898355-4095
LINE ITEM NUMBER: 0010
$600001 \mathrm{EA}$

COST/UNIT:

TOTAL COST

$\$ 1,150.00$
$\$ 1,150.00$

FOR FURTHER INFORMATION:

ELOY N. GIRON

ALBUQUEROUE

(505) $844-2746$

NM 87185

TO FREEZE EQUIPMENT:

ELOY N. GIRON

ALBUQUEROUE

(505) $844-2746$

NM 87185

LOCATION OF EQUIPMENT:

SANDIA NATIONAL LABORATORIES

PROPERTY REAPPLICATION DIVISION 7617

PROPERTY REAPP

NM 87123 
EFFECTIVE DATE: 07/01/94 EXPIRATION DATE: 07/31/94

CODE EXCESS PROPERTY DESCRIPTION

REFERENCE MUMBER : 1069

7025 IBM CPU XT SN-51439

REPORT NUMBER: $894609-9091$

REFEREMCE NUMBER : 1070

7025 IBM PC-XT CPU, SN-64183545160

REPORT NUMBER: $894609-9091$
No of

COND UNITS UNIT

ACQUISITION PRICE

$500001 \mathrm{EA}$

COST/UNIT:

TOTAL COST:

$\$ 6,441.00$

$\$ 6,441.00$

LINE ITEM NUMBER: 32

$4 \quad 00001$ EA

COST/UNIT:
TOTAL COST:

$\$ 3,624.00$

$\$ 3,624.00$

FOR FURTHER INFORMATION:

DAVID MITCHELL

AIKEN

(803) $725-9540$

SC 29802

TO FREEZE EQUIPMENT:

DAVID MITCHELL

AIKEN

SC 29802

803) $725-9540$

LOCATION OF EQUIPMENT:

WESTINGHOUSE

SAVANMAH RIVER SITE

AIKEN

SC 29802 
EFFECIIVE DATE: $07 / 01 / 94$ EXPIRATION DATE: $07 / 31 / 94$

\section{COOE EXCESS PROPERTY DESCRIPTION}

REFERENCE NUMBER : 1071

7025 IBM XI CPU HARD DRIVE SN-6095-6545160

REPORT NUMBER: $894609-9090$

REFERENCE NUMBER : 1072

7025 IBM XT CPU, SN-51602020131

REPORT NUMBER: $894609-9090$

ELIGIBLE EQUIPMENT

No of

COND UNITS UNIT

ACQUISITION PRICE

LINE ITEM NUMBER: 36

$600001 \mathrm{EA}$

COST/UNIT:
TOTAL COST:

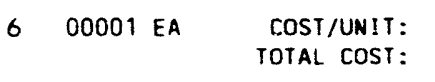

LINE ITEM NUMBER: 33
CONTACIS(S)

$\$ 1,708.00$

$\$ 1,708.00$

FOR FURTHER INFORMATION:

DAVID MITCHELL

AIKEN

SC 29802

(803) $725-9540$

TO FREEZE EQUIPMENT:

DAVID MITCHELL

AIKEN

SC 29802

(803) $725-9540$

LOCATION OF EOUIPMENT :

MESTINGHOUSE

SAVANMAH RIVER SITE

AIKEN

SC 29802

FOR FURTHER INFORMATION:

DAVID MITCHELL

AIKEN

SC 29802

(803) $725-9540$

TO FREEZE EOUIPMENT:

DAVID MI TCHELL

AIKEN

SC 29802

(803) $725-9540$

LOCATION OF EQUIPMENT:

WEST INGHOUSE

SAVANHAH RIVER SITE

AIKEN

SC 29802 
EFFECIIVE DATE: 07/01/94 EXPIRATION DATE: $37 / 31 / 94$

FSC

COOE EXCESS PROPERTY DESCRIPTION

REFERENCE NUMBER : 1073

7025 IBM XT CPU, SN-5160202013

REPORT NUMBER: $894609-9090$

REFERENCE NUMBER : 1074

7025 IBM XT CPU, SN-51604256573

REPORT NUMBER: $894609-9090$
NO OF

COND UNITS UNIT

ACQUISITION PRICE

$600001 E A$

COST/UNIT:
TOTAL COST:

$\$ 1,530.00$

$\$ 1,530.00$

LINE ITEM NUMBER: 34

$600001 E A$

COST/UNIT:
IOTAL COST:

LINE ITEM NUMBER: 35
COnIACT(S)

FOR FURTHER INFORMATIOH:

DAVID MITCHELL

AIKE

703) $725-9540$

TO FREEZE EQUIPMENT

DAVID MITCHELL

AIKEN

(803) $725-9540$

LOCATION OF EOUIPMENT

MESTINGHOUSE

SAVANMAH RIVER SITE

AIKEN

SC 29802

OR FURTHER INFORMATION

DAVID MITCHELL

(803) $725-9540$

SC 29802

TO FREEZE EQUIPMENT

DAVID MITCHELL

AIKEN

(803) $725-9540$

LOCATION OF EOUIPMENT :

UEST INGHOUSE

SAVAMMAH RIVER SITE

AIKEN

SC 29802 
EFFECTIVE DATE: 07/01/94 EXPIRATION DATE: 07/31/94

FSC

\section{COOE EXCESS PROPERTY DESCRIPTION}

REFERENCE MUMBER : 1075

7025 IBM XT CPU, SN-51602020664

REPORT NUMBER: $894609-9090$

REFERENCE NUMBER : 1076

7025 IBM XXT CPU HARD DRIVE SN-60956545160

REPORT NUMBER: $894609-9090$

ELIGIBLE EOUIPMENT

no of

COND UNITS UWIT

ACOUISITION PRICE

$600001 \mathrm{EA}$ COST/UNIT:
TOTAL COST:

LINE ITEM MUMBER: 37

600001 EA

COST/UNIT: TOTAL COST:

LINE ITEM NUMBER: 38
Contacts (S)

$\$ 1,530.00$

FOR FURTHER IMFORMATIOW:

DAVID MITCHELL

AIKEN

(803) $725-9540$

TO FREEZE EQUIPMENT :

DAVID MITCHELL

AIKEN

(803) $725-9540$

SC 29802

LOCATION OF EQUIPMENT:

WESTINGHOUSE

SAVAMMAH RIVER SITE

AIKEN

SC 29802

$\$ 1,882.00$
$\$ 1,882.00$

FOR FURTHER INFORMATION:

DAVID MITCHELL

AIKEN

(803) $725-9540$

TO FREEZE EQUIPMENT:

DAVID MITCHELL

AIKEN

SC 29802

(803) $725-9540$

LOCATION OF EQUIPMENT:

MESTINGHOUSE

SAVAMMAH RIVER SITE

AIKEN

SC 29802 
EFFECTIVE DATE: 07/01/94 EXPIRATION DATE: $07 / 31 / 94$

FSC

\section{CODE EXCESS PROPERTY DESCRIPTION}

\section{REFERENCE NUMBER : 1077}

7025 LAPTOP

\# 8391326334 BARCOOE 850365

REPORT NUMBER: $895529-4235$

LINE ITEM NUMBER: 0015

REFERENCE NUMBER : 1078

7025 LAPTOP

836122734 BARCODE 851142

X94-04-078 MANUF. ZENITH SERIAL \#9 00001 EA

FOR FURTHER INFORMATION :

WILLIS J. MILLER

CINCINMATI

(513) $738-6630$

TO FREEZE EQUIPMENT:

WILLIS J. MILLER

(513) $738-6630$

LOCATION OF EQUIPMENT

U.S. DOE/FERMCO

7400 WILLEY ROAD

FERNALD

OH 4503 
EFFECIIVE DATE: 07/01/94 EXPIRATION DATE: $07 / 31 / 94$

FSC

COOE EXCESS PROPERTY DESCRIPTION

REFERENCE NUMBER : 1079

7025 LAPTOP

* 617330908 barcooe 851028

REPORT NUMBER: $895529-4233$

X94-04-088 MANUF. ZENITH SERIAL

COND UNITS UNIT

90001 EA

COST/UNIT:
TOIAL COST:

LINE ITEM MUMBER: 0006

X94-04-169 MANUF. TOSHIBA SERIAL 900001 EA

COST UNIT:
TOTAL COST:

LAPTOP
\# O2743578 BARCOOE 854889
LINE ITEM NUMBER: 0017

\section{ACQUISITION PRICE $\quad$ CONTACT(S)}

$\$ 1,650.00$ FOR FURTHER IMFORMATION:

$\$ 1,650.00$ WILLIS J. MILLER

CIMCIMMAII

(513) $738-6630$

OH 45239-8704

TO FREEZE EQUIPMENT:

WILLIS J. MILLER

CINCIMMAII

OH $45239-8704$

(513) $738-6630$

LOCATION OF EOUIPMENT:

U.S. DOE/FERACO

U.S. DOE/FERICO

FERMALD

OH 45030

FOR FURTHER INFORMATION:

HILLIS J. MILLER

CINCIMMATI

(513) $738-6630$

OH 45239-8704

TO FREEZE EOUIPMENT:

WILLIS $J$. MILLER

cImeImenall

ON $45239-8704$

(513) $738-6630$

LOCATION OF EOUIPMEMT:

U.S. DOE/FERMCO

7400 UILLEY ROAD

FERMALD

OH 45030 
EFFECTIVE DATE: 07/01/94 EXPIRATION DATE: 07/31/94

FSC

CODE EXCESS PROPERTY DESCRIPTION

REFERENCE MUMBER : 1081

7025 LAPTOP

* 08614640 barcooe 856318

X94-04-206 MAMUF. TOSHIBA SERIAL

cono units uni

ACOUISITIOU PRICE

$\$ 3.595 .00$

COST/UNIT:
TOTAL COST:

$900001 \mathrm{EA}$
REPORT MUMBER: 895529-4232

LIME ITEM MUMBER: 0022
REFERENCE MUMBER : 1082

7025 MISC COMPUTER ITEMS, 2EA - STORAGE TRAY, 10 CARTRIDGE TAPES TEA - MP TAPE BACKUP, 40N EG

ZEA - MOUSE PEN, DARTEK

IEA - SPCOLER BUFFER, LASER JET $\|$

5 CARDS - HP 1000 CARD, X.25 NETWORK

WULTIFUWCTIOU CAPD 88

1286

EGA VIDEO

WLTI-EGA

\& 6-PAC CARD
$4 \quad 00001$ LT

Cost/unIT:
TOTAL COST:

$\$ 2.025 .00$

FOR FURTHER INFORMATIOW:

JEMEIFER IMHOFF

MEST MIFFLIM

(412) $476-5328$

PA 15122-0109

TO FREEZE EOUIPMEWT:

JEMUIFER IMAOFF

WEST MIFFLIN

(412) $476-5328$

PA 15122-0079

LOCATIO OF EOUIPUEMT

BETIIS ATOMIC POUER Laboratory

BETIIS ATONIC POAER LABCRATORY

S14 PIIISBURGH/MCKEESPORT BLVD 
EFFECTIVE DATE: $07 / 01 / 94$ EXPIRATION DATE: $07 / 31 / 94$

FSC

COOE EXCESS PROPERTY DESCRIPIION

\section{REFERENCE MUMBER : 1083}

7025 MONITOR

W/A SERIAL \# 04029816M089

BAR COOE 854880

REPORT MUMBER: $895529-4109$

LINE ITEM MUMBER: 0008

\section{REFERENCE NUMBER : 1084}

7025 MONITOR

* $7153151 \mathrm{ML}$ bARCOOE 853022

X94-04-103 MANUF. COMPAO SERIAL

$00001 \mathrm{EA}$

TOTAL COST:

LINE ITEM MUMBER: 0017

Mo of

Cono units unt

$\cos 1 /$ Un $1 T$

TOTAL COST:

REPORT MUMBER: $895529-4233$
ACOUISITION PRICE

\section{$\$ 5.687 .00$ \\ $\$ 5,687.00$}

HILLIS J. MILLER
CIMCIMATI
(513) $738-6630$

TO FREEZE EQUIPMENT:

WILLIS $J$. MILLER

CIMcImair

(513) $738-6630$

OAf $45239-870$

IOCATIOU OF EOUIPNEMT:

U.S. DOE/FERACO

7400 UILLEY ROAD

FERMAID

OH 45030

FOR FURTHER IMFORMATION:

WILLIS J. MILLER

CINCIMNATI

(513) $738-6630$

OH $45239-870$

TO FREEZE EQUIPMEMT:

WILLIS $J$. MILLER

CIMCImuatI

On 45239-8704
(513) $738-6630$

LOCATION OF EQUIPHEWT:

U.S. DOE/FERAMCO

7400 WILLEY ROAO

FERUALD

OH 45030 
EFFECTIVE DATE: 07/01/94 EXPIRATION DATE: 07/31/94

FSC

COOE EXCESS PROPERTY DESCRIPIION

REFERENCE MUMBER : 1085

7025 MONITOR E-MACHINE, SN-08128

REPORT MUMBER: $894609-9091$

REFERENCE MUMBER : 1086

7025 MONITOR E-MACHINE, SN-97350

REPGRT MUMBER: $894609-9091$

ELIGIBLE EOUIPMENT

no of

COMD UNITS UNIT

ACQUISITIOA PRICE

$7 \quad 00001 E$

COST/UNIT:
IOTAL COST:

LIME ITEM MUMBER: 20

700001 EA

COST/UNIT:

TOTAL COSI:

LIME ITEM MUMBER: 21
$\$ 1.102 .00$
$\$ 1.102 .00$

DAVIO

(803) $725-9540$

TO FREEZE EOUIPMENT

DAVID MITCHELL

AIKEN

(803) $725-9540$

SC 29802

OCATION OF EOUIPHEMT:

LESTINGHOUSE

SAVAMEAH RIVER SITE

SC 29802

$1,102.00$

s. 102.00

FOR FURTHER INFORMATION:

DAVID MITCHELL

(803) $725-9560$

SC 29802

TO FREEZE EOUIPMENT:

DAVID MITCHELL

AIKEM

(803) $725-9540$

LOCAIION OF EQUIPMENT

LESTIMGHOUSE

SAVAMUAH RIVER SITE

SAVAMEAH

SC 29802 
EFFECTIVE DATE: 07/01/94 EXPIRATION DATE: 07/31/94

\section{COOE EXCESS PROPERTY DESCRIPIION}

REFERENCE NUMBER : 1087

7025 MONITOR E-MACHINE, SN-10895

REPORT NUMBER: $894609-9091$

REFERENCE NUMBER : 1088

7025 MONI TOR IBM, SN-1021360

REFORT NUMBER: $894609-9091$

ELIGIBLE EQUIPMENT

No of

COND UNITS UNIT

$700001 \mathrm{EA}$

COST/UNIT:
TOTAL COST:

LINE ITEM NUMBER: 22

$400001 E A$

COST/UNIT:
TOTAL COST:

LINE ITEM NUMBER: 19
ACQUISITION PRICE

$\$ 1,120.00$

$\$ 1,120.00$

$\$ 1,014.00$

$\$ 1,014.00$

DAVIDEN

AIKEN MITCHELL

803) $725-9540$

TO FREEZE EQUIPMENT:

DAVID MITCHELL

AIKEN

(803) $725-9540$

LOCATION OF EQUIPMENT:

WEST INGHOUSE

SAVANNAH RIVER SITE

AIKEN

SC 29802
SC 29802

8902 
EFFECTIVE DATE: 07/01/94 EXPIRATION DATE: 07/31/94

FSC

\section{COOE EXCESS PROPERTY DESCRIPTION}

REFERENCE MUMBER : 1089

TO25 NOWITOR MAC PLUS, SN-F606541M0001A

REPORT MUMBER: $894609-9090$

LINE ITEM NUMBER: 56

$$
\text { NO OF }
$$

COND UNITS UNIT

ACQUISITION PRICE

$600001 \mathrm{EA}$

COST/UNIT:
TOTAL COST:

$\$ 1.611 .00$

$\$ 1,611.00$

OAVID MITCHELL INFRMATION:

DAVID MITCHELL

AIKEN

SC 29802

TO FREEZE EQUIPMENT :

DAVID MITCHELL

AIKEN

SC 29802

(803) $725-9540$

LOCATION OF EQUIPMENT:

WESTINGHOUSE

SAVAMNAH RIVER SITE

AIKEN

SC 29802

REFERENCE MUMBER : 1090

7025 MONITOR MAC PLUS, SN-F6312X3MOCŨ̃ IÂ

REPORT NUMBER: $894609-9090$
LINE ITEM NUMBER: 58

\section{EA COST/UNIT: \\ TOTAL COST:}

$\$ 1.611 .00$

$\$ 1,611.00$

DAVID MITCHELL

AIKEN

SC 29802

TO FREEZE EQUIPMENT:

DAVID MITCHELL

AIKEN

(803) $725-9540$

SC 29802

LOCATION OF EQUIPMENT:

WESTINGHOUSE

SAVANMAH RIVER SITE

AIKEN

SC 29802 
EFFECTIVE DATE: $07 / 01 / 94$ EXPIRATION DATE: $07 / 31 / 94$

FSC

\section{CODE EXCESS PROPERIY DESCRIPTION}

REFERENCE NUMBER : 1091

7025 MONITDR MAC PLUS, SN-F6335R2M0001A

REPORT NUMBER: $894609-9090$

REFERENCE NUMBER : 1092

7025 MONITOR MAC PLUS, SN-F606553M0001A

REPORT NUMBER: $894609-9090$
ELIGIBLE EQUIPMENT

$$
\text { NO OF }
$$

COND UNITS UNIT

600001 EA

COST/UNIT: TOTAL COST:

LINE ITEM NUMBER: 59

600001 EA

COST/UNIT:
TOTAL COST:

LINE ITEM NUMBER: 60
ACOUISITION PRICE

$\$ 1,611.00$

$\$ 1,611.00$

DAVID MITCHELL

AIKEN $725-9540$

SC 29802

TO FREEZE EOUIPMENT :

DAVID MITCHELL

AIKEN

(803) $725-9540$

LOCATION OF EQUIPMENI:

WESTINGHOUSE

SAVANNAK RIVER SITE

AIKEN

SC 29802
$\$ 1,611.00$ $\$ 1,611.00$
FOR FURTHER INFORMATION:

DAVID MITCHELL

AIKEN

SC 29802

TO FREEZE EOUIPMENT:

DAVID MITCHELL

AIKEN

(803) $725-9540$

SC 29802

LOCATION OF EOUIPMENT:

WESTINGHOUSE

SAVANNAH RIVER SITE

AIKEN

SC 29802 
EFFECIIVE DATE: $07 / 01 / 94$ EXPIRATION DATE: $07 / 31 / 94$

COOE EXCESS PROPERTY DESCRIPIION

REFERENCE NUMBER : 1093

7025 MONITOR MFG: HEWLETT PACKARD MODEL: 132798 TYPE: COLOR MONITOR MFG: HEWLETT PACKARD MODEL:
ACO DATE: $1 / 84$ REF NO: $53550-14$

REPORT NUMBER: $890531-4103$

LINE ITEM NUMBER: 0120

REFERENCE NUMBER : 1094

7025 MONITOR MFR: E-MACHINES, MODEL 1008, "BIG PICTURE", S/N BP-M1914, ACQ. 400001 EA D ATE: 6/88, APPROX. WT: 25 LABS., $16^{\prime \prime W} \times 14^{\prime \prime L} \times 15^{\prime \prime H}$.

REPORT NUMBER: $899105-4083$

LINE ITEM NUMBER: 0026

$500001 \mathrm{EA}$
NO OF

COND UNITS UNIT

ACQUISITION PRICE

COST/UNIT: TOTAL COST:

$\$ 4,851.00$ $\$ 4,851.00$

MANCY J. SITTON

RICHLAND

(509) 376.5108

WA 99352

TO FREEZE EOUIPMENT:

NANCY J. SITION

RICHLAND

(509) $376-5108$

LOCATION OF EQUIPMENT:

WESTINGHOUSE HANFORD COMPANY ESS\&S, 1167A BLDG/1100 AREA

RICHLAND
COST/UNIT: TOTAL COSI:
$\$ 1.815 .00$ $\$ 1.815 .00$
FOR FURTHER INFORMATION:

JANET F. BROOKS

IVERMORE

(510) $294-2186$

TO FREEZE EQUIPMENT:

JANET $F$. BROOKS

LIVERMORE

(510) $294-2186$

CA $94550-0969$

LOCATION OF EQUIPMENT:

SANDIA NATIONAL LABORATORIES

PROPERTY REAPPLICATION DEPT - 8532-1

LIVERMORE

CA $94550-0969$ 
EFFECTIVE DATE: $07 / 01 / 94$ EXPIRATION DATE: $07 / 31 / 94$

FSC

\section{COOE EXCESS PROPERTY DESCRIPTION}

REFERENCE NUMBER : 1095

7025 MONITOR MFR: SILICON GRAPHICS, MODEL TF56705KW-S6, S/N 204002326, ACQ. D ATE: 11/92, APPROX. WT: 30 LBS., 16"W $\times 18^{\prime \prime} \mathrm{L} \times 16^{\prime \prime H}$.
NO OF

COND UNITS UNII

$00001 \mathrm{EA}$

(6)

TOTAL COST:

LINE ITEM NUMBER: 003
REPORT NUMBER: $899105-4083$
REFERENCE NUMBER : 1096

7025 MONITOR MFR: SUN SYSTEMS, MODEL C, S/N 832AR0176, ACQ. DATE: 7/1/92, 5 0000i EA AP PROX. WT: 30 LBS., 16"W X 18"L X $16 " \mathrm{H}$.

REPORT NUMBER: $899105-4083$
LINE ITEM NUMBER: 0033
COST/UNIT: TOTAL COST:
$\$ 5,000.00$

$\$ 5,000.00$
$\$ 2,150.00$
$\$ 2,150.00$
FOR FURTHER INFORMATION:

JANET F. BROOKS

LIVERMORE

(510) $294-2186$

TO FREEZE EQUIPMENT:

JANET F. BROOKS

LIVERMORE

(510) $294-2186$

CA $94550-0969$

LOCATION OF EQUIPMENT:

SANDIA NATIONAL LABORATORIES

PROPERTY REAPPLICATION DEPT. 8532-1

LIVERMORE

CA $94550-096$ 
EFFECTIVE DATE: $07 / 01 / 94$ EXPIRATION DATE: $07 / 31 / 94$

$\operatorname{tsc}$

\section{COOE EXCESS PROPERTY DESCRIPIION}

REFERENCE NUMBER : 1097

7025 MONITOR MFR: SUN MICROSYSTEMS, MOOEL M19P114, S/N 1008CP6780, ACQ.

DATE: $7 / 90$, APFROX. WT: 50 LBS., 18"W $\times 16^{\prime \prime L} \times 18^{\prime \prime H}$

REPORT NUMBER: $899105-4083$

LINE ITEM NUMBER: 0034

REFERENCE NUMBER : 1098
7025 MONITOR MFR: SUN MICROSYSTEMS, MODEL C, S/N B22F0918, ACO. DATE: $3 / 90,700001$ EA APPROX. WT: 50 LBS. 18"W $\times 20^{\prime \prime L} \times 18^{\prime \prime} \mathrm{H}$.

REPORT NUMBER: $899105-4083$

LINE ITEM NUMBER: 0035

No of

$00001 \mathrm{EA}$ COND UNITS UNIT

ACQUISITION PRICE

$\$ 1,020.00$

COST/UNIT: TOTAL COST:

$\$ 1,020.00$

JANET F. BROOKS

(510) $294-2186$

CA $94550-0969$

TO FREEZE EQUIPMENT:

JANET $F$. BROOKS

LIVERMORE

(510) 294-2186

LOCATION OF EQUIPMENT

SANDIA NATIONAL LABORATORIES

PROPERTY REAPPLICATION DEPT. $8532-1$

LIVERMORE

CA $94550-0969$

$\begin{aligned} \text { COST/UNIT: } & \$ 1,800.00 \\ \text { TOTAL COST: } & \$ 1,800.00\end{aligned}$

FOR FURTHER INFORMATION:

JANET F. BROOKS

LIVERMORE

CA $94550-0969$

TO FREEZE EQUIPMENT:

JANET $F$. BROOKS

LIVERMORE

(510) $294-2186$

CA $94550-0969$

LOCATION OF EQUIPMENT:

SANDIA NATIONAL LABORATORIES

PROPERTY REAPPLICATION DEPT. 8532-1

LIVERMORE

CA $94550-0969$ 
EFFECTIVE DATE: 07/01/94 EXPIRATION DATE: $07 / 31 / 96$

FSC

\section{COOE EXCESS PROPERTY DESCRIPTION}

REFERENCE NUMBER : 1099

7025 MONITOR, SOMY TRINITRON, MDL GDM-1901-12, S/N 200023026, HIGH RESOLUTION, COLOR, GRAPHIC DISPLAY, REC 12/86, 19" SCREEN, 125 LB

REPORT NUMBER: $892401-4080$

LINE ITEM NUMBER: 006
NO OF

COND UNITS UNIT

$4 \quad 00001 E A$

COST/UNIT: TOTAL COST:

$\$ 3,510.00$ $\$ 3,510.00$

FOR FURTHER INFORMATION:

JENNIFER IMHOFF

WEST MIFFLIN

(412) $476-5328$

PA 15122-0109

TO FREEZE EQUIPMENT :

JENNIFER IMHOFF

WEST MIFFLIN

(412) $476-5328$

PA 15122-0079

LOCATION OF EQUIPMENT

BETTIS ATOMIC PONER LABORATORY

814 PITTSBURGH/MCKEESPORT BLVD

WEST MIFFLIN

PA 15122-0079 COST/UNIT:
TOTAL COST:
FOR FURTHER INFORMATION: JENNI FER IMHOFF WEST MIFFLIN (412) $476-5328$

TO FREEZE EQUIPMENT

JENNIFER IMHOFF

WEST MIFFLIN

(412) $476-5328$

PA 15122-0079

LOCATION OF EQUIPMENT :

BETTIS ATOMIC POUER LABORATORY

814 PITTSBURGH/MCKEESPORT BLVD

WEST MIFFLIN

PA 15122-0079 
EFFECTIVE DATE: 07/01/94 EXPIRATION DATE: 07/31/94

FSC

\section{COOE EXCESS PROPERTY DESCRIPTIO}

REFERENCE MUMBER : 1101

7025 PC MFR: COMPAO MCOEL 2551 S/N 4904AM1B2708, ACO. DATE: 3/89, APP ROX. WT: 30 LBS., $20^{\prime \prime} \mathrm{W} \times 17^{\prime \prime} \mathrm{L} \times 6^{\prime \prime} \mathrm{H}$.

REPORT MUMBER: $899105-4083$

LIHE ITEM NUMBER: 0038

REFERENCE MUMBER : 1102

7025 PC MFR: COMPAO, MOOEL 2570, S/N 4713AJ2B0027, ACQ. DATE: 8/87, APP ROX. WT: 40 LBS., $20 " \mathrm{~W} \times 17^{\prime \prime L} \times 6^{\prime \prime H}$.

REPORT MUMBER: $899105-4083$

LINE ITEM NUMBER: 0039
No of

COND UNITS UNIT

ACQUISITION PRICE

400001 EA

COST/UNIT: TOTAL COST:

\section{$\$ 3,000.00$} $\$ 3,000.00$

\section{JAMET F. BROOKS \\ LIVERMORE}

FOR FURTHER INFORMATION

CA $94550-0969$

(510) $294-2186$

TO FREEZE EOUIPMENT:

JANET $F$. BROOKS

LIVERMORE

(510) $294-2186$

CA $94550-0969$

LOCATION OF EQUIPMENT:

SANDIA MATIONAL LABORATORIES

PROPERTY REAPPLICATION DEPT. 8532-1

LIVERMCRE

CA $94550-0969$
600001 EA COST/UNIT:

TOTAL COST:
$\$ 2,274.00$ $\$ 2,274.00$
FOR FURTHER INFORMATIOM:

JANET F. BROOKS

CA 94550.0969

(510) $294-2186$

TO FREEZE EQUIPMENT:

JANET F. BROOKS

IVERMORE

(510) $294-2186$

CA $94550-0969$

LOCATION OF EOUIPMENT :

SANDIA NATIONAL LABORATORIES

PROPERTY REAPPLICATION DEPT. 8532-1

LIVERMORE

CA $94550-0969$ 
EFFECTIVE DATE: 07/01/94 EXPIRATION DATE: 07/31/94

\section{COOE EXCESS PROPERTY DESCRIPIION}

REFERENCE NUMBER : 1103

7025 PC MFR: HP MODEL 45925A, S/N 2628A06622, ACO. DATE: 6/86, APPROX. WT: 500001 EA 30 LBS. 17"W $\times 15^{\prime \prime L ~} \times 7 " H$.

REPORT NUMBER: $899105-4083$

LINE ITEM NUMBER: 0061

\section{REFERENCE NUMBER : 1104}

7025 PC MFR: IBM, 5155, S'N 0063473-5155, ACQ. DATE: 10/84, APPROX. HT: 604400001 EA LBS. 20"W X $\times$ " $\times$. $\times 17^{\prime \prime H . ~}$

REPORT NUMBER: $899105-4083$

INE ITEM NUMBER: 0048

COND UNITS UNI
No of

ACQUISITION PRICE

COST/UNIT:

TOTAL COST:

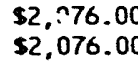

JANET $F$. BROOKS
LIVERMORE

(510) $294-2186$

TO FREEZE EQUIPMENT:

JANET $F$. BROOKS

LIVERMORE

(510) $294-2186$

CA $94550-0969$

LOCATION OF EQUIPMENT

SAMDIA NATIONAL LABORATORIES

PROPERTY REAPPLICATION DEPT 8532-1

LIVERMORE

CA $94550-0969$
COST/UNIT:

TOTAL COST:
$\$ 1,620.00$

\$i, 620.00
FOR FURTHER INFORMATION:

JANET F. BROOKS

LIVERMORE

(510) $294-2186$

CA $94550-0969$

TO FREEZE EQUIPMENT:

JAMET $F$. BROOKS

LIVERMORE

(510) $294-2186$

CA $94550-0969$

LOCATION OF EOUIPMENT

SANDIA NATIONAL LABORATORIES

SANDIA NATIONAL LABORATORIES $8532-1$

PROPERTY REAPPLICATION DEPT. $8532-1$
LIVERMORE
CA $94550-0969$ 
EFFECTIVE DATE: 07/01/94 EXPIRATION DATE: $07 / 31 / 94$

FSC

CODE EXCESS PROPERIY DESCRIPIION

REFERENCE NUMBER

7025 PC MFR: IBM, MODEL 5150, S/N 10668145150, ACQ. DATE: 11/86, APPROX. WT: 30 LBS., $20^{\circ " W} \times 16^{\prime \prime} \mathrm{L} \times 6^{\prime \prime} \mathrm{H}$.

REPORT NUMBER: $899105-4083$

LINE ITEM NUMBER: 0040

REFERENCE NUMBER : 1106

7025 PC MFR: IBM, MOOEL 5150, S/N 08265515150, ACO. DATE: 4/84, APPROX. WT: 4 00001 EA 30 LBS., 20 "W $\times 16^{\prime \prime L ~} \times 6^{\prime \prime H}$.

REPORT NUMBER: $899105-4083$

LINE ITEM NUMBER: 0041

No of

COND UNITS UNIT

ACQUISITION PRICE

$\$ 1,720.00$

$\$ 1,720.00$

TOTAL COST:

JAMET F. BROOKS
LIVERMORE

(510) $294-2186$

CA $94550-0969$

TO FREEZE EQUIPMENT:

JANET $F$. BROOKS

LIVERMORE

(510) $294-2186$

LOCATION OF EOUIPMENT:

SANDIA MATIONAL LABOPATORIES

PROPERTY REAPPLICATION DEPT. 8532-1

LIVERMORE

CA $94550-0969$

COST/UNIT:

TOTAL COST:
$\$ 1,600.00$

$\$ 1,600.00$
FOR FIJRTHER INFORMATION:

JANET F. BROOKS

LIVERMORE

(510) $294-2186$

CA $94550-0969$

TO FREEZE EQUIPMENT :

JAMET $F$. BROOKS

LIVERMORE

(510) $294-2186$

CA $94550-0969$

LOCATION OF EQUIPMENT

SANDIA NATIONAL LABORATORIES

PROPERTY REAPPLICAIION DEPT 8532-1

LIVERMORE

CA 94550-0969 
EFFECTIVE DATE: 07/01/94 EXPIRATION DATE: $07 / 31 / 94$

FSC

CODE EXCESS PROPERTY DESCRIPIION

REFERENCE NUMBER : 1907

7025 PC MFR: IBM MODEL 5150, S/N 10907695150, ACQ. DATE: 9/84, APPROX. WT: 4 00001 EA 30 LBS., $20 " \mathrm{H} \times 16^{\prime \prime L} \times 6^{\prime \prime H}$.

REPORT NUMBER: $899105-4083$

LINE ITEM NUMBER: 0042

REFERENCE NUMBER : 1108

7025 PC MFR: IBM, MODEL 5150, S/N 07527365150 , ACQ. DATE: 3/84, APPROX. WT: 400001 EA 30 LBS. 20"W X $16^{\prime \prime L} \times 6^{\prime \prime H}$.

REPORT NUMBER: $899105-4083$

LINE ITEM NUMBER: 0043
No OF

COND UNITS UNIT

ACQUISITION PRICE

COST/UNIT:

IOTAL COST:

$\$ 1,620.00$

$\$ 1,620.00$

FURTHER INFORMATION:

JANET F. BROOKS

LIVERMORE

CA $94550-0969$

(510) $294-2186$

In FREEZE EOUIPMENT:

JANE; $F$. BROOKS

LIVERMORE

CA $94550-0969$

(510) $294-2186$

LOCATION OF EQUI PMENT:

SANDIA NATIOALLL

PROPERTY REAPPLICATION DEPT. 8532-1

LIVERMORE
COST/UNIT:
TOTAL COST:
$\$ 1,600.00$

$\$ 1,600.00$

FOR FURTHER INFORMATION:

JANET F. BROOKS

LIVERMORE

(510) $294-2186$

CA $94550-0969$

TO FREEZE EOUIPMENT:

JANET F. BROOKS

LIVERMORE

CA $94550-0969$

(510) $294-2186$

LOCAIION OF EQUIPMENT:

PROPERTY REAPPLICATION DEPT. 8532-

LIVERMORE

CA $94550-0969$ 
DOE USED ENERGY RELATEO LABORATORY EOUIPMENT GRANT PROGRAM

EFFECTIVE DAIE: 07/01/94 EXPIRATION DATE: 07/31/94

FSC $\quad$ NO OF

COOE EXCESS PROPERTY DESCRIPTION

REFERENCE MUMBER : 1109

T025 PC MFR: IPM MOOEL 515J S/N 08267585150, ACQ. DATE: 3/84, APPROX. WT: 400001 EA 30 LBS., 20"W $\times 16^{\prime \prime L} \times 6^{\prime \prime H}$.

REPORT MUMBER: $899105-4083$

LINE ITEM NUMBER: 0044

RE FERENCE NUMBER : 1110

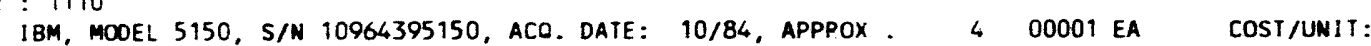

WI: 30 LBS., $20^{\prime \prime W} \times 16^{\prime \prime L} \times 6^{\prime \prime H}$.

REPORT MUMBER: $899105 \cdot 4083$

LINE ITEM NUMBER: 0045
COND UNITS UNIT

ACOUISITION PRICE

COST/UNIT: TOTAL COST:

$\$ 1,600.00$ $\$ 1,600.00$ TOTAL COST:

$\$ 1,620.00$ $\$ 1,620.00$ JANET F. BROOKS LIVERMORE

CA $94550-0969$

TO FREEZE EOUIPMENT:

JANET F. BROOKS

LIVERMOR

(510) $294-2186$

CA $94550-0969$

LOCATION JF EOUIPMENT :

SAMDIA I:ATIOMAL LABORATORIES

PROPERIY REAPPLICATION DEPT. 8532-1

LIVERMORE

CA $94550-0969$ 
EFFECTIVE DATE: $07 / 01 / 94$ EXPIRATION DATE: $07 / 31 / 94$

FSC

COOE EXCESS PROPERTY DESCRIPTION

REFERENCE MUMBER : 1111

7025 PC MFR. IBM, MOOEL 5150, S/N 0568475, ACQ. DATE: 5/84, APPROX. WT: 35 PC MFR: IBM, MOOEL 5150
LBS., - TH $\times 16^{\prime \prime L} \times 6 " \mathrm{H}$.

REPORT NUMBER: $899105-4083$

LINE ITEM NUMBER: 0046
No of

COMD UNITS UNIT

ACOUISITION PRICE

$500001 \mathrm{EA}$

COST/UNIT: IOTAL COST:

$\$ 1,600.00$ $\$ 1,600.00$

s1.600.00

REFERENCE NUMBER : 1112

7025 PC MFR: IBM, MODEL 5150, S/N 06299925150, ACO. DATE: 2/84, APPROX. WT: 4 O0001 EA 30 LBS., $20^{\circ \prime} \times 16^{\prime \prime} \mathrm{L} \times 6^{\prime \prime} \mathrm{H}$.
COST/LNIT: TOTAL COST: $\$ 1,578.00$
$\$ 1,578.00$ 
EFFECTIVE DATE: 07/01/94 EXPIRATION DATE: $07 / 31 / 94$

\section{FSC \\ COOE EXCESS PROPERTY DESCRIPTION}

REFERENCE MUMBER : 1113

7025 PC MFR: IBM, MOOEL 5150, S/N 0361670, ACQ. DATE: 3/86, APPROX. HT: 30

LBS. . 20" $\mathrm{W} \times 16^{\prime \prime} \mathrm{L} \times 5^{\mathrm{H}} \mathrm{H}$

REPORT NUMBER: $899105-4083$

LINE ITEM MUMBER: 0050

REFERENCE MUMBER : 1114

7025 PC MFR: IBM, MOOEL 5160, S/N 60370855160, ACO. DATE: 5/85, APPROX. UT: 5 00001 EA

30 LBS. . 20" $\mathrm{H} \times 16^{\mathrm{ML}} \times 5^{\mathrm{M}} \mathrm{H}$.

REPORT NUMBER: $899105-4083$

LINE ITEM MUMBER: 0051

Mo of

COND UMITS UNIT

$600001 \mathrm{EA}$

ACOUISITION PRICE

COST/UNIT:

TOTAL COST:

600.00

$$
\begin{aligned}
& \text { JANET F. BROOKS } \\
& \text { LIVERUORE }
\end{aligned}
$$

Ca $94550-0969$

IO FREEZE EOUIPMENT:

JMET F. BROOKS

JAMET F.

(510) $294-2186$

Ca $94550-0969$

\section{LOCATION OF EOUIPNENT:}

SAMOIA MATIOMAL LABORATORIES

PROPERTY REAPPLICATIOA DEPT. 8532

LIVERMORE

ca $94550-0969$

COST/UNIT:

TOTAL COST:
$\$ 1.620 .00$

$\$ 1.620 .00$
FOR FURTHER IMFOAMATION:

JANET F. BROOKS

LIVERMORE

(510) $294-2186$

CA $94550-0969$

TO FREEZE EQUIPMEMT:

JANET F. BROOXS

LIVERMORE

(510) $294-2186$

CA 94550-0969

LOCATIOW OF EOUIPWENT:

SMDOIA MATIOMAL LABORATORIES

PROPERTY REAPPLICATION DEPT. 8532-1

LIVERMORE

CA $94550-0969$ 

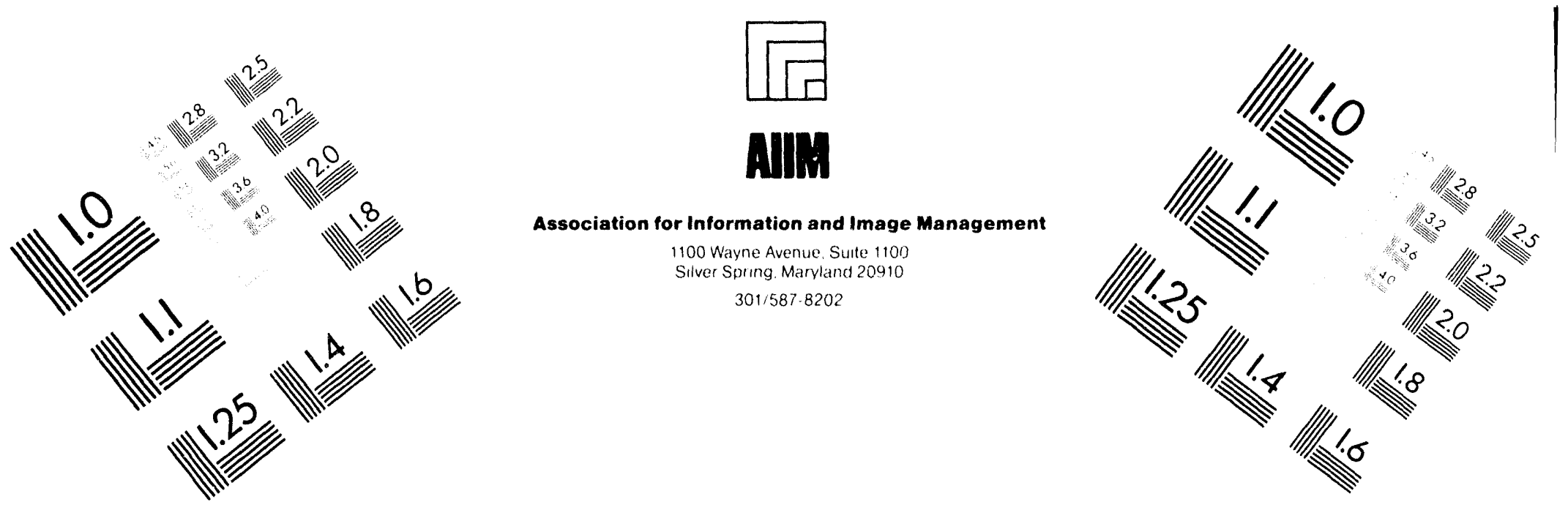

\section{Centimeter}

|

Inches
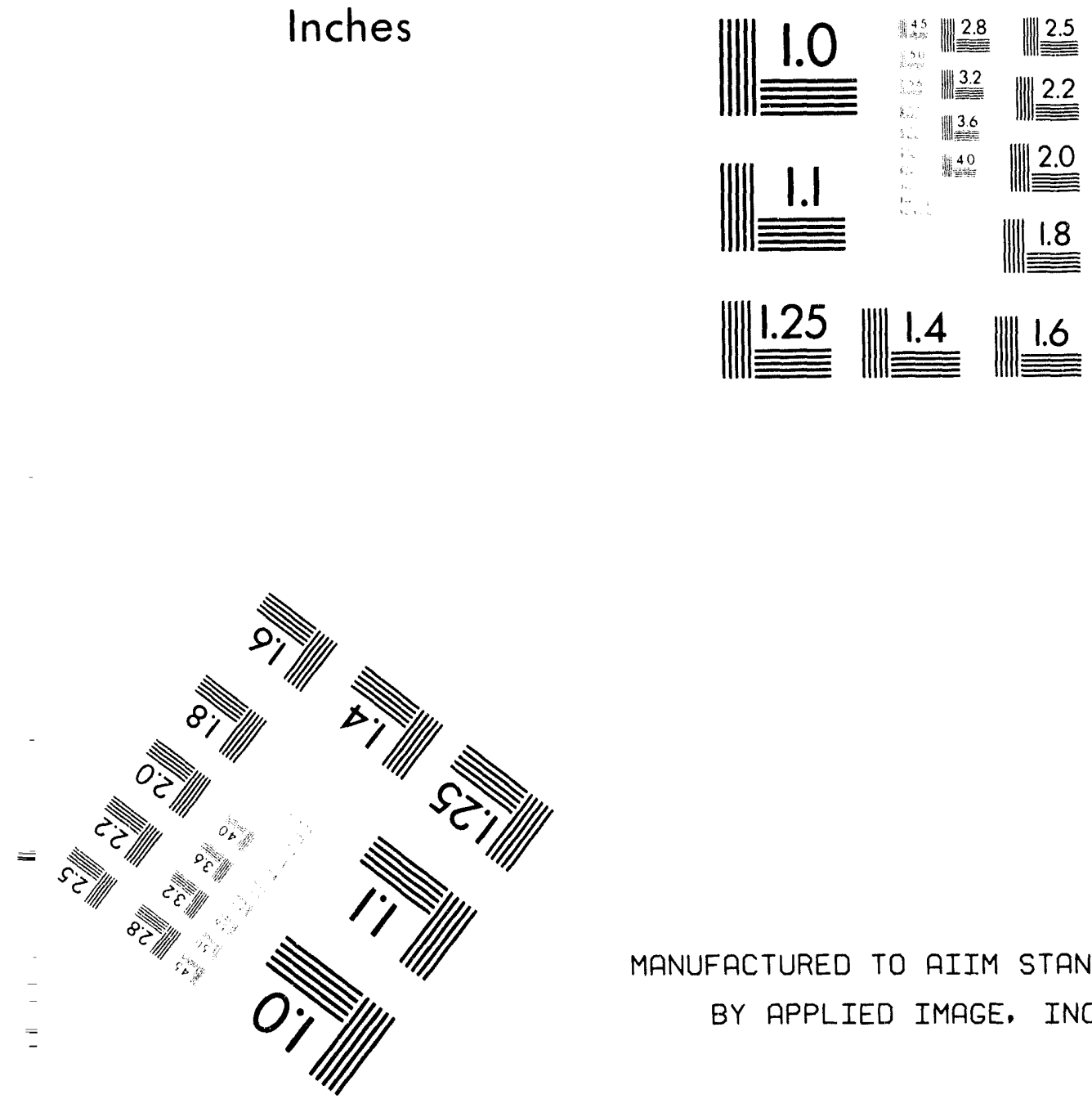

MANUFACTURED TO AIIM STANDARDS

BY APPLIED IMAGE. INC.

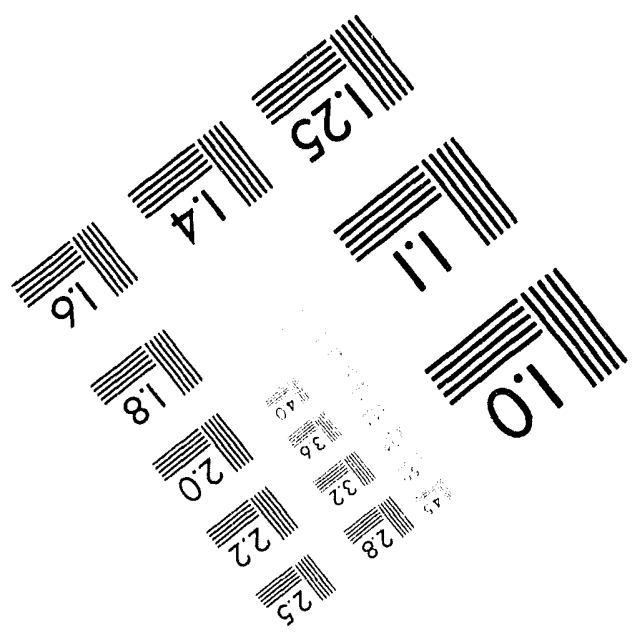



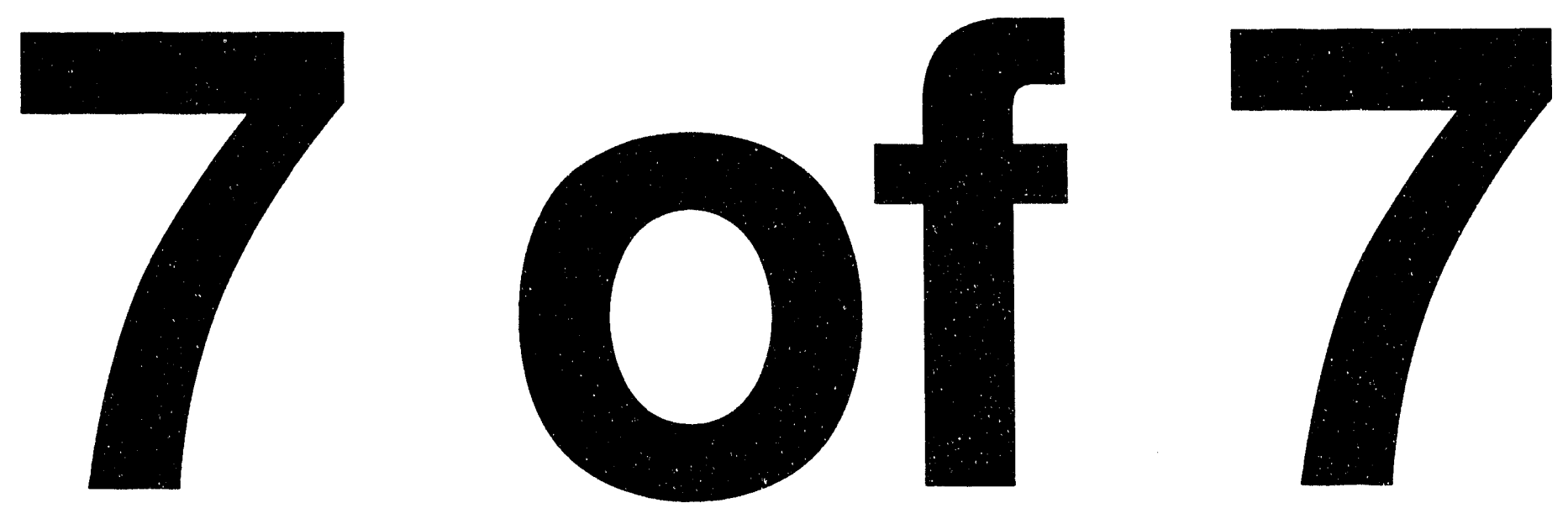
EFFECTIVE DATE: 07/01/94 EXPIRATION DATE: $07 / 31 / 94$

FSC

CODE EXCESS PROPERTY DESCRIPIION

REFERENCE NUMBER : 1115

7025 PC MFR: IBM, MODEL 5150, S/N 10789005150, ACO. DATE: 8/84, APPROX. WT: 500001 EA 35 LBS., 20"W X $16^{\prime \prime L} \times 6^{\prime \prime H}$.

REPORT NUMGER: $899105-4083$

LINE ITEM NUMBER: 0052

REFERENCE NUMBER : 1116

7025 PC MFR: IBM, MODEL 5150, S/N 09098755150, ACO. DATE: 4/84, APPROX. WT: 5 00001 EA 35 LBS. , 20"W $\times 16^{\prime \prime} \mathrm{L} \times 6^{\prime \prime H}$

\section{NO OF}

COND UNITS UNIT

ACQUISITION PRICE

COST/UNIT:

TOIAL COST:

$\$ 1,843.00$

$\$ 1,843.00$

JANET F. BROOKS
LIVERMORE

(510) $294-2186$

CA 94550-0969

TO FREEZE EQUIPMENT:

JANET F. BROOKS

LIVERMORE

CA $94550-0969$

(510) $294-2186$

LOCATION OF EQUIPMENT :

LABORATORIES

PROPERTY REAPPLICATION DEPT. 8532-1

LIVERMORE

CA $94550-0969$

COST/UNIT: TOTAL COST:
$\$ 1,600.00$

$\$ 1.600 .00$
OR FURTHER INFORMATION:

JANET F, BROOKS

IVERMORE

(510) $294-2186$

IO FREEZE EQUIPMENT:

JANET F. BROOKS

LIVERMORE

(510) $294-2186$

CA $94550-0969$

LOCATION OF EOUIPMENT:

SANDIA NATIONAL LABORATORIES

PROPERTY REAPPLICATION DEPT. 8532 -

LIVERMORE

CA $94550-096$ 
EFFECTIVE DATE: $07 / 01 / 94$ EXPIRATION DATE: $07 / 31 / 94$

\section{FSC}

COOE EXCESS PROPERTY DESCRIPIION

REFERENCE NUMBER : 1117

7025 PC MFR: IBM, MOOEL 8580-111, S/N CB55-5043702, ACO. DATE: 4/89, AP PC MFR: IBM, MODEL $8580-111$, S/N CBSS
PROX. WT: 50 LBS., 7"W X 19 "1. $\times 23^{\prime \prime H}$.

REPORT NUMBER: $899105-4083$

LINE ITEM NUMBER: 0054

REFERENCE NUMBER : 1118

7025 PC MFR: IBM, MOOEL 8580-111, S/N CB55-5031365, ACQ. DATE: 4/89, AP PROX. WT: 50 LBS., 7"W X 19 "'L $\times 23 " \mathrm{H}$

REPORT NUMBER: $899105-4083$

LINE ITEM NUMBER: 0055

LINE ITEM NUMBR: 0054

NO OF

COND UNITS UNIT

ACQUISITION PRICE

COST/UNIT:

IOTAL COST:

$\$ 8,150.00$

$\$ 8,150.00$

(n)

$400001 E A$

\section{COST/UNIT: \\ TOTAL COST:}

$\$ 8,150.00$
$\$ 8,150.00$
CONTACTSS)

FOR FURTHER INFORMATION :

JANET F. BROOKS

LIVERMORE

CA $94550-0969$

(510) $294-2186$

TO FREEZE EQUIPMENT:

JAMET $F$. BROOKS

IVERMORE

(510) $294-2186$

CA $94550-0969$

LOCATION OF EQUIPMENT:

SANDIA NATIONAL LABORATIRIES

PROPERTY REAPPLICATION DEPT. 8532-1

LIVERMORE

CA $94550-0969$

FOR FURTHER INFORMATION:

JANET F. BROOKS

LIVERMORE

CA $94550-0969$

(510) $294-2186$

TO FREEZE EOUIPMENT:

JANET F. BROOKS

CA $94550-0969$

LOCATION OF EOUIPMENT:

SANDIA NATIONAL LABORATORIES

PROPERTY REAPPLICATION DEPT. 8532-1

LIVERMORE

CA $9: 4550-0969$ 
EFFECTIVE DATE: 07/01/94 EXPIRATION DATE: $07 / 31 / 94$

\section{$\cos$}

REFERENCE NUMBER : 1119

7025 PC MFR: IEM, MODEL 5170 , S/N 5170-7099601, ACQ. DATE: 10/80, APPRO X. 500001 EA PC MFR: 1 BM, MOOEL 5170, S/N 5170
WT: 35 LBS., $21{ }^{\prime \prime W} \times 17{ }^{\prime \prime L} \times 6^{\prime \prime H}$.

REPORT NUMSER: $899105-4083$

LINE ITEM NUMBER: 0056

REFERENCE NUMBER : 1120

7025 PC MFR: IGM, MODEL 51666061, SIN 5060519, ACQ. DATE: 6/84, APPROX. WT: 400001 EA 30 LBS., 20"W $\times 16^{\prime \prime L} \times 6^{\prime \prime H}$.

NO OF
COND UNITS UNIT

ACQUISITION PRICE

COST/UNIT: TOTAL COST

$\$ 2.425 .00$ $\$ 2,425.00$

$$
\begin{aligned}
& \text { LIVERMORE } \\
& \text { (510) } 294.2
\end{aligned}
$$

(510) $294 \cdot 2186$

CA $94550-0969$

TO FREEZE EQUIPMENT:

JANET F. BROOKS

LIVERMORE

(510) $294-2186$

CA $94550-0969$

LOCATION OF EQUIPMENT:

SANDIA NATIONAL LABORATORIES

PROPERTY REAPPLICATION DEPT. 8532-1

LIVERMORE

CA $94550-0969$
COST/UNIT:

OTAL COST:
$\$ 2,389.00$ $\$ 2,389.00$
FOR FURTHER INFORMATION:

JANET F. BROOKS

IIVERMORE

CA $94550-0969$

(510) $294-2186$

TO FREEZE EQUIPMENT:

JANET F. BROOKS

IVERMORE

CA $94550-0969$

(510) $294-2186$

LOCATION OF EQUIPMENT:

SANOIA NATIONAL LABORATORIES

PROPERTY REAPPLICATION DEPT. 8532-1

LIVERMORE

CA $94550-0969$ 
EFFECIIVE DATE: 07/01/94 EXPIRATION DATE: $07 / 31 / 94$

FSC

\section{COOE EXCESS PROPERTY DESCRIPTION}

7025 PC MER: IBM, MODEL $5150,5 / N$

30 LBS., 20"W X 16"L $\times 6^{\prime \prime H}$

REPORT NUMBER: $899105-4083$

LINE ITEM NUMBER: 0059

REFERENCE NUMBER : 1122

7025 PC, PORTABLE MFR: IBM, MOOEL 5155, S/N 0023699-5155, ACQ. DATE: 11/87, 4 O0001 EA APPRO X. WT: 60 LBS., $20 " \mathrm{~W} \times 8$ "L $\times 17 " \mathrm{H}$

LINE ITEM NUMBER: 0047
No OF

COND UNITS UNI

ACQUISITION PRICE

1.843 .00

$\$ 1,843.00$

OTAL COST:

COST/UNIT:

IOTAL COSI :

$\$ 1,9: 5.00$

$\$ 1,925.00$

$1,925.00$

HHER INFORMATION:

LIVERMORE

CA $94550-0969$

TO FREEZE EQUIPMENT:

JANET F. BROOKS

LIVERMORE

(510) $294-2186$

CA $94550-0969$

OCATION OF EQUIPMENT

SANDIA NATIONAL LABORATORIES

PROPERTY REAPPLICAIION DEPT. 8532-1

LIVERMORE

CA $94550-0969$ 
EFFECTIVE DATE: 07/01/94 EXPIRATION DATE: $07 / 31 / 94$

FSC

\section{COOE EXCESS PROPERTY DESCRIPTION}

REFERENCE NUMGER : 1123

7025 PERSONAL COMPUTER 69192, MANUF: IBM MODEL $5160088 \mathrm{~S} / \mathrm{N} 004354453$

1 ASSY., INCLUDES MONITOR AND KEYBOARD. 20 MEG HARO DRIVE. SIZE: 20"W $X$ O" $F / R \times 20^{\prime \prime H}$ EST HT: 45 LBS

MFGR YR: 1987

REFERENCE NUMBER : 1126

7025 PERSONAL COMPUTER 62421, MANUF: IBM MODEL 5160087 S/N 5308320

1 ASSY., INCLUDES MONITOR AND KEYBOARD. 10 MEG. HARD DRIVE. SIZE: $20^{\prime \prime}$

× 16"F/R' X 20"H EST WT: 45 LBS

MFGR YR: 1984

REPORT NUMBER: $896404-4126$

LINE ITEM NUMBER: 0008
NO OF

COND UNITS UNIT ACOUISITION PRICE

$500001 \mathrm{EA}$

COST/UNIT:

TOTAL COST:

$\$ 1,596.00$

BARBARA HOLMES, REP.
KANSAS CITY

MC $64 \$ 41-6159$

TO FREEZE EQUIPMENT:

BARBARA HOLMES, REP.

KANSAS CITY

(816) $997-2976$

MO $64141-6159$

LOCATION OF EQUIPMENT

GLLIEDSIGNAL, INC.

2000 EAST 95 TH STREET

KANSAS CITY

MO $64131-3095$

$500001 E A$

\section{COST/UNIT:}

TOTAL COST:

$\$ 3.007 .00$

$\$ 3,007.00$
FOR FURTHER INFORMATION:

KANSAS CITY

(816) $997-2976$

TO FREEZE EOUIPMENT:

BARBARA HOLMES, REP.

KANSAS CITY

(816) $997-2976$

LOCATION OF EOUIPMENT:

ALLIEDSIGNAL, INC.

2000 EAST OSTH STREET

KANSAS CITY

MO 64131-3095 
EFFECTIVE DATE: $07 / 01 / 94$ EXPIRATION DATE: $07 / 31 / 94$

FSC

\section{COOE EXCESS PROPERTY DESCRIPIION}

REFERENCE MUMBER : 1125

7025 PERSONAL COMPUTER 66961 , MANUF: IBM MODEL 5160078 S/N 6203776 PERSONAL COMPUTER 60961 , MANUF: IBM MODEL

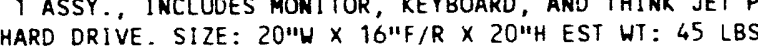

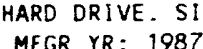

REPORT NUMBER: $896404-4126$

LINE ITEM NUMBER: 0010

REFERENCE NUMBER : 1126

7025 PERSONAL COMPUTER 69193, MANUF: IBM MOOEL 5160088 S/N 004041546
I ASSY

1 ASSY. INCLUDES MONITOR, KEYBOARD AND OUIET JET

DRIVE. SIZE: 2014
MFGR YR: 1987

REPORT NUMBER: $896404-4126$

LINE ITEM NUMBER: 0011
NO OF

COND UNITS UNIT

ACQUISITION PRICE

500001 EA COST UNIT:
TOTAL COST:

$\$ 1,492.00$

$\$ 1,492.00$

1.492 .00

FOR FURTHER INFORMAT
BARBARA HOLMES, REP.

KANSAS CITY

(816) $997-2976$

TO FREEZE EQUIPMENT:

BARBARA HOLMES, REP.

KANSAS CITY

(816) $997-2976$

LOCATION OF EOUIPMENT:

ALLIEDSIGNAL, INC.

2000 EAST 95TH STREET

KANSAS CITY
$500001 E A$

COST/UNIT:

TOTAL COST:

$\$ 1,596.00$
FOR FURTHER INFORMATION:

BARBARA HOLMES, REP.

KANSAS CITY

MO 64141-6159

(B16) $997-2976$

TO FREEZE EQUIPMENT:

BARBARA HOLMES, REP

KANSAS CITY

MO $64141-6159$

LOCATION OF EQUIPMENT:

ALLIEDSI GNAL, INC.

2000 EAST 95TH STREET

KANSAS CITY

MO $64131-3095$ 
EFFECTIVE DATE: $07 / 01 / 94$ EXPIRATION DATE: $07 / 31 / 94$

\section{FSC \\ NO OF}

\section{COOE EXCESS PROPERTY DESCRIPTION}

REFERENCE NUMBER : 1127

7025 PERSONAL COMPUTER 62460, MANUF: IBM MOOEL 5150 S/N 1029175
1 ASSY. INCLUDES MONITOR AND KEYBOARD, AND QUIET JET PRINTER. 20 MEG.

1 ASSY., INCLUDES MONITOR AND KEYBOARD,

HARD DRIVE. SIZE: $20^{\prime \prime} \times 16^{\prime \prime F} / R \times 20^{\prime \prime} \mathrm{H}$

EST WT: 45 LBS MFGR YR: 1984

REPORT NUMBER: $896404-4126$

LINE ITEM NUMBER: 0012

REFERENCE NUMBER : 1128

7025 PERSONAL COMPUTER 66973, MANUF: IBM MODEL 5160078 S/N 6202238

1 ASSY., INCLUDES MONITOR AND KEYBOARD. 256K RAM. HARD DRIVE HAS BEEN

REMOVED. SIZE: $16^{\prime \prime W} \times 15^{\prime \prime F / R} \times 20^{\prime \prime H}$ EST WT: 45 LBS

MFGR YR: 1986

REPORT NUMBER: $896404-4126$

LINE ITEM NUMBER: 0020

\section{COND UNITS UNIT ACOUISITION PRICE CONTACT(S)}

5 D0001 EA COSTIUNIT: $\quad \begin{aligned} & \$ 1,229.00 \\ & \end{aligned}$ TOTAL COST:

$\$ 1,229.00$

BARBARA HOLMES, REP.

KANSAS CITY

(816) $997-2976$

TO FREEZE EQUIPMENT :

BARBARA HOLMES, REP.

KANSAS CITY

(816) $997-2976$

LOCATION OF EOUIPMENT:

ALLIEDSIGNAL, INC.

2000 EAST 95TH STREET

KANSAS CITY

MO 64131-3095
COST/UNIT: TOTAL COST:
FOR FURTHER INFORMATION:

BARBARA HOLMES, REP.

KANSAS CITY

MO 64141-6159

(816) $997-2976$

TO FREEZE EQUIPMENT :

BARBARA HOLMES, REP.

KANSAS CITY

(816) $997-2976$

MO 64141-6159

LOCATION OF EQUIPMENT:

ALLIEDSIGNAL, INC.

2000 EAST 95TH STREET

KANSAS CITY

MO 64131-3095 
EFFECTIVE DATE: $07 / 01 / 94$ EXPIRATION DATE: $07 / 31 / 94$

FSC

COOE EXCESS PROPERIY DESCRIPTION

REFERENCE NUMBER : 1129

7025 PERSONAL COMPUTER 67809, MANUF: IBM MOOEL 5170068 S/N 00526338

PERSONAL COMPUTER 67809, MANUF: IBM MOOEL S170068. HARD DRIVE HAS BEEN

1 ASSY.. INCLUDES MON IS CE 67812 EXTERNAL DISK DRIVE, MFE. IOMEGA.

SIZE: $20^{\prime \prime W} \times 16^{\prime \prime F} / R \times 28^{\prime \prime H}$ EST WT: 45 LBS MFGR YR: 1986

REPORT NUMBER: $896404-4126$

LINE ITEM NUMBER: 0021

REFERENCE NUMBER : 1130

PERSONAL COMPUTER 72539 MANUF: HEHLETT PACKARD MODEL $459450 \mathrm{~S} / \mathrm{N}$ $2821 V 10580$

1 ASSY. INCLUDES MONITOR AND KEYBOARD. 6LOK RAM, HARD DRIVE HAS BEEN

MFGR YR: 1988

REPORT NUMBER: $896404-4126$

LINE ITEM NUMBER: 0022
800001 EA

COST/UNIT:

TOTAL COST:

$\$ 5,200.00$

NO OF

COND UNIIS UNIT $\quad$ ACOUISITION PRICE

$\$ 4,100.00$

TOTAL COST:

(816) $997-2976$

In FREEZE EQUIPMENT:

GARBARA HOLMES, REP.

KANSAS CITY

LCATION OF EOUIPMENT:

ALLIEDSIGNAL, INC.

2000 EAST 95TH STREET

KANSAS CITY

MO $64131-3095$

FOR FURTHER INFORMATION :

BARBARA HOLMES, REP.

KANSAS CITY

(816) $997-2976$

MO 64141-6159

TO FREEZE EQUIPMENT:

BARBARA HOLMES, REP.

KANSAS CITY

MO $64141-6159$

(816) 997-2976

LOCATION OF EOUIPMENT

ALLIEDSIGNAL, INC.

2000 EAST 95TH STREET

KANSAS CITY 
FSC

\section{COOE EXCESS PROPERTY DESCRIPTION}

REFERENCE NUMBER : 1131

7025 PERSONAL COMPUTER 72505, MANUF: HEWLETT PACKARD MOOEL 45945D S/N $2821 V 10145$

1 ASSY. INCLUDES MONITOR AND KEYBOARD. 640K RAM, HARD DRIVE HAS BEEN REMOVED. SIZE: $17^{\prime \prime W} \times 15^{\prime \prime F / R} \times 20^{\prime \prime H}$ EST WT: 50 LBS

MFGR YR: 1988

REPORT NUMBER: $896404-4126$

LINE ITEM NUMBER: 0023

\section{REFERENCE NUMBER : 1132}

7025 PERSONAL COMPUTER 72525 , MANUF: HEWLETT DACKARD MODEL 459450 S/N $2821 V 10618$

1 ASSY INCLUDES MONITOR AND KEYGOARD. 64OK RAM. HARD DRIVE HAS BEEN

REMOVED. SIZE: $17 " W \times 15^{\prime \prime F} / R \times 20 " H$ EST WT: 50 LBS

MFGR YR: 1988

LINE ITEM NUMBER: 0024
No of
800001 EA
COND UNITS UNIT

\section{ACOUISITION PRICE} COST/UNIT:
TOTAL COST:

$\$ 3,200.00$ $\$ 3,200.00$
CONTACT(S)

FOR FURTHER INFORMATION:

BARBARA HOLMES, REP.

KANSAS CITY

(816) $997-2976$

MO $64141-6159$

TO FREEZE EQUIPMENT: BARBARA HOLMES, REP.

XANSAS CITY

M0 $64141-6159$

(816) $997-2976$

LOCATION OF EQUIPMENT:

ALLIEDSIGNAL, INC.

2000 EAST 95TH STREET

KANSAS CITY

MO 64131-3095
$800001 E A$ TOTAL COST
$\$ 5,200.00$
FOR FURTHER INFORMATION:

BARBARA HOLMES, REP.

KANSAS CITY

(816) $997-2976$

TO FREEZE EQUIPMENT: BARBARA HOLMES, REP.

KANSAS CITY

MO 64141-6159

(816) $997-2976$

LOCATION OF EQUIPMENT :

ALLIEDSIGNAL, INC.

2000 EAST 95TH STREET

KANSAS CITY

MO 64131-3095 
EFFECTIVE DATE: $07 / 01 / 94$ EXPIRATION DATE: $07 / 31 / 94$

FSC

\section{CODE EXCESS PROPERTY OESCRIPTION}

REFERENCE NUMBER : 1133

7025 PERSONAL COMPUTER 72529, MANUF: HEWLETT PACKARD MODEL 45945D S/N

2821 VI0232 1 ASSY., INCLUDES MONITOR AND KEYBOARD. 640K RAM, HARD DRIVE HAS BEEN REMOVED. SIZE: $17^{W} \mathrm{~W} \times 15^{\prime \prime F} / \mathrm{R} \times 20^{\prime \prime H}$ EST WT: 50 LBS

MFGR YR: 1988

REPORT NUMBER: $896404-4126$

LINE ITEM NUMBER: 0025

REFERENCE NUMBER : 1134

7025 PERSONAL COMPUTER 72528 , MANUF: HEWLETT PACKARD MODEL $459450 \mathrm{~S} / \mathrm{N}$ $2821 V 10154$

1 ASSY. INCLUDES MONITOR AND KEYBOARD. 64OK RAM, HARD DRIVE HAS BEEM REMOVED. SIZE: $17^{\prime \prime W} \times 15^{\circ \mathrm{F}} / \mathrm{R} \times 20^{\prime \prime H}$ EST WT: $50 \mathrm{LBS}$

REMOVED. MFGR YR: 1988

No of

COND UNITS UNIT

ACQUISITION PRICE

$800001 \mathrm{EA}$

COST/UNIT: TOTAL COST:

$\$ 5,200.00$

BARBARA HOLMES, REP.
KANSAS CITY

(816) $997-2976$

MO $64141-6159$

TO FREEZE EQUIPMENT:

BARBARA HOLMES, REP

KANSAS CITY

(816) $997-2976$

LOCATION OF EQUIPMENT :

ALLIEDSIGNAL, INC.

2000 EAST 95TH SIREE

KANSAS CITY

COST/UNIT:

TOTAL COST:
$\$ 5.200 .00$

$\$ 5,200.00$
FOR FURTHER INFORMATION:

BARBARA HOLMES, REP.

KANSAS CITY

(816) $997-2976$

TO FREEZE EQUIPMENT:

BARBARA HOLMES, REP.

KANSAS CITY

(816) $997-2976$

LOCATION OF EOUIPMENT:

ALLIEDSIGNAL, INC.

2000 EAST 95TH STREE

KANSAS CITY

MO 64131-3095 
EFFECTIVE DATE: $07 / 01 / 94$ EXPIRATION DATE: $07 / 31 / 94$

FSC

\section{COOE EXCESS PROPERTY DESCRIPTION}

REFERENCE NUMBER : 1135

7025 PERSONAL COMPUTER 62219. MANUF: IBM MOOEL $5150160 \mathrm{~S} / \mathrm{N} 1108828$

1 ASSY. INCLUDES MONITOR AND KEYBOARD. 4 MEG. MENORY. SIZE: $20 \mathrm{WW} X$

16 "F/R X 18"K EST HT: 45 LBS

MFGR YR: 1984

REPORT NUMBER: $896404-4126$

LINE ITEM NUMBER: 0029

REFERENCE NUMBER : 1136

7025 PERSONAL COMPUTER 66405, MANUF: 1BM MODEL 5150068 S/N 2026182

1 ASSY INCLUDES MONITOR, KEYBOARD AND HAS A 30 MEG. HARD DRIVE. SIZE:

20"W X 16"F/R X 20"H EST WT: 45 LBS

MFGR YR: 1986

REPORT NUMBER: $896404-4120$

LINE ITEM NUMBER: 0045
NO OF

COND UNITS UNIT

ACQUISITION PRICE

$\$ 1,397.00$

$00001 E A$

COST/UNIT:

TOTAL COST:

$\$ 1,397.00$

BARBARA HOLMES, REP

MO 64141-6159

(816) $997-2976$

TO FREEZE EOUIPMENT:

BARBARA HOLMES, REP.

KANSAS CITY

(816) $997-2976$

MO 64141-6159

LOCATION OF EQUIPMENT:

ALLIEDSIGNAL, INC.

2000 EAST 95TH SIREET

KANSAS CITY

MO 64131-3095

400001 EA

COST/UNIT:

TOTAL COST:

$\$ 1,394.00$

$\$ 1,394.00$

FOR FURTHER INFORMATION :

BARBARA HOLMES, REP.

KANSAS CITY

16) $997-2976$

TO FREEZE EQUIPMENT:

BARBARA HOLMES, REP.

KANSAS CITY

MO $64141-6159$

(816) $997-2976$

LOCATION OF ECUIPMENT:

ALLIEDSIGNAL, INC.

ALLIEDSIGNAL, INC.

KANSAS CITY 
EFFECTIVE DATE: $07 / 01 / 94$ EXPIRATION DATE: $07 / 31 / 94$

\section{CODE EXCESS PROPERTY DESCRIPTION}

REFERENCE NUMBER : 1137

7025 PERSONAL COMPUTER 69194, MANUF: IBA MODEL 5160088 S/N 004041701

1 ASSY., INCLUDES MONITOR AND KEYBOARD. 20 MEG. HARD DRIVE. SIZE: 20"W

$\times 16^{\prime \prime F} / R^{\prime} \times 20^{\prime \prime H}$ EST WT: 45 LBS

MFGR YR: 1987

REPORT NUMBER: $896404-4126$

LINE ITEM NUMBER: 0046

\section{REFERENCE NUMBER : 1138}

7025 PERSONAL COMPUTER 58493, MANUF: IBM MODEL 5150-164 S/N 317233

1 ASSY, INCLUDES MONITO , KEYBOARD AND PRINTER. SIZE: $20^{\prime \prime} \mathrm{W} \times 16^{\prime \prime F} / \mathrm{R} \times$

6"H EST WT: 30 LBS

6"H EST HT: 30

LINE ITEM NUMBER: 0047
NO OF

COND UNITS UNIT

ACQUISITION PRICE

$500001 \mathrm{EA}$

COST/UNIT:

TOTAL COST:

$\$ 1,596.00$

$\$ 1,596.00$

BARBARA HOLMES, REP.

KANSAS CITY

(816) 997-2976

TO FREEZE EQUIPMENT:

BARBARA HOLMES, REP.

KANSAS CITY

(816) $997-2976$

LOCATION OF EQUIPMENT:

ALLIEDSIGNAL, INC.

2000 EAST 95TH STREET

KANSAS CITY

$800001 \mathrm{EA}$

COST/UNIT:

TOTAL COST:

$\$ 1,473.00$

$\$ 1,473.00$

FOR FURTHER INFORMATION:

BARBARA HOLMES, REP.

KANSAS CITY

MO $64141-6159$

(816) $997-2976$

TO FREEZE EQUIPMENT:

BARBARA HOLMES, REP.

KANSAS CITY

(816) $997-2976$

LOCATION OF EQUIPMENT:

ALL IEDSIGNAL, INC.

2000 EAST 95TH STREET

KANSAS CITY

MO $64131-3095$ 
EFFECTIVE DATE: $07 / 01 / 94$ EXPIRATION DATE: $07 / 31 / 94$

FSC

COOE EXCESS PROPERTY DESCRIPIION

REFERENCE NUMBER : 1139

7025 PERSONAL COMPUTER 65102, MANUF: IBM MODEL 5160068 S/N 6428808 1 ASSY.. INCLUDES MONITOR AND KEYBOARD. 1 MEG. HARD DRIVE. SIZE: $25 \mathrm{HW}$ 25"F/R X 26"H EST WT: 45 LBS

MFGR YR: 1986

REPORT NUMBER: $896404-4126$

LINE ITEM NUMBER: 0066

\section{REFERENCE NUMBER : 1140}

7025 PERSONAL COMPUTER 73761, MANUF: IBM MODEL 8560-041 S/N $72-8185903$ 1 ASSY., INCLUDES MONITOR AND KEYBOARD. 1 MEG., HARD DRIVE. SIZE: 25 "W X 25"F/R X 25"H EST WT: 45 LBS

MFGR YR: 1988
ELIGIBLE EQUIPMENT
NO OF

COND UNITS UNIT

$600001 \mathrm{EA}$
ACQUISITION PRICE

TOTAL COST:

COST/UNIT:
TOTAL COST:

\section{$\$ 1,763.00$ \\ $\$ 1,763.00$}

CONTACT(S)

FOR FURTHER INFORMATION:

BARBARA HOLMES, REP.

KANSAS CITY

MO $64141-6159$

816) 997-2976

TO FREEZE EOUIPMENT: BARBARA HOLMES, REP.

KANSAS CITY

(816) $997-2976$

MO 64141-6159

LOCATION OF EQUIPMENT:

ALLIEDSIGNAL, INC.

2000 EAST 95TH STREET

KANSAS CITY

MO $64131-3095$

$600001 \mathrm{EA}$

COST/UNIT:
TOTAL COST:

$\$ 2,858.00$

$\$ 2,858.00$

FOR FURTHER INFORMATION

BARBARA HOLMES, REP.

KANSAS CITY

(816) 997-2976

MO $64141-6159$

TO FREEZE EQUIPMENT: BARBARA HOLMES, REP.

KANSAS CITY

MO 64141-6159

(816) $997-2976$

LOCATION OF EOUIPMENT:

ALLIEDSIGNAL, INC.

2000 EAST 95TH STREET

KANSAS CITY 
EFFECTIVE DATE: $07 / 01 / 94$ EXPIRATION DATE: $07 / 31 / 94$

FSC

\section{COOE EXCESS PROPERTY DESCRIPTION}

REFERENCE NUMBER : 1141

7025 PERSONAL COMPUTER 73814, MANUF: IBM MODEL 8550-031 S/N 72-7011902

1 ASSY., INCLUDES MONITOR WHICH IS BAD, AND KEYBOARD. 1 MEG. HARD

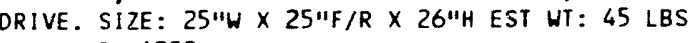

MFGR YR: 1988

REPORT NUMBER: $896404-4126$

LINE ITEM NUMBER: 0068

$$
\text { No of }
$$

COND UNITS UNIT

ACQUISITION PRICE

$600001 \mathrm{EA}$

COST/UNIT: TOTAL COST:

$\$ 2,858.00$

$\$ 2,858.00$

BARBARA HOLMES, REP.
KANSAS CITY

MO $64141-6159$

TO FREEZE EQUIPMENT:

BARBARA HOLMES, REP.

KANSAS C! TY

(816) $997-2976$

LOCATION OF EOUIPMENT:

ALLIEDSIGNAL, INC.

2000 EAST 95TH STREET

KANSAS CITY

MO $64131-3095$

$\$ 2,858.00$

COST/UNIT:

TOTAL COST:

$\$ 2,858.00$

FOR FURTHER INFORMATION:

BARBARA HOLMES, REP.

KANSAS CITY

MO $64141-6159$

25"F/R X 26"H EST WT: 45 LBS

MFGR YR: 1988

REPORT NUMBER: $896404-4126$

LINE ITEM NUMBER: OC69
TO FREEZE EQUIPMENT:

BARBARA HOLMES, REP.

KANSAS CITY

(816) $997-2976$

MO 64141-6159

LOCATION OF EQUIPMENT:

ALLIEDSIGNAL, INC.

2000 EAST 95TH STREET

KANSAS CITY

MO $64131-3095$ 
EFFECTIVE DATE: $07 / 01 / 94$ EXPIRATION DATE: $07 / 31 / 94$

FSC

COOE EXCESS PROPERTY DESCRIPIION

REFERENCE NUMBER : 1143

7025 PRINTER

SERIAL \# 2706A28292 BARCODE 850541

X94-04-036 MANUF. HEWLETT PACKARD

COND UNITS UNIT

$900001 \mathrm{EA}$

COST/UNIT:
TOIAL COST:

LINE ITEM NUMBER: 0007

REPORT NUMBER: $895529-4235$

REFERENCE NUMBER : 1144

7025 PRINTER

\# 193 PLUS SERIAL \# 603A1021853

X94-04-053 MANUF. OKIDATA MODEL 900001 EA

COST/UNIT:

TOTAL COST:

BARCODE 850389
REPORT NUMBER: $895529-4235$

LINE ITEM NUMBER: 0016

\section{ACUISITION PRICE}

$\$ 1,463.00$
$\$ 1,463.00$

$\$ 1,800.00$

$\$ 1,800.00$

\section{CONTACT(S)}

FOR FURTHER INFORMATION:

WILLIS J. MILLER

CINCINNATI

(513) $738-6630$

OH 45239-8704

TO FREEZE EQUIPMENT:

WILLIS J. MILLER

CINCINNATI

(513) $738-6630$

OH $45239-8704$

LOCATION OF EQUIPMENT:

U.S. DOE/FERMCO

7400 WILLEY ROAD

FERNALD

$\mathrm{OH} 45030$

FOR FURTHER INFORMATION:

WILLIS J. MILLER

CINCINNATI

(513) $738-6630$

OH 45239-8704

TO FREEZE EQUIPMENT:

WILLIS J. MILLER

CINCINNATI

OH 45239-8704

LOCATION OF EQUIPMENT:

U.S. DOE/FERMCO

7400 WILLEY ROAD

FERNALD

$\mathrm{OH} 45030$ 
EFFECTIVE DATE: 07/01/94 EXPIRATION DATE: 07/31/94

FSC

\section{COOE EXCESS PROPERTY DESCRIPTION}

REFERENCE NUMBER : 1145

7025 PRINTER

05017039 BARCODE 851013

X94-04-087 MANUF EPSON SERIAL

REPORT NUMBER: $895529 \cdot 4233$

LINE ITEM NUMBER: 000

\section{REFERENCE NUMBER : 1146}

7025

851038
$900001 \mathrm{EA}$

NO OF

COND UNITS UNIT

ACOUISITION PRICE

COST/UNIT: TOTAL COST:

\section{$\$ 1,500.00$}

$\$ 1,500.00$

(c)

$900001 E A$

\section{COST/UNIT:} IOTAL COST:
$\$ 2.059 .00$ $\$ 2,059.00$

FOR FURTHER INFORMATION:

WILLIS J. MILLER

CINCINNATI (513) $738-6630$

TO FREEZE EQUIPMENT:

WILLIS J. MILLER

REPORT NUMBER: $895529-6233$

LINE ITEM NUMBER: 0008

(513) $738-6630$

LOCATION OF EOUIPMENT :

U.S. DOE/FERMCO

7400 HILLEY ROAD

FERNALD

OH 45030
OH 45239-8704

OH 45239-8704 
EFFECTIVE DATE: 07/01/94 EXPIRATION DATE: 07/31/94

FSC $\quad$ NO OF

CODE EXCESS PROPERTY DESCRIPTION

REFERENCE NUMBER : 1147

T025 PRINTER X94-04-092 MANUF. HEWLETT PACKARD SER:AL \# 2531 J04422 BARCODE 851044

REPOR! NUMBER: $895529-4233$

LINE ITEM NUMBER: 0009

\section{REFERENCE NUMBER : 1148}

7025 PRINTER

X94-04-093 MANUF. HEWLETT

PACKARD SERIAL \# 2531 J09257 BARCODE 851046

REPORT NUMBER: $895529-4233$

LINE ITEM NUMBER: 001
COND UNITS UNIT

\section{ACOUISITION PRICE}

900001 EA

COST/UNIT: IOTAL COST:

\section{$\$ 2,059.00$} $\$ 2,059.00$ WILLIS J. MILLER
CINCINNATI (513) $738-6630$

TO FREEZE EQUIPMENT

WILLIS J. MILLER

CINCINNATI

(513) $738-6630$

OH $45239-8704$

LOCATION OF EQUIPMENT:

U.S. DOE/FERMCO

7400 WILLEY ROAD

FERNALD

$\mathrm{OH} 45030$

OH $45239-8704$

$\$ 2,007.00$

WILLIS J. MILLER

(513) $738-6630$

TO FREEZE EQUIPMENT

WILLIS J. MILLER

CINCINMAT!

(513) $738-6630$

OH $45239-8704$

LOCATION OF EQUIPMENT:

U.S. DOE/FERMCO

7400 WILLEY ROAD

FERMALD

$\mathrm{OH} 45030$
OH 45239-8704 
EFFECTIVE DATE: $07 / 01 / 94$ EXPIRATION DATE: $07 / 31 / 94$

\section{COOE EXCESS PROPERTY DESCRIPIION}

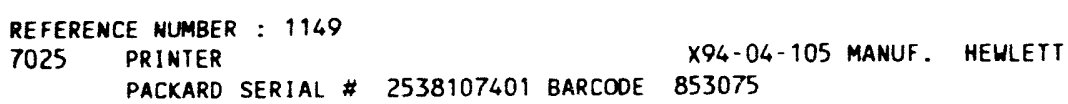

PACKARD SERIAL \# 2538107401 BARCOOE 853075

REPORT NUMBER: $895529-4233$

LINE ITEM NUMBER: 0018

REFERENCE NUMBER : 1150

TO25 PRINTER

\# 49000 BARCODE 852563

X94-04-122 MANUF. OKIDATA SERIAL 900001 EA

REPORT NUMBER: $895529-4233$

LINE ITEM NUMBER: 0027
COST/UNIT: TOTAL COST:

$\$ 1,837.00$

$\$ 1,837.00$

No OF

90001 EA COST/UNIT: $\$ \$ 1,500.00$ TOTAL COST:

$\$ 1,500.00$

WILLIS J. MILLER
CINCINAATI

CINCINNATI

OH 45239-8704

TO FREEZE EQUIPMENT:

WILLIS J. MILLER

CINCINNATI

(513) $738-6630$

OH 45239-8704

LOCATION OF EQUIPMENT :

U.S. DOE/FERMCO

7400 WILLEY ROAD

FERNALD

OH 45030

FOR FURTHER INFORMATION:

WILLIS \&. MILLER

CINCINNATI

(513) $738-6630$

OH 45239-8704

TO FREEZE EQUIPMENT:

HILLIS J. MILLER

CINCINNATI

(513) $738-6630$

OH 45239-8704

LOCATION OF EQUIPMENT

U.S. DOE/FERMCO

7400 WILLEY ROAD

FERNALD

OH 45030 
DOE USED ENERGY RELATED LABORATORY EQUIPMENT GRANT PROGRAM

EFFECTIVE DATE: $07 / 01 / 94$ EXPIRATION DATE: $07 / 31 / 94$
FSC
COOE EXCESS PROPERTY DESCRIPTION

7025 PRINTER

\# 48802 barcooe 852566

X94-04-123 MANUF. OKIDATA SERIAL

COND UNITS UNIT

900001 EA

REPORT NUMBER: $875529-4.233$

LINE ITEM NUMBER: 0028

\section{REFEREMCE NUMBER : 1152}

7025 PRINTER 852019
REFERENCE NUMBER : 1151
$900001 \mathrm{EA}$

COST/UNIT:

TOTAL COST
$\$ 2.102 .00$

$\$ 2.102 .00$
FOR FURTHER INFORMATION:

WILLIS J. MILLER

CINCINNATI

(513) $738-6630$

OH 45239-8704

TO FREEZE EQUIPMENT:

WILLIS $\mathrm{J}$. MILLER

CINCINNATI

(513) $738-6630$

OH 45239-8704

LOCATION OF EOUIPMENT:

U.S. DOE/FERMCO

7400 WILLEY ROAD

FERNALD

OH 45030 
EFFECIIVE DATE: $07 / 01 / 94$ EXPIRATION DATE: $07 / 31 / 94$

COOE EXCESS PROPERTY DESCRIPTION

PEFERENCE MUMBER : 1153

7025 PRINTER

X94-04-159 MANUF. HEWLETT
PRINTER

DEDART NUMBER: $895529-4232$

LINE ITEM NUMBER: 0012

REFERENCE NUMBER : 1154

7025 PRINTER

* 603A1025608 BARCOOE 856419

X94-04-207 MANUF. OKIDATA SERIAL 900001 EA

COST/UNIT:

TOTAL COST:
$\$ 1,800.00$

$\$ 1,800.00$

FOR FURTHER INFORMATION:

WILLIS J. MILLER

CINCINNAT

(513) $738-6630$

OH 45239-8704

TO FREEZE EQUIPMENT:

WILLIS J. MILLER

CINCINMATI

OH 45239-8704

REPORT NUMBER: $895529-4232$

LINE ITEM NUMBER: 0023

(513) $738-6630$

LOCATION OF EQUIPMENT:

U.S. DOE/FERMCO

7400 WILLEY ROAD

FERNALD

OH 45030 
EFIECTIVE DATE: 07/01/94 EXPIRATION DATE: 07/31/94

\section{COOE EXCESS PROPERTY DESCRIPIION}

REFERENCE NUMBER : 1155

7025 PRINTER 57553 MANUF: EPSON MOOEL MX100 S/N 331040

I EACH, SIZE: 22"W X 15"F/R $\times$ 6"H EST WT: 10 LBS MFGR YR: 1982

REFORT NUMBER: $896404-4126$

LINE ITEM NUMBER: 0074

\section{REFERENCE NUMBER : $i 156$}

PRINTER 65150, MANUF: HEWLETT PACKARD MOOEL 2686 A S/N 2602.25856

1 EACH, OPTION 300 . SIZE: 20 "W $\times 18^{\prime \prime F} / R \times 12^{\prime \prime H}$ EST WT: 60 LBS MFGR YR:

1986
No of

COND UNIIS UNIT

ACQUISITION PRICE

$600001 \mathrm{EA}$

COST NUNIT:

TOTAL COST:

$\$ 1.198 .00$

$\$ 1,198.00$

THER INFORMATION:

BARBARA HOLMES, REP.

KANSAS CITY

MO 64141-6159

(816) $997-2970$

TO FREEZE EQUIPMENT:

BARBARA HOLMES, REP.

BARBARA HOLLY

(816) $997-2976$

LOCATION OF EQUIPMENT:

ALLIEDSIGNAL, INC.

2000 EAST 95TH STREE

KANSAS CITY

140 64131-3095

$500001 E A$
COST/UNIT:

TOTAL COST:
$\$ 2,680.00$

$\$ 2,680.00$
FOR FURTHER INFORMATION:

BARBARA HOLMES, REP.

KANSAS CITY

(816) $997-2976$

MO 64141-6159

TO FREEZE EOUIPMENT:

BARBARA HOLMES, REP.

KANSAS CITY

Mo $64141-6159$

REPORT NUMBER: $896404-4126$

LINE ITEM NUMBER: 0009
816) $997-2976$

LOCATION OF EOUIPMENT:

ALLIEDSIGNAL, INC.

2000 EAST 95 TH STREET

KANSAS CITY

MO $64131-309$ 
EFFECTIVE DATE: $07 / 01 / 94$ EXPIRATION DATE: $07 / 31 / 94$

\section{FSC}

REFERENCE MUMBER : 1157

7025 PRINTER 68336, MANUF: IBM MOOEL 3812 S/N 000016537

1 EACH. SIZE: $20^{\prime \prime W} \times 21 " \mathrm{~F} / \mathrm{R} \times 14^{\prime \prime} \mathrm{H}$ EST WT: 200 LBS MFGR YR: 1987

ELIGIBLE EOUIPMENT
OND UNITS Un:

$400001 \mathrm{EA}$
ACOUISITION PRICE

COST/UNIT:

TOTAL COST:

LINE ITEM MUMBER: 0055
REPORT NLMBER: $896404-4126$

REFERENCE NUMBER : 1158

7025 PRINTER EPSON LO- 1500
LINE ITEM NUMBER: 3

\section{$500001 \mathrm{EA}$

\author{
COST/UNIT: \\ TOTAL COST:
}

REPORT NUMBER: $894609-9090$
$\$ 1,050.00$
$\$ 1,050.00$

FR FURTHER INFORMATION:

DAVID MITCHELL

AIKEN

AIKEN $725-9540$

SC 29802

TO FREEZE EQUIPMENT:

DAVID MITCHELL

DAIKEN

sr 29802

(803) $725-9540$

LOCATION OF EOUIPMENT:

WESTINGHOUSE

SAVANMAH RIVER SITE

AIKEN

SC 29802

MO $64141-6159$

MO 64141-6159 
EFFECIIVE DATE: $07 / 01 / 94$ EXPIRATION DATE: $07 / 31 / 94$

FSC
COOE EXCESS PROPERTY DESCRIPTION

\section{REFERENCE NUMBER : 1159}

7025 PRINTER HENERAL COMPUTER LASERURITER, SN-A004783H

REPORT NUMBER: $894009-9090$

REFERENCE NUMBER : 115

7025 PRINTER HEWLEIT PACKARD OUIE JET SN-273050

REPORT NUMBER: $894609-9090$

IINE ITEM NUMBER: 31 ELIGIBLE EQUIPMENT

LINE ITEM NUMBER: 32

No of

COND UNITS UNIT

ACQUISITION PRICE

$600001 E A$

COST/UNIT:

TOTAL COST:

$\$ 1,440.00$

$\$ 1.440 .00$

.440 .00

DAVID MITCHELL

AIKEN

(803) $725-9540$

SC $<9802$

TO FREEZE EOUIPMENT.

DAVID MITCHELL

AIKEN

(803) $725-9540$

LOCATION OF EQUIPMENT:

WESTINGHOUSE

SAVANHAH RIVER SITE

AIKEN

SC 29802

600001 EA

COST/UNIT:
TOTAL COST:

$\$ 1,250.00$
$\$ 1,250.00$

FOR FURTHER INFORMATION:

DAVID MITCHELL

AIKEN

(803) $725-9540$

SC 29802

TO FREEZE EQUIPMENT :

DAVID MITCHELL

AIKEN $725-9540$

SC 29802
LOCATION OF EQUIPMENT:

WESTINGHOUSE

SAVANMAH RIVER SITE

AIKEN

SC 29802 
EFFECIIVE DATE: 07/01/94 EXPIRATION DATE: 07/31/94

\section{COOE EXCESS PROPERTY DESCRIPIION}

REFERENCE NUMBER : 1161

7025 PRINTER HP, SN-2336J15218

REPORT NUMBER: $894609-9091$

LINE ITEM NUMBER: 35

\section{REFERENCE NUMBER : 1162}

7025 PRINTER MFG: INTEFRAL DATA MODEL: 132 TYPE: MATRIX ACO DATE: $11 / 82$ REF NO: $53550-10$

REPORT NUMBER: $890531-4103$
No of

COND UNITS UNIT

ACQUISITION PRICE

50000 I EA

COST/UNIT:

$500001 E A$

COST/UNIT:
TOTAL COST:

$\$ 1,245.00$

FOR FURTHER INFORMATION:

NANCY 3 . SITTON

RICHLAND

(509) $376-5108$

WA 99352

TO FREEZE EQUIPMENT:

NANCY J. SITTON

RICHLAND

WA 99352

(509) $376-5108$

LOCATION OF EQUIPMENT :

WESTINGHOUSE HANFORD COMPANY

ESS\&S, 1167A BLDG $; 1100$ AREA

ESS\&S, 1167A BLDG; 1100 AREA
RICHLAND 99352 
EFFECTIVE DATE: 07/01/94 EXPIRATION DATE: $07 / 31 / 94$

\section{COOE EXCESS PROPERTY DESCRIPTION}

REFERENCE NUMBER : 1103

7025 PRINTER MFG: TALARIS SYSTEMS MOOEL: T810SNF ACO DATE: 9/85 REF NO: $53550-01$

REPORT NUMBER: $800531-4103$

LINE IIEM NUMBER: 0124

REFERENCE NUMBER : 1164

7025 PRINTER MFG: TALARIS SYSTEMS MODEL: TB1OSNF ACO DATE: $9 / 85$ REF NO: $53550-04$

REPORT NUMBER: $890531 \cdot 4103$

LINE ITEM NUMBER: 0127
No of

COND UNIIS UNIT ACOUISITION PRICE

$900001 \mathrm{EA}$

COST/UNIT:

IOTAL COST:

$\$ 2,965.00$

965.00

NRNCY J. SITTON
RICHLAND
(509) $376-5108$

WA 99352

TO FREEZE EQUIPMENT:

NANCY J. SITTON

RICHLAND

(509) $376-5108$

LOCATION OF EQUIPMENT:

WESTINGHOUSE HANFORD COMPANY

ESSES, 1167A BLDG/1100 AREA

RICHLAND

WA 99352

$500001 \mathrm{EA}$

COST/UNIT:

TOTAL COST:

$\$ 2,965.00$

$\$ 2,965.00$

FOR FURTHER INFORMATION:

NANCY J. SITTON

RICHLAND

WA 99352

(509) $376-5108$

TO FREEZE EOUIPMENT:

NANCY J. SITTON

RICHLAND

(509) $376-5108$

LOCATION OF EQUIPMENT:

WESTINGHOUSE HANFORD COMPANY

ESSES, 1167A BLOG/1100 AREA

RICHLAND

WA 99352 
EFFECTIVE DATE: $07 / 01 / 94$ EXPIRATION DATE: $07 / 31 / 94$

\section{FSC CODE EXCESS PROPERTY DESCRIPTION \\ No of}

REFERENCE NUMBER : 1165

7025 PRINTER MFR. DATA PRODUCTS, MOOEL SPG8072 PROPERTY \#X-16778
LINE ITEM NUMBER: 0014

\section{$400001 \mathrm{EA}$ COST/UNIT:}

REFERENCE NUMBER : 1166

7025 PRINTER MFR. LASER, MOOEL RASCOL II PROPERTY \#C4364

REPORT NUMBER: $898264-4130$
COND UNITS UNIT

ACQUISITION PRICE

$400001 \mathrm{EA}$

COST/UNIT: TOTAL COST :

\section{$\$ 1,355.00$}

$\$ 1,355.00$
CONTACT(S)

FOR FURTHER INFORMATION:

REBECCA FINCH

GRAND JUNCTION

(303) 248-6634

TO FREEZE EQUIPMENT :

REBECCA FINCH

GRAND JUNCTION

(303) 248-6634

CO $81502-5504$

LOCATION OF EQUIPMENT:

RUST GEOTECH

2597 B $3 / 4$ ROAD

GRAND JUHCTION

Co $81502-5504$

CO $81502-5504$

\section{$\$ 9.441 .00$
$\$ 9.441 .00$}

FOR FURTHER INFORMATION:

REBECCA FINCH

GRAND JUNCTION

(303) $248-6634$

CO $81502-5504$

TO FREEZE EQUIPMENT :

REBECCA FINCH

GRAND JUNCTION

(303) 248-6634

CO $81502-5504$

LOCATION OF EQUIPMENT:

RUST GEOTECH

2597 B 3/4 ROAD

GRAND JUNCTIION

CO 81502-5504 
DOE USED ENERGY RELATED LABORATORY EQUIPMENT GRANT PROGRAM

EFFECTIVE DATE: $07 / 01 / 94$ EXPIRATION DATE: $07 / 31 / 94$ FSC

\section{COOE EXCESS PROPERTY DESCRIPTION}

REFERENCE NUMBER : 1167

7025 PRINTER MFR. NEC, MOOEL 8815 PROPERTY \#X-4284

REPORT NUMBER : $898264-4130$

REFERENCE NUMBER : 1168

7025 PRINTER MFR. NEC, MODEL 7715 PROPERTY \#X-16302

REPORT NUMBER: $898264-4130$
ELIGIBLE EQUIPMENT
No of

COND UNITS UNIT

ACQUISITION PRICE

$700001 \mathrm{EA}$

COST/UNIT: TOTAL COST:

LINE ITEM NUMBER: 0005

$700001 \mathrm{EA}$

COST/UNIT:
TOTAL COST:

$\$ 2,083.00$

$\$ 2,083.00$

LINE ITEM NUMBER: 0013
$\$ 1,573.00$

$\$ 1,573.00$
FOR FURTHER INHORMATION:

REBECCA FINCH

(303) 248-6634

CO 81502-5504

TO FREEZE EQUIPMENT:

REBECCA FINCH

GRAND JUNCTION

(303) 248-6634

Co $81502-5504$

OCATION OF EQUIPMENT:

RUST GEOTECH

2597 B $3 / 4$ ROAD

GRAND JUNCTIION

CO $81502-5504$

FOR FURTHER INFORMATION:

REBECCA FINCH

GRAND JUNCTION

(303) $248-6634$

CO $81502-5504$

TO FREEZE EQUIPMENT:

REBECCA FINCH

GRAND JUNCTION

(303) 248-6634

CO $81502-5504$

OCATION OF EQUIPMENT :

RUST GEOTECH

2597 B 3/4 ROAD

GRAND JUNCTION

CO $81502-5504$ 
EFFECTIVE DATE: $07 / 01 / 94$ EXPIRATION DATE: $07 / 31 / 94$

FSC

CODE EXCESS PROPERTY DESCRIPTION

REFERENCE NUMBER : 1169

7025 PRINTER MFR. TEXAS INSTRUMENTS, MODEL T1810 PROPERTY \#S-10917

REPORT NUMBER: $898264-4130$

LINE ITEM NUMBER: 0004

REFERENCE NUMBER : 1170

7025 PRINTER MFR. TOSHIBA, MOOEL P351 PROPERTY \#X-12828

500001 EA

COST/UNIT:
TOTAL COST:

LINE ITEM NUMBER: 0006

NO OF

COND UNITS UNIT

400001 EA

COST/UNIT: TOTAL COST:

REPORT NUMBER : $898264-4130$
ACQUISITION PRICE

$\$ 1,735.00$

$\$ 1.735 .00$

REBECCA FINCH

(303) 248-6634

TO FREEZE EQUIPMENT :

REBECCA FINCH

GRAND JUNCTION

(303) 248-6634

LOCAYION OF EQUIPMENT:

RUST GEOTECH

2597 B 3/4 ROAD

GRAND JUNCTION

CO 81502-5504
$\$ 1,119.00$

$\$ 1,119.00$
FOR FURTHER INFORMATION:

REBECCA FINCH

GRAND JUNCTION

(303.2 248-663.4

TO FREEZE EQUIPMENT:

REBECCA FINCH

GRAND JUNCTION

CO $81502-5504$

(303) 248-6634

LOCATION OF EOUIPMENT:

RUST GEOTECH

2597 B 3/4 ROAD

GRAND JUNCTION

CO $81502-5504$ 
EFFECTIVE DATE: 07/01/94 EXPIRATION DATE: $07 / 31 / 94$

\section{FSC \\ COOE EXCESS PROPERTY DESCRIPTION}

REFERENCE NUMBER : 1171

7025 PRINTER MFR. TOSHIBA, MODEL P-341 PROPERTY \#X-12285

ELIGIBLE EQUIPMENT

No OF

COND UNITS UNIT

$700001 \mathrm{EA}$

ACQUISITION PRICE

\section{COSTIUNIT:
TOTAL COST:}

TOTAL COST:

LINE ITEM NUMBER: 0007

$400001 \mathrm{EA}$
COST/UNIT: TOTAL COST:
REFERENCE NUMBER : 1172
7025 PRINTER MFR. TOSHIBA, MODEL P351 PROPERTY \#X-13096

REPORT NUMBER: $898264-4130$
LINE ITEM NUMBER: 0008
$\$ 1,070.00$ $\$ 1,070.00$

\section{$\$ 1,159.00$ $\$ 1,159.00$}

(1)

$\underline{\operatorname{CONTACT}(S)}$

FOR FURTHER INFORMATION:

REBECCA FINCH

GRAND JUNCTION

(303) 248-6634

CO 81502-5504

TO FREEZE EQUIPMENT:

REBECCA FINCH

GRAND JUNCTION

(303) 248-6634

CO $81502-5504$

LOCATION OF EQUIPMENT:

RUST GEOTECH

2597 B 3/4 ROAD

GRAND JUNCTION

CO $81502-5504$

FOR FURTHER INFORMATION:

REBECCA FINCH

GRAND JUNCTION

(303) 248-6634

CO $81502-5504$

TO FREEZE EQUIPMENT:

REBECCA FINCH

GRAND JUNCTION

(303) 248-6634

CO $81502-5504$

LOCATION OF EQUIPMENT:

RUST GEOTECH

2597 B 3/4 ROAD

GRAND JUNCTION

CO $81502-550$ 
EFFECTIVE DATE: 07/01/94 EXPIRATION DATE: 07/31/94

FSC

COOE EXCESS PROPERTY DESCRIPTION

REFERENCE NUMBER : 1173

7025 PRINTER MFR. TOSHIBA, MODEL P-351 PROPERTY \#X-13287

REPORT NUMBER: $898264-4130$

LINE ITEM NUMBER: 0009

No of

COND UNITS UNIT

ACQUISITION PRICE

400001 EA

COST/UNIT:

TOTAL COST:

$\$ 1,398.00$

$\$ 1,398.00$

\section{REBECCA FINCH}

GRAND JUNCTION

Co $81502-5504$

(303) 248-6634

TO FREEZE EQUIPMENT:

REBECCA FINCH

GRAND JUNCTION

(303) 248-6634

LOCATION OF EQUIPMENT:

RUST GEOTECH

2597 B $3 / 4$ ROAD

GRAND JUNCTION

CO $81502-5504$
700001 EA COST/UNIT:

TOTAL COST:
$\$ 1,398.00$

$\$ 1,398.00$
FOR FURTHER INFORMATION :

REBECCA FINCH

GRAND JUNCTION

GRAND JUNCTION

CO $81502-5504$

TO FREEZE EQUIPMENT :

REBECCA FINCH

GRAND JUNCTION

(303) 248-6634

Co 81502-5504

LOCATION OF EQUIPMENT:

RUST GEOTECH

2597 B 3/4 ROAD

GRAND JUNCTION

CO 81502-5504 
EFFECTIVE DATE: 07/01/94 EXPIRATION DATE: $07 / 31 / 94$
FSC

\section{CODE EXCESS PROPERTY DESCRIPTION}

REFERENCE NUMBER : 1175
7025 PRINTER, HP LASERJET PLUS, MDL 2686A, S/N 2602J65810, TYPE 15980, MFG $10 / 86$
ELIGIBLE EQUIPMENT
NO OF

COND UNITS UNIT

$600001 \mathrm{EA}$

COST/UNIT:

TOTAL COST

$\$ 2,677.00$

$\$ 2,677.00$
REPORT NUMBER: $892401-4080$

LINE ITEM NUMBER: 0045
REFERENCE NUMBER : 1176

7025 PRINTER, HP LASERJET PLUS, MDL 2686A, S/N 2602J65804, MFG 10/86
$600001 E A$

$\operatorname{COST/UNIT:}$

TOTAL COST
$\$ 2,677.00$

$\$ 2,677.00$
FOR FURTHER INFORMATION:

JENNIFER IMHOFF

WEST MIFFLIN

(412) $476-5328$

PA $15122-0109$

TO FREEZE EQUIPMENT:

JENN I FER IMHOFF

WEST MIFFLIN

(412) $476-5328$

PA 15122-0079

LOCATION OF EOUIPMENT:

BETIIS ATOMIC POWER LABORATORY

814 PITTSBURGH/MCKEESPORT BLVD

WEST MIFFLIN

PA 15122-0079 
EFFECTIVE DATE: $07 / 01 / 94$ EXPIRATION DATE: $07 / 31 / 94$ ELIGIBLE EQUIPMENT

FSC

7025 PRINTER, HP LASERJET MDL 2686A, S/N 2602J65806, MFG 10/86
COOE EXCESS PROPERTY DESCRIPTION

REFERENCE NUMBER : 1177

NO OF

COND UNITS UNIT

ACQUISITION PRICE

$400001 \mathrm{EA}$

\section{COST/UNIT:}

TOTAL COST:

\section{$\$ 2,677.00$}

$\$ 2,677.00$

REPORT NUMBER: $892401-4080$

LIME ITEM NUMBER: 0060

REFERENCE HUMBER : 1178

7025 PRINTER, HP MDL 2671G, S/N 2342A17617

REPORT NUMBER: $892401 \cdot 4080$

INE ITEM NUMBER: 0019

\section{EA \\ COST/UNIT:
TOTAL COST:}

$\$ 1,640.00$
$\$ 1,640.00$

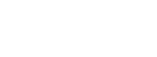

CONTACT(S)

FOR FURTHER INFORMATION:

JENHIFER IMHOFF

WEST MIFFLIN

PA 15122-0109

(412) $476-5328$

TO FREEZE EQUIPMENT:

JENWIFER IMHOFF

WEST MIFFLIN

(412) $476-5328$

PA 15122-0079

LOCATION OF EOUIPMENT:

BETTIS ATOMIC POWER LABORATORY

814 PITTSBURGH/MCKEESPORT BLVD

WEST MIFFLIN

A $15122-0079$

FOR FURTHER INFORMATION:

JENM I FER IMHOF $F$

WEST MIFFLIN

PA 15122-0109

(412) $476-5328$

TO FREEZE EOUIPMENT:

JENNI FER IMHOF

UESI MIFFLIN

(412) $476-5328$

PA 15122-0079

LOCATION OF EQUIPMENT:

BETTIS ATOMIC POWER LABORATORY

814 PITTSBURGH/MCKEESPORT BLVD

WEST MIFFLIN

PA 15122-0079 
EFFECTIVE DATE: 07/01/94 EXPIRATION DATE: $07 / 31 / 94$

FSC

\section{COOE EXCESS PROPERTY DESCRIPTION}

REFERENCE NUMBER : 1179

7025 PRINTER, LASERJET MFG. HP MODEL \# HP2686A SERIAL \#2550J14560 EGSG $10 \quad 010914-00$

YEAR ACO 1986 DOE \#00005209.00

REPORT NUMBER: $898289-4049$

LINE ITEM NUMBER: 1128

\section{REFERENCE NUMBER : 1180}

7025 PROPRINTER II 1590 PO. MANUF: IBM MOOEL N/A S/N N/A

1 LOT CONSISTING OF 6 EACH SIZE: $16^{\prime 1 \mathrm{~W}} \times 12^{\prime \prime} / \mathrm{F} / \mathrm{R} \times 5^{\prime 1 H}$ EST WT: 5 LBS

MFGR YR: N/A
No of

COND UNITS UNIT

ACOUISITION PRICE

$4 \quad 00001$ EA

COST/UNIT:

IOTAL COST:

$\$ 2,006.65$

$\$ 2,006.65$

G.A. ELVEY
GOLDEN

(303) $465-3765$

CO 80402-0464

TO FREEZE EOUIPMENT:

G.A. ELVEY

GOLDEN

(303) $465-3765$

LOCATION OF EQUIPMENT.

EGEG ROCKY FLATS, INC.

BUILDING 0ZO-BROAMFIELO FACILITY

BROOMFIELD

CO 80020 $\begin{array}{rrr}400001 \mathrm{LT} & \text { COST/UNIT: } & \$ 1,800.00 \\ & \text { TOTAL COST: } & \$ 1,800.00\end{array}$

$\$ 1,800.00$

FOR FURTHER INFORMATION :

BARBARA HOLMES, REP.

KANSAS CITY

(816) $997-2976$

MO $64141-6159$

IO FREEZE EOUIPMENT:

BARBARA HOLMES, REP.

KAMSAS CITY

(816) 997-2976

LOCATION OF EOUIPMENT:

ALLIEDSI GNAL, INC

2000 EAST 95IH STREET

KANSAS CITY 
EFFECTIVE DATE: 07/01/94 EXPIRATION DATE: 07/31/94

\section{COOE EXCESS PROPERTY DESCRIPTION \\ REFERENCE MUMER : 1181 \\ 7025 RT GRAPHICS LORKSTATION BOX 82845, MANUF: IBM MODEL 5085 O2A S/N $73440 \mathrm{~mm}$ \\ No of \\ COND UNITS UNIT} 1 EACH. SIZE: $22^{\prime \prime} W \times 22^{\prime \prime F} / R \times 13^{\prime \prime H}$ EST UT: 10 LBS MFGR YR: 1988

REPORT MUMBER: $896404-4126$

LINE ITEM NUMBER: 0002

REFERENCE NUMBER : 1182

7025 SCS1, CARD/1000, HP 1T62-0002, 12016-80005, $12 \times 8 \times 1 / 2^{\prime \prime}$

REPORT MUMBER: $892401-4080$

LINE ITEM MUNBER: 0020
$400001 \mathrm{EA}$

ACOUISIIIION PRICE

COST/UNIT: TOTAL COST:

$\$ 10,037 . \mathrm{mn}$

$\$ 10,037.00$

KAMSAS CITY

$14064141-6159$

TO FREEZE EOUIPMENT:

BARBARA HOLMES, REP.

KARBARA HOLY CITY

(816) $997-2976$

M0 64141-6159

LOCATION OF EOUIPAENT:

ALLIEDSIGMAL, INC

2000 EAST 95 IH STREE

KANSAS CITY

mo 64131-3095

$\$ 2,000.00$

$\$ 2,000.00$
FOR FURTHER INFORMATION :

JENWIFER IMHOFF

WEST MIFFLIN

(412) $476-5328$

PA 15122-0109

TO FREEZE EQUIPMENT:

JEMNIFER IMHOFF

LEST MIFFLIN

(412) $476-5328$

PA 15122-0079

LOCATION OF EQUIPMENT:

BETIIS ATOAIC POAER LABORATORY

814 PIITSBURGH/MCKEESPORT BLV

UESI MIFFLIN

PA 15122-0079 
EFFECTIVE DATE: 07/01/94 EXPIRATION DATE: $07 / 31 / 94$ ELIGIBLE EOUIPMENT

\section{FSC \\ COOE EXCESS PROPERTY DESCRIPTION}

REFERENCE MUMBER : 1183

7025 SYSTEM SUN 427 TAPE DRIVE, SN-902F1444 CPU, SN-851H0819, MONITOR, KEYBOARD AND MOUSE
No of

COND UNITS Unit

ACOUISITION PRICE

$4 \quad 00001 \mathrm{EA}$

COST/UNIT:

TOTAL COST:

$\$ 13,994.00$

$\$ 13,994.00$
LINE ITEM MUMBER: 65

$500001 \mathrm{EA}$

\section{COST/UNIT: \\ TOTAL COST:}

$\$ 1,752.00$
$\$ 1.752 .00$
CONTACT(S)

FOR FURTHER INFORMATION:

DAVID MITCHELL

AIKEN

SC 29802

(803) $725-9540$

IO FREEZE EOUIPMEMT:

DAVID MITCHELL

AIKEN

(803) $725-9540$

SC 29802

LOCATION OF EOUIPMENT :

MESTINGHOUSE

SAVAMEMAH RIVER SITE

AIKEN

SC 29802

7025 TABLET MFG: HEWLETT PACKARD MOOEL: 9111A ACO DATE: 12/83 REF MO: $53550-09$

REPORT NUMBER: $890531-4103$

LINE ITEM MUmBER: 0132
FOR FURTHER IMFORMATION:

NAMCY J. SITTOW

RICHLAMD

(509) 376.5108

WA 99352

TO FREEZE EQUIPMENT:

MANCY $J$. SITTOW

RICHLAMD

(509) 376.5108

LOCATION OF EQUIPMEMT:

WESTINGHOUSE HANFORD COMPANY

ESS\&S, 1167A BLDG/1100 AREA

RICHLAND 
EFFECTIVE DATE: 07/01/94 EXPIRATION DATE: 07/31/94

FSC

\section{CODE EXCESS PROPERTY DESCRIPIION}

REFEREMCE MUMBER : 1185

7025 TAPE BACKUP EXIERMAL BACK UP IAPE DRIVE FOR COMPUTERS, DRIVE USES DATA

CARTRI DGE 120VAC, $60 \mathrm{HZ}$ MAYMARD, MS $1101 \mathrm{~A}, 62002000$.

REPORT MUMBER: $898355-4096$

LINE ITEM MUMGER: 0018

\section{REFERENCE MUMBER : 1186}

7025 TAPE BACKUP EXTERMAL BACK UP TAPE DRIVE FOR COMPUTERS. DRIVE USES DATA 7 D0001 EA

CARTR IOGE. IOOVAC, $60 \mathrm{HZ}$. MAYMARD, MS1101A, 61902000 mo of

COMD umits unit

ACQUISITIOU PRICE

COST/AMIT:

TOTAL COST:

00001 EA

$\$ 1,050.00$

FOR FURTHER INFORMATION:

ELOY N. GIRON

ALBUOUEROUE

(505) $844-2746$

87185

TO FREEZE EOUIPMENT:

ELOY N. GIROW

AL BUOUEROUE

(505) $844-2746$

47185

LOCATION OF EOUIPMENT:

ELOY N. GIROW

PROPERTY REAPPLICAIION DIV. 7617

PROPERTY REAPPLICATION DIV.
ALBUDUEROUE

COST/UNIT:

TOTAL COST:
$\$ 1,050.00$

$\$ 1,050.00$

FOR FURTHER IMFCRMATION

ELOY N. GIROM

MLBUAUEROU:

(505) $844-2746$

87185

TO FREEZE EOUIPMENT:

ELOW N-GIRON

ALBUAUEROUE

(505) $846-2746$

87185

LOCATION OF EOUIPMENT:

ELOY M. GIROW

PROPERTY REAPPLICATION DIV. 7617

PROPERTY REAPPLICATION DIV. P617 87123 
DOE USED EMERGY RELATED LABORATORY EOUIPMENT GRANT PROGRAM ELIGIBLE EOUIPMENT

EFFECTIVE DATE: 07/01/94 EXPIRATION DATE: 07/31/94

\section{COOE EXCESS PROPERTY DESCRIPTION}

REFERENCE MUMBER : 1187

7025 IAPE DRIVE EXTERNAL TAPE DRIVE FOR AN IBM PC, USES A TAPE CARTRIDGE.

120VAC, 60 HZ. 6157002, SER. 2603896.

REPORT NUMBER : $898355-4096$

LINE ITEM MUMBER: 0016

REFERENCE MUMBER : 1188

LONG, 1600 OR 6250 BPI, 9 TRACK D 400540 EA

TAPE, MAGNETIC, $1 / 2$ TAPES PER CARTON, 36 CARTON ON SKID, 1600LB

REPORT MUMBER: $892401 \cdot 4080$

LINE ITEM NUMBER: 0030

$600001 \mathrm{EA}$
NO of

COND UNITS UNIT

ACOUISITION PRICE

COST/UNIT:
TOTAL COST:

$\$ 1,320.00$

$\$ 1,320.00$

\section{ELOY N. GIRO
ALBUQUEROUE}

(505) $84,4-2746$

87185

TO FREEZE EQUIPMEMT:

ELOY N. GIROM

ALBUOUEROUE

(505) $844-2746$

MM 87185

LOCATION OF EQUIPMENT:

ELOY N. GIRON

PROPERTY REAPPLICATION OIV 7617

PROPERTY REAPPLICATION DIV. 7617

Min 87123
COST/UNIT:

TOTAL COST:

$\$ 10.00$ $\$ 5,400.00$
FOR FURTHER IMFORMATION

JENMI IFER IMHOFF

WEST MIFFLIN

(412) $476-5328$

PA 15122-0109

TO FREEZE EQUIPMENT:

JENWI FER IMHOFF

LEST MIFFLIN

PA 15122-0079

(412) $476-5328$

LOCATION OF EOUIPMENT:

BETTIS ATOMIC POAER LABORATORY

B14 PITTSBURGH/MCKEESPORT BLVD

814 PITTSBURGH/MCKEESPORT BLV $15122-0079$ 
EFFECTIVE DATE: $07 / 01 / 94$ EXPIRATION DAIE: $07 / 31 / 94$

FSC

\section{COOE EXCESS PROPERTY DESCRIPTION}

DEFERENCE MUMBER : 1189

7025 TERMIMAL MFG: SELENAR MOOEL: 100XL TYPE: VIDEO ACO DATE: $3 / 86$ REF NO: $53550-14$

REPORT MLABER: $890531-6103$
LINE ITEM MUMBER: 0121

\section{no of \\ COND UNITS UNIT}

$5 \quad 00001 \mathrm{EA}$ COST/UNIT:
TOTAL COST:
REFERENCE MUMBER : 1190

7025 TERMIMAL MFG: TEKTRONIX MODEL: CT8500 ACO DATE: 12/83

REF NO: $53550-15$
$500001 \mathrm{EA}$ COST/UNIT:
TOTAL COST

LIME ITEM MUMBER: 0122
REPORT NUMBER: $890531-4103$

\section{ACQUISITIOA PRICE}

$\$ 1,540.00$
MANCY J. SITTOW

RICHLANO

(509) $376-5108$

HA 99352

TO FREEZE EOUIPMENT:

MANCY J. SITTOW

RICHLAND

(509) $376-5108$

LOCATION OF EOUIPMENT

WESTIMGHOUSE HANFORD COMPANY

ESS\&S, 1167A BLDG/1100 AREA

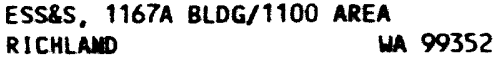

$\$ 1,890.00$

FOR FURTHER IMFORMATION :

MANCY J. SITTON

(509) $376-5108$

HA 99352

TO FREEZE EQUIPMEMT:

NANCY J. SITTON

RICHLAND

(509) 376-5108

LOCATION OF EOUIPMENT:

LESTINGHOUSE HANFORD COMPANY

ESS\&S, 1167A BLDG/1100 AREA

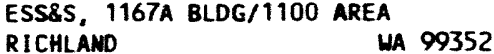


EFFECTIVE DATE: $07 / 01 / 94$ EXPIRATION DATE: $07 / 31 / 94$

\section{COOE EXCESS PROPERTY DESCRIPTION}

REFERENCE MUMBER : 119

7025 TERMIMAL MFG: TEKTRONIX MODEL: 4107 TYPE: VIDEO ACO DATE: 2/85 REF NO: 29764-01

REPORT NUMBER: $890531-4103$ ELIGIBLE EQUIPMENT

NO OF

COND UNITS UNIT

$500001 \mathrm{EA}$

\section{ACQUISITION PRICE}

COST/UNIT: TOTAL COST:

LINE ITEM NUMBER: 0123

REFERENCE NUMBER : 1192

7025 TERMINAL MFR: DEC, MODEL VT100-AA, S/N ABAI514, ACQ. DATE: 4/85, TERMINAL MFR: DEC, MOOEL VT 100-AA, S/N ABAI
APPROX. WT: 30 LBS., $18^{\prime \prime} \times 15^{\prime \prime L} \times 15^{\prime \prime}$.

$600001 \mathrm{EA}$
COST/UNIT: TOTAL COST:
$\$ 2,977.00$

$\$ 2.977 .00$
FOR FURTHER INFORMATION:

JANET F. BROOKS

LIVERMORE

(510) $294-2186$

TO FREEZE EQUIPMENT:

JANET F. BROOKS

LIVERMORE

(510) $294-2186$

LOCATION OF EQUIPMENT:

SAMDIA MATIOMAL LABOPATORIES

SANOIA MATIOMAL LABORATORIES

PROPERTY REAPPLICATION DEPT. 8532-1 
EFFECTIVE DATE: 07/01/94 EXPIRATION DATE: $07 / 31 / 94$

FSC

\section{COOE EXCESS PROPERTY DESCRIPIION}

REFERENCE NUMBER : 1193

7025 TERMINAL MFR: DEC, MODEL VT 100-AA, S/N ABU3601, ACQ. DATE: 4/86, APPROX. WT: 30 LBS., $18^{\prime \prime} \mathrm{W} \times 15^{\prime \prime L} \times 15^{\prime \prime} \mathrm{H}$.

REPORT NUMBER: $899105-4083$

LINE I IEM NUMBER: 0063

REFERENCE NUMBER : 1194

7025 TERMINAL MFR: DEC, MODEL VT240-B, S/N AB70902E13, ACQ. DATE: 6/87, APPRO $X$. WT: 60 LBS., $18^{\prime \prime W} \times 15^{\prime \prime L} \times 16^{\prime \prime H}$.

$400001 \mathrm{EA}$

COST/UNIT:

OTAL COST:

$\$ 1.935 .00$

$\$ 1,935.00$

FOR FURTHER INFORMATION:

ANET F. BROOKS

IVERMORE

CA $94550-0969$

TO FREEZE EQUIPMENT :

JANET $F$. BROOKS

(510) $294-2186$

CA $94550-0969$

ANET $F$. BROOKS

(510) $294-2186$

JAMET $F$. BROOKS

(510) $294-2186$

CA $94550-0969$

EQUIPMENT

NATIONAL LABORATORIES

PROPERTY REAPPLICATION DEPT. 8532-

LIVERMORE

CA $94550-0969$

LOCATION OF EQUIPMENT :

SANDIA NATIONAL LABORATORIES

PROPERTY REAPPLICATION DEPT. 8532-1

LIVERMORE

CA $94550-0969$ 
EFFECTIVE DATE: 07/01/94 EXPIRATION DATE: $07 / 31 / 94$

\section{COOE EXCESS PROPERTY DESCRIPTION}

REFERENCE NUMBER : 1195

7025 TERMINAL MFR: DEC, MODEL VS240-A, S/N AB42601RK1, ACQ. DATE: 11/87, APPR OX. WT: 40 LBS., 18"W X 14"L X 14"H.

REPORT NUMBER: $899105-4083$

LINE ITEM NUMBER: 0065

REFERENCE NUMBER : 1196
7025 TERMINAL MFR: DEC, MODEL VT100-AA, S/N ABM3453, ACO. DATE: $8 / 82$.

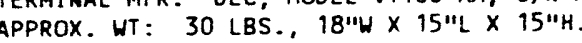

$400001 E A$

COST/UNIT:

TOTAL COST:

$\$ 1,505.00$

No of COND UNITS UNIT COST/UNIT:
TOTAL COST:

$\$ 1,400.00$ $\$ 1,400.00$

(1)

FOR FURTHER INFORMATION:

JANET $F$. BROOKS

LIVERMORE

CA $94550-0969$

(510) $294-2186$

TO FREEZE EQUIPMENT:

JANET F. BROOKS

LIVERMORE

(510) $294-2186$

CA $94550-0969$

LOCATION OF EQUIPMENT

IA NATIONAL LABORATORIES

PROPERTY REAPPLICATION DEPT. $8532-1$

LIVERMORE

CA $94550-0969$ 
EFFECTIVE DATE: $07 / 01 / 94$ EXPIRATION DATE: $07 / 31 / 94$

\section{FSC \\ COOE EXCESS PROPERTY DESCRIPIION}

REFERENCE MUMBER : 1197

7025 TERMINAL MFR: DEC, MOOEL VT100-AA, S/N ABS7496, ACQ. DATE: $10 / 83$, APPROX. WT: 25 LBS. 18 "W X 15"L $\times 15 " \mathrm{H}$.

REPORT NUMBER: $899105-4083$

LIME ITEM NUMBER: 0067

REFERENCE NUMBER : 1198

7025 TERMINAL MFR: DEC, MODEL VT100-AA, S/N WF70930, ACQ. DATE: $3 / 83$, TERMINAL MFR: 25 LBS. 18 "H X $15^{\prime \prime L} \times 15^{\prime \prime H}$. COST/UNIT: TOTAL COST:

LINE ITEM NLMBER: 0068

COND UNITS UNIT

TOTAL COST:
No of

ACQUISITION PRICE

$\$ 1,515.00$

$\$ 1,515.00$

FOR FURTHER INFORMATION:

JANET F. BROOKS

LIVERMORE

(510) $294-2186$

CA $94550-0969$

TO FREEZE EQUIPMENT :

JANET F. BROOKS

LIVERMORE

(510) $294-2186$

CA $94550-0969$

LOCATION OF EQUIPMENT:

SANDIA NATIONAL LABORATORIES

PROPERTY REAPPLICATION DEPT. $8532-1$

PROPERTY REAPPLICATION DEPT. 8532-1
LIVERMORE CAA $94550-0969$

REPORT NUMBER: $899105-4083$ 


\section{COOE EXCESS PROPERTY DESCRIPTION}

REFERENCE NUMBER : 1199 7025 TERMINAL MFR: TEKTRONIX, MODEL 4105, S/N B026956, ACQ. DATE: $5 / 84$ APPRO $X$. WT: 40 LBS., $17^{\prime \prime} \mathrm{W} \times 20^{\prime \prime L} \times 14^{\prime \prime} \mathrm{H}$

REPORT NUMBER: $899105-4083$

LINE ITEM NUMBER: 0069

REFERENCE NUMBER : 1200

7025 TERMINAL MFR: TEKTRONIX, MOOEL 4025A, S/N B066575, ACQ. DATE: 10/82,

APP ROX HT. 50 LBS. . 18"W $\times 20^{\prime \prime L} \times 13^{\prime \prime H}$.

REPORT NUMBER: $899105-4083$

LINE ITEM NUMBER: 0070
NO OF

COND UNITS UNIT

ACQUISITION PRICE

$4 \quad 00001 \mathrm{EA}$

COST/UNIT:

TOTAL COST:

$\$ 2,999.00$

$\$ 2,999.00$
CONTACT(S)

FOR FURTHER INFORMATION:

JANET $F$. BROOKS

CA $94550-0969$

(510) $294-2186$

TO FREEZE EQUIPMENT:

JANET F. BROOKS

(510) 294-2186

CA $94550-0969$

LOCATION OF EQUIPMENT:

SANDIA NATIONAL LABORATORIES

PROPERTY REAPPLICATION DEPT. $8532-1$

LIVERMORE

CA $94550-0969$

$\$ 4,868.00$

FOR FURTHER INFORMATION:

JANET F. BP.OOKS

CA $94550-0969$

TO FREEZE EQUIPMENT:

JANET $F$. BROOKS

LIVERMORE

(510) $294-2186$

CA $94550-0969$

LOCATION OF EQUIPMENT:

SANDIA NATIONAL LABORATORIES

PROPERTY REAPPLICATION DEPT $8532-1$

LIVERMORE

CA $94550-0969$ 


\section{EFFECIIVE DATE: $07 / 01 / 94$ EXPIRATION DATE: $07 / 31 / 94$}

FSC

\section{COOE EXCESS PROPERTY DESCRIPTION}

REFERENCE NUMBER : 120

ORKSTATION, HAS A 19" DIAGONAL COLOR SCREEN WITH A DEG AUSS CONTROL HAS A STD KEYBRD. 12OVAC, $60 \mathrm{HZ}$. DEC, VSSXBAA, SER. HY853011.

REPORT NUMBER: $898355-4096$

LINE ITEM NUMBER: 0021

REFERENCE NUMBER : 1202

7025 VECTRA, HP RS/25C, INTEL 386/25, MDL D2020A, S/N 2925A03654, CONF 02060 A , MFG 6/89, $20 \times 8 \times 20^{\prime \prime}, 8 L B$

400001 EA TOTAL COST:

6 OONOE EA COST/UNIT: TOTAL COST:

REPORT NUMBER: $892401-4080$
INE ITEM NUMBER: 0026

\section{UNIIS UNIT ACOUISITION PRICE CONTACTSS}

$\$ 2,000.00$

$\$ 2,000.00$

(1)

TO FREEZE EQUIPMENT :
ELOY N. GIRON

ELOY N. GIRON

(505) $844-2746$

LOCATION OF EQUIPMENT:

ELOY N. GIRON
PROPERTY REAPPLICATION DIV. 7617

ALBUQUERQUE

NM 87123
$\$ 3,500.00$

$\$ 3,500.00$
FOR FURTHER INFORMATION:

JENNIFER IMHOFF

WEST MIFFLIN

(4i2) $476-5328$

PA 15122-0109

TO FREEZE EQUIPNENT :

JENNIFER IMHOFF

WEST MIFFLIN

(412) $476-5328$

PA 15122-0079

LOCATION OF EQUIPMENT:

BETTIS ATOMIC POWER LABORATORY

814 PIITSBURGH/MCKEESPORT BLVD

WEST MIFFLIN

PA 15122-0079 
EFFECTIVE DATE: 07/01/94 EXPIRATION DATE: $07 / 31 / 94$

FSC

COOE EXCESS PROPERTY DESCRIPTION

REFERENCE NUMBER : 1203

7025 VECIRA, HP RS/20, INTEL 386,20, MDL D $1612 \mathrm{~A}$, S/N 2824A06286, MFG 8/88, 20400001 EA $\times 8 \times 2 O^{\prime \prime}, 20 \mathrm{LB}$

REPORT NUMEER: $892401-4080$

LINE ITEM NUMBER: 0027

REFERENCE NUMBER : 1204

7025 VECTRA, HP RS/25C, INTEL 386/25, MDL D2020A, S/N 2925A03670, MFG 6/89,
$20 \times 8 \times 20^{\circ}, 20 L B$

REPORT NUMBER: $892401-4080$

LINE ITEM NUMBER: 0028
UNIIS UNIT ACOUISITION PRICE CONTACTSS)

\section{COST/UNIT: $\quad \$ 3,500.00$}

TOTAL COST:

$\$ 3,500.00$

OR FURTHER INFORMATION:

JENNIFER IMHOFF

UEST MIFFLIN

PA 15122-0109

(412) $476-5328$

TO FREEZE EQUIPMENT :

JENNI FER IMHOFF

WEST MIFFLIN

PA 15122-0079

(412) $476-5328$

LOCATION OF EQUIPMENT:

BETTIS ATOMIC POWER LABORATORY

814 PITTSBURGH/MCKEESPORT BLVD

WEST MIFFLIN

PA $15122-0079$
COST/UNIT:

TOTAL COST:

$\$ 3,500.00$

$\$ 3,500.00$
FOR FURTHER INFORMATION:

JENNIFER IMHOF

WEST MIFFLIN

PA 15122-0109

TO FREEZE EQUIPMENT:

JENN I FER IMHOF

WEST MIFFLIN

(412) $476-5328$

PA 15122-0079

LOCATION OF EQUIPMENT:

BETTIS ATOMIC PONER LABORATORY

814 PITTSBURGH/MCKEESPORT BLVD

WEST MIFFLIN

PA 15122-0079 
EFFECTIVE DAIE: $07 / 01 / 94$ EXPIRATION DATE: $07 / 31 / 94$

\section{FSC}

COOE EXCESS PROPERTY DESCRIPTION

REFERENCE MUMBER : 1205

7025 VECTRA, HP RS/20, INTEL 386/20, MDL D1612A, S/N 2824A04417, MFG 6/88, 20 $\times 8 \times 20{ }^{\prime \prime}, 20 \mathrm{LB}$

REPORT NUMBER: $892401-4080$

LINE ITEM NUMBER: 0029

\section{REFERENCE MUMBER : 1206}

MRT STATION SHOWS 16 COLOR FROM A PALETTE OF 4096, 1376 $\times 1024$

ADDRESSABILITY 19" DISPLAY 4 MBYTES SYSTEM MEMORY. 120VAC, $60 \mathrm{HZ}$.

TEKTRONIX, $4317 / 04$, SER. BO40435.

REPORT NUMBER: $898355-4095$

LINE ITEM NUMBER: 0059

No of

COND UNITS UNIT

ACQUISITION PRICE

COST/UNIT:

TOTAL COST:

$\$ 3,500.00$

$\$ 3,500.00$

(4)

WEST
TO FREEZE EQUIPMENT:
JEWHIFER IMHOF
WEST MIFFLIN

(412) $476-5328$

PA 15122-0079

LOCATION OF EQUIPMENT:

BETTIS ATOMIC ROUER LABDORATORY

814 PITTSEURGH/MEKEESPORT BLVD

WEST MIFFLIN

PA 15122-0079

$5 \quad 00001$ EA

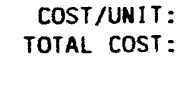

COST/UNIT:
FOR FURTHER INFORMATION:

ELOY N. GIRON

ALBUQUERQUE

(505) $844-2746$

NM 87185

TO FREEZE EQUIPMENT:

ELOY N. GIRO

ALBUIBUEROUE

(505) 844-2746

87185

LOCATION OF EQUIPMENT:

SAMDIA NATIONAL LABORATORIES

PROPERTY REAPPLICATION DIVISION 7617

ALBUQUERQUE NM 87123 
EFFECTIVE DATE: $07 / 01 / 94$ EXPIRATION DATE: $07 / 31 / 94$

FSC

COOE EXCESS PROPERTY DESCRIPIION

REFERENCE MUMBER : 1207

7035 PRINTER MFR: NEC, MODEL NO. PINURITER P7, S/N 501194495, ACQ. DATE: PRINTER MFR: NEC, MODEL NO. PINURITER P7, S/N
8/8 7. APPROX. WT: 30 LBS., $24^{\prime} W \times 14^{\prime} \mathrm{L} \times 7^{\prime} \mathrm{H}$.

REPORT NUMBER: $899105-4082$

LINE ITEM NUMBER: 0036

REFERENCE NUMBER : 1208

7035 PRINTER MFR: A PPROX. WT: 40 LBS., $17 . \mathrm{W} \times 21$. $\mathrm{L} \times 7 \% \mathrm{H}$.

REPORT NUMBER : 899105-4082
NO OF

COND UNITS UNIT

ACQUISITION PRICE

$400001 \mathrm{EA}$

COST/UNIT:

TOTAL COST:

$\$ 1,200.00$

$\$ 1,200.00$

(2)

\section{TO FREEZE EQUIPMENT: \\ LYNN E. SHACKELFOOT \\ LIVERMORE}

(510) $294-3067$

LOCATION OF EQUIPMENT:

SANDIA MATIONAL LABORATORIES

PROPERTY REAPPLICATION DEPT 8532-1

LIVERMORE

CA $94550-0969$
COST/UNIT: TOTAL COST:
$\$ 3,412.00$

$\$ 3,412.00$
FOR FURTHER INFORMATION:

IYNM E. SHACKELFOOT

LIVERMORE

(510) $294-3067$

CA $94550-0969$

TO FREEZE EQUIPMENT:

LYMN E. SHACKELFOOT

LIVERMORE

CA $94550-0969$

LOCATION OF EQUIPMENT:

SAMDIA MATIOMAL LABORATORIES

PROPERTY REAPPLICATION DEPT. 8532-1

LIVERMORE

CA $94550-0969$ 
EFFECIIVE DATE: $07 / 01 / 94$ EXPIRATION DATE: $07 / 31 / 94$

COOE EXCESS PROPERTY DESCRIPTIOH.

REFERENCE NUMBER : 1209

7035 PRINTER MFR: TEXTRONIX, MODEL NO $4612,5 / N$ B010538, ACO. DATE: $5 / 82,60001$ EA A PPROX. WT: 50 LBS.: $17 . \mathrm{W} \times 21 . \mathrm{L} \times 7 . \mathrm{H}$

REPORT NUMBER: $899105-408$

LINE ITEM NUMBER: 0039

REFERENCE NUMBER : 1210

7035 PRINTER MFR: IEXTRONIX, MODEL NO. 4612, S/N B102274, ACQ. DATE: 9/84, 4 O0001 EA A PPROX. WT: 50 LBS., $171 \mathrm{~W} \times 211 \mathrm{~L} \times 7 . \mathrm{H}$.

REPORT MUMBER: $899105-4082$

LINE ITEM MUMBER: 0040

COND UNITS UNIT
No of

ACQUISITION PRICE

COST/UNIT:

TOTAL COST:

$\$ 3.543 .00$

53.53 .00

$$
\begin{aligned}
& \text { LYNN E. } \\
& \text { LIVERMORE } \\
& \text { (510) } 294-3
\end{aligned}
$$

(510) $294-3067$

TO FREEZE EOUIPMENT

LYMN E. SHACKELFOOT

LIVERMORE

(510) $294-3067$

LOCATION OF EQUIPMENT:

SAMDIA MATIONAL LABORATORIES

SAMDIA MATIONAL LABORATORIES

LIVERMORE

CA $94550-0969$
COST/UNIT:

TOTAL COST:
$\$ 2,995.00$
$\$ 2,995.00$
FOR FURTHER INFORMATION:

LYWM E. SHACKELFOOT

LIVERMORE

(510) $294-3067$

CA $94550-0969$

TO FREEZE EOUIPMENT:

LYMM E. SHACKELFOOT

LIVERMORE

(510) $294-3067$

CA $94550-0969$

LOCATIOA OF EOUIPMENT

SAMDIA MATIOMAL LABORATORIES

PROPERTY REAPPLICATION DEPT B532-1

LIVERMORE

CA $94550-0969$ 
EFFECTIVE DATE: $07 / 01 / 94$ EXPIRATION DATE: $07 / 31 / 94$

FSC

COOE EXCESS PROPERTY DESCRIPTION

COMD UWITS UNIT

REFERENCE NUMBER : 1211

RE35 PRINIER MFR: IEXTRONIX, MOOEL NO 4612, S/N 8011970, ACQ. DATE: $2 / 84,4$ 00001 EA A PPROX. WT: $50 \mathrm{LBS} ., 17 . \mathrm{W} \times 211 \mathrm{~L} \times 7 \% \mathrm{H}$.

REPORT MUMBER: $899105-4082$

LINE ITEM NUMBER: 0041

REFERENCE NUMBER : 1212

7035 PRINTER MFR: TEXTRONIX, MOOEL NO. 4612, S/N B011997. ACE. DATE: 4/86, 4 00001 EA A PPROX. WT: $50 \mathrm{LBS}$., $17 . \mathrm{W} \times 21 . \mathrm{L} \times 7 . \mathrm{H}$.

REPORT NUMBER: $899105-4082$

LINE ITEM MUMBER: 0042
ACOUISITION PRICE
COST/UNIT:

TOTAL COST:

$\$ 2,995.00$
$\$ 2,995.00$

and

CONTACt(S)

FOR FURTHER IMFORMATION:

LYMUE E. SHACKELFOOT

LIVERMORE

(510) $294-3067$

CA $94550-0969$

TO FREEZE EQUIPHENT:

LYMN E. SHACKEL FOOT

LIVERMORE

(510) $294-3067$

LOCATIOW OF EQUIPMENT:

SANDIA MUTIOMAL LABORATORIES

PROPERTY REAPPLICATIOA OEPT 8532-1

LIVERMORE

ca $94550-0960$

COST/UNIT:

TOTAL COST:

$\$ 2.995 .00$

FOR FURTHER IMFORMATION:

LYMU: E. SMACKEL FOOI

LIVERMORE

(510) $294-3067$

Ca $94550-0969$

TO FREEZE EQUIPUEMT:

LYMU E. SHACXEL FOOT

LIVERTORE

(510) $296-3067$

LOCATION OF EOUIPMEMT:

SAMDIA MAIIOMAL LABORATORIES SADDIA MAIIOMAL LABORATORIES

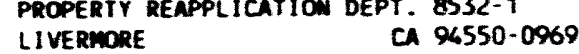


EFFECTIVE DATE: 07/01/94 EXPIRATION DATE: 07/31/94

FSC

COOE EXCESS PROPERTY DESCRIPIION

REFERENCE MUMBER : 1213

7035 PRINTER, B/W DST MAXRIX MFR: HEWLETT PACKARD, MOOEL MO. 2671G, S/M 2327 A15604, ACO. DATE : 8/83, APPROX. WT: 20 LBS., $16^{\prime} \mathrm{W} \times 16^{\circ} \mathrm{L} \times 4^{\circ} \mathrm{H}$

REPORT MUMBER: $899105-4082$

LIME ITEM MUMBER: 0028

REFERENCE MUMBER : 1214

7035 PRIMTER, COLOR IMK-JET MFR: TEKTRONIX, MOOEL MO. 4696, S/N J023345, ACO. DATE: 3/87. A PPROX. WT: $30 \mathrm{LBS}$., $21 . \mathrm{W} \times 14 . \mathrm{L} \times 6 . \mathrm{H}$. wo of

COMD UNIIS Uni

ACOUISIIIOU PRICE

$\$ 1.032 .00$

$\$ 1,032.00$

costhint:

cost/umit:

TOTAL COST
$\$ 1.382 .00$

$\$ 1,382.00$

FOR FURTHER IMFOOMATION

LYMM E. SHACKELFOOT

LIVERMARE

(510) $296-3067$

CA $94550-0969$

TO FREEZE EOUIPMEMT:

I VUW E. SHACKELFOOT

LIVERMORE

(510) $294-3067$

CA $94550-0069$

LOCATIOW OF EOUIPMENT:

SADIA MATIOUAL LABORATORIES

PRDPERTY REAPPI ICATIOA DEPT. 8532-1

PROPERTY REAPPLICAIIOU DEPI. 8532-1 
EFFECIIVE DATE: 07/01/94 EXPIRATION DATE: 07/31/94

FSC

COOE EXCESS PROPERTY DESCRIPIION

REFERENCE MUMBER : 1215

TO3S PRINTER, FXIOO MFR: ESPON, MOOEL NO. P1OFA, S/M 143336, ACO. DATE: 4/85. APPRO X. WT: $30 \mathrm{LBS} ., 26 . \mathrm{W} \times 16 \cdot \mathrm{L} \times 6 . \mathrm{H}$.

REPORT NUMBER: $899105-4082$

LIME ITEM MUMBER: 0027

\section{REFERENCE NUMBER : 1216}

TO3S PRINTER

A5200CGM0156. ACO. DATE: 6/85, APPROX. UT: $80 \mathrm{LBS} . .17$ W $\times 19 . \mathrm{L} \times$ $12 \cdot \mathrm{H}$

$600001 \mathrm{EA}$

COST/UNIT:

TOTAL COST:

CosT/UNIT: TOTAL Cost:

\subsection{1 .00 $\$ 2,341.00$}

55.324 .00 $\$ 5.324 .00$ (5)

FOR FURTHER INFORMATION:

L TUM E. SHACKELLOOT

ca 94550.0969

TO FREEZE EOUIPMENT

I IMU E. SHACKELFOOI

(510) $294-3067$

REPORT MUMBER: $899105-4082$

LIME ITEM MUMBER: 0019

LOCATION OF EOUIPMENT

SANDIA MATIOMAL LABORATORIES

PROPERTY REAPPLICAIION DEPT. 8532-1

LIVERMORE

CA $94550-0969$ 
EFFECTIVE DATE: 07/01/94 EXPIRATION DATE: 07/31/94

FSC

COOE EXCESS PROPERTY DESCRIPIIOM

REFERENCE MMBER : 1217

7035 PRINTER LASER JET MFR: HEULETT PACKARD, MCOEL MO. 2686A, S/M 2550113829, ACO. DATE : 4/86, APPROX. WT: 70 LBS.. 19.W X $16.1 \times 12 \cdot \mathrm{H}$

ELIGIBLE EQUIPMEN 
EFFECTIVE DATE: $07 / 01 / 94$ EXPIRATION DATE: $07 / 31 / 94$

FSC

COOE EXCESS PROPERTY DESCRIPTION

REFERENCE MUMBER : 1219

7035 PRINTER, LASER JET MFR: HEWLETT PACKARD, MOOEL NO. 2686A, S/M

$2602 \mathrm{~J} 62605$, ACQ. DATE : 1/87. APPROX. WT: 80 LBS., $19^{\prime} \mathrm{W} \times 16^{\prime} \mathrm{L} \times 12^{\prime} \mathrm{H}$

REPORT NUMBER: $899105-4082$

LINE ITEM NUMBER: 0032

REFERENCE NUMBER : 1220

7035 PRINTER, LASER JET MFR: HEWLETT PACKARD, MODEL NO. 2686A, S/N
$2602 J 49093$. ACO. DATE : 9/86. APPROX. WT: 80 LBS., $19 \cdot \mathrm{W} \times 16^{\prime} \mathrm{L} \times 12^{\prime} \mathrm{H}$.

$4 \quad 00001$ EA
$\cos T / U N 1 T$
TOTAL COST:

No of

COND UNITS UNIT

COST/UNIT:

TOTAL COST:

$\cos (\cos 20$

ACQUISITION PRICE

$\$ 2,345.00$

$\$ 2,345.00$ 
EFFECTIVE DATE: $07 / 01 / 94$ EXPIRATION DATE: $07 / 31 / 94$

FSC

\section{COOE EXCESS PROPERTY DESCRIPTION}

REFERENCE NUMBER : 122

7035 PRINTER, LETTERPRINTER 100 MFR: DIGITAL, MODEL NO. LA100-ZA, S/N PNV8249, ACO. DATE: 2/85, APPROX. WT: 25 LBS., 22 'W $\times 16^{\prime} \mathrm{L} \times 11^{\prime} \mathrm{H}$.

REPORT NUMBER: $899105-4082$

LINE ITEM NUMBER: LAS
NO OF

COND UNITS UNIT

ACQUISITION PRICE

$500001 \mathrm{EA}$

COST/UNIT: TOTAL COST:
$\$ 1,115.00$ $\$ 1,115.00$

LYNN E. SHACKELFOOT

LIVERMORE

(510) $294-3067$

CA $94550-0969$

TO FREEZE EQUIPMENT

LYNM E. SHACKELFOOT

(510) $294-306$

CA $94550-0969$

LOCATION OF EQUIPMENT:

SANDIA NATIONAL LABORATORIES

PROPERTY REAPPLICATION DEPT. 8532-1

LIVERMORE

CA $94550-0969$

REFERENCE NUMBER : 1222

RACK W/EQUIPMENT, SCANHER, CLABRATOR, POWER SUPPLY, MFR: HP, ZI-

TECH/ELGAR, S/N 3495A/9819/3495A

ACO. DATE: 6/79, APPROX. WT: 250 LBS., $22 \cdot \mathrm{W} \times 36^{\prime} \mathrm{L} \times 72 \cdot \mathrm{H}$.

$400001 \mathrm{EA}$

COST/UNIT:

TOTAL COST:

$\$ 17,010.00$

$\$ 17,010.00$

FOR FURTHER INFORMATION:

LYNN E. SHACKELFOO

LIVERMORE

CA $94550-0969$

TO FREEZE EQUIPMENT:

LYNN E. SHACKELFOOT

LIVERMORE

(510) 294-3067

CA $94550-0969$

REPORT MUMBER: $899105-4082$

LINE ITEM NUMBER: 0049

LOCATION OF EQUIPMENT:

SANDIA NATIONAL LABORATORIES

PROPERTY REAPPLICATION DEPT. 8532-1

LIVERMORE

CA $94550-0969$ 
EFFECTIVE DATE: 07/01/94 EXPIRATION DATE: $07 / 31 / 94$

COOE EXCESS PROPERTY DESCRIPIION

REFERENCE NUMBER : 1223 \begin{tabular}{l}
7035 RACK W/EQUIPMENT, OSCILLOSRAPH/AMPLIFIER/PAICH PANEL MFR: BELL + \\
HOWELL/AMER METAL, MOOEL NO. HR3000, S/N 2036, ACO. DATE: \\
\hline $11 / 67$,
\end{tabular} HOWELL/AMER METAL, MOOEL NO. HR3O00, S/N 2036
APPROX. WT: 500 LBS., $23^{\prime} \mathrm{H} \times 36^{\prime} \mathrm{L} \times 80^{\prime} \mathrm{H}$.

REPORT NUMBER: $899105-4082$

LINE ITEM NUMBER: 0050

REFERENCE NUMBER : 1224

RACK H/EQUIPMENT, POWER SUPPLY/SPECTROMETER/GENERATOR PLUS MFR: KEVEX

作

O/79, APPROX WT: 200 LBS., $23 \cdot \mathrm{W} \times 23 . \mathrm{L} \times 42 \cdot \mathrm{H}$.

REPORT NUMBER: $899105-4082$

LINE ITEM NUMBER: 0051
NO OF

COND UNITS UNIT

ACOUISITION PRICE

400001 EA

COST/UNIT:

TOTAL COST:

$\$ 21,449.00$

21.449 .00

(510) $294-306$

CA $94550-0969$

TO FREEZE EQUIPMENT:

IYNN E. SHACKELFOOT

LIVERMORE

510) $294-3067$

OCATION OF EQUIPMENT:

SAMDIA MATIOMAL LABORATORIES

SANDIA NATIONAL LABORATORIES

PROPERTY REAPPLICATION DEPT
LIVERMORE
CA $94550-0969$
$500001 \mathrm{EA}$

TOTAL COS

$\$ 21.705 .00$

$\$ 21,705.00$
FOR FURTHER INFORMATION:

LYNN E. SHACKELFOOT

LIVERMORE

510) $294-3067$

CA $94550-0969$

TO FREEZE EQUIPMENT:

LYNN E. SHACKELFOOT

LIVERMORE

(510) $294-3067$

CA $94550-0969$

LOCATION OF EQUIPMENT:

SANDIA MATIONAL LABORATORIES

SANDIA NATIONAL LABORATORIES $8532-9$

LIVERMORE

CA $94550-0969$ 
EFFECTIVE DATE: $07 / 01 / 94$ EXPIRATION DATE: $07 / 31 / 94$

FSC

CODE EXCESS PROPERTY DESCRIPTION

REFERENCE NUMBER : 1225

7035 RACK U/EOUIPMENT PRINTER, PC/CHASSIC, SCANNER DATA, DISC DRIVE MFR: DIG EOUIP. MODEL NO. LASORA/BA23A/2204A, S/N TCF8178A/WF5 3005515, ACQ. EQUIP. MOOEL NO. LASORA/BA23A/2204A, S/N TCF8178A/WFS
DATE: $7 / 82$, APPROX WT: 300 LABS., $22^{\prime} \mathrm{W} \times 36^{\circ} \mathrm{L} \times 80^{\prime} \mathrm{H}$. ELIGIBLE EQUIPMENT

NO OF

COND UNITS UNIT

ACQUISITION PRICE

COST/UNIT:

TOTAL COST

$\$ 8,368.00$

REPORT NUMBER: $899105-4082$

LINE ITEM NUMBER: 0052

REFERENCE MUMBER : 1226

7035 RACK W/EOUIPMENT PROCESSOR MCA/AMPLIFIER PLUS/A/D CONYERTER MFR:

RACK H/EQUIPMENT, PROCESSOR MCA/AMPLIFIER PLUS/A/D CONVERTER MFR:
CANBERRA, MOOEL NO. SERIES $90, \mathrm{~S} / \mathrm{N} 8077 / 6892994$, ACQ. DATE: 4/86, APPROX

WT: $300^{\circ}$ LABS., $23^{\circ} \mathrm{W} \times 36^{\circ} \mathrm{L} \times 72^{\prime} \mathrm{H}$.

$400001 \mathrm{EA}$

COSTIUNIT:

TOTAL COST:

$\$ 17,354.00$

$\$ 17,354.00$

FOR FURTHER INFORMATION:

LYNN E. SHACKELFOOT

LIVERMORE

(510) 294-3067

CA $94550-0969$

TO FREEZE EOUIPMENT

LYNN E. SHACKELFOOT

IVERMORE

CA $94550-0969$

(510) 294-3067

LOCATION OF EQUIPMENT:

SANDIA NATIONAL LABORATORIES

PROPERTY REAPPLICATION DEPT. $8532-1$

LIVERMORE

CA $94550-096$ 
EFFECTIVE DATE: $07 / 01 / 94$ EXPIRATION DATE: $07 / 31 / 94$

\section{COOE EXCESS PROPERTY DESCRIPTION}

REFERENCE NUMBER : 1227

7035 RATEMETER/AMPLIFIER PLUS MFR: EG+G ORTEC/TENNELEC, MODEL NO. $434 / T C$

$250 \mathrm{~A}$

S/N 219, ACO. D ATE: 1/72, APPROX. WT: 70 LBS., $19 . \mathrm{W} \times 18 . \mathrm{L} \times 9 . \mathrm{H}$.

REPORT NUMBER: $899105-4082$

LINE ITEM NUMBER: 0046

REFERENCE NUMBER : 1228

7035 TAPE DRIVE MFR: SUN MICROSYSTEM, MODEL EXP- 2

S/N 016G0407, ACQ. DATE: 3/9 O, APPROX. WT: 25 LBS., $71 \mathrm{~W} \times 20^{\circ} \mathrm{L} \times$ S.H.

600001 EA
COST/UNIT: TOTAL COST:
$\$ 4,200.00$ $\$ 4,200.00$
NO OF

COND UNITS UNIT

ACQUISITION PRICE

COSTIUNIT:
TOTAL COST:

$\$ 2,508.00$ $\$ 2,508.00$

to

CONTACT(S)

FOR FURTHER INFORMATION:

LYNN E. SHACKELFOOT

LIVERMORE
(510) $294-3067$

CA $94550-0969$

TO FREEZE EQUIPMENT:

LYNN E. SHACKELFOOT

LIVERMORE

(510) $294-3067$

CA $94550-0969$

LOCATION OF EQUIPMENT:

SANDIA NATIONAL

PROPERTY REAPPLICATION DEPT - 8532-1

LIVERMORE

CA $94550-0969$

FOR FURTHER INFORMATION:

LYNN E. SHACKEL FOOT

LIVERMORE
(510) $294-3067$

CA $94550-0969$

TO FREEZE EQUIPMENT:

LYNN E. SHACKELFOOT

LIVERMORE

CA $94550-0969$

(510) 294-3067

LOCATION OF EOUIPMENT

SANDIA MATIOMAL LABORATORIES

PROPERTY REAPPLICATION DEPT. 8532-1

LIVERMORE

CA $94550-0969$ 
EFFECTIVE DATE: 07/09/94 EXPIRATION DATE: $07 / 31 / 94$

\section{COOE EXCESS PROPERTY DESCRIPTION}

REFERENCE NUMBER : 1229

7050 PRINTER THINK JET MFG: HEHLETT PACKARD

MDL: $2225 \mathrm{~A}$

SN: 2448530740

TAG \#S 1979

ACQ : JAN 85

REPORT NUMBER: $893604-4137$

ELIGIBLE EQUIPMENT

$$
\text { NO OF }
$$

COND UNITS UNIT

$4 \quad 00001$ EA
ACOUISITION PRICE

\section{COST/UNIT:} TOTAL COST:

LINE ITEM NUMBER: 0005
REFERENCE NUMBER : 1230

7110 CHALKBOARD, 36X60"28GA PORC STEEL ( 9 EACH), MFR. GREENSTEEL, M ODEL 7110-61871, ACO. YR. $93, E 37814$
400001 EA

\section{COST/UNIT: \\ TOTAL COST:}

$\$ 530.06$ $\$ 530.06$
FOR FURTHER INFORMATION:

JOANNE BREZNIK

LIVERMORE

(510) $424-4101$

TO FREEZE EQUIPMENT:

JOANNE BREZNIK

LIVERMORE

CA 94550

REPORT NUMBER: $899102-4163$

LINE ITEM NUMBER: 0040
(510) $424-4101$

LOCATION OF EQUIPMENT:

LAURENCE LIVERMORE NATIONAL LAB

EXCESS PROPERTY SECTION, L-696

LIVERMORE 
EFFECTIVE DATE: $07 / 01 / 94$ EXPIRATION DATE: $07 / 31 / 94$

\section{CODE EXCESS PROPERTY DESCRIPIION}

REFERENCE NUMBER : 1231

7110 CHALKBOARD, 48X96", 28 GA PORC STEEL ( 9 EACH), MFR. GREENSTEEL, MODEL CHALKBOARD, 48X96", 28 GA PORC
$7110-61869$, ACO. YR. $93, E 37824$

REPORT NUMBER: $899102-4163$

LINE ITEM NUMBER: 0046

REFERENCE NUMBER : 1232

DRAFTING TABLE TOP W/VINYL COVER (2 EACH), MFr. HAMILTON, MOOE L 7110 59191
NO OF

COND UNITS UNIT

ACQUISITION PRICE

$4 \quad 00001 E A$

COST/UNIT:

TOTAL COST:

$\$ 648.00$

$\$ 648.00$

JOANNE BREZNIK
LIVERMORE

(510) $424-4101$

CA 94550

TO FREEZE EQUIPMENT:

JOANNE BREZNIK

LIVERMORE

CA 94550

(510) $424-4101$

LOCATION OF EQUIPMENT:

ALRENCE LIVERMORE MATIONAL LAB

EXCESS PROPERTY SECTION, L-696

LIVERMORE

CA 94550

REPORT NUMBER: 899102-4163

LINE ITEM NUMBER: 0045
400001 EA COST/UNIT:

TOTAL COST:
$\$ 612.00$

$\$ 612.00$
FOR FURTHER INFORMATION:

JUANNE BREZNIK

LIVERMORE

(510) $424-410$

CA 94550

TO FREEZE EQUIPMENT:

JOANHE BREZNIK

LIVERMORE

CA 94550

(510) $424-4101$

LOCATION OF EQUIPMENT:

LAWRENCE LIVERMORE NATIONAL LAB

EXCESS PROPERTY SECTION, L-696

LIVERMORE

CA 9455 
EFFECTIVE DATE: $07 / 01 / 94$ EXPIRATION DATE: $07 / 31 / 94$

FSC

COOE EXCESS PROPERTY DESCRIPTION

REFEREMCE NUMBER : 1233

7110 DRAFTING TABLE

\#260037 BARCODE 854603

REPORT MUMBER: $895529-4232$

LINE ITEM NUMBER: 0027

REFERENCE NUMBER : 1234

7110 DRAFTING TABLE

* 26C038 BARCOOE 854604

X94-04-278 MANUF. MAYLINE SERIAL 7 00001 EA

REPORT NUMBER: $895529-4232$

LINE ITEM NUMBER: 0028
COST/UNIT:

TOTAL COST:

$\$ 3,000.00$

TOTAL COST:

\section{UNITS UHIT ACOUISITION PRICE}

COST/UNIT: $\quad \$ 3,000.00$

$\$ 3,000.00$

FOR FURTHER INFORMATION:

WILLIS J. MILLER

CINCINMATI

(513) $738-6630$

OH 45239-8704

TO FREEZE EOUIPMENT:

WILLIS $J$. MILLER

CINCINMATI

(513) $738-6630$

LOCATION OF EQUIPMENT:

U.S. DOE/FERMCO

7400 WILLEY ROAD

FERMALD

OH 45030

FOR FURTHER INFORMATION:

WILLIS J. MILLER

CINCINNATI

(513) $738-6630$

OH 45239-8704

TO FREEZE EQUIPMENT :

WILLIS J. MILLER

CINCIMMATI

(513) $738-6630$

OH $45239-8704$

LOCATION OF EOUIPMENT:

U.S. DOE/FERMCO

WILODEY ROAD

FERNALD

OH 45030 
EFFECTIVE DATE: 07/01/94 EXPIRATION DATE: 07/31/94

FSC

COOE EXCESS PROPERTY DESCRIPTION

REFERENCE NUMBER : 1235

7110 DRAFTING TABLE

* 224253 BARCOOE 854602

REPORT HUMBER : $895529 \cdot 4232$

LINE ITEM NLMBER: 0029

REFERENCE NUMBER : 1236

7110 ORAFTING TABLE

* 226256 barcooe 85460 ;

X94-04-280 MANUF. MAYLINE SERIAL 7 00001 EA

COST UNAT:

OTAL COST:

\section{TOTAL COST:}

\section{ACQUISITION PRICE}

$\$ 3,000.00$

$\$ 3,000.00$
$\$ 33.000 .00$

FOR FURTHER INFORMATION:

WILLIS J. MILLER

CINCINNATI

(513) $738-6630$

OH $45239-8704$

TO FREEZE EOUIPMENT:

WILLIS J. MILLER

CINCINMATI

24 45239-8704

REPORT MUMBER : $895529-4232$

LINE ITEM NUMBER: 0030

LOCATION OF EOUIPMENT:

U.S. DOE/FERMCO

7400 WILLEY ROAD

FERMALD

OH 45030 
EFFECTIVE DATE: $07 / 01 / 94$ EXPIRATION DATE: $07 / 31 / 94$

REFERENCE NUMBER : 1237

7110 TABLE, DRAFTING, PEDES, 19" HT 60037-1/2 (3 EA), MFR. MAYLINE, MOOEL TABLE, DRAFTING, PEDES, $19 "$ HT $60 \times 3$
$7110-62718$, ACO. YR., $93, E 37828$

REPORT NUMBER: $899102-4163$

\section{FSC COOE EXCESS PROPERTY DESCRIPIION}

No of

COND UMIIS UNIT

ACOUISITION PRICE

$400001 \mathrm{EA}$

COST/UNIT: TOTAL COST:

$\$ 2.958 .57$ $\$ 2,958.57$

$$
\text { LIVERMORE }
$$

(510) $424-4101$

CA 94550

TO FREEZE EQUIPMENT:

JOANME BREZNIK

LIVERMORE

CA 94550

(510) $424-4101$

LOCATION OF EQUIPMENT

LAURENCE LIVERMORE NATIOMAL LAB

EXCESS PROPERTY SECTION, L-696

LIVERMORE

REFERENCE NUMBER : 1238

7435 COMP ACC EXPANDR UMI SYSTEM BOARD 8087 640KB MEMORY KEYBOARD PARALLEL

PRINTER VIDEO DI SPLAY UNIT 2 DISKETIE DRIVES ASYNC COMMUNICATIONS

$600001 \mathrm{EA}$

COST/UNIT:

TOTAL COST:

$\$ 2,373.00$

373.00

CLOCK/CALENDAR I FIXED DISK DRIVE. 12OVAC, 60 HZ. IBM.

REPORT NUMBER: $898355-4096$

LINE ITEM NUMBER: 0040
FOR FURTHER IMFORMAIION:

ELOY N. GIRON

ALBUOUEROUE

(505) $844-2746$

NM 87185

TO FREEZE EOUIPMENT

ELOY N. GIRON

87185

(505) $844-2746$

LOCATION OF EOUIPMENT

ELOY N. GIROW

PROPERTY REAPPLICATION DIV 7617

ALBUOUERQUE 
EFFECTIVE DATE: 07/01/94 EXPIRATION DATE: $07 / 31 / 94$

\section{COOE EXCESS PROPERTY DESCRIPIION}

PEFERENCE MUMBER : 1239

7435 COMPUTER, PERSONAL XT COMPUER WARALLEL PORT ANO VIDEO PORT. 12OVAC, 60 HZ. AT\&T, PC6300, SER. $0285751^{\circ}$.

REPORT NUMBER : $898355-4096$

LINE ITEM NUMBER: 0041

REFERENCE MUMBER : 1240

7435 COMPUTER, PERSONAL 286 COMPUTER 2 5-1/4" DISK DRIVES HARD DRIVE, 2 RS120VAC, $60 \mathrm{HZ}$. IBM, 5170339.7197645

LINE ITEM MUMBER: 0042
No or

$\$ 1.445 .00$

COST/UNIT:

TOTAL COST:

$\$ 1,445.00$

60001 EA

(2)


EFFECTIVE DAIE: 07/01/94 EXPIRATION DATE: $07 / 31 / 04$

FSC

COOE EXCESS PROPERTY DESCRIPIION

REFERENCE MMBER : 1241

7435 COMPUTER, PERSONAL 286 COMPUTER 640KB RAM 2 5-1/4" DISK DRIVES, HARD DRIVE, SERIAL P ORT, PARALLEL PORT, AMD VIDEO PORT. 12OVAC, $60 \mathrm{HZ}$. HEH PACK, 45 959C. SER. 2805A112.

REPORT MUMBER: $898355-4096$

LINE ITEM MUMBER: 0043

REFEREMCE MUMBER : 1242

COMUTER PERSOUAL SYSTEM BOARO 640 KB MEMCQY KEYBRD PARALLEL PRINTER,

VIDEO DISPLAY UIT, I DISKETIE DRIVE, ASYMC COMMUMICATION ALT SYMC

$600001 \mathrm{EA}$ COMmInICATI ONS, I FIXED OISK DRIVE. COMPAO, SER. MOME.

REPORT MUMBER : 898355-4096

LIME ITEM MUMBER: 0046

wo of

600001 EA
COMD UNITS UNIT

ACOUISITION PRICE

COST/UNIT:

TOTAL COST:

$\$ 3,142.00$

$\$ 3.142 .00$

(2)

\section{TO FREEZE ECUIPMEMT: \\ ELOT M. GIROM \\ MLUCUERCAE}

(505) $844-2746$

LOCATION of EQUIPMENT:

ELON M. GIROW

PeOPERTY REAPPI ICATIO DIV 7617

ALEUCUERQue

in 87123
COST/NAIT:

rotal cost:
$\$ 2,000.00$

$52,000.00$

$\$ 2,000.00$
FOR FURTHER IMFORMATIOW:

ELOY N. GIROW

ALBuareraue

(505) $844-2746$

m 87185

TO FREEZE EOUIPMEWT:

ELOY N. GIROW

(505) $844-2746$

87185

LOCATIOO OF EQUIPAEMT:

ELOT W. GIROM

PROPERTY REAPPLICATIOO DIV. 7617

alsucueroue

in 87123 
EFFECTIVE DATE: 07/01/96 EXPIRATION DATE: $07 / 31 / 94$

\section{COOE EXCESS PROPERTY DESCRIPIION}

REFERENCE MMBER - 1243

7635 COMPUTER PERSOUAL XI MACHIME HITH MATH COPROCESSOR, 604K BASE MEMORY, DO EXTEMDE M EMOQY MO HAPD DRIVE CGA ADAPIER ABOOH VIDEO NDORESS AORB: $(5-1$ 14") FLOPY DRIVE I PARALLEL PORT AMO 1 SERIAL PORT 120 VAC, $69 \mathrm{HZ}$., IBM, 5150176, SER. 1071027.

ELIGIBLE EOUIPHEMT
REPORT NUMBER: $898355-4096$

LIME ITEM MUMBER: 0045 mo of

COND Uirs unI

$600001 \mathrm{EA}$

cost/unit:

TOTAL COST:

$\$ 1,843.00$

$\$ 1,843.00$

(1)

\section{ELOY N. GIROW \\ ALBucter}

FOR FURTMER IMFOEMAIIOW:

(SOS) $844-2746$

87185

TO FREEZE EOUIPHEWT:

ELOT W. GIROW

ALEUCUERCUE

(505) $844-2746$

87185

LOCATION OF ECUIPNEWT:

ELOY M. GIROM

PROPERIY REAPPLICATIOW DIV. 761

al BuOUEROUE

REFERENCE MUMBER : 1246

7435 COHPUTER PERSOUAL SYSTEM BOARD 8087 640KB MEMORY PARALLEL PRINTER,

VIDEO DISPLAY UT IT 2 DISKETIE ORIVES ASYMC COMUUMICATIOWS ALT ASYMC

6 O0001 EA

cost/unit

TOTAL COST:

$\$ 2.065 .00$

VIDEO DISPLAY UN IT, 2 DISKETIE DRIVES, I2OVAC, $60 \mathrm{HZ}$. COMP

FOR FURTHER INFORMATIOW:

ELOY N. GIROM

AL BUOUEROUE

(505) $846-2746$

87185

TO FREEZE EOUIPUEMT:

ELOT M. GIROW

ALBUCUEROUE

(505) $844-2746$

LOCATION OF ECUIPMENT:

ELOY M. GIROM

PROPERTY REAPPLICATION DIV. 7617

ALBUOUERTUE

87123 
EFFECTIVE DATE: 07/01/94 EXPIRATION DATE: $07 / 31 / 94$

FSC

\section{COOE EXCESS PROPERTY DESCRIPTION}

REFERENCE MUMBER : 1245

7435 COMPUTER, PERSOMAL 286 AT MACHINE 287 MATH COPROCESSOR 640K BASE MEMOR 2688K EXIEND ED MEMORY VGA VIDEO ADAPTER AOOOH VIDEO MDORESS. 12OVAC. $60 \mathrm{HZ}$. 1BM, 5170,080765 .

REPORT MUMBER: $898355-4096$

LINE ITEM MUMBER: 0047

REFERENCE MUMBER : 1246

7435 COMPUTER, PERSONAL SYSTEM BOARD GLOKB MEMORY KEYBRD PARALLEL PRINTER, VIDEO DISPLAY UNIT, I DISKETIE DRIVE. ASYNC COMUMICATIONS ALT ASYMC COMMUNICAT IONS, I FIXED DISK DRIVE. ATST.

REPORT MUMBER: $898355-4096$

LINE ITEM MUMBER: 0048
No of

COND UNITS UNII

ACOUISITIOW PRICE

$600001 \mathrm{EA}$

COST/UNIT: TOTAL COST:

$\$ 3,581.00$ $\$ 3,581.00$

FOR FURTHER IMFORMAIIOW:

ELOY N. GIRON

AL BUOUEROLE

(505) $844-2746$

87185

TO FREEZE EOUIPMENT:

ELOY N. GIROW

ALBUOUEROUE

(S05) $844-2746$

m7185

LOCATION OF EOUIPMENI :

ELOY N. GIROW

PROPERTY REAPPLICATION DiV. 7617

ALBUOUEROUE

FOR FURTHER IMFORMAIION:

ELOY N. GIRO

ALBUOUEROUE

(505) $844-2746$

Mm 87185

TO FREEZE EOUIPMENT:

ELOY N. GIKOM

ALBUOUEROUE

87185

LOCATION OF EOUIPMENT:

ELOY $M$. GIROM

PROPERTY REAPPLICATION DIV. 7617

ALBUOUEROUE

87123 
EFFECTIVE DATE: $07 / 01 / 94$ EXPIRATION DATE: $07 / 31 / 94$

\section{FSC \\ CODE EXCESS PROPERTY DESCRIPIION}

\section{REFERENCE NUMBER : 1247}

7435 COMPUTER, PERSONAL 286 COMPUTER, 2 5-1/4" DISK DRIVES 21 MB HARD SERIAL PORT, PARALLEL PORT. AND EGA VIOEO PORT. 12OVAC, $60 \mathrm{HZ}$. IBM, 5150166, SER. 1725894.

REPORT NUMBER: $898355-4096$

LINE ITEM NUMBER: 0049

REFERENCE NUMBER : 1248

640 KB OF RAM, FIXED DISK A FLOPPY DRIVE, SERIAL PORT AND PARAL LEL PORT. 120VAC, 60 HZ. ATST, PC6300, SER. 019051.

$7 \quad 00001$ EA

COST/UNIT: TOTAL COST:

\section{ACQUISITIOA PRICE}

COST/UNIT:

TOTAL COST :

$\$ 1,694.00$
$\$ 1,694.00$

CONTACTS

\section{FOR FURTHER IMFORMAIION : \\ ELOY N. GIROA \\ ALBUOUEROUE \\ (505) $844-2746$ \\ 87185 \\ TO FREEZE EQUIPMENT: \\ ELOY N. GIRON \\ ALBUEUERQUE \\ Mn 87185 \\ (505) $844-2746$ \\ LOCATION OF EQUIPMENT: \\ ELOY N. GIROW \\ PROPERTY REAPPLIICATION DIV. 7617}

ALBUOUERQUE

NM 87123

REPORT NUMBER: $898355-4096$

LINE ITEM NUMBER: 0050

FOR FURTHER INFORMATION :

ELOY N. GIRON

ALBUOUEROUE

(505) $844-2746$

TO FREEZE EOUIPMEMT:

ELOY N. GIRON

ALBUOUEROUE

(505) $844-2746$

87185

LOCATION OF EOUIPMENT:

ELOY N. GIRON

PROPERTY REAPPLICATION DIV. 7617

PROPERTY REAPPLICATION DIV. 7617 
EFFETTIVE DATE: $07 / 01 / 94$ EXPIRATION DATE: $07 / 31 / 94$

FSC

CODE EXCESS PROPERTY DESCRIPTION

REFERENCE MUMBER : 1249

7435 COMPUTER, PERSONAL SYSTEM BOARD 8087 640KB MEMORY. 120VRC, $60 \mathrm{HZ}$. IBM, 600001 EA 5170, SER. 5157122.

REPORT NUMBER: $898355-6096$

LINE ITEM NUMBER: 0051
NO OF

COND UNITS UNIT $\quad$ ACQUISITION PRICE

COST/UNIT:

TOTAL COST:

$\$ 3,466.00$ $\$ 3,466.00$

ELOY N. GIRON

ALBUQUERQUE

(505) $844-2746$

NFF 87185

TO FREEZE EQUIPMENT:

ELOY N. GIRON

ALBUQUERQUE

(505) $844-2746$

NM 87185

LOCATION OF EQUIPMENT:

ELOY N. GIRON

PROPERTY REAPPLICATION DIV. 7617

ALBUQUERQUE

NM 87123

REFERENCE NUMBER : 1250

7435 COMPUTER, PERSONAL 286 AT MACHINE (HPVECTRA) 287 MATH COPROCESSOR, 636K

BASE MEMORY, 1536K EXTENDED MEMORY, VGA VIDEO ADATER AOCOH VIDEO ADDRESS

$A: 1(5-1 / 4 ")$ FLOPPY DRIVE B:I(5-1/4K") FLOPPY DRIVE, I PARALLEL

600001 EA

$\operatorname{cosT/UNIT:}$

TOTAL COST:

$\$ 1.761 .00$

$\$ 1.761 .00$

FOR FURTHER INFORMATION:

ELOY N. GIRON

ALBUQUERQUE

(505) $844-2746$

NM 87185

TO FREEZE EQUIPMENT:

ELOY N. GIRON

ALBUQUERGUE

(505) $844-2746$

NM 87185

LOCATION OF EQUIPMENT:

ELOY N. GIRON

PROPERTY REAPPLICATION DIV. 7617

ALBUQUERQUE

NM 87123 
EFFECIIVE DATE: $07 / 01 / 94$ EXPIRATION DATE: $07 / 31 / 94$

FSC

\section{COOE EXCESS PROPERTY DESCRIPIION}

REFERENCE NUMBER : 1251 COMPUTER, PERSONAL 286 COMPUTER HITH 640KB RAM 5-1/4" DISK DRIVE HARD
DRIVE, 2 SERIA L PORTS, PARALLEL PORT AND VIDEO PORT. 120VAC, 60 HZ. IBM, 5160,3000174

REPORT NUMBER: $898355-4096$

LINE ITEM NUMBER: 0053

\section{REFERENCE NUMBER : 1252}

7435 COMPUTER, PERSONAL SYSTEM BOARD 640KB MEMORY KEYBRD, PARALLEL PRINTER,

VIDEO DISPLAY UNIT, I DISKETTE DRIVE, ASYNC COMMUNICATIONS, 1 FIXED DISK DRIVE. I2OVAC, 60 HZ. AT\&T, PC6300, SER. 24239.

REPORT NUMBER: $898355-4096$

LINE ITEM NUMBER: 005

$$
\text { NO OF }
$$

COND UNITS UNIT

ACQUISITION PRICE

CONTACTSS

$700001 \mathrm{EA}$

COST/UNIT:

TOTAL COST:

$\$ 1,544.00$

$\$ 1,544.00$

ELOY N. GIRON

(505) $844-2746$

NM 87185

TO FREEZE EQUIPMENT

ELOY N. GIRON

ALBUQUERQUE

(505) $844-2746$

NM 87185

LOCATION OF EQUIPMENT

ELOY N. GIRON

PROPERTY REAPPLICATION DIV. 7617

ALBUQUERQUE

\section{$600001 \mathrm{EA}$ COST/UNIT: \\ TOTAL COST:}

$\$ 2,103.00$

FOR FURTHER INFORMATION

ELOY N. GIRON

ALBUQUERQUE

NM 87185

TO FREEZE EQUIPMENT:

ELOY N. GIRON

ALBUQUERQUE

(505) $844-2746$

NM 87185

LOCATION OF EQUIPMENT

ELOY N. GIRON

PROPERTY REAPPLICATION DIV. 7617

ALBUQUERQUE

NM 87123 
EFFECTIVE DATE: 07/01/94 EXPIRATION DATE: $07 / 31 / 94$

FSC

\section{COOE EXCESS PROPERTY DESCRIPIION}

\section{REFERENCE NUMBER : 1253}

7435 COMPUTER, PERSONAL XT COMPUTER 64OKB RAM 2 5-1/4" DISK DRIVE, HARD DRIVE, SERIAL POR T, PARALLEL PORT, AND VIDEO PORT. 12OVAC, $60 \mathrm{HZ}$. IBM, 5160,4252785

REPORT NUMBER: $898355-4096$

LINE ITEM NUMBER: 0055

\section{REFERENCE NUMBER : 1254}

7435 COMPUTER, PERSONAL 286 COMPUTER WITH 1024 KB RAM 2 5-1/4" DISK DRIVES HARD DRIVE, 2 SERIAL PORT, 2 PARALLEL PORT, AND VIDEO PORT. 12OVAC, 60 HZ. IB M, 5170339, 7198484 .

REPORT NUMBER: 898355-4096

LINE I TEM NLMBER: 0056
NO OF

COND UNITS UNIT

ACQUISITION PRICE

$600001 \mathrm{EA}$

$\operatorname{COST/UNIT:}$

TOTAL COST:

$\$ 1,964.00$

$\$ 1,964.00$

\section{OROY $M$ THER INFORMATION: \\ ELOY N. GIRON}

ALBUQUERQUE

(505) $844-2746$

NM 87185

TO FREEZE EQUIPMENT :

ELOY N. GIRON

ALBUQUERQUE

(505) $844-2746$

NM 87185

LOCATION OF EQUIPMENT:

ELOY N. GIRON

PROPERTY REAPPLICATION DIV. 7617

ALBUQUEROUE

MM 87123

\section{$00001 \mathrm{EA}$ \\ COST/UNIT: \\ TOTAL COST:}

$\$ 3,932.00$

$\$ 3,932.00$

FOR FURTHER INFORMATION:

ELOY N. GIRON

ALBUQUERQUE

(505) $844-2746$

NM 87185

TO FREEZE EQUIPMENT:

ELOY N. GIRON

ALBUQUERQUE

(505) 844-2746

NM 87185

LOCATION OF EQUIPMENT :

ELOY N. GIRON

PROPERTY REAPPLICATION DIV. 7617

ALBUQUERQUE 
FSC

\section{COOE EXCESS PROPERTY DESCRIPTION}

REFERENCE NUMBER : 1255

7435 COMPUTER, PERSONAL 286 COMPUTER WITH 1024 KB RAM 3-1/2" DISK DRIVE, 5

1/4" DISK DRIV E, HARD DRIVE, VIDEO PORT, SERIAL PORT, AND PARALLEL

PORT. 120VA C, $60 \mathrm{HZ}$. HEW PACK, 45945C, SER. 2752A077.

REPORT NUMBER: $898355-4096$

LINE ITEM NUMBER: 0057

\section{REFERENCE NUMBER : 1256}

7435 COMPUTER, PERSONAL DIGITAL COMPUTER, HITH 2 5-1/4" DISK DRIVES, HARD

DRIVE, WITH POR TS FOR VIDEO COMMI AND PR1. 120VAC, $60 \mathrm{HZ}$. DEC,

PC380AA, SER. KA 819044
$\$ 3.774 .00$ $\$ 3,774.00$

\section{ELOY N. GIRON}

FOR FURTHER INFORMATION:

(505) $844-2746$

NM 87185

TO FREEZE EQUIPMENT:

ELOY N. GIRON

ALBUQUEROUE

(505) $844-2746$

NM 87185

LOCATION OF EQUIPMENT:

ELOY N. GIRON

PRCPERTY REAPPLICATION DIV. 7617

ALBUGUERQUE

NM 87123

FOR FURTHER INFORMATION:

ELOY N. GIRON

ALBUQUERQUE

NM 87185

(505) $844-2746$

TO FREEZE EQUIPMENT :

ELOY N. GIRON

ALBUQUEROUE

(505) $844-2746$

LOCATION OF EQUIPMENT:

ELOY N. GIRON

PROPERTY REAPPLICATION DIV. 7617

ALBUQUERQUE

NM 87123 


\section{EFFECTIVE DATE: $07 / 01 / 94$ EXPIRATION DATE: $07 / 31 / 94$}

FSC

\section{CODE EXCESS PROPERTY DESCRIPTION}

REFERENCE NUMBER : 125

7435 COMPUTER, PERSONAL 386 COMPUTER WITH 3712KB RAM 5-1/4" DISK DRIVE, 3 1/2" DISK DRIVE, SERIAL PORT, PARALLEL PORT, AND VIDEO PORT. 12OVAC, $60 \mathrm{HZ}, 40$, SER. 4826AT2B.

REPORT NUMBER: $898355-4096$

LINE ITEM NUMBER: 0059

REFERENCE NUMBER : 1258

7435 COMPUTER, PERSONAL 386 AT MACHINE(HPVECTRA) 636K BASE MEORY, 5120K

EXTENDED MEMORY $\checkmark$ GA VIDEO ADAPTER AOOOH VIDEO ADDRESS 1:A $2 M(5-1 / 4 ")$

FLOPPY DRIVE CMOS CLOCK 1 PARALLEL PORT 1 SERIAL PORT. 12OVAC, $60 \mathrm{HZ}$

HEW PA CK, D141B, SER. 3012A153.

REPORT MUMBER: $898355-4096$

LINE ITEM NUMBER: 0060
COND UNITS UNIT

$600001 \mathrm{EA}$

COST/UNIT:

TOTAL COST

ACQUISITION PRICE

$\$ 3,673.00$ $\$ 3,673.00$

ALBUOUERQUE
(505) $844-2746$

TO FREEZE EQUIPMENT:

ELOY N. GIRON

ALBUQUERQUE

(505) $844-2746$

LOCATION OF EQUIPMENT:

ELOY N. GIRON

PROPERTY REAPPLICATION DIV. 7617

ALBUQUERQUE

NM 87123

$600001 \mathrm{EA}$

COST/UNIT:

TOTAL COST:

$\$ 1,875.00$

$\$ 1,875.00$

FOR FURTHER INFORMATION:

ELOY N. GIRON

ALBUQUERQUE

(505) $844-2746$

NM 87185

TO FREEZE EQUIPMENT:

ELOY N. GIRON

ALBUQUERQUE

(505) $844-2746$

NM 87185

LOCATION OF EQUIPMENT

ELOY N. GIRON

PROPERTY REAPPLICATION DIV. 7617

ALBUQUERQUE

NM 87123 
EFFECTIVE DATE: $07 / 01 / 94$ EXPIRATION DATE: $07 / 31 / 94$

FSC

\section{CODE EXCESS PROPERTY DESCRIPTION}

REFERENCE NUMBER : 1259

7435 COMPUTER PERSONAL 386 COMPUTER WITH 3712 KB RAM 3-1/2" DISK DRIVE, HARD DRIVE, 2 PA RALLEL PORT, SERIAL PORT AND VIDEO PORT. I20VAC, $60 \mathrm{HZ}$. SPERRY G YR, DCP5800, SER. 39146175.

REPORT NUMBER: $898355-4096$

LINE ITEM NUMBER: 0061

\section{REFERENCE NUMBER : 1260}

7435 MICROCOMPUTER MFR: APPLE

MOD:

SER: NONE PCO6120 PRC-40012

REPORT NUMBER: $899109-4101$

INE ITEM NUMBER: 0001
No of

COND UNITS UNIT

ACOUISITION PRICE

COST/UNIT:

TOTAL COST:

$\$ 15,698.00$

$\$ 15,698.00$

$00001 \mathrm{EA}$

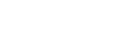

TO FREEZE EQUIPMENT:
ELOY N. GIRON

ELOY N. GIRON

(505) $844-2746$

NM 87185

LOCATION OF EQUIPMENT:

ELOY N. GIRON

REAPPLICATION DIV. 7617

ALBUOUEROUE

NM 87123
500001 EA

$$
\begin{aligned}
& \text { COST/UNIT: } \\
& \text { TOTAL COST: }
\end{aligned}
$$

$\$ 3,544.00$
$\$ 3,544.00$
FOR FURTHER INFORMATION:

MATTIE PACE

STANFORD

(415) $926-2231$

CA 94309

TO FREEZE EQUIPMENT:

MATIIE PACE

(415) $926-2231$

LOCATION OF EQUIPMENT:

LOCATION OF EQUIPMENT: STANFORD LINEAR ACCEL

2575 SAND
MENLO PARK

CA 94025 
EFFECTIVE DATE: $07 / 01 / 94$ EXPIRATION DATE: $07 / 31 / 94$

FSC

\section{COOE EXCESS PROPERTY DESCRIPTION}

REFERENCE NUMBER : 1261

7435 MICROCOMPUTER MFR: APPLE

MOD: A2S2065P

SER: 147926 PC10822 PRC-40012

REPORT NUMBER: $899109-4101$

LINE ITEM NUMBER: 0002

REFERENCE NUMBER : 1262

7435 MICROCOMPUTER MFR: I B M

MOD: 5150

SER: 0515160 PC06823 PRC-40012

REPORT NUMBER: $899109-4101$
No $\mathrm{OF}$

COND UNITS UNIT

ACQUISITION PRICE

$500001 \mathrm{EA}$

COST/UNIT:

TOTAL COST:

$\$ 3,544.00$

$\$ 3,544.00$

MATTIE PACE
STANFORD

(415) $926-223$

CA 94309

TO FREEZE EQUIPMENT:

MATTIE PACE

STANFORD

CA 94309

(415) $926-2231$

LOCATION OF EQUIPMENT:

STANFORD LIMEAR ACCELERATOR CENTER

2575 SAND HILL ROAD

MENLO PARK

CA 94025

$500001 E A$

COST/UNIT:

TOTAL COST:

$\$ 1,843.00$

$\$ 1,843.00$

FURTHER INFORMATION:

MATIIE PACE

STANFORD
(415) $926-2231$

CA 94309

TO FREEZE EQUIPMENT:

MATTIE PACE

CA 94309

(415) $926-2231$

LOCATION OF EOUIPMENT:

STANFORD LINEAR ACCELERATOR CENTER 2575 SAND HILL ROAD

MENLO PARK

CA 94025 
EFFECTIVE DATE: $07 ; 01 / 94$ EXPIRATION DATE: $07 / 31 / 94$

FSC

\section{COOE EXCESS PROPERTY DESCRIPTION}

\section{REFERENCE NUMBER : 1263}

7435 MICROCOMPUTER MFR: I B M

MOO: 5160

SER: 539228725160 PC10086 PRC-40012

REPORT NUMBER: $899109-4101$

REFERENCE NUMBER : 1264

7435 MICROCOMPUTER MFR: I B M

MOD: 5160

SER: 51744435160 PC11124 PRC-40012

REPORT NUMBER: $899109-4101$
No of

COND UNITS UNIT

ACQUISITION PRICE

$500001 E A$

COST/UNIT:

TOTAL COST:

\section{$\$ 2,829.00$}

$\$ 2,829.00$

FOR FURTHER INFORMATION:

MATTIE PACE

STANFORD

(415) $926-2231$

CA 94309

TO FREEZE EQUIPMENT:

MATTIE PACE

STANFORD

(415) $926-2231$

LOCATION OF EOUIPMENT:

STANFORD LINEAR ACCELERATOR CENTER

2575 SAND HILL ROAD

MENLO PARK

CA 94025
$500001 E A$

$$
\begin{aligned}
& \text { COST IUNIT: } \\
& \text { TOTAL COST: }
\end{aligned}
$$

LINE ITEM NUMBER: 0005
$\$ 3,496.50$
$\$ 3,496.50$
FOR FURTHER INFORMATION:

MATTIE PACE

STANFORD

(415) $926-2231$

CA 94309

TO FREEZE EQUIPMENT:

MATTIE PACE

STANFORD

CA 94309

(415) $926-2231$

LOCATION OF EQUIPMENT:

STANFORD LINEAR ACCELERATOR CENTER

2575 SAND HILL ROAD

MENLO PARK

CA 94025 
EFFECTIVE DATE: $07 / 01 / 94$ EXPIRATION DATE: $07 / 31 / 94$

FSC

\section{COOE EXCESS PROPERTY DESCRIPTION}

REFERENCE NUMBER : 1265

7435 MICROCOMPUTER MFR: 1 B

MOO: 5160

SER: 54981815160 PC11119 PRC-40016

REPORT NUMBER: $899109-4117$

REFERENCE NUMBER : 1266

7435 MICROLINE PRINTER MFR: APPLE

MOD: 237420 PC02258 PRC-40013

REPORT NUMBER: $899109-4101$
NO OF

COND UNITS UNIT

ACQUISITION PRICE

500001 EA

COST/UNIT: TOTAL COST:

LINE ITEM MUMBER: 0002

$500001 \mathrm{EA}$

\section{COST/UNIT: \\ TOTAL COST:}

LINE ITEM NUMBER: 0007
$\$ 2,999.00$ FOR FURTHER INFORMATION:

$\$ 2,999.00$ MATIIE PACE

STANFORD

(415) $926-2231$

CA 94309

TO FREEZE EDUIPMENT:

MATTIE PACE

STANFORD

CA 94309

(415) $926-2231$

LOCATION OF EQUIPMENT:

STANFORD LIMEAR ACCELERATOR CENTER 2575 SAND HILL ROAD

CA 94025

MENLO PARK
$\$ 1,100.00$

$\$ 1,100.00$

si.100.00

FOR FURTHER INFORMATION:

MATTIE PACE

(415) $926-2231$

CA 94309

TO FREEZE EQUIPMENT:

MATTIE PACE

STANFORD

CA 94309

(415) 926-2231

LOCATION OF EQUIPMENT:

STANFORD LINEAR ACCELERATOR CENTER 2575 SAND HILL ROAD

MENLO PARK

CA 94025 
EFFECTIVE DATE: $07 / 01 / 94$ EXPIRATION DATE: $07 / 31 / 94$

FSC

CODE EXCESS PROPERTY DESCRIPIION

REFERENCE NUMBER : 1267

7435 MICROTAPE DRIVE MFR: APPLE

MOO: DISK II

SER: NONE PCO6121 PRC-40012

REPORT NUMBER: $899109-4101$

LINE ITEM NUMBER: 0006

REFERENCE NUMBER : 1268

7435 MONIOR PC 1411 DIAGONAL SCREEN, 256 COLORS FROM A PALETTE OF 4.096 6ONOX 8514001 , SER. 1031249 .

LINE ITEM NUMBER: 0063
NO OF

COND UNITS UNIT

$500001 E A$

\section{COST/UNIT:
TOTAL COST:}

600001 EA

\section{COSTIUNIT \\ TOTAL COST}

$\$ 1.070 .00$ $\$ 1.070 .00$

FOR FURTHER INFORMATION:

ELOY N. GIRON

ALBUOUEROUE

(505) $844-2746$

WM 87185

TO FREEZE EOUIPMENT:

ELOY N. GIRON

ALBUOUEROUE

(505) $844-2746$

NM 87185

LOCATION OF EOUIPMENT:

ELOY N. GIRON

PROPERTY REAPPLICATION DIV. 7617

AL BUOUEROUE

87123 
EFFECTIVE DATE: 07/01/94 EXPIRATION DATE: 07/31/94

FSC

COOE EXCESS PROPERTY DESCRIPTION

REFERENCE MUMBER : 1269

7435 MONITOR, PC 19" DIAGOMAL SCREEN, 16 COLORS FROM A PALEITE OF 64, $8 \times 14$ WONITOR, PC 19" DIAGOMAL SCREEN, 16 COLORS FROM A PALETTE OF $64,8 \times 14$ CHA RACTER MATRIX 640X350 GRAPHICS $640 \times 200$ AND $320 \times 200$ GRAPHICS IN EM ULATION MODES. 12OVAC, 60 HZ. NEC, JC2001V, 8XC

REPORT MUMBER: $898355-4096$

LINE ITEM MUMBER: 0064

REFEREMCE MUMBER : 1270

PLOTIER, PC MEDIA SIZE IS $8.5 \times 11 \%$. PLOTTER USES 2 FIBER IIP PENS, MAS 5

CHAR ACTER SETS, USES HP-1B INTERFACE. T2OVAC, 48-66HZ. HEU PACK, 747

$600001 \mathrm{EA}$ OA, 23084363

No of

$600001 \mathrm{EA}$
COND UNITS UWIT

ACQUISITION PRICE COST/UHIT:
TOTAL COST:

$\$ 2,193.00$ $\$ 2,193.00$

\section{ELOY K. GIRON \\ ALBUOUEROUE}

URTHER IMFORMATION:

(505) $844-2746$

87185

TO FREEZE EQUIPMEMT:

ELOY W. GIROW

ALBUCUEROUE

m 87185

LOCATIOW OF ECUIPNENT:

EtOY $N$. GIRON

PROPERTY REAPPLICATION OIV. 7617

ALBUOUEROUE

4. 87123
COST/UNIT: TOTAL COST:
$\$ 1,055.00$ 51.055 .00
FOR FURTHER INFORRATION:

ELOY N. GIRO

ALBUDUEROUE

(505) $844-2746$

87185

TO FREEZE EOUIPWEWT:

ELOY N. GIROM

ALBUDUEROLE

(505) 846-2746

m 87185

LOCATIOM OF EOUIPMENT:

ELOY M. GIROW

PROPERTY REAPPLICATION OIV. 7617

albuOUEROUE

87123 
EFFECTIVE DATE: 0T/01/94 EXPIRATION DATE: 07/31/94

FSC

COOE EXCESS PROPERTY DESCRIPTION

REFERENCE MUMBER : 127

7435 PLOTIER PC MEDIA SIZE IS $8.5 \times 111$, USES SIX FIBER IIP PEM UHICH ARE AUTO PLOTTER, PC MEDIA SIZE IS 8.5X11", USES SIX FIBER TIP PEN WHICH ARE AUTO 7 .

wo of

cono wirs witr

ACOUISITION PRICE

$600001 \mathrm{EA}$ cost Nunit TOTAL COST:

$\$ 1.895 .00$

$\$ 1.895 .00$

REPORT MUMBER: $898355-6096$

LINE ITEM MUMBER: 0066

\section{REFERENCE MUMER : 1272}

7435 PLOTIER, PC MEDIA SIZE IS $8.5 \times 11$ " USES SIX FIBER TIP PEN UHICH ARE AUTO

CAPP ING HAS STD INTERFACES RS-232-C. 120VAC, 48-66HZ. HEW PACK, 7475

A, 2641 v027.

REPORT MUMBER: $898355-4096$

LINE ITEM MUMBER: 006

\section{Do001 EA COST/UNIT:}

IOTAL COST:

$\$ 1.270 .00$

$\$ 1,270.00$
FOR FURTHER INFORMIIOM:

ELOY W. GIRO

Albuaieroue

(505) 844-2746

111 87185

TO FREEZE EOUIPMENT

ELOY M. GIRO

MLUCUEROEE

(505) 846-2746

14 8718

LOCATION of EOUIPMENT:

PLOP W. GIROW

Al BuOUEROUE

(4) 87123


EFFECTIVE DATE: 07/01/94 EXPIRATION DATE: 07/31/94

\section{conp units unit}

COOE EXCESS PROPERTY DESCRIPTION

PEFERENCE MUMBER : 1273

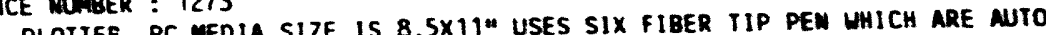
PLOTER, DC MEDIA SI2E 266,19390

$600001 \mathrm{EA}$

cost/untr:
rotal cost:

ACOUISIIIOO PRICE

$\$ 1.269 .00$

$\$ 1,269.00$

REPORT MUMBER: $898355-4096$

LIME ITEM MUMBER: 0068

REFERENCE MUMBER : 1274

REFERENCE MUMBER :
7435 PLOITER, PC MEDIA SIZE IS $8.5 \times 11$ ". PLOTTER USES 2 FIBER TIP PENS, HAS 5
CHARA CTESS SET USES

$600001 \mathrm{EA}$

OA, 2644 VO26.
COST/UNIT:

TOTAL COST:
$\$ 1,850.00$

$\$ 1,850.00$
LINE ITEM MUMBER: 0069

FOR FURTMER IMFCRMATION:

ELOY M. GIROM

ALBUCUEROUE

(505) $846-2746$

TO FREEZE ECUIPAENT :

ELOY M. GIROW

MLUCUE

(505) 846-2746

m 87185

ELOY $*$. GIROW

PROPERTY REAPPLICATION DIV 7617

ALBUOUEROUE 
EFFECTIVE DATE: $07 / 01 / 94$ EXPIRATION DATE: $07 / 31 / 94$

COOE EXCESS PROPERTY DESCRIPTION

REFERENCE MUMBER : 1275

7435 PLOITER PC MEDIA SIZE IS $8.5 \times 11^{\prime \prime}$, USES SIX FIBER TIP PENS UHICH ARE AUTO CAP PING, HAS STD INTERFACES RS-232. 12OVAC, $60 \mathrm{HZ}$. HEW PACK, $7475 A, 2325 A 298$

ELIGIBLE EOUIPMENT

638

no of

COND UMITS UMIT

$600001 \mathrm{EA}$

cosicumit TOTAL COST:

LINE ITEM MUMBER: 0070
REPORT NUMBER: $898355-4096$

REFERENCE NUMBER : 1276

7435 PRINTER PC THE 2686 A LASER PRINTING SYSTEM OPERATES AT 45 PAGES PER MINUTE O N PLAIN $8.5 \times 1 \%$ FANFOLD PAPER. 120VAC, 60 HZ. HEW PACK, 2686A, 24 J07930.

600001 EA

COST/UNIT:

TOTAL COST:

\section{ACQUISITION PRICE}

$\$ 1.269 .00$
$\$ 1.269 .00$

INFORMATION:

ELOY M. GIROM

(505) $846-2746$

87185

TO FREEZE EQUIPNENT:

ELOY $N$. GIROW

ALBUCUEROUE

(505) $844-2746$

NM 87185

LOCATION OF EQUIPMENT:

ELOY N. GIROW

PRDPERTY REAPPLICATIOA DIV. 7617

ALBUOUEROUE

in 87123

REPORT MUMBER: $898355-4096$

LINE ITEM MUMBER: 0071 
EFFECTIVE DATE: 07/01/94 EXPIRATION DATE: 07/31/94

\section{COOE EXCESS PROPERTY DESCRIPTION}

FERENCE NUMBER : 1277

7460 VIEMER MICROFICHE READER/PRINTER 1983 3M CO., MODEL 800, S/N 314561

$(N, B C, 0000832)$
NO OF

COND UNITS UNIT

ACQUISITION PRICE

$400001 \mathrm{EA}$

COST/UNIT:

TOTAL COST:

LINE ITEM NUMBER: 0003

REFERENCE NUMBER : 1278

7490 LETTERING MACHINE MFR: KROY, MODEL NO. 8OK, S/N 20220, ACQ. DATE:

LEITERING MACHINE MFR: KROY, MOOEL NO.
2/84, APP.OXX. HT: 40 LBS., $21{ }^{\prime} \mathrm{W} \times 26^{\prime} \mathrm{L} \times \mathrm{S} 2^{\prime} \mathrm{H}$.

$600001 \mathrm{EA}$

COST/UNIT:

TOTAL COST:

$\$ 1.809 .00$

$\$ 1,809.00$
$\$ 1,078.37$

$\$ 1,078.37$

CONTACT(S)

\section{FOR FURTHER INFORMATION:}

TOM JENKS

(605) $353-9217$

SD 57350

TO FREEZE EQUIPMENT:

TOM JENKS

HURON

(605) 353-9217

SD 57350

LOCATION OF EQUIPMENT

UESTERM AREA POUER ADMINISTRATION WESTERN AREA POWER ADMINISTRATION 11O IOWA N.E.

SD 57350

FOR FURTHER INFORMATION:

LYNN E. SHACKELFOOT

LIVERMORE

(510) $294-3067$

CA $94550-0969$

TO FREEZE EQUIPMENT

LYNN E. SHACKELLOOT

LIVERMORE

(510) $294-3067$

CA $94550-0969$

LOCATION OF EQUIPMENT:

SANDIA NATIONAL LABORATORIES

PROPERTY REAPPLICATION DEPT. 8532-1

LIVERMORE 
EFFECTIVE DATE: 07/01/94 EXPIRATION DATE: 07/31/94

\section{COOE EXCESS PROPERTY DESCRIPTION}

REFERENCE NUMBER : 1279 S/N: 1212
7510 COUNTER TIME INTERVAL MFR: HEWLETT PACKARD MODEL: 5379A

ELIGIBLE EQUIPMENT

$$
\text { No of }
$$

COND UNITS UNIT

$4 \quad 00001 \mathrm{EA}$ COST/UNIT:
TOTAL COST:

LINE ITEM NUMBER: 0004
REFERENCE NUMBER: 1280
8615 FUME HOOD MFR:

3 DO LBS MFR: DURALAG, MOOEL

REPORT NUMBER: $899105-4081$

LINE ITEM NUMBER: 0053
COST/UNIT:

OTAL COST:
$\$ 2,209.00$ $\$ 2,209.00$

\section{$\$ 1,188.00$} $\$ 1,188.00$
CONTACT(S)

FOR FURTHER INFORMATION:

CYNDI DELONG

LAS VEGAS

(702) 295-6753

NV 89193-8521

TO FREEZE EQUIPMENT:

CYNDI DELONG

LAS VEGAS

(702) $295-6753$

NV 89193-8521

LOCATION OF EQUIPMENT:

REYNOLDS ELECTRICAL \& EHGINEERIHG CO

NEVADA TEST SITE

MERCURY

NV 89023

FOR FURTHER INFORMATION:

IYNN E SHACKEL FOOT

LIVERMORE

LIVERMORE

CA $94550-0969$

TO FREEZE EQUIPMENT:

LYNN E. SHACKELFOOT

LIVERMORE

CA $94550-0969$

(510) $294-3067$

LOCATION OF EQUIPMENT:

LOCATION OF EOUIPMENT:

SANDIA NATIONAL LABORATORIES

PROPERTY REAPPLICATION DEPT. $8532-1$
LIVERMORE
CA $94550-0969$ 
EFFECTIVE DATE: $07 / 01 / 94$ EXPIRATION DATE: $07 / 31 / 94$

\section{FSC \\ COOE EXCESS PROPERTY DESCRIPTION}

REFERENCE NUMBER : 1281

9999 GRAPHIC TAPE 66102113 , MANUF: N/A MCOEL N/A S/N N/A

2 ROLLS OF $150 \mathrm{FT}$. EACH OF GRAPHIC TAPE FOR TRI METAL VACUUM FURNANCE.

SIZE: 8"U $\times$ 8"F/R $\times$ 5"H EST WT: 2 LBS

MFGR YR: $N / A$

REPORT MUMBER: $896404-4124$

LINE ITEM NUMBER: 0032

$$
\text { NO OF }
$$

COND UNITS UNIT

ACQUISITION PRICE

CONTACTSS)
$100001 \mathrm{EA}$ COST/UNIT: $\$ 2,085.00$ TOTAL COST:
FOR FURTHER INFORMATION:

BARBARA HOLMES, REP.

KANSAS CITY

MO 64141-6159

TO FREEZE EQUIPMENT:

BARBARA HOLMES, REP.

KANSAS CITY

MO 64141-6159

LOCATION OF EQUIPMENT

ALLIEDSIGNAL, INC.

2000 EAST 95TH STREET

KANSAS CITY

MO $64131-3095$ 


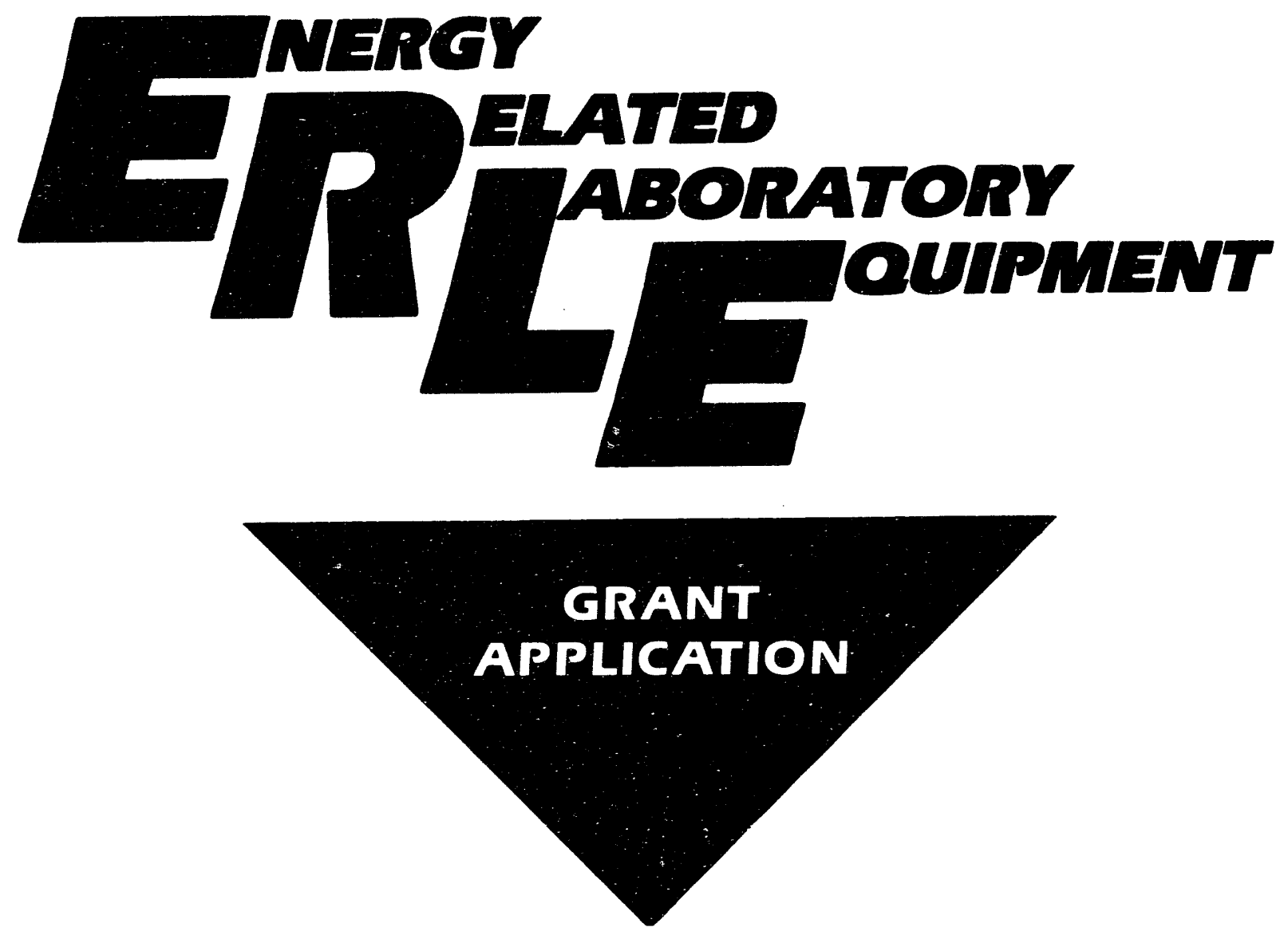




\section{OMB Disclosure Statement}

Public reporting burden for this collection of information is estimated to average two hours per response, including the time for reviewing instructions, searching existing data sources, gathering and maintaining the data needed, and completing and reviewing the collection of information. Send comments regarding this burden estimate or any other aspect of this collection of information, including suggestions for reducing this burden, to Office of Information Resources Management Policy, Plans, and Oversight, AD-241.2 - GTN, Paperwork Reduction Project (1910-1400), U.S. Department of Energy, 1000 Independence Avenue, S.W. Washington, DC 20585; and to the Office of Management and Budget (OMB), Paperwork Reduction Project (1910-1400), Washington, DC 20503.

\section{APPLICANT INFORMATION}

Name:

Department:

City:
Telephone: ( Address:

\section{(}

\section{FOR DOE OFFICE USE ONLY}

Proposal Number:

Area Assurance of Compliance of CRA:

Action:

Grant

Loan

Date Received:

OE Code:

Grant Number:

\section{EQUIPMENT REQUESTED}

(1) PROPOSED OBJECTIVES. Provide detailed information on the piece(s) of equipment requested from a Used Energy Related Laboratory Equipment (ERLE) Excess Property Listing (SF-120). '-ist items exactly as they appear in the Used Energy Related Laboratory Equipment Catalog or on the EEGADS Data Base or attach a photocopy of items as listed in the catalog. Be sure to include: (a) the Record Number, (b) FSC code, (c) report number, and (d) the line item number for each item requested.

DOE staff contacted to "freeze" the equipment (Name/Date):

ERLE Catalog month/year:

\begin{tabular}{|c|c|c|c|c|c|}
\hline Item & $\begin{array}{c}\text { Record } \\
\text { Number }\end{array}$ & $\begin{array}{c}\text { FSC } \\
\text { Code }\end{array}$ & $\begin{array}{c}\text { Report } \\
\text { Number }\end{array}$ \\
\hline & $\begin{array}{c}\text { Line Item } \\
\text { Number }\end{array}$ & $\begin{array}{c}\text { Acquisition } \\
\text { Cost }\end{array}$ \\
\hline & & & \\
\hline
\end{tabular}

(Note: Attach additional pages as required.) 


\section{ENERGY RELATED SCIENCE ENGINEERING EDUCATION PROGRAM}

(2) Describe how the equipment will be used in this program and describe any specific experiments, uses, and projects in which the equipment will be used. Be specific.

(a) How will the requested equipment be used in this program?

(b) Describe any specific experiments, uses, and projects in which the equipment will be used. 
For each course of which the proposed equipment would be a part, please provide the following information:

(3) COURSE NUMBER AND TITLE:

DESCRIPTION:

NEW COURSE: YES [ ] NO [ ] ANNUAL ENROLLMENT:

HOURSMEE.K:

EDUCATION LEVEL: $\quad$ Undergraduate: $\quad \begin{array}{llllllll} & 1 & 2 & 3 & 4 & \text { Graduate: } & \text { M.S. } & \text { Ph.D. }\end{array}$

(4) GRADUATE DEGREES AWARDED (Please show the number and level of degrees awarded within the area to be supported for the last five years.)

Number of Degrees Awarded:

M.S.:

Ph.D.:

(3) COURSE NUMBER AND TITLE:

DESCRIPTION:

NEW COURSE: YES [ ] NO [ ] ANNUAL ENROLLMENT:

EDUCATION LEVEL: $\quad$ Undergraduate: $\quad \begin{array}{llllllll} & 1 & 2 & 3 & 4 & \text { Graduate: } & \text { M.S. } & \text { Ph.D. }\end{array}$

(4) GRADUATE DEGREES AWARDED (Please show the number and level of degreos awarded within the area to be supported for the last five years.)

Number of Degrees Awarded: M.S.: Ph.D.:

(3) COURSE NUMBER AND TITLE:

DESCRIPTION:

NEW COURSE: $\quad$ YES [ ] NO [ ] ANNUAL ENROLLMENT:

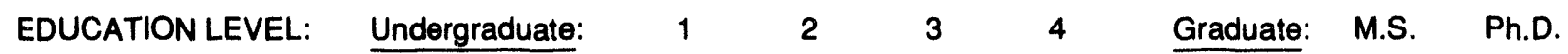

(4) GRADUATE DEGREES AWARDED (Please show the number and level of degrees awarded within the area to be supported for the last five years.)

Number of Degrees Awarded:

M.S.: Ph.D.: 


\section{PARTICIPATING FACULTY}

(5) DATA ON PRESENT FACULTY. For each faculty member participating in the proposed laboratory program, please provide the following information:

Name:

Degree(s):

Brief Background:

Name:

Degree(s):

Brief Background:

Name:

Degree(s):

Brief Background:

PRIOR GRANTS

(6) Please provide information on any prior federal educational assistance grant for equipment (financial or otherwise) to the same or to a related department. Explain any relationship to the currently requested equipment and to the proposed program. 


\section{CERTIFICATIONS}

(7) Please provide the following information on certifications:

Statement A. The terms and conditions as set forth below will be accepted as a part of arly grant that is awarded. The statement should be attached to this sheet of the proposal directly below Statement $A$.

Statement B. The institution will abide by the "U.S. Department of Energy, ERLE Grant Drug-Free Workplace Requirements Certification." 


\section{CERTIFICATION STATEMENT A: TERMS AND CONDITIONS}

When a grant is executed, the following Terms and Conditions will apply:

(1) The term "grantee" as used herein means the educational institution receiving this grant. The term "DOE" means the "United States Department of Energy" and its duly authorized representatives. The phrase "persons acting on behalf of the DOE" includes authorized contractors of the DOE and their duly authorized representatives. The term "equipment" as used herein means the used items described on the face hereof as the item(s) being granted. The term "DOE Facility" means the laboratory, plant, or office, operated by or on behalf of the DOE, in possession and/or responsible for each equipment item shown on the face thereof.

(2) The grantee understands that the DOE may fulfill its obligations under this grant through any DOE Facility(ies).

(3) The grantee agrees to pay for all eost of packing, normal and special handling crating and shipping, and agrees to coordinate the costs and any payments with the DOE Facility.

(4) The grantee will be responsible for any repair and modification costs to any equipment received under this grant.

(5) The grantee agrees to provide shipping instructions to and arrange for the payment of shipping costs with the cognizant DOE Facility(ies) within one week from acceptance of this grant.

(6) Neither the Government, DOE, nor persons acting on behalf of the DOE make any warranty or other representation expressed or implled, that the equipment granted under this program will accomplish the results for which it is requested or intended.

(7) The grantee hereby releases and agrees to hold the Government, DOE, or persons acting on behalf of the DOE harmless from any and all liability of every kind and nature whatsoever resulting from the receipt, shipping, installation, operation, handling, use and maintenance of the equipment after said equipment is physically removed from the DOE Facility(ies).

(8) The grantee will utilize the granted equipment primarily for energy-related instructional purposes in regularly scheduled laboratory and instructional courses and associated activities.

(9) Title to the equipment being granted hereunder shall vest in the grantee upon delivery of said equipment FOB carrier at the DOE Facility.

(10) The grant of any nuclear equipment granted hereunder does not relieve the grantee from complying with the Atomic Energy Act of 1954, as amended, and the regulations issued pursuant thereto, including any requirements for permits and licenses, with respect to such equipment. (See especially, regulations of the Nuclear Regulatory Commission, Chapter 1, Title 10, Code of Federal Regulations.)

(11) The disposition of any patents or inventions or discoveries resulting from the use of the equipment granted hereunder shall be the

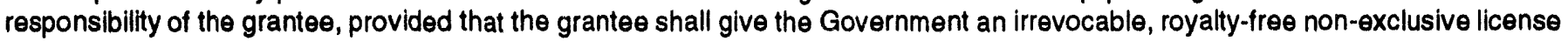
for the use of such inventions, or discoveries for governmental purposes. The grantee hereby waives all claims for damages under Section 183 of Title 35 U.S. Code by reason of the imposition of any secrecy order on any patent application, and also any claim for just compensation or award, under the Atomic Energy Act of 1954, as amended, with respect to any invention or discovery made or conceived in the course of or in connection with work performed with the granted equipment.

(i2) The grantee agrees to comply with the DOE's Financial Assistance Regulations, 10 Code of Federal Regulations Part 600, a copy of which has been furnished to the grantee.

(13) At the end of the first year of use of the equipment, the institution agrees to provide the DOE with a report on the use of equipment; such reports will describe (a) any new courses instituted as a result of the grant of the equipment; (b) existing course which have beon expanded as a result of the grant of the equipment; (c) research activities, e.g., thesis titles, journal articles, sponsored research, etc.; and (d) other ways the equipment has been used to enhance course, e.g., experiments, demonstrations, etc.

(14) The grantee in signing this grant accepts the grant, its terms, conditions and acknowledges that this agreement and the application referenced herein comprise the total agreement between the grantee and the DOE.

Organization Name

Name and Title of Authorized Representative 


\section{CERTIFICATION STATEMENT B: DRUG-FREE WORKPLACE}

This certification is required by the Drug-Free Workplace Act of 1988 (Pub. L. 100-690, Title V, Subtitle D), and is implemented through additions to the Debarment and Suspension regulations, published in the Federal Reglster on January 31, 1989.

An organizational applicant certifies that it will provide a drug-free workplace by:

(1) Publishing a statement notifying employees that the unlawful manufacture, distribution, dispensing, possession, or use of a controlled substance is prohibited in the grantee's workplace and specifying the actions that will be taken against employees for violation of such prohibition.

(2) Establishing a drug-free awareness program to inform employees about:

(a) the dangers of drug abuse in the workplace;

(b) the grantee's policy of maintaining a drug-free workplace;

(c) any available drug counseling, rehabilitation, and employee assistance program; and

(d) the perialties that may be imposed upon employees for drug abuse violations occurring in the workplace.

(3) Making it a requirement that each employee to be engaged in the performance of the grant be given a copy of the statement required by paragraph (1).

(4) Notifying the employee in the statement required by paragraph (1) that, as condition of employment under the grant, the employee will:

(a) abide by the terms of the statement; and

(b) notify the employer of any criminal drug statute conviction for a violation occurring in the workplace not later than five days after such conviction.

(5) Notifying the agency within ten days after receiving notice under subparagraph 4(b) from an employee or otherwise receiving actual notice of such conviction.

(6) Taking one of the following actions, within 30 days of receiving notice under subparagraph $4(\mathrm{~b})$, with respect to any employee who is so convicted:

(a) taking appropriate personnel action against such an employee, up to and including termination; or

(b) requiring such employee to participate satisfactorily in a drug abuse assistance or rehabilitation program approved for such purposes by a Federal, State, or local health, law enforcement, or other appropriate agency.

(7) Making a good faith effort to continue to maintain a drug-free workplace through implementation of paragraphs $1,2,3,4,5$, and 6.

Place of Performance: The applicant shall insert in the space provided below the site(s) for the performance of work done in connection with the specific grant (street address, city, county, state, zipcode).

This assurance is given in connection with any and all financial assistance from the Department of Energy after the date this form is signed. This includes payments after such date for financial assistance approved before such date. The applicant recognizes and agrees that any such assistance will be extended in reliance on the representations and agreements made in this assurance, and the United States shall have the right to seek judicial enforcement of this assurance. This assurance is binding on the applicant, its successors, transferees, and assignees, and on the authorized official (or individual applicant, as appropriate) whose signature appears below.

Organization Name 

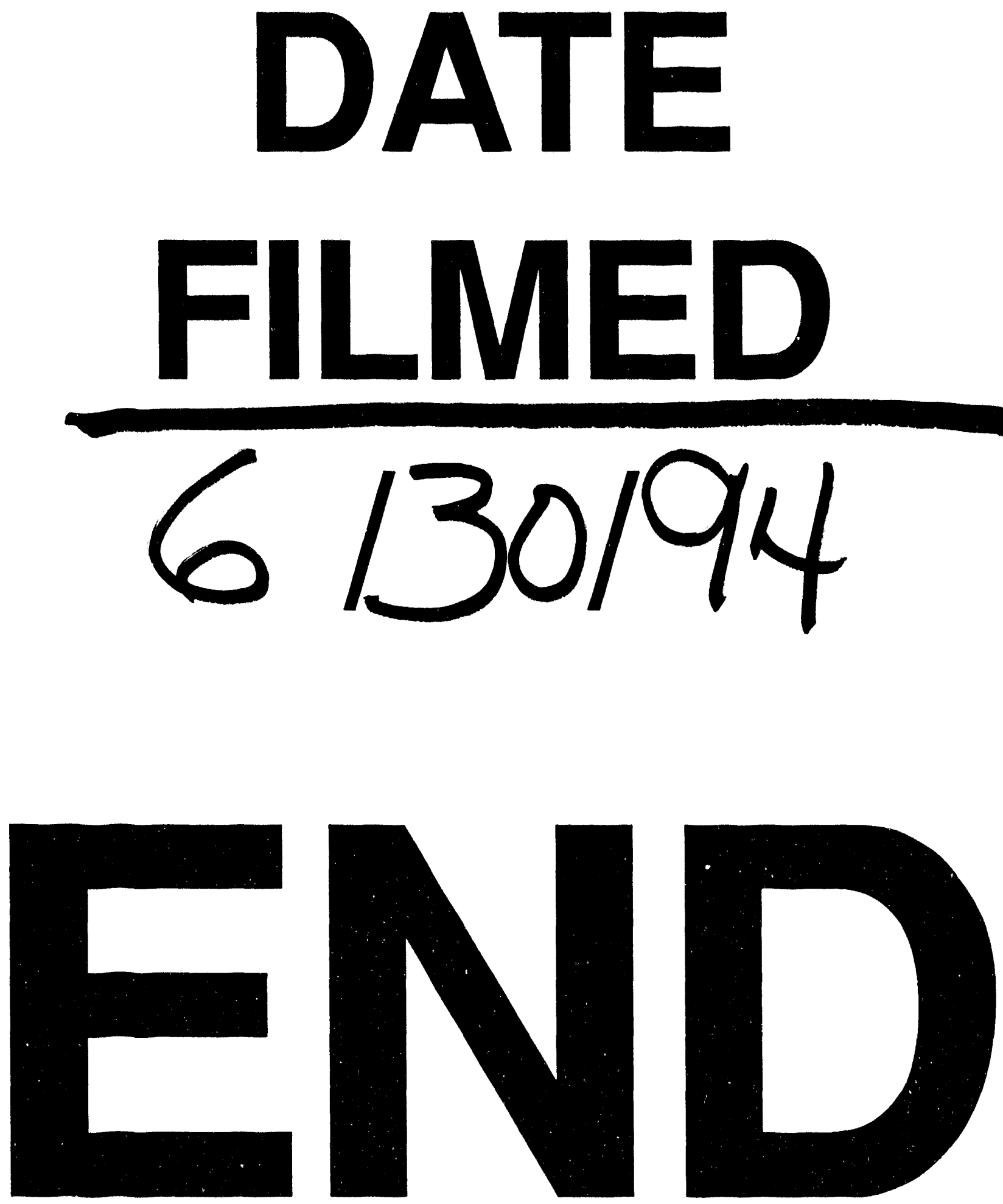


$$
\longrightarrow
$$

